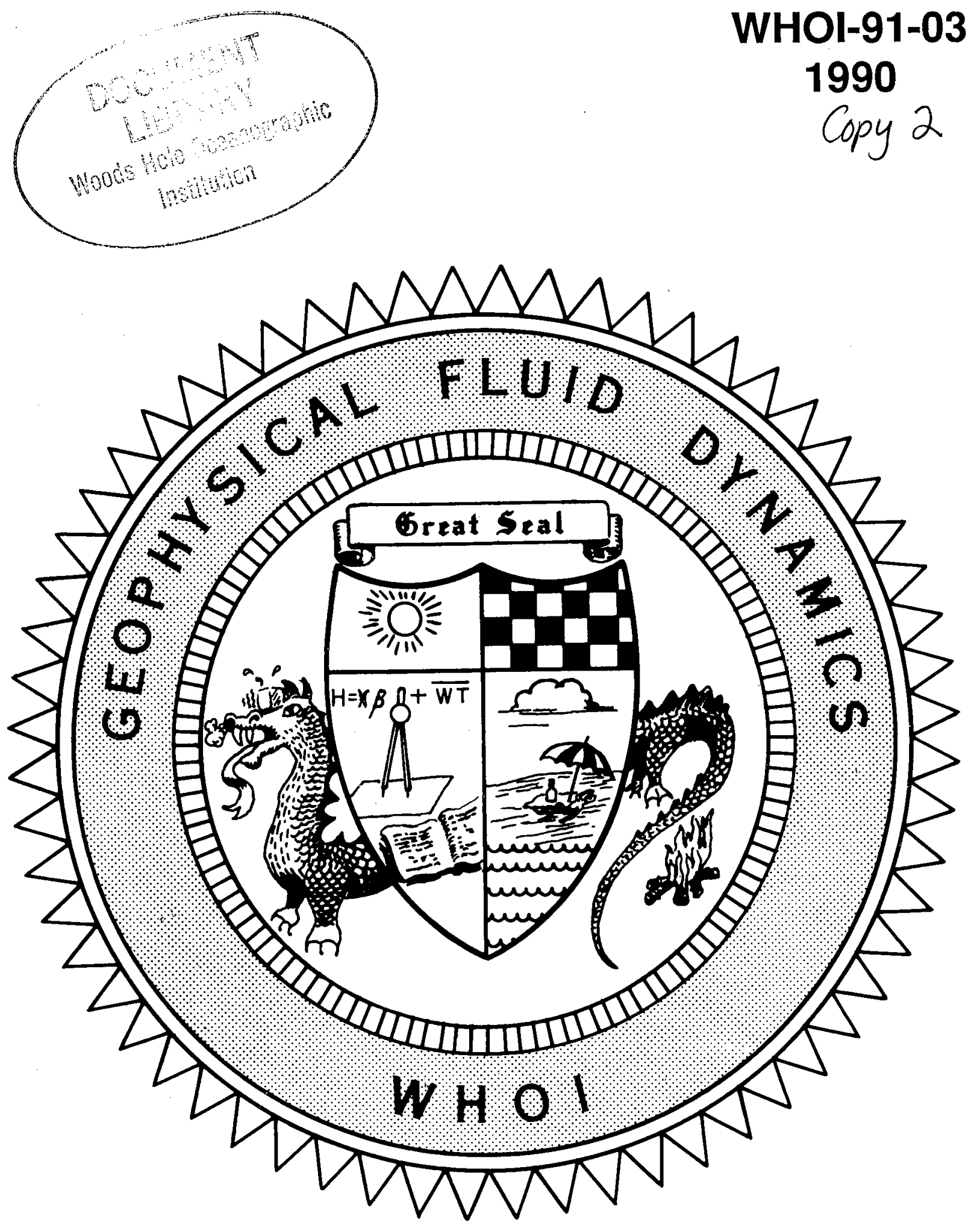

Course Lectures Abstracts of Participants Fellows Project Reports 


\title{
WHOI-91-03
}

\section{Summer Study Program \\ in \\ Geophysical Fluid Dynamics \\ Stellar Fluid Dynamics}

\author{
by \\ Rick Salmon, Director, \\ Edited by \\ Barbara Ewing DeRemer \\ Woods Hole Oceanographic Institution \\ Woods Hole, Massachusetts 02543
}

October 1990

\section{Technical Report}

Funding was provided by the National Science Foundation

through Grant No. OCE 8901012.

Reproduction in whole or in part is permitted for any purpose of the United States Government. This report should be cited as Woods Hole Oceanog. Inst. Tech. Rept., WHOI-91-03.

Approved for public release; distribution unlimited.

\section{Approved for Distribution:}

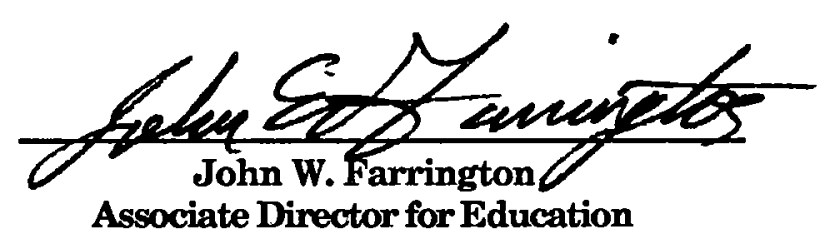





\section{PREFACE}

The 1990 program on "Stellar Fluid Dynamics" marked our deepest penetration into astrophysical fluid dynamics since 1963 . Introductory lectures by Ed Spiegel and JeanPaul Zahn, with a supplement on solar MHD by Steve Childress, paved the way for more specialized lectures on solar oscillations (Balmforth), radiatively-driven stellar winds (Owocki), and neutron stars (Arons). Norm Lebovitz gave us a beautiful synthesis of the theory of polytropes, and Leon Golub challenged our theoretical impulses with the latest $\mathrm{x}$ ray images of the solar corona. There was considerable focus on stellar convection (Zahn, Stein, Ghosal), and on flows with strong magnetic fields.

As usual, the lecture subjects ranged considerably beyond the special topic of the summer, with GFD filling its traditional role as a clearinghouse for new ideas among the many fields concerned with rotating, differentially-heated fluids. Some of these topics (symmetry groups, wavelets, negative energy modes) seem about to burst upon the fluid mechanics scene, while others, such as the flow on a three-dimensional sphere in fourdimensional space, may yet be a few years away.

This was a year in which many familiar faces were absent or tardy, and some new ones appeared. Former fellows Andrew Gilbert and Andrew Woods joined the staff, and newcomer Phil Morrison, who gave us added breadth in the direction of theoretical plasma physics, seemed to enjoy discussing everything with everybody. Our nine fellows (from the USA, Canada, England, Germany, and Ireland) came to us from diverse backgrounds in astronomy, mathematics, physics, and fluid dynamics; all seemed to thrive in the interdisciplinary atmosphere of Walsh Cottage.

1990 was also the year that computers came to Walsh Cottage -- with a vengeance. Thanks to a generous gift from the Mellon Foundation, we were able to buy or borrow two Sun workstations, a laser printer, and two personal computers. After a bumpy start that nearly overwhelmed the director, we were rescued by the computer expertise and generous assistance of Glenn Flierl and Steve Meacham.

Once again, we gratefully acknowledge the support of the National Science Foundation and the Office of Naval Research, and the capable assistance of Jake Peirson and his staff in the Education Office of the Woods Hole Oceanographic Institution. Special thanks go to Barbara Ewing-DeRemer, our administrative assistant and editor, who kept things running smoothly in the cottage.

Rick Salmon, 1990 director 


\section{GFD Participants}

The Fellows

$\begin{array}{lll}\text { Neil Balmforth } & \text { UK } & \text { University of Cambridge } \\ \text { George Bell } & \text { USA } & \text { Woods Hole Oceanographic Institution } \\ \text { Colm-Cille Caulfield } & \text { Eire } & \text { University of Cambridge } \\ \text { Brian Chaboyer } & \text { Canada } & \text { Yale University } \\ \text { Richard Kerswell } & \text { UK } & \text { Massachusetts Institute of Technology } \\ \text { Stefan Linz } & \text { Germany } & \text { Universitat des Saarlaudes } \\ \text { Nathan Platt } & \text { USA } & \text { Brown University } \\ \text { Wendy Welch } & \text { USA } & \text { University of Washington } \\ \text { Eric Won } & \text { USA } & \text { Columbia University }\end{array}$

The Staff and Visitors

$\begin{array}{ll}\text { James L. Anderson } & \begin{array}{l}\text { Stevens Institute of Technology } \\ \text { Jonathan Arons }\end{array} \\ \text { Antoine Badan } & \begin{array}{l}\text { University of California, Berkeley } \\ \text { Centro de Investigación Cientifica y de Educación Superior }\end{array} \\ \text { Stephen Childress } & \text { New York University } \\ \text { Glenn Flierl } & \text { Massachusetts Institute of Technology } \\ \text { Andrew Fowler } & \text { University of Oxford } \\ \text { Sandip Ghosal } & \text { Columbia University } \\ \text { Andrew Gilbert } & \text { University of Cambridge } \\ \text { Dan Givoli } & \text { Technion } \\ \text { Leon Golub } & \text { Harvard University } \\ \text { Jackson Herring } & \text { National Center for Atmospheric Research } \\ \text { Rainer Hollerbach } & \text { University of California, San Diego } \\ \text { Glenn Ierley } & \text { Michigan Technical University } \\ \text { Joseph B. Keller } & \text { Stanford University }\end{array}$


Norman Lebovitz

Albert Libchaber

Willem Malkus

Philip J. Morrison

Stan Owocki

Michael Proctor

David Rose

Robert Rosner

Rick Salmon

Engelbert Schucking

Andrew Soward

Edward Spiegel

Robert Stein

Melvin Stern

Olivier Thual

George Veronis

John Weiss

Nigel Weiss

John A. Whitehead

Andrew Woods

Jean-Paul Zahn
University of Chicago

University of Chicago

Massachusetts Institute of Technology

University of Texas

University of Delaware

University of Cambridge

New York University

University of Chicago

University of California, San Diego

New York University

The University, Newcastle upon Tyne

Columbia University

Michigan State University

Florida State University

National Center for Atmospheric Research

Yale University

Aware Inc.

University of Cambridge

Woods Hole Oceanographic Institution

University of California, San Diego

Observatoire Midi-Pyrénees, Toulouse 


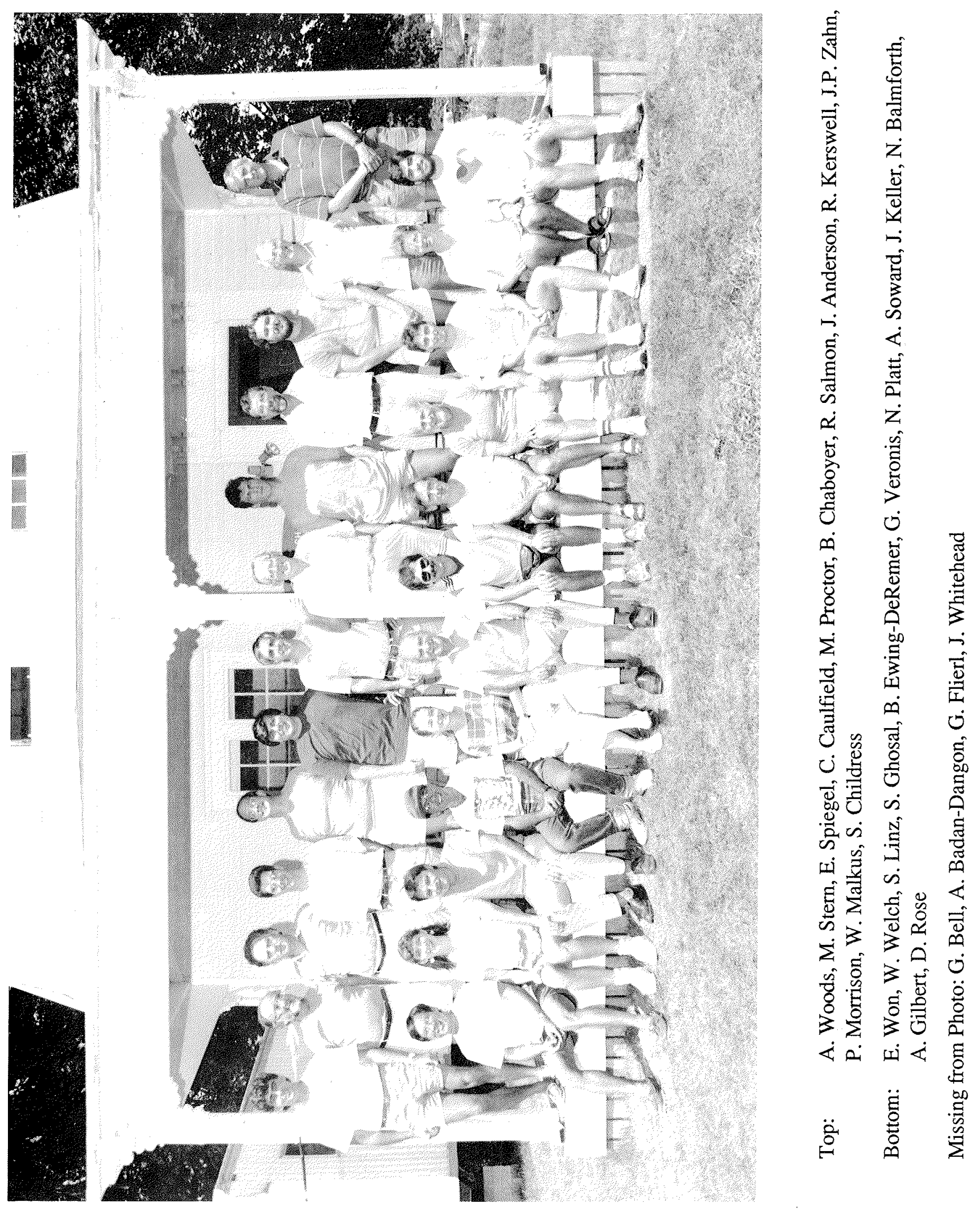




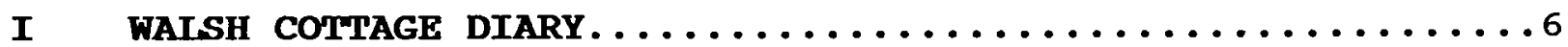

II PHOTO: OUR PRINCIPAL IECTURERS,

Jean-Paul Zahn \& Edward spiegel..............11

\section{IECTURES}

Stellar Fluid Dynamics I: E. Spiegel...............12

Stellar Fluid Dynamics II: E. Spiegel...............22

Stellar Fluid Dynamics III: E. Spiegel.................

Stellar Fluid Dynamics IV: E. Spiegel..............43

Stellar Fluid Dynamics V: Rotating Stars, J.P. Zahn.....52

Stellar Fluid Dynamics VI: Rotating Stars, J.P. Zahn.....70

Stellar Fluid Dynamics VII: Rotating Stars, J.P. Zahn....82

Stellar Fluid Dynamics VIII: Rotating Stars, J.P. Zahn...88

Stellar Fluid Dynamics IX: Rotating Stars, J.P. Zahn....100

Stellar Fluid Dynamics X: E. Spiegel...............112

IV SPECIAL LECTURES BY STEPHEN CHILDRESS:

An Introduction to Solar MHD (Summary).......... 125

v PHOTO: Observing volcanic eruptions on the porch

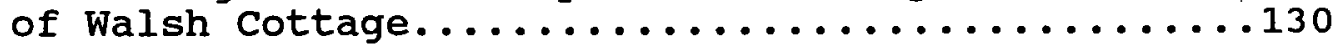




\section{ABSTRACTS OF SEMINARS}

Relativistic Fluctuation-Dissipation Theorems, Radiative Hydrodynamics and Galaxy Formation James Anderson

Solar Acoustic Oscillations

N.J. Balmforth

Inviscid Models Associated with Vortex Reconnection Stephen Childress

Rossby Wave Radiation from strong Eddies Glenn R. Flierl

Convection and chaos Andrew C. Fowler

On Thermonuclear Convection Sandip Ghosal

On Fast Dynamo Action in steady Chaotic Flow

A.D.Gilbert, S. Childress, and U. Frisch

Artificial Boundary Conditions for Wave Problems in Unbounded Domains Dan Givoli

Very High Resolution Solar x-ray Imaging Leon Golub

Coherent structures and statistical Theory of Turbulence Jackson Herring

A modal $\alpha^{2}$-Dynamo in the Limit of Asymptotically Small Viscosity

Rainer Hollerbach 
VI ABSTRACTS OF SEMINARS (continued)

Dynamics of Localized structures with Galilean Invariance Christian Elphick, G.R. Ierley, Oded Regev and E.A. Spiegel

Fast Reaction, slow Diffusion, Curve shortening and Harmonic Mapsi

Joseph B. Keller

Polytropes and Their Perturbations Norman R. Lebovitz

Interface Dynamics: Playing with Symmetries A. Libchaber

Some Aspects of Convection in Binary Fluid Mixtures Stefan J. Linz

Hydromagnetic Instability Due to Elliptical Flow David $W$. Hughes and

Willem V.R. Malkus

The Free Energy "Principle", Negative Energy Kodes and stability

P. J. Morrison

Radiatively Driven stellar winds Stan Owocki

Chaos and Noise in Dynamical Systems with Slow Invariant Subspaces

Michael R. E. Proctor

Calculating Transient Coronal Loops David W. Rose

Blocking a Barotropic shear Flow Melvin Stern 
VI ABSTRACTS OF SEMINARS (continued)

\section{A Fluid Mechanicist's Introduction to Lie Symmetry Groups and Partial Differential Equations Rick Salmon}

Vortices in Tight Embrace

E. L. Schucking and

E. A. Spiegel

$\alpha \omega-$ Galactic Dynamos

A. M. Soward

The Catastrophe Structure of Thermohaline Convection olivier Thual

Simulations of Solar Convection Bob Stein and Ake Nordlund

Instability of Flow with Temperature-Dependent Viscosity: A Model of Magma Dynamics $J \cdot A$. Whitehead and

Karl R. Helfrich

A Long Laboratory salt Finger George Veronis

Applications of Compactly supported Wavelets to the Numerical Solution of Partial Differential Equations John Weiss

Traveling Waves and Oscillations in Compressible Magnetoconvection Nigel Weiss

Hodeling Mesogranules and Exploders on the Sun Nigel Weiss 
VI ABSTRACTS OF SFIINARS (continued)

Fluid Mechanics and Melting Andrew W. Woods

Convective Penetration in stellar Interiors Jean-Paul Zahn

\section{FELLOWS PROJECT REPORTS}

The structure and stability of Rapidly Rotating Polytropes Neil J. Balmforth

The Nonlinear Evolution of a Perturbed Axisymmetric Eddy George I. Bell

The Rise and Fall of Buoyant Plumes

C. P. Caulfield

Transport of a Chemical in stellar Radiative Zones Brian Chaboyer

Magnetic Flux Tubes and Convection Richard Kerswell

Naturally Driven Dispersion in Tilted Porous Layers Stefan J. Linz

Behavior of a Fifth Order System of ODE's with Intermittency Nathan Platt

Difussion in Poiseuille Flow Wendell T. Welch

Classification of similarity solutions of the Two-Dimensional Convection Equations Eric C. Won 


\section{Walsh Cottage Diary}

\begin{tabular}{|c|c|c|c|}
\hline June 18 & Monday & & Arrivals. Opening ceremonies. \\
\hline June 19 & Tuesday & $10: 00$ & $\begin{array}{l}\text { Ed Spiegel } \\
\text { Stellar Fluid Dynamics I }\end{array}$ \\
\hline \multirow[t]{2}{*}{ June 20} & Wednesday & $10: 00$ & $\begin{array}{l}\text { Ed Spiegel } \\
\text { Stellar Fluid Dynamics II }\end{array}$ \\
\hline & & $14: 00$ & $\begin{array}{l}\text { Steve Childress } \\
\text { Introduction to Solar MHD (Part 1) }\end{array}$ \\
\hline \multirow[t]{2}{*}{ June 21} & Thursday & $10: 00$ & $\begin{array}{l}\text { Ed Spiegel } \\
\text { Stellar Fluid Dynamics III }\end{array}$ \\
\hline & & $14: 00$ & $\begin{array}{l}\text { Steve Childress } \\
\text { Introduction to Solar MHD (Part 2) }\end{array}$ \\
\hline June 22 & Friday & $10: 00$ & $\begin{array}{l}\text { Ed Spiegel } \\
\text { Stellar Fluid Dynamics } I V\end{array}$ \\
\hline June 25 & Monday & $10: 00$ & $\begin{array}{l}\text { Jean-Paul Zahn } \\
\text { Stellar Fluid Dynamics } V\end{array}$ \\
\hline \multirow[t]{2}{*}{ June 26} & Tuesday & $10: 00$ & $\begin{array}{l}\text { Jean-Paul Zahn } \\
\text { Stellar Fluid Dynamics VI }\end{array}$ \\
\hline & & $13: 30$ & $\begin{array}{l}\text { Jonathan Arons } \\
\text { Radio Pulsars }\end{array}$ \\
\hline \multirow[t]{3}{*}{ June 27} & Wednesday & $10: 00$ & $\begin{array}{l}\text { Jean-Paul Zahn } \\
\text { Stellar Fluid Dynamics VII }\end{array}$ \\
\hline & & $13: 30$ & $\begin{array}{l}\text { Jonathan Arons } \\
\text { Radiation Gas Dynamics of Accretion } \\
\quad \text { onto Neutron Stars }\end{array}$ \\
\hline & & $17: 30$ & GFD 11, Fisheries 17 \\
\hline \multirow[t]{2}{*}{ June 28} & Thursday & $10: 00$ & $\begin{array}{l}\text { Jean-Paul Zahn } \\
\text { Stellar Fluid Dynamics VIII }\end{array}$ \\
\hline & & $14: 30$ & $\begin{array}{l}\text { Andrew Fowler } \\
\text { Convection and chaos }\end{array}$ \\
\hline
\end{tabular}




\begin{tabular}{|c|c|c|c|}
\hline June 29 & Friday & $10: 00$ & $\begin{array}{l}\text { Jean-Paul Zahn } \\
\text { Stellar Fluid Dynamics IX }\end{array}$ \\
\hline July 2 & Monday & 10:00 & $\begin{array}{l}\text { Ed Spiegel } \\
\text { Stellar Fluid Dynamics X }\end{array}$ \\
\hline \multirow[t]{2}{*}{ July 3} & Tuesday & 10:00 & $\begin{array}{l}\text { Neil Balmforth } \\
\text { Solar Oscillations }\end{array}$ \\
\hline & & $17: 30$ & GFD 8, New Alchemy 22 \\
\hline July 4 & Wednesday & & Holiday \\
\hline July 5 & Thursday & $10: 00$ & $\begin{array}{l}\text { Stan Owocki } \\
\text { Radiatively-driven Stellar Winds (Part 1) }\end{array}$ \\
\hline July 6 & Friday & $10: 00$ & $\begin{array}{l}\text { Stan Owocki } \\
\text { Radiatively-driven Stellar Winds (Part 2) }\end{array}$ \\
\hline July 9 & Monday & $10: 00$ & $\begin{array}{l}\text { Norman Lebovitz } \\
\text { Polytropes and their Perturbations }\end{array}$ \\
\hline July 10 & Tuesday & $10: 00$ & $\begin{array}{l}\text { Andrew Gilbert } \\
\text { On Fast Dynamo Action in Steady Chaotic Flow }\end{array}$ \\
\hline \multirow[t]{2}{*}{ July 11} & Wednesday & $10: 00$ & $\begin{array}{l}\text { Joe Keller } \\
\text { Fast Reaction and Slow Diffusion }\end{array}$ \\
\hline & & $13: 30$ & $\begin{array}{l}\text { Rick Salmon } \\
\text { Application of Lie Symmetry Groups } \\
\text { to Partial Differential Equations (Part 1) }\end{array}$ \\
\hline \multirow[t]{2}{*}{ July 12} & Thursday & $10: 00$ & $\begin{array}{l}\text { David Rose } \\
\text { Calculating Transient Coronal Loops }\end{array}$ \\
\hline & & $17: 30$ & GFD 7, Marine Policy 9 \\
\hline July 13 & Friday & $10: 00$ & $\begin{array}{l}\text { Rick Salmon } \\
\text { Application of Lie Symmetry Groups } \\
\text { to Partial Differential Equations (Part 2) }\end{array}$ \\
\hline July 16 & Monday & $10: 00$ & $\begin{array}{l}\text { Melvin Stern } \\
\text { Blocking a Barotropic Shear Flow }\end{array}$ \\
\hline July 17 & Tuesday & $10: 00$ & $\begin{array}{l}\text { Andrew Woods } \\
\text { Fluid Mechanics and Melting }\end{array}$ \\
\hline
\end{tabular}




\begin{tabular}{|c|c|c|c|}
\hline \multirow[t]{2}{*}{ July 18} & Wednesday & 10:00 & $\begin{array}{l}\text { Jack Whitehead } \\
\text { Instability of Flow in a Slot } \\
\text { with Temperature-dependent Viscosity }\end{array}$ \\
\hline & & $17: 30$ & GFD 2, Geology and Geophysics 15 \\
\hline July 19 & Thursday & 10:00 & $\begin{array}{l}\text { Nigel Weiss } \\
\text { Travelling Waves and Oscillations in Compressible } \\
\text { Magnetoconvection }\end{array}$ \\
\hline July 20 & Friday & 10:00 & $\begin{array}{l}\text { Stefan Linz } \\
\text { Convection in Binary Fluid Mixtures }\end{array}$ \\
\hline July 23 & Monday & 10:00 & $\begin{array}{l}\text { Leon Golub } \\
\text { High-resolution Observations of the Solar } \\
\quad \text { Magnetic Field }\end{array}$ \\
\hline July 24 & Tuesday & 10:00 & $\begin{array}{l}\text { Jack Herring } \\
\text { Coherent Structures and Statistical } \\
\text { Turbulence Theory }\end{array}$ \\
\hline July 25 & Wednesday & 10:00 & $\begin{array}{l}\text { Nigel Weiss } \\
\text { Modelling Mesogranules and Exploders } \\
\quad \text { on the Sun }\end{array}$ \\
\hline \multirow[t]{2}{*}{ July 26} & Thursday & 10:00 & $\begin{array}{l}\text { Jean-Paul Zahn } \\
\text { Penetrative Convection in Stars }\end{array}$ \\
\hline & & $17: 50$ & GFD 5, Ocean Engineering 14 \\
\hline July 27 & Friday & 10:00 & $\begin{array}{l}\text { Jim Anderson } \\
\text { Fluctuation-dissipation in Radiative Transport }\end{array}$ \\
\hline \multirow[t]{2}{*}{ July 30} & Monday & 10:00 & $\begin{array}{l}\text { Phil Morrison } \\
\text { The Free Energy Principle, Negative Energy Modes, } \\
\text { and Stability (Part 1) }\end{array}$ \\
\hline & & 14:00 & $\begin{array}{l}\text { Dan Givoli } \\
\text { Artificial Boundary Conditions for Wave Problems } \\
\quad \text { in Unbounded Domains }\end{array}$ \\
\hline July 31 & Tuesday & 10:00 & $\begin{array}{l}\text { Rainer Hollerbach } \\
\text { An } \alpha^{2} \text { Dynamo in the Limit of } \\
\text { Asymptotically Small Viscosity }\end{array}$ \\
\hline August 1 & Wednesday & $10: 00$ & $\begin{array}{l}\text { Andrew Soward } \\
\alpha-\omega \text { Galactic Dynamos }\end{array}$ \\
\hline August 2 & Thursday & 10:00 & $\begin{array}{l}\text { Michael Proctor } \\
\text { The Effect of Noise on Dynamical Systems } \\
\quad \text { with Slow Invariant Subspaces }\end{array}$ \\
\hline
\end{tabular}


17:30 GFD 35, Administration 11

August $3 \quad$ Friday

10:00 Phil Morrison

The Free Energy Principle, Negative Energy Modes, and Stability (Part 2)

August $6 \quad$ Monday $\quad 10: 00 \begin{aligned} & \text { Albert Libchaber } \\ & \text { Interface Dynamics }\end{aligned}$

August $7 \quad$ Tuesday $\quad$ 10:00 Steve Childress

Inviscid Problems Related to Vortex Reconnection

August 8 Wednesday 10:00 Engelbert Schucking

Two Vortices in Tight Embrace

August 9 Thursday 10:00 Sandip Ghosal

On Thermonuclear Convection

17:50 GFD 19, Facilities 15

August 10 Friday 10:00 Olivier Thual

The Catastrophe Structure of

Thermohaline Convection

August 13 Monday

10:00 Glenn Ierley

Dynamics of Localized Structures with Galilean Invariance

August 14 Tuesday

10:00 John Weiss

Application of Compactly Supported Wavelets to Partial Differential Equations

August 15 Wednesday 10:00 Glenn Flierl

Rossby Wave Radiation from Strong Vortices

August 16 Thursday 10:00 George Veronis

A Long Laboratory Salt Finger

17:30 GFD 20, Biology 11

August 17 Friday

10:00 Robert Stein

Simulatiions of Solar Convection

August 20 Monday 10:00 Colm-cille Caulfield

The Rise and Fall of Buoyant Plumes

11:15 Wendy Welch

Diffusion in Poiseuille Flow

13:30 Bryan Chaboyer

Transport in Stellar Radiative Zones 
The Stability of Axisymmetric Vortices

11:15 Stefan Linz

Convection in Tilted Porous Layers

13:30 Nathan Platt

Behavior of a Fifth-order System of Ordinary Differential Equations with Intermittency

15:00 Fellows 39, Staff 23

August $22 \quad$ Wednesday

10:00 Neil Balmforth

The Structure and Stability of Rapidly Rotating Polytropes

11:15 Richard Kerswell

Magnetic Flux Tubes and Convection

13:30 Eric Won

Symmetries and Similarity Solutions of the Two-Dimensional Convection Equations

August 24 Friday

Farewell. 


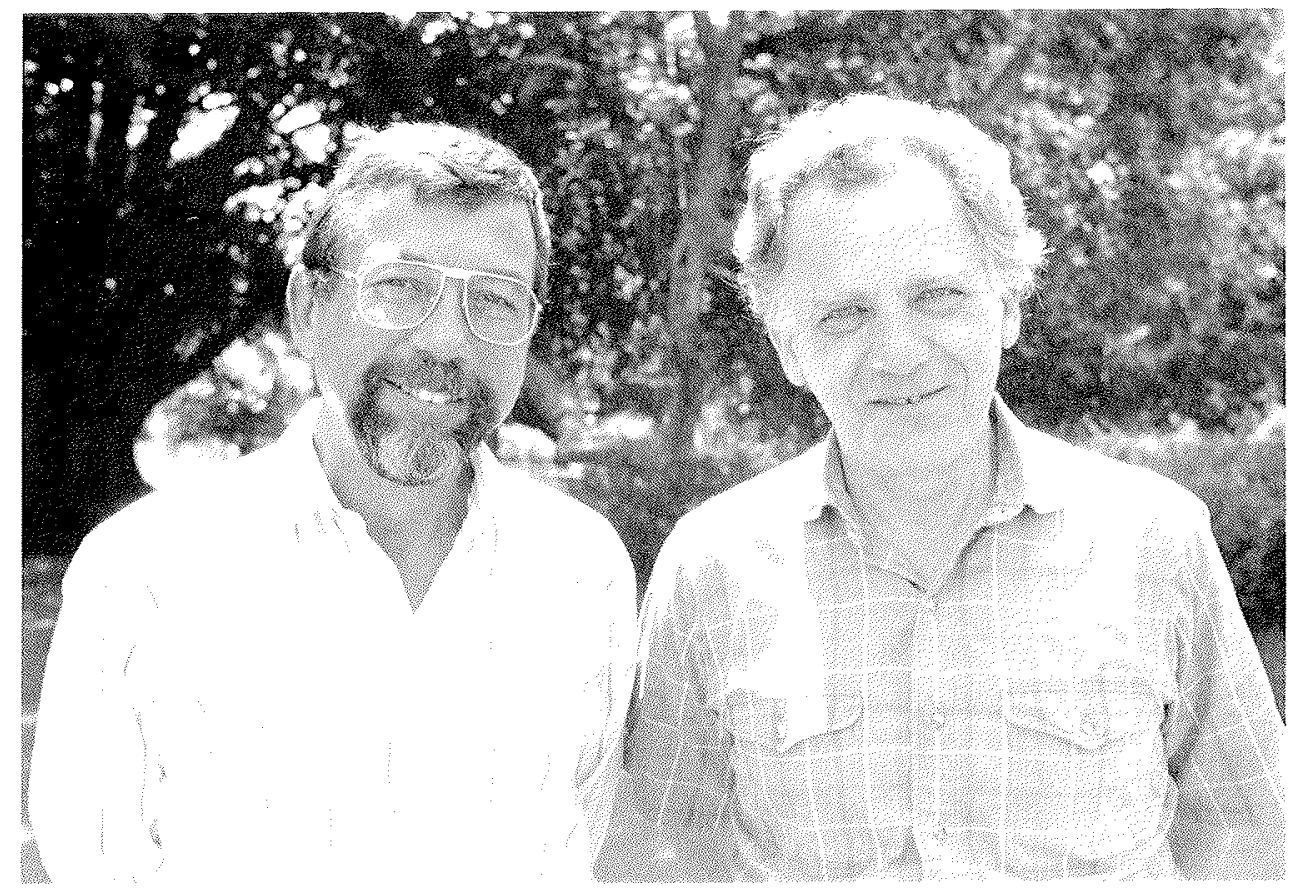

OUR PRINCIPAL IECTURERS,

Jean Paul Zahn and Edward Spiegel

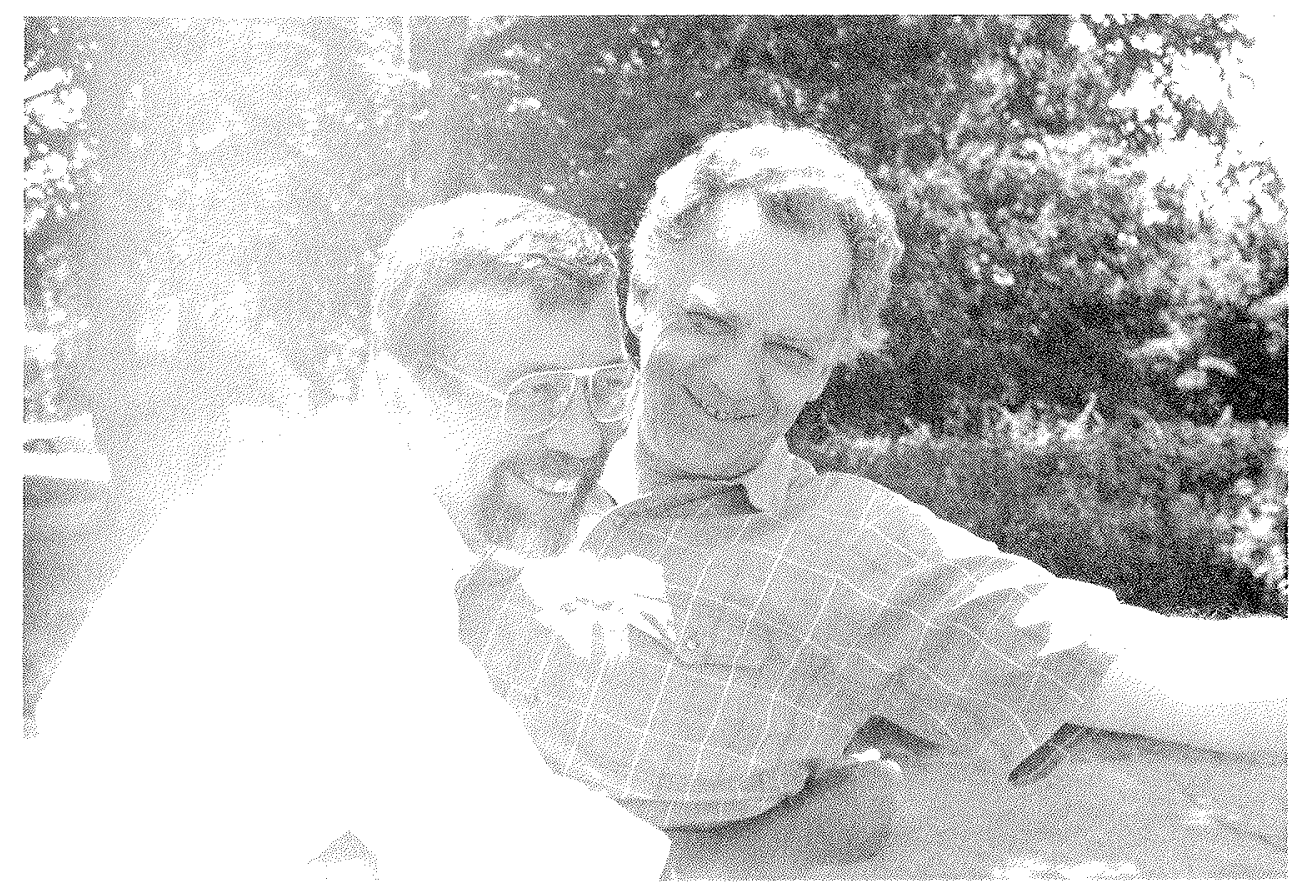




\title{
STELLAR FLUID DYNAMICS
}

\author{
E.A. Spiegel \\ Astronomy Department \\ Columbia University \\ New York, NY 10027 \\ Lecture I
}

\section{Astrophysical Fluid Dynamics}

Astrophysics is a branch of astronomy, so when I say "astronomers" in these lectures, I include astrophysicists. In fact, astronomy is mostly astrophysics so I think that this is a good characterization. On the forefront of astronomy, the job is to isolate the relevant physics in different kinds of celestial objects with a view to modeling. It is only after some agreement about the nature of the objects has been reached that careful studies are warranted. In good astrophysics, physical arguments, often based on rough estimates, are the key to success.

As more phenomena in astronomical objects are recognized to be fluid dynamical, the need for intuitive understanding of such processes grows. In this school, we have practiced the development of understanding fluid processes by careful analysis of simple models. Often, over the decades, practitioners have felt that such studies may not be relevant to their disciplines, but more and more, the language of G.F.D. inquiry is heard in discussions by these same practitioners. The reason that these words have acquired increased meaning and comprehensibility comes from their careful elucidation in simple pilot studies. That, at any rate, is the credo of Walsh College.

Astronomers have studied what they call cosmical gas (or aero-) dynamics for years. That is astrophysics in which the physics is fluid dynamics. What we shall discuss here is to be called Astrophysical Fluid Dynamics to emphasize that the aim is, like that of GFD, to extract relevant models that are sufficiently simple to be analyzed in detail by whatever means are needed. The results of such analysis are to be used to inform astrophysical studies but, as the name implies, our subject here is fluid dynamics and it is an end in itself. No apologies need be made for that.

Modern developments in the mathematical sciences are also affecting the progress in astronomy. In astromathematics, the object is to parallel the work in astrophysics by using general mathematical ideas to isolate mathematical processes that elucidate the behavior of a cosmic object. For example, if we suspect that a galactic pattern is engendered by an instability, we need not decide exactly which instability it is in order to begin to write down an equation to describe such a pattern. If we can isolate a suitable equation for the purpose by qualitative mathematical arguments, we can later go back and argue about which among possible physical processes is responsible for the instability. A.F.D. is a good source of examples of this approach. 
In the lectures sketched here, the concentration is on stellar fluid dynamics. That fits in nicely with the Astro in A.F.D. There will be no attempt to provide a systematic course on the background astrophysics, though some introductory material will be given as the need seems to arise. The main aim is to introduce some fluid dynamical problems that seem peculiarly stellar. Afterwards, the lectures of Jean-Paul Zahn about rotating stars will restore sweet reason.

\section{$2 \quad$ Large Scale Structures}

Our vision of the the universe has gone through many revisions over the past few millenia. In the decades of the forties and fifties it was generally assumed that the universe is homogenous and isotropic. That people were uncomfortable with this idealization is attested by the name they gave it: the Cosmological Principle. Today, though wider possibilities are considered and the situation seems more confused, there is real improvement as the outlook has become more Copernican. We are not going to discuss cosmology here, but it seems worth saying just a few words about some of the salient features to place the stellar situation in context.

It now seems reasonably certain that the visible matter in the universe constitutes only a few percent of the total mass. This visible mass is concentrated in galaxies that appear to be arranged in a hierarchical distribution. A simple way to think about the distribution of galaxies is to imagine that they lie on a fractal set, as has long been conjectured (see the books of Peebles or of Mandelbrot). Such a set has lacunae, voids where there are no galaxies, and the galaxian distribution is rather filamentary. The dimension of the fractal is a matter of debate (see Thieberger, et al., in "The Ubiquity of Chaos", AAAS 1990, S. Krasner, ed.). What matters is that the galaxies appear to be markers in a cosmic flow about which our only knowledge is that locally (in space and time) it is an expansion. What happens elsewhere in spacetime is not known except by speculations that form a sort of mathematical theology of some charm. We do not know the nature of the invisible matter.

The galaxies themselves consist of stars, gas and dust in differing mixtures. They have diverse morphologies that are matched to these melanges. Our galaxy, the Milky way, has a mass of approximately $10^{12} \mathcal{M}_{\odot}$ of visible matter where $\mathcal{M}_{\odot}$ is the sun's mass $\approx 2 \times 10^{33}$ $\mathrm{gm}$. Much of this is in a disk that is suspected of being embedded in an invisible halo of ten or a hundred times this mass. We are not going to worry about the cosmic flow nor about the circulations in galaxies. Our attention is to be focussed on the fluid dynamics within stars. Before we get to that, let us review briefly the fluid dynamics itself.

\section{Fluid Dynamics}

The work usually discussed in this course has to do with GFD, and that is only a special case of AFD. So although many in the audience know fluid dynamics, we still ought to 
set down a simple version of it in a somewhat general way. It will not be necessary, however, to derive the Boussinesq approximation. Still, we ought to mention a question that does occupy astrophysicists wanting to think about cosmic fluid dynamics. Is a simple continuum a valid description of the astrophysical plasmas with which they generally have to deal? There is a lot of discusion of this point and it involves comparing mean free paths to the scales of motion. In fact, even when those comparisons do not seem to support the use of the fluid picture, it has often been used with the defence that collective interaction resulting from long range em and gravitational forces makes for fluid behavior. Let us skip all that dreary stuff and simply adopt the fluid model to describe motions in stars.

Another point that must be mentioned in the interests of respectablity is that, in most astrophysical circumstances, length scales are so large that the Reynolds number is generally astronomical. Hence turbulent flows are the rule in this subject. In the face of that remark, sensible people would change subjects, but we are not among them and have been here for thirty years and more trying to work around this dilemma that natural scientists face in thinking about fluid motions. But this problem may make it clear why we are sometimes schematic in AFD.

So consider this simple equation for the momentum balance of a fluid:

$$
\frac{d \mathbf{u}}{d t}=\mathbf{F}
$$

where the velocity field $\mathbf{u}$ depends on $\mathbf{x}$ and $t, d / d t=\partial_{t}+\mathbf{u} \cdot \nabla$ is the material derivative and $\mathbf{F}$ is a body force per unit mass. For the simplest case, we take $\mathbf{F}$ to be conservative. Then we have a special case of inviscid fluid dynamics with $\mathrm{F}=-\nabla \mathcal{V}$.

Consider some simple models for $\mathcal{V}$ :

(1) $\mathcal{V}=V(\mathbf{x}, t)$ - specified potential only;

(2) $\mathcal{V}=V(\mathbf{x}, t)+h(\rho)$ - includes local coupling of the fluid with itself ( $h$ is a point function of $\rho)$;

(3) $\mathcal{V}=V(\mathbf{x}, t)+h(\rho)+H[\rho]-$ with both local and nonlocal couplings of the density field ( $H$ is a functional of $\rho$ ).

For a conservative force, as assumed, Kelvin's Circulation Theorem gives the permanence of irrotationality. Hence, if the flow is initially irrotational, there exists a function $\phi$ such that $\mathbf{u}=\nabla \phi$. It follows that

$$
\phi_{t}+\frac{1}{2}(\nabla \phi)^{2}+\mathcal{V}=0
$$

This is Bernoulli's Theorem in fluid mechanics and the Hamilton-Jacob equation in classical mechanics.

We shall also assume the kinematic condition that mass is conserved:

$$
\rho_{t}+\nabla \cdot(\rho \mathbf{u})=0 \text {. }
$$

For an irrotational, barotropic fluid we have $H=0$. Then, on letting $\rho=R^{2}$ and $\psi=R e^{i \phi}$, we can write the equations of motion and continuity concisely as

$$
i \psi_{t}=-\frac{1}{2} \nabla^{2} \psi+\left(V+h(\rho)+\frac{\nabla^{2} R}{2 R}\right) \psi
$$


Other interesting cases exist, such as the choice $H[\rho]=-\nabla^{2} R / 2 R$, which leads to the nonlinear Schrödinger equation

$$
i \psi_{t}=-\frac{1}{2} \nabla^{2} \psi+\left[h\left(|\psi|^{2}\right)+V(\mathbf{x}, t)\right] \psi
$$

\section{Expanding Coordinate System}

Most people in this audience know all about transforming to rotating coordinates, so instead of going back over this, let us look at another transformation that is common in astrophysics - the transformation to expanding coordinates. This is a device that has been much used in cosmology and also in the study of fluid dynamics in pulsating stars. Flow in an expanding system is a pleasant introduction to AFD since this choice of coordinates leads to fictitious forces in complete analogy to those of rotating fluids.

Inspired by Hubble's law, we set $\mathbf{u}=H(t) \mathbf{x}$ with

$$
\frac{d \rho}{d t}=-\rho \nabla \cdot \mathbf{u}
$$

and obtain, for a homogeneous system,

$$
\frac{\dot{\rho}}{\rho}=-3 H
$$

Hence, $H=D_{t}\left[\ln \left(\rho_{0} / \rho\right)^{\frac{1}{3}}\right]$, where $D_{t}=d / d t$. We introduce the scale factor $R(t)$ defined by $R^{-3}(t)=\rho / \rho_{0}$ where $\rho_{0}$ is the density of a fiducial epoch. Set $\mathbf{x}=R \mathbf{y}$ and let $\mathbf{v}=R \dot{\mathbf{y}}$, where the dot means time derivative. Then it is possible to show that $\mathbf{v}=\dot{\mathbf{x}}-H \mathbf{x}$ (where $H=\dot{R} / R$ ) and $\mathbf{v} R(t)=$ const, which is the analogue of the conservation of angular momentum. That is, $R(t)$ increases with time, causing $|\mathbf{P}|=|m \mathrm{v}|$ to decrease. Qualitatively, the image is that the wavelength conjugate to $\mathbf{P}$ increases. The analogous effect for photons is called the cosmological red shift.

The possibilites for $R(t)$ (in the simplest Newtonian models) are shown in figure 1 for various energy densities. Against this background, we want to write the equations of motion. However, we shall follow the order of the lectures as they were given and defer those equations to Lecture II since the questions at this point caused a non-negligible deflection in the direction of Lecture I.

\section{The Sun}

"The sun is round like a ball or an orange," is what first graders were taught fifty years ago. Later, it was necessary to make this statement more quantitiative, but we need not go into that. Let us consider a spherically symmetric hydrostatic sun. Then an estimate of the central temperature can be derived:

$$
\frac{d p}{d r}=-\rho g
$$




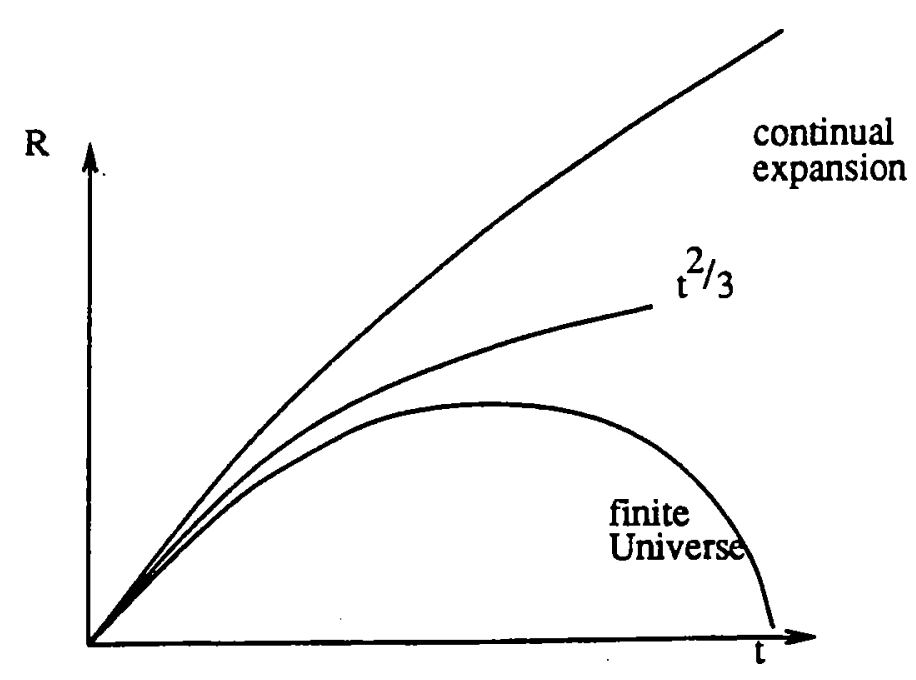

Figure 1: Scale factor as a function of time.

where $g=G \mathcal{M} / r^{2}$ and, for a perfect gas, with $\mathcal{R}$ as gas constant and $\mu$ as mean molecular weight, we have

$$
p=\frac{\rho \mathcal{R} T}{\mu}
$$

Hence in order of magnitude, we have $p \sim G \mathcal{M} \rho / R$, where $R$ is the star's radius, and so

$$
\frac{\rho \mathcal{R} T}{\mu} \sim \frac{G \mathcal{M} \rho}{R} \Rightarrow \mathcal{R} T \sim \frac{G \mathcal{M} \mu}{R},
$$

up to factors of order unity. For the sun this gives $T \sim 10^{7} \mathrm{~K}$ at depth.

Then from the measured rate of emission of luminous energy per unit time - the luminosity - we can determine the thermal time of the star, the so-called Kelvin-Helmholtz time scale. From the balances already given, we see that the potential energy has the magnitude $G \mathcal{M}^{2} / R$ and that this is comparable to the thermal energy in the star. Hence the thermal time scale may be estimated as

$$
\tau_{K H}=\frac{G \mathcal{M}^{2}}{R L} .
$$

For the sun, $L_{\odot} \approx 4 \times 10^{33} \mathrm{ergs} / \mathrm{sec}$, so $\tau_{K H}=30$ million years. Since the time scale for adjusting any hydrostatic imbalance is short compared to $\tau_{K H}$ - the acoustic travel time across a solar radius is about an hour - the assumption of hydrostatic balance is well justified.

Simple considerations explain the global (dare we say structural?) stability of the sun. The heat source is provided by nuclear reactions in the core where hydrogen fuses into 
helium. For particles to have sufficient energy to overcome Coulomb repulsion so that the nuclei can interact strongly, the temperature must be high enough. If the temperature increases, the energy production by nuclear fusion correspondingly increases and the star expands and cools. A temperature is reached at which the energy loss matches that produced in the core. However, at the small scales, there may be instabilities that do not profoundly modify the mean structure.

\section{Radiation}

In hot stars, we must allow for the effects of the pressure of the radiation, $p_{\text {rad }}=\frac{1}{3} a T^{4}$, where $a$ is a constant. The radiation pressure is comparable to the gas pressure when $a T^{4} \sim \mathcal{R} \rho T$. This occurs for $T^{3} / \rho \sim \mathcal{R} / a$. On the other hand, we have already seen that $\mathcal{R} T \sim G \mathcal{M} / R$ and $\rho \sim \mathcal{M} / R^{3}$. We combine these estimates and find that the mass at which radiation pressure and gas pressure are of the same order is $\mathcal{M} \sim 40 \mathcal{M}_{\odot}$. Above this mass, radiation pressure dominates, but we never see any stars with masses much in excess of about $60 \mathcal{M}_{\odot}$. Some think that this is a result of vibrational instabilities related to radiation pressure.

Radiation coming up from within a star may be compared to a fluid fowing through a porous medium. The radiation produces a force of levitation on the stellar material and, in the hot stars, this force may compete with the gravitational force. The situation resembles that in a fluidized bed. There a fluid flows through a porous medium made of particles that are not attached to each other. When the drag per particle equals the mean weight of the particles the bed of particles expands and turns into a fluid. In the stellar case, the porous medium is already a fluid (a gas or plasma) but there is also a critical case where the levitating force of the outflowing radiation compensates the weight of the particles.

The bolometric luminosity of a star, $L$, is its total rate of emission of light. When this luminosity is in excess of a cerain critical value, the Eddington luminosity, $L_{E}$, the radiative force per unit mass of stellar material exceeds the gravitational force in the outer layers. It is generally presumed that the material in a star that found itself in this situation would be blown away. This does not happen when a bed of particles is first fluidized since the drag force per particle diminishes when the density of particles decreases. In the stellar case, normal stars with masses in excess of about $60 \mathcal{M}_{\odot}$ exceed the Eddington limit.

\section{Surface Properties}

From the hydrostatic picture we can derive a number of observable properties of stars. Because of the complexities of the microphysics, these derivations require numerical integrations. But we can at least see what is involved physically. Let us leave out rotation and magnetic fields for this purpose. 
Knowing the central temperature of the star, we can estimate that the outward radiative flux, in the absence of convection, is $\sim K T / R$ where $K$ is the radiative conductivity and $R$ is the radius. Here we are supposing that the surface temperature is much less than $T$, the temperature in the deep interior. If we assume simple dependences of $K$ on the state variables, such as power laws, we can derive the dependence of the luminosity on the bulk properties such as mass and chemical composition.

Notice that these derivations require no statements about the source of energy. If the source were turned off, the qualitative aspects we have just discussed would hardly be changed. However, if we want the static state to last for much more than a thermal time, we do need an energy source, and that is provided by thermonuclear reactions. These tend to be energy sensitive, so there is some fine tuning in the central conditions as the hydrostatics comes into accord with the nuclear reaction rates.

Another delicate problem in the hydrostatic stellar structure theory is the determination of surface conditions. This theory tells us the luminosity and the radius of a star, given its chemical composition and a few plausible simplifying conditions. If the star radiated like a blackbody of radius $\mathrm{R}$ and temperature $T_{e}$ ( $e$ for effective) then

$$
L=4 \pi R^{2} \sigma T_{e}^{4} .
$$

The actual surface temperature is not far from this. Indeed the operational definition of temperature is ambiguous in the surface layers, which are clearly out of thermal equilibrium, so $T_{e}$ could provide one definition of surface temperature. By the way, we have just passed into the subject called stellar atmospheres, as distinct from stellar structure theory, which we have been discussing so far, however loosely.

The meshing of these two main topics of stellar physics is like the interaction of oceanography and meteorology and the key observation that must be explained by this joining of forces is the so-called H-R diagram. This is a plot of $\log L$ vs. some observed spectral property of stars that measures $T_{e}$. We shall not have time to go into this spectral lore, even though it is the backbone of classical astrophysics. When I was a student, we often were handed spectra on exams and asked to read off (in effect) the surface temperature. We shall bypass this process so, in figure 2 , the abscissa is $T_{e}$ straightaway, with $T_{e}$ decreasing to the left in accordance with astophysical tradition.

The full calculations show that, for a given chemical composition, assumed uniform throughout the star, and with no rotation, the equilibria form a one parameter family of solutions, with the mass as parameter. The locus of this family depends on the values of parameters such as the chemical composition, but for reasonable choices, one finds that a large majority of stars lie on a curve like that shown. Of course, the real observations show some revealing additional details (many of which are understood in terms of stellar evolution and the development of chemical inhomogeneities), but the main point is that newly born stars do fall on this main sequence, as it is called. The maximum mass is at the upper left and it decreases downward along the sequence. At the upper end, $L$ goes like a large power of $\mathcal{M}, 5$ or so. The development and verification of all this is the stuff of stellar evolution theory, which we shall not go into here. 


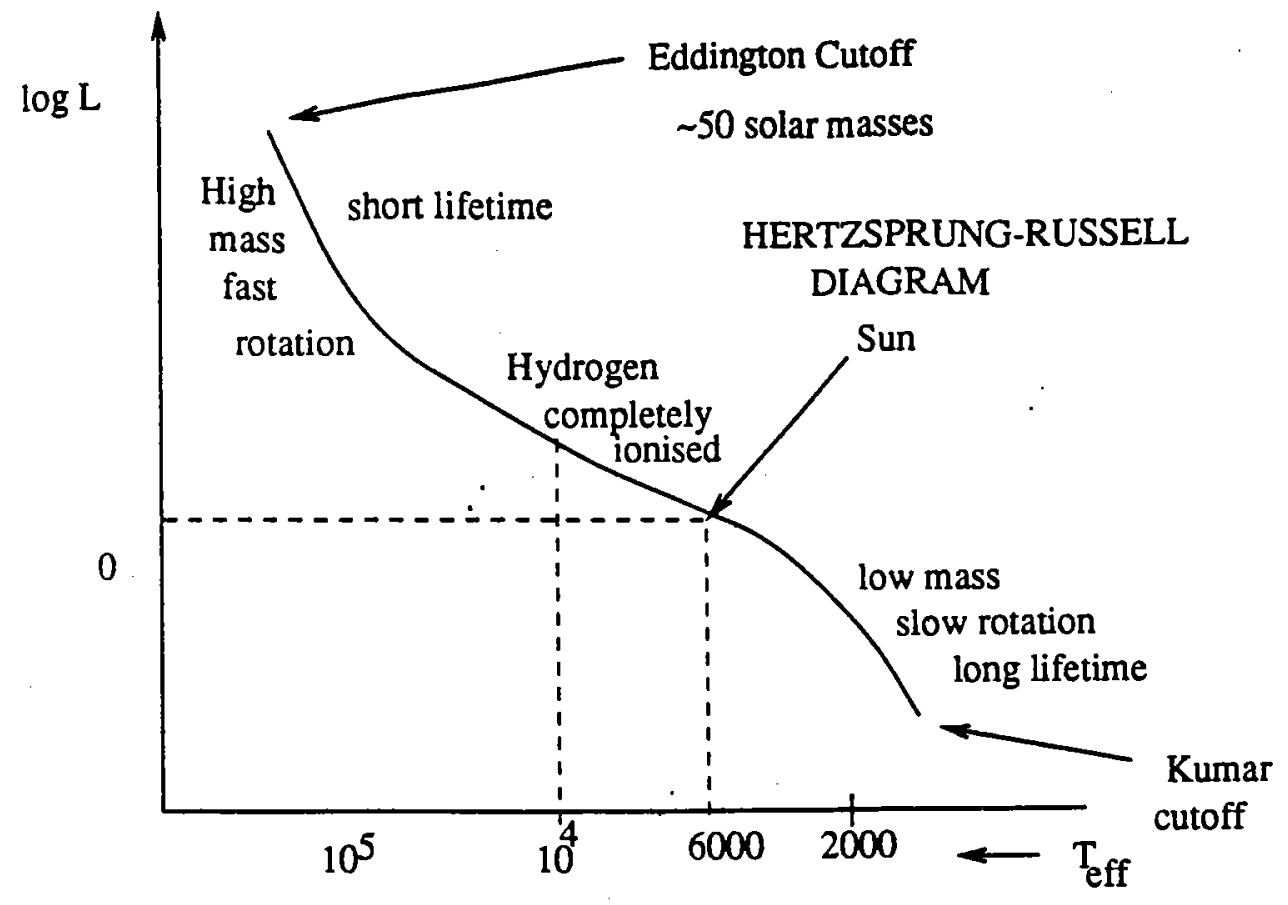

Figure 2: Schematic H-R

We have shown only a section of the theoretical main sequence in the figure, which corresponds to what is actually oberved. As we have suggested, the upper cutoff is probably connected to radiative processes, such as the Eddington limit. In any case, for large masses, the lifetime on the main sequence is small, as is easily estimated. The energy available from nuclear reactions is some slight fraction of the rest energy $\mathcal{M c}^{2}$. Divide this by $L$ and you get a time which is millions of years at the top of the main sequence. This is very short compared to the similar estimate for the sun, which runs to tens of billions of years.

Another feature of the observations that is not really understood is the so-called luminosity function. This is the number of stars at each luminosity and it increases with decreasing $L$. This is a direct consequence of the mass spectrum of newly formed stars. For small enough masses, the central temperature is so low that there are effectively no nuclear reactions. That Kumar limit is somewhere below $0.1 \mathcal{M}_{\odot}$. Those unproductive stars include the so-called brown dwarfs. Their numbers remain a mystery that the space telescope was suppposed to dispel.

\section{Convection}

In computing the main sequence shown in figure 2 , there are already a lot of difficulties to be surmounted. Not only do we need to deal with the microphysics of ionization, opacity, energy generation and radiative transfer, we have to do something about convection. When we write the hydrostatic equation, since the stars are typically composed of perfect 
gas, this is coupled to temperature and to an energy transport equation. So we need to compute the radial temperature gradient. Once we have this, we can check for convective instability. A static model is locally susceptible to convection when the radial gradient of specific entropy (essentially what meteorologists call the potential temperature) is negative. That is, for constant molecular weight,

$$
\frac{d S}{d r}=\frac{C_{p}}{T}\left(\frac{d T}{d r}+\frac{g}{C_{p}}\right) .
$$

Here we are assuming that we are in the outer layers so that the gravitational attraction is constant. When this gradient is negative, we confront convective instability.

In stars, when the the gradient is convectively unstable, the resulting Rayleigh number is typically astronomical, because of the great length scales involved. In fact, it is very hard to obtain the static solutions for stars before convection is allowed for, unless you compute them yourself, since astronomers never publish them. It is therefore not a simple matter to find out what the conventional Rayleigh numbers in stars really are. In any case, stars are so nonBoussinesq that the meaning of the Rayleigh number has to be thought about a bit as well.

When astronomers do encounter unstable entropy gradients, they frequently replace them by zero gradients, the neutrally stable value, and compute the model that way. This use of the notion of convective equilibrium goes back to the last century and it has not greatly wavered. The problem in putting the whole region of convection onto a simple adiabat is that some stellar models, such as that for the sun, are sensitive to which adiabat is chosen. So algorithms have been devised for the purpose. Whether you regard this as science or voodoo depends on your background and goals. As you can readily imagine, the main delicacy hinges on the treatment of the boundary layers. Let us leave this astrophysical skeleton in its closet.

What is found theoretically is that strong convective instability occurs in the outer layers of stars in the lower half of the main sequence. For example, it is believed that the outer one third (in radius) of the sun is vigorously convective. That convection occurs largely because of the high opacity (low thermal conductivity) of partially ionized hydrogen and to some extent because of its high specific heat.

In the upper half of the main sequence, the hydrogen is fully ionized, so convection is not strong at all, though almost all stars do have some surface convective instability. On the other hand, these hot stars have interior nuclear reactions which are temperature sensitive. This promotes strong temperature gradients and convective cores are the rule in the upper main sequence. We shall not have time to get much involved in thermonuclear convection. Our interest will be confined to the fluid dynmics of the outer layers of stars.

Observations suggest that there is strong fluid dynamical activity in the envelopes of both hot ("early") and cool ("late") stars. The cool stars have vigorously convective envelopes. But in the very hot stars, which tend to rotate rapidly $\left(R \Omega^{2} \sim g\right)$, the profiles of spectral lines show the evidence of line broadening (through Doppler effects) by motions with speeds that may even be supersonic. All sorts of other clues point to vigorous activity 
in those hot stellar envelopes, and we will offer some suggestions about the causes in lecture 3. In the intermediate case of the so-called A-stars, the fluid dynamical activity is relatively low. This permits certain peculiarities, as astronomers call them, to manifest themselves.

Notes submitted by N. Platt and R. Kerswell. 


\section{Lecture II}

\section{Expanding Flows}

Our opening example of AFD is the study of a fluid in expansion. The simplest case is that of a uniform, unbounded medium such as cosmologists study. Even for cosmology, this is an oversimplification. The universe is filled with radiation, which is observed to be extremely isotropic with an almost perfect black body radiation spectrum at $T \sim 3 \mathrm{~K}$. According to standard big bang cosmology, this radiation consists of photons emitted from the early universe as orginally predicted by Gamow's students Alpher and Herman in the 1940's. We shall leave out the effects of this radiation, which were pronounced in the early days of whatever cosmic event we are going through just now. But we note in passing that the great degree of isotropy of this radiation attests to the great homogeneity of our universe out to large distances. That is the basis for thinking that a uniform Eulerian flow makes a good model. However, the seemingly fractal distribution of the visible galaxies points to a chaotic Lagrangian flow.

We return to the description of expanding coordinates begun in Lecture $I$ and consider a fluid with velocity $u=H(t) x$. From conservation of mass we have, for homogeneous density,

$$
\frac{\partial \rho}{\partial t}=-\rho \nabla \cdot \mathbf{u}
$$

which gives

$$
\frac{\dot{\rho}}{\rho}=-\nabla \cdot u=-3 H
$$

Thus,

$$
B=\frac{d}{d t} \ln \left[\frac{\rho}{\rho_{0}}\right]^{-1 / 3}
$$

and we define $R(t) \equiv\left(\rho / \rho_{0}\right)^{-1 / 3}$, which is called the scale factor. Two objects initially separated by a distance $r_{0}$ at time $t=t_{0}$ will have a separation at time $t$ of $r=R(t) r_{0}$, where we have set $R\left(t_{0}\right)=1$.

Now consider conservation of momentum in an isentropic self-gravitating fluid:

$$
\partial_{\mathfrak{t}} \mathbf{u}+\mathbf{u} \cdot \boldsymbol{\nabla} \mathbf{u}=-\nabla V
$$

where $V$ is the gravitational potential. There is no term corresponding to pressure or entropy gradients because the fluid is homogeneous. Substitution of $\mathbf{u}=E(t) \mathbf{x}$ yields

$$
\left(\dot{H}+B^{2}\right) \mathrm{x}=-\nabla V
$$

On taking the divergence of the above equation and using Poisson's equation we get

$$
3\left(\dot{H}+H^{2}\right)=-4 \pi G \rho,
$$


which also gives an equation for $R(t)$,

$$
\ddot{R}=-\frac{4}{3} \pi G \rho_{o} / R^{2} .
$$

To obtain the peculiar momentum of a particle, such as a galaxy, we introduce the coordinate transformation

$$
\mathbf{x}=R(t) \tilde{\mathbf{x}} .
$$

We differentiate this and solve for

$$
R \dot{\tilde{\mathbf{x}}}=\dot{\mathbf{x}}-H \mathbf{x},
$$

which is the velocity relative to the expanding background. Multiply this by $R$ and differentiate to obtain

$$
\frac{d}{d t}\left(R^{2} \dot{\overline{\mathbf{x}}}\right)=R\left(\dot{\overline{\mathrm{x}}}-\frac{\vec{R}}{R} \mathrm{x}\right) \text {. }
$$

From equation (7), we see that, if the gravity of the uniform background was the only force acting on the particle, then the right hand side of equation (10) would be zero. Then we would get $R^{2} \dot{\tilde{x}}=$ constant and so the peculiar velocity (for a particle of constant mass) decreases like $R^{-1}$. Thus, with respect to an expanding background, the momentum (P) will decrease,

$$
\mathbf{P}=\frac{\mathbf{P}_{\mathbf{o}}}{R} \text {. }
$$

This result clarifies the way an expanding gas cools by analogyg with the way an expanding gas loses angular momentum. Thus, $R^{2} \dot{\dot{x}}$ is like the angular momentum of a particle and the coordinate velocity $\dot{\overline{\mathbf{x}}}$ is analogous to an angular velocity.

The Boltzmann distribution is $f(P) \sim e^{-E / k T}$ where $E$ is the energy, given by $E=$ $\left(P^{2}+m^{2}\right)^{1 / 2}$ (the speed of light has been set equal to one), $k=$ Boltzmann's constant and $T=$ temperature. Thus, for a non-relativistic particle, $E \approx m^{2}+\mathrm{P}^{2} /(2 m)$ and so for the momentum distribution to be time independant (recall equation (11)), we must have $T \sim R^{-2}$. For relativistic particles (in particular photons), $m \ll<$, hence $E \sim P$. Thus, for $f(P)$ to be time independant we must have $T \sim R^{-1}$. We see that relativistic and non-relativistic particles in the universe cool at different rates. Therein lies the means to understand why the background radiation is at only $3 K$. On the other hand, there is no explanation here for the large peculiar motions in the gas of galaries (a few hundreds of $\mathrm{km} / \mathrm{sec}$ ).

For more general motions, we want to go into coordinates sugested by this simple flow. Starting from the usual Euler equation

$$
\rho D_{\mathbf{z}} \mathbf{u}=-\nabla p-\rho \nabla V,
$$

where $D_{t}=\partial_{t}+\mathbf{u} \cdot \nabla$, we may transform into the expanding coordinates given by (8). We set $\tilde{\mathbf{u}}=\dot{\tilde{\mathbf{x}}}$, whence $R \tilde{\mathrm{u}}=\mathbf{u}-H \mathbf{x}$. Again, the quantity $\overline{\mathrm{u}}$ is a coordinate velocity, analogous to an angular velocity, so the physical velocity is $R \bar{u}$. 
We may also transform $p, \rho$ and $V$, but we shall not take time for this here. The main point is that the left hand side of the Euler equation, in expanding coordinates, becomes

$$
\tilde{D}_{\mathrm{t}}+2 H \tilde{\mathrm{u}}+\dot{H} \tilde{\mathrm{x}}+\bar{\nabla}\left(\frac{1}{2} H^{2} \overline{\mathrm{x}}^{2}\right)
$$

where $\bar{D}_{\mathfrak{t}}=\partial_{\mathfrak{t}}+\tilde{\mathbf{u}} \cdot \tilde{\nabla}$ and $\tilde{\nabla}=R \nabla$. We have three fictitious forces in this transform of the inertial term, in the sense that a fictitious force is a real force that cannot be felt by an inertial observer. There is the cosmic drag, $H \overline{\mathbf{u}}$, the expanding analogue of the Coriolis force. However, it is antiparallel to the peculiar motion, and represents a dissipative term. The other two terms are also familiar looking to students of rotating fluids: there is a term like a centrifugal potential and an analogue of the Euler force which comes when the rigidly rotating frame you go into has a time dependent rotation rate. This kind of dynamics has not been much explored as yet, but it arises in stars as well as cosmology. For example, in a radially pulsating star, we have a periodic $R(t)$ and can ask what happens to the criterion for the onset of convection (Poyet and S., Astron. J., 1979).

\section{Stars}

\subsection{Stellar Evolution}

There are several stages in the evolution of a star. For most of its lifetime, a star burns hydrogen in its core, converting it into helium. This permits a simple estimate of stellar lifetimes. For example, the Sun has a total mass of $2 \times 10^{33} \mathrm{~g}, 10 \%$ of which is in a core hot enough for burning. The amount of energy released per gram of hydrogen is approximately $1 \%$ of its rest-mass energy, $m_{B} c^{2}$. Thus the hydrogen-burning lifetime of the Sun is

$$
\tau=\frac{2 \times 10^{33} \times 0.1 \times 0.01 m_{H} c^{2}}{L_{\odot}} \sim 10^{10} \mathrm{yr} .
$$

where $L_{\odot}=4 \times 10^{33} \mathrm{erg} / \mathrm{sec}$.

After the hydrogen in the core is exhausted, the core slowly contracts until the temperature increases to the point where the helium begins to burn. The star will go through successive burning and contracting stages, each time burning heavier elements. The final stage reached depends on the total mass of the star. The highest mass stars are able to convert silicon into iron. As no exothermic nuclear reaction involving iron is possible, no further central nuclear reactions occur. Continued contraction heats the core until iron breaks down endothermically. The temperature drops, and so does the pressure. The star collapses supersonically until the core density becomes large. The envelope rebounds and the star becomes a supernova, according to one version of the story. There is a lot of fluid dynamics in this but most of it is in the minds of the Crays. 


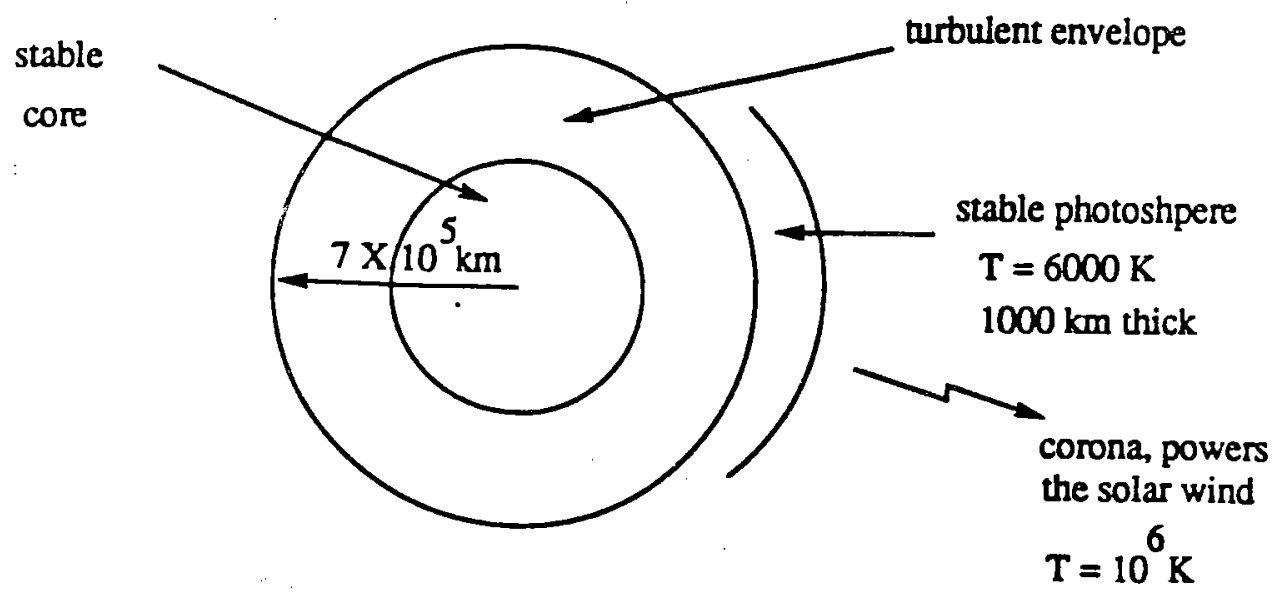

Figure 1: Schematic diagram of the Sun

\subsection{The Solar Atmosphere}

In the outer layers of the Sun, hydrogen is partially ionized, hence there are free electrons which can be captured by a neutral hydrogen atom. As the binding energy of the second electron to $\mathrm{H}$ is only $0.7 \mathrm{eV}$, a passing photon can be easily absorbed in a free-free transition. This makes for very high opacities. So radiation is an inefficient means of heat transfer in the solar atmosphere and convection must occur.

However, within $1000 \mathrm{~km}$ of the surface (the place where the depth measured in photon mean free paths is of order unity), the sun's atmosphere is convectively stable. This place of transition roughly defines the photosphere where most of the sunlight we see originates. From there outward, the temperature increases and we go into the hot $\left(2 \times 10^{6} \mathrm{~K}\right)$ corona that envelops the sun. The corona is not hydrostatic; it is expanding to feed the solar wind. A schematic diagram of the Sun is shown in figure 1 , which suggests that a $1 \frac{1}{2}$ layer model (as in the ocean) might be useful for analyzing the solar atmosphere.

\subsection{Equations of a Simple Stellar Atmosphere}

Let us charactereize an atmosphere as the portion of a star where the gravitational acceleration $(g)$ can be safely considered to be constant. In a cartesian coordinate system, where $z$ is in the vertical direction, the equation of hydrostatic balance is

$$
\frac{d p}{d z}=-g \rho
$$

where $p=$ pressure and $\rho=$ density. Assuming an ideal gas, we have

$$
p=\frac{k}{\bar{m}} \rho T
$$


where $\bar{m}=$ mean molecular mass, usually written $\mu m_{H}$, where $m_{H}$ is the mass of unit atomic weight. We sometimes write the gas constant as $\mathcal{R}=k / m_{B}$, which permits us to leave the mean molecular weight, $\mu$, in evidence. For the purposes of this discussion we shall assume constant $\mu$, though it may in reality depend on the state of the material or its age.

Thus, we have two equations in three unknowns $(p, \rho$ and $T)$. In the simplest case, we assume $T=$ constant and obtain

$$
\begin{aligned}
& p=p_{m} e^{-z / B} \\
& \rho=\rho_{m} e^{-2 / B}
\end{aligned}
$$

where

$$
H=\frac{\mathcal{R} T}{\mu g} .
$$

Thus, in an undisturbed atmosphere that consists of an ideal gas at constant temperature, the pressure and density decay exponentially with height.

To investigate the time-varying pressure and density fields, let us consider each field as having a static component and a much smaller time-dependent component:

$$
\begin{aligned}
& p=p_{0}(z)+p^{\prime}(x, t) \\
& \rho=\rho_{0}(z)+\rho^{\prime}(x, t)
\end{aligned}
$$

where $p_{0}$ and $\rho_{0}$ are given by the static solutions shown above in equations (17) and (18). These perturbations are assumed to evolve isentropically and since $S=c_{v} l n\left(p / \rho^{\tau}\right)$, we have

$$
\frac{D p}{D t}=c^{2} \frac{D \rho}{D t}
$$

where $c^{2} \equiv \gamma p_{0} / \rho_{0}=\left(\gamma k T_{0}\right) / \bar{m}, \gamma=c_{p} / c_{0}$ and we are still assuming the ideal gas law. Substituting these pertubations into the momentum and continuity equations and neglecting second order terms yields:

$$
\rho_{\circ} \frac{\partial u}{\partial t}=-\frac{\partial p^{\prime}}{\partial x}, \quad \rho_{\circ} \frac{\partial v}{\partial t}=-\frac{\partial p^{\prime}}{\partial y}, \quad \rho_{\circ} \frac{\partial w}{\partial t}=-\frac{\partial p^{\prime}}{\partial z}-\rho^{\prime} g
$$

and

$$
\frac{\partial \rho^{\prime}}{\partial t}+\left\{\frac{\partial\left(\rho_{0} u\right)}{\partial x}+\frac{\partial\left(\rho_{0} v\right)}{\partial y}+\frac{\partial\left(\rho_{0} w\right)}{\partial z}\right\}=0
$$

where $u, v$ and $w$ are the velocities in the $x, y$ and $z$ directions respectively. From equation (22) we also have

$$
\frac{\partial p^{\prime}}{\partial t}-\rho_{\circ} g w=\gamma \frac{p_{0}}{\rho_{0}}\left(\frac{\partial \rho^{\prime}}{\partial t}+w \frac{\partial \rho_{0}}{\partial z}\right) .
$$

Now we can eliminate $u, v$ and $w$ from equations (23), (24) and (25) to yield the following equations in $p^{\prime}$ and $\rho^{\prime}$ only:

$$
\frac{\partial^{2} \rho^{\prime}}{\partial t^{2}}-\nabla^{2} p^{\prime}=g \frac{\partial \rho^{\prime}}{\partial z}
$$




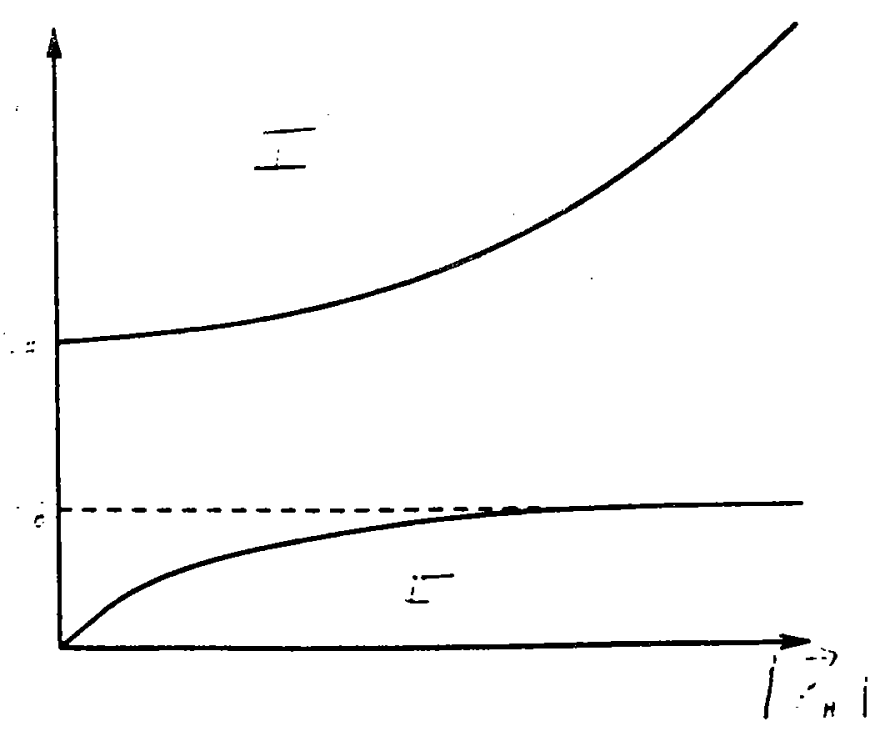

Figure 2: Dispersion relation for Lagrangian perturbation with $T=$ constant

and

$$
\frac{\partial^{2}}{\partial t^{2}}\left[p^{\prime}-\gamma \frac{p_{0}}{\rho_{0}} \rho^{\prime}\right]=(\gamma-1)\left(\frac{\partial p^{\prime}}{\partial z}+g \rho^{\prime}\right) .
$$

Let us look for solutions of the form

$$
\left(\begin{array}{c}
\rho^{\prime} \\
p^{\prime}
\end{array}\right)=\left(\begin{array}{c}
\bar{\rho} \\
\bar{p}
\end{array}\right) \cdot \exp \left[i\left(\kappa_{H} \cdot x-\omega t+\kappa z\right)-z / 2 H\right]
$$

where $\bar{\rho}$ and $\bar{p}$ are amplitudes, $\kappa_{H}$ is a two-dimensional horizontal wave number, $\mathbf{x}$ is a two-dimensional position vector, $\omega$ a frequency and $\kappa$ is a vertical wave number. We have choosen the exponential variation in $z$ to conserve wave energy. Substitution into equations (26) and (27) yields the following dispersion relation (Lamb 1924):

$$
\omega^{4}-\left[\left(\kappa_{B}^{2}+\kappa^{2}\right) c^{2}+\omega_{i}^{2}\right] \omega^{2}+c^{2} \kappa_{H}^{2} \omega_{b}^{2}=0
$$

where $\omega_{i}^{2} \equiv \gamma g /(2 H)$ and $\omega_{b}^{2} \equiv \frac{\gamma-1}{\gamma} \frac{g}{H}$. This yields two sets of relations between $\omega$ and $\kappa_{H}$, as shown in figure 2. In group I, $\omega^{2}=\left(\kappa_{B}^{2}+\kappa^{2}\right) c^{2}+\omega_{1}^{2}$, so that $|\omega| \geq \omega_{1}$ and in the limit as $\left|\kappa_{B}\right| \rightarrow \infty$, these high frequency waves have $\omega= \pm \kappa_{B} c$. Thus they are just non-dispersive sound waves, and are referred to as p-modes in astrophysics. In group II, $\omega^{2}=c^{2} \kappa_{H}^{2} \omega_{b}^{2} /\left[\left(\kappa_{H}^{2}+\kappa^{2}\right) c^{2}+\omega_{i}^{2}\right]$, so that $|\omega| \leq \omega_{b}$. In the limit as $\left|\kappa_{H}\right| \rightarrow \infty$, we have $\omega= \pm \omega_{b}$, which are simply gravity waves (called g-modes).

We have considered only isentropic perturbations to the static state, neglecting thermal effects. Since $S=c_{0} \ln p-c_{0} \gamma \ln \rho, \frac{D S}{D t}=0$ implies that

$$
\frac{D p}{D t}=\gamma \frac{p}{\rho} \frac{D \rho}{D t}=\gamma \frac{p_{o}+p^{\prime}}{\rho_{o}+\rho^{\prime}} \frac{D \rho}{D t} .
$$

To first order, then we have

$$
\frac{D p}{D t}=c^{2} \frac{D \rho}{D t}
$$


This is a Lagrangian perturbation and it yields two families of solutions as discussed. $A$ rough and ready approach that gives just the sound waves is $\frac{\partial S}{\partial t}=0$ (an Eulerian perturbation).

As we are also interested in the velocity of such wave solutions, we could have eliminated $p^{\prime}$ and $\rho^{\prime}$ from equations (23), (24) and (25). Rotating the $\hat{x}$ axis into the direction of propagation (i.e. letting $v=0$ ) and introducing $\omega=v o r t i c i t y=\nabla \times u$ and $\chi=$ compressibility $=\nabla \cdot \mathbf{u}$ we obtain (Lamb, 1924)

and

$$
\frac{\partial^{2} \chi}{\partial t^{2}}=c^{2} \nabla_{1}^{2} \chi+\left(\frac{d c^{2}}{d z}-\gamma g\right) \frac{\partial \chi}{\partial z}+g \hat{z} \cdot \nabla \times \omega
$$

$$
\frac{\partial^{2} \omega}{\partial t^{2}}=-\left[\frac{d c^{2}}{d z}+(\gamma-1) g\right] \nabla \times(\hat{z} \chi)
$$

where

$$
\nabla_{1}^{2}=\frac{\partial^{2}}{\partial x^{2}}+\frac{\partial^{2}}{\partial z^{2}}
$$

In the case of an isothermal atmosphere, these equations can be solved to yield horizontal traveling wave solutions of the form $u=e^{(\gamma-1) g s / c^{2}} f(c t-x)$ and $\omega=0$.

Remaining issues which may be important are the effects of non-linearity and dissipation which have been neglected in this discussion. A further complication is the consideration of a polytropic atmosphere in which temperature varies linearly with height, $T(z)=\beta z$. In this case we find two familes of solutions which are somewhat similar to the isothermal case, as shown in figure 3 .

This is a very superficial introduction to these matters and more details can be found in Lamb's book. In the case of the sun such waves are now being observed and the measured frequencies are used in connection with some knowledge of the radial amplitude distribution to learn much about the internal structure and rotation of the sun. Yet we still know rather little about these modes. How and when do they go unstable when dissipation is included? Why do they seem to be so weakly nonlinear? How do they couple to the turbulent convection?

\section{References}

Lamb, H. 1924 Hydrodynamics, $5^{\text {th }}$ ed. (Cambridge: Cambridge University Press).

Notes submitted by Wendy Welch and Brian Chaboyer 


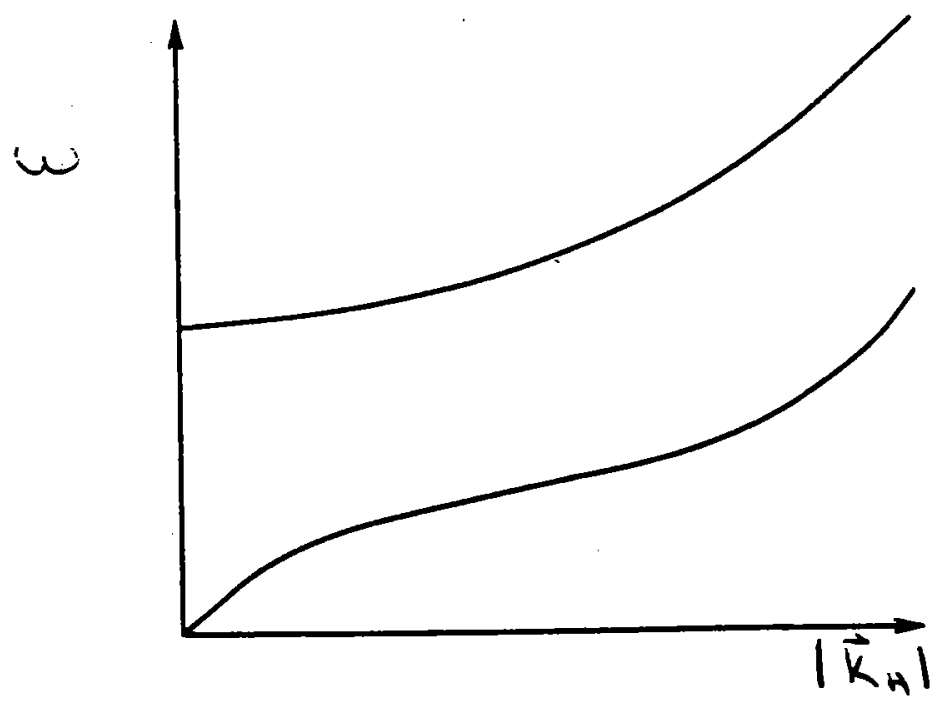

Figure 3: Schematic dispersion relation for $T=\beta z$ 


\section{Lecture III. Photogasdynamics}

\section{The equations of photohydrodynamics}

The picture to be built up involves thinking of the outward diffusion of photons through the outer layers of the star as a fluid of photon particles moving outward through a porous medium. The radiation field might be derived from the full equations of electromagnetism. However, quantum fluctuations and relativistic effects are not important in a typical stellar atmosphere, and a corpuscular picture where the radiation is represented as a fluid of photon particles will be described here. We will also ignore polarization, that is spin.

The matter field is described by the velocity $u$, the density $\rho$, and the pressure $p$, while the radiation field is characterized by the flux $F$, the energy density $E$, and the pressure tensor. $\mathcal{P}$. The equations of momentum, continuity, and thermal energy of the field of matter are (i.e. Hsieh and Spiegel, 1976; Mihalas and Mihalas, 1984)

$$
\begin{aligned}
\rho \frac{D \mathbf{u}}{D t} & =-\nabla p-g \rho \hat{z}+\rho \frac{\kappa+\sigma}{c} \overline{\mathbf{F}} \\
\frac{D \rho}{D t} & =-\rho \nabla \cdot \mathbf{u} \\
\rho c_{v} \frac{D T}{D t}-\frac{D p}{d t} & =-\rho \kappa c(\bar{S}-\bar{E})
\end{aligned}
$$

where $\kappa$ is the mean absorption coefficient and $\sigma$ is the scattering coefficient. The velocity of the matter is measured with respect to an inertial frame, preferably the one in which the star is at rest, call it the star frame. Then $\bar{E}, \bar{S}$, and $\bar{E}$ are the radiation flux, source function and energy density of the radiation field measured in the local rest frame of the matter. These are simply related to $F, S$, and $E$, the same quantities measured in the star frame, and which are the flux, source function and energy density that are normally referred to in the radiative transfer theory of a static medium. The transformation equations relating these quantities measured in the two frames are (to leading order in 
$|\mathbf{u}| / c)$

$$
\begin{aligned}
\overline{\mathbf{F}} & =\mathbf{F}-E \mathbf{u}-\mathcal{P} \cdot \mathbf{u}, \\
\bar{E} & =E-\frac{2 \mathbf{u} \cdot \mathbf{F}}{c^{2}} \\
\bar{S} & =S=a T^{4}
\end{aligned}
$$

In addition, the equations governing the field of matter (1)-(3) require an equation of state,

$$
p=\frac{\mathcal{R}}{\mu} \rho T
$$

and the specification of the absorption and scattering coefficients $\sigma(\rho, p)$ and $\kappa(\rho, p)$.

The final term in the momentum equation (1) represents the momentum imparted to the matter per unit time from the radiation field, i.e. a radiation pressure gradient.

The fluid equations for the radiation are derived by taking frequency integrated moments of the transfer equation. The radiative equations of motion are:

$$
\begin{aligned}
\frac{\partial E}{\partial t}+\nabla \cdot \mathbf{F} & =\rho \kappa(S-E)-\rho\left(\frac{\kappa-\sigma}{c}\right) \mathbf{u} \cdot \mathbf{F}, \\
\frac{1}{c^{2}} \frac{\partial \mathbf{F}}{\partial t}+\nabla \cdot \mathcal{P} & =-\rho\left(\frac{\kappa+\sigma}{c}\right) \mathbf{F}+\frac{\rho \kappa}{c}(S-E) \mathbf{u}
\end{aligned}
$$

The source function $S$ simply represents the emission of radiation by the fluid, and $E$ the absorption. The final term in equation (8), which governs the evolution of the energy density of the radiation field, is the rate of work done by the radiation upon the matter field. Equation (9) expresses the evolution of the momentum density of the fluid (and is the counterpart of conservation of momentum for the matter field). The first term on the right hand side of (9) represents the force of matter upon the radiation fluid, whereas the second term indicates how a net loss of energy by absorption creates an additional force upon the radiation fluid, through the concomittant momentum exchange.

The system of equations for the radiative fluid are completed when an approximation or closure for the pressure tensor $\mathcal{P}$ is specified. Here we shall use,

$$
\mathcal{P}=\frac{1}{3} E I+\frac{1}{c^{2}}\left[u F+F u-\frac{2}{3}(u \cdot F) I\right]+\mathcal{T}
$$


where $\mathcal{I}$ is the identity tensor and $\mathcal{T}$ is the radiative viscosity tensor satisfying $\operatorname{Tr}(\mathcal{T})=0$. The quantity in square brackets in (10) is a transformation correction due to the motion of the material.

The isotropic part of $\mathcal{P}$ provides the dominant contribution to the pressure tensor in regions where the photon mean free path is small, i.e. the medium is optically thick. Neglecting all but this term yields

$$
\mathcal{P}=\frac{1}{3} E \mathcal{I}
$$

This isotropic approximation to $\mathcal{P}$ is known as the Eddington approximation. When the photon mean free path is large, the medium is "optically thin" and the anisotropic components of $\mathcal{P}$ may become important.

When the region where the radiation and matter fields interact is plane parallel and the particle fluid is motionless, the equations reduce to what has been called the Milne problem (Chandrasekhar, 1960). In this case the full radiative transfer equations can be solved exactly.

The derivation of the photohydrodynamic equation suggests that there may be a more natural approximation to the problem than the Eddington approximation. Essentially, the Eddington approximation assumes that the pressure tensor $\mathcal{P}$ is isotropic in the rest frame of the star. The derivation of the equations takes account of the transformation between this frame and the rest frame of the moving matter. There may be a different frame, which one might call the "radiative frame", in which, if one assumes $\mathcal{P}$ to be isotropic, the exact solution is better approximated. If one considers general transformations in the Milne problem, and subsequently implements the Eddington approximation in these frames, one finds that there exist two frames, one subsonic and one supersonic (with respect to $c / \sqrt{3}$ ) in which the exact solution for a particular moment, is produced by this means. However, whether this result is unique to the Milne problem, and whether either choice of one of these frames is sensible is not evident.

\section{Hydrostatics and linear stability}

As an example, consider a plane-parallel atmosphere in hydrostatic balance

$$
\mathbf{F}=F_{0} \hat{z} ; S=E=a T^{4} ; p=\Re \rho T
$$




$$
\begin{aligned}
& \frac{d p}{d z}=-\rho g+\left(\frac{\kappa+\sigma}{c}\right) F_{0} \rho=-\rho g . \\
& \frac{d E}{d z}=-3 \rho\left(\frac{\kappa+\sigma}{c}\right) F_{0}
\end{aligned}
$$

A simple solution is obtained by neglecting variations in ionization (so that $\Re=\mathcal{R} / \mu$ is constant) and supposing that the atmosphere is hot enough that $\sigma$ (scattering) dominates $\kappa$ (absorption). The distribution of temperature with height is illustrated in Figure 1. As

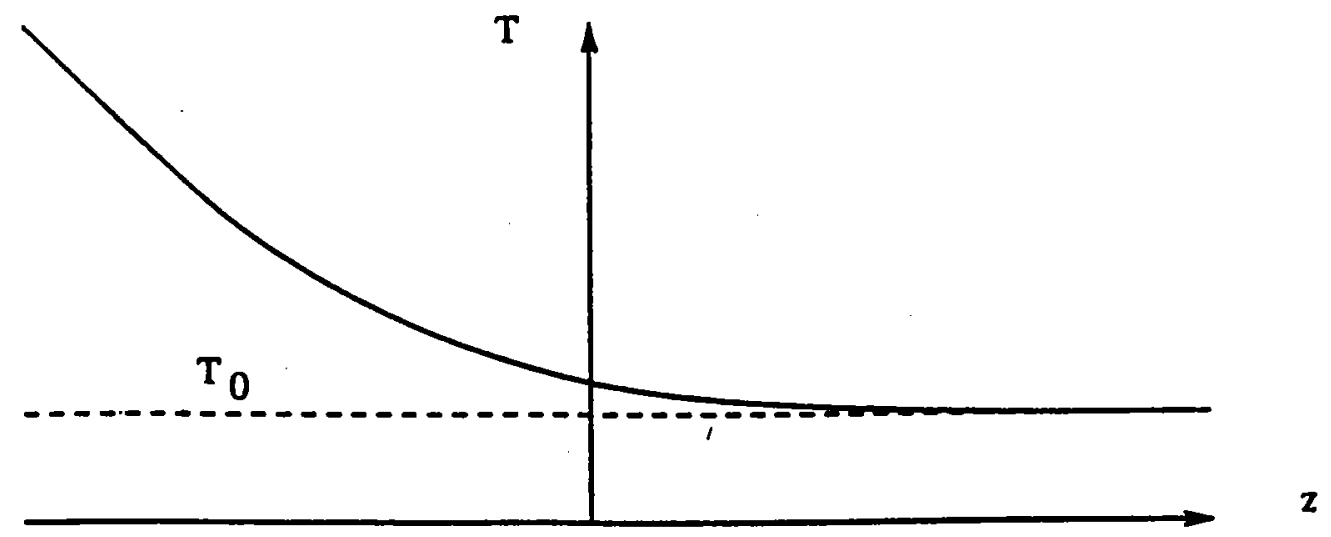

Figure 1: The temperature distribution with height for the idealized model atmosphere. At depth, the structure is polytropic while, high in the atmosphere, the temperature declines exponentially to $T_{0}$ (note that the scale height is approximately $R T / g_{\text {.) }}$.

the radiation pressure gradient increases, the effective gravitational constant $g$. decreases. The case $g_{*}=0$ corresponds to the Eddington limit. Beyond this point the atmosphere levitates. The hydrostatic solution for $g_{*}<0$ has a density which increases with height. A star above the Eddington limit would presumably have its surface layers blown off by radiation pressure. Yet rare stars exist that appear to exceed the Eddington limit.

The stability of this solution may be examined by perturbing the hydrostatic equilibrium and solving the resulting linear equations for the perturbation. In this example, instabilities occur when we omit the viscous terms and absorption. Though dissipation may push such linear instabilities to nearly the Eddington limit, they may occur nonlinearly in stellar conditions and produce fluid dynamical activity, such as "photon bubbles", to be discussed shortly. 


\section{The heat equation in the absence of fluid motion}

If the material velocity vanishes, the photohydrodynamic equations for the thermal energy of the matter field, and the radiation field, become

$$
\begin{aligned}
\rho c_{v} \frac{\partial T}{\partial t} & =-\rho \kappa c(S-E), \quad S=a T^{4}, \\
\frac{\partial E}{\partial t}+\nabla \cdot F & =\rho \kappa c(S-E), \\
\frac{1}{c^{2}} \frac{\partial F}{\partial t}+\frac{1}{3} \nabla E & =-\rho\left(\frac{\kappa+\sigma}{c}\right) \mathrm{F} .
\end{aligned}
$$

Since the travel times for light across the distances of interest are large for stars (one says that $c \rightarrow \infty$ ), this can be simplified. We see, first of all, that $E \approx S$. Moreover, the term $c^{-2} F_{t}$ is small. In first approximation it may be neglected and this gives an approximation for $F$, which we use to approximate $F_{t}$ in this equation. Then we obtain the radiative heat equation for $T$ (Unno and S., P.A.S.J., 1966), which, after we take certain liberties, becomes

$$
\left(4 a T^{3}+\rho c_{v}\right) \frac{\partial T}{\partial t}-\frac{c_{v}}{3 \rho(\kappa+\sigma) \kappa} \nabla^{2} \frac{\partial T}{\partial t}=\frac{4 a c T^{3}}{3 \rho(\kappa+\sigma)} \nabla^{2} T
$$

In the limit where the photon mean free path is large - that is, $\rho^{2} \kappa(\kappa+\sigma) \ll 1-$ equation (18) reduces to

$$
\frac{\partial T}{\partial t} \propto-\left(T-T_{0}\right)
$$

where $T_{0}$ is a constant of integration chosen as the equilibrium temperature. In this optically thin (or transparent) limit, disturbances decay according to Newton's law of cooling.

When the mean free path is small $\left(\rho^{2} \kappa(\kappa+\sigma) \gg 1\right),(18)$ reduces to

$$
\frac{\partial T}{\partial t} \propto \nabla^{2} T
$$

In the optically thick or opaque limit, the decay of disturbances is governed by a diffusion equation. 


\section{Photon Bubbles}

\subsection{The analogy with fluidized beds}

Consider a collection of small particles (e.g. sand) resting on a porous plate. A fluid (e.g. air) is forced up through the plate and particles.

When the flux of fluid through the plate is low, the particles are relatively unaffected, and act like a porous medium. This can be thought of as analogous to the diffusion of a photon gas through a stellar atmosphere. In fact, the equation governing the motion of the fluid is of the form

$$
\frac{D p}{D z}=-\lambda F
$$

where $p$ is the fluid pressure, $\lambda$ is the porosity of the particles, and $F$ is the vertical fluid flux. This is just D'Arcy's law, and it is qualitatively similar to the equations that result in certain problems in radiative transfer.

As the flux of fluid increases, the drag on each particle due to the diffusing fluid also increases. When the drag per particle exceeds the weight of the particle the whole bed is levitated. Levitation expands the bed, allowing the fluid to move more freely between the particles which diminishes the drag. The bed is then said to be fluidized (Davidson and Harrison, 1963). Quicksand is a familiar example of this phenomenon.

If the fluid density, $\rho_{f}$, is much less than the density of an individual particle, $\rho_{p}$, bubbles of fluid appear and rise through the bed of particles. These bubbles presumably are generated by instability of the fluidized bed.

The bubbles can ascend to collapse at the surface of the bed, which has the appearance of a boiling liquid. The bubbles in fluidized beds are kidney-shaped (see Fig. 2) in vertical cross section. Overall, the motion of the bubbles mixes the particles. However, the particles with the bighest drag are carried upward with the bubbles. This process is called elutriation, and may be exploited in industrial applications for separating particles with different drag coefficients. Perhaps in stars we can think of photoelutriation.

\subsection{Photoconvection}

The analogy with fluidized beds suggests that there be bubbles filled with photons percolating through a stellar atmosphere. Even without the benefit of the analogy, astrophysicists have speculated on this possibility in radiatively dominated situations. Indeed, the 


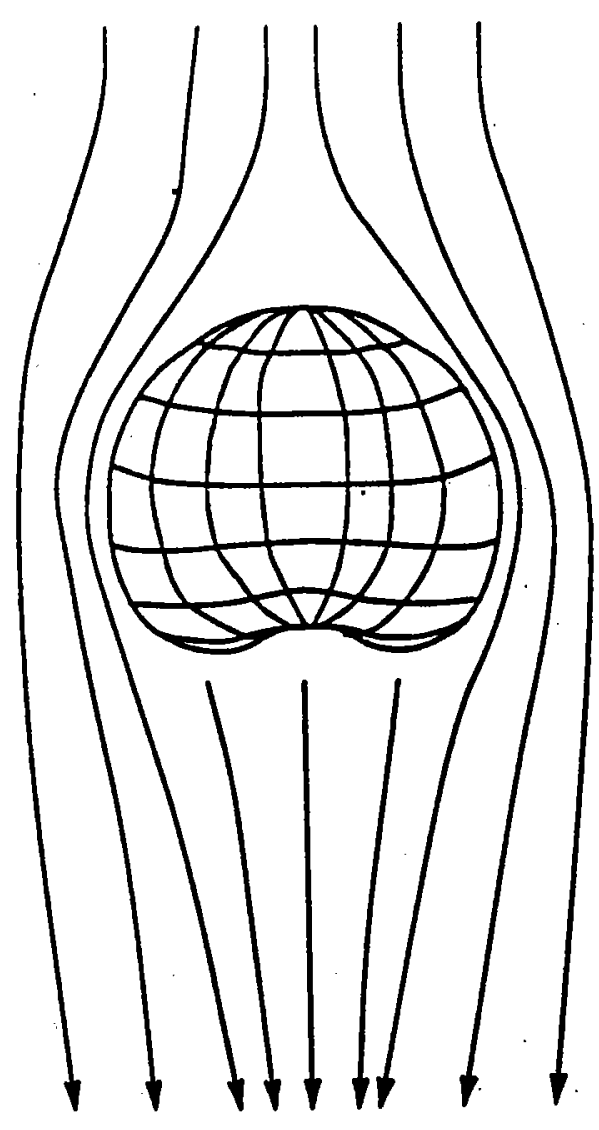

Figure 2: A kidney shaped bubble in a fluidized bed.

analogy is not perfect; the instability of a fluidized bed is caused by the density dependence of the drag coefficient. This is not usually a significant process in the astrophysical cases. Moreover, the bubbles in the fluidized bed are maintained by fluid that typically cannot be absorbed by the particles, which is not the case for photons in the stellar case.

Numerical calculations suggest that linear instabilities in hot stellar atmospheres are suppressed by dissipative processes except for stars near the Eddington limit (Marzec, 1976). If linear instability may occur for $\mathcal{E}=L / L_{E}$ in excess of some critical value, $\varepsilon_{c}$, it is possible that the bifurcation is subcritical and nonlinear instabilities may occur for some reasonable stellar conditions with $\varepsilon<\varepsilon_{\mathrm{c}}$ that would give rise to bubbles. 


\subsection{Bubble theory}

For the simple theory of photon bubbles we consider the fate of a spherical hole carved out of a stellar atmosphere. For the case of pure scattering in the Eddington approximation, we have $\mathbf{F} \propto \nabla E$ and, for radiative equilibrium, we assume, $\nabla \cdot \mathbf{F}=0$. For a coordinate system centered on the center of the hole and with $\theta$ measured from the vertical symmetry axis, we find

$$
\mathbf{F}=F_{0} \nabla\left[\cos \theta\left(r-\frac{r_{0}^{3}}{r^{2}}\right)\right]
$$

where $r_{0}$ is the radius of the sphere. We are here assuming that the Eddington approximation holds also within the bubble, where the photons scatter from the surface and are isotropized. We have neglected absorption.

The radiation field about the hole is distorted in much the same way as an electric field is affected by a conducting sphere in electrostatics. The originally uniform flux $\mathbf{F}=F_{0} \hat{z}$ suffers a dipole distortion. The distorted field lines produce an additional force on the matter. The external force density $f$ is

$$
\mathbf{f}=-\rho g \hat{z}+\frac{\rho \sigma}{c} \mathbf{F}=-\rho g_{*} \hat{z}-\rho \nabla \phi
$$

where $g_{*}$ is the effective gravity defined by (13) and $\phi$ is the potential of the dipole distribution

$$
\phi=\frac{F_{0} c}{\sigma r_{0}^{3}} \frac{z}{r^{3}}
$$

Figure 3 illustrates the distorting field $\nabla \phi$.

The force $f$ produces a fluid circulation which causes the bubble to rise, and to deform. If $V \hat{z}$ is the velocity of the bubble, let $\mathbf{v}=\mathbf{u}-V \hat{z}$ be the flow field around the bubble in a frame of reference translating with it. If we assume that the bubble remains nearly spherical, then we may take $v$ to be the incompressible flow around a spherical obstacle. Thus,

$$
v=V \nabla\left[z\left(1+\frac{r_{0}}{2 r^{3}}\right)\right]
$$

The flow $\mathbf{v}$ is irrotational, and satisfies Bernoulli's Law. This may be used to estimate the upward speed $V$ of the bubble. Bernoulli's Law specifies that

$$
h=-g_{*} z-\phi-\frac{1}{2}|v|^{2}
$$




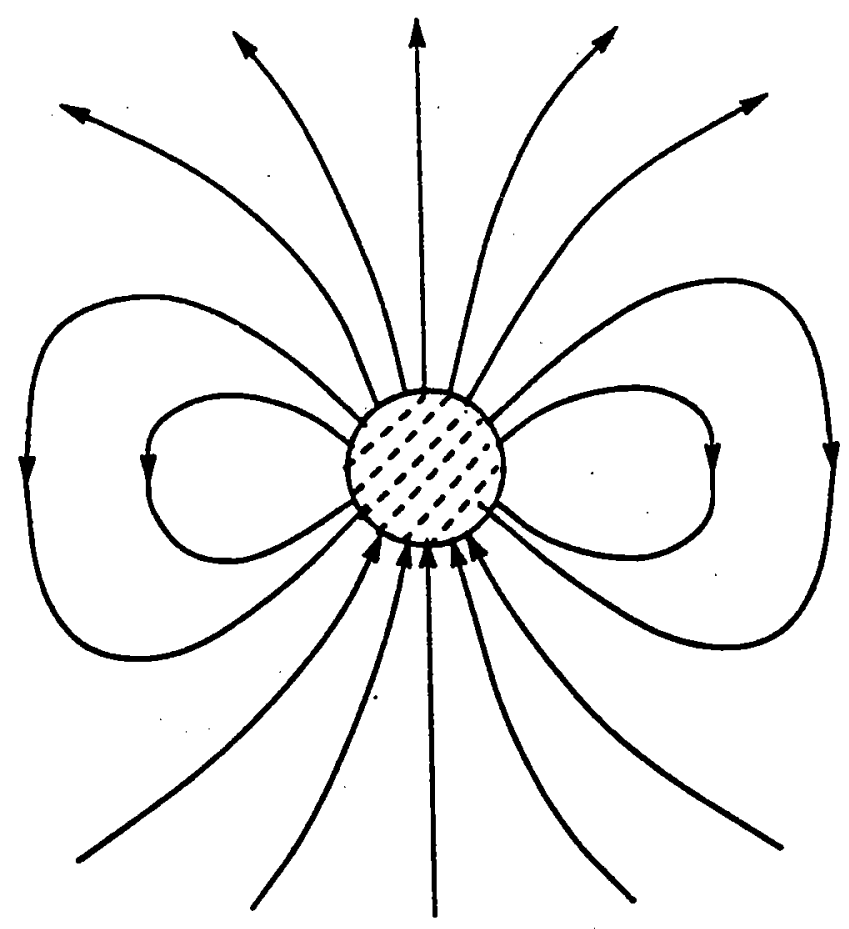

Figure 3: The field lines for the additional dipolar force caused by the presence of the bubble. 
is constant along streamlines. Taking into account the relevant boundary conditions (Spiegel, 1976) gives the result

$$
\therefore \quad V=\frac{2}{3} \sqrt{g r_{0}}
$$

On the lower half of the bubble, the fluid dynamical pressure $|v|^{2} / 2$ must be balanced by an accompanying distortion of the bubble. This distorted shape is indicated in figure 5 , and in the fluidized beds, gives rise to the kidney shaped bubbles.

At the surface the bubble will burst with an effluence of the photons contained within it. Thus excess flux from bursting bubbles may produce shot noise in the stellar luminosity. The atmospheric vortices that we shall describe below could even produce bright spots. When the bubble bursts, particles may become supersonically ejected. Such phenomena may be the basis for the intense hydrodynamic activity of these early stars. The problem is to confirm the existence of photon bubbles.

\section{The effects of rotation}

Hot stars are fast rotators and the interplay of rotational and radiative dynamical processes is likely to be central to understanding their fluid dynamics. Rotation alone is already a significant modulator of stellar fluid dynamics. Its effect upon an initially spherical object is to decrease the polar radius $R_{\text {pole }}$ and increase the equatorial radius $R_{\text {eq. }}$. The difference between these radii is

$$
\frac{\Delta R}{R_{\text {eq }}}=\frac{R_{\text {eq }}-R_{\text {pole }}}{R_{\text {eq }}} \sim \frac{\Omega^{2}}{\Omega^{2}+G_{\rho}}
$$

where $\rho$ is some mean density.

The rotation induces a distortion of the surfaces of constant temperature; the pole and equator exhibit a temperature difference of approximately

$$
\Delta T / T \sim \Delta R / R
$$

From the perfect gas law, we estimate the pressure difference to be

$$
\Delta p \sim \Re \rho \Delta T \sim p \frac{\Delta R}{R} .
$$

This must drive an "astrostrophic" zonal flow $u$, such that

$$
\Delta p \sim \rho u \Omega .
$$


Therefore the magnitude of this thermal wind is

$$
u \sim \frac{g \Delta R}{\Omega R}
$$

where the surface pressure $p$ has been replaced by $\rho g R$ according to estimate based upon vertical hydrostatics.

When the rotation is very fast, as in many hot young stars, equatorial centrifugal accelerations compare to $g$. Thus $g / \Omega \sim R \Omega=V_{\text {eq. }}$ Therefore the flow produces an equatorial acceleration and, since the Reynolds numbers of such flows are large, instabilities are likely. We expect vortex formation, as on major planets. Because of the complications invloved in the radiative flows, these vortices may may serve as conduits for rapid escape of radiations from within and produce powerful emergent beams (Dowling and $\mathrm{S}$., in press).

\subsection{Vortices in hot atmospheres}

Consider a vortex in a polytropic atmosphere. If the vortex is strong enough, we may for a qualitative first look, ask what it will be like without the effect of rotation. Under hydrostatic balance the vertical and horizontal pressure gradients of a steady vortex satisfy

$$
\frac{d p}{d z}=\rho g, \quad \frac{d p}{d r}=\frac{\rho v^{2}}{r},
$$

where $z$ increases in the downward direction. If the specific enthalpy is $h$, then

$$
h(r, z)=g z+f(r)
$$

where $f(r)$ must satisfy

$$
\frac{d f}{d r}=\frac{v^{2}}{r}
$$

For a standard vortex of the form

$$
v=\left\{\begin{array}{ll}
v_{0} r / r_{0} & \text { if } r<r_{0} \\
v_{0} r_{0} / r & \text { if } r>r_{0}
\end{array},\right.
$$

we have

$$
f=v_{0}^{2}\left\{\begin{array}{ccc}
r^{2} / 2 r_{0}^{2}-1 & \text { if } r<r_{0} \\
-r_{0}^{2} / 2 r^{2} & \text { if } r>r_{0}
\end{array}\right.
$$




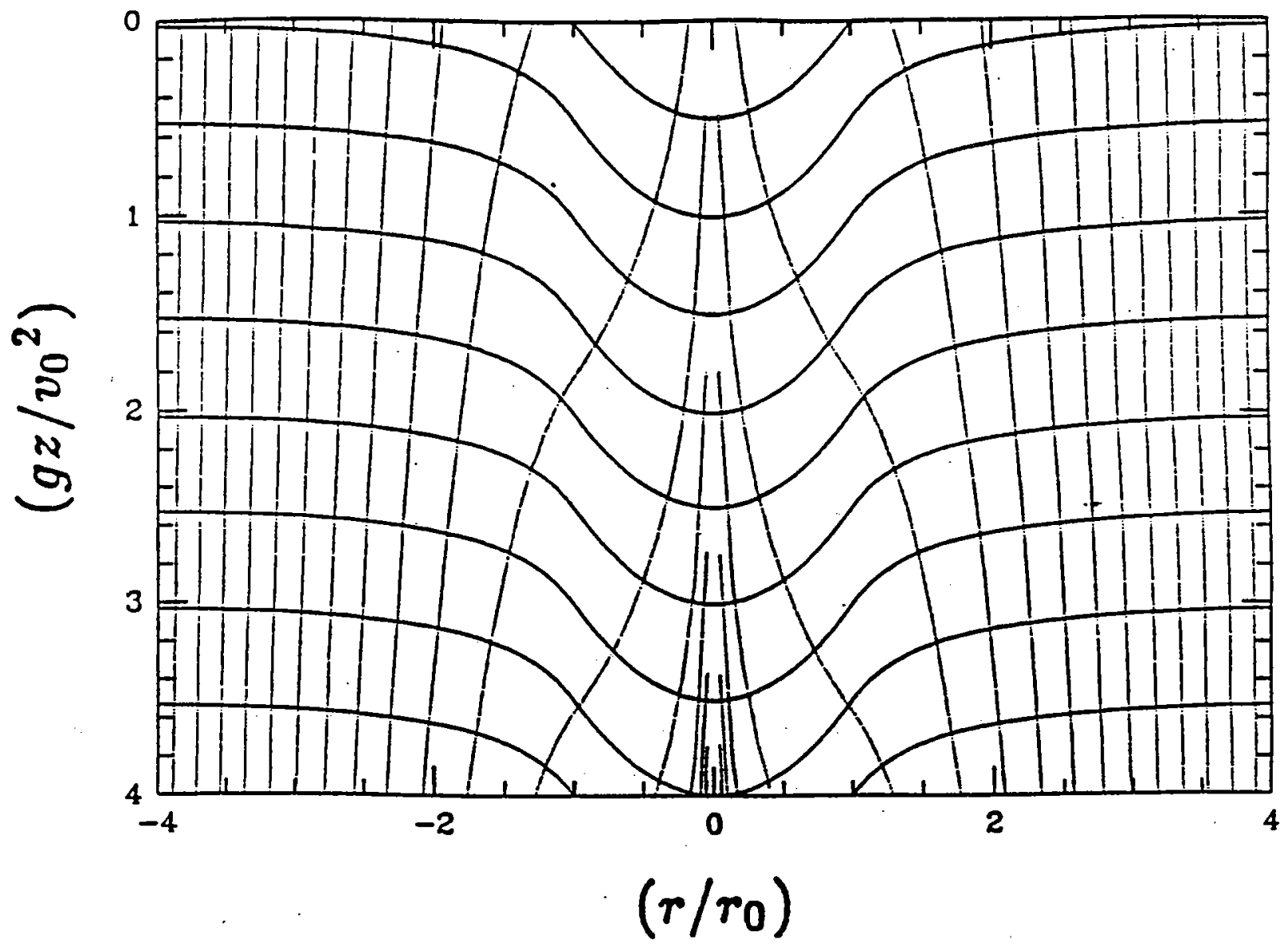

Figure 4: The isotherms in a vertical section through the axis of an atmospheric vortex. The dotted lines indicate the streamlines of the radiative flux as it is focussed into the vortex.

The vortex deforms the stellar surface from the plane $z=0$ into

$$
z=\frac{v_{0}^{2}}{g}\left\{\begin{array}{ccc}
r^{2} / 2 r_{0}^{2}-1 & \text { if } r<r_{0} \\
-r_{0}^{2} / 2 r^{2} & \text { if } r>r_{0}
\end{array} .\right.
$$

Therefore there is a depression of the surface. The maximum depth of the depression is $v_{0}^{2} / g$.

The isotherms $h=$ constant in a vertical section through the axis of the vortex are shown in figure 5. The fluid at the center of the vortex is now cooler than that outside it. The radiative flux is now focussed into the vortex and consequently the depression of the surface appears bright. Thus it forms a "starspot". The focussing of the flux is also shown in figure 4. Therefore, upon the surface of hot stars there may be bright spots, in contrast to the dark spots observed upon cooler stars such as the sun.

Finally, we note that this mechanism may allow a star to exist above the Eddington limit: the vortices channel photons along their axes and therefore reduce the pressure of radiation over the remainder of the surface. 


\section{References}

1. Chandrasekhar, S. (1960). Radiative Transfer (New York, Dover).

2. Davidson, J.F. and Harrison, D. (1963). Fluidized Particles (Cambridge University Press).

3. Hsieh, S.-H. and Spiegel, E.A. (1976). Ap. J., 207, 244.

4. Marzek, C.M. Thesis. Columbia University, Dept of Physics (1976).

5. Mihalas, D. and Mihalas, B.W. (1984). Radiation Hydrodynamics.

6. Spiegel, E.A. (1976). In Problems in Stellar Convection, ed. E.A. Spiegel and J.-P. Zahn, Lecture notes in Physics 71 (Springer-Verlag, Berlin).

7. Dowling, T.E. and Spiegel E.A. (1990). In Nonlinear problems in astrophysics, ed. R. Buchler and S. Gottesman (N.Y. Acad. Sci.).

Prepared by N.J. Balmforth and G. Bell. 


\section{Lecture IV. Solar AFD}

\section{Introduction}

A good white light photograph of the sun reveals that the solar surface is covered with a time-dependent pattern of granules, a cellular arrangement of bright patches. The sizes of these granules range down to the resolution limit of observations, about 300 $\mathrm{km}$. Individual granules may last ten minutes or more, depending on how deformed they are allowed to get and still be considered to be the original granule. Spectroscopic observations show that the brighter (presumably hotter) portions of the granules are rising. The granulation is normally thought to be a manifestation of thermal convection.

The films are the best way to get some feeling for the phenomenon. They were made at the Pic du Midi Observatory (thanks to T. Rouddier for providing that one) and from the observations made in space (blessings on G.W. Simon for that one). They have been processed to bring out the granulation by the group at Lockheed (Alan Title, and his colleagues), which means, in particular, that the vigorous acoustic oscillations of the solar atmosphere have been filtered out. The speeds of the granules themselves (a few tenths $\mathrm{km} / \mathrm{sec}$ ) are well below the local sound speed of close to $10 \mathrm{~km} / \mathrm{sec}$. The morphological details of the granulation are too complicated to repeat in these notes.

Spectroscopic observations showing the velocity component toward the earth at each point on the solar surface reveal other structures. The most significant are the supergranules. These are cell-like structures about $20,000 \mathrm{~km}$ across consisting of horizontal motions flowing from central upwellings. The outflow velocity is about $0.5 \mathrm{~km} / \mathrm{sec}$, comparable to the equatorial rotation speed of the whole sun. But the lifetime of one of these supergranules is about a day, compared to the rotation period of one month.

Another sort of observation made of the solar surface is the magnetic field, which tends be quite ropy. The main concentrations of field are at the vertices where the supergranules meet. It is believed that the intersticial field strengths run to 1700 gauss. The magnetic field thus forms a large pattern outlining the supergranules. Moreover, emission by ionized calcium is particularly strong where the field is strong (probably because of plasma affects generated by the fields) and a calcium emission network clearly outlines the supergranulation.

There is a wealth of further structure in the solar observations, but there is no time to go into such details. This particular selection was made because of the belief that the phenomena I have mentioned are direct manifestations of the strong convection that is thought to power various signs of fluid activity on the sun. One other observed process ought to mentioned - the sunspots.

That same white light photograph of the sun, exposed to bring out the granulation, will often show dark spots (comparable in size to supergraniules). Other observational techniques reveal that in these spots there are fields of a few thousand gauss. Such fields can inhibit the convective motions and lower the emergent heat flux. That will already cause some darkening, but there is more to the story of spot structure. The degree of 


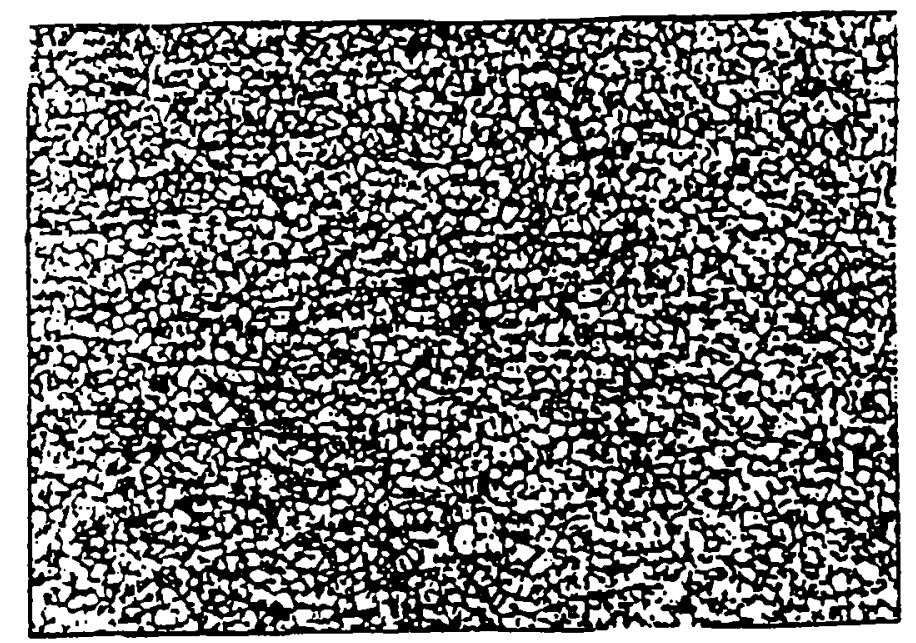

Figure 1: Solar Granulation

spottedness of the sun varies on a time scale of 11 years, perhaps chaotically. We shall come back to this at the end of the lecture.

\section{Solar Convection}

Energy is generated in the core of the sun (as in most stars) and it appears largely in the form of radiation. It takes a photon about 30 million years to escape from the deep interior of the sur. The hydrostatic equations, together with the transport equation for the radiation, then lead to a static model that gives the march of the state variables, temperature, density and specific entropy, through the star. For a perfect gas, the specific entropy is

$$
S=C_{v} \log \frac{p}{\rho^{\gamma}}
$$

where $C_{v}$ is the specific heat at constant volume and $\gamma$ the ratio of specific heats. So, if we use the equation of state for a perfect gas, we get

$$
\frac{d S}{d r}=\frac{C_{p}}{T}\left(\frac{d T}{d r}+\frac{g}{C_{p}}\right)
$$

where $g=G \mathcal{M}_{r} / r^{2}$ and $\mathcal{M}_{r}$ is the mass interior to a sphere of radius $r$.

This can be understood by displacing a parcel of fluid in the vertical direction by an. amount $d r$. Its energy change consists of an internal energy perturbation of $C_{p} d T$ plus a change of potential energy amounting to $g d r$. So the total energy change agrees with $T d S$ and, when it is negative, we have instability. This criterion for the onset of convection is called the Schwarzschild criterion, after one of the many who derived it for themselves, going back at least to the middle of the nineteenth century. 
Starting from the center, the entropy gradient from static models is positive most of the way out to the edge of the sun, where it goes strongly negative, and stays that way nearly to the edge. The final reversal back to a positive entropy gradient occurs at about one photon mean free path from the outside. This is schematically shown in the figure. I can produce only a schematic figure since I know of no modern calculation of the specific entropy distribution of a static solar model. It appears that all programs that calculate solar models contain an algorithm that replaces the unstable region and some of the underlying stable region by an effectively neutral region. Any reasonable algorithm that replaces the static entropy portions that are either stable or neutral is bound to produce a model in which the neutral zone is larger than the original unstable zone.

All stars with $T_{e}$ less than about $8,000 \mathrm{~K}$ have extensive outer zones of convective equilibrium. The cause is the ionization of hydrogen, the major constituent of stellar material. In the hot stars, hydrogen is completely ionized throughout. In the cool stars, there is nearly neutral hydrogen close to the surface. The relatively few atoms that are ionized release electrons that find themselves in a sea of hydrogen atoms. An electron near an atom polarizes it so that there is a weak attraction between them. The spectrum of interaction energies is almost always continuous. So any passing photon can cause a change in this energy and thus be absorbed or deflected. In other words, partially ionized hydrogen is very opaque. A strong temperature gradient is therefore needed to force the radiation through it. At the same time, the ability of the material to soak up energy into ionization causes $C_{p}$ to be large. So we get convection zones in the outer regions of cool stars.

There is no means of calculating a conventional Rayleigh number without a good static model, but estimates from the fully convective model suggest that the Rayleigh number is truly astronomical, probably in excess of $10^{20}$. So when the convection starts, it is certainly turbulent. That is why only the local criterion is considered. The idea then is to replace the unstable region by one of convective neutrality where the entropy gradient is locally zero. As I have already said, this device is bound to make the convective zone deeper than the original unstable zone. The thickness of this zone (fixed by the choice of a free parameter in the algorithm) is adjusted to make the solar radius come out right. Recent developments in acoustic sounding of the sun have permitted fine tuning of the models and there is now some confidence that the depth of the solar convection zone is $300,000 \mathrm{~km}$. Presumably, there will be boundary layers on this zone, especially at the top. Estimates of the depth of the upper boundary layer are of the same order as the size of large granules.

In fact, there is no general agreement on what physics determines the length scales and time scales of the granules and supergranules. It is not even certain that the granules are primarily driven by buoyancy. The large scale shears in the supergranules may play a role in their formation. Magnetic feedbacks may have a role in determining the preferred sizes of either supergranules or granules. We do not know how these processes couple to the large scale circulation seen on the solar surface. We can reach for analogies to laboratory convection or to numerical simulations, but even those are not really under- 
stood. However, at this moment, the simulations, experiments and solar observations are all moving forward quickly, so this is a good time to be thinking of these things.

\section{Solar Rotation}

Observational evidence suggests that stars condense out of the interstellar medium. As the protostar contracts, it spins faster. The object is probably turbulent and the angular velocity is roughly constant throughout its bulk. To become something resembling a star, it must shed considerable angular momentum. This it does through a combination of magnetic stresses and mass expulsion. There may be an ambient disk left behind in the contraction from which a solar sytem may form. In the end, if there is to be an object resembling a main sequence star formed, it ought not to have an angular velocity too much in excess of $\sqrt{2 G M / R^{3}}$, corresponding to a rotation period of about a day for the sun. It is a problem to get rid of the excess angular momentum, so that most newly formed (single) stars will be pressed up against this limiting angular velocity. However, most aged, cool solar-type stars have rotation periods significantly longer than a day and it is concluded that they must have lost angular momentum since arriving on the main sequence.

There is a mass flow of about $10^{-13} \mathcal{M}_{\odot} / y r$. The deeper causes are not understood, but something is heating the very outer, highly tenuous solar layers to a temperature of $2 \times 10^{6} \mathrm{~K}$. The leading candidate is plasma instability. In any case, the material is so ionized that it emits inefficiently and has trouble getting rid of the energy, so it is forced to expand to avoid thermal runaway. In fact, it expands right off the sun and makes a thermal wind. The magnetic field lends a certain rigidity to the flow and it therefore decouples from the sun at a greater angular velocity than that of the surface. This makes the solar wind efficient at removing angular momentum.

Suppose you had a bucket of water suspended by a rope and spinning. If you put some holes in the side of the bucket how fast would it slow down? Suppose that you stuck some pipes into the holes so that the water had to go out a distance before leaving the system. The water would leave at a greater distance from the side of the bucket and, even if the rate of loss of mass were the same as before, the rate of angular momentum loss would be greatly enhanced. That may give you some qualitative notion of how the magnetic field works in making the sun slow down more quickly. If the sun were to somehow maintain rigid rotation, then the half life of its angular momentum would be about $5 \times 10^{9} \mathrm{yr}$. This agreement with the age of the sun may not be a coincidence.

If the sun is approximately rigid, we can derive the law of its angular velocity. Suppose that the outflow remains nearly rigid out to some distance $R_{A}$. This distance has to do with the strength of the magnetic field that is pulled out with the flow and is estimated to be about $100 R_{\odot}$. Then

$$
\mathcal{M} R_{\odot}^{2} \frac{d V}{d t}=\dot{\mathcal{M}} R_{A}^{2} V
$$

where $V=R_{\odot} \Omega$. Though $\dot{M} \sim-10^{-13} \mathcal{M}_{\odot} /$ yr today, we do not know whether it is 
constant. It is almost surely affected by the magnetic field strength. The magnetic field is likely to be produced by dynamo action that is dependant on rotation. So other things being equal, we assume that

$$
\frac{\dot{\mathcal{M}} R_{A}^{2}}{\mathcal{M} R^{2}}=-\alpha\left(\frac{V}{V_{o}}\right)^{n}
$$

where $\alpha$ has dimensions of $[\text { time }]^{-1}$. This gives

$$
V^{n}=\frac{V_{0}^{n}}{1+\alpha n t}
$$

This law of angular velocity has a couple of free parameters, but for large $t$, we see that it suggests that the half life of stellar angular velocity is $n / t$. This explains the agreement we have already noted for the solar case if $n \approx 1$.

Of course, the sun is not rigid. So we have a classical sort of spin down problem with the surface layers being slowed down, including the highly turbulent convection zone. The remaining question is whether the momentum can be extracted from the interior layers at the required rate. This stratified spin down problem with its attendant stability problem has not been solved. Not even the formulation is generally agreed upon. But now, with new solar soundings, we have measurements of zonal flows, so theories of the solar circulation now have some reasonably hard facts to confront. The main issue, one that still is not decided, is whether the whole sun has spun down to the surface rotation rate. This question was the center of controversy twenty years ago because a rapidly rotating core could result in a small quadrapole moment to the sun's mass distribution. This would modify the sun's gravitational field and have a small effect on the precession of the perihelion of Mercury. Thus the rotation rate of the sun's core might be relevant to tests of the theory of general relativity. This is not a lively possibility today, but there are other interesting questions that are affected by the possibility of fast rotation of the solar core (a possiblity still defended at Yale), such as the prospect of dynamo action.

\section{Solar Cycle}

In 1843 Schwabe suggested that the sunspot number varied with a period of about ten years. The sunspot number as officially defined is a measure of the coverage of the sun by relatively dark areas associated with strong magnetic fields. The solar magnetic variation is complicated in its details, but the main feature is that the total varies on a time scale of eleven years in a way that looks rather chaotic. If this is a chaotic process, it is also spatio-temporal. Active regions of spottedness first appear at solar latitudes $\pm 37^{\circ}$ and these peaks of activity drift equatorward.

The latitudinal bands of activity are dominated by large spot groups typically about $10^{5} \mathrm{~km}$ apart. The groups are mainly pairs of spots with opposite magnetic polarities as if they were simply the two feet of an enormous magnetic arch. Throughout each band, the leading spots will tend to have the same magnetic polarity, 'so we can imagine that each band is the trace of a huge magnetic serpent girding the sun. The polarity of the 


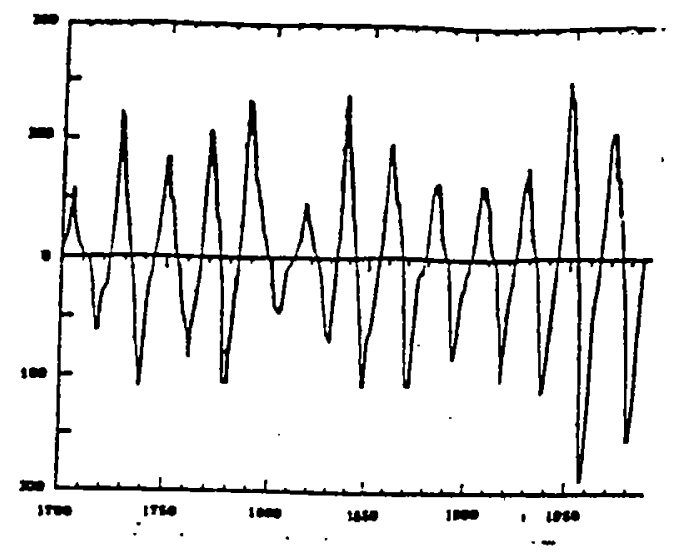

Figure 2: The annual mean sunspot number in the Bracewell style

leading spots is different in the northern and southern bands. About seven years after these first appear, at about the same time in the north and south, and they have drifted about a third of the way to the equator, the bands from the previous cycle arrive at the equator and apparently disappear. The bands at midlatitude continue their equatorward drift for another four years or so, when their successors appear at $\pm 37^{\circ}$. Again the new wave continues equatorward, and so forth, and the new bands have the opposite polarity, with respect to leading and following spots, from their predecessors. The cycle could be therefore said to have a twenty-two year time scale. On the other hand the waves of activity take eighteen years to go from the latitude of first appearance to the equator. If (as Proctor and I suspect) there are only four solitary waves, which are virtually indetectable when they move away from the equator, the cycle consists of simple round trips in which a wave of solar activity takes eighteen years to go to the equator and four to return.

There are a number of questions that this zeroth order characterization raises about the solar activity. waves. What is their nature? What happens to them when they reach the equator? What generates them? What determines their characteristics, speed, width, amplitude? These are questions about dynamo theory and they are at the heart of solar research. In this introductory spirit, I shall not pursue further details, but turn to the lumped case, which is the study of the temporal variation of the total sunspot number, leaving the spatial behavior out of account.

In figure 2 we show the annual mean sunspot number since 1700 in a representation designed to allow for the magnetic cycle, where the negative of the number of spots is shown on every other cycle, after a suggestion of Bracewell. If we use information from before 1700, as Eddy has done, we get another view, that of figure 3.

We see that during the time of Newton there were virtually no spots. A confirmation of this result of historical scholarship comes from the study of the abundance of $C^{14}$ as a function of time using tree rings and cores from the poles. The idea is that, in times of strong solar activity, the solar wind pulls out magnetic field with it. This reaches out to the earth and shields it from cosmic rays, which would otherwise make $C^{14}$. So the periods of high $C^{14}$ abundance are times of low solar activity. At any rate, the data, so interpreted, confirm the Maunder minimum, or Newton intermission. More than this, they suggest that such intermissions are recurrent, that is, that solar activity is intermittent.

Naturally, there are some attempts to model such things, though the number of care- 


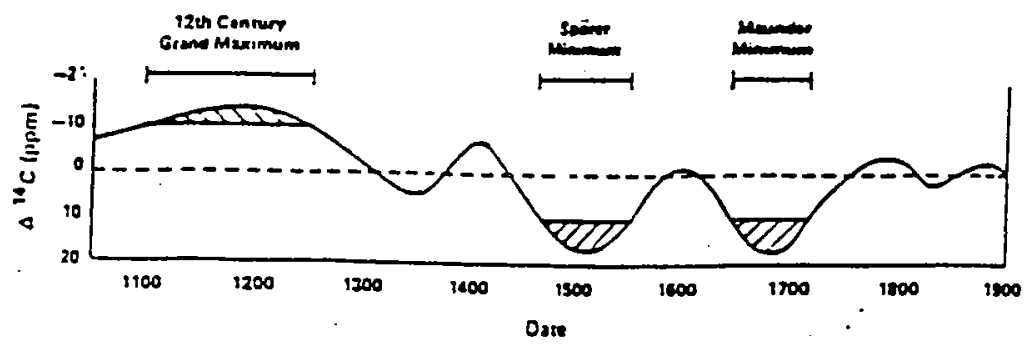

Figure 3: Variation of the concentration of ${ }^{14} C$ after Eddy.

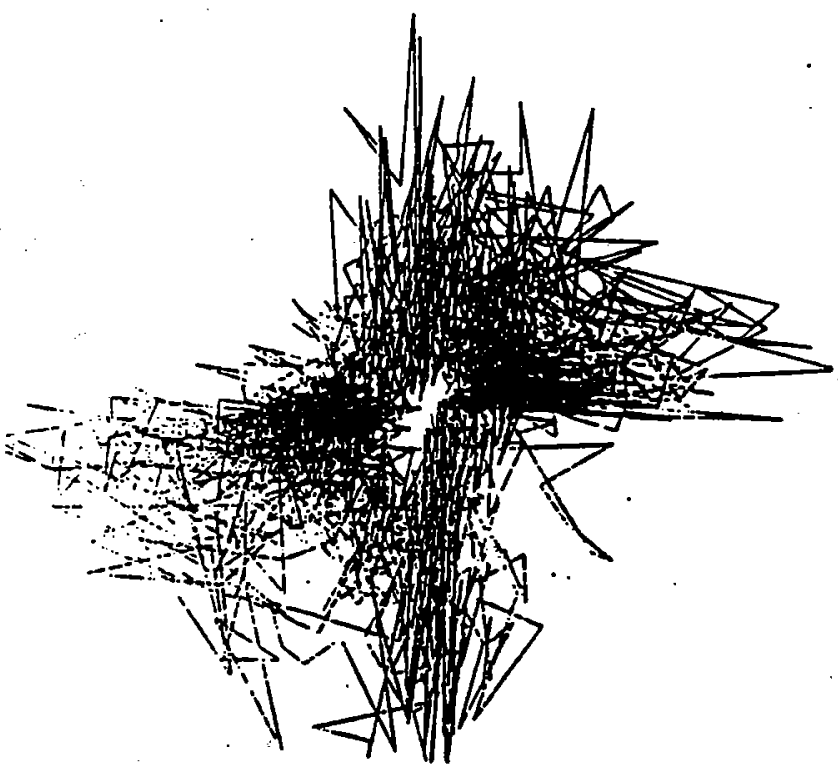

Figure 4: Projection of the three-d phase portrait of the solar cycle

fully observed cycles seems a bit too small for the purpose. The most direct way to proceed is to attempt to construct a phase portrait of the solar cycle. This can be done from $N\left(t_{i}\right)$, the number of spots as a function of time. Using a tabulation of these data, kindly provided by J.A. Eddy, A.N. Wolf and I used the reconstruction trick proposed by Ruelle and others. This is done by taking the column of $N_{i}$ and making making a copy of it displaced by about three years. A third copy displaced by the same amount then gave us a listing of triplets, loosely analogous to $N, \dot{N}, \bar{N}$, which could be plotted as a trajectory in a three-dimensional phase space. Such a plot, projected onto a plane, looks like nothing more than a scatter diagram. But put it into a computer and rotate it around (in three space) till it looks like something, and you get figure 4. There does seem to be some structure; indeed too much to model. The daily variations in these data are too rich. The idea is to try to model a simpler version, by smoothing the data. Indeed, you can smooth the thing till it looks like a limit cycle. But, with somewhat less smoothing, say on a time scale of a. year and half, using the Bracewell trick, you get something that has the general shape of a Lorenz attractor as shown. This does not have any intermittency of the solar kind. . 
The simplest reasonable kind of model seems to be one with about dimension five, such as Childress used to model dynamos some years ago. But here is one that has the bowtie look of a Lorenz attractor and gives the right kind of intermittency:

$$
\begin{aligned}
& \ddot{x}=-x^{3}-2 x y+\lambda x-\epsilon \nu \dot{x} \\
& \dot{y}=-y^{3}-x^{2}+\lambda y-\epsilon \nu \dot{y} \\
& \dot{\lambda}=-\epsilon\left[\lambda+a\left(x^{2}+y^{2}-1\right)\right]
\end{aligned}
$$

The idea of this model for the solar cycle goes back to discussions with D.W. Moore. We thought that there could be two dynamo processes in the sun. One is a general solar dynamo going on continually, with a second, subconvective dynamo generating the solar activity, but strongly coupled to the convective zone. This model is a loose representation of that picture. For vanishing $x$ and $\dot{x}$ the system is just the well-known Lorenz system (transformed to the Walsh Cottage version). The reason for that was Malkus' suggestion that a dynamo could nicely be modeled that way (see also Rurmaikin's discusion of solar dynamoes). The other two degrees of freedom are meant to represent a subconvective oscillation. At any rate, we get from this primitive idea some strong intermittency and some healthy chaotic oscillations (see the report by Platt, in this volume).

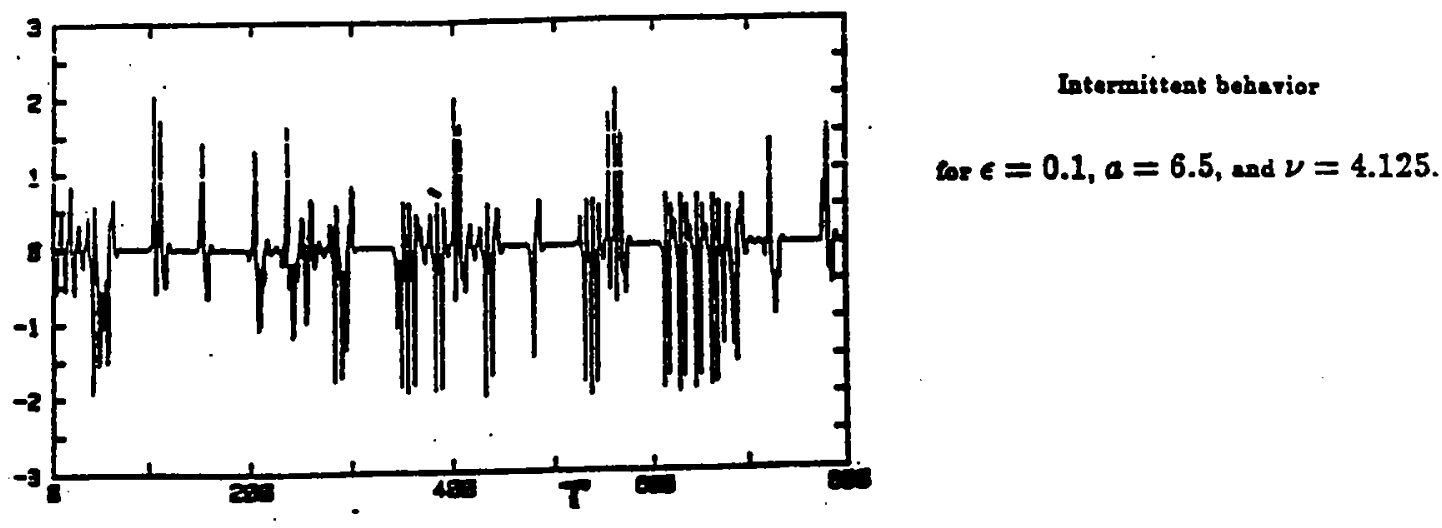

\section{References:}

R.Kippenhahn and A.Weigert, Stellar Structure and Evolution. Springer-Verlag, Berlin 1989.

M.Stix, The Sun-An Introduction, Springer-Verlag, Berlin 1989.

G.Newkirk Jr. and K.Frazer, "The Solar Cycle”, Phys.Today, 35, Vol. 4, pp.25-33 (1982).

A. Ruzmaikin, Comm. Astrophys. and Space Sci.. 
A well-smoothed (v 1 year)
solar attractor.

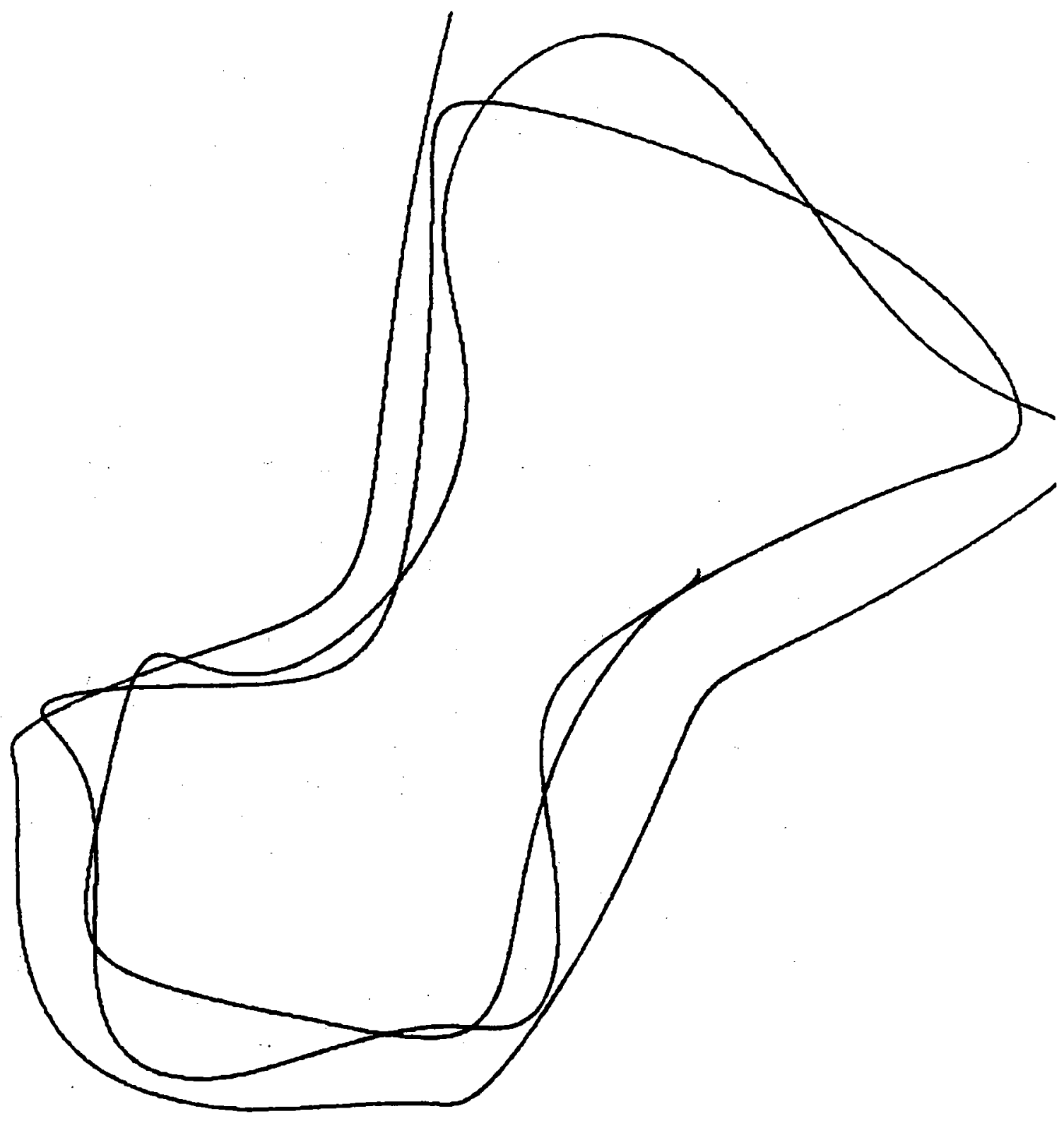

51 


\section{Lecture V. Rotating Stars}

\section{Observations}

The sun forms the best known example of a rotating star. Galileo was the first to observe the drift of sunspots on the solar surface in the 17 th century. He noticed that a spot took about 14 days to cross the solar disc, and that this was roughly the same whether the spot passed through the center of the disc or along a shorter path at some distance from the equator. The rate of rotation also seemed non-uniform with motion appearing to slow as the spot approached the solar limb. Galileo recognised this as an effect of foreshortening which would result if and only the spots were near the solar surface.

Scheiner, a Jesuit priest, observed that the sun took 27 days to complete a rotation. He discovered the differential rotation of the sun by more accurately mapping the passage of sunspots at different latitudes. Spots farther from the solar equator were found to move with a slower velocity. It was also known that the sun was tilted by $\approx 5 \%$ to the earth's axis. In 1643-48, Hevelius managed to map the surfaces of the moon and the sun by engraving their projected images on a tinplate. Such were the accuracy of these mappings that modern techniques can be used to analyse the differential rotation. The results differ from present day readings.

For other stars, however, more sophisticated techniques are required. The most commonly used is the analysis of spectral lines. For a non-rotating star, the presence of certain elements in the stellar atmosphere will produce dark absorption lines in the stellar emission spectrum.

If the star is rotating however and radiation is sampled from both receding and approaching sides of the stellar disc, then the absorption line will be both red and blue shifted. As a result the absorption line will broaden and flatten.

The width of the band is proportional to $v \sin i$ where $i=$ inclination of the stellar axis to the line of vision and $v=$ equatorial velocity. Unfortunately $i$ is unknown. If the stellar surface is uniformly illuminated the absorption line would have an elliptical shape. However the flattening at the bottom indicates the darkening at the stellar limb. 

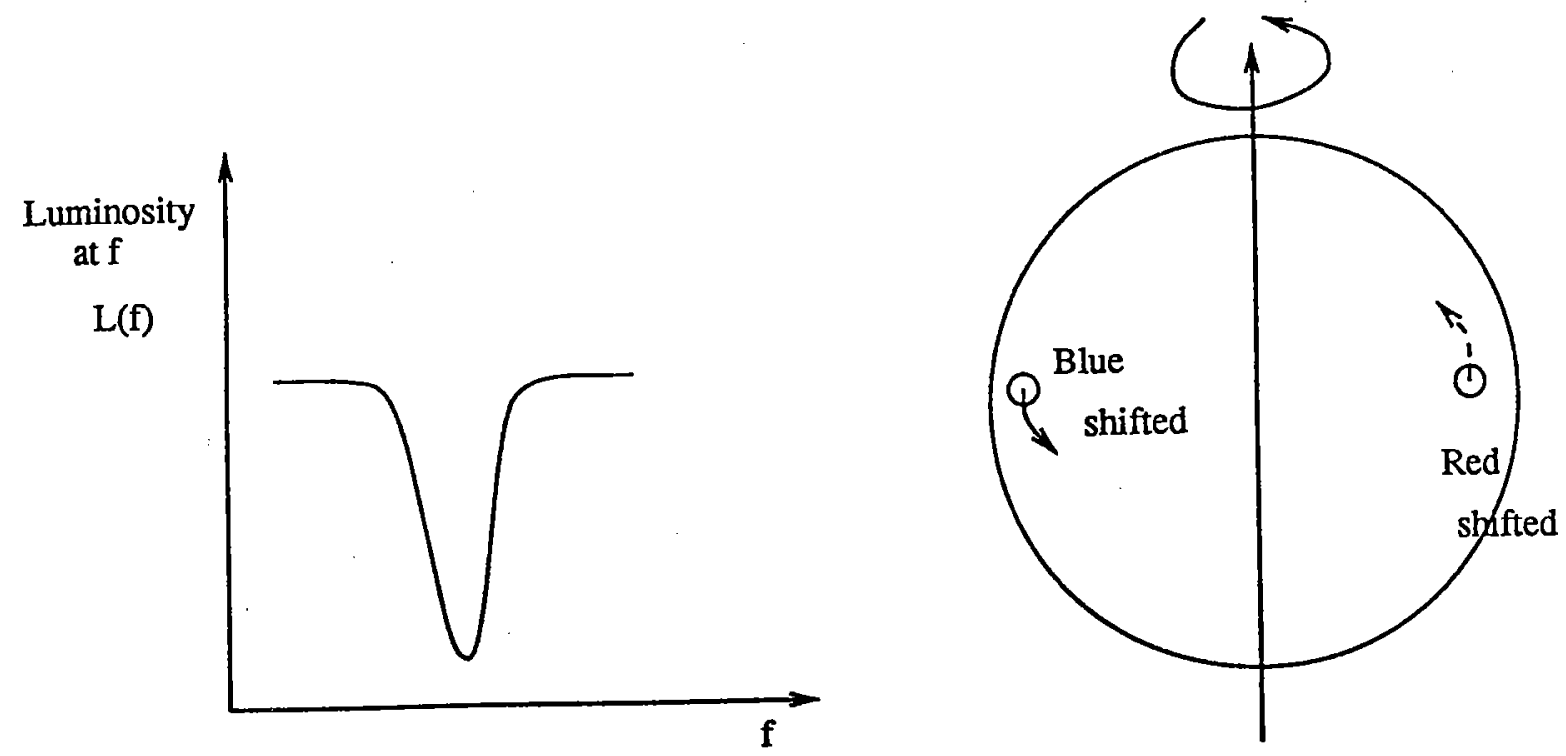

Figure 1: an absorption dip
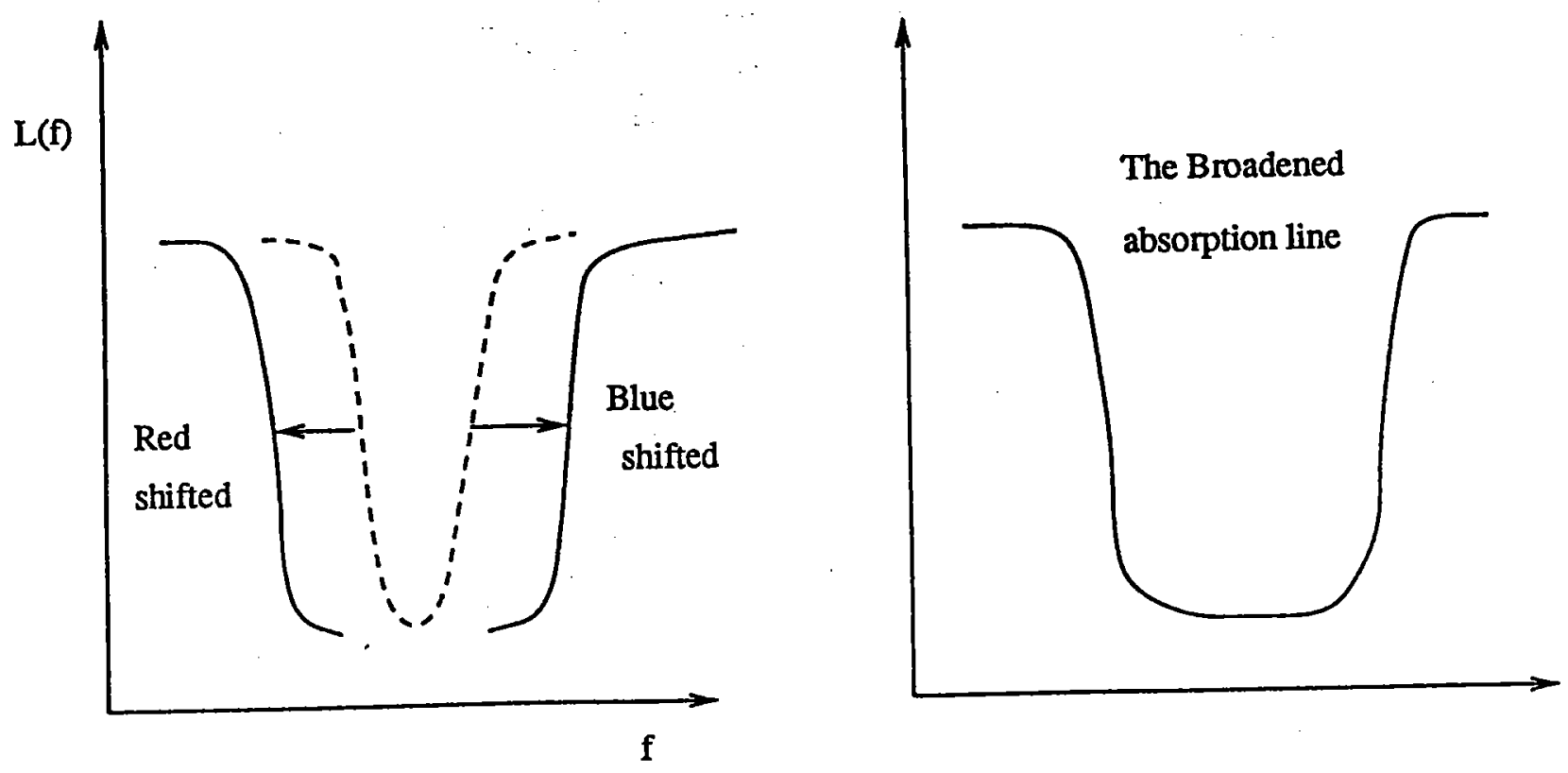

Figure 2: doppler broadening 


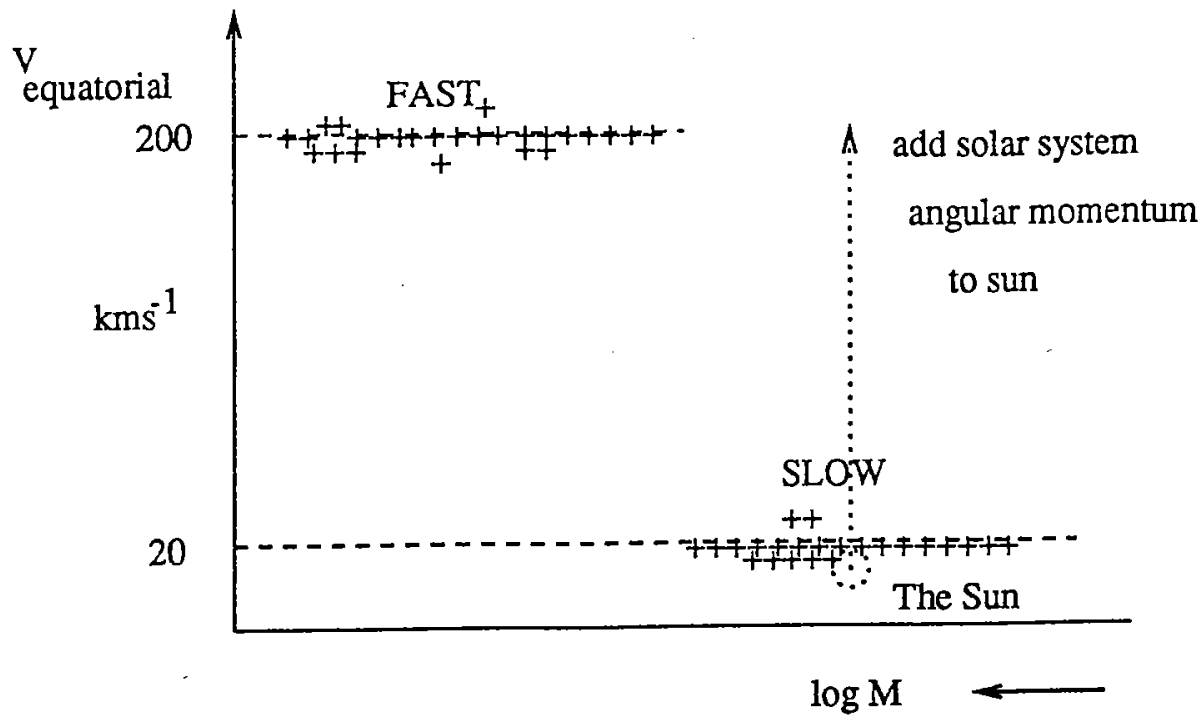

Figure 3: Rotational velocities vs $\log M$

There are ways to obtain more information about the stellar rotation if the surface exhibits non-uniformities. For example tracking a patch of different chemical composition (or other irregularities that appear periodically) across the surface can yield more specific information about the rotation speeds at particular latitudes. However this approach is not without hazards. Care must be taken to ensure that variations are not a result of luminosity changes or of stellar pulsations. Sometimes frequency splitting due to the Coriolis force may be seen, much like the zeeman effect.

Equatorial velocities tend to have two orders of magnitude; either fast at $200 \mathrm{kms}^{-1}$ or slow below $20 \mathrm{kms}^{-1}$ (e.g. the sun corresponds to $2 \mathrm{kms}^{-1}$ ). Massive stars all are fast rotators whereas low mass stars are slow rotators.

It appears all star loose most of their angular velocity as they age via combination of magnetic braking and direct mass loss. For our sun, it was suggested earlier that the process had been predominantly mass loss in the form of orbiting planets. The solar angular momentum consists of $\frac{1}{100}$ th of the total solar system value. If the sun was to retrieve the total angular momentum of the solar system it would be a fast rotator. But observations of stellar clusters do not support this explanation. 


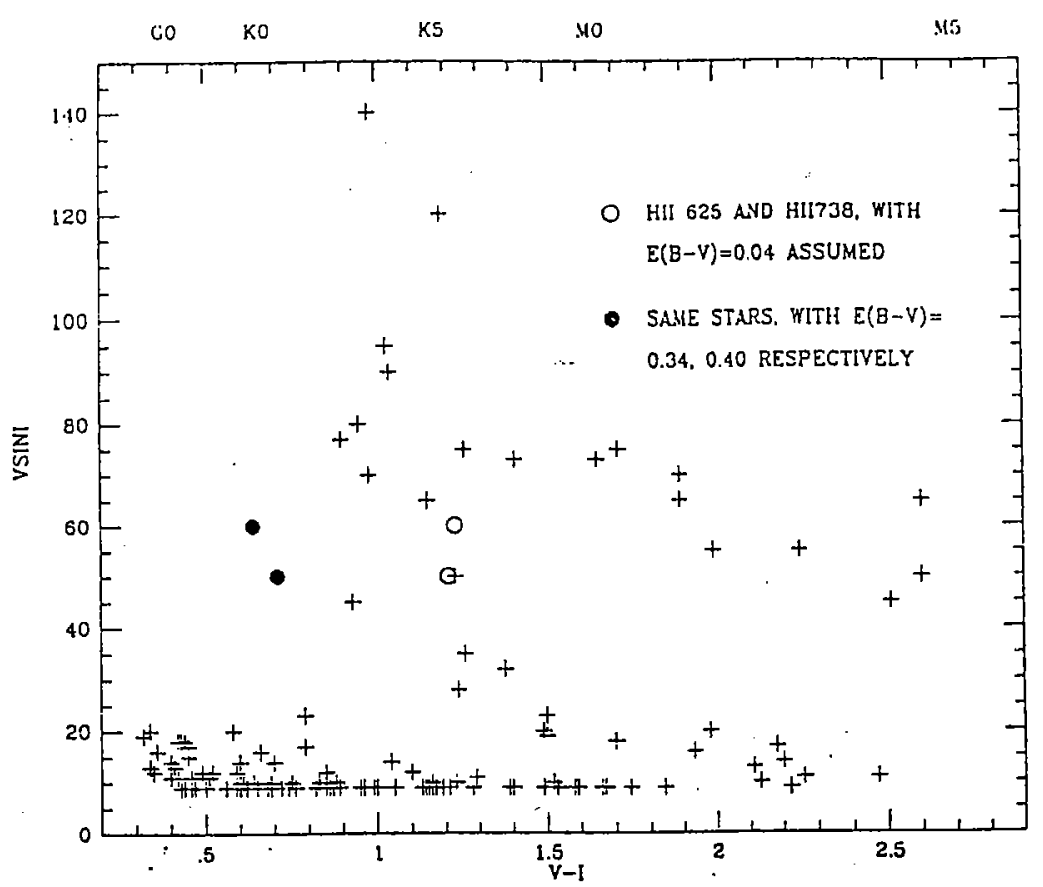

Fia. 6.-Distribution of spectroscopic rotational velocities as a function of reddening-eorrected $r-1$ color for the Pleiades. HII 62S and HII 738 are show iwice, sorresponding to the two aiternative reddening corrections.

Figure 4: average age 10E8 years

A star cluster allows a large number $\left(10^{5} / 10^{6}\right)$ of stars, of similar ages, to be studied. A plot of $v \sin i$ versus an indicator of mass yields fig. 4 for a cluster of average age $10^{8}$ years.

Notice the high degree of scatter although there are the beginnings of accumulation at $v \sin i \approx 20 \mathrm{kms}^{-1}$. An older cluster, with average age $\approx$ $5 \times 10^{8}$ years exhibits considerable slow down (note the change of vertical scale).

It seems that the "slow down" time scale is $\approx 500 \times 10^{6}$ years. Nothing can be said about the direction of rotation. The formation of stars is thought to be such a turbulent process that any large scale galactic vorticity is lost and hence no uniform star rotation direction should be expected. Calcium emission lines can be used to infer direction however this requires much effort. The orientation of the stellar axis to the line of sight seems such that $\sin i$ is randomly distributed.

Stars are found to reach a state where their rotational velocity is dependent only on their mass, regardless of initial conditions. A star typically loses most of its angular momentum in its pre-main sequence period.

The above Hertzsprung-Russell diagram demonstrates how well stellar 


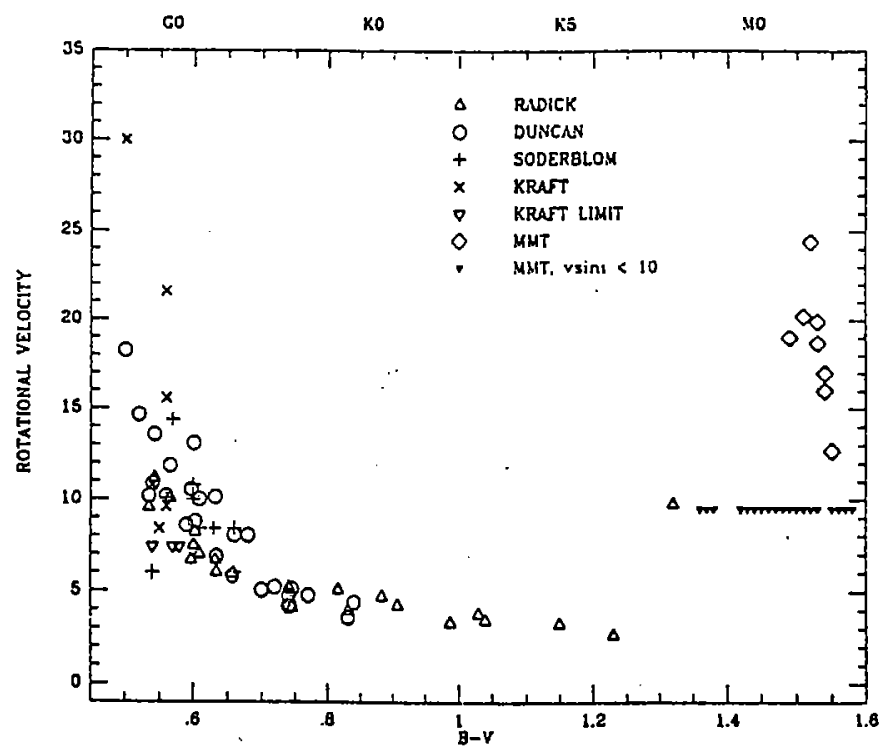

FIG. 2.-Distribution of rotational velocities in the Hyades as a function of $\boldsymbol{B}-\boldsymbol{V}$ color (or spectral type). Spectroscopic rotational velucities have been corrected for inclination effects assuming a random distribution of 2xial orientations. Stars with rotational velocity limits of $u$ sin $i<10 \mathrm{~km} s$ bluer than $B-V=1.3$ have been omitted for clarily.

Figure 5: average age 5x10E8 years

evolution can be predicted purely from the mass. In particular the final or long time ( $10^{9}$ years) value for the equatorial velocity is independent of the initial angular momentum. The main process thought to produce this effect is the braking by magnetic stellar winds. Kawaler in "Angular Momentum loss in Low-Mass stars" astrophysical journal vol 333 p236-247 (1988) produces a simple expression of angular momentum loss by this process, and applies it to evolutionary stellar models. The basic assumption in magnetic braking is that escaping matter does not possess the angular momentum corresponding to the stellar surface but to a distance, called the Alfven radius $r_{A}$, far above the surface. In a simplified view, the magnetic lines of force act as a lever arm, which out to $r_{A}$, forces the escaping material to rotate rigidly with the star. Beyond $r_{A}$ the field becomes too weak to enforce rigid rotation and the angular momentum of the escaping matter is conserved. The loss of angular momentum $\mathrm{dJ}$ corresponding to a spherical shell of mass $\mathrm{dM}$ crossing $r_{A}$ during the time interval $d t$ is

$$
\frac{d J}{d t}=\frac{2}{3} \frac{d M}{d t} R^{2} \Omega\left[\left(\frac{r_{A}}{R}\right)_{\text {radial }}\right]^{2}
$$




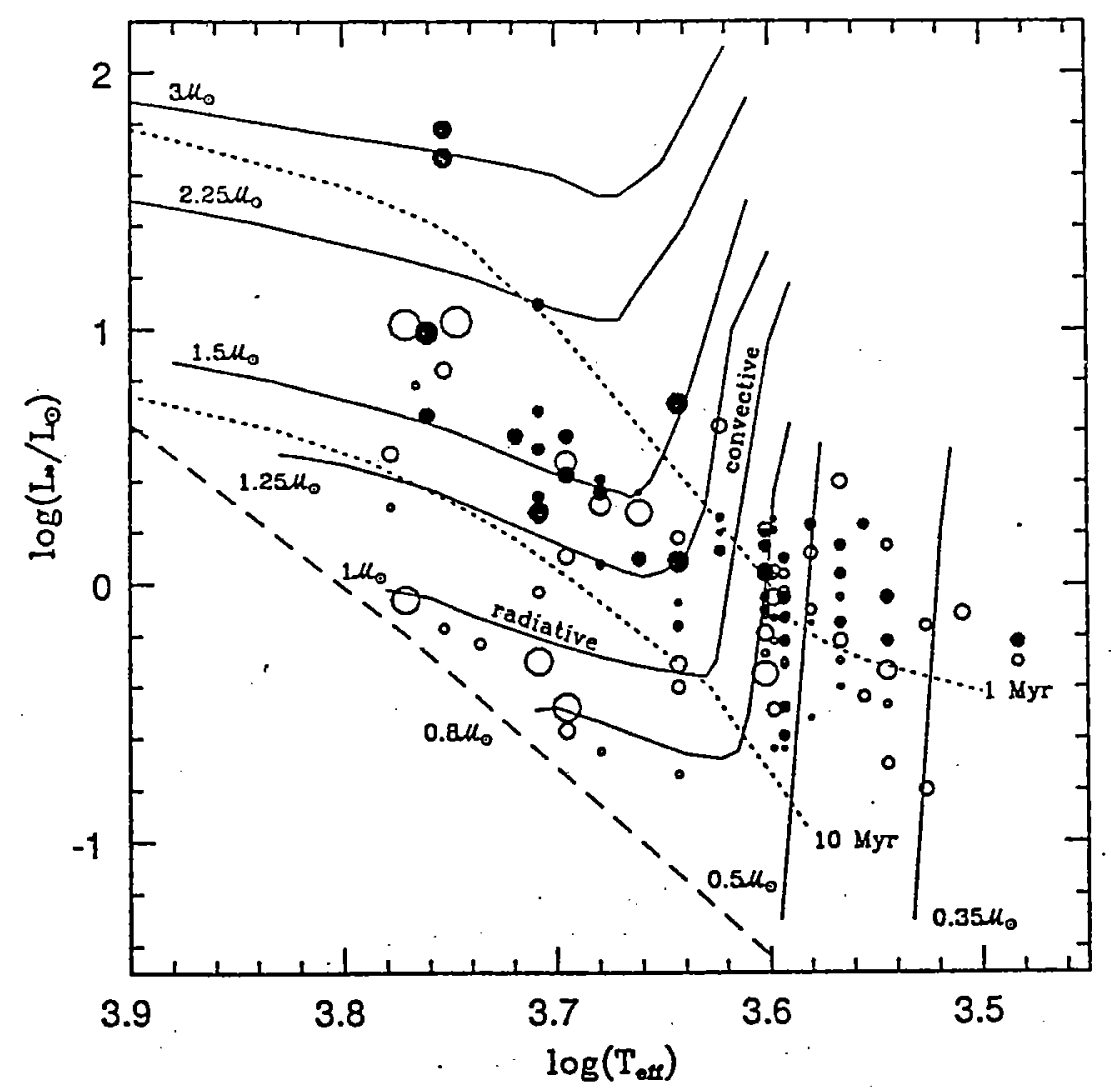

Figure 1. Rotational velocities of low-mass pre-main sequence stars in the H-R diagram. Öpen and dark circles represent $T$ Tauri stars with $\mathrm{H}_{\alpha}^{\prime}$ equivalent widths smaller and greater than $10 \AA$, respectively, and the circle area is proportional to usini in the range from less than 10 to about $100 \mathrm{~km}$

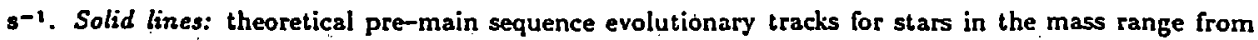
0.35 to $3 \mathrm{M}_{\odot}$ (from Cohen and Kuhi 1979). Dotted lines: isochrones corresponding to an age of $10^{5}$ and $10^{7}$ years, respectively. Dashed line: theoretical zero-age main sequence.

Figure 6: Hertzsprung-Russell diagram 
for a radial field geometry and

$$
\frac{d J}{d t}=\frac{2}{3} \frac{d M}{d t} R^{2} \Omega\left[\left(\frac{r_{A}}{R}\right)_{d i p o l e}\right]
$$

for a dipole field geometry. Here $\mathrm{R}$ is the stellar radius and $r_{A}$ is the Alfven radius to which corotation of stellar wind is maintained. The field strength determines the value $\frac{r_{A}}{R}$ while the field geometry at $r_{A}$ governs the power of $\frac{r_{A}}{R}$ in the equation. Kawaler uses:-

$$
\frac{d J}{d t}=\frac{2}{3} \frac{d M}{d t} R^{2} \Omega\left[\left(\frac{r_{A}}{R}\right)_{\text {radial }}\right]^{n}
$$

where $n=2$ is radial field geometry and $n=3 / 7$ for a dipole field. He also assumes the simplification that the total field strength is proportional to the rotation rate to some power.

$$
B_{0}=k_{B}\left(\frac{R}{R_{0}}\right)^{-2} \Omega^{a}
$$

Combining

$$
\frac{d J}{d t}=-k_{w} \Omega^{1+4 a n / 3}\left(\frac{R}{R_{o}}\right)^{2-n}\left(\dot{m}_{14}\right)^{1-2 n / 3}\left(\frac{m}{m_{o}}\right)^{-n / 3}
$$

Here $\dot{m}_{14}=\frac{d M}{d t}$ in units of $-10^{-14} M_{o} y r^{-1}$, and $k_{w}, k_{v}, k_{B}, n \& a$ are free parameters allowing fitting of the model. He uses $a=1 \&$ allows $n$ to vary. To relate $\mathrm{J}$ to $v_{\text {eq }}$ he uses 2 cases A \& B. In case A the star is assumed to be uniformly rotating i.e. the angular momentum is evenly distributed, whereas in case $\mathrm{B}$ a convective layer is assumed steadily rotating and below this each spherical shell retains its own angular momentum. The asymptotic result $v_{e q} \sim t^{-3 a n / 4}$ is achieved for long time. His results are as follows:-

The most interesting plot is, however, below.This clearly shows the independence of the final equatorial velocity on the initial angular momentum for both cases.

Kawaler shows that the simple formulation of angular momentum loss by the magnetic winds, when applied to evolving pre main-sequence and mainsequence models, is adequate qualitatively to explore the spin down of low mass stars. The rotation velocity, at times $\approx 10^{9}$ years, is independent of the initial J. 


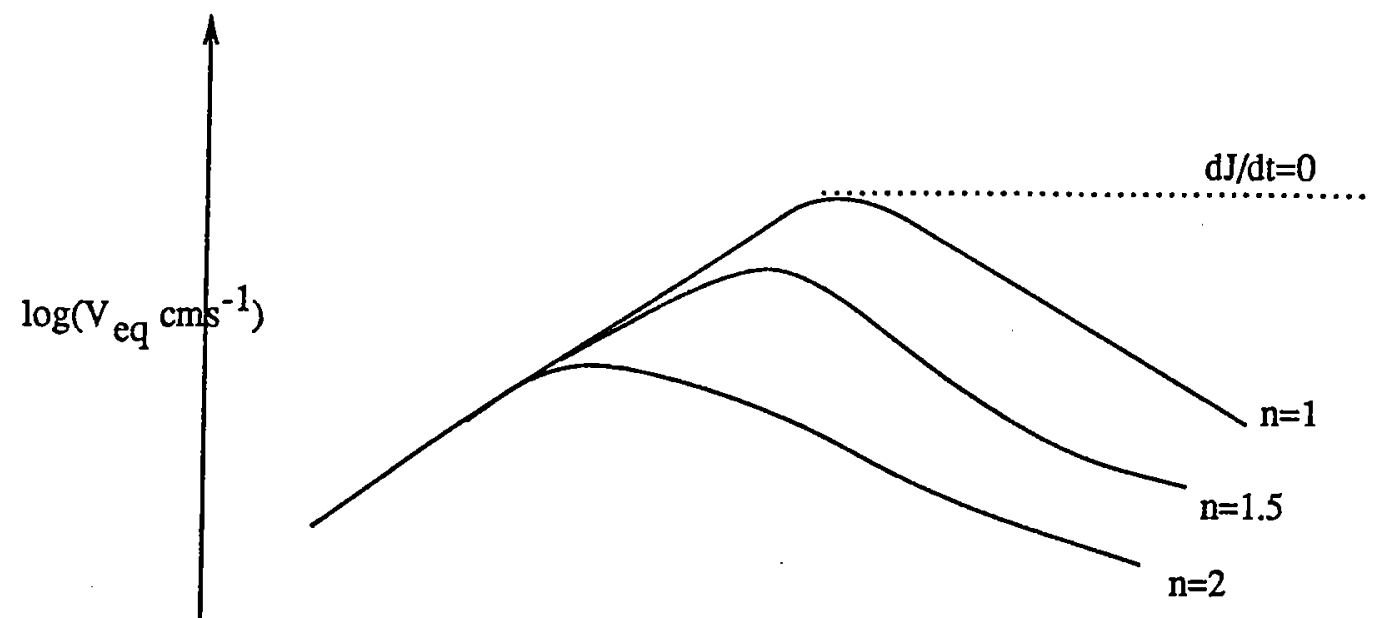

CASE A

1.0 solar mass

$\log t$

Figure 7: case A

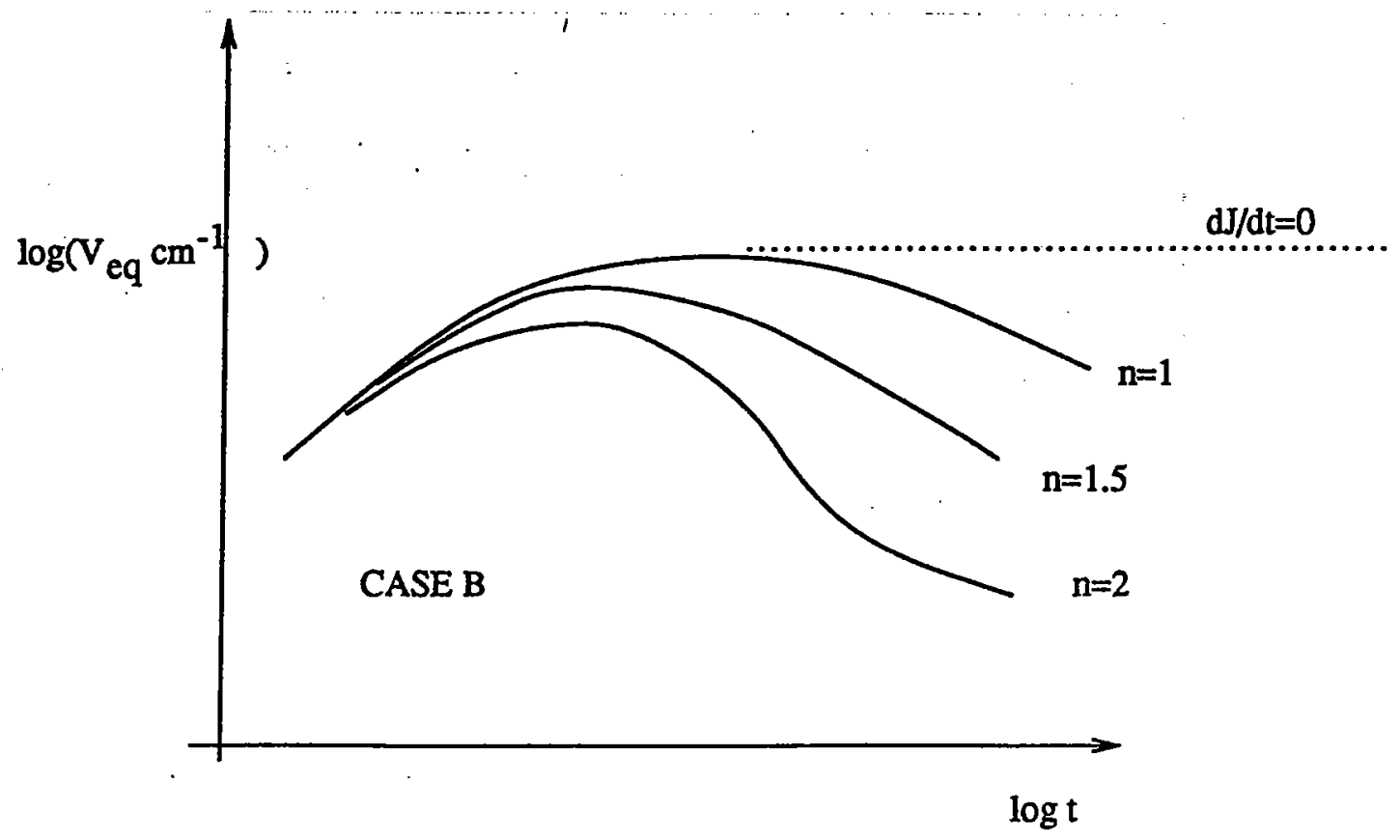

Figure 8: case B 


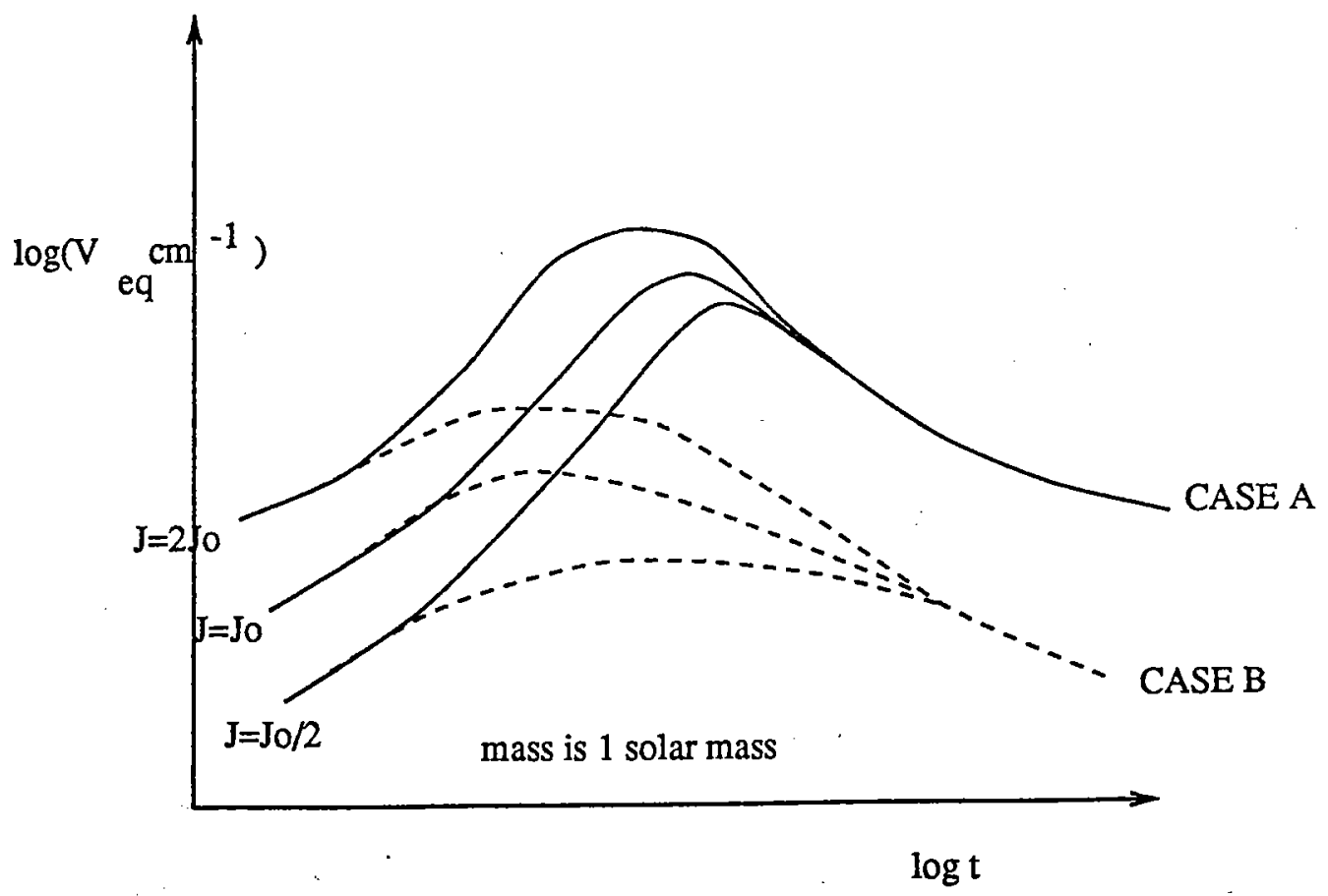

Figure 9: different angular momentum initial conditions

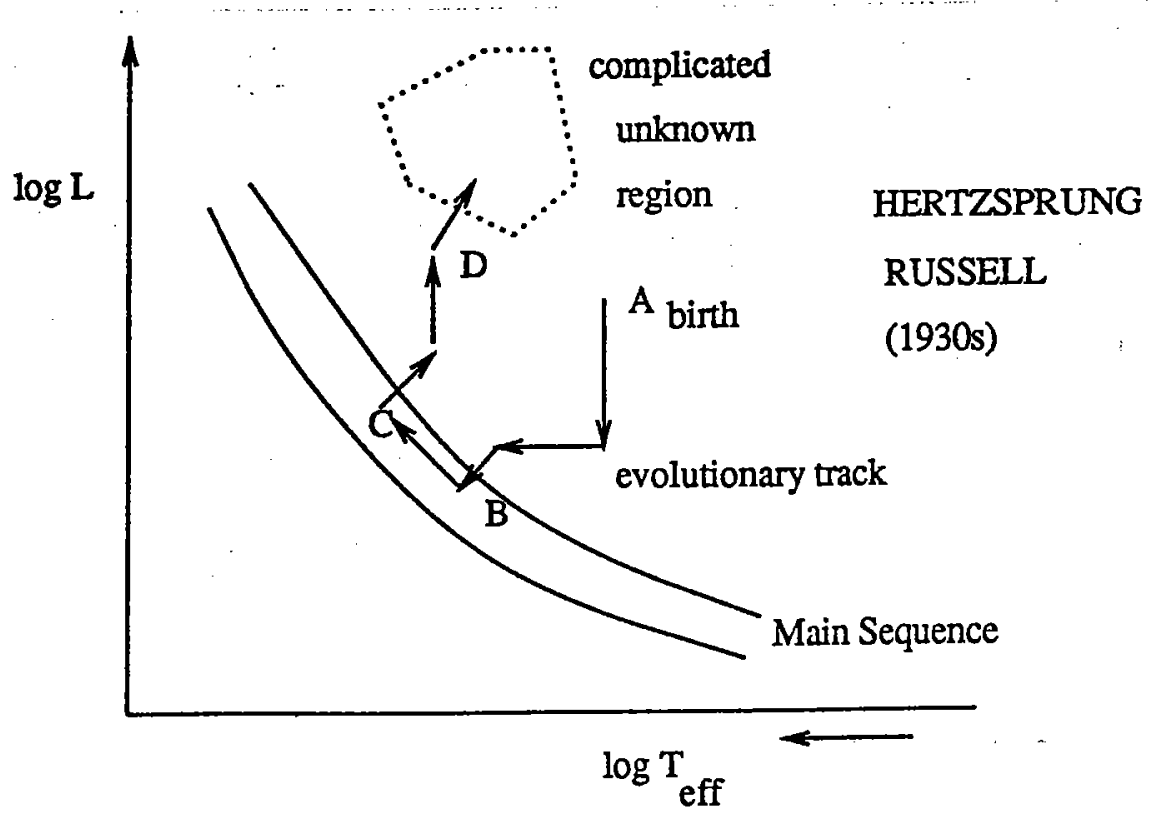

Figure 10: Evolutionary track 
In summary the evolution of a star appears to be adequately characterised by only the mass for most of its lifetime (ABCD). One would expect dependence on the angular velocity $\Omega$. However as illustrated above, after the star has lost most of its angular momentum (at the beginning of its lifetime on the main-sequence) $\Omega$ settles to a value which only depends on the mass $M$ and not on the initial conditions. Thus if evolution depends on $M \& \Omega$ then $E_{\text {evolution }}=E(M, \Omega)=E(M, \Omega(M))=\hat{E}(M)$. Eventually the star leaves the main-sequence having burnt most of its $\mathrm{H}_{2}$ to $\mathrm{He}$. The resulting loss of homogeneity produces a subsequent evolution too rich to be described simply by the mass.

Low mass stars consist of a radiative interior, which is unmixed and where nuclear burning takes place, and a surrounding mixed convective zone. Attempts to model the radiative interior as a 1-D problem ( depth as the independent variable ) have proved inadequate. It appears that differential rotation, which is intimately coupled to the magnetic field, is a crucial process in the dynamics and must be included in any description. 


\subsection{General Properties and The Virial Theorem}

In an inviscid, self-gravitating fluid with no associated magnetic field, the equation of motion is

$$
\rho \frac{D}{D t} \mathbf{v}=-\nabla p-\rho \nabla \phi
$$

where $\phi$ is the gravitational potential, that is

$$
\mathbf{g}=-\nabla \phi
$$

We define cartesian and cylindrical frames of reference in the following way

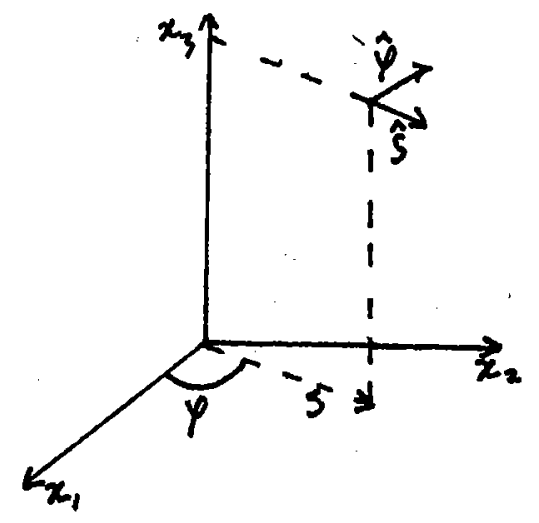

fig. 2.1: Cartesian and Cylindrical Coordinate Systems.

i.e. the $x_{i}$ are the cartesian coordinates, and $s, x$, and $\varphi$ are the cylindrical polar coordinates with unit vectors as shown. We can also define

$$
\mathrm{x}=\left(x_{1}, x_{2}, x_{3}\right)^{T} .
$$

In terms of cartesian coordinates, (1) is

$$
\rho \frac{D}{D t} v_{i}=-\frac{\partial}{\partial x_{j}} p-\rho \frac{\partial}{\partial x_{j}} \phi .
$$

We multiply this equation by $x_{i}$ and integrate over the volume of interest, e.g. the whole volume of the star. Considering the right hand side of (1a), we see

$$
\int_{V} \rho x_{i} \frac{D}{D t} v_{j} d V=\int_{V} \frac{D}{D t}\left(\rho x_{i} v_{j} d V\right)-\int_{V} \rho \frac{D}{D t} x_{i} v_{j} d V-\int_{V} x_{i} v_{j} \frac{D}{D t}(\rho d V)
$$

where

$$
d V \equiv d x_{1} d x_{2} d x_{3}
$$

By definition,

$$
\frac{D}{D t} x_{i}=v_{i}
$$


and, by conservation of mass, since the mass of a parcel of fluid cannot change as it moves with the fluid,

Thus

$$
\frac{D}{D t}(\rho d V)=0
$$

where $K_{i k}$ is defined as

$$
\begin{aligned}
\int_{V} \rho x_{i} \frac{D}{D t} v_{j} d V & =\int_{V} \frac{D}{D t}\left(\rho x_{i} v_{j} d V\right)-\int_{V} v_{i} v_{j} \rho d V \\
& =\frac{d}{d t} \int_{V} x_{i} v_{j} \rho d V-2 K_{i k}
\end{aligned}
$$

$$
1 / 2 \int_{V} v_{i} v_{j} \rho d V
$$

(Note that $K \equiv K_{i i}$ is the kinetic energy of the system).

Now, if the volume of integration $V$ contains all the ,mass of the system, which we assume is the case if the volume contains the entire mass of the star, then

$$
\phi \equiv-G \int_{V} \frac{\rho\left(\mathbf{x}^{\prime}, t\right)}{\left|\mathbf{x}-\mathbf{x}^{\prime}\right|} d V^{\prime}
$$

where $G$ is the gravitational constant, and $d V^{\prime}$ is analogous to $d V$ above. Thus,

$$
\int_{V} \rho x_{i} \frac{\partial}{\partial x_{j}} \phi d V=G \int_{V} \int_{V} \frac{\rho(\mathbf{x}, t) \rho\left(\mathbf{x}^{\prime}, t\right) x_{i}\left(x_{j}-x_{j}^{\prime}\right)}{\left|\mathbf{x}-\mathbf{x}^{\prime}\right|} d V d V^{\prime}
$$

Now, if we define

$$
W_{i j} \equiv-1 \text { over } 2 G \int_{V} \int_{V} \frac{\rho(\mathbf{x}, t) \rho\left(\mathbf{x}^{\prime}, t\right) x_{i}\left(x_{j}-x_{j}^{\prime}\right)\left(x_{i}-x_{i}^{\prime}\right)}{\left|\mathbf{x}-\mathbf{x}^{\prime}\right|^{3}} d V d V^{\prime}
$$

then

$$
\int_{V} \rho x_{i} \frac{\partial}{\partial x_{j}} \phi d V=-W_{i j} \text { and } W \equiv W_{i i}=1 / 2 \int_{V} \rho \phi d V,
$$

$W$ being the gravitational potential energy.

Finally, the last term we need to consider is

$$
\begin{aligned}
\int_{V} x_{i} \frac{\partial}{\partial x_{j}} p d V & =-\int_{V} \frac{\partial}{\partial x_{j}}\left(x_{i} p\right) d V-\delta_{i j} \int_{V} p d V \\
& =-\int_{S} x_{i} p n_{j} d S+\delta_{i j} \int_{V} p d V
\end{aligned}
$$

by Gauss' Divergence theorem, where $\mathbf{n}$ is the unit normal to the boundary $S$ of the volume $V$. But by definition $p$ is zero there. Therefore, collating all the terms of the integration of (1a), we see that

$$
\frac{d}{d t} \int_{V} x_{i} v_{j} \rho d V=2 K_{i j}+W_{i j}+\delta_{i j} \int_{V} p d V
$$


where all the tensors on the right hand side are symmetric over their indices. Thus, if we multiply across by the alternating tensor $\epsilon_{i j k}$, we see that the right hand side of (2) is zero, and the left hand side, in vector notation, becomes

$$
\frac{d}{d t} \int_{V} \mathbf{x} \times \mathbf{v} \rho d V=0
$$

Physically, this equation states that angular momentum is conserved globally.

In the presence of a magnetic field, there is a Lorentz force, and so (1) becomes

$$
\rho \frac{D}{D t} \mathbf{v}=\nabla p-\rho \nabla \phi+\mathbf{j} \times \mathbf{B},
$$

where $\mathbf{j}$ is current and $\mathbf{B}$ is magnetic field. In this case

$$
\frac{d}{d t} \int_{V} \mathbf{x} \times \mathbf{v} \rho d V=\int_{V} \mathbf{x} \times(\mathbf{j} \times \mathbf{B}) \rho d V,
$$

i.e. in general there may be a nonzero torque, depending on the magnetic field structure. that

Returning to (2), from the symmetry over the indices of the right hand side, we see

$$
\frac{d}{d t} \int_{V} x_{i} v_{j} \rho d V=\frac{d}{d t} \int_{V} x_{j} v_{i} \rho d V
$$

Therefore

$$
\frac{d}{d t} \int_{V} x_{i} \frac{D}{D t} v_{j} \rho d V=\frac{d}{d t} \int_{V} x_{j} \frac{D}{D t} v_{i} \rho d V=\frac{1}{2} \frac{d^{2}}{d t^{2}} \int_{V} x_{i} x_{j} \rho d V .
$$

Thus, if we define the tensor

$$
I_{i j} \equiv \int_{V} x_{i} x_{j} \rho d V
$$

we see that

$$
I_{i i} \equiv I=\int_{V}|\mathbf{x}|^{2} \rho d V,
$$

the moment of inertia. Thus (2) can be written in the form

$$
\frac{1}{2} \frac{d^{2}}{d t^{2}} I_{i j}=2 K_{i j}+W_{i j}+\delta_{i j} \int_{V} p d V,
$$

which is known as the Virial equation.

If we take the trace of (5) we obtain

$$
\frac{1}{2} \frac{d^{2}}{d t^{2}} I=2 K+W+3 \int_{V} p d V,
$$

which expresses the energy balance of the system. We assume that the system of interest is made up of an ideal gas in a steady state. In the stellar context, deviations from the 
ideal gas law are often quite small, and the evolution of inertia is quite small compared to timescales of kinetic energy such as the rotation period. Thus these assumptions are often quite reasonable. For a perfect gas

$$
p / \rho=R T,
$$

where $R$, the gas constant, is defined as $\kappa / \bar{m}$, where $\bar{m}$ is the mean mass of the particles. Also, in terms of specific heat capacities at constant pressure $\left(c_{p}\right)$ and volume $\left(c_{v}\right)$

$$
c_{p}-c_{v}=c_{v}\left(c_{p} / c_{v}-1\right) \equiv c_{v}(\gamma-1)=R \text {. }
$$

Thus (6) may be written as

$$
\frac{1}{2} \frac{d^{2}}{d t^{2}} I=2 K+W+3(\gamma-1) U_{T}
$$

where

$$
U_{T} \equiv \int_{V} c_{v} T \rho d V
$$

is the total thermal energy, which must be a positive quantity. In the case of an approximately steady state, the right hand side of (7) is assumed zero, and so

$$
2 K+W+3(\gamma-1) U_{T}=0 \text {. }
$$

This equation shows the balance of energy in the star system. $W$ is negative, and the other two terms are positive ( $c_{p} \geq c_{v}$ always). If we initially ignore macroscopic kinetic energy, we see that, under contraction, gravitational energy must change into thermal energy. However, in fast rotating stars, $U_{T}$ may be considered small, yet positive. Clearly $K$ can never be greater than $W / 2$. But, under contraction, conservation of angular momentum requires the star system to spin faster. Thus excess kinetic energy must be lost in some way, i.e. radiated. Nevertheless, this is a gross simplification of the true picture. An increase in thermal energy causes an expansion that will vary both $W$ and $K$. So a parameter of interest is the ratio of kinetic energy $K$ to the absolute magnitude of the gravitational potential energy $|W|$.

A rough measure of this for a star of radius $R_{0}$ and rotation rate $\Omega$ is $I \Omega^{2} /|W|$. Now

$$
I \sim 4 \pi \rho \int_{0}^{R_{0}} r^{4} d r=3 M R_{0}^{2} / 5 \quad\left(M=4 / 3 \pi \rho R_{0}^{3}\right)
$$

and

$$
|W| \sim G M / 2 R_{0}
$$

Thus

$$
I \Omega^{2} /|W| \sim \Omega^{2} R_{0}^{3} / G M,
$$

the oblateness of the star. This is usually quite small (in the case of the sun the value is $\sim 2 \times 10^{-5}$ ) but may approach higher values (of about 0.1 ) in fast rotators. If we include a magnetic field,we also see that, in stellar terms, the magnetic energy is small compared to the gravitational energy. 


\subsection{Hydrostatic Equilibrium}

Let us thus investigate the implications of the following assumptions:

i) the star is isolated in space, and rotates about a fixed axis (which, without loss of generality, we assume to be the $z$ axis) with angular velocity $\Omega(s, z)$;

ii) the system is stationary in an inertial frame, i.e. we have purely rotational motion (often referred to as hydrostatic motion);

iii) dissipative forces may be neglected;

iv) no electromagnetic force is acting on the star.

With these assumptions, the equation of motion becomes

$$
0=-\frac{1}{\rho} \nabla p-\nabla \phi+\hat{\mathbf{s}} s \Omega^{2}(s, z) .
$$

If we now take the curl of (9), we eliminate the gravitational term and so

$$
0=-\nabla \frac{1}{\rho} \times \nabla p+\nabla\left[s \Omega^{2}(s, z)\right] \times \hat{\mathbf{s}} .
$$

Thus, if $\Omega$ is purely a function of $s$ the second term in (10) is zero. Thus, for (10) to be satisfied, surfaces of equal pressure (isobars) and equal density (isopycnals) must coincide. This is referred to as a barotropic state. In a barotropic state, since $\Omega$ is purely a function of $s$, we may rewrite (9) as

$$
0=-\frac{1}{\rho} \nabla p-\nabla \phi+\nabla \int_{0}^{s} s^{\prime} \Omega^{2}\left(s^{\prime}, z\right) d s^{\prime},
$$

where

$$
\int_{0}^{s} s^{\prime} \Omega^{2}\left(s^{\prime}, z\right) d s^{\prime}
$$

is referred to as the centrifugal potential. Now we see that we can define a "total" or effective gravitational potential $\Psi$ that takes into account the effect of rotation, where

$$
\Psi \equiv \phi-\int_{0}^{s} s^{\prime} \Omega^{2}\left(s^{\prime}, z\right) d s^{\prime},
$$

and

$$
g_{e} \equiv-\nabla \Psi
$$

is called the effective gravity.

(As a sideline, in the absence of a barotropic assumption, an effective gravity is often defined as

$$
g_{e} \equiv-\nabla \phi+\hat{\mathbf{s}} s \Omega^{2}(s, z) .
$$

In this case, we see from (9) that effective gravity, defined in this way, is always orthogonal to isobars.) 


\subsection{Differential Rotation and Kinetic Energy}

We have already alluded to the effect of the principle of conservation of angular momentum on the evolution of the rotational energy. Let us now consider this more closely in the case of a spherically symmetric rotating star of given mass and radius. If we consider this star in a cylindrical coordinate frame as defined above, the angular momentum $J$ is given by

$$
J=\int_{V} s^{2} \Omega(s, z) \rho d V
$$

If we now perturb an initial constant rotation rate $\Omega_{0}$ to

$$
\Omega(s, z)=\Omega_{0}+\delta \Omega(s, z)
$$

we see that the kinetic energy of the new configuration is given by

$$
K=1 / 2 \int_{V} s^{2} \Omega^{2} \rho d V=1 / 2 \int_{V} s^{2} \Omega_{0}^{2} \rho d V+1 / 2 \int_{V} s^{2} \delta \Omega^{2} \rho d V+\Omega_{0} \int_{V} s^{2} \delta \Omega \rho d V .
$$

But

$$
J=\int_{V} s^{2} \Omega_{0} \rho d V+\int_{V} s^{2} \delta \Omega \rho d V=J_{0}
$$

thus

$$
\int_{V} s^{2} \delta \Omega \rho d V=0
$$

and

$$
K=K_{0}+1 / 2 \int_{V} s^{2} \delta \Omega^{2} \rho d V
$$

Thus any perturbation to uniform angular rotation can only increase kinetic energy, and differential rotation may be considered as a storage of kinetic energy. This has an important consequence, since any dissipation within the system will tend to reduce the kinetic energy, and hence we have a tendency to uniform rotation. 
Now, in the barotropic case, we have

$$
\begin{aligned}
\frac{1}{\rho} \nabla p & =-\nabla \Psi \\
\text { i.e. } \frac{1}{\rho} d p & =-d \Psi .
\end{aligned}
$$

Now, since on a surface of constant $\Psi, d \Psi=0$, we see that $d p=0$ there, i.e. the surface is also a surface of constant $p$. We have already demonstrated that the surfaces of constant $\rho$ and $p$ coincide. These relationships are usually signified by writing $\rho$ and $p$ purely as functions of $\Psi$, though of course, $\Psi$ as a function of $p$ conveys the same information.

Alternatively, if we allow the rotation rate to vary along its rotation axis (i.e. let $\Omega$ be a function of $z$ as well as $s$ ) we have what is known as a baroclinic star. The curl of (9) becomes

$$
0=-\nabla \frac{1}{\rho} \times \nabla p+\hat{\varphi} \frac{d}{d z}\left[s^{2} \Omega(s, z)\right] .
$$

The second term quantifies the effect of differential rotation on the hydrostatic equilibrium of the fluid. In this case, isobars and isopycnals are orientated at an angle, which is a characteristic of baroclinicity.

In summary, barotropes are distinguished by $\frac{d \Omega}{d z}=0, p(\Psi), \rho(\Psi)$, where $\Psi$ is defined by $(10)$.

\subsection{The Von Zeipel Paradox}

In the stellar context, variations of rotation rate along $z$ are often relatively small, and so, as a first approximation, we investigate the implications of a state of thermal equilibrium on a barotropic star. If we assume that a barotropic star is in strict radiative equilibrium, the radiative flux $F$ and the nuclear reaction rate $\epsilon_{N}$ are related by

$$
\nabla . \mathbf{F}=\rho \epsilon_{N}
$$

(This is just the evolution equation for specific entropy in thermal equilibrium.) We will also assume that we are deep enough in the star so that it is optically thick. Then $F$ is given by the Eddington approximation, i.e.

$$
\mathbf{F}=-\chi \nabla T
$$

where $\chi \equiv \chi(\rho, T)$ is the radiative conductivity. Let us assume that the star has uniform chemical composition, so that the temperature is a function of $\rho$ and $p$. Thus in such a star, all dependent variables can be expressed in terms of $\Psi$, the effective gravitational potential defined by (10). Thus (13) can be rewritten as

$$
0=\nabla \cdot\left(\chi(\Psi) \frac{d T}{d \Psi} \nabla \Psi\right)+\rho \epsilon_{N}
$$

or

$$
\left[\frac{d}{d \Psi}\left(\chi \frac{d T}{d \Psi}\right)(\nabla \Psi)^{2}\right]=-\left[\left(\chi \frac{d T}{d \Psi}\right) \nabla^{2} \Psi+\rho \epsilon_{N}\right]
$$


If we now make the further assumption of uniform rotation, (i.e. assume $\Omega=\Omega_{0}$, aconstant), we see that the right hand side of (16) is purely a function of $\Psi$, and hence is constant on level surfaces of $\Psi$, since by definition,

$$
\nabla^{2} \Psi=2 \Omega_{0}^{2}-4 \pi G \rho .
$$

However, effective gravity $g_{e}(\equiv-\nabla \Psi)$ is not a constant on level surfaces in general, and thus (16) can only be satisfied if

$$
\chi \frac{d T}{d \Psi} \equiv C \quad \text { a constant }
$$

and, from (17),

$$
\epsilon_{N}=4 \pi G C\left(1-\frac{\Omega_{0}^{2}}{2 \pi G \rho}\right) .
$$

This solution is highly unphysical, since $\epsilon_{N}$ required to maintain radiative equilibrium is virtually depth independent except near the surface, where it is a function of the rotation rate. Also, the model breaks down in the limit of a surface of zero density. This is known as Von Zeipel's Paradox, who first studied this problem in 1924. Various extensions of this paradox to more general rotation laws have been made, and so it is necessary to allow a barotropic star to depart from thermal equilibrium, i.e considering the specific entropy equation,

$$
\begin{aligned}
\rho T\left(\frac{\partial S}{\partial t}+\mathbf{u} . \nabla S\right) & =-\nabla . \mathbf{F}+\rho \epsilon_{N} \\
& =\rho \epsilon_{\Omega} \neq 0
\end{aligned}
$$

The rotation thus acts as if it generated sources and/or sinks of energy, with an energy generation rate $\epsilon_{\mathbf{\Omega}}$ per unit mass. The presence of this source term on the right hand side of (20) implies either that the specific entropy will be locally modified or that motions will occur, or indeed a combination of these two effects.

Notes submitted by Richard Kerswell and Colm-cille Caulfield. 


\section{LECTURE VI}

\section{Governing Equations; Characteristic Time-Scales}

As shown in the previous lecture, we cannot have a rotating star in thermodynamic and hydrostatic equilibrium. Therefore, we must solve the full set of equations:

$$
\begin{aligned}
\rho\left[\frac{\partial \vec{v}}{\partial t}+(\vec{v} \cdot \nabla) \vec{v}\right] & =-\nabla p-\rho \nabla \phi+\nabla \cdot \overrightarrow{\vec{\tau}} \\
\frac{\partial \rho}{\partial t}+\nabla \cdot(\rho \vec{v}) & =0 \\
\rho T\left[\frac{\partial S}{\partial t}+\vec{v} \cdot \nabla S\right] & =\nabla \cdot \chi \nabla T+\rho \epsilon_{n}+[\text { viscous dissipation of heat }] \\
\rho\left[\frac{\partial c_{i}}{\partial t}+\vec{v} \cdot \nabla c_{i}\right] & =\text { sources }+\operatorname{sinks}+\nabla \cdot D \nabla c_{i}
\end{aligned}
$$

where $\vec{v}=3$-dimensional velocity relative to an inertial frame (i.e. including velocity due to rotation of the star), $\overrightarrow{\vec{\tau}}=$ viscous stress tensor, $\chi=$ conductivity, $\epsilon_{n}=$ nuclear reaction rate, $D=$ diffusivity, and $c_{i}=$ concentration of substance $i$. (Thus there will be an equation of the form (4) for each substance.) In addition, we have Poisson's Law, describing the gravitational field:

$$
\nabla^{2} \phi=4 \pi G \rho
$$

and finally, we have the equation of state:

$$
p=p\left(\rho, T, c_{i}\right)
$$

and likewise:

$$
\epsilon_{n}=\epsilon_{n}\left(\rho, T, c_{i}\right)
$$

To solve these equations, we need to separate scales, both spatially and temporally. Spatially, we have two natural scales: microscopic and macroscopic. For instance, in the convection zone of a star, diffusion of energy occurs primarily on a microscopic scale (and a fast time scale) and is governed by the following flux equation:

$$
\vec{F}_{\mathrm{e}}=\rho C_{p} D_{t} T \cdot \nabla S
$$

Temporally, there is a wide range of scales, as illustrated by Fig. 1. The smaller time scales correspond to hydrostatic balance, rotation, and convection. We are interested in the larger time scales, corresponding to large scale thermal and nuclear adjustments. Thus we can neglect terms in the above equations which correspond to fast time scales, i.e. $\partial \rho / \partial t$ and $\partial \vec{v} / \partial t$. This is because density and velocity changes are much faster than 


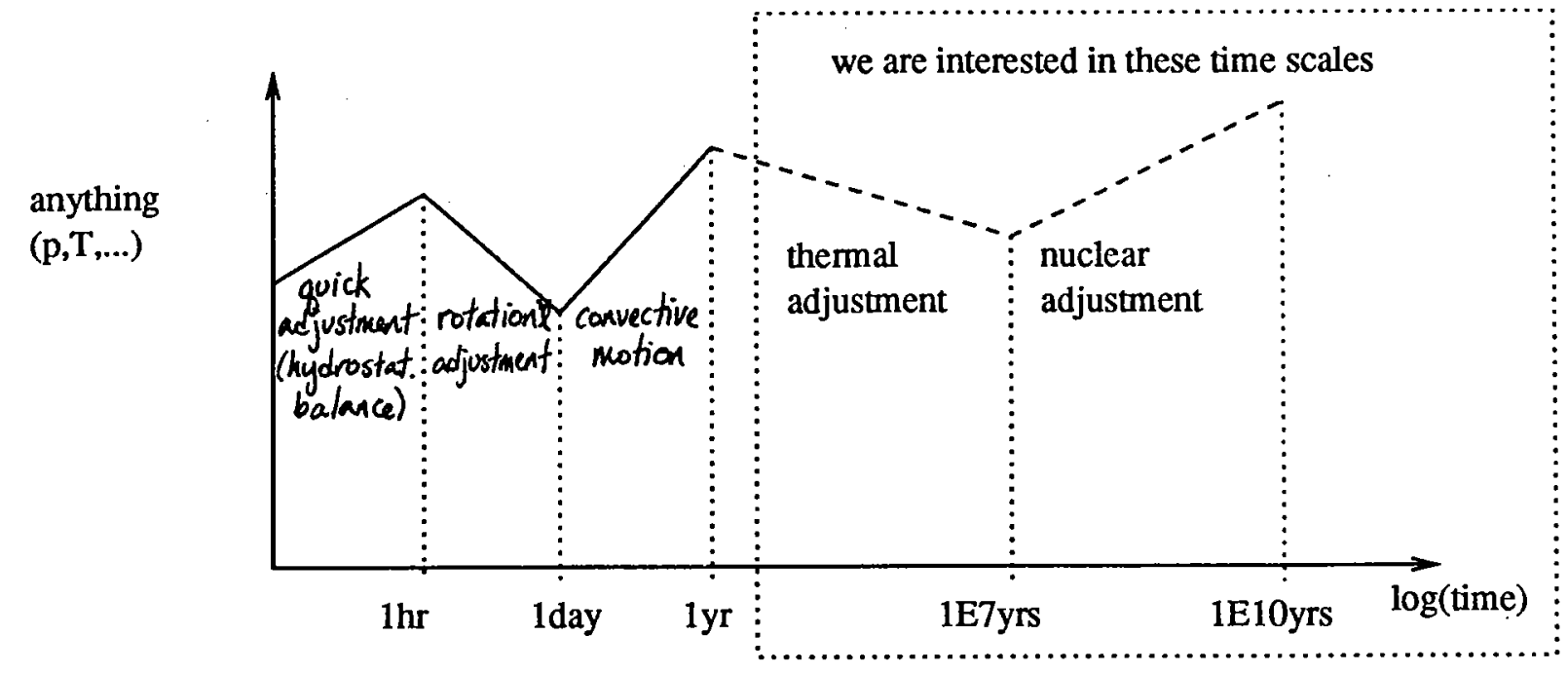

Figure 1: Time Scales of Motions in a Star

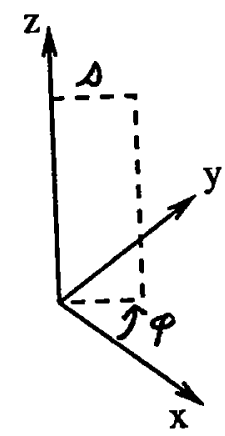

Figure 2: Axisymmetric Coordinate System

entropy and concentration. (Entropy changes are related to average temperature changes and concentration changes are due to nuclear reactions, both of which occur on slow time scales, as shown by Fig. 1.)

Therefore, let us rewrite the above equation set. We assume hydrostatic balance and axisymmetry (on these large time scales; see Fig. 1) and we make the anelastic assumption $(\partial \rho / \partial t=0$, as explained above). Furthermore, we split the velocity into meridional $(\vec{u})$ and azimuthal components: $\vec{v}=\vec{u}(s, z)+\hat{\phi}\left(s \Omega+u_{\phi}\right)$, where $\Omega=$ average rotation rate of the star (considered constant). Using these assumptions we arrive at:

$$
\begin{aligned}
-2 \Omega \rho u_{\phi} \hat{s} & =-\nabla p-\rho \nabla \psi+(\nabla \cdot \overrightarrow{\vec{\tau}})_{m} \\
\rho\left[\frac{\partial}{\partial t}\left(s u_{\phi}\right)+\vec{u} \cdot \nabla\left(s^{2} \Omega+s u_{\phi}\right)\right] & =\nabla \cdot\left[\rho \nu s^{2} \nabla\left(\frac{u_{\phi}}{s}\right)\right] \\
\nabla \cdot \rho \vec{u} & =0 \\
\rho T\left[\frac{\partial S}{\partial t}+\vec{u} \cdot \nabla S\right] & =\nabla \cdot(\chi \nabla T)+\rho \epsilon_{n}+v i s c \text { diss of heat } \\
\rho\left[\frac{\partial c_{i}}{\partial t}+\vec{u} \cdot \nabla c_{i}\right] & =\text { sources }+ \text { sinks }+\nabla \cdot\left(D \nabla c_{i}\right)
\end{aligned}
$$


where $\psi=\phi-\frac{1}{2} s^{2} \Omega^{2}$ and $(\nabla \cdot \overrightarrow{\vec{\tau}})_{m}=$ viscous stress in the meridional plane.

This set of equations has only three time-derivatives and thus should be relatively easy to solve if the transport coefficients of mass (D), momentum $(\nu)$, and energy $(\chi)$ plus the nuclear reaction rate $\left(\epsilon_{n}\right)$ are prescribed. However, identifying these coefficients can be difficult, because they must quantify transport in turbulent motion.

\section{Waves}

Before solving the set of equations for long time scales, let us investigate the effects of leaving $\partial \rho / \partial t$ and $\partial \vec{v} / \partial t$ in place. The momentum equation then is:

$$
\frac{\partial \vec{u}}{\partial t}+2 \vec{\Omega} \times \vec{u}=-\frac{1}{\rho} \nabla p-\nabla \phi
$$

where $\vec{u}=3$-dimensional velocity relative to a rotating reference frame and $\vec{\Omega}=$ rotation rate of the star. Note that we have neglected the stress term $\nabla \cdot \overrightarrow{\vec{\tau}}$ of (1) because the viscosity is assumed to be small.

Now, taking the curl of (14) yields:

$$
\frac{\partial}{\partial t}(\nabla \times \vec{u})-2 \Omega \frac{\partial \vec{u}}{\partial z}-\frac{2 \Omega}{\rho}(\vec{u} \cdot \nabla \rho) \hat{z}=-\nabla\left(\frac{1}{\rho}\right) \times \nabla p .
$$

We have used the anelastic assumption $\partial \rho / \partial t=0$ to give $\nabla \cdot \vec{u}=-\frac{1}{\rho}(\vec{u} \cdot \nabla \rho)$ and the vector identity:

$$
\nabla \times \vec{\Omega} \times \vec{u}=\vec{u} \cdot \nabla \vec{\Omega}-\vec{\Omega} \cdot \nabla \vec{u}+\vec{\Omega}(\nabla \cdot \vec{u})-\vec{u}(\nabla \cdot \vec{\Omega})
$$

where the first and last terms vanish because we consider $\vec{\Omega}$ to be spatially constant.

In the special case of zero stratification $(\nabla \rho=0)$, equation (15) describes so-called inertial waves. When $\nabla \rho \neq 0$, there is a strong coupling between those waves and gravity (or internal) waves. Only purely horizontal waves do not feel the stratification (since then $\vec{u} \cdot \nabla \rho=0$ ); we consider them next.

Writing (14) in spherical coordinates, projecting everything onto a horizontal plane (see Fig. 3) yields:

$$
\begin{aligned}
& \frac{\partial u_{\theta}}{\partial t}-2 \Omega \cos \theta u_{\phi}=-\frac{1}{\rho r} \frac{\partial p}{\partial \theta} \\
& \frac{\partial u_{\phi}}{\partial t}+2 \Omega \cos \theta u_{\theta}=-\frac{1}{\rho r \sin \theta} \frac{\partial p}{\partial \phi}
\end{aligned}
$$




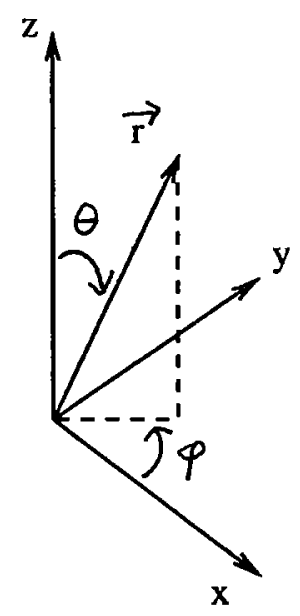

Figure 3: Spherical Coordinate System

Eliminating the pressure then yields:

$$
\frac{\partial}{\partial t}\left[\frac{\partial}{\partial \theta}\left(\sin \theta u_{\phi}\right)-\frac{\partial u_{\theta}}{\partial \phi}\right]-2 \Omega_{o} \sin ^{2} \theta u_{\theta}=0
$$

Now we look for toroidal solutions in terms of Legendre Polynomials and Fourier modes in the form:

$$
\begin{aligned}
u_{\theta} & =\frac{i m P_{n}^{m}(\cos \theta)}{\sin \theta} \exp [i(m \phi-\sigma t)] \\
u_{\phi} & =-\frac{\partial P_{n}^{m}(\cos \theta)}{\partial \theta} \exp [i(m \phi-\sigma t)]
\end{aligned}
$$

(Note that this velocity field is divergence-free.) The solutions obey the following dispersion relation:

$$
[\sigma n(n+1)+2 \Omega m] P_{n}^{m}(\cos \theta) \exp [i(\phi-\sigma t)]=0
$$

or:

$$
\frac{\sigma}{m}=\text { wave speed }=-\frac{2 \Omega}{n(n+1)}
$$

These solutions are analogous to planetary waves in oceanography.

\section{Convective-Type Instabilities}

Returning once more to the case of long time scales (where we disregard $\partial \rho / \partial t$ and $\partial \vec{v} / \partial t$ ), we recognize that we have the following feedback loop: Instabilities, then, play a key role 
large time scale evolutions

(approx. 1E8 years)

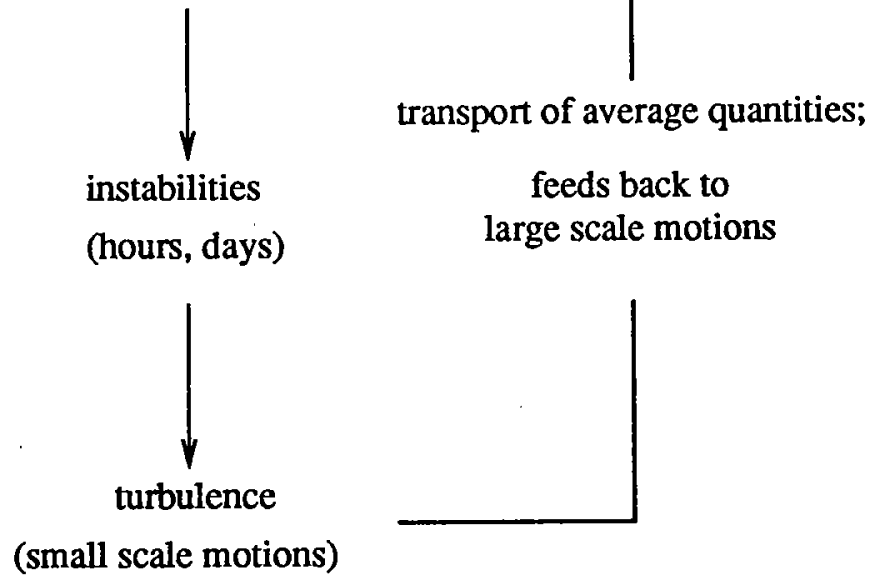

Figure 4: Feedback Loop of Instabilities

in the long time scale motions of a star. Let us therefore consider the different kinds of instabilities which can arise in the system.

\subsection{Double Diffusive Instabilities}

First, consider a positive entropy gradient in a non-rotating star. (See Figure 5.) If a parcel of matter is disturbed from its original position $A$ to position $B$, then it tends to go back to $A$, since the surrounding environment has higher temperature and lower density. Thus an oscillation develops in the system. If the star is homogeneous then the parcel will oscillate with a buoyancy frequency:

$$
N^{2}=\frac{1}{c_{p}} \frac{d S}{d r}=\frac{g}{H_{p}}\left[\left(\frac{\partial \ln T}{\partial \ln p}\right)_{\text {adiabatic }}-\frac{\partial \ln T}{\partial \ln p}\right]
$$

If $N^{2}<0$ then we have instability (negative entropy gradient), while if $N^{2}>0$ then the system is in a state of stable equilibrium. If the medium is chemically stratified (i.e. we have a gradient of a molecular weight which acts as a restoring force) the buoyancy frequency has two components

$$
N^{2}=N_{T}^{2}+N_{\mu}^{2}
$$

where $N_{T}^{2}$ is as before and $N_{\mu}^{2}=g / H_{p}(\partial \ln \mu / \partial \ln p)$. Here, $\mu$ is the molecular weight.

Let us examine the effect of dissipation. In a system with entropy stratification, insta- 


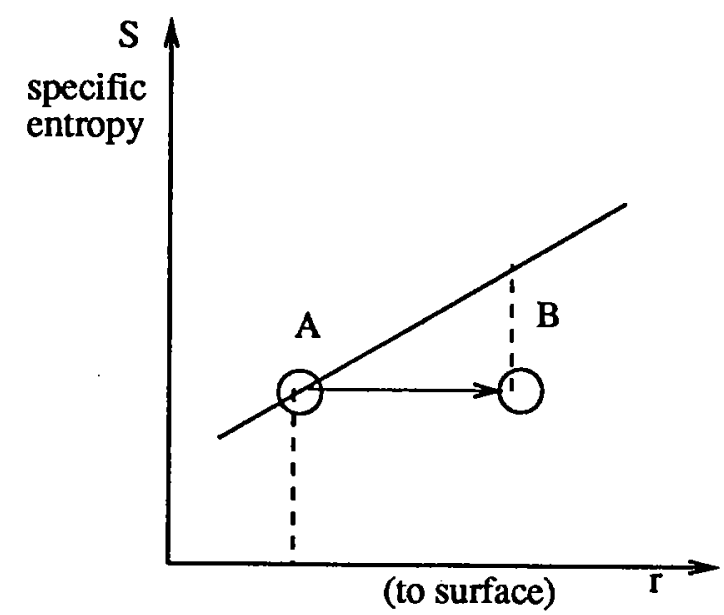

Figure 5: Positive entropy gradient

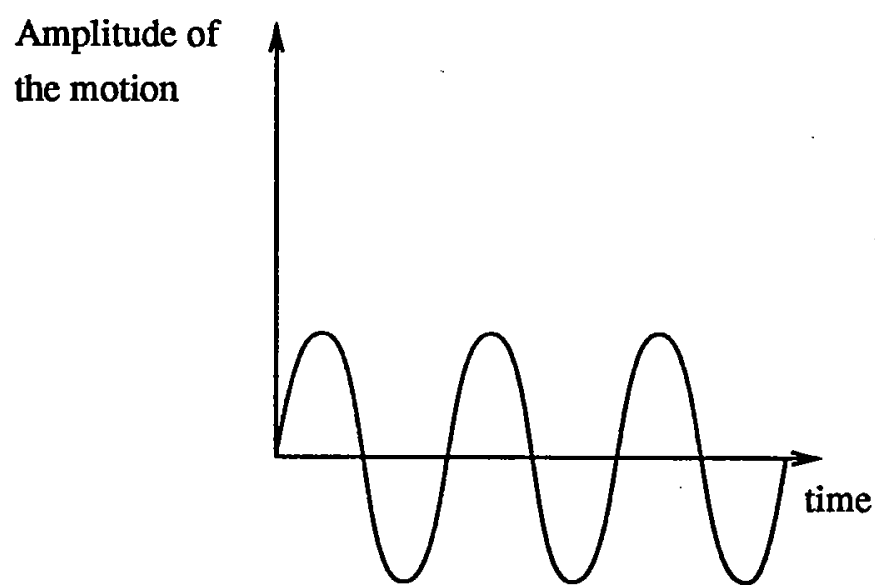

Figure 6: Stable oscillatory state

bility occurs if

$$
N^{2}<-\frac{\text { const }}{t_{\nu} t_{d}}
$$

where $t_{\nu}$ is a viscous diffusion time and $t_{d}$ is a thermal diffusion time. If $N^{2}>0$ and $N_{\mu}^{2}$ is negligible then there are two cases according to $t_{d} \gg \frac{1}{N}$ or $t_{d} \sim \frac{1}{N}$. If $t_{d} \gg \frac{1}{N}$ then the system is in a stable oscillatory state. (See Figure 6.)

If $t_{d} \sim \frac{1}{N}$ then the oscillations tends to die out. (See Figure 7.) Thus, if thermal diffusion is fast enough (i.e. $t_{d}$ small enough ) then it weakens the restoring force, damping out the motion.

The case when $N^{2}>0$ and $N_{T}^{2}$ and $N_{\mu}^{2}$ have opposite signs is interesting because time scales for diffusion of matter and temperature are quite different. This leads to a double- 


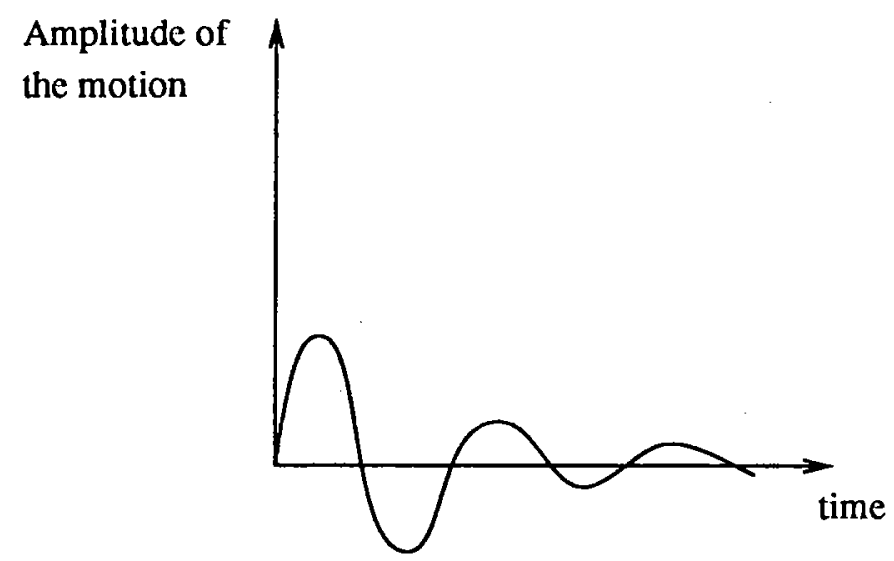

Figure 7: Oscillations die out

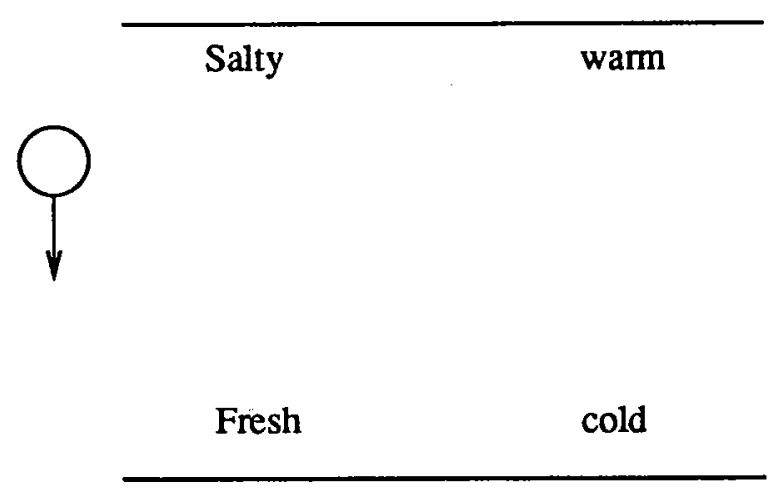

Figure 8: Salt warm water on top of cool fresh water

diffusive instability well known in Geophysical Fluid Dynamics, but generally ignored in Astrophysical Fluid Dynamics.

First consider $N_{T}^{2}>0$ and $N_{\mu}^{2}<0$. As an oceanographic example, suppose we have salty warm water on top of cool fresh water. (See Figure 8.) If the salinity exceeds some threshold value in the top layer then we have the famous salt fingers instability. There is a stellar analogy with a binary star system in which one member, having burned all the hydrogen in its core, throws off helium-rich matter to its partner. The companion star gains heavier matter and experiences double-diffusive instability.

If $N_{T}^{2}<0$ and $N_{\mu}^{2}>0$ (for example a destabilizing thermal gradient and a stabilizing salinity gradient) then oscillations grow in the system and we have thermohaline convection or overstability; in astrophysics this may occur in the convection zone of massive stars, and is called semi-convection. (See Figure 9.) 


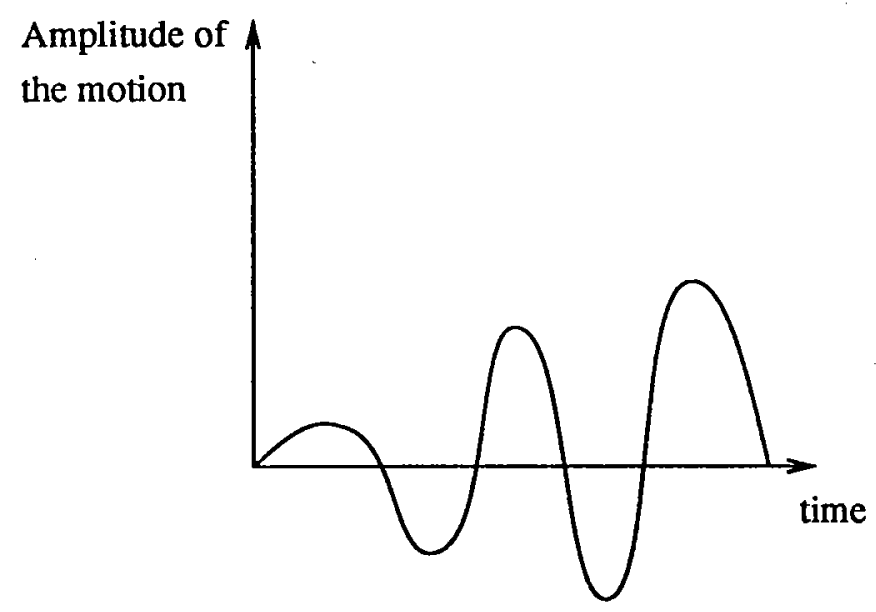

Figure 9: Overstability

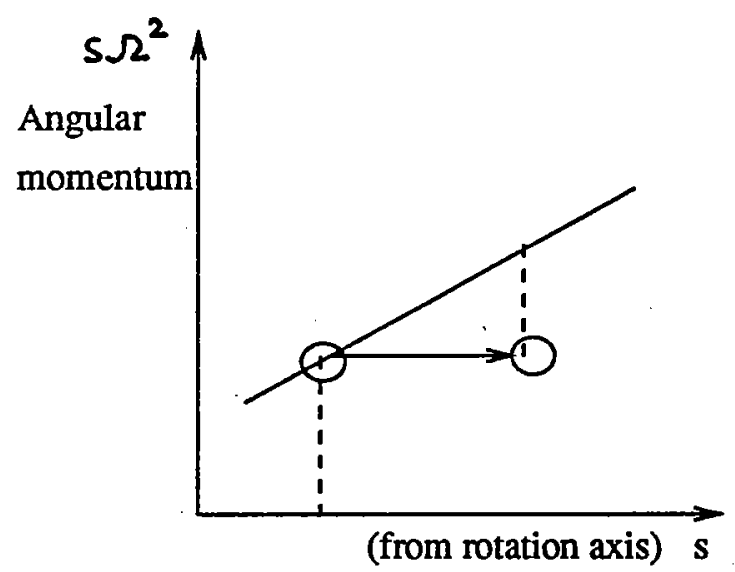

Figure 10: Positive angular momemtum gradient

\subsection{Baroclinic Instabilities}

Now, let us add rotation to the system. First consider an angular momentum gradient (by analogy with the entropy gradient in the non-rotating case). (See Figure 10.) If we try to displace a parcel of matter as shown above, the conservation of angular momentum will act as a restoring force. Similarly to the case of a non-rotating star, we define the Rayleigh frequency by

$$
N_{\Omega}^{2}=\frac{1}{s^{3}} \frac{d}{d s}\left(s^{2} \Omega\right)^{2}
$$

As before we have stability if $N_{\Omega}^{2}>0$ and instability if $N_{\Omega}^{2}<0$.

Now, we turn our attention to a system which has both rotation and stratification. 
Assume $\Omega=\Omega(s, z)$. Then taking the curl of hydrostatic equation:

$$
0=-\frac{1}{\rho} \nabla P+\nabla \phi+\hat{s}\left(s \Omega^{2}\right)
$$

we arrive at

$$
0=-\nabla\left(\frac{1}{\rho}\right) \times \nabla P+\nabla\left(s \Omega^{2}\right) \times \hat{s}
$$

For a perfect gas we have

$$
T d s=c_{p} d T-\frac{d p}{\rho}
$$

Thus,

$$
T d S=c_{p} d T-\frac{\mathcal{R}}{c_{p}} d \ln P
$$

This implies

$$
\frac{1}{c_{p}} d S=\left(1-\frac{\mathcal{R}}{c_{p}}\right) d \ln P-d \ln \rho
$$

Substituting this last equation into equation (29) yields:

$$
\frac{1}{c_{p}} \frac{\nabla S}{\rho} \times \nabla P=\nabla\left(s \Omega^{2}\right) \times \hat{s}
$$

We can approximate the above equation by

$$
\frac{1}{c_{p} \rho}\left|\frac{d S}{d n} \| \frac{d P}{d n}\right| \sin \alpha=\frac{\partial}{\partial z}\left(s \Omega^{2}\right)
$$

where $n$ is outward normal and $\alpha$ is a baroclinic angle between $\frac{d S}{d n}$ and $-\frac{d P}{d n}$. Using hydrostatic balance we obtain

$$
N^{2} \sin \alpha=\frac{\partial}{\partial z}\left(s \Omega^{2}\right)=\frac{g}{c_{p}} \frac{d S}{d r} \sin \alpha
$$

Note that if $\Omega$ increases towards the equator then $\nabla S$ points outward as shown in Figure 11. Rotating this picture and choosing surfaces of constant entropy and pressure we obtain Figure 12. Two possible scenarios for the displaced parcel of matter are displayed. In case (1) we have stability because $d S / d r>0$. In case (2) the displaced parcel of matter is warmer and lighter than its environment and tends to move away due to buoyancy. Thus the shaded region in Figure 12 is unstable. However, for the case of an axisymmetric 


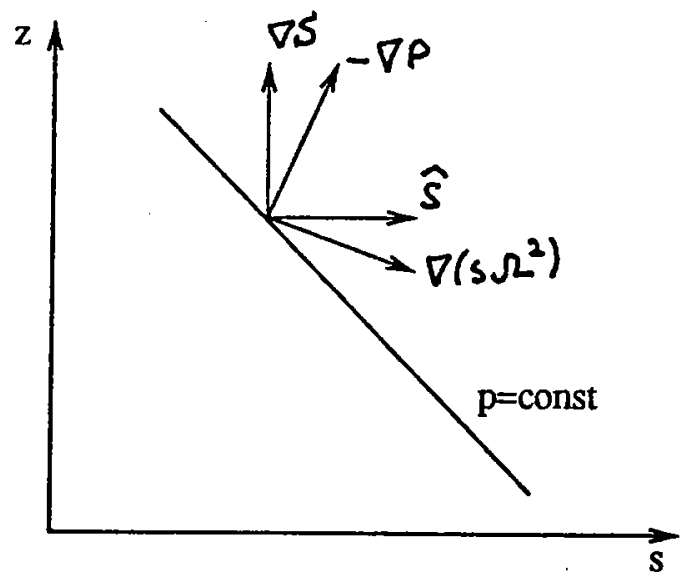

Figure 11: Rotation and stratification

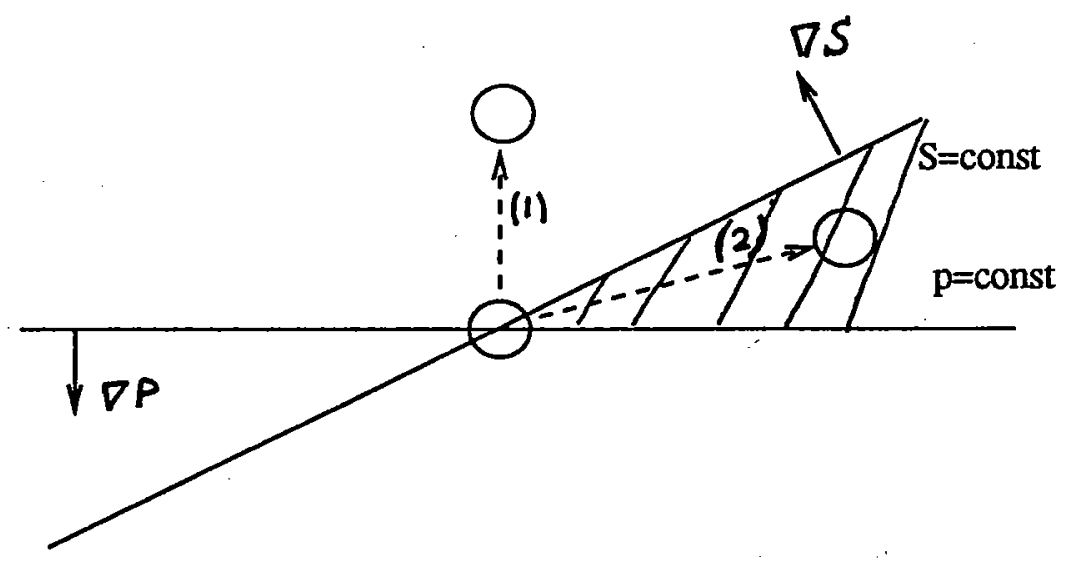

Figure 12: Rotation and stratification 


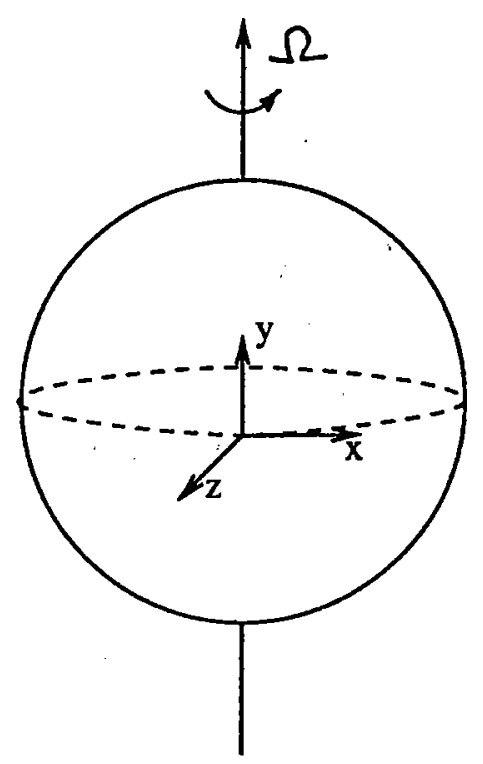

Figure 13: Coordinate system

displacement, the system is still stable if $N_{\Omega}^{2}>0$, since type (2) displacements are ruled out by the conservation of angular momentum.

If we add dissipation with $\nu \ll \kappa$, the system is unstable when $N_{\Omega}^{2}<\frac{\nu}{\kappa} N^{2}$, where $N^{2}$ is a buoyancy frequency described earlier.

Another instability occurs in axisymmetric system when $N^{2} \frac{\nu}{\kappa}<\left|s \frac{\partial \Omega^{2}}{\partial z}\right|$. This instability is called the Goldreich-Schubert-Fricke instability.

For non-axisymmetric displacements, the necessary condition for linear instability is that $\partial \Pi / \partial y=0$ in the domain where $\Pi$ is the potential vorticity:

$$
\Pi=(2 \Omega+\nabla \times \mathbf{u}) \frac{\nabla S}{\rho}
$$

and $\frac{\partial}{\partial y}$ is the derivative in the latitudinal direction ${ }^{1}$ (see Figure 13). Thus, we have instability if there is an inflection point in the longitudinal direction of the velocity field. It can be shown ${ }^{2}$ that

$$
\frac{\partial \Pi}{\partial y}=\left(\beta-\frac{\partial^{2} U}{\partial y^{2}}\right)-\frac{1}{\rho} \frac{\partial}{\partial z} \frac{f^{2}}{N^{2}}\left(\rho \frac{\partial \mathbf{u}}{\partial z}\right)
$$

where $\beta=\frac{\Omega}{\cos \theta}, f=\Omega \sin \theta$. Thus, the necessary condition for instability $d \Pi / d y=0$ is analogous to the Rayleigh criterion for parallel shear flows. 


\section{References}

[1] J. Pedlosky, Geophysical Fluid Dynamics, Springer-Verlag, 2nd edition, (1987)

[2] J.-P. Zahn, Instability and Turbulence in Rotating Objects, Cource 8, Astrophysical Fluid Dynamics, Elsevier Science Publishers B.V., (1989)

Notes submitted by N. Platt and W. Welch 


\section{Lecture VII.}

\section{Shear Instabilities}

\section{Introduction}

Upon the main sequence, where they spend most of their life burning hydrogen, stars can be roughly divided into two classes. The low mass stars (with masses less than $1.6 M_{\odot}$ ) have convective envelopes and mostly rotate slowly. Higher mass stars have convective cores and usually rotate comparatively quickly. Within these convection zones, the velocity of the flows can become quite large, and in some cases approaches the speed of sound. Consequently the timescale upon which quantities are advected throughout the convection zone is very short (of the order of months) in comparison to the typical times upon which thermal or evolutionary adjustments take place. In the radiative regions of the star, motions generated by the rotation are much slower. These motions (instabilities) can, however, transport chemical elements and momentum and are therefore important for the slow adjustments and evolution of the star. In fact their effect can be embodied within the transport coefficients (such as viscosity and diffusion) that occur in the fluid dynamic equations governing the adjustments of the star.

Our primary concern are shear flows, since these are common in differentially rotating stars. Instabilities in shear flows were first investigated by Rayleigh (1880). He showed that a necessary condition for the existence of a linear instability within the mean flow of an inviscid fluid is simply that there be an inflexion point in the velocity profile in the direction of the shear (as illustrated in figure 1). This result was extended by Fjortoft (1950) who showed that if there was a shear in the background flow, then for linear instability, the vorticity must be locally enhanced at the inflexion point.
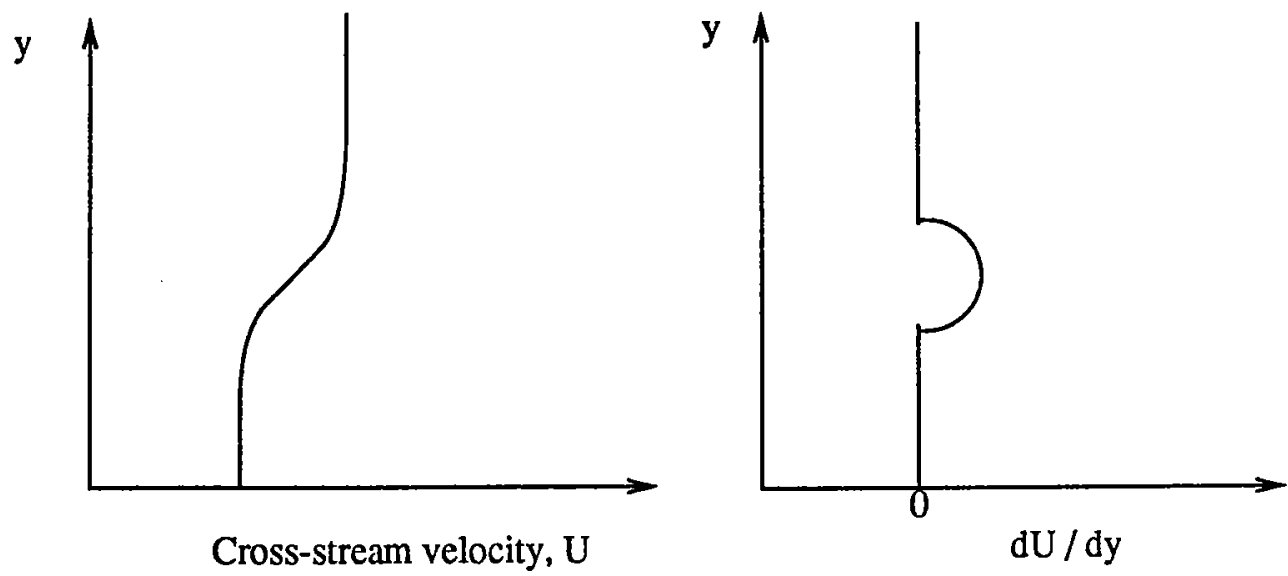

Figure 1. An unstable shear flow. Shown are the cross-stream velocity profile and the vorticity. 


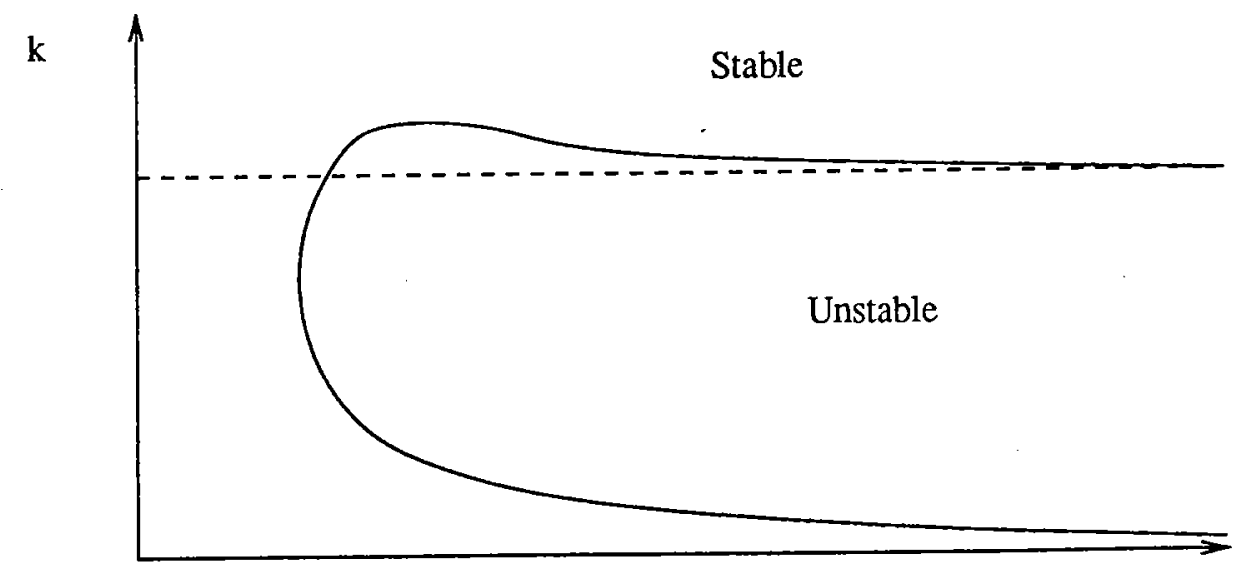

$\operatorname{Re}$

Figure 2: The range of linearly unstable horizontal wavenumbers as a function of the Reynolds number of the flow $R e$. The background flow has no shear.

This flow is unstable to small perturbations. If the perturbation has the dependance $e^{i k x}$ upon the coordinate in the direction of the flow, $x$, then the perturbation is unstable provided $k d<O(1)$ where $d$ is the characteristic scale of the velocity inflexion, and the mean flow has no background shear. That is, there exist long-wave instabilities.

When the fluid is viscous, then the instability must also compete against dissipation. The range of linearly unstable wavenumbers as a function of the effective Reynolds number $R e=|d U / d z| d^{2} / U$, is shown in figure 2. (In this example there is no background shearing motion.)

\section{Poiseuille Flow}

Plane Poiseuille flow describes the flow of fluid between two plane-parallel plates. The mean velocity profile is parabolic and is strongly influenced by the presence of the boundaries. In fact it is the presence of these boundaries that causes the flow to become linearly unstable at a Reynolds number of $R \simeq 6000$. Experimentally the onset of instability occurs at critical Reynolds number of $R_{c} \simeq 1000$. This is indicative that there are finite amplitude instabilities at lower values of $R$. Such instabilities can be examined by developing the velocity potential of the perturbation to the mean flow $U(y, t)$ as a set of discrete modes:

$$
\mathbf{u}=\hat{\mathbf{x}} U(y, t)-\nabla \times \hat{\mathbf{z}} \Psi(x, y, t)
$$

with

$$
\Psi(y, t)=\sum_{n=1}^{N} \psi_{n}(y, t) e^{i n k x} .
$$

This expansion has the advantage that the variation of the perturbation in the direction of the shear can be analyzed in detail at the expense of retaining only a few modes to describe the variation in the direction of the mean flow. Of course one still has to specify the wavenumber $k$, but it can be treated as a free parameter and varied to estimate its effect 


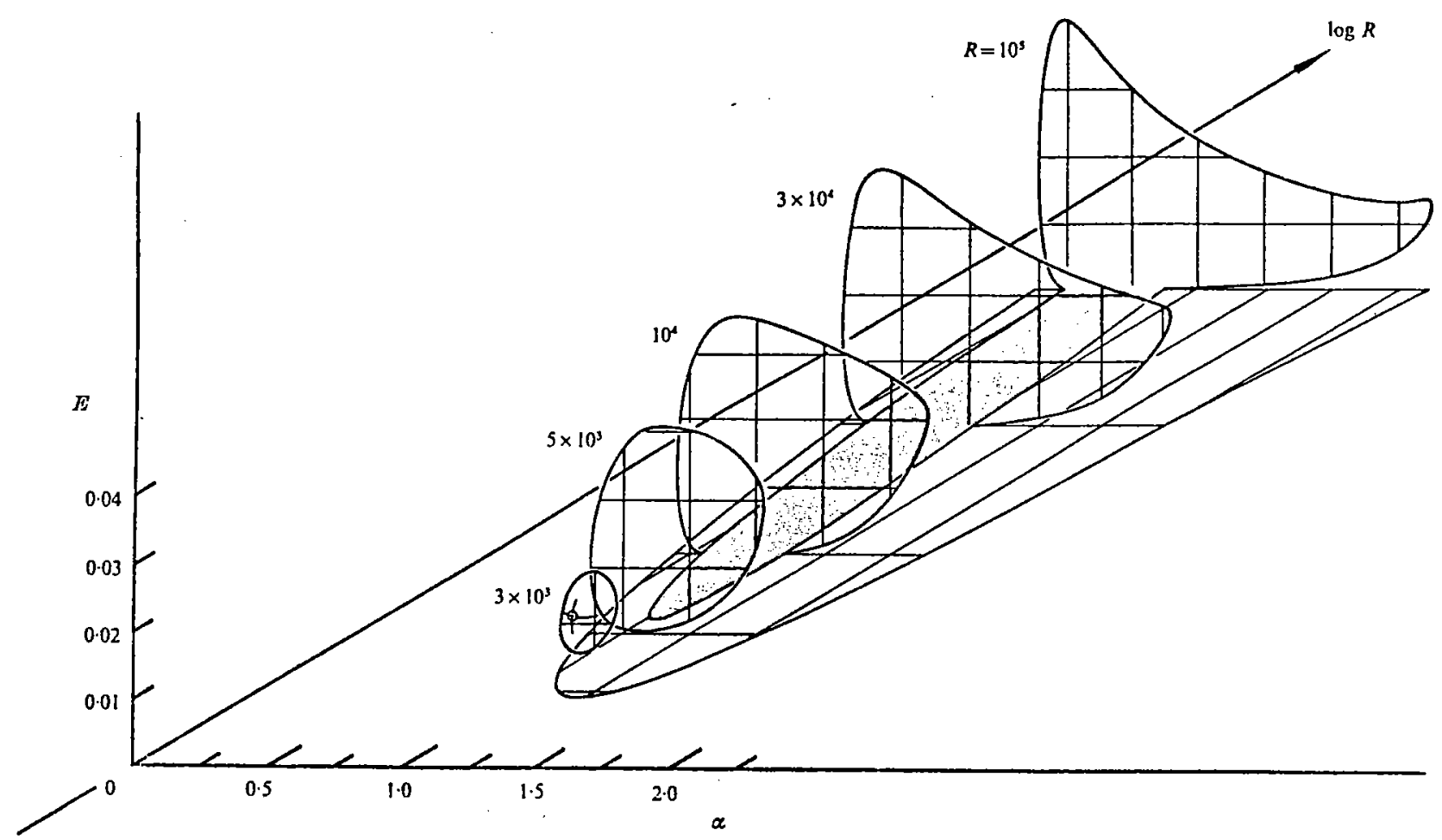

FIGUne 2. Illustrating tho solution surface on which finite amplitude steady solutions are found in the one-mode approximation (case I). The vertical co-ordinate is the disturbance energy $E$ [cf. (2.22)]. The linear instability loop and the region of metestability for case $I$ shown in figuro 1 are indicated in the $E=0$ plane.

Figure 3:

upon the solutions. A reasonable choice of its value might be that value that corresponds to the critical Reynolds number. The nonlinear system derived for the case $N=2$ was investigated by Zahn et al. (1974). They found that this system does indeed exhibit finite amplitude instabilities at Reynolds numbers below the critical value for the onset of linear instability. In fact the critical Reynolds number was found to be reduced to $\sim 3000$. The range of unstable solutions with a given amplitude as functions of the wavenumber $k$ and the Reynolds number is indicated in the three-dimensional plot shown in figure 3 (taken from $\mathrm{Zahn}$ et al., 1974). The nonlinear solutions that figure 3 is derived from can in some cases be described by stationary waves in some Galilean frame moving in the direction of the flow with speed $c$. These are, however, not observed in the experiments, suggesting that even these are unstable to some other solutions. A further analysis by Orszag and Kells (1980) seems to confirm this; These two-dimensional solutions are unstable to three-dimensional perturbations. This reduces the critical Reynolds number even further and brings tolerable agreement between theory and experiment. 


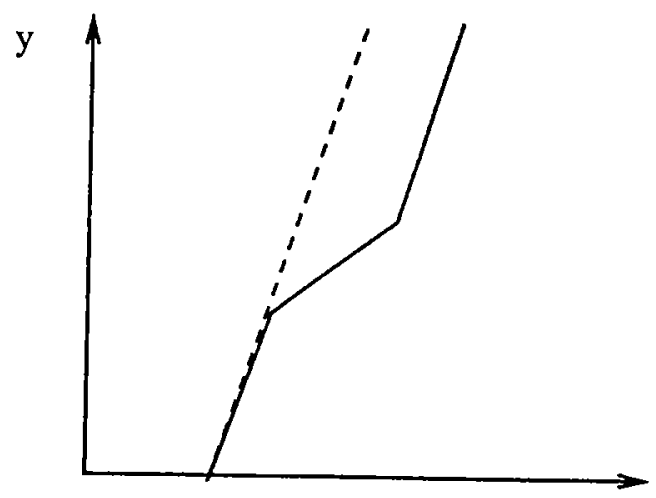

Cross-stream velocity, $\mathrm{U}$

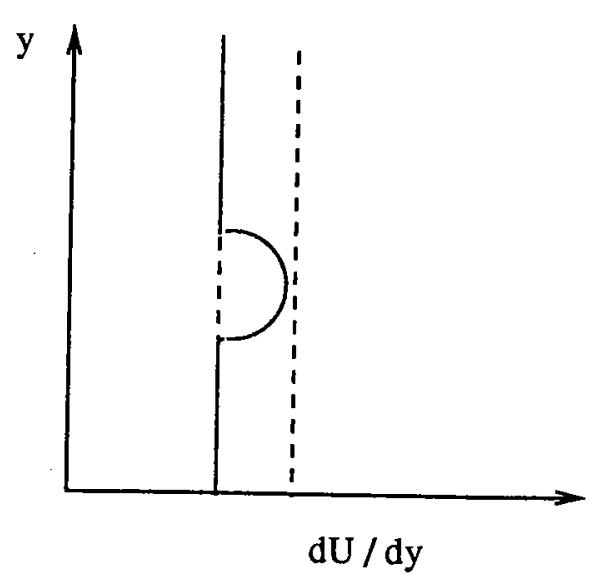

dU $/ \mathrm{dy}$

Figure 4: Couette flow and a peruturbed Couette flow containing an inflexion point.

\section{Plane Couette Flow}

Couette flow describes the motion of a fluid with a uniform shear transverse to the direction of the flow (figure 4). Theoretically this flow is linearly stable, but it is found to be unstable in the laboratory. When the flow also contains a superposed inflexion point, as illustrated in figure 4 , the flow unsurprisingly becomes linearly unstable.

Lerner and Knobloch (1987) has considered the inviscid case and finds unstable wavenumbers with $k<\epsilon / d$, where $\epsilon$ is the fractional increase in the vorticity. Since the growth rate of the instability must exceed the rate of viscous dissipation one expects instability for

$$
\sigma \simeq|d U / d y| \epsilon>k \nu / d^{2}
$$

If the characteristic scale of the perturbation is $L=\pi / k$ then these conditions can be rewritten in terms of the effective Reynolds number of the flow,

$$
R e=|d U / d y| \epsilon^{2} L^{2} / \nu>(L / d)^{2},
$$

and $\pi d / L<\epsilon$. These conditions define a region of the plane $\epsilon-(d / L)$ in which instabilities exist (figure 5). There exists a critical value of $\epsilon$ for the flow which scales as $R e^{-1 / 3}$ (Dubrulle and Zahn, 1990). A finite-amplitude analysis of Couette flow has yet to be performed.

\section{Stabilization through Stratification}

Under certain conditions shear flows can become stabilized. When the fluid is stratified in the direction of the shear, a necessary condition for linear stability can be written in terms of the Richardson number

$$
R i=\frac{N^{2}}{|d U / d y|^{2}}>\frac{1}{4}
$$




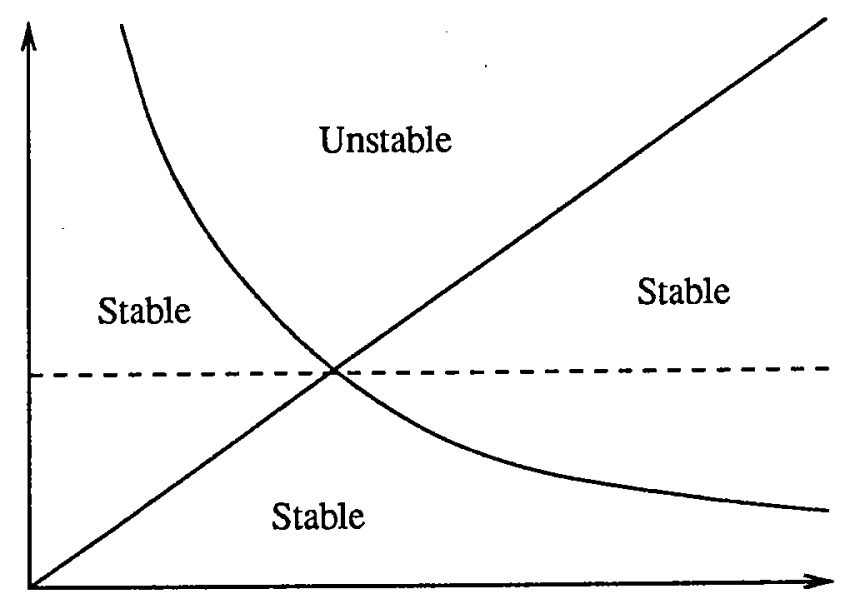

$\mathrm{d} / \mathrm{L}$

Figure 5: The range of linearly unstable perturbations with a characteristic scale $L$ shown as a region in the $\epsilon-(d / L)$ plane.

where $N$ is the buoyancy or Brunt-Väisallä frequency and the fluid is stratified in the direction of $y$. Therefore stratification can loosely be thought of as providing a stabilization (but see Thorpe, 1969).

However, stratification is not always felt by the instability. If the characteristic timescale upon which the disturbance is smoothed out by the diffusion of heat, $\kappa / d^{2}$ (where $\kappa$ is the diffusivity), is shorter than the characteristic timescale of the perturbation, $|d U / d y|^{-1}$, then the stratification is not felt by the perturbation. Therefore some criterion concerning the diffusion timescale must be introduced into equation (5). If the Peclet number is written as

$$
P e=|d U / d y| d^{2} / \kappa
$$

then the necessary condition can be written as

$$
R i=\frac{N^{2}}{|d U / d y|^{2}}[\operatorname{Max}(1, P e)]^{-1}>O(1)
$$

If we introduce the Reynolds number $R=|d U / d y| d^{2} / \nu$, then where the Peclet number exceeds one, the condition becomes

$$
\frac{N^{2}}{|d U / d y|^{2}}(\nu / \kappa) R>O(1)
$$

Since instability will only occur when the Reynolds number exceeds a critical value $R_{c}$, the complete condition for stability is

$$
\frac{N^{2}}{|d U / d y|^{2}}(\nu / \kappa) R_{c}>O(1)
$$


Another possible restoring force that may cause stabilization in stars is that provided by rotation. The analogue to equation (5), the necessary condition for stability in the rotating fluid would be

$$
R i=\frac{N_{\Omega}^{2}}{|d U / d y|^{2}}>O(1)
$$

where $N_{\Omega}$ is now the Rayleigh frequency. However, in contrast with a stratification, it appears that the coriolis force is not capable of stabilizing the flow.

\section{References}

1. Dubrulle, B., and Zahn, Z.-P. (1990). Submitted to J. Fluid Mech..

2. Fjortoft, R. (1950). Geofys. Publ., 17.

3. Lerner, J., and Knobloch, E. (1988). J. Fluid Mech., 198, 117.

4. Orszag, S.A., and Kells, L.C. (1980). J. Fluid Mech., 86, 159.

5. Rayleigh (1880). Scientific Papers, 1, 474 (Cambridge university press).

6. Thorpe, S.A. (1969). J. Fluid Mech., 36, 673.

7. Zahn, J.-P., Toomre, J., Spiegel, E.A., and Gough, D.O. (1974). J. Fluid Mech., 64, 319.

Prepared by N.J. Balmforth and Brian Chaboyer 
Lecture VIII.

\section{Shear Flow Instability and the Transition to Turbulence}

\section{Taylor-Couette Flow}

The Taylor-Couette experiment provides another example of shear flow instability. In a classic paper, G. I. Taylor (1923) described experimental and analytical results concerning the flow between two coaxial cylinders of radii $R_{1}$ and $R_{2}$, with $R_{1}<R_{2}$, rotating with angular velocities $\Omega_{1}$ and $\Omega_{2}$, respectively. We begin our analysis of such an experiment by looking for steady solutions. We will use cylindrical coordinates where $s$ denotes radius, $\phi$ azimuthal angle and $z$ displacement along the axis. The radial symmetry of the problem suggests that we look for solutions where the pressure $p$ and angular rotation, rate $\Omega$ depend only upon $s$, and where the other two components of the velocity are zero. The $\hat{s}$ and $\hat{\phi}$ components of the Navier-Stokes equations then simplify to

$$
\begin{aligned}
\frac{d p}{d s} & =\rho s \Omega^{2} \\
\frac{1}{s^{2}} \frac{d}{d s}\left(s^{3} \frac{d \Omega}{d s}\right) & =0
\end{aligned}
$$

Equation (2) has solutions of the form $s^{n}$. The general solution is

$$
\Omega(s)=\frac{k_{1}}{s^{2}}+k_{2}
$$

where the constants $k_{1}$ and $k_{2}$ are determined by the boundary conditions $\Omega\left(R_{1}\right)=\Omega_{1}$ and $\Omega\left(R_{2}\right)=\Omega_{2}$. In particular

$$
k_{2}=\frac{\Omega_{2} R_{2}^{2}-\Omega_{1} R_{1}^{2}}{R_{2}^{2}-R_{1}^{2}} .
$$

\subsection{The Rayleigh condition for linear inviscid stability}

If we ignore viscosity, there is a simple condition for instability of the flow (3) to small perturbations. This condition is the analog (in a cylindrical geometry) of the well known Rayleigh inflection point criterion for planar shear flows. If the angular momentum $\left|s^{2} \Omega(s)\right|$ increases with increasing $s$, then a fluid parcel displaced in radius experiences a 
restoring force, otherwise it is pushed further away from its initial radius. Mathematically, the Rayleigh condition (necessary for stability) is that

$$
\Omega \frac{d}{d s}\left(s^{2} \Omega\right)=2 s \Omega k_{2} \geq 0, \text { for all } s \text { in the interval } R_{1}<s<R_{2} .
$$

Since vorticity $\omega=d / d s\left(s^{2} \Omega\right) / s$, this condition is equivalent to the statement that $\omega(s)$ and $\Omega(s)$ have the same sign everywhere in the flow.

If the cylinders are rotating in opposite directions, then $\Omega(s)$ changes sign between them, and since $k_{2}$ is constant (5) is not satisfied and thus the flow is unstable. If the cylinders are rotating in the same direction $\left(\Omega_{1} \geq 0, \Omega_{2} \geq 0\right)$ the Rayleigh condition is satisfied if and only if $k_{2}>0$, or

$$
\Omega_{2} R_{2}^{2}>\Omega_{1} R_{1}^{2}
$$

Therefore, when the inner cylinder is at rest $\left(\Omega_{1}=0\right)$, the Rayleigh condition is satisfied whereas if the outer cylinder is at rest $\left(\Omega_{2}=0\right)$, the Rayleigh condition is not satisfied and the flow is unstable.

We note two important limitations of the above analysis. First, the Rayleigh condition (5) is not sufficient for stability. The above analysis applies only to axisymmetric disturbances, and the flow may be linearly unstable to more general types of disturbances. Second, the Reynolds number of the flow was assumed to be infinite, and nonzero viscosity may stabilize flows that do not satisfy (5). Despite these limitations, the Rayleigh condition proves a simple rule of thumb for stability in the case of large Reynolds number.

\subsection{Two types of instability}

Figure 1 summarizes the stability regimes for Taylor-Couette flow. The Rayleigh criterion (6) gives the inviscid linear stability boundary $\Omega_{2} R_{2}^{2}=\Omega_{1} R_{1}^{2}$. The region of linear instability, including viscosity, was calculated from theory by Taylor (1923). This region is eventually entered as the velocity of the inner cylinder increases. Experimentally, when the flow enters the region of linear instability it undergoes a transition into a different type of steady flow. Taylor vortices appear, which are radially symmetric circulations similar in appearance to convection cells (Figure 2). The agreement between the theoretical and experimental linear stability boundaries is excellent. As the velocity of the inner cylinder is increased further several more transitions occur until the motion is three dimensional and eventually turbulent.

By deriving a bound on the total energy of the flow, Joseph \& Munson (1970) showed that in the hatched region of Figure 1, the flow was stable to perturbations of arbitrary amplitude. However, the flow is not stable to finite amplitude disturbances in the entire region of linear stability. In fact, as early experiments demonstrated (Wendt, 1933), a completely different kind of instability from that seen by Taylor can occur. Wendt 


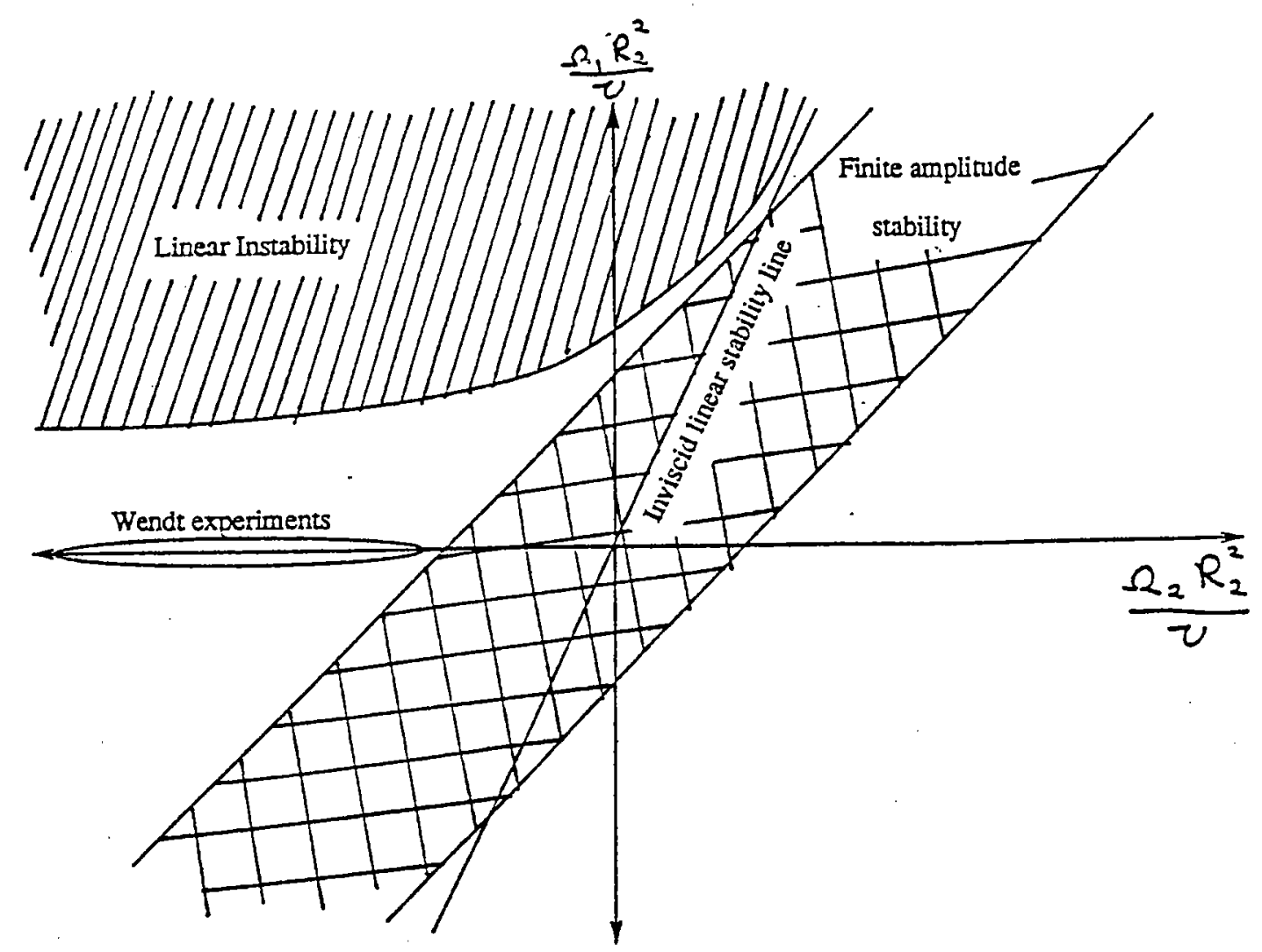

Figure 1: Summary of stability regimes for Taylor-Couette flow.

conducted an experiment in which the inner cylinder was at rest (in this regime the flow (3) is always linearly stable). At he increased the speed of the outer cylinder, he observed a transition near Reynolds number $R=R_{t}$ to a turbulent flow which was intermittent in space. When the outer cylinder was then slowed down the instability persisted until $R$ dropped below $R_{c}$, where $R_{c}<R_{t}$. From this we can deduce that the instability requires a finite kick, or is a subcritical instability, although Wendt himself did not give such an explanation.

Coles (1965) obtained the subcritical instability by starting in the linearly un-

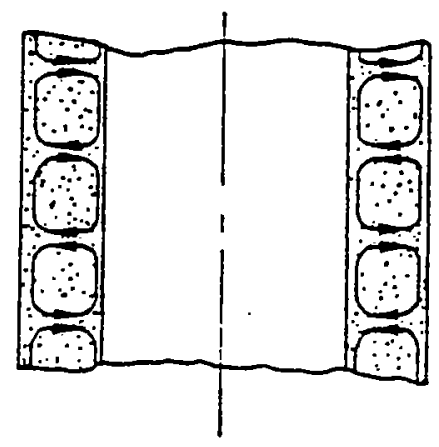

Figure 2: Taylor vortices between coaxial cylinders. 
stable region and then slowly varying the rotation rates $\Omega_{1}$ and $\Omega_{2}$ in such a way that the instability was always present yet the inner cylinder eventually was brought to rest. Experimental results support the hypothesis that the subcritical instability is found only above the critical Reynolds number $R_{c}$, which has been estimated experimentally as

$$
R_{c}=\frac{\Omega_{2} R_{2}\left(R_{2}-R_{1}\right)}{\nu} \approx 2000
$$

It is interesting that this value is close to the critical Reynolds number for plane Couette flow.

\section{Geostrophic Turbulence}

The standard view of three dimensional turbulence is that the energy of the flow moves from large scales to small scales where it is eventually dissipated by viscosity. However, the large scale motions of planetary atmospheres and oceans are to leading order two dimensional and geostrophic (i.e. horizontal pressure gradient balanced by the Coriolis force). The energy cascade of geostrophic flows differs greatly from the energy cascade characteristic of three dimensional turbulence, and in this section we examine the relevance of the theory of geostrophic turbulence (Rhines, 1975; Pedlosky 1987) to stellar dynamics.

In a two dimensional geostrophic turbulence, the peak of the energy spectrum shifts in time towards a wavenumber $1 / R_{d}$, where $R_{d}$ is called the Rossby deformation radius and is defined by

$$
R_{d} \equiv \frac{N H}{f}
$$

Here $N$ is the Brunt-Väisälä frequency and $H$ is the pressure scale height. The parameter $f \equiv 2 \Omega \sin \theta$, called the effective Coriolis parameter, is the component of the planetary vorticity $\Omega \hat{z}$ normal to the planetary surface at latitude $\theta$. The Rossby deformation radius is very large near the equator and approaches zero at the poles, but for the mid-latitude regions of the earth, $R_{d} \sim 100 \mathrm{~km}$ for the oceans and $R_{d} \sim 1000 \mathrm{~km}$ for the atmosphere. For many stars, the Rossby deformation radius at mid-latitudes is on the order of the radius of the star $r$ (if the stratification is felt, i.e. Péclet number much larger than unity). Thus the common oceanic and atmospheric approximation that $f$ is constant (or linear) over a small portion of the surface will be invalid in general, and evidently we must include from the start the full spherical geometry of the problem.

The argument of the last paragraph leads us to believe that if geostrophic eddies exist in stars, their horizontal length scale $L$ may be comparable to the stellar radius $r$. Let $U$ be a typical velocity in such an eddy. If the turnover time of the eddy $U / L$ is greater than a rotation period, the eddy can excite Rossby waves-the characteristic low frequency 
waves common in the earth's atmosphere and oceans. Presumably if $U / L$ is comparable to the frequency of a Rossby wave, the eddy will disperse as a packet of Rossby waves. Thus, a persistent geostrophic eddy must satisfy

$$
\frac{U}{L}<\frac{\beta l}{l^{2}+n^{2}+\left(L / R_{d}\right)^{2}},
$$

where $l$ and $n$ are the horizontal wavenumbers of the Rossby wave. The parameter $\beta$ is defined by

$$
\beta=\frac{2 \Omega \cos \theta}{r}=\frac{1}{r} \frac{\partial f}{\partial \theta} .
$$

When $R_{d} \ll r, \beta$ is approximately constant in a region around the latitude $\theta=\theta_{0}$ (this is the case in atmospheric and oceanic situations). However, for a star $R_{d} \sim r$, and $\beta$ varies considerably over the problem. In particular, $\beta$ goes to zero at the poles, which are regions where Rossby waves cannot exist. Once again, a complete treatment of the problem is more complex as it must take into account the sphericity of the star, but the analysis here at least gives us an idea of what may be expected.

\subsection{Dissipation of Energy}

We now address the problem of the dissipation of energy in the horizontal modes. It is clear, though that eddies and waves dissipate energy differently. Where there are eddies, one can form a diffusivity from the largest length and velocity scales and reasonably expect this diffusivity to damp out the eddies. In waves, which can transport momentum, however, dissipation is much weaker, and may also occur in wave breaking. Thus in order to know how energy is dissipated at any given point in the flow, we must know in which regimes to expect eddies and in which regimes to expect waves.

A clue is given by the Rossby number $R o=u / 2 \Omega l$. The momentum equation for a rotating fluid contains the sum of advection and Coriolis terms:

$$
(\mathbf{u} \cdot \nabla) \mathbf{u}+\mathbf{2} \mathbf{\Omega} \times \mathbf{u}
$$

Letting $l$ be a characteristic length, we can estimate the magnitude of the advection term by $u^{2} / l$ and the magnitude of the Coriolis term by $2 \Omega u$. Their quotient, the Rossby number, then tells us the relative importance of advection vs. Coriolis forces for the flow. For example, on small length scales $R o>1$ and we expect the Coriolis force to be negligible. In these regimes, then, the flow should exhibit three-dimensional character and an inertial cascade leading to viscous dissipation. When $R o<1$ rotation is important and the flow should posess anisotropic character and presumably waves. Hopfinger, Browand, and Gagne devised an experiment which demonstrates these different flow patterns on different scales 


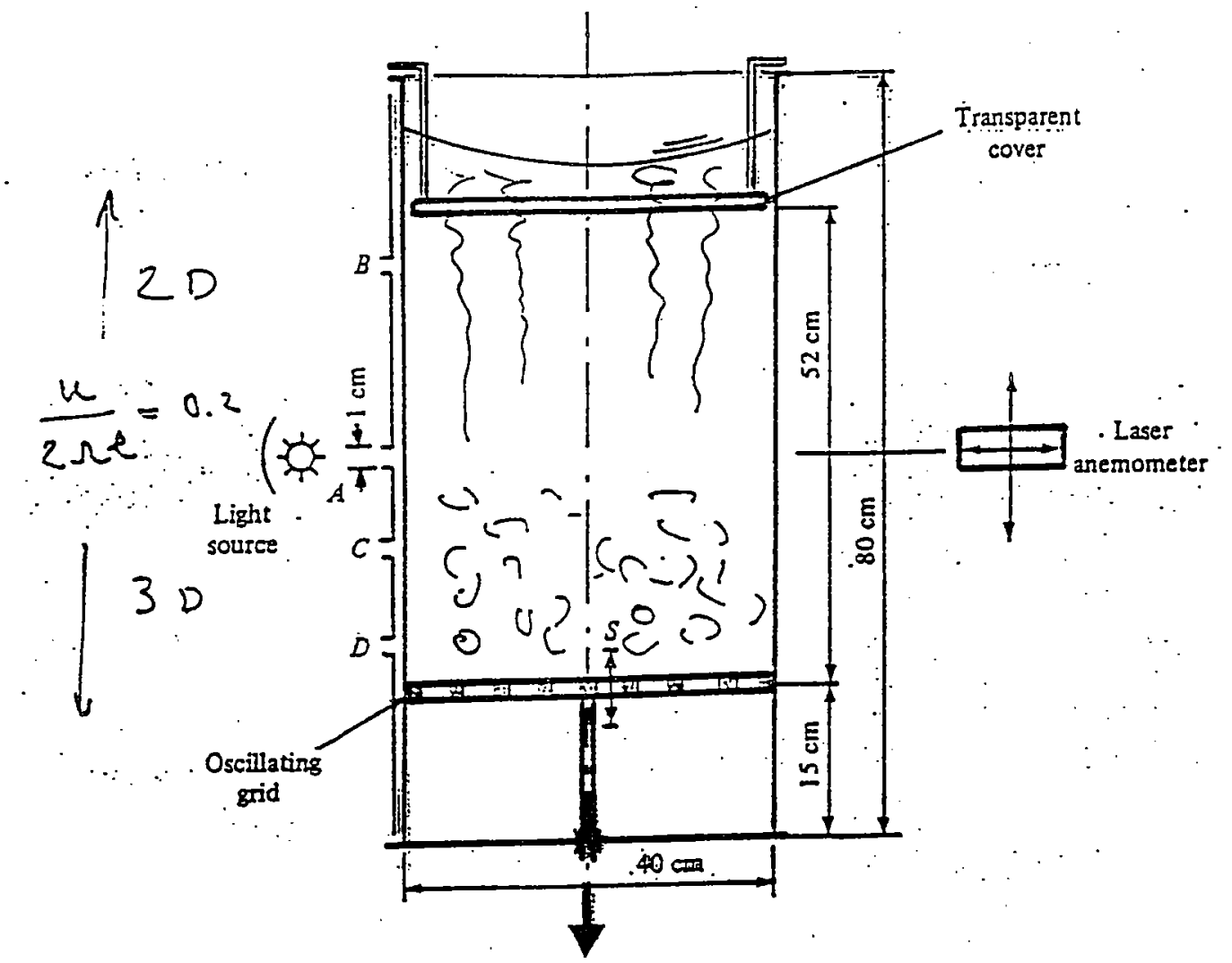

Figure 3: Schematic of the experimental set-up of Hopfinger, Browand, and Gagne with results.

in a rotating fluid. Their set-up, diagrammed with their findings in Fig. 3, consisted of a circular cylindrical tank which could be rotated about its axis. They introduced energy into the system by oscillating a grid close to the bottom end of their tank. They found turbulent eddies near the oscillating grid and oscillatory disturbances toward the other end of the tank, since the turn-over rate scales as $u / l \sim z^{-2}$, the Rossby number decreases with height. The transition from eddies to waves occurred at the critical Rossby number $R o_{\text {crit }}=0.2$, with eddies in the region where $R o>0.2$ and waves in the region where $R o<0.2$ which agrees with our previous arguments.

\subsection{Stabilizing Effect of a Composition Gradient}

Before we resume our discussion of stars, let us briefly discuss an experiment by Stillinger, Mellard, and van Atta. They maintained a salinity gradient in a rectangular tank which they disturbed at one end with an oscillating grid (Fig. 4). Recall that for our purposes in stars, a salinity gradient has the same characteristics as a molecular weight gradient provifded by elements heavier than hydrogen.

Fig. 5 summarizes their results with the following scales also plotted: dissipation scale, characteristic length scale, and the Ozmidov scale, which is the scale for which $u / l=N_{\mu}$, where $N_{\mu}$ is the buoyancy frequency. Their results show the usual progression of eddies near the grid, a transition region with fewer and fewer eddies and more and more wave characteristics, followed by waves only beyond a certain point. What is surprising, 


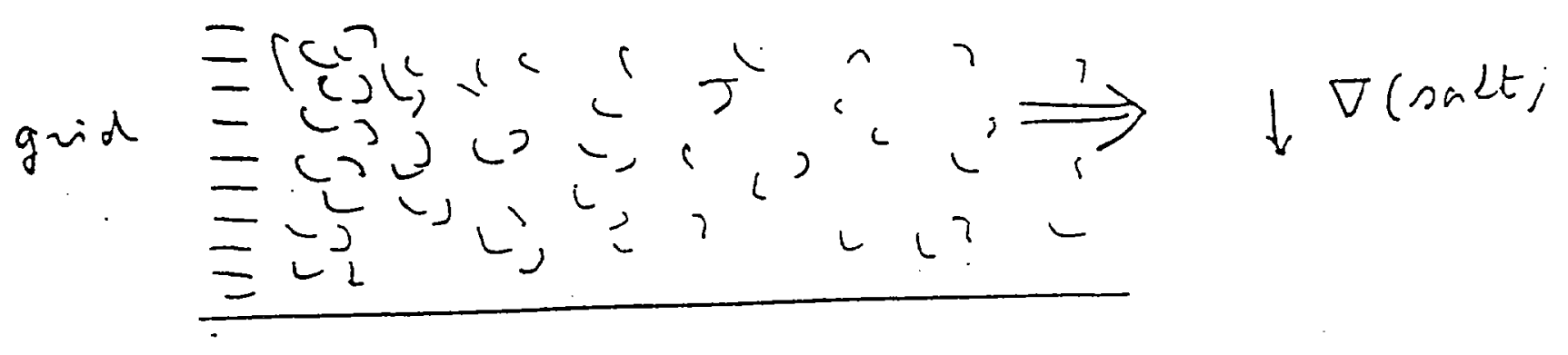

Figure 4: Schematic of experimental set-up of Stillinger et. al., with qualitative results.

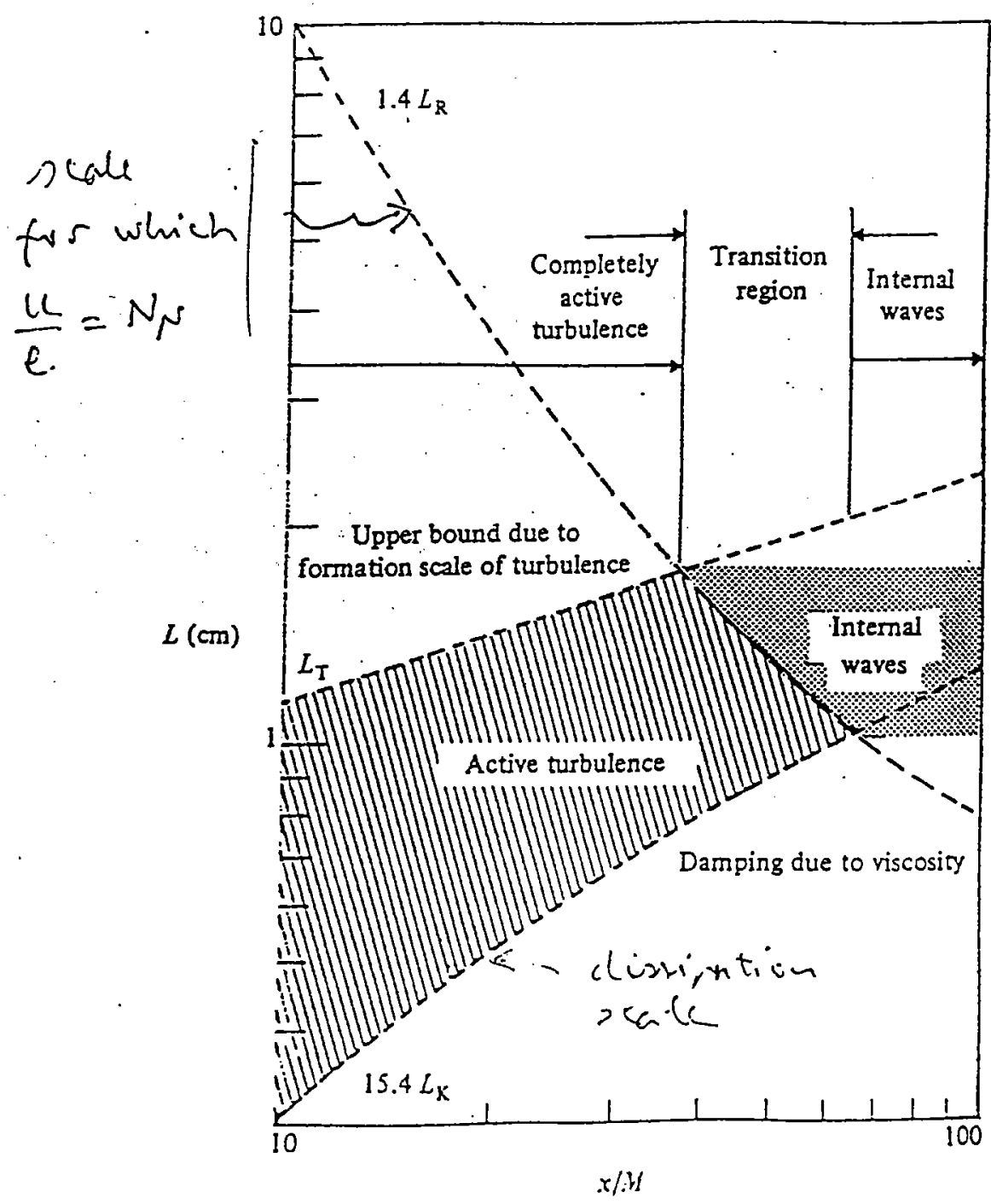

Figure 5: Evolution map for homogenous turbulence in a stably stratified fluid with $L_{T} \ll$ $L_{R}$ initially. 


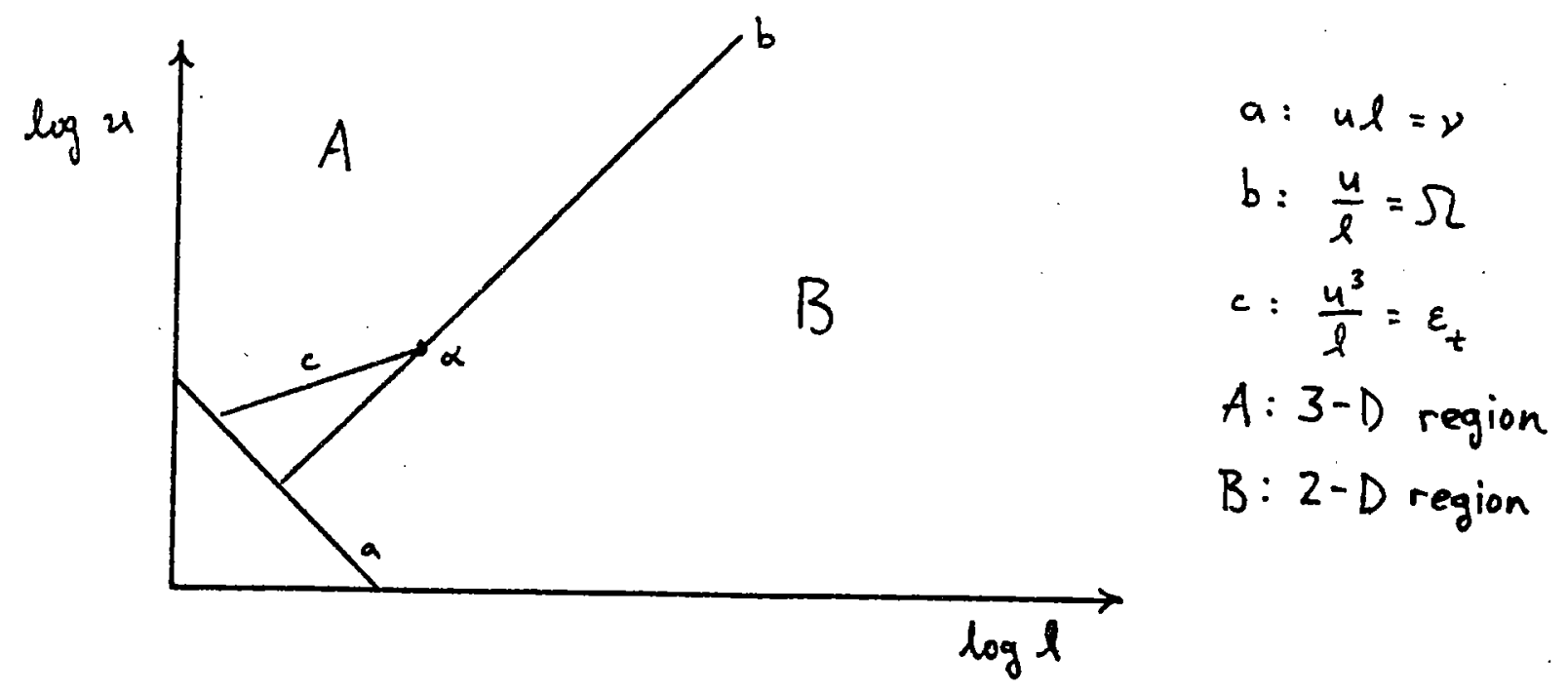

Figure 6: Regions of eddies of size $l$ and velocity $u$.

though, is that the eddy-only turbulent region is so short, and that the wave-only region begins so soon. Thus, the experiment shows that a salinity gradient (i. e. mass gradient in stars) has an anisotropic effect on the flow. Indeed the group reports that the velocity $u \sim z^{-1}$ which means that (since length $l \sim z$ ) $u / l \sim z^{-2}$.

\subsection{Vertical Diffusivity in Rotating Stars}

Let us now use these ideas to begin to build a theory of rotating stars. We will begin by determining the eddy diffusivity. Fig. 6 is a graph showing the distribution of eddies of size $l$ and velocity $u$. Line a, along' which $u l=\nu$, we will call the viscous dissipation line. We are interested in the region above the viscous dissipation line where $R e>1$. Line $\mathrm{b}$, along which $u / l=\Omega$, we will call the Rossby line. The Rossby line divides the region above the viscous dissipation line into two regions: region $A$ above the Rossby line where $R o>1$, and region $\mathrm{B}$ below the Rossby line where $R_{0}<1$. In region A we expect three-dimensional turbulence obeying $u^{3} / l=\epsilon_{t}$, where $\epsilon_{t}$ is the generation rate of turbulent kinetic energy. This relation, which is equivalent to Kolmogorov's $k^{-5 / 3}$ law, is in Fig. 6 as line e, which we will naturally call the Kolmogorov line. In region B, where Coriolis forces make the motion two-dimensional, we expect geostrophic turbulence, or waves. It is clear from the above that the velocity $u$ and the length $l$ are the important parameters. The eddy diffusivity should be their product when they are largest in region A. This is clearly at the intersection of the Rossby line with the Kolmogorov line which is marked as $\alpha$ in Fig. 6. At $\alpha, u=\sqrt{\epsilon_{t} / \Omega}$ and $l=\sqrt{\epsilon_{t} / \Omega^{3}}$. The vertical diffusivity, $D_{\mathrm{v}}$, is thus

$$
D_{\mathbf{v}}=\frac{\epsilon_{t}}{\Omega^{2}}
$$


In the presence of a molecular weight gradient, the Rossby line is replaced by a similar line where $u / l=N_{\mu}^{2}$, when $N_{\mu}^{2}>\Omega^{2}$, and the vertical diffusivity is then

$$
D_{\mathrm{v}}=\frac{\epsilon_{t}}{N_{\mu}^{2}}
$$

\section{Thermal Imbalances in a Barotropic Stars}

Now that we understand how rotation affects instabilities, let us begin to consider its effect on the star as a whole. Recall that although rotating stars can be in hydrostatic equilibrium, since rotation acts as if it generates sources and sinks of energy, radiative equilibrium cannot be achieved, and therefore

$$
\begin{aligned}
\rho T\left(\frac{\partial S}{\partial t}+\mathbf{u} \cdot \nabla S\right) & =-\nabla \cdot \mathbf{F}+\rho \epsilon_{n} \\
& =\rho \epsilon_{\Omega} \neq 0
\end{aligned}
$$

where $\mathbf{F}=-\chi \nabla T$ is the heat flux and $\epsilon_{n}$ is the energy generation rate per unit mass. We will compute $\epsilon_{\Omega}$ in the manner we have done before, except that we will restrict our attention to the barotropic case since this is the only case in which we can express $\epsilon_{\Omega}$ in closed form.

For a barotropic star, the Laplacian of the total potential $\Psi$ is

$$
\nabla^{2} \Psi=-4 \pi G \rho+\frac{1}{s} \frac{d}{d s}\left(\Omega^{2} s^{2}\right)
$$

(Recall that in the barotropic case $S, \rho, \chi, \epsilon_{n}$, etc. are functions of $\Psi$ only). Furthermore

$$
\mathbf{F}=-\chi\left(\frac{d T}{d \Psi}\right) \nabla T
$$

Recalling that $\Omega$ is a function of $s$ only (see Lecture 1, Hydrostatic Equilibrium), we now split the rotational term above into a mean part $\left\langle\Omega^{2}\right\rangle$ over a level surface, which is a function of $\Psi$ only, and its fluctuating part $\left(\Omega^{2}\right)^{\prime}$, which depends also on the latitude:

$$
\frac{1}{s} \frac{d}{d s}\left(\Omega^{2} s^{2}\right)=2\left\langle\Omega^{2}\right\rangle+2\left(\Omega^{2}\right)^{\prime}
$$

In the same way we shall expand the square of the effective gravity:

$$
(\nabla \Psi)^{2}=\left\langle g^{2}\right\rangle+\left(g^{2}\right)^{\prime}
$$


Likewise, the right hand side of eq. 11 can be written

$$
\begin{aligned}
-\nabla \cdot \mathbf{F}+\rho \epsilon= & \left\langle-\nabla \cdot \mathbf{F}+\rho \epsilon_{n}\right\rangle+(-\nabla \cdot \mathbf{F})^{\prime} \\
= & \left\langle\frac{d}{d \Psi}\left(\chi \frac{d T}{d \Psi}\right)\left\langle g^{2}\right\rangle+\left(\chi \frac{d T}{d \Psi}\right)\left(-4 \pi G \rho+2\left\langle\Omega^{2}\right\rangle\right)+\rho \epsilon_{n}\right\rangle \\
& +\left(\frac{\left(g^{2}\right)^{\prime}}{\left\langle g^{2}\right\rangle} \frac{d}{d \Psi}\left(\chi \frac{d T}{d \Psi}\right)\left\langle g^{2}\right\rangle+2\left(\Omega^{2}\right)^{\prime}\left(\chi \frac{d T}{d \Psi}\right)\right)^{\prime} .
\end{aligned}
$$

Note that the $\chi(d T / d \Psi)$, which was constant before, is now in the barotropic case a function of $\Psi$, which we will call $c(\Psi)$. We thus deduce the vector equation

$$
\chi(\Psi) \nabla T=c(\Psi) \nabla \Psi
$$

of which we take the flux over an equipotential surface $\Sigma(\Psi)$. The integral of the left hand term is

$$
\iint_{\Sigma} \chi(\Psi) \nabla T d S=-L(\Psi)
$$

where $L(\Psi)$ is the luminosity, i. e. the total energy traversing that surface, and that of the right hand term is

$$
\begin{aligned}
c(\Psi) \iint_{\Sigma} \nabla \Psi \cdot d S & =c(\Psi) \iiint_{\mathcal{V}} \nabla^{2} \Psi d V \\
& =c(\Psi)\left(-4 \pi G M(\Psi)+2\left\langle\Omega^{2}\right\rangle \mathcal{V}(\Psi)\right)
\end{aligned}
$$

where $M(\Psi)$ and $\nu(\Psi)$ are the mass and the volume contained by the equipotential surface. Hence

$$
c(\Psi)=\frac{L}{4 \pi G M} \frac{1}{1-\Lambda}
$$

where $L, M$, and $\Lambda=\frac{\left\langle\Omega^{2}\right\rangle}{2 \pi G \phi}$ (the oblateness) are functions of $\Psi$. With this value for $c(\Psi)$, and recalling that the mean part of eq. 13 is zero, we get the following expression for the energy generation rate $\epsilon_{\mathbf{\Omega}}$ which is due to the rotation:

$$
\epsilon_{\Omega}=\frac{\left(g^{2}\right)^{\prime}}{\left\langle g^{2}\right\rangle}\left(\frac{L}{M} \frac{\left(1-\frac{\left\langle\Omega^{2}\right\rangle}{2 \pi G_{\rho}}\right)}{1-\Lambda}-\epsilon_{n}\right)-\frac{\left(\Omega^{2}\right)^{\prime}}{2 \pi G \rho} \frac{L}{M} \frac{1}{1-\Lambda} .
$$

Let us examine the sign of $\epsilon_{\Omega}$ in a uniformly rotating star $\left((\Omega)^{\prime}\right)$. In most of the star the nuclear reaction rate, $\epsilon_{\Omega}$, is negligible. The sign of eq. 14 is then governed by two terms: the $\left(g^{2}\right)^{\prime}$ term which is varies with latitude, and the $1-\left\langle\Omega^{2}\right\rangle / 2 \pi G \rho$ term which is a function of depth alone. Since gravity is stronger at the poles and weaker at the equator, it is clear from eq. 12 that $\left(g^{2}\right)^{\prime}>0$ near the poles and $\left(g^{2}\right)^{\prime}<0$ near the equator (since $\left\langle g^{2}\right\rangle$ is 


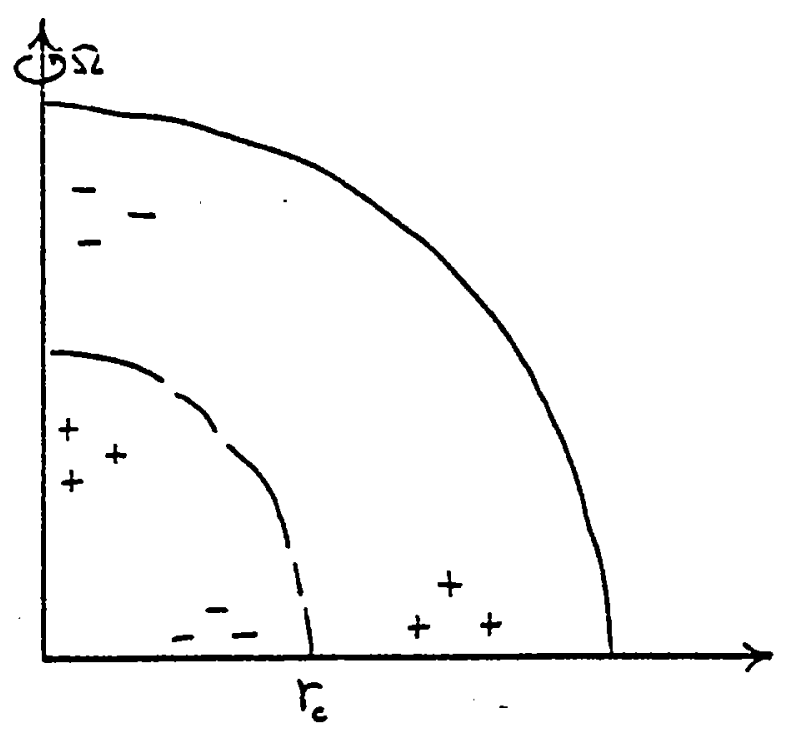

Figure 7: Regions of sources $(+)$ and sinks $(-)$ of rotational energy within a rotating barotropic star. At $r_{c},\left\langle\Omega^{2}\right\rangle / 2 \pi G \rho=1$.

constant). Thus, at a fixed depth, $\epsilon_{\Omega}$ changes sign as one moves from pole to equator. Deep enough within the star the term $\left\langle\Omega^{2}\right\rangle / 2 \pi G \rho$ is small, and so $1-\left\langle\Omega^{2}\right\rangle / 2 \pi G \rho>0$. As one moves outward to the star's surface the density decreases, and at a certain distance from the center, $r_{c},\left\langle\Omega^{2}\right\rangle / 2 \pi G \rho=1$. For $r>r_{c}, 1-\left\langle\Omega^{2}\right\rangle / 2 \pi G \rho<0$. This is summarized schematically in Fig. 7 where "+" represents sources and "-" represents sinks. Since the radial component of the meridional circulation is proportional to $\epsilon_{\Omega}$, the star is divided into two separated circulation cells: an inner one and an outer one. This separation is indeed exhibited in the results of a numerical study conducted by G. G. Pavlov and D. G. Yakolev (Fig. 8).

And finally, let us remark that the term, $\left\langle\Omega^{2}\right\rangle / 2 \pi G \rho$, whose sign was responsible for the division of the rotating barotropic star into inner and outer circulation cells regions (eq. 14), gives rise to a singular perturbation: It is inversely proportional to density $\rho$, and is second order in the oblateness $\Lambda$. Since $\left(g^{2}\right)^{\prime}$ is first order in $\Lambda$, the $1 / \rho$ dependence of $\epsilon_{\Omega}$ means one must be extremely careful at the star's edge, since a first order theory in $\Lambda$ will not exhibit the outer convection cell. It is unfortunate that a whole literature missed this phenomenon.

FIG. 8. Metdional circulation in a s:at in the upper part of the main sequence wlith $\Omega=$ const. The dash-dor Une denotes the flow Interface $\rho=\rho_{\text {.: }}$ : at the "dead" polnts (heavy markdng) the flow veloctry equals zero; $C$ ) convective core.

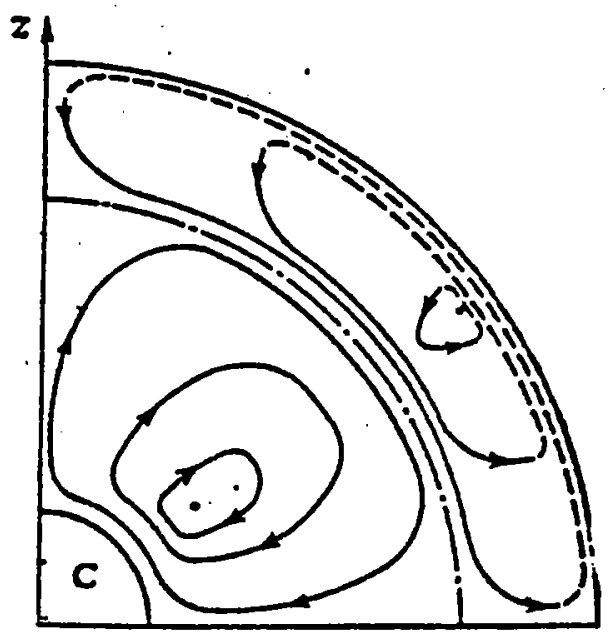




\section{References}

- Coles. JFM 211965 p. 385.

- Hopfinger, Browand, and Gagne. JFM 1251982 p. 505.

- Joseph, D. D. and Hung, W. 1971,Arch. Ration. Mech. Anal. 44, 1.

- Joseph \& Munson. JFM 431970 p. 545.

- G. G. Pavlov and D. G. Yakolev, 1978, Sov. Astron. 22, 595.

- Pedlosky, Geophysical Fluid Dynamics, Springer-Verlag 1987.

- Rhines. JFM 691975 p. 417.

- Stillinger, Mellard, and van Atta. JFM 1311983 p. 91.

- Wendt. Ing. Arch. 41933 p. 577

Notes submitted by George Bell and Eric Won. 
Lecture IX.

\section{Flow Between the Sun's Convection and Radiation Zones and Transport of Chemicals}

\section{Introduction}

In the last lecture we started the discussion of the large scale behavior in space and the long time behavior of stars and pointed out that their basic driving mechanism is the Stokes term and that we deal with a situation of nonthermal equilibrium. Here we want to discuss the influence of stress where we have in particular the sun in mind. Remember that in the convection zone nearly all heat transport is carried by convection. Radiation plays a negligible role. The differential rotation (i.e. that the equator rotates about $30 \%$ faster than the poles) in the convection zone is enforced by Reynolds stresses. We know today from helioseismology that differential rotation goes through the whole convection zone and that in contrast the radiation zone appears to rotate uniformly. This information comes from measurements of the eigenfrequencies of acoustic waves. The splitting of the eigenfrequencies due to Coriolis forces can nowadays be measured with depth and latitude. The latter indicates that no differential rotation takes place in the radiative core of the sun. Thus between the convection zone and the radiation zone there must be a layer in which the switching to differential rotation in the sun's convection zone takes place. Within this boundary layer, which is just below the convection zone, additional circulations have to compensate the jumps in angular momentum. Since the of the convection zone is slower than of the radiation zone over most latitudes, one has to expect Ekman layer like circulation between both similar to Taylor vortices as shown in Fig.1.1.

From observations follows that in this boundary layer seems to be a jump in the angular velocity, but that the average angular momentum varies smoothly. Thus there seems to be no net torque, only a differential torque imposed by by the convection zone. In this lecture we first discuss in more mathematical detail the processes leading to flows in the boundary layer between radiation and convection zone. After that we shortly discuss the transport of a chemical and close the lecture with an application of these results to the Lithium transport. 
Fig.1.1. Convection and radiation zone and meridional circulations between both.

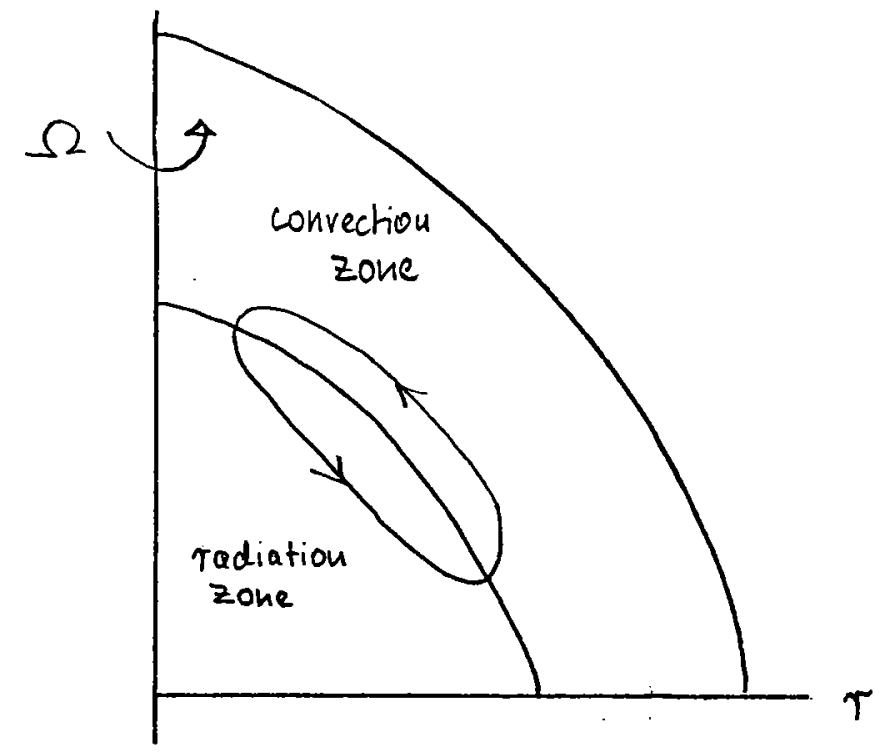

fig. 2.1: Spherical Coordinate System.

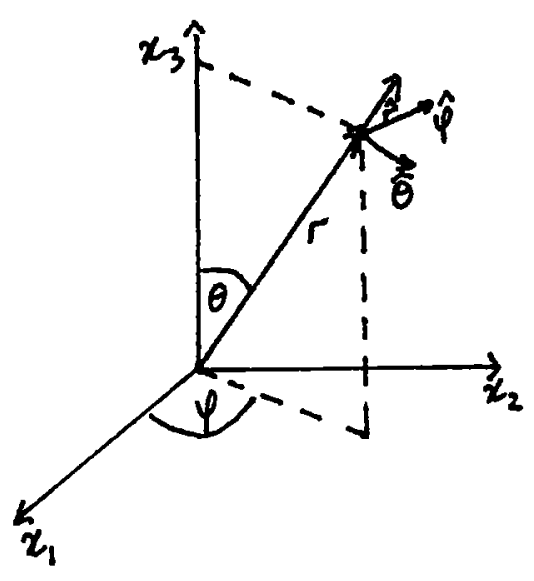




\section{Circulations below a Convective Envelope}

As before, we make the following assumptions:

i) the fluid is in hydrostatic equilibrium;

ii) the fluid motion is axisymmetric (i.e. motion is independent of azimuthal angle);

iii) the anelastic approximation may be made, i.e. the partial time derivative of the density in the equation of mass conservation may be neglected, so that it becomes

$$
\nabla \cdot(\rho \mathbf{u})=0 ;
$$

iv) the Rossby number of the fluid motion is much less than one. (The Rossby number, $R_{0}$, is a measure of the effect of rotation on a fluid motion of a certain scale. The simplest case to consider is that of a body of fluid rotating with constant period $\Omega^{-1}$. A fluid motion scale $L$ and characteristic velocity $U$ has a natural timescale, or period, $L / U$. If this timescale is much less than the timescale of the rotation $(1 / \Omega)$, then the rotation will play a very important role in the evolution of the system. Thus, if we define $R_{0}$ as the ratio of these two timescales, i.e.

$$
R_{0} \equiv \frac{U}{2 \Omega L},
$$

then in the case of $R_{0} \ll 1$, rotation effects are likely to be important).

Above we described the flow in a cylindrical coordinate system. A perhaps more natural system is that of spherical polar coordinates $r, \theta$, and azimuthal angle $\phi$ (see fig. 2.1).

We assume that the oblateness of a spheroid of interest (e.g. the sun), is sufficiently small to allow us to treat the radial direction as perpendicular to horizontal surfaces without introducing significant errors. We are interested in stationary solutions. In this case, we split all the dependent variables into two parts, one part that is a pure function of $r$, that we know a priori, and an (assumed) perturbative part that is a function of both $r$ and $\theta$, i.e.

$$
\begin{aligned}
p(r, \theta) & =p_{0}(r)+\epsilon p_{1}(r, \theta), \\
\rho(r, \theta) & =\rho_{0}(r)+\epsilon \rho_{1}(r, \theta), \\
\Omega(r, \theta) & =\Omega_{0}(r)+\epsilon \Omega_{1}(r, \theta), \\
T(r, \theta) & =T_{0}(r)+\epsilon T_{1}(r, \theta), \\
(\epsilon & \ll 1) .
\end{aligned}
$$

In a steady state of constant rotation, the fluid velocity is defined by the relation

$$
\mathbf{u}=\mathbf{r} \times \mathbf{\Omega},
$$

and since

$$
\boldsymbol{\Omega} \equiv \hat{\mathbf{r}}(\Omega \cos \theta)-\hat{\theta}(\Omega \sin \theta),
$$

then the unperturbed velocity has only one nonzero component, which is in the azimuthal direction and is given by the following relation:

$$
\bar{u}_{\phi}=r \Omega \sin \theta .
$$


Thus, in the perturbed case, if we wish to consider small deviations from solid body rotation, we consider a velocity vector

$$
\mathbf{u}=\epsilon\left(u_{\mathbf{r}}, u_{\theta}, u_{\phi}\right)^{T},
$$

and we assume that, to first order, $u_{\phi}$ has an analogous form to the unperturbed flow, i.e.

$$
u_{\phi}=r \Omega_{1}(r, \theta) \sin \theta .
$$

We now consider, in spherical polar coordinates, the axisymmetric equations of motion for such a small velocity perturbation. Remembering that we have assumed axisymmetry, and hydrostatics, the equations of motion and mass conservation, assuming viscosity remains constant, can be expanded in powers of $\epsilon$. We make the further assumption that the scale over which the vertical velocity changes is very much smaller than the scale over which the density changes. In this case we treat density as constant except in the buoyancy term in the momentum equation. This is known as the Boussinesq approximation. The only zero order terms in $\epsilon$ occur in the radial component of the momentum equation, and express hydrostatic balance, i.e.

$$
\frac{\partial}{\partial r} p_{0}=\rho_{0} g .
$$

For simplicity, we shall ignore all terms except the $\nabla^{2} u$ terms in the viscous drag, and we assume that the viscosity $\nu$ is constant. In this case, the governing equations, reduce to a linear set. The momentum equation, when decomposed into $r, \theta$ and $\phi$ coordinate directions respectively, becomes, remembering that all terms are independent of $\phi$,

$$
\begin{aligned}
-2 \Omega_{0} \Omega_{1} r \sin ^{2} \theta= & -\frac{1}{\rho_{0}} \frac{\partial}{\partial r} P_{1}+g \frac{\rho_{1}}{\rho_{0}} \\
& +\frac{\nu}{r^{2}}\left[\frac{\partial}{\partial r}\left(r^{2} \frac{\partial}{\partial r} u_{r}\right)+\frac{1}{\sin \theta} \frac{\partial}{\partial \theta}\left(\sin \theta \frac{\partial}{\partial \theta} u_{r}\right)\right] ; \\
-2 \Omega_{0} \Omega_{1} r \sin \theta \cos \theta= & -\frac{1}{\rho_{0} r} \frac{\partial}{\partial \theta} P_{1} \\
& +\frac{\nu}{r^{2}}\left[\frac{\partial}{\partial r}\left(r^{2} \frac{\partial}{\partial r} u_{\theta}\right)+\frac{1}{\sin \theta} \frac{\partial}{\partial \theta}\left(\sin \theta \frac{\partial}{\partial \theta} u_{\theta}\right)\right] ; \\
2 \Omega_{0}\left(u_{r} \sin \theta+u_{\theta} \cos \theta\right)=\nu & {\left[\frac{\sin \theta}{r^{3}} \frac{\partial}{\partial r}\left(r^{4} \frac{\partial}{\partial r}\left[\Omega_{1}\right]\right)\right.} \\
& \left.+\frac{1}{r \sin ^{2} \theta} \frac{\partial}{\partial \theta}\left(\sin ^{3} \theta \frac{\partial}{\partial \theta} \Omega_{1}\right)\right]
\end{aligned}
$$

The equation for the conservation of mass becomes

$$
\frac{1}{r} \frac{\partial}{\partial r}\left(r^{2} \rho_{0} u_{r}\right)+\frac{\rho_{0}}{\sin \theta} \frac{\partial}{\partial \theta}\left(\sin \theta u_{\theta}\right)=0 .
$$

For closure, we also require a balance of heat. We assume that conductivity or opacity is constant, and also that we can ignore the effect of $\epsilon_{N}$, the nuclear reaction rate as a 
source of heat. If furthermore, there are no sources or sinks of heat of any kind, there must be a balance between the advection and diffusion of heat. We define a characteristic lengthscale of entropy advection

$$
H \equiv H_{p}\left[\left(\frac{\partial \ln T}{\partial \ln P}\right)_{a d}-\left(\frac{\partial \ln T}{\partial \ln P}\right)\right]^{-1},
$$

where subscript ad denotes an adiabatic motion, and $H_{P}$ is the pressure scale height. Then, to first order in $\epsilon$, heat balance requires

$$
\frac{T_{0} u_{r}}{H}=\frac{\kappa}{r^{2}}\left[\frac{\partial}{\partial r}\left(r^{2} \frac{\partial}{\partial r} T_{1}\right)+\frac{1}{\sin \theta} \frac{\partial}{\partial \theta}\left(\sin \theta \frac{\partial}{\partial \theta} T_{1}\right)\right] .
$$

Equations 2.2-6 constitute a set of five equations in the five unknown perturbative parts of the dependent variables, i.e. $\rho_{1}, T_{1}, \Omega, u_{r}$, and $u_{\theta}$. We then look for separable solutions for $r$ near to $R$ but within the radiative zone (i.e. $r \leq R$ ) of the form (in the example of pressure)

$$
P_{1} \propto P(\theta) \exp (-k \xi)
$$

where

$$
\xi \equiv R-r,
$$

and $k$, the inverse of a "scale height" is assumed "large" in some sense, defined as

$$
R k \gg 1 \text {. }
$$

In general, for a variable $V$ of this form,

$$
\frac{\partial}{\partial r} V=k V
$$

We see simple scaling for balance in (2.5) implies that

$$
R k u_{\mathrm{r}} \sim u_{\theta} .
$$

Thus

$$
u_{r} \ll u_{\theta} \text {. }
$$

We now investigate the implications of scalings (2.7) and (2.8). In (2.6), since $R k$ is large, we may ignore the second term on the right hand side, and so we have a balance

$$
\frac{u_{r}}{H}=\kappa k^{2} \frac{T_{1}}{T_{0}} .
$$

Now, if we have a perfect gas, to first order we may approximate $\rho_{1} / \rho_{0}$ as $T_{1} / T_{0}$. Since in (3), the effect of differential rotation must be sufficiently strong to balance the latitudinal pressure gradient, the effective balance is

$$
-2 \Omega_{0} \Omega_{1} R \sin \theta \cos \theta=-\frac{1}{\rho_{0} R} \frac{\partial}{\partial \theta} P_{1} .
$$


Therefore, the Coriolis term is negligible in (2.2), which thus reduces to simple hydrostatic balance, i.e.

$$
\frac{1}{\rho_{0}} k P_{1}=g \frac{T_{1}}{T_{0}}
$$

Only in the equation in the azimuthal direction (i.e. (2.4)) is viscosity important at this order. Remembering (2.7) and (2.8), we see that the most significant balance in (2.4) is

$$
2 u_{\theta} \Omega_{0} \cos \theta=\nu k^{2} \Omega_{1} R \sin \theta .
$$

Collating all these results, we see that the problem reduces to solving the mass conservation equation for the $\theta$ dependence of $u_{\theta}$, i.e.

$$
R k u_{r}+\frac{1}{\sin \theta} \frac{\partial}{\partial \theta}\left(\sin \theta u_{\theta}\right)=0
$$

with the scaling relations (2.9-12). Now (2.9) and (2.11) imply

$$
u_{r}=\frac{\kappa k^{3} H}{\rho_{0} g} P_{1}
$$

If we differentiate (2.13) with respect to $\theta$, and use (2.14), we see that the terms in $u_{r}$ cancel, and

$$
\frac{\partial}{\partial \theta}\left[\frac{1}{\sin \theta} \frac{\partial}{\partial \theta}\left(\sin \theta u_{\theta}\right)\right]+\frac{\kappa k^{4} R H}{\rho_{0} g} \frac{\partial}{\partial \theta} P_{1}=0 .
$$

But, using (2.10) and (2.12), we see that

$$
\begin{aligned}
\frac{\partial}{\partial \theta} P_{1} & =2 \rho_{0} \Omega_{0} \Omega_{1} R^{2} \sin \theta \cos \theta \\
& =\rho_{0} \Omega_{0} R^{2} \sin \theta \cos \theta\left(\frac{2 \Omega_{0} u_{\theta} \cos \theta}{\nu k^{2} \Omega_{1} R \sin \theta}\right) \\
& =\frac{4 \rho_{0} \Omega_{0}^{2} R u_{\theta} \cos ^{2} \theta}{\nu k^{2}}
\end{aligned}
$$

Thus combining (2.15) and (2.16),

$$
\frac{\partial}{\partial \theta}\left[\frac{1}{\sin \theta} \frac{\partial}{\partial \theta}\left(\sin \theta u_{\theta}\right)\right]+\left[\frac{4 \kappa k^{2} R^{2} H \Omega_{0}^{2}}{\nu g}\right] \cos ^{2} \theta u_{\theta}=0
$$

or

$$
\frac{\partial}{\partial \theta}\left[\frac{1}{\sin \theta} \frac{\partial}{\partial \theta}\left(\sin \theta u_{\theta}\right)\right]+\lambda^{2} \cos ^{2} \theta u_{\theta}=0
$$

where

$$
\lambda^{2}=\frac{4 \kappa k^{2} R^{2} H \Omega_{0}^{2}}{\nu g}=\left(\frac{2 \Omega_{0} k R}{N r^{1 / 2}}\right)^{2}
$$


and $\operatorname{Pr}$ is the Prandtl number, (the ratio of molecular to thermal diffusivities), and $N$ is the Brunt-Vaïsäla frequency.

If we make the substitution $\cos \theta=x$, and $\sin \theta u_{\theta}=f(x)$, we see that we impose the boundary conditions that $f( \pm 1)=0$ and

$$
\frac{d}{d x}=-\frac{1}{\sin \theta} \frac{d}{d \theta}=-\frac{1}{\left(1-x^{2}\right)^{1 / 2}} \frac{d}{d \theta},
$$

and (2.17) reduces to

$$
-\left(1-x^{2}\right)^{1 / 2} \frac{d}{d x}\left(-\frac{d}{d x} f\right)+\frac{\lambda^{2} x^{2}}{\left(1-x^{2}\right)^{1 / 2}} f=0
$$

or

$$
\frac{d^{2}}{d x^{2}} f+\frac{\lambda^{2} x^{2}}{\left(1-x^{2}\right)} f=0
$$

with

$$
f( \pm 1)=0 \text {. }
$$

Now, (2.18) is an equation for the $\theta$ dependence of $u_{\theta}$. We have already assumed that the $r$ dependence is of the form $\exp (-k \xi),(\xi=R-r)$. So, from (2.12), we obtain an expression for the differential rotation $\Omega_{1}$, namely.

$$
\Omega_{1}=\frac{2 u_{\theta} \Omega_{0} \cos \theta}{\nu R k^{2} \sin \theta}=\Delta \Omega \exp (-k \xi) \frac{x}{1-x^{2}} f(x)
$$

using (2.18) and defining

$$
\Delta \Omega \equiv \frac{2 \Omega_{0}}{\nu k^{2}}
$$

the scaled "variability" in the rotation rate.

Now from (2.13), we see that

$$
\begin{aligned}
R u_{r} & =-\frac{1}{k \sin \theta} \frac{\partial}{\partial \theta}\left(\sin \theta u_{\theta}\right) \\
& =\frac{1}{k} \frac{d}{d x} f \exp (-k \xi) \\
& =\frac{\nu(k R)}{2}\left(\frac{\Delta \Omega}{\Omega_{0}}\right) \exp (-k \xi) \frac{d}{d x} f, \\
& =\frac{2 \Omega^{2} H \kappa(k R)^{3}}{g \lambda^{2}}\left(\frac{\Delta \Omega}{\Omega_{0}}\right) \exp (-k \xi) \frac{d}{d x} f
\end{aligned}
$$

using (2.17) and (2.21). Similarly, we may write

$$
\begin{aligned}
R \sin \theta u_{\theta} & =R \exp (-k \xi) f(x) \\
& =\frac{\nu(k R)^{2}}{2}\left(\frac{\Delta \Omega}{\Omega_{0}}\right) \exp (-k \xi) f(x) \\
& =\frac{2 \Omega^{2} H \kappa(k R)^{3}}{g \lambda^{2}}\left(\frac{\Delta \Omega}{\Omega_{0}}\right) \exp (-k \xi) f(x)
\end{aligned}
$$


Let us now consider the physical implications of this solution. Firstly, the entire solution is driven at $\xi=0$, and by the definition of $\xi$, we only consider solutions penetrating downwards into the radiative interior. This model does not attempt to describe the complex motions in the convective zone, although if the porosity is large, matching with models utilising such concepts as eddy viscosity is at least consistent along the boundary, since from (2.24-25), radial velocities can be considered to be driven by either viscosity, $\nu$, or thermal diffusivity, $\kappa$.

If we turn our attention to the form of the solutions for $f$ i.e. the $\theta$ dependence of the velocity field satisfying (2.18-19), we start from the assumption that there is an equatorial plane of symmetry. In that case we investigate odd solutions for $f$. Without going into the full solution, we see that, for small $x$, the solution approaches

$$
f(x) \sim x-\frac{\lambda^{2} x^{5}}{20} .
$$

We may roughly sketch the solution curve thus:

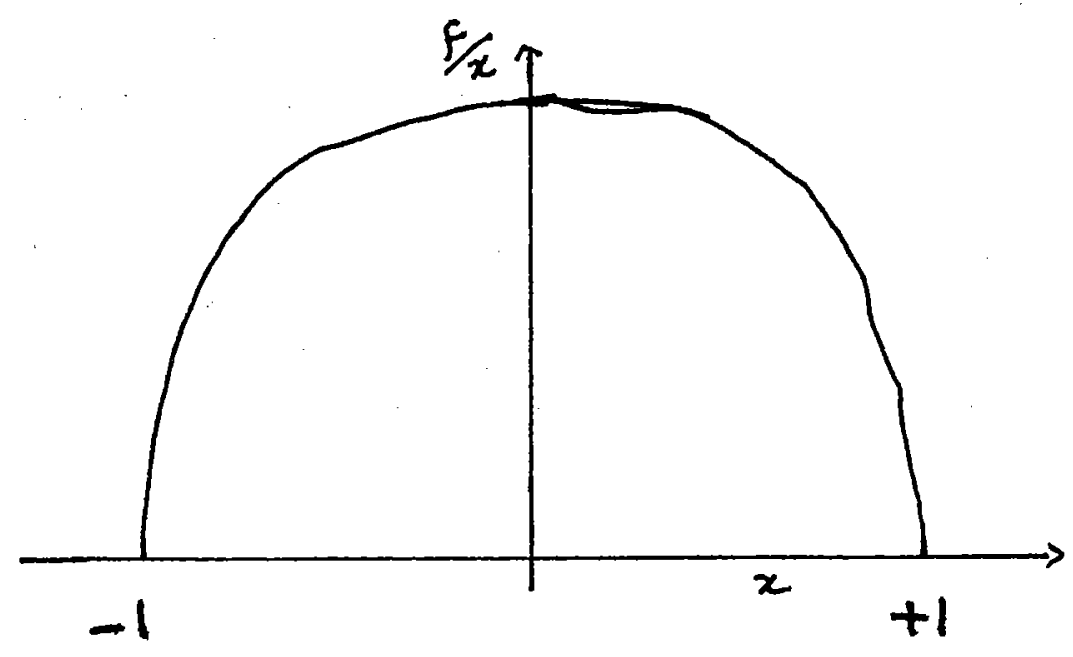

fig 2.2: Sketch of Solution Curve.

Thus we have a circulation near the interface with the convective zone, which may be considered to be a thermal boundary layer. However, closer to the core, motions driven by heat sinks and sources ( see the Eddington-Sweet circulation above) overcome the effects of differential rotation. We can compare timescales for the two separate circulations. We already know that the timescale for the Eddington-Sweet circulation can be taken as

$$
t_{E S} \sim t_{K H}\left(\frac{G M}{\Omega^{2} R^{3}}\right),
$$

where $t_{K H}$, the Kelvin-Helmholtz timescale is $R^{2} / \kappa$. Thus

$$
t_{E S} \sim \frac{g R}{\kappa \Omega^{2}} \sim \frac{N^{2} R H}{\kappa \Omega^{2}} .
$$


From (2.23), a typical timescale of the motions due to differential rotation is

$$
\begin{aligned}
\frac{1}{R u_{r}} & =\frac{g \lambda^{2}}{2 \Omega^{2} H \kappa(k R)^{3}}\left(\frac{\Delta \Omega}{\Omega_{0}}\right)^{-1} \exp (-k \xi) \frac{d}{d x} f \\
& \sim\left(\frac{N^{2} R H}{\kappa \Omega^{2}}\right)\left(\frac{\Omega_{0} \exp (-k \xi)}{\Delta \Omega(k R)^{3} R H} \frac{d}{d x} f\right) \\
& \sim t_{E S}\left(\frac{\Omega_{0} \exp (-k \xi)}{\Delta \Omega(k R)^{3} R H} \frac{d}{d x} f\right)
\end{aligned}
$$

In the Sun

$$
\frac{\Delta \Omega}{\Omega} \sim 0.3 .
$$

Thus, when $\xi$ is small, this process due to differential rotation may be important. But in the above equation, $k R$ is large (as assumed in the model), and hence the effect of the circulation driven by the differential rotation rapidly drops. Higher modes, i.e. those modes that occur with larger $\lambda$, have even narrower shells of effect. Thus a cellular rotation structure will be restricted to a region very close to the interface between the convective and radiative zones. At larger depths, the Eddington-Sweet circulation must dominate. However, this model is extremely simplistic. The differential rotation is unstable, and the turbulent motions generated by such instabilities give rise to an eddy viscosity which depends on depth. This problem is then no longer linear. Although the $\theta$ dependence can still be obtained by the above method, the full equations must be solved in $r$ for the vertical dependence, assuming, as usual, that viscosity does not vary with latitude. This approach is, nevertheless, beyond the scope of these lectures. 


\section{Transport of a chemical}

When inhomogeneities develop in a star, one can ask for the effects of varying chemical composition. The mass balance equation for a chemical with concentration $c$ reads

$$
\frac{\partial}{\partial t}(\rho c)+\nabla \cdot(\rho c \mathbf{U})=\nabla \cdot\left(D_{t} \rho \nabla c\right)
$$

Here the second term on the left hand side represents the advection of the chemical, the right hand side the difussion with $D_{t}$ being the total diffusivity. A concentration flux due to advection through the surface $\Sigma$

$$
\phi=\int_{\Sigma} \rho c \mathbf{u} \cdot \mathbf{d} \mathbf{S}
$$

can only be nonzero if $c$ varies over $\Sigma$. Let $\Sigma$ be a horizontal surface at radius $r$. Then the total concentration at a radius $r$ can be decomposed in the mean concentration $c_{0}(r)$ and fluctuations $\delta c(r, \theta)$

$$
c(r, \theta)=c_{0}(r)+\delta c(r, \theta)
$$

representing the vertical changes of $c(r, \theta)$ at radius $r$. In general, horizontal and vertical anisotropies due to concentration are possible. In the sun the horizontal transport is likely to be much more effective than the one in the vertical, due to the instabilities generated by differential rotation. The basic problem in the following will be to estimate $\delta c$. We follow along the lines of G.I. Taylor in his discussion of the diffusion in a pipe, but in contrast to that work we have here no mean velocity. The equations for the mean vertical transport equation following from concentration field equation is given by

$$
\frac{\partial}{\partial t}\left(\rho c_{0}\right)+\frac{1}{r^{2}} \frac{d}{d r}\langle\rho U \delta c\rangle=\frac{1}{r^{2}} \frac{d}{d r}\left(D_{\nu} \rho r^{2} \frac{d c_{0}}{d r}\right)
$$

Here $d_{\nu}$ denotes the vertical diffusivity and the brackets denote averaging over a level surface. The second term on the right hand side of eq. (3.4) represents the advection controlled by the transport of $\delta c$. We know from Lecture 8 that

$$
D_{\nu} \sim \frac{\epsilon_{t}}{\Omega^{2}}
$$

where $\epsilon_{t}$ is the turbulent viscous dissipation,which depends on the amount of differential rotation $\delta \Omega$. To estimate the strength of the advection term in eq. (3.4) we need an estimation of $\delta c$ which can be obtained as follows:

One multiplies eq. (3.1) written in terms of $\delta c$ and carries out lateral averaging. Then one finds

$$
\frac{1}{2} \frac{\partial}{\partial t}\left\langle\rho(\delta c)^{2}\right\rangle+\left\langle\delta c \rho U_{r} \frac{d}{d r} c_{0}\right\rangle+\langle\delta c \rho \mathrm{U} \cdot \nabla \delta c\rangle=\left\langle\delta c \nabla \cdot\left(D_{t} \rho \nabla \delta c\right)\right\rangle
$$

Assuming that 
- horizontal diffusion is very strong, i. e. that $D_{t}$ can be replaced by $D_{H}$,

- $|\delta c| \ll d c_{0} / d r$, and

- quasi-stationarity holds,

the second term on the left hand side of eq. (3.6) is equal to the right hand side of eq. (3.6). Replacing $\nabla$ by $\frac{1}{R}$ leads finally to

$$
|\delta c| \sim-\left|U_{\digamma}\right| \frac{d c_{0}}{d r} \frac{R^{2}}{D_{H}}
$$

where $|\ldots|$ denotes the maximum. The important point is that $|\delta c|$ is proportional to the radial concentration gradient. Inserting the above formula into the advective part of the mean vertical transport equation gives

$$
\frac{1}{r^{2}} \frac{d}{d r} r^{2}\langle\rho \mathrm{U} \delta c\rangle \simeq \frac{1}{r^{2}} \frac{d}{d r}\left(D^{*} \rho r^{2} \frac{d c_{o}}{d r}\right)
$$

with

$$
D^{*} \sim \frac{\left|U_{r}\right|^{2} R^{2}}{D_{H}} .
$$

Thus the transport of a chemical can be written in form of a diffusion equation with a diffusion constant $D^{*}$, which shows that the Eddington Sweet circulation actually becomes a diffusive process. For more details concerning the estimation of the maximum value of $\delta c$ we refer to the fellow contribution of Brian Chaboyer.

\section{Transport of Lithium}

Let us finally discuss an experimental result which supports the existence of transport below the convection zone. Lithium is burned typically at temperatures around $210^{6} \mathrm{~K}$, thus well in the radiation zone of the sun. If there would not exist a motion below the convection zone, whose bottom is at temperatures of $10^{6} \mathrm{~K}$, would keep its original abundance. But the observations show that it is depleted by a factor of about $10^{3}$. The same mild transport seems to occur in other stars. In Fig. 4.1 the abundance of Lithium for Hyades dwarfs is shown as function of the surface temperature (or the mass of the stars). Also there Lithium is transported to the surface-probably due meridional circulation. The deep well for $T_{\text {eff }}$ about $6700 \mathrm{~K}$ is one up to now not well explained feature. 


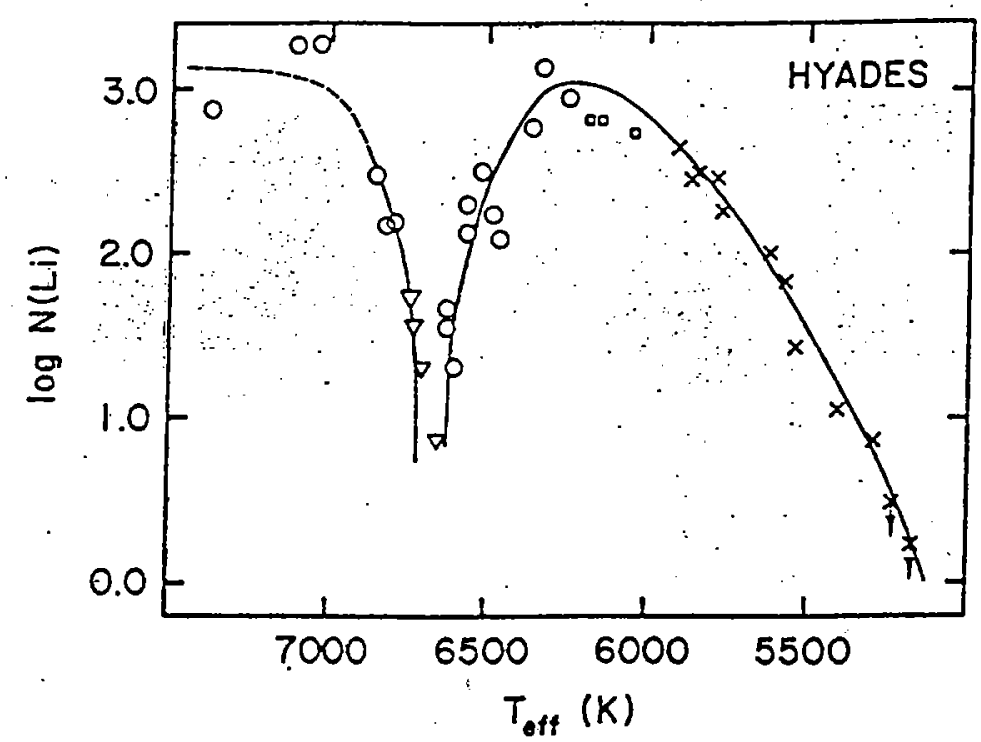

Fig. 4.1. - Lithium abundance [on the scale of $\log N(\mathrm{H})=12.00$ ] for the Hyades dwarfs as a function of effective temperature. The open circles and open rriangles correspond to detections and upper timits. respectively. The crosses are the $G$ dwarf datz of Cayrel et al. (1984). The small open squares are abundances from equivalent widths of Duncan and Jones (198j) from spectri taken at Lick Observatory.

Notes submitted by Stefan Linz and Colm-cille Caulfield. 


\section{Lecture X. Vibrational Instabilities}

\section{Unstable Stars}

Though most stars are changing slowly and can be considered hydrostatic, they also fluctuate about the hydrostatic state with diverse time scales and amplitudes. Probably, every star has some variability that can be detected by sufficiently careful and extensive observations. Astronomers have qualitatively classified many kinds of variability, and the members of each class are called $X$-type variables, where $X$ is the name of the star that is the prototype of the class of variables. We would require too much time to go into all the classes and what they do, so we will simply mention some of the favorites. These are best indicated by their place in the $\mathrm{H}-\mathrm{R}$ diagram.

Figure 1 is a sketch of the H-R diagram showing the zero age main sequence (ZAMS) which represents the end of the contraction phase and the start of nuclear burning in the core. The point 1 , the Kumar limit represents the lowest possible mass $\left(\sim 0.05 \mathcal{M}_{\odot}\right)$ for which the temperature at the core is hot enough to initiate nuclear burning of hydrogen. There may exist stars below this limit, and their possible existence is suggested by the the dashed line, which possibly continues on to JUpiter. Point 2 marks the place on the main sequence where stars first encounter the Eddington limit. At the critical mass represented by point $2,\left(\sim 70 \mathcal{M}_{\odot}\right)$ the outward radiative force on the matter at the surface of the star balances the gravitational attraction. If more matter is added to the star, the luminosity is raised and the excess matter is thought to be blown away by the radiative force.

The most important stellar variability occurs in the nearly vertical strip shown in the figure. This is called the Cepheid instability strip, after the Cepheid variables that lie in the upper portion of the instability strip. These stars vary nearly periodically in luminosity and surface velocity, as a result of an instability that we shall describe presently. The Cepheid variables, whose periods are measured in days and weeks, have played a very important role in the subject because their luminosities are well correlated with their periods of variation. They can be used to gauge the distance to any stellar system in which they can be observed, once the period-luminosity law is calibrated. That turned out to be a difficult matter because the stars in the lower part of the strip, with shorter periods, are not the same as the classical Cepheids, but they were the only lind near enough to us for a luminosity to be well determined. There were some false steps made on this account that had ramifications for estimates of the scale of the universe. It is also interesting that the strip reaches down to the location of the sun in the diagram. Indeed, the sun also is subject to overstabilities but, the sun's gravest modes are not unstable, in contrast to the case of the classical Cepheid variables.

The stars well above the main sequence and cooler than Cepheids also tend to be variable, but they are not periodic. Much less is understood about these semi-regular and irregular variables than about the Cepheids. There are several other kinds of variation throughout the diagram. For example, the stars in the neighborhood of the $Z$ in $Z A M S$ vary for unkown causes. These $\beta$ Canis Majoris stars are especially interesting in GFD 


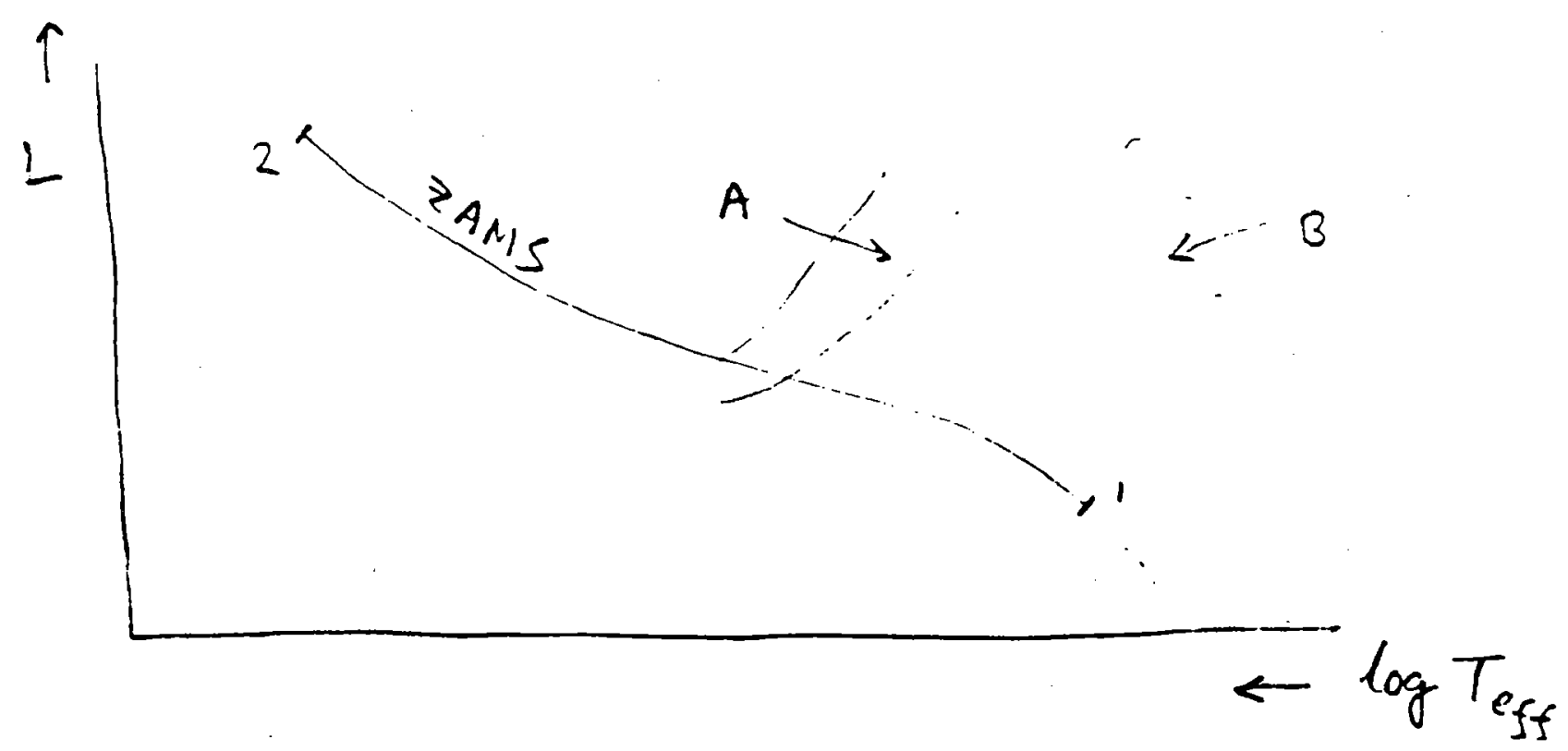

Figure 1: The H-R digram and the instability strip

because they have an oscillatory double-diffusive instability in progress, with helium as the slow diffuser.

Another evident kind of variability is that associated with the solar cycle, and it is interesting in having a time scale measured in decades. Hints of similar variations have been detected in cool stars. It is not yet clear what fixes the time scale of the solar variability. This is in stark contrast to the simpler variation of the Cepheids, whose time scale is the travel time of a sound wave across the star. The solar variability has a time scale that may be set by the travel time of the wave of activity that moves from midlatitudes to the equator. Proctor and I believe that this wave is an envelope of overstable dynamo waves, which suggests that its propagation speed is controlled indirectly by the turbulent processes that produce the subconvective dynamo.

\section{The $\kappa$-mechanism.}

When a star expands a bit, it cools, but it is not clear whether the increased area or the decreased temperature wins in determining its modified emergent radiation. Depending on thermal effects or phases, this could go either way. If the star radiates more when it is cool, the perturbation will die away, but if energy is put in during the hot phase, that is destabilizing. Of course such remarks are too vague to be of help in understanding stellar instability, and there is no very simple physical argument for explaining it. We shall instead summarize a pedagogical model of N.H. Baker (1966) that clarifies the physical mechanism responsible for the pulsational overstability in Cepheids. The mechanism 
arises in fluctuations in the opacity $\kappa$ and was proposed by Eddington.

In a spherical distribution of matter, the mass $\mathcal{M}_{r}$ contained in a sphere of radius $r$ is a monotonically increasing function of $r$. If the star remains radial, $\mathcal{M}_{r}$ is a Lagrangian coordinate and we will use it as independent variable with $0<\mathcal{M}_{\mathbf{r}}<\mathcal{M}$. The equation describing how I varies with $\mathcal{M}$ is

$$
\frac{\partial r}{\partial \mathcal{M}_{r}}=\frac{1}{4 \pi r^{2} \rho}
$$

where $\rho\left(\mathcal{M}_{r}, t\right)$ is the density. The equation of motion for a thin shell of mass $d \mathcal{M}_{\mathrm{r}}$ is

$$
\frac{\partial p}{\partial \mathcal{M}_{r}}=-\frac{1}{4 \pi r^{2}}\left(\frac{G \mathcal{M}_{r}}{r^{2}}+\frac{\partial^{2} r}{\partial t^{2}}\right)
$$

where $p\left(\mathcal{M}_{r}, t\right)$ is the gas pressure. For an ideal gas, the energy equation for the fluid between $\mathcal{M}_{r}$ and $\mathcal{M}_{r}+d \mathcal{M}_{r}$ is

$$
\frac{\partial L}{\partial \mathcal{M}_{r}}=-C_{p} \frac{\partial T}{\partial t}+\frac{\delta}{p} \frac{\partial p}{\partial t}
$$

where $L\left(\mathcal{M}_{r}, t\right)$ is the luminosity, $T\left(\mathcal{M}_{r}, t\right)$ is the absolute temperature, $C_{p}$ is the specific heat per unit mass at constant pressure and

$$
\delta=-\left(\frac{\partial \ln \rho}{\partial \ln T}\right)_{p}
$$

The condition for radiative equilibrium is

$$
L=-\frac{64 \pi^{3} a c \sigma^{4} T^{3}}{3 \kappa} \frac{\partial T}{\partial \mathcal{M}_{r}}
$$

where $a, c, \sigma$ are fundamental constants and $\kappa$ is the opacity. Equation (2.4) is a good approximation only in the optically thick region of the star and under conditions of local thermodynamic equilibrium. We will assume (2.4) to be valid even in the outer envelope as we are only interested in a qualitative understanding. Lastly, we have the ideal gas law

$$
p=\frac{k}{\mu m_{B}} \rho T
$$

where the mean molecular weight $\mu$ is assumed constant and $k$ is the Boltzmann's constant.

\subsection{One-zone approximation}

Equations (2.2) to (2.5) can be solved under suitable boundary conditions when $\frac{\theta}{\partial t}=0$ and this gives the static state(s) of the star $\left(p_{0}, p_{0}, T_{0}, r_{0}\right)$, with the luminosity $L_{0}$. We assume small perturbations from the static state:

$$
r=r_{0}\left(1+r^{\prime}\right)
$$




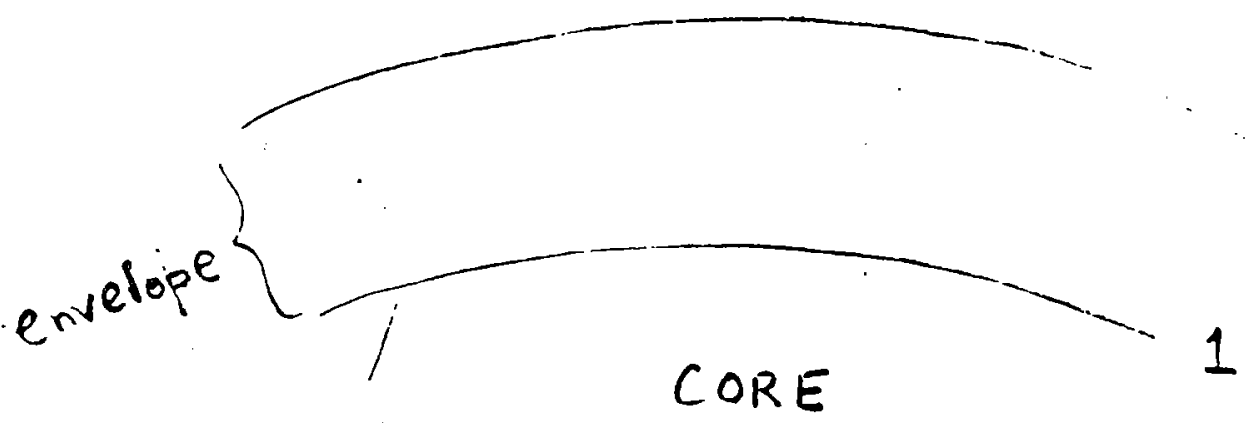

Figure 2: The one-zone model.

$$
\begin{gathered}
T=T_{0}\left(1+T^{\prime}\right) \\
\rho=\rho_{0}\left(1+\rho^{\prime}\right) \\
p=p_{0}\left(1+p^{\prime}\right) \\
L=L_{0}+l^{\prime}
\end{gathered}
$$

Normally, these equations are solved by finite differencing, each element in that scheme corresponding to a shell of mass of the star. In Baker's qualitative model, one simply writes the equation for a single mass shell as if it were the whole envelope. The instability mechanism in Cepheids arises due to opacity variations in a relatively shallow outer envelope containing a very small fraction of the total mass of the star. Since the motion is almost entirely in this envelope we write our equations only for the envelope, assuming that the region below the boundary 1 is motionless. Also, the variation of $p^{\prime}, \rho^{\prime}$ and $T^{\prime}$ with $\mathcal{M}_{r}$ is neglected in the envelope. We cannot however neglect the variation of $l^{\prime}$ because $\frac{\partial l^{\prime}}{\partial \mathcal{M}^{\prime}}$ is really the term that drives the oscillations. Therefore we will make the approximation

$$
\frac{\partial l^{\prime}}{\partial \mathcal{M}_{r}}=\frac{l^{\prime}-l_{1}}{2 m}
$$

where $l^{\prime}$ is the luminosity fluctuation at some "halfway point" in the envelope, $l_{1}$ is the value of $l^{\prime}$ at the boundary 1 and $m$ is the total mass in the envelope. Since the core does not participate in the oscillations, the luminosity at the boundary 1 does not change, so that $l_{1}=0$. Therefore $(2.7)$ becomes

$$
\frac{\partial l^{\prime}}{\partial \mathcal{M}_{r}}=\frac{l^{\prime}}{2 m}
$$




\subsection{Perturbation equations}

On substituting equations (2.6) into (2.2) and retaining only terms linear in the primed quantities we get

$$
\frac{d^{2} r^{\prime}}{d t^{2}}=g\left(4 r^{\prime}+p^{\prime}\right)
$$

where $g=\frac{G M}{r_{0}^{2}}$ and we have used the fact that the static pressure vanishes on $\tau_{0}$. This says that the dynamics of the envelope is driven by pressure fluctuations and perturbations of gravitational potential. To understand how this works, we need to look at the coupling to the thermodynamics.

In the one-zone approximation (2.7), linearization of (2.3) gives

$$
\frac{l^{\prime}}{2 m}=-T_{0} C_{\mathrm{p}} \frac{d T^{\prime}}{d t}+\delta \frac{d p^{\prime}}{d t}
$$

Linearization of (2.4) gives

$$
\frac{l^{\prime}}{L_{0}}=-\frac{\kappa_{\rho}}{\kappa_{0}} \rho^{\prime}-\left(\frac{\kappa_{T}}{\kappa_{0}}-4\right) T^{\prime}
$$

where

$$
\begin{aligned}
& \kappa_{\rho}=\frac{\partial \kappa}{\partial \rho}\left(\rho_{0}, T_{0}\right) \\
& \kappa_{T}=\frac{\partial \kappa}{\partial T}\left(\rho_{0}, T_{0}\right) \\
& \kappa_{0}=\kappa\left(\rho_{0}, T_{0}\right) .
\end{aligned}
$$

The linearized form of (2.5) is

$$
p^{\prime}=\rho^{\prime}+T^{\prime}
$$

and of the continuity equation (2.1) is

$$
3 r^{\prime}-\rho^{\prime}=0
$$

If nothing else, this set of equations shows why it is not simple to characterize the conditions for vibrational instability of a star. The thermodynamic interdependences are just too rich. However, (2.11) is the key; it shows how the couplings are mediated by the dependences of $\kappa$ on $\rho$ and $T$. There is a certain loose analog to instabilities due to negative differential resistivities in all this.

Let $r_{1}$ be the the characteristic radius of the envelope, and set

$$
r^{\prime} \approx r_{1} / 2
$$

where $r_{1}$ is the outer radius. Similarly (2.13) gives

$$
\rho^{\prime}=\frac{3 r_{1}}{2}
$$


Equations (2.15), (2.12), (2.11), (2.10) and (2.9) can be combined into a single equation for $r_{1}^{\prime}$ :

$$
\frac{d^{3} r_{1}}{d t^{3}}+A \frac{d^{2} r_{1}}{d t^{2}}+B \frac{d r_{1}}{d t}+C r_{1}^{\prime}=0
$$

where

$$
\begin{gathered}
A=\frac{L_{0}}{2 m\left(\delta-T_{0} C_{p}\right)}\left(\frac{\kappa_{T}}{\kappa_{0}}-4\right) \\
B=\frac{8 \delta-11 T_{0} C_{p}}{2\left(\delta-T_{0} C_{p}\right)} \\
C=\frac{L_{0}}{4 m\left(\delta-T_{0} C_{p}\right)}\left(3 \frac{\kappa_{p}}{\kappa_{0}}+11\left(\frac{\kappa_{T}}{\kappa_{0}}-4\right)\right)
\end{gathered}
$$

If we look for solutions in the form $r_{1} \sim e^{\text {st }}$ then (2.16) gives

$$
s^{3}+A s^{2}+B s+C=0
$$

Equation (2.17) has three roots. As one crosses the Cephied instability strip due to the specific way the opacity derivatives $\kappa_{p}, \kappa_{T}$ change, the two complex roots move across the imaginary axis to the real positive half of the complex plane. This represents the onset of overstability as envisioned by Eddington (1925). However, for a reliable numerical solution of the Cepheid instability problem, forty years were needed.

\section{Convective Overstabilities}

The onset of overstability is usually a subtle process, but the one that is crucial to the Cepheid instability is too complicated to be real fun. A simpler case of overstability occurs when the energy source is differential buoyancy. The first instance was magnetoconvection (studied by W.B. Thompson and by S. Chandrasekhar) and later came the rotating case (Chandrasekhar). The simplest example is in double diffusive convection as suggested by Melvin Stern and demonstrated by George Veronis. The mechanism in this kind of overstability is generic in convection with restoring forces (Moore and Spiegel, 1966); the irreversiblity of the oscillations that Cowling spoke of in the mhd case plays a role in all cases.

Consider a fluid parcel of mass $m$ moving through the star, but neglect the effect of the parcel on the star. Assume that there is a mechanism such as Coriolis force or a stable concentration gradient that makes for an oscillatory behavior. If we allow for this mechanism by a generic restoring force, $r$ per unit mass, the equation of vertical motion is

$$
m \frac{d^{2} z}{d t^{2}}=-g\left(m-m_{0}\right)+m r(z)
$$

where the mass displaced by the parcel is

$$
m_{0}=m \rho_{0} / \rho
$$


and $\rho_{0}(z)$ is the prescribed background density.

We use the Boussinesq equation of state,

$$
\rho=\rho_{-}\left[1-\alpha\left(T-T_{-}\right)\right]
$$

where $\rho$. and $T$. are constants. After some manipulations using the Boussinesq ideas and setting

$$
\theta=T-T_{0}(z)
$$

we reduce the equation of motion to the form

$$
\frac{d^{2} z}{d t^{2}}=g \alpha \theta+r(z) \text {. }
$$

This is somewhat easier to grasp than the equivalent equation for stellar pulsation (2.9) partly because there is less going on in this case, but also because of the simplifications allowed in the Boussinesq case. For a fuller treatment, we should include friction.

We adopt Newton's law of cooling to describe the thermal effects:

$$
\frac{d T}{d t}=-q\left(T-T_{0}\right)
$$

We have

$$
\frac{d T}{d t}=\frac{d}{d t}\left(T-T_{0}+T_{0}\right) \cong \frac{d}{d t}\left(\theta+T_{0}\right)=\frac{d \theta}{d t}-\beta \frac{d z}{d t}
$$

where $-\beta(z)=d T_{0} / d z$. Then (3.6) becomes

$$
\frac{d \theta}{d t}=\beta \frac{d z}{d t}-q \theta
$$

The third order problem posed here has the same linear structure as that for the stellar pulsation case. However, in this simpler case, we have included nonlinear effects in the $z$-dependences of $\beta$ and $r$. Using the leading order terms in their Taylor series, we get a nonlinear model for the development of instability. With suitable choices of parameters, we find a chaotic behavior (Moore and Spiegel, 1966). In a similar way, the analysis of the previous section can be extended to the nonlinear regime (Baker, Moore and Spiegel, Astron J., 1966) with chaotic results. Thus the occurence of aperiodic stellar oscillations may have a natural explanation. But we need to be able to extract the nonliear equations more reliably. We turn to that question next.

\section{Amplitude equations}

Let the state of the star be denoted by a column vector $U$ whose elements are the density $\rho$, the temperature $T$, the components of velocity and whatever fields are necessary to uniquely specify the conditions in the star. The general equation describing the star can generally be written in the form

$$
\partial_{t} \mathbf{U}=F(\mathbf{U}, \partial)
$$


where $t$ is time, $\partial$ denotes spatial derivatives and $F$ expresses the full stellar dynamical behavior - it is a flow in state space. It is understood that boundary and initial conditions are attached to $\mathbf{F}$ and $\partial_{t}$, respectively.

A steady state of the star is given by $U_{0}(x)$ where

$$
F\left(\mathbf{U}_{0}(\mathbf{x}), \partial\right)=0
$$

\subsection{The linear problem}

The perturbation equation about the static state can be written in terms of the new variable

$$
\mathbf{u}(x, t)=\mathrm{U}(x, t)-\mathrm{U}_{0}(x) .
$$

We split the right hand side of (4.1) into linear and strictly nonlinear parts:

$$
\partial_{t} \mathbf{u}=\mathcal{L}(\partial, \lambda) \mathbf{u}+\mathcal{N}(\mathbf{u})
$$

where $\mathcal{L}$ is a linear operator and $\mathcal{N}$ is a nonlinear operator (which can in general depend on space derivatives) and $\lambda$ represents all the parameters describing the star (such as its total mass). It is useful to first study the associated linear problem obtained by omittng $\mathcal{N}(u)$ from (4.4):

$$
\partial_{t} \mathbf{u}=\mathcal{L} \mathbf{u}
$$

We look for solutions of the form

$$
\mathbf{u}(\mathbf{x}, t)=\mathbf{v}(\mathbf{x}) e^{s t}
$$

Then (4.4a) gives an eigenvalue problem for determining $s$ and $v$.

$$
\mathcal{L} \mathbf{v}=\mathbf{s v} \text {. }
$$

We assume here that (4.6) has a discrete spectrum $s_{1}, s_{2}, \ldots$ with eigenvectors $\psi_{1}, \psi_{2}, \ldots$ If $\mathcal{L}$ is selfadjoint then the eigenvectors form a basis. (In case of degeneracy one can construct an orthogonal basis in the subspace corresponding to the degenerate eigenvalue.) However, $\mathcal{L}$ is in general not selfadjoint and there may exist a root(s) of algebraic multiplicity $n$, with $\mathrm{m}$, the number of eigenvectors corresponding to it, less than $\mathrm{n}$. In such a case, we need additional vectors to span the subspace (Friedman, 1956).

To find additional basis vectors, we look for solutions of the form

$$
\mathrm{u}(x, t)=\sum_{k=0}^{l} \psi_{k}(x) t^{k} e^{a t} A_{k}
$$

where $l \leq n$. As illustration, consider a case with $n=2$ and $m=1$. Suppose we have already found the one eigenvector $\psi_{1}(x)$ such that $\mathcal{L} \psi_{1}=s \psi_{1}$. We look for a solution in the form

$$
\mathrm{u}(x, t)=\phi_{0}(x) e^{s t} A_{0}+\phi_{1}(x) t e^{s t} A_{1}
$$


where $\psi_{1}(x)$ is unknown. Substituting into the associated linear equation we find

$$
\begin{gathered}
\left(s-L_{00}\right) A_{0}-A_{1} t L_{10}=0 \\
A_{0} L_{01}+A_{1}\left(t L_{11}-t s-1\right)=0
\end{gathered}
$$

where

$$
\left(\begin{array}{ll}
L_{00} & L_{01} \\
L_{10} & L_{11}
\end{array}\right)
$$

is the matrix representation of $\mathcal{L}$ in the basis $\left(\psi_{0}, \psi_{1}\right)$.

The terms with different powers of $t$ vanish separately, so from (4.9) we find $L_{00}=s$ and $L_{10}=0$. Similarly, from (4.10), we get $L_{11}=s$ and $A_{1}=A_{0} L_{01}$. Therefore there is a solution (4.8) of the form

$$
\mathbf{u}(x, t)=A_{0} e^{s t}\left(\psi_{0}+L_{01} t \psi_{1}\right)
$$

which, on substitution into the linear equation, gives us the additional information that

$$
A_{0} \mathcal{L} \psi_{0}=A_{0} s \psi_{0}+A_{1} \psi_{1} .
$$

The matrix representation of $\mathcal{L}$ is then of the form

$$
\left(\begin{array}{ll}
s & q \\
0 & s
\end{array}\right)
$$

where $q=A_{1} / A_{0}$.

This procedure has an obvious generalization to the case $m, n>1$. If one succeeds in applying this procedure to each degenerate subspace then the matrix representation of $\mathcal{L}$ is in general

$$
\left(\begin{array}{cccc}
B_{1} & 0 & 0 & \ldots \\
0 & B_{2} & 0 & \ldots \\
0 & 0 & B_{3} & \ldots \\
\ldots & \ldots & \ldots & \ldots
\end{array}\right)
$$

where $B_{1}, B_{2}$ etc. are blocks each having the form

$$
\left(\begin{array}{ccccc}
\sigma & A & 0 & 0 & \cdot \\
0 & \sigma & B & 0 & \cdot \\
0 & 0 & \sigma & C & \cdot \\
\cdot & \cdot & \cdot & \cdot & .
\end{array}\right)
$$

One can find another representation of the above matrix called the companion form or the Jordan-Arnold form, that is sometimes more convenient. In the $3 \times 3$ case it has the form

$$
\left(\begin{array}{lll}
0 & 1 & 0 \\
0 & 0 & 1 \\
\alpha & \beta & \gamma
\end{array}\right)
$$


Figure 3: Distribution of eigenvalues at marginality

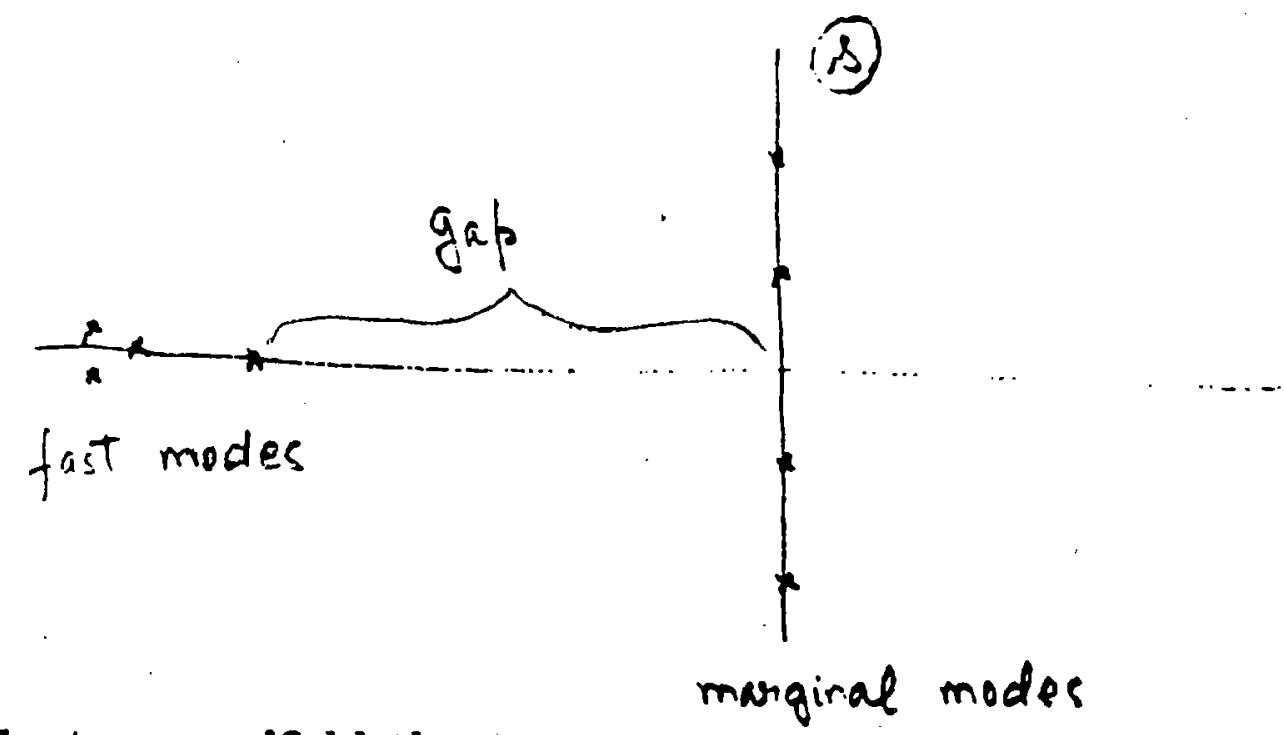

\subsection{Center manifold theory}

If you have a mix of reacting chemicals or nuclei, the rapidly reacting species go quickly to the equilibrium abundances dictated by the local instantaneous conditions. This equilibrium is then evolved according to the behavior of the slowly reacting species. In the case of instability theory, a similar situation prevails. If you expand the state vector $U$ in terms of the normal modes, you get a set of ordinary differential equations for these modes and these are of the form of reaction equations. The slowly evolving modes will control the situation here just as the slowly reacting species dictate the nature of the equilibrium.

The slow modes in this case are those with low growth rates and low damping rates. These are modes that are nearly marginal. This statement can be made precise when we have modes that are exactly marginal for some values of the parameters. Suppose that the distribution of eigenvalues $s$ in the complex plane has the following special structure for some value(s) of the parameter(s) $\lambda=\lambda_{0}$ : a finite number $\mathrm{n}$ of the modes are on the imaginary axis while all the others are bounded away from the imaginary axis for small $\left|\lambda-\lambda_{0}\right|$. The modes away from the imaginary axis can, for many purposes be on either side of it, but we shall consider the simplest case here where there are slow modes with $\Re(s)=0$ and fast modes with $\Re(s)<-\alpha$, where $\alpha>0$.

We denote the fast modes by $\phi_{j}$ and the slow modes by $\psi_{j}$, and make the decomposition

$$
u(x, t)=\sum_{i} B_{i} \phi_{i}(x)+\sum_{j} A_{j}(t) \psi_{j}(x) .
$$

Substituting (4.11) into (4.4) we find the equations for $A_{i}$ and $B_{j}$

$$
\frac{d A_{i}}{d t}=\mathcal{M}_{i j} A_{j}+f_{i}(\mathbf{A}, \mathrm{B})
$$




$$
\frac{d B_{i}}{d t}=\mathcal{K}_{i j} B_{k}+g_{i}(\mathbf{A}, \mathbf{B})
$$

Here the matrices $\mathcal{M}$ and $\mathcal{K}$ are given by linear theory and $f_{i}$ and $g_{j}$ are strictly nonlinear functions of the amplitudes in the expansions. By the very construction of this situation, we know that $\mathcal{K}$ is invertible, so (4.13) can be rewritten as

$$
\mathbf{B}=\mathcal{K}^{-1} \dot{\mathrm{B}}-\mathcal{K}^{-1} \mathbf{g}(\mathbf{A}, \mathbf{B}) .
$$

We expect that, after a short transient, the fast modes will have equilibrated and thereafter the system will change slowly. Hence, we take advantage of this to construct a series of approximations based on (4.14). In first approximation, we have

$$
\mathbf{B}=\mathbf{0} \text {. }
$$

This is called the Galerkin approximation. To get the next order we put $\mathbf{B}=0$ on the right hand side of (4.14). Then

$$
\mathrm{B}=-\mathcal{K}^{-1} \mathrm{~g}(0, \mathrm{~B})
$$

This is called the adiabatic approximation. One can continue this iteration procedure substituting (4.16) into (4.14) to get the next order of approximation, and so on. We can then in principle determine a function

$$
B=B(A)
$$

This function defines an invariant subspace of the state space (coordinatized by the coefficients in its expansion in eigenfunctions) in which the system moves after the transients. This is the center manifold.

If we substitute (4.17) into (4.13) we have

$$
\dot{\mathbf{A}}=\mathcal{M A}+\mathbf{f}(\mathbf{A}, \boldsymbol{B}(\mathbf{A})) \text {. }
$$

Thus, the dynamics of the system is reduced from an infinite dimensional state space down to motion in a subspace of finite dimension. This is what we did in the previous section, but the present approach is more reliable. Equation (4.18) is sometimes called an amplitude equation.

\subsection{Normal forms}

The linear portion of (4.18) is determined only by the number of instabilities and their degeneracies. In each case, we want to use (4.18) for a neighborhood of parameter space near to $\lambda_{0}$. The forms of the matrix $\mathcal{M}$ for each case are standard, so we need only to compute the nonlinear terms. Can we not also find a standard form for the nonlinear amplitude equation? If so, for each configuration of instabilities, we shall have a single equation to study to get some idea of the temporal behavior. 
Supose that after the reductions to the center manifold, we have derived an amplitude equation like (4.18) with conceivably complicated nonlinear terms as in (4.18). Suppose that this can be written as

$$
\dot{\mathrm{B}}=\mathcal{M} \mathrm{B}+\mathbf{\Gamma}(\mathrm{B})
$$

where $\Gamma$ is arbitrary, but we shall assume that it is analytic.

We want to put (4.19) into a standard or normal form by means of transformation

$$
\mathbf{B}=\mathbf{X}+\Psi(\mathbf{X})
$$

where $\Psi$ is a strictly nonlinear function. This will not change the linear part of (4.19), but it will modify the nonlinear terms. Let us assume the new equation is

$$
\dot{\mathbf{X}}=\mathcal{M} \mathbf{X}+\mathbf{G}(\mathbf{X})
$$

where $G$ is some standard form of the nonlinear terms. We will see that the forms that are allowed are determined by the linear theory.

Substituting (4.21) in (4.19) we find

$$
\mathcal{F} \Psi=\Gamma(X+\Psi)-G \partial_{X} \Psi-G
$$

where

$$
\mathcal{F}=\mathcal{M} \mathbf{X} \partial_{\mathbf{X}}-\mathcal{M} .
$$

The operator $\mathcal{F}$ is determined by the linear problem and it is in fact the Lie derivative with respect to the vector field $\mathcal{M X}$.

We shall not give details (see Spiegel, 1985) but sketch the main idea, which is that if we want a particular $G$ we can see whether that choice makes (4.22) soluble so that there is a $\Psi$ that does the job. To check this we need the so-called solvability conditions on (4.22). For this we need an inner product on state space, which we shall assume has been defined. Then we can define an adjoint operator $\mathcal{F}^{t}$ by

$$
(\mathcal{F} \Psi, \Phi)=(\Psi, \mathcal{F} \dagger)
$$

where $(\cdot, \cdot)$ denotes the inner product. Now any $\bar{\Psi}$ such that

$$
\mathcal{F}+\bar{\Psi}=0
$$

is orthogonal to the left side of (4.24) and we conclude that

$$
\left(\Gamma(X+\Psi)-G \partial_{X} \Psi-G, \bar{\Psi}\right)=0 .
$$

The conditions (4.26) (there will generally be several adjoint null vectors) can be used to fix $\mathbf{G}$. Normally, one proceeds perturbatively, expanding in $\mathbf{X}$ and gets $\mathbf{G}$ and $\Psi$ term by term. Thus, for any stability configuration, provided the degree of instabilty is not pronounced, the form of the amplitude equation can be studied. 


\subsection{The end}

For the case of convective instabilities such as we encounter in cool star atmospheres, these amplitude expansions will not be very helpful by themselves. The instabilities are too strong and the linear modes are far to the right of the imaginary axis. We have to imagine that the turbulence has renormalized the situation back to a slightly unstable state, in the large. Then the motions that develop against the turbulent background can be studied with amplitude equations.

The simplest situations have only one instability, the direct instability that gives the so-called pitchfork bifurcation and overstability that flowers into the Hopf bifurcation. For double instabilities, there are four simple possibilities: two direct instabilities, two overstabilities, one of each, and an overstability whose frequency can vanish as the marginal condition is approached. This last gives rise to what may be called the Bogdanov bifurcation. All these cases produce either periodic or quasiperiodic behavior.

When three instabilities occur, then chaotic behavior can develop as in the example of the oscillating fluid parcel. The normal forms for these cases have all been written down to leading order. Buchler and collaborators have been systematically applying them to stellar studies.

\section{References:}

·1] Arneodo A., Coullet P.H., Spiegel E.A.,1985, Geophys. Astrophys. Fluid Dynamics, Vol.31, page 1.

[2] Baker N., 1963, Simplified models for cepheid instability, Plenum Press, Stellar Evolution.

[3] Carr J., Applications of Centre Manifold Theory, Springer-Verlag, Applied Mathematical Sciences 35 (1981)

[4] Coullet P.H., Spiegel E.A., 1983, SIAM J. Appl. Math., Vol. 43, No. 4, page 776.

[5] Eddington A.S., 1882, The internal constitution of the stars, Cambridge University Press.

[6] Friedman B, 1956, John Wiley and Sons, New York, Principles and techniques of applied mathematics.

[7] Kippenhahn R., Wiegert A., Springer-Verlag, Stellar structure and evolution.

[8] Moore D.W., Spiegel E.A., 1966, Ap.J, Vol 143, page 871.

[9] Spiegel E.A., Cosmic Arrhythmias, in Chaotic Behavior in Astrophysics, R. Buchler, J. Perdang and E.A. Spiegel, eds. (Reidel, Dordrecht), 91, 1985. 


\title{
An introduction to solar MHD (Summary)
}

\author{
Stephen Childress
}

\section{Basics}

Astrophysical fluid dynamics must generally include the possibility of electromagnetic fields since the fluid is often in the plasma state, currents can flow, and magnetic and electrostatic forces may be important. Astrophysical systems are also large, so that Reynolds numbers of the form $U L / \eta$, where $U$ and $L$ are characteristic velocity and length scales and $\eta$ is the diffusivity of some physical quantity, are typically very large. The turbulent phenomena which result involve complicated interactions between fluid and magnetic field, solar magnetism being the most directly observable product of these interactions.

The solar magnetic field is characterized by two complementary properties: On the one hand, there is a global structure connected with the well-known 22 year cycle. Every period of roughly 11 years produces a new round of magnetic activity (most notably in the appearance of sunspots) and culminates in a reversal of polarity. This periodicity implies an active process of renewal of magnetic activity, with production of fresh field (at least in the observable surface layers) its main consequence. We note that this solar dynamo cycle is only roughly periodic, and indeed essentially turned off for the 70 year "Maunder minimum" beginning in 1645, and in other similar epochs.

On the other hand, the magnetic field is, in the small, "rough" and intermittent. Perhaps the most expressive adjective is "fibrillated". Instead of being a smooth vector field, the magnetic field $\mathbf{B}(\mathbf{x}, t)$ in the photosphere (and presumably also in the convection zone) has a filamentary structure where intense tubes of flux are embedded in regions of much smaller field. As a result, peak fields on the Sun reach several thousand gauss, while the spatially-averaged field is only about one gauss. 
We thus see two lines of enquiry into solar magnetic phenomena, one in the large and dealing with the cycle as a whole, the other in the small and focusing on specific magnetic features such as the fibril structure or definite objects such as sunspots, flares, and prominences. Solar flares, in particular, are noteworthy for the energy released. Flares are apparently magnetic in origin and have a variety of configurations. Parker has suggested that smaller versions of flares, in the form of tangential discontinuities in the magnetic field, may play a significant role in the heating of the solar corona up to $10^{6}$ degrees Kelvin.

The minimal system needed to discuss these phenomena mathematically are the equations of one-fluid, ideal magnetohydromagnetics (MHD). These equations describe a perfectly conducting, generally compressible gas. To allow for resistive effects (such as solar flares), we can relax the condition of infinite conductivity. Then Ohm's law takes the form, in the simplest MHD model,

$$
\mathbf{J}=\sigma[\mathbf{E}+\mathbf{u} \times \mathbf{B}]
$$

where we have introduced customary symbols for current, electric, and velocity fields, and $\sigma$ is the electrical conductivity of the material. We also have the equations of Ampère and Faraday, and the solenoidal property of the magnetic induction field:

$$
\begin{gathered}
\nabla \times \mathbf{E}=-\partial \mathbf{B} / \partial t, \\
\mu \mathbf{J}=\nabla \times \mathbf{B}, \nabla \cdot \mathbf{B}=0 .
\end{gathered}
$$

Here $\mu$ is the magnetic permeability. For most problems of interest in astrophysics the displacement current may be neglected in Ampère's law (the time scale of events being large compared to the transit time of light across the system), an approximation we have already included in equation (3). This has the effect of filtering out the electromagnetic radiation.

These equations combine to yield, for any given velocity field $\mathbf{u}(\mathbf{x}, t)$, a kinematic equation of the magnetic field,

$$
\partial \mathbf{B} / \partial t-\nabla \times(\mathbf{u} \times \mathbf{B})-1 / R m \nabla^{2} \mathbf{B}=0 .
$$

Here we have gone over to dimensionless variables and introduced the magnetic Reynolds number $R \mathrm{~m}$. In the solar photosphere and generally in astrophysical MHD, $R m$ is a large parameter ( with values generally greater than 
$10^{5}$ ). Here we refer to estimates based upon the molecular, not the turbulent value of the diffusivity. Thus equation (4), which is the MHD induction equation, is in astrophysical problems highly singular in the distribution of resistive effects, and indeed, and we have already noted, one sees this physical property in the fibrillation of the solar magnetic field. We may take the existence of flux ropes and sheets as evidence of small dissipation.

In MHD the principal new dynamical effect is the Lorentz force which appears on the right of the momentum equation

$$
\rho(d \mathbf{u} / d t)+\nabla p=\mathbf{J} \times \mathbf{B}
$$

Using a vector identity, we see that this new force is a sum of a pressure gradient and the effective tension in the lines of force,

$$
\mathbf{J} \times \mathbf{B}=\mu^{-1}\left[\mathbf{B} \cdot \nabla \mathbf{B}-\nabla B^{2} / 2\right]
$$

This effective tension results in new wavelike phenomena, typified by the shear Alfvèn waves.

If diffusive effects are expelled from equation (4) (by setting $R m=\infty$ ) we then recover the kinematic equation for a magnetic field carried by a perfect conductor. One usually then refers to the magnetic field as "frozen" into the conductor; indeed it is only in this ideal case that one has a firm grasp of magnetic lines of force as entities which are moved about in a prescribed manner. In this case the evolution of the magnetic field reduces to the geometry of material lines under the Lagrangian map determined by the velocity field $u$. Lagrangian coordinates $\mathbf{x}(\mathbf{a}, t)$ are defined by

$$
d \mathbf{x} / d t=\mathbf{u}(\mathbf{x}(\mathbf{a}, t), t), \mathbf{x}(\mathbf{a}, 0)=\mathbf{a} .
$$

If $J_{i j},=\partial x_{i} / \partial a_{j}$ is the Jacobian of the Lagrangian map, then the magnetic field transforms according to

$$
B_{i}(\mathbf{x}(\mathbf{a}, t), t)=J_{i j} B_{j}(\mathbf{a}, 0) .
$$

A consequence of this is the conservation of flux: if $S$ is a material surface, then

$$
d / d t \int \mathbf{B} \cdot \mathrm{d} \mathbf{S}=0 .
$$


This allows field intensity to be increased by compression or by stretching of fluid elements. In two dimensions, such stretching also leads to folding and ultimately to highly sheared magnetic structures which are rapidly dissipated. In three dimensions, however, models such as the "stretch-twist-fold" map suggest that field reinforcement can occur and a dynamo process realized.

Even for systems with a large $R m$ the effects of dissipation thus remain locally important. A good example of this is the "flux expulsion" from 2-D eddies and from other (even 3-D) fluid motions which tend to produce highly sheared structures.

Most models of solar processes rely heavily on the approximation of frozen-in fields. The early work by Babcock on the solar cycle, and subsequent improvements by Leighton, suggest a global geometry for the solar magnetic cycle, but a detailed understanding of the dynamo process is lacking, owing primarily to the ambiguous status of certain physical mechanisms (especially the "alpha effect") which are often invoked in kinematic dynamo theory, but can be mathematically analyzed only in a few models.

\section{Fast dynamos}

The Lagrangian viewpoint is useful in describing unsteady kinematic dynamos consisting of sudden fast movements interspersed within epochs of zero fluid motion. The epochs of "stasis" allow diffusion to smooth out the field up to some small length scale. Using this technique for maps of the unit cube into itself, we can construct "fast" dynamos which amplify magnetic field exponentially at a rate which, for large $R m$, is independent of $R m$ and therefore of the order of the "eddy turnover time" $L / U$.

These fast dynamos are also thought to occur in steady three-dimensional flows containing regions of Lagrangian chaos. The reason for this is the exponential line stretching which occurs in the chaotic parts, as measured e.g. by the Liapunov exponent for the flow. This stretching amplifies magnetic field in the ideal limit. The question then arises: is the folding up of the field so severe that shear dissipation cancels the amplification process? In one example, Andrew Gilbert has found that dynamo action survives robustly. This example utilizes a "chaotic web" to extract a simple map along the separatrices of a periodic array of eddies. Equation (8) is used to compute the flux of field through a small element of the chaotic region, as a function 
of time. Exponential growth is observed in the chaotic region but not in the regions of integrable flow. The field becomes highly intermittent and fibrillated as time progresses and there is thus good reason to suppose that true turbulence will always generate a field such as we see on the Sun.

In summary, there are some special cases where we can compute the evolution of magnetic fields in the kinematic sense, which suggest that a magnetic field such as we see on the Sun is very likely in astrophysical turbulence. However little is known about the physics of small-scale structure, about the corresponding dynamical problems, or about the global organization of these weakly dissipative processes.

\section{References}

Childress, S. and Strauss, H.R. , Lecture Notes on Solar MHD, Courant Institute, Spring 1990.

Parker, E.N. Cosmical Magnetic Fields, Clarendon Press, Oxford, 1979.

Priest, E.R. Solar Magnetohydrodynamics, Riedel Publishing Company, 1984. 


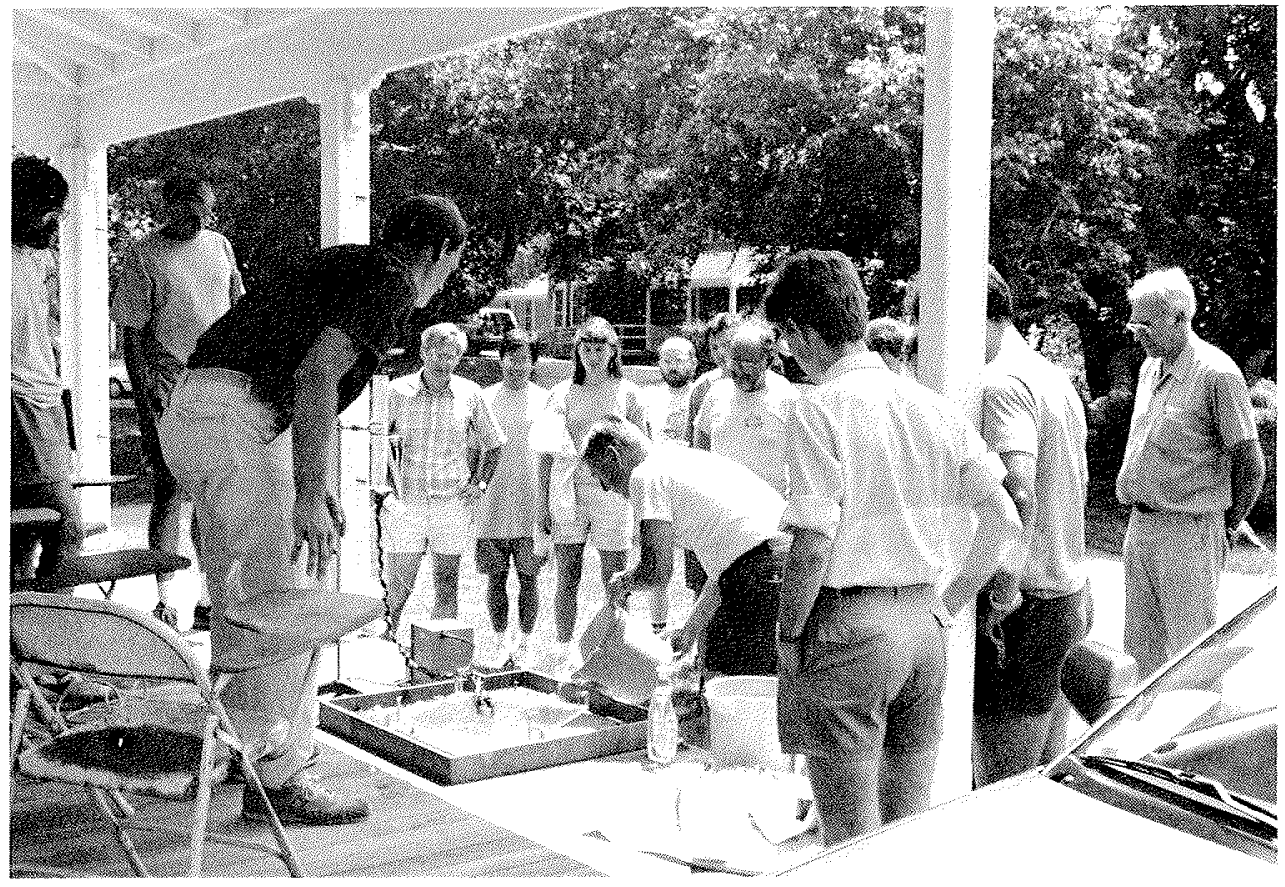

$\mathrm{V}$ Observing volcanic eruptions on the porch of Walsh cottage 
Relativistic Fluctuation-Dissipation Theorems, Radiative Hydrodynamics and Galaxy F'ormation

\author{
James L. Anderson \\ Stevens Institute of Technology \\ Hoboken, New Jersey, 07030
}

\title{
ABSTRACT
}

The origin of structure in the universe continues to be a mafor subject of research. The original suggestion of Newton that structure arose in an Inltially uniform distribution of matter from gravitational instability, now called Jean's Instab1lity, is still considered to be the basic underlying source of this structure. The main difficulty with applying this idea to the problem of galaxy formation is that, in an expanding universe, instabilities grow only as a power ( roughly 2/3) of the time rather than exponentially due to the competition between the collapse of a fluctuation that exceeds the Jean's mass and the expansion of the universe. Thus thermal fluctuations that arise after the era of decoupling of matter and radiation will. not have enough t. Ime to evolve into galactic concentrations in the time between decoupling and the present. At the same time, It was difflcult to see how the significantly larger density fluctuations (on the order of percents) needed to make galaxies on that time scale could have arisen naturally. One possibility which has been put. forward is that density fluctuations arose in the very early universe due to quantum fluctuations in the H1gg's fleld present during a hypothetical "Inflationary" phase of the universe's evolution. However, that proposal has run into the difficulty that the fluctuations predicted by the colemanWelnberg potential used in the "new inflationary" scenar10 are 4-5 orders of magnitude too big.

An alternate proposal was put forth by Saslaw (1968) who suggested that there might be a signiflcant enhancement of thermal fluctuations due to the long-range nature of the gravitational interaction. In particular, Saslaw was able to show, using thermodynamic arguments, that these enhanced fluctuations have a pronounced peak in thelr spectrum at the Jean's wavelength. Following on Saslaw's suggestion, SImon (1970) made use of a fluctuation-dissipation theorem for the Navier-Stokes equations derived by Landau and Lifshitz (1963) to show that such a spectrum would arise if one added fluctuating forces to these equations. However, both Saslaw's and Simon's treatments were nonrelativistic and hence could not be applied to fluctuations that would have arisen before the era of decoupling. While such fluctuations might have had enough time to evolve into galaxies at the present time, they would have had to survive a perlod of acoustic damping just prior to decoupling. Welnberg (1971) Invest1gated this dampling and found that if the density of the present untverse is $10^{-30} \mathrm{~g} / \mathrm{cm}^{3}$ then galaxy sized fluctuations would be damped by a factor of $10^{-71}$ and that only cluster sized fluctuations and larger would survive through the decoupling era. In a somewhat, denser universe however, galaxy sized fluctuations might just manage to survive if they were sufficlently large to begin with. However, Weinberg offered no mechanism for such fluctuations prior to decoupling.

As a preliminary to an 1nvestigation of the origin and growth of fluctuations prior to the decoupling era, we have developed a relativistic generalization of the Landau-Lifshitz fluctuation-dissipation theorems for a 
one-component relativistic fluid (1990). In particular we have determined the relation between the fluctuating forces that have been added to the relativistic Navier-Stokes equations and the coefficients of bulk and shear viscosity and thermal conductivity appearing in these equations. Since however, prior to decoupling, radiation played an important role, it is necessary to extend these results to a multi-component system. We have used the equations of radiative hydrodynamics derived by us (1976) for this purpose. We hope to use these result.s to study whether the density fluctuations produced by these fluctuating forces could have survived beyond the decoupling era to become galaxios.

\section{References}

Anderson, J.L. 1976, Cen. Rele \& Gray, 7, 53

Anderson, J.L., Nowotny, E. 1990, Bhysica A 163, 501

Landau, L.D., If Ifhitz,E.M. 1963, Fluid Mechanics, Pergamon Press, Oxford

Saslaw, W.C. 1.968, Mon. Not. Rey. Astron. Soc. 141, 1

Simon, R. 1970, Astron. \& Astrophys. 6, 151

Weinberg,S. 1971, Astrophys. J. 168, 127 


\title{
SOLAR ACOUSTIC OSCILLATIONS
}

\author{
N.J. Balmforth \\ Institute of Astronomy, \\ University of Cambridge, \\ Madingley Road, Cambridge, CB3 OHA, \\ England.
}

The measurements of the oscillations of the sun have now reached considerable precision: literally thousands of individual oscillations have been identified and many of their frequencies have been determined to accuracies of less than a percent.

Theoretical modelling of the linear, adiabatic pulsations of solar models has reproduced these frequency measurements to a surprising level of accuracy. There are small, but nevertheless significant differences (e.g. Christensen-Dalsgaard, 1989). This seems to suggest that these theoretical adiabatic pulsations describe solar oscillations fairly well. Indeed, it appears that the differences between the observed frequencies and the theoretical adiabatic pulsation frequencies are largely caused by differences between the basic structure of the sun and that of the theoretical model intended to represent it.

The formulation of the linear, adiabatic pulsation problem can be used to develop inversion techniques to infer how the structure of a particular theoretical model differs from that of the sun. This has been very successful in helping to refine the modelling of solar structure. Using these methods, the agreement between observed and theoretical pulsation frequencies has been improved. The observed frequencies, however, do not compose a complete mode set. This leads to resolution and uniqueness problems in the theoretical inversion. The observed frequencies also correspond only to pulsations that do not propagate substantially into the central regions of the sun. This severely restricts the depth to which information can be extracted about the sun. Extensive observations are planned in the coming decade and may alleviate these problems (for example, the "SOHO" space mission and the "GONG" ground-based observatories). Another theoretical problem is 
the error that is introduced when the pulsations are treated as adiabatically propagating waves in the turbulent super-adiabatic boundary layer of the solar convection zone. In fact, this is probably where much of the remainder of the discrepancy between observation and theory has its origin.

Inversion methods can also be applied to infer information about the rotation rate of the sun. In a spherically symmetric star the axial symmetry gives rise to a degeneracy in the azimuthal order of the pulsation modes. Any non-axially symmetric phenomenon that affects the oscillations will break this symmetry and remove the degeneracy. This is exactly analogous to the instance where a magnetic field lifts the degeneracy of the energy levels of an atom and produces Zeeman splitting. The rotation of the sun is responsible for lifting this degeneracy in the solar oscillations and produces a splitting of the mode frequencies. Since the extent of the splitting depends in some integral fashion upon the depth-dependent rotation curve, the information contained in the pulsations that penetrate to different depths inside the sun can be used to measure some features of this rotation curve.

The power of the solar oscillations is concentrated within an envelope in frequency about $3 \mathrm{mHz}$. This envelope appears to be largely independent of the horizontal wavenumber of the modes (with the possible exception of modes that propagate predomantly horizontally throughout the sun Christensen-Dalsgaard and Gough, 1982; Libbrecht et al., 1985; Rhodes, 1990). This suggests that oscillations are generated where the modes propagate almost vertically, i.e. near the surface of the sun in the turbulent convective boundary layer. Under the conditions that prevail in these regions, it is extremely difficult to model nonadiabatic linear pulsations, principally because there exists no reliable description of turbulent convection. Nevertheless, there are indications that the acoustic oscillations are intrinsically stable, but driven stochastically and nonlinearly by turbulence (Stein, Nordlund and Kuhn, 1989; Kumar and Goldreich, 1989; Balmforth and Gough, 1990): the modes become the manifestation of acoustic noise, generated by turbulent convection, in a resonant acoustic cavity. If this is true, then the modes react like damped simple harmonic oscillators under the influence of a temporally random forcing. The power spectrum of the oscillators is approximately Lorenztian, and the half widths at half maximum of the peaks are just the damping rates of the modes. These line widths have been measured for the sun (Libbrecht, 1988), and are qualitatively similar to recent theoretical nonadiabatic linear pulsation calculations (Balmforth and Gough, 1990).

Most recently it has been discovered that the solar oscillation frequencies 
are changing with the solar cycle. The dependence of the frequency change upon the absolute frequency of the modes is indicative that the oscillations are being affected by changes in the turbulent convective boundary layer (Libbrecht and Woodward, 1990). Therefore the frequency change may be used as a direct probe of the principal effects of the solar cycle upon the stratification of the sun. This effect is surprisingly superficial. It can also be modelled by simple theoretical calculations that change the efficacy of convective energy transport, which is a potential consequence of the build up of magnetic flux in the convection zone. However, these calculations directly contradict the observations of the change in the solar luminosity over the solar cycle (Woodward and Hudson, 1983). Thus the solar structure does not appear to be undergoing a global (latitudinally invariant) change over the solar cycle, unless the direct effect of the magnetic forces is responsible for changing the pulsation frequencies.

\section{REFERENCES}

1. Balmforth, N.J., and Gough, D.0., 1990. To appear in the proceedings of the conference held in Versailles, May, 1989, entitled Inside the sun; to be published in Solar Physics.

2. Christensen-Dalsgaard, J., 1989. In Seismology of the sun and sun-like stars,ed. V. Domingo and E. Rolfe, ESA.

3. Christensen-Dalsgaard, J., and Gough, D.0., 1982. Monthly Notices Royal Astronomical Society, 198, 141.

4. Libbrecht, K.L., 1988. Astrophysical Journal, 334, 510.

5. Libbrecht, K.L., Popp, B.D., Kaufman, J.M., and Penn, M.J., 1985. Nature, 323, 235.

6. Libbrecht, K.L., and Woodward, , 1990. Nature, in press.

7. Kumar, P., and Goldreich, P., 1989. Astrophysical Journal, 342, 558.

8. Rhodes, E., 1990. In a presentation at the Institute for Theoretical Physics, Santa Barbara, as part of the programme on Helioseismology, January 1990 to July 1990.

9. Stein, R.F., Nordlund, A., and Kuhn, J., 1989. In Seismology of the sun and sun-like stars,ed. V. Domingo and E. Rolfe, ESA.

10. Woodard, M.W., and Hudson, H.S., 1983. Nature, $305,589$. 


\title{
Inviscid models associated with vortex reconnection
}

\author{
Stephen Childress
}

The question of global regularity of 3D Euler flow has implications for the kinds of mechanisms available for viscous reconnection of vortex lines. Conversely, studies of vortex reconnection might be suggestive of the most singular Euler flows. A simple model equation studied by Constantin, Lax, and Majda has the form $\omega_{t}=\omega H(\omega)$ where $H$ is the Hilbert transform and $\omega$ is a function of $x, t$. This equation, whose initial-value problem may be solved exactly, supports "dipole-like" singularities across which $\omega$ changes sign. Thus in this one-dimensional analog the singularity is closely associated with viscous cancellation described by $w_{t}=w^{2} / 2+\nu w_{x x}$ where $w=H(\omega)+$ $i \omega$.

In real 3D Euler flows no clear evidence of a singularity exists. Using the vortex-reconnection criterion, the Taylor-Green initial condition is not especially appropriate, since the early phase of the motion compresses vortex lines connecting the eddies into layers of one sign. Vortex reconnection would be enhanced for the initial velocity $\left(\psi_{y},-\psi_{x}, 0\right)$ where $\psi=\sin x \sin y f(z)$ near a double zero of $f(z)$. On the other hand the work of Pumir and Siggia on singularity development from anti-parallel vortex tubes deals with essentially a vortex reconnection geometry. The inviscid development of the vortices involves considerable $2 \mathrm{D}$ distortion of the cores. Their recent computations utilizing mesh refinement have found at most exponential growth of vorticity, however. Viscous simulation of reconnection at tube Reynolds numbers of order 1000 shows /brid a weaker compression of the vortices toward thin cores. The "bridging" process occurs in a layer formed by the compression of the cores onto the plane of symmetry. Saffman has devised a model for the "snap-back" of the bridged or rejoined tubes, which accounts for the enhanced straining induced by the axial flow along the tubes away from 
the reconnection region. This nonlinear feedback is caused by the viscous annihilation of the axial low-pressure cores. The time for viscous reconnection depends upon the flow causing it. Simple straining-flow models of viscous reconnection suggest that, with at most exponential of vorticity, the time grows with Reynolds number $R e$ like $\log R e$. For singularities consistent with the Beale-Kato-Majda estimate e.g. maximum vorticity growing like $\left(t^{*}-t\right)^{-1}$, reconnection is complete by time $t^{*}$.

A model for singularities/reconnection utilizing evolution of a bilayer of anti-parallel vorticity has a certain attraction since core distortion tends to eliminate tubes as discrete objects with well-defined circulations. A thinlayer model based upon evolution from perturbations of a triangular jet $(u, v, w)=(0, V(x), 0), V(x)=\omega_{0}\left(-|x| / \epsilon+\chi_{0}\right)$ was developed. The thinlayer limit involves fixed $O(1)$ variables $x / \epsilon, z / \epsilon$ for small $\epsilon$. Letting $x, z$ now denote these stretched variables, and $\mathbf{u}, \mathbf{w}$ similarly denoting stretched velocity components, the resulting system is

$$
\begin{gathered}
D v=0, \quad u_{x}+v_{y}+w_{z}=0 \\
D\left(w_{x}-u_{z}\right)+v_{x} w_{y}-v_{y} w_{x}+u_{z} v_{y}-v_{z} u_{y}=0 .
\end{gathered}
$$

On the boundary $x= \pm \chi(y, z, t)$ of the vortical region we have

$$
u=\chi_{t}+w \chi_{z}, u=\phi_{x}, v=0, w=\phi_{z},
$$

where $\phi$ is harmonic in $x, z$ in the exterior and vanishes at infinity. The twodimensional version of this problem leads to the well-known nonlinear wave equation for $\chi: \chi_{t}+\omega_{0} \chi \chi_{y}=0$. Various thin-layer initial conditions have been considered. Two anti-parallel elliptic patches were simulated, using contour dynamics, by David Dritschel. The patches develop into a highlydistorted "T"-shaped structure, resembling the distorted vortex cores in the Pumir-Siggia model.

The 3D structure of the above thin-layer model is still intact, and we have focused first on a truncation which assumes

$$
v=\omega(y, z, t)(-|x|+\chi(y, z, t)), w=w(y, z, t),
$$

and that $w=H(u)$ on the boundary, where $H$ is the Hilbert transform in $z$. This model reduces to the system describing volume conservation,

$$
\chi_{t}+(V \chi)_{y}+(w \chi)_{z}=0
$$


where $V \equiv \omega \chi / 2$ is the average $y$-velocity over the layer, an assumed equation for $\omega$,

$$
\omega_{t}+V \omega_{y}+w \omega_{z}=\omega w_{z}
$$

and the Hilbert connection between $u$ on the boundary and $w$. Since the last two equations imply $V_{t}+2 V V_{y}+w V_{z}=0$, we see that the system admits an integral $V=V_{0}=$ constant. By examining the linear stability of the triangular jet in the various approximations, we find that the thin layer model perserves the "varicose", neutrally stable waves, but that the simplified model, restricted to the integral surface $V=V_{0}$, expels the 2D Kelvin-Helmholtz waves which distort the cores of vorticies. We thus obtain a system

$$
\chi_{t}+(w \chi)_{z}=0, w=-H\left(\chi w_{z}+V_{0} \chi_{y}\right) .
$$

David Olson has studied the evolution of pertubations of $\chi_{0}$ in this model. Although the results are preliminary and have been obtained only at modest resolution, the indication are that high-low pairs form and propagate. The low steadily deepens while the high remains at about fixed amplitude. Since $2 V_{0}=\omega \chi$ is fixed, singularities now correspond to zeros of $\chi$. We think of the vicinity of such a point as a "hot spot" were reconnection is heightened. We thus envisage introducing in such a region a local viscous boundary layer on the plane of symmetry, where the extent of reconnection can be assessed. 


\section{Rossby Wave Radiation from Strong Eddies \\ Glenn R. Flierl \\ MIT}

Models of steady nonlinear eddies in geophysical flows often require conditions which seem not to match well with eddies which have been studied in the atmosphere or ocean. For example, consider a two layer quasigeostrophic model with mean flows $\bar{U}_{1}, \bar{U}_{2}$ in the upper and lower layers respectively. For a steadily propagating eddy, the motions in the far field are a linear combination of the solutions to

$$
\nabla^{2} \Phi=\left(K^{2} / R_{d}^{2}\right) \Phi
$$

with the two values of $K^{2}$ satisfying the equation

$$
\left[K^{2}+\frac{\beta R_{d}^{2}-\left(\bar{U}_{2}-c\right) /(1+\delta)}{\bar{U}_{1}-c}\right]\left[K^{2}+\frac{\beta R_{d}^{2}-\left(\bar{U}_{1}-c\right) \delta /(1+\delta)}{\bar{U}_{2}-c}\right]=\left(\frac{\delta}{1+\delta}\right)^{2}
$$

with $\delta$ being the ratio of the upper layer depth to the lower layer depth. Analysis of this equations shows that solutions are confined (both $K^{2}$ values positive) for $c>\bar{U}_{1}$ and $c>\bar{U}_{2}$. Thus isolated eddies must travel eastward with respect to both mean flows. This is not generally observed. Also, isolated eddies must satisfy have vanishing angular momentum $\iint \delta \mathbf{r} \times \mathbf{v}_{1}+\mathbf{r} \times \mathbf{v}_{2}=0$. Again, it is hard to justify this as applying to Gulf Stream Rings or features such as the Red Spot.

Thus, we must consider cases in which one or more of the exterior fields have a wavelike or radiating character. We then would not expect to find steady state solutions, but we can look for situations in which the exchange of energy between a strong eddy and the Rossby wave field is relatively weak. Then the eddy would be relatively long-lived and might be thought to be a steady state solution. It is important to understand the rate at which energy is lost from the eddy structure and how the wave field might affect the evolution of the eddy.

As a first example, we consider the case in which one mode is trapped and one wavelike - in the absence of mean flows, this would correspond to a situation in which the eddy is moving westward with a velocity $c \leq-\beta R_{d}^{2}$, so that the baroclinic mode is not wavelike, but the barotropic mode is. We look first at just the baroclinic mode in isolation. The vanishing net angular momentum theorem implies that either the speed is exactly the long wave speed or the baroclinic angular momentum must vanish $\left(\iint \phi_{B C}=0\right)$. In the former case, there is a solution with streamfunction decaying as $r^{-1} \sin \theta$ which includes a strong axisymmetric monopolar component. (This solution has, of course, no net angular momentum because the flow is purely baroclinic and therefore $\delta \mathbf{v}_{1}+\mathbf{v}_{2}=0$.)

But this eddy does not satisfy the two layer equations exactly: the nonlinear interactions generate barotropic flow and that, in turn, alters the baroclinic flow field. Analysis of the equations in the limit where $\delta$ is small show that the barotropic streamfunction satisfies

$$
\left[\nabla^{2}-\frac{\beta}{c}\right] \phi_{B T}=\left[\nabla^{2}-\frac{1}{R_{d}^{2}}-\frac{\beta}{c}\right] \phi_{B C}
$$


which is the equation for topographically forced waves. The right hand side vanishes in the exterior (streamlines connected to infinity) but not in the interior. Since $c<0$, the barotropic equation has radiating solutions; therefore, we must satisfy radiation conditions. This leads to an asymmetry with most of the barotropic field extending to the east of the eddy. The asymmetric part of the field has a southward flow near the eddy (for an anticyclonic baroclinic circulation), forcing the eddy to move southward. In addition, the baroclinic energy decays as

$$
\frac{\partial}{\partial t} \frac{1}{2} \iint\left[\left|\nabla \phi_{B C}\right|^{2}+\left|\phi_{B C}\right|^{2} / R_{d}^{2}\right]=-\frac{\delta}{(1-\delta)^{2}} \iint\left(\frac{\partial}{\partial x} \phi_{B T}\right)\left[\beta-c \nabla^{2}+\left(c / R_{d}\right)^{2}\right] \phi_{B C}
$$

The right hand side has no contribution from the exterior part of the eddy, but has a negative contribution, proportional to the energy itself, from the interior region. Thus we have decay of the baroclinic eddy on a time scale inversely proportional to the layer depth ratio, $\delta$.

As a second example, consider a barotropic vortex pair oriented so that it will move westward. In this case, the only mode present is radiating, rather than trapped. When the flow speeds in the vortex pair, which are proportional to the eddy velocity, $U$, are large compared to the Rossby wave speed, $\beta \ell^{2}$, we can solve by expanding in a small parameter $\epsilon=\beta \ell^{2} / U$. We solve by matched asymptotic expansions: in the far field, the flow varies on a large scale, $X=\epsilon^{1 / 2} x$, and the dominant balance is linear. We look at the solution which is singular near the origin (and therefore will match with the decaying field of the interior dipole) together with the free waves necessary to satisfy the radiation condition. As we approach the eddy, the far field reduces to the $r^{-1} \sin \theta$ characteristic of the dipole plus a weak $r^{2} \sin 2 \theta$ strain field arising from the Rossby wave field.

The interior equations are then examined at various orders in $\epsilon$ - in particular, the order 1 and $\epsilon$ terms lead to a steadily propagating solution which can be matched to the singular part of the far field. The $\epsilon^{3 / 2}$ terms bring in a slow time dependence and a linear equation for the perturbation to the dipole streamfunction. We use two solvability conditions to demonstrate that the enstrophy in the dipole is conserved in the presence of the strain field, while the energy decays. We add an assumption that the eddy maintains the form of a Batchelor/ Lamb modon; this seems necessary because we cannot solve the perturbation equation in detail. Presumably a full solution would allow us to determine the evolution of the functional relationship between the potential vorticity and streamfunction. With this assumption, we can show that the speed is fixed, but the radius decays and the amplitude decreases as $t^{-1 / 2}$.

Finally, we consider the problem of an equivalent barotropic monopolar eddy. Here, neither of the approaches above works. The eddy is radiating in the only available mode, but is not coupled weakly. Nor is the wave scale significantly different form the eddy scale; both are order of the deformation radius. The integral theorem suggests that the speed should be near the long wave speed, as do numerical experiments. If $c=-\beta R_{d}^{2}$, the system is at the boundary between trapped and wavelike; however, it can still radiate because of adjustment to the initial state. Analysis of the resultant radiation pattern (using an adhoc and unjustifiable linearization) suggests that an anticyclonic vortex should have a southward velocity proportional to $t$ for small times and to $1 / t$ for long times as the wave field is gradually left behind. 
In summary, we have explored a number of models of strong eddies which decay because of loss of energy to radiating modes. In some cases these losses could be made up for by other mechanisms, such as the absorption of incoming waves or vortices; in other situations, the waves simply provide a natural (and non viscous) decay mechanism. While steady state solutions provide much information about the dynamics, these unsteady solutions may be more relevant to many strong oceanic and atmospheric phenomena.

\title{
CONVECTION AND CaAOS
}

\author{
Andrew C. Fowler
}

University of Oxford

Abstract. We discuss two types of chaotic behaviour exhibited by high Prandtl number convection, that is 'phase chaos' and plumes. In mantle convection, these differing aspects of the motion find their expression in the migration of subduction zones and hot spots, respectively. The analysis of plumes in a single convection cell can be attempted in the framework of Howard's 'bubble' model of convection, using an asymptotic analysis based on a similar method applied to the Lorenz equations. In the partial differential equation case, this leads us in principle to an approximate Poincare map for the flow. However, we find that Howard's assumption of differing time scales for the processes of growth and flow instability is in error, and (for a single developing plumel the thermal regime is likely to be periodic. For the case of a large aspect ratio, where multiple plume development can take place, the corresponding Poincare map should lead to a chaotic distribution of plumes in space and time.

For cellular convection, we give a synopsis of the recent scaling theory of the Chicago group. There is a mean flow in the cell, fuelled by thermal plumes which erupt from the boundary layer as they are advected across the cell. The theory is strictly applicable, however, only to Prandtl numbers of $0(1)$.

Cellular ('phase') chaos at large Rayleigh number can be modelled using a set of ordinary differential equations for variables which describe the size and location of slowly varying convection cells. The differential equations are parametrised using quasi-stationary boundary layer theory. The same method can in principle be extended to three dimensions, and represents a paradigm for the study of time-dependent motions of the earth's lithospheric plates. 


\title{
On thermonuclear convection
}

\author{
Sandip Ghosal
}

\begin{abstract}
Dilke and Gough (1972) suggested that some g-modes in the sun might be overstable, triggering periodic mixing of the solar core. This according to the authors might account for the observed deficiency of neutrinos coming from the sun and also explain the ice ages. Unno [6], with the help of a quasiadiabatic analysis using the work integral, concludes that $l \sim 3$ or 4 and $n \sim 1$ are the modes most likely to be unstable. Instability of these modes have been looked for numerically but no firm conclusion has yet been reached. Unno's quasiadiabatic analysis is valid provided the time scale of $\mathrm{g}$-mode oscillations is much shorter than the thermal time scale. This condition is clearly satisfied for the low 1 low $n$ modes. However, for the low 1 high $\mathbf{n}$ modes, the horizontal wavenumber is small and the g-mode oscillation period approaches zero. We consider a two layer model with the lower layer containing temperature-dependent heat sources (representing the layer of $\mathrm{He}^{3}$ in the solar core.) This model should always be stable according to the quasiadiabatic analysis. But it is found that in the limit of large horizontal scales, thermal instability affects the spatial structure of the g-modes (which in this limit have long time scales) and induce a monotonic instability.

The degree of the temperature dependence of the heat sources is described by a parameter $\mathrm{E}$. The critical $\mathrm{E}$ for the onset of thermonuclear convection depends on the boundary conditions assumed, but it is found to be in the same general range as the values of $E$ in the solar core. A nonlinear analysis is made and it is found that the onset of thermonuclear convection is described by a subcritical bifurcation in $\mathrm{E}$. This kind of qualitative analysis suggests that there might be long and narrow "shellular" convection patterns in the solar core confined mostly in the region of the $H e^{3}$ layer. If this is true, it will have the following effect on our calculation of the neutrino luminosity of the sun: the rate of generation of heat in the layer we are considering can be represented as $\left\langle T^{m}\right\rangle$ where $\mathrm{m}$ is some integer $\sim 5$ and $\langle>$ denotes horizontal average. $\langle T\rangle$ is the temperature distribution computed from the spherically symmetric standard solar model. Now $\left\langle T^{m}\right\rangle$ is greater than $\langle T\rangle^{m}$, therefore the luminosity of the new model with the shellular convection is a bit larger than that of the standard solar model. In order to bring the luminosity into agreement with the observed luminosity the central temperature of the model must be decreased. Since the neutrino luminosity $L_{\nu}$ of the high energy neutrinos come from a region very close to the centre, $L_{\nu} \sim T_{c}^{n}$ where $T_{c}$ is the central temperature and $n \sim 16$. Therefore a small change in $T_{c}$ produces a significant change in $L_{\nu}$. A numerical estimate shows that in order to reduce $L_{\nu}$ to half its value one needs about a 40 percent variation of the temperature over the length of a cell (for the $l=1$ modes.) The possible effects of rotation have not been studied yet. It is important to know whether this kind of convection that has been demonstrated qualitatively is really present in the sun even if it turns out that the solar neutrino problem has a different origin; complete understanding of the macroscopic aspects of the problem is essential if one for example wants to set some bounds on the neutrino mass.
\end{abstract}




\section{References}

[1] Dilke F.W.W. and Gough D.O. Nature 240, no.5379, p262, Dec1 1972.

[2] Defouw R.J., thesis (1970)

[3] Defouw R.J., Ap.J 160, 659 (1970)

[4] Field G.B., Ap.J., 142, 531 (1965)

[5] Unno W.,Osaki Y.,Ando H.,Saio H.,Shibahashi H., "Nonradial oscillations of stars", University of Tokyo press, 1989.

[6] Unno W.,Publ. Astron. Soc. Japan, 27, 81-99, (1975).

[7] Boury A., Gabriel M., Noels A., Scuflaire R., Ledoux P., Astron. and Astrophys. 41, $279-285(1975)$

[8] Christen-Dalsgaard J., Dilke F.W.W., Gough D.O., Mon. Not. R. Astron. Soc. 169, $429-445$ (1974)

[9] Defouw R.J., Siquig R.A., Hansen C.J., Ap.J. 184, 581-586 (1973)

[10] Dziembowski W., Sienkiewicz R., Acta Astr. 23, 273 (1973)

[11] Saio H., Ap.J. 240, 685 (1980)

[12] Rosenbluth N.M., Bahcall N.J., Ap.J. 184, 9-16 (1973)

[13] Saio H., Cox J.P., Hansen C.J., Astron. and Astrophys. 85, 263-264 (1980)

[14] Schwarzschild M., Harm R., Ap.J. 184, 5-8 (1973)

[15] Shibahashi H., Osaki Y., Unno W., Publ. Astron. Soc. Japan, 27, 401-410 (1975)

[16] Spiegel E.A. and Veronis G., Ap.J., 131, 442 (1960)

[17] Parker E.N., Ap.J., 117, 431 (1953)

[18] Merryfield W.J., Toomre J., Gough D.0., Ap.J. 353, 678-697 (1990) 


\title{
ON FAST DYNAMO ACTION IN STEADY CHAOTIC FLOW
}

\author{
A.D. Gilbert, D.A.M.T.P., Cambridge University, U.K., \\ S. Childress, C.I.M.S., New York University, U.S.A. \\ \& U. Frisch, Observatoire de Nice, France.
}

It is now understood how the motion of a conducting fluid can amplify weak magnetic field (see, for example, Moffatt 1978 for a review). Although kinematic dynamo theory is generally believed to explain the existence of terrestrial and solar magnetic fields, many fascinating questions remain. One interesting problem is to understand the rapid rate of magnetic field generation in the sun. This led to the following distinction (Vainshtein \& Zeldovich 1972): a dynamo is called "fast" if the magnetic energy grows on a convective time-scale when the magnetic Reynolds number is very large, as is the case for the sun. If the growth of the field occurs on a diffusive time-scale, which is very large in the sun, or on some intermediate time-scale, the dynamo is called "slow". Chaotic flows are prime candidates for fast dynamo action since they stretch vectors exponentially; in the absence of magnetic diffusion, the magnetic energy grows exponentially on the convective timescale. However a chaotic flow folds as well as stretches field, and this generally leads to intense dissipation of field when weak diffusion is introduced. This can dramatically reduce the growth of field and even kill it completely, an example being when the flow is planar.

We have considered dynamo action in a steady chaotic flow which is modelled on the $A B C$ flow for $A=B=1$ and $C \ll 1$ (see, for example, Dombre, Frisch, Greene, Hénon, Mehr \& Soward 1986). The model flow is constructed in such a way that the motion of particles and magnetic field vectors can be calculated exactly when there is no diffusion (Gilbert \& Childress 1990). The model flow contains a chaotic web and field is stretched exponentially. We calculated the evolution of field and found evidence that field is folded constructively in the sense that the average field in a volume grows exponentially in time. This provides evidence for fast dynamo action since the principal effect of weak diffusion is to smear field over space, destroying fine structure while leaving mean field behind (Finn $\&$ Ott 1988). We have also obtained preliminary results for the original $A B C$ flows for the case $A=B=C=1$, which indicate constructive folding of magnetic field and suggest fast dynamo action. Research is now underway to include the effects of weak diffusion in our calculations.

\section{References}

Dombre, T., Frisch, U., Greene, J.M., Hénon, M., Mehr., A. \& Soward, A.M., J. Fluid Mech., 167, 353 (1986).

Finn, J.M. \& Ott, E., Phys. Fluids, 31, 2992 (1988).

Gilbert, A.D. \& Childress, S. Phys. Rev. Lett., submitted (1990).

Moffatt, H.K., "Magnetic fluid generation in electrically conducting fluids," Cambridge University Press (1978).

Vainshtein S.I. \& Zeldovich, Ya.B., Sov. Phys. Usp., 15, 159 (1972). 


\title{
ARTIFICIAL BOUNDARY CONDITIONS FOR WAVE PROBLEMS IN UNBOUNDED DOMAINS
}

\author{
by \\ Dan Givoli \\ Dept. of Aerospace Engineering \\ Technion, Haifa 32000 , Israel
}

\begin{abstract}
When solving numerically a wave problem in a unbounded domain, one first has to make the computational domain finite by introducing an artificial boundary. Then an appropriate boundary condition must be imposed on this artificial boundary, so that waves coming out of the computational domain are transmitted through the boundary without giving rise to spurious reflection. A considerable amount of work has been done to devise such non-reflecting artificial boundary conditions. In this talk the various approaches are reviewed, usually leading to local approximate boundary conditions. Then a new method is presented, which is the result of joint work with Joseph B. Keller. In this method we choose the artifcial boundary to be a circle or a sphere, and we derive an exact nonlocal boundary condition on this boundary. Thus, the original problem in the unbounded domain is replaced by another problem in a finite domain which has exactly the same solution there. The finite element presented which demonstrate the superiority of the exact nonlocal boundary condition over approximate local ones.
\end{abstract}




\title{
Very High Resolution Solar X-ray Imaging
}

\author{
Leon Golub \\ Smithsonian Astrophysical Observatory \\ 60 Garden Street, Cambridge MA 02138 USA
}

\section{Magnetic Fields and Coronal Emission}

Study of the solar corona has a relatively short history - only 150 years - with a handful of major milestones along the way (Table I). Briefly, following the rather late recognition that the Sun has a corona at all, the big breakthrough came with the realization that the coronal temperature is of order $10^{6} \mathrm{~K}$. This high temperature explains the possibility of a corona extending to heights of several Solar radii above the photosphere; it also has the further implication that, because thermal conductivity is so efficient, an unconstrained corona will expand outward, producing a solar wind; this was subsequently observed when satellites were placed into orbit and could measure the particle flux directly. The last major milestone was the realization that there exists a close connection between regions of enhanced emission in the lower corona and locations on the solar surface at which strong magnetic field regions have emerged from the interior.

\section{Table 1. Milestones in Coronal Physics}

1842: Total eclipse across S. Europe - first serious study of corona 1850's: Photography of corona and its spectrum 1930: Development of coronagraph 1939: Identification of $\lambda 6374 \mathrm{~A}$ as $\mathrm{Fe} \mathrm{X}$ 1940's: Solar radio astronomy begins 1946: Availability of UV, XUV and x-ray observations from space 1958: Unconstrained hot corona will expand - solar wind 1960's: Connection between enhanced coronal regions and magnetic fields

The connection between coronal activity and magnetic fields is most easily seen when the corona is viewed on-disk, as can be done in X-rays (Figure 1). It is clear that the corona is brightest when there is newly emerged magnetic flux and weaker when the field has diffused across the surface. The open, unconstrained corona, which is connected with high-speed solar wind streams, Bartels M-regions, and recurrent geomagnetic substorms, is associated mainly with coronal holes (Krieger, Timothy and Roelof 1973), which are large areas of the Solar surface dominated by a single magnetic polarity and within which the magnetic field is open to interplanetary space.

The relation between $\mathrm{B}$ and $\mathrm{X}$-ray emission is more than just qualitative; the view which developed out of the Skylab analysis was that the magnetic field played an active role in the coronal heating process (Rosner et al. 1978). From this view it is possible to obtain scaling relations among observable quantities (Golub et al., 1980) which can then be compared to the actual data. There is, however, no unanimity about this view, and theories which consider the B-field to have a more passive role are still being explored. For instance, a popular idea in the 60 's was that closed loops would trap Alfven waves, and this view is once again being explored in a quantitative manner today. 

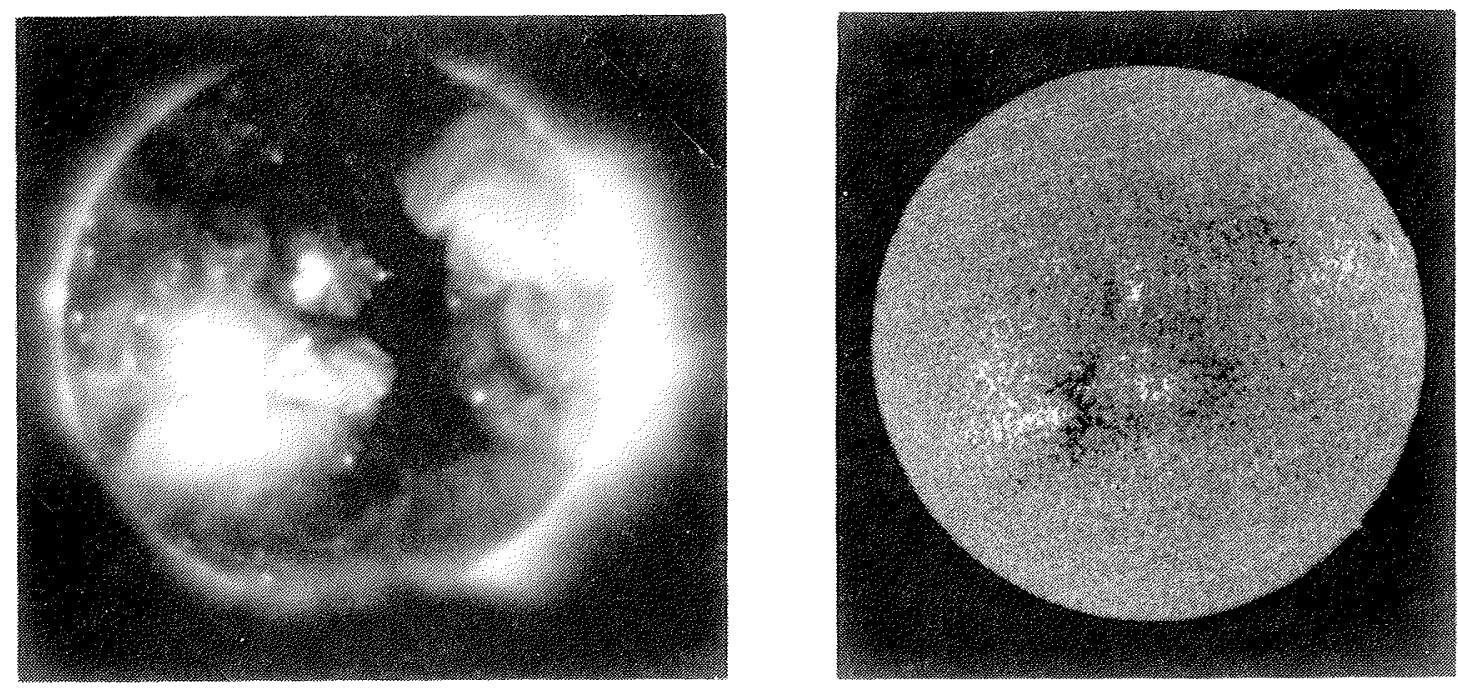

Figure 1. Skylab soft $\mathrm{x}$-ray image of the corona compared with KPNO magnetogram on the same day. Bright regions of enhanced coronal emission are clearly associated with the surface locations at which strong bipolar magnetic fields have emerged.

No matter what heating mechanism is invoked, it must explain the strong correlation between the intensity of coronal emission and the strength of the magnetic field. However, the correlation is not a simple one because, as we will show below, high temperature plasma is not seen above sunspot umbrae, which are the strongest magnetic field regions. Clearly, there is more involved than just the strength of the magnetic field or the length of the loop, and we must consider the footpoint boundary conditions as well when we attempt to model the formation and heating of coronal structures (Rosner and Golub 1990).

\section{X-ray Detection and Recording Methods}

The two basic instrumental challenges involved in X-ray observations of the corona are formation of an image and recording of the image. Each of the tasks can be divided into two major methods: X-ray imaging has been accomplished for many years by grazing incidence optics, which have the advantage that they are proven reliable, that they can be used at short wavelengths and that they. work over a broad wavelength range. The alternative technique which we are using, is multilayer coating of figured optics, which permits X-rays to be reflected at normal incidence. This technique has the advantage that image quality, in terms of optical figure quality, aberrations and scattering, is substantially improved. Also, the optics are relatively low cost and lightweight, and they work over a narrow wavelength band, thereby providing some spectroscopic capability free of charge.

For image recording one can either use photographic emulsions or electronic (TV-type) array detectors. The latter have progressed significantly in recent years (Kalata and Golub 1988), but for an object like the Sun they still are not wholly adequate. This is because the Sun has a large angular diameter and we are now achieving subarcsecond resolution. In order to fully utilize the information in a $1 / 2$ arcsecond resolution image, one needs a pixel size of $1 / 4$ arcsecond (actually 0.22 arcsec). With an angular diameter of $\simeq 2500$ arcsec if we want to image portions of the corona projecting above the limb, the number of pixels needed is $10,000 \times 10,000$, i.e., $10^{8}$ pixels per image or $10^{9}$ bits per image. 
There are some methods available by which this large image format could be achieved, but their costs far exceed the budgetary constraints of a rocket program or even most astronomy satellite missions. We are therefore using film to record the image, while also developing an electronic X-ray imaging detector which operates at broadcast-quality TV resolution. For photographic emulsions, the sensitivity is roughly inversely proportional to the square of the resolution; there is thus a tradeoff between resolving power and exposure time. In a rocket flight the observing is about 5 minutes, so that exposure time is a major consideration; the number of photons per second reaching the focal plane translates directly into a limiting resolution for the experiment.

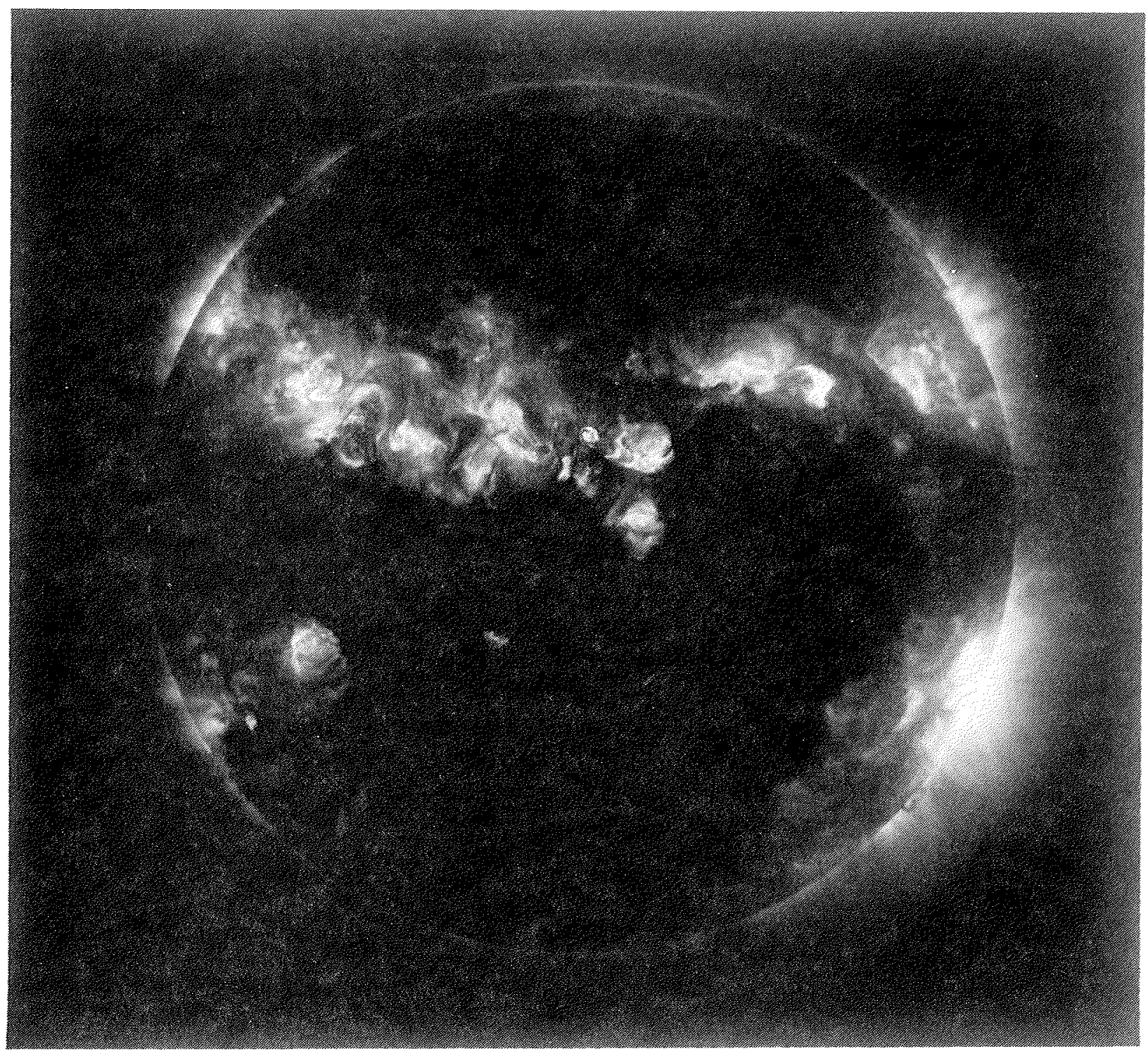

Figure 2. X-ray image of the Solar corona obtained by the NIXT sounding rocket payload, 11 September 1989; data are recorded at $63.5 \mathrm{~A}$ in the coronal emision lines of $\mathrm{Mg} \mathrm{X}$ and Fe XVI. 
The NIXT payload uses a $25 \mathrm{~cm}$ diameter, $\mathrm{f} / 8$ mirror at prime focus, coated to reflect 63.5 A x-rays. The passband includes coronal emission lines of Fe XVI and Mg X, formed at temperatures of $3 \times 10^{6}$ and $1 \times 10^{6} \mathrm{~K}$, respectively (Golub et al. 1990). This passband permits imaging of the quiet corona as well as active regions; also, as we describe in sec. 4 below, plasma at flare temperatures is recorded by this instrument in a particularly effective manner.

\section{Loop Atmospheres}

The new high resolution data (Figure 2) reveal three major features of importance for models of coronal formation and heating (Golub et al. 1990): i) the coronal loops which were observed in previous grazing incidence studies are now resolved into more numerous thinner, i.e., higher aspect ratio ("spaghetti" model) loops; ii) the x-ray loops seen in the corona maintain a fairly constant cross-section for most of their length, then taper rapidly at their endpoints, terminating in small patches of bright chromospheric emission, either in regions of enhanced network or in penumbral brightenings; iii) there are no hot loops terminating within sunspot umbrae: the corona directly above sunspots is dark and is surrounded by hot, bright loops which originate in strong magnetic field regions outside of the spots themselves.

\section{Flares}

The multilayer coated mirrors which we used in obtaining the new data have the property that they will reflect and focus any $63.5 \mathrm{~A}$ x-rays which enter the telescope. The central passband wavelength was chosen because of the Fe XVI emission coronal emission line, so that active regions will be most effectively imaged. However, the high temperature $\left(10^{7} \mathrm{~K}\right)$ plasma produced in flares will emit continuum at all soft $\mathbf{x}$-ray wavelengths, and the portion of this continuous spectrum which falls within the NIXT passband will be imaged just as are any other 63.5 A photons. We are thus able to observe not only the active regions which produce flares, but also the flares which occur in those regions.

Moreover, the fact that it is continuum rather than line radiation which is imaged has, by chance, the desireable property that the amount of radiation in the multilayer passband is, per unit emission measure, sharply reduced. This effect largely cancels the observationally problematic fact that, in a flare, the emission measure is greatly enhanced at all wavelengths, reaching a maximum at the higher temperatures. However, because the NIXT response is lower at high temperatures, the flare is only an order of magnitude brighter in our images than are active regions, even though the emission measure in the flare is over a thousand times greater than that of active regions. We are thus able to observe both the flare and the surrounding fainter regions in the same image; in this we are also helped by the extremely low scattering of multilayer mirrors, which keeps the bright emission localized away from the fainter features. It is for these reasons that flares have the beneficial property of looking distinctly unspectacular in the NIXT data. 
There are two striking new features visible in the $x$-ray data: the main body of the flare consists of a single bright arch of emission, comprising about most of the total; a 3-D display of the flare intensity is shown in Figure 5 to illustrate this point. The second feature is that there is bright $\mathrm{x}$-ray emission at the location of the two $\mathrm{H} \alpha$ ribbons, and each of the $\mathrm{x}$-ray ribbons contains structure within it. Examination of the development of the flare in $\mathrm{H} \alpha$ explains the latter observation: each of the flare ribbons is itself observed to be a small two-ribbon event, so that what we are observing is a pair of two-ribbon flares within the larger two-ribbon event. Since we have only this one observation it is too soon to tell whether this type of complication is characteristic of flares in general or peculiar to this one event. However, the $\mathrm{H} \alpha$ development of this flare was not particularly unusual, so it appears that our new ability to see the fainter $\mathrm{x}$-ray structure simultaneous with the brighter structure is really yielding a new view of flares.

During the brief flight of the NIXT payload, a second flare started in an active region at the west limb. In $\mathrm{H} \alpha$ the flare is seen as a small spray of material beginning at about 16:36 UT. As often happens in such events, the material observed in the $\mathrm{H} \alpha$ ejection disappears as it leaves the Solar surface; however, since the event is transverse to the line of sight, it is not likely that the material is Doppler shifted out of the passband. Instead, we are now able to establish through the $\mathrm{x}$-ray observations that the material disappears from $\mathrm{H} \alpha$ because it is heated: in x-rays we observe an event which is co-spatial and nearly co-temporal with the $\mathrm{H} \alpha$ event. The main difference is a small time delay between the two, in that the evolution of the event in $\mathrm{x}$-rays is about $1 / 2$ minute behind the $\mathrm{H} \alpha$ event. Moreover, the $\mathrm{x}$-ray ejection appears to be a hollow cone of hot material, as would be the case if a layer on the outside of the ejected chromospheric material is being heated as it rises. A detailed study of this event is currently in progress (Herant et al., in preparation).

\section{references}

Golub, L., Herant, M., Kalata, K., Lovas, I., Nystrom, G., Pardo, F., Spiller, E. and Wilczynski, J.: 1990, Nature 344, 842.

Golub, L., Maxson, C., Rosner, R., Serio, S. and Vaiana, G.S.: 1980, Ap. J. $238,343$.

Kalata, K. and Golub, L.: 1988, Proc. SPIE 982, 64.

Krieger, A.S., Timothy, A.F. and Roelof, E.C.: 1973, Solar Phys. 29, 505.

Rosner, R. and Golub, L.: 1990, J. Geoph. Res. (in press).

Rosner, R., Golub, L., Coppi, B. and Vaiana, G.S.: 1978, Ap. J. $222,317$.

Spiller, E.: 1990, Opt. Eng. 29, 609. 


\title{
Coherent Structures and Statistical Theory of Turbulence
}

\author{
Jackson Herring \\ National Center for Atmospheric Research*, Boulder, Colorado 80307, U.S.A.
}

The statistical theory of turbulence (in the form of equations of motion for the twopoint covariances, such as Kraichnan's, 1959 DIA) may be viewed as the logical avenue to single-point equations for Reynolds stress and energy dissipation (Leslie, 1973; Herring, 1973; Yoshizawa, 1980). For example, far from boundaries a typical closure for the Reynolds stress $\left\langle u_{i} u_{j}\right\rangle \equiv R_{i j}(\mathbf{x}, t)$ is (Hankalic and Launder, 1972):

$D R_{i j} / D t=-\left(c_{I} / \tau\right)\left(R_{i j}-(2 / 3) \delta_{i j} E\right)+c_{D} \partial_{k}(E / \epsilon)\left\{\left(R_{k n} R_{i j, n}+R_{j n} R_{i k, n}+R_{i n} R_{j k, n}\right\}+\cdots\right.$

In (1), D/Dt $\equiv \partial_{t}+\left\langle u_{i}\right\rangle \partial_{i}, E$ is the kinetic energy $=(1 / 2) R_{i i}$, and $\tau$ the turbulence time-scale $E / \epsilon, \epsilon$ being the energy dissipation whose equation of evolution begins as

$$
D \epsilon / D t=-c_{\epsilon} \epsilon / \tau+\cdots
$$

Equations (1) and (2) lead-in their simplest form-to mixing length, or $\kappa-\epsilon$ estimates for heat and momentum transport. Coefficients $\left(c_{I}, c_{D}, c_{\varepsilon}\right)$ specify rates at which the state of homogeneity $\left(c_{D}\right)$, isotropy $\left(c_{I}\right)$, and time-scaling $\left(c_{\epsilon}\right)$ are approached in the absence of forcing. They may be "derived" from two-point closures by making the usual WKB approximation, assuming $\left.R \equiv \mathbf{x}_{1}+\mathbf{x}_{2}\right)$ slow, and $\rho \equiv(\mathbf{1} / 2)\left(\mathbf{x}_{1}-\mathbf{x}_{2}\right)$ fast. Effects of the fast variables then show up in (1) and (2) in determining the coefficients that are in reality spectral integrals (see Yoshizawa, 1980). Hence, it is important to assess under what conditions such equations may have sufficient validity to be useful guides in inferring heat and momentum transport. Generally, consolidating of the flow into isolated structures is inhospitable to the underlying near-Gaussian assumptions made to arrive at the two-point formalism. This talk, then, mainly concerns some examples of flow in which such structures may play an important role.

We examine first the simple problem of the decay of isotropic turbulence starting from initial conditions in which the energy (and enstrophy $\Omega \equiv \int_{0}^{\infty} p^{2} E(p) d p$ ) is mainly at large scales and for which viscous effects are negligible. The question is whether the flow will remain of bounded variation for a finite time, or will-as predicted by some of the simpler statistical theories (such as the eddy-damped Markovian quasi-normal theory, e,g., Lesieur, 1988, pp.92-94)-explode, with $\Omega \rightarrow \infty$ at a time $t_{c} \sim 6 \Omega(0)$. Recent numerical simulation results for random initial conditions are discussed, which suggest that $\Omega$ increases only exponentially. We argue that such regularity suggests quasi-twodimensional small scales (á $l a$ Pumir and Siggia, 1990), and that the tendency is toward an exotic state unrelated to real turbulence. For example, an exponential growth of $\Omega(t)$ implies an exponential decrease of $\left\langle(\partial u / \partial x)^{3}\right\rangle /\left(\left\langle(\partial u / \partial x)^{2}\right\rangle\right)^{3 / 2} \equiv S(t)$. This follows from the simple isotropic relation (see e.g., Lesieur op. cit. p. 93, equation (7-5)):

$$
d \Omega(t) / d t \sim S(t) \Omega^{3 / 2}
$$

* The National Center for Atmospheric Research is sponsored by the National Science Foundation. 
Experimentally $S \sim .5$, for developed turbulence. The explanation of the failure of the closure in this case lies in the spacial separation of regions of strong vorticity $(\omega)$ from strong strain $\mathcal{S}$. This is readily seen from (3), and the relation (see again Lesieur, op. cit. p. 93 , equation $(7-2))$

$$
d \Omega(t) / d t=\left\langle\left(\omega_{i} \mathcal{S}_{i j} \omega_{j}\right)\right\rangle
$$

Some numerical data is presented that illustrates these points.

Next, we examine the question of "Eulerization", by which we mean that there exist patches in the flow in which the nonlinear terms are strongly reduced from their nominal Gaussian value. (The discussion is drawn from Chen et al., 1989). Such "Eulerized" regions are thought to be related, but perhaps not synonymous with, coherent structures. One measure of the importance of such a region is

$$
\left.\left\langle(\partial \mathbf{u} / \partial t)^{2}\right\rangle /\left\{(\partial \mathbf{u} / \partial t)^{2}\right\rangle\right\}_{G}
$$

where $\{\cdot\}_{G}$ means that the moments of $\mathbf{u}$ entering $\left(\partial_{t} \mathbf{u}\right)^{2}$ are evaluated as if $\mathbf{u}$ were Gaussian. The comparisons of DNS with closure (in the form of the DIA, Kraichnan, 1959) are in reasonable agreement, both predicting a reduction by about a factor of 2 in the mean-squared Eulerian acceleration, as compared to its Gaussian evaluation.

Finally we consider two-dimensional turbulence, which exhibits an extreme tendency to organize itself into isolated vortices if left to decay. We examine such structures and the failings of the statistical theory, as described in Herring and McWilliams (1984). We may get at the issue of separateness of vorticity and strain by examining-in the mean field approximation-the short time development of the two-point Reynolds stress in the presence of a structured mean field, which represents-in an admittedly vague way-the large scales of the flow. The spectrum of the Reynolds stress is specified in a compact representation in which $R_{i j}(\mathbf{k}) \Rightarrow\left\{\left\langle\left|u_{1}+i u_{2}\right|^{2}\right\rangle \equiv U_{0}(k),\left\langle\left(u_{1}-i u_{2}\right)^{2}\right\rangle \equiv U_{2}(k)\right\}$. (These are just $\sim$ the first two angular harmonics of the stream function, Herring, 1975). Then for $U_{0}(k), U_{2}(k)$ :

$$
\begin{gathered}
(D / D t+2 \nu(k)) U_{0}(k)=\mathcal{S}^{*}(1+k \partial / \partial(4 k)) U_{2}(k)+\cdots \\
(D / D t+2 \nu(k)) U_{2}(k)=i \zeta U_{2}+(1 / 2+k \partial / \partial(4 k)) U_{0} \cdots \\
\mathcal{S} \equiv\left\langle-\partial_{1} u_{1}+\partial_{2} u_{2}+i\left(\partial_{2} u_{1}+\partial_{1} u_{2}\right)\right\rangle \\
\zeta=\left\langle-\partial_{2} u_{1}+\partial_{1} u_{2}\right\rangle
\end{gathered}
$$

If we now assume an additional term in the RHS of (6) representing a linear return to isotropy $\left(-\mu U_{2}(k)\right)$, and that this term balances the mean vorticity "tumbling" term, we may eliminate $U_{2}(k)$ in (5), and find:

$$
(D / D t+2 \nu(k)) U_{0}=|S|^{2} \mu /\left(\mu^{2}+\zeta^{2}\right)(1+k \partial / \partial(4 k))(1 / 2+k \partial / \partial(4 k)) U_{0}(k)
$$

We remark that setting the RHS of $(9)$ zero $\Rightarrow E_{0}(k)=2 \pi k U_{0}(k) \sim k^{-3}$. Notice that regions of excess strain $\mathcal{S}$ (over vorticity $\zeta$ imply rapid transfer (to ever smaller scales), whereas regions where $|\zeta|>>|\mathcal{S}|$ are stable. This is similar to the "Weiss" criterion (Weiss, 1981) for discriminating zones of stability in two dimensional flow. Weiss' criterion 
is simply $\mathcal{S}^{2}<\zeta^{2}$ ) implies stability. In three dimensions, it is the second invariant of $\mathcal{S}$, proportional to the pressure variance: this point has been explored by Hunt (1988) and by Nelkin and Tabor (1990).

If we now try to integrate ( 7 ) into homogeneous turbulence theory, we have a fundamental problem in that for homogeneous flows, there is no way to discriminate between strain and vorticity, at least at the level of second-order moments. Thus, the mechanism for discriminating between stable and active regions is lost, unless we have equations that involve more than second-order moments and associated Green's functions.

\section{References}

Chen, H. D., J. R. Herring, R. M. Kerr, and R. H. Kraichnan, 1989: Non-Gaussian statistics in isotropic turbulence. Phys. Fluids A. 1(11), 1844-54.

Hanjalic, K., and B. E. Launder 1972: A Reynolds stress model of turbulence and its application to thin shear flows. J. Fluid Mech., 52, 609-638.

Herring, J. R., 1973: Statistical turbulence theory and turbulence phenomenology. In Proc. Langley Working Conf. on Free Turbulent Shear Flows. NASASP321, Langley Research Center, Langley, VA (available from NTIS as N73-2815415GA).

Herring, J. R., 1975: Theory of two-dimensional anisotropic turbulence. J. Atmos. Sci., 32, 2254-2271.

Herring, J. R., and J. C. McWilliams, 1985: Comparison of direct numerical simulation of two-dimensional turbulence with two-point closure: The effects of intermittency. $J$. Fluid Mech., 158, 229-242.

Hunt, J. C. R., A. A. Wray, and P. Moin 1988: Eddies streams and convergence zones in turbulent flows. In Studying Turbulence Using Numerical Simulation Database II; Proceedings of the 1988 Summer Program, Center for Turbulence Research. Report CTR-S88, December 1988, NASA Ames Research Center-Stanford University.

Kraichnan, R. H., 1959: The structure of isotropic turbulence at very high Reynolds numbers. J. Fluid Mech., 5, 497-543.

Lesieur, M., 1987: Turbulence in Fluids, Martinus and Nijhoff Publishers, Dordrecht, The Netherlands.

Leslie, D. C., 1973: Developments in the Theory of Turbulence, Clarendon Press, Oxford.

Nelkin, M. and M. Tabor, 1990: Time correlations and random sweeping inb isotropic turbulence. Phys. Fluids, 2, 81-83.

Pumir, A. and E. Siggia, 1990: Collapsing solutions to the 3-D Euler equations Phys. Fluids $A$ 2, 220-241.

Yoshizawa, A., 1980: Statistical theory for Boussinesq turbulence. J. Phys. Soc. Japan, 48, 647-652.

Weiss, J., 1981: The dynamics of enstrophy transfer in two-dimensional hydrodynamics. La Jolla Institute Report, La Jolla, CA. 123 pp. 


\section{A MODAL $\alpha^{2}$-DYNAMO IN THE LIMIT OF ASYMPTOTICALLY SMALL VISCOSITY}

\section{Rainer Hollerbach}

Inst. of Geophys, and Planet. Physics

Scripps Institution of Oceanography

La Jolla, CA 92093 USA

The macrodynamic equilibration of $\alpha^{2}$-dynamos in the limit of asymptotically small viscosity is considered. In the momentum equation, the primary balance of forces is between the Lorentz force and the Coriolis force. However, if one considers the integrated Iorentz torque on geostrophic contours, the Coriolis force is incapable of balancing this torque, and so if there is such a net torque it must be balanced by viscosity. As a result, in the limit of asymptotically small viscosity, the geostrophic flow induced by this torque can become quite large. The resulting scaling of the field, namely as some positive power of the viscosity, means that in the limit of vanishing viscosity one has no dynamo.

Taylor (1963) proposed that there simply is no such net torque, that the flow distorts the field in precisely such a way that there is no torque, and showed that this requirement in fact uniquely determines the flow. Thus, viscosity is not relevant or even present in this framework, and so the equilibration is inviscid, as geophysical scaling arguments suggest it ought to be. The difficulty with this approach is that in general, that is for most choices of $\alpha$, the linear kinematic eigensolution does generate a net torque, and so at least for some small range of supercritical forcing viscosity must be important.

However, as the forcing becomes more and more supercritical and hence the nonlinear coupling between the flow and the magnetic field becomes more and more important, it is conceivable, as hypothesized by Malkus \& Proctor (1975), that the flow will tend toward just the "eigenflow" required in Taylor's development. That this approach to the Taylor state does in fact occur, at least in an infinite planar geometry, was subsequently verified by Soward \& Jones (1983). It is this transition from the viscously controlled regime to the inviscid Taylor regime, and the subsequent equilibration, that we explore in this work, this time in a spherical gecmetry. 
The dynamo presented here is an expansion in the free decay modes of the magnetic field. The momentum equation is expanded as an asymptotic series, making use of a simplified functional form for the dissipation, and yields explicit expressions for the leading order geostrophic and next order ageostrophic velocities. With these expressions substituted back into the induction equation, a set of modal amplitude equations is derived and solved for a variety of choices of $\alpha$.

All $\alpha$ 's investigated yielded Taylor solutions. For some choices of $\alpha$ the Taylor state is approached in a smooth progression starting from the linear eigensolution, but for other choices it requires a finite amplitude jump. In the latter case the solution track leading to the Taylor state can be either stable or unstable, and this affects where the transition from the viscous regime to the inviscid regime takes place. In the asymptotic limit the subsequent equilibration is indeed independent of viscosity, as envisioned by Malkus \& Proctor, and depending on the choice of $\alpha$ it can be either steady-state or oscillatory.

\section{REFERENCES}

Malkus, W. V. R. and Proctor, M. R. E., "The macrodyramics of $\alpha$-effect dynamos in rotating fluids", J. Fluid Mech. 67, 417-443 (1975).

Soward, A. M. and Jones, C. A., " $\alpha{ }^{2}$-Dynamos and Taylor's constraint", Geophys. \& Astrophys. Fluid Dynam. 27, 87-122 (1983).

Taylor, J. B., "The magnetohydrodynamics of a rotating fluid and the Earth's dynamo problem", Proc. Roy. Soc. London A $274,274-283$ (1963). 


\title{
DYNAMICS OF LOCALIZED STRUCTURES VITH GALILEAM INVARIANCE
}

Christian Elphick, G. R. Ierley, Oded Regev and E. A. Spiegel

\begin{abstract}
We consider a nonlinear partial differential equation having both translational and Galilean invariance arising in the Kapitza problem (Benney, 1964). Under suitable conditions, Hopf bifurcation in extended systems, to leading order, is represented by this phase equation. We study the interaction of the localized structures that are formed in such systems both numerically and by means of an effective particle approach. The dynamics of a pair of interacting localized structures produces an interesting scattering problem with the possibility of capture into one of an infinite number of discrete bound states. For the many-body problem the localized structures organize themselves into patterns displaying spatial chaos.
\end{abstract}




\title{
Fast reaction, slow diffusion, curve shortening and harmonic maps1
}

\author{
Joseph B. Keller \\ Departments of Mathematics and Mechanical Engineering \\ Stanford University
}

\begin{abstract}
The reaction-diffusion. problem

$$
u_{t}=\varepsilon \Delta u-\varepsilon^{-1} V_{u}(u), \quad u(x, 0, \varepsilon)=g(x), \quad \partial_{n} u=0 \text { on } \partial \Omega
$$
\end{abstract}

for a vector $u(x, t, \varepsilon)$ is considered in a domain $\Omega \in R^{m}$. An asymptotic solution is constructed for $\varepsilon$ small. It shows that at each $x, u$ tends quickly to a minimum of $V(u)$. When $V$ has several minima, $u$ tends to a piecewise constant function. Boundary layer expansions are constructed around the resulting surfaces of discontinuity or fronts. Each front is found to move along its normal with a constant velocity determined by the discontinuity $[V]$ in $V$ across it. When $[V]=0$, the front's normal velocity is $\varepsilon \kappa$, where $\kappa$ is its mean curvature. The motion of fronts in this manner is studied for arcs in the plane which are normal to $\partial \Omega$ at their endpoints, and for fronts which are closed curves. It is shown a front can shrink to a point in a finite time or tend to a locally shortest diameter of $\Omega$. In the latter case, a nonconstant steady state $u(x, \infty, \varepsilon)$ results.

Then the asymptotic behavior of $u$ is determined for $\varepsilon$ small when $f(u)=0$ on a connected manifold $M$ of stable equilibrium points. It is found that $u$ tends rapidly to $M$, being driven by reaction. Then $u$ evolves slowly by diffusion restricted to $M$. It tends ultimately to a limit that is a harmonic map of $\Omega$ into $M$. Next, the case where $f(u)$ has stable equilibrium points on two manifolds $M_{1}$ and $M_{2}$ is treated. In this case a front develops in $\Omega$. It separates the regions where $u$ is close to $M_{1}$ from the regions where $u$ is close to $M_{2}$. For $f(u)=V_{u}(u)$ a boundary layer solution is constructed for $u$ near the front, and the velocity of the front is found to be proportional to the jump in $V$ across it, to leading order in $\varepsilon$. When $V(u)$ has the same value on $M_{1}$ and $M_{2}$, this term is zero and the front velocity is $\varepsilon$ times its mean curvature. The case of a spherically symmetric potential $V(|u|)$ and the case $M=S^{1}$ are presented to illustrate the results.

1 This lecture is based upon the following two papers which were published last year: Jacob Rubinstein, Peter Sternberg and Joseph B. Keller, SIAM J. Appl. Math. 49, 116$133,1722-1733(1989)_{i}$ 


\title{
POLYTROPES AND THEIR PERTURBATIONS
}

\author{
Norman R. Lebovitz \\ The University of Chicago
}

The classical theory of polytropes is that of spherically symmetric, self-gravitating figures of equilibrium in which the pressure-density relation is given by the formula $p=K p^{1+1 / n}$. This theory is summarized and the limitation to the range $0<n<5$ is derived in a simple manner via an identity due to Pohozaev.

For $n \rightarrow 5$ the figures tend to infinite central condensation, i.e., the ratio of central density to mean density becomes infinite. This limit therefore models the late stages of stellar evolution. For this purpose a perturbation theory based on $\varepsilon=5-n$ as the perturbation parameter, wherein the singular model of infinite central condensation is the unperturbed solution, is described. The formal theory is shown to have certain convenient properties, but the relation of the formal, asymptotic solutions to the exact solutions remains unsettled.

Rotational perturbations of polytropes have been discussed in the literature over a long period of time. Here the idea is that of using the angular velocity as a small parameter to obtain approximate solutions of the equations governing rotating stars. First some generalities regarding axially symmetric rotating masses are discussed, and then the theory of rotating polytropes is summarized. Defects in this theory are noted and certain possible modifications are suggested to try to correct them. Among these are a coordinate stretching, as implicitly employed in Clairaut theory, and a systematic version of an idea of Monaghan and Roxburgh employing different approximations in inner and outer regions, which might be able to extend the perturbation theory to large rotation rates. 


\title{
Interface Dynamics: Playing with Symmetries
}

\author{
A. Libchaber \\ The Research Institute \\ University of Chicago
}

\begin{abstract}
The directional growth of a nematic phase into the isotropic one of a liquid crystal is presented. Three lengthscales define the problem:

$l_{D}=D / V$, diffusion length, where $D$ is the impurity diffusion coefficient and $V$ the velocity of the interface. This is the destabilizing term.

$l_{T}=(m \Delta C) / G$, thermal length, where $G$ is the thermal gradient in $\mathrm{deg} \mathrm{cm}^{-1}$ and $m \Delta C$ the temperature jump across the solidus--liquidus region ( $m$ is the slope of the liquidus).
\end{abstract}

$l_{C}=\gamma L$, capillary length, where $\gamma$ is the interface tension and $L$ the latent heat.

$l_{T}$ and $l_{C}$ are stabilizing terms. The onset of a wavy pattern corresponds to $l_{D} l_{T}$. When $l_{D}$ or $l_{T}$ become comparable to $l_{C}$ the interface restabilizes. One thus defines and measures a stability tongue.

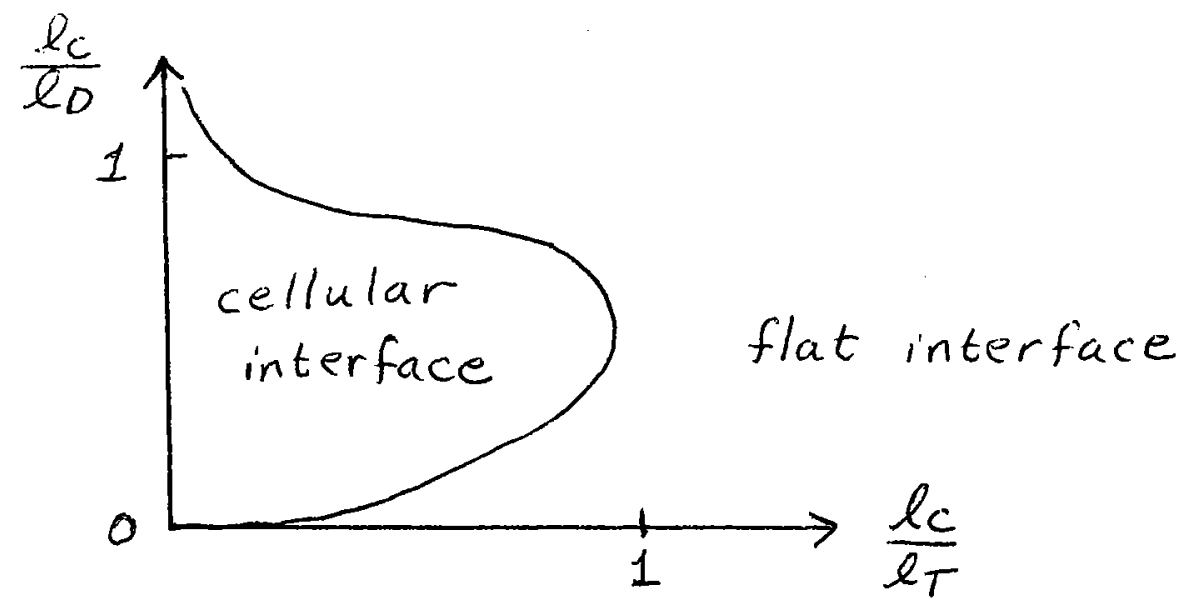

For small velocities the bifurcation to a cellular interface is supercritical. Secondary instabilities develop beyond this critical velocity. They correspond to a spontaneous breaking of translational invariance, leading to travelling waves with sources and sinks. For larger velocities, drops of tilted cells appear, breaking the parity invariance $x \rightarrow-x$. For even higher velocities time symmetry is broken.

We have presented an overview of this problem, where a rich variety of bifurcations can be studied. A simple model with two coupled modes of wavenumber 9 and 29 mimic the observations. 


\title{
Some Aspects of Convection in Binary Fluid Mixtures
}

\author{
Stefan J. Linz \\ FR.11.1 Theoret. Physik, Universitaet des Saarlandes \\ D-66 Saarbruecken, West-Germany
}

The onset of convection and the weakly nonlinear convective behavior of binary fluid mixtures in a Rayleigh-Benard setup has attracted growing interest in the last few years. In this seminar we reviewed some of our results [1-8] obtained in the last years. The system we consider is a horizontal layer of a binary fluid mixture enclosed between two parallel, impermeable plates. The gravitational field acts vertically. The system is described by the field equations for velocity, temperature, and concentration of the lighter component of the mixture. Temperature and concentration field are cross-coupled via the Soret effect (temperature fluctuations can drive concentration fluctuations) and the Dufour effect (concentration diffusion currents can drive temperature fluctuations). Between the boundaries there is a vertical temperature gradient.

Focussing on liquid mixtures (where the Dufour effect is ignorable) we derived a generalized Lorenz model [1] assuming idealized free-slip boundary conditions for the velocity field, but realistic impermeable boundary conditions for the concentration field. The model based on a Galerkin approximation allows two-dimensional standing and propagating roll patterns. The stability analysis $[1,2]$ shows that, near the codimension-two point where stationary and oscillatory instabilities compete, there is a gap in the critical wave numbers and consequently the zero Hopf frequency limit cannot be reached. The weakly nonlinear solutions were discussed: steady overturning convection $[1,2,4]$, traveling wave solutions $[3,4]$, standing wave solutions [4] and their stability. Beyond that, transport properties of traveling wave solutions were elucidated [3,4]: they generate heat, concentration, and mass currents and-via Reynolds stresses-a mean flow in the horizontal direction. These properties were later confirmed in a numerical simulation [9]. The changes of the stability thresholds caused by realistic no-slip boundary conditions were presented [7].

The influence of non-Oberbeck-Boussinesq effects for small Soret coupling and the effect of barodiffusion on the stability of the conductive state [5] were discussed.

We estimated that in gaseous mixtures the Dufour effect is no longer ignorable [6]. As an aside we presented a slightly different system where the temperature at the top and bottom boundary are equal and a vertical concentration gradient is applied. There the Dufour effect can generate stationary and oscillatory instabilities for large enough Dufour coupling [6]. This seems to be possible in gaseous mixtures. Returning to the RayleighBenard like system we studied the changes of the stability of the conductive state caused by the additional influence of the Dufour effect in gaseous mixtures [8].

Finally we reviewed briefly some of our results [4] on the convective behavior if there is in addition a porous medium between the boundaries. In particular we showed that there are no supercritical traveling wave solutions. 
[1] S.J.Linz,M.Luecke,Phys.Rev.A35,3997 (1987).

[2] S.J.Linz,M.Luecke,Springer Series in Synergetics 41,292 (1988).

[3] S.J.Linz,M.Luecke,H.W.Mueller,J.Niederlaender,Phys.Rev.A38,5727 (1988).

[4] S.J.Linz,Ph.D. Thesis, Universitaet Saarbruecken (1989).

[5] S.J.Linz,M.Luecke,Phys.Rev.A36,3505 (1987).

[6] S.J.Linz,Phys.Rev.A40,7175 (1989).

[7] W.Hort,S.J.Linz,M.Luecke, in Nonlinear Evolution of spatio- temporal Structures in Dissipative Continuous Systems (F.H.Busse,L.Kramer eds.), NATO ASI Series B (1990) and in preparation.

[8] O.Lhost,S.J.Linz,H.W.Mueller,submitted for publication.

[9] W.Barten,M.Luecke,W.Hort,M.Kamps,Phys.Rev.Lett.63,376 (1989). 


\title{
HYDROMAGNETIC INSTABILITY DUE TO ELLIPTICAL FLOW
}

\author{
David W. Hughes \\ Department of Applied Mathematics and Theoretical Physics \\ University of Cambridge \\ and \\ Willem V. R. Malkus \\ Department of Mathematics \\ MIT
}

An unbounded region of elliptical flow gives rise to three-dimensional instabilities which are exact solutions, (Bayly, 1986). The simplest and fastest growing of such solutions is the "spin-over" mode, (Waleffe, 1988, 1990). This "spin-over" mode is observed to be a principal instability in bounded laboratory flows (Gledzer et al., 1975, Malkus, 1989). The search for hydromagnetic consequences of these instabilities include the G.F.D. study by Brazell 1987 and the work of Craik 1988. In both of these latter papers it was concluded that an initial magnetic field was not amplified by the growing three-dimensional mode, but merely advected. An apparent exception to this finding was the special case of constant electric current parallel to the constant vorticity of the elliptic flow. This basic state permitted exponential growth of both the fluid spin-over mode and its magnetic counterpart, for any departures of the basic state from magnetic-kinetic energy equipartion (Bush 1988). The energy sources and equilibration of this unique solution are considered here. The complete equations for the disturbance vorticity $\boldsymbol{\omega}$ and disturbance current $j$ are written

$$
\begin{gathered}
\dot{\omega}=D:(\omega-\alpha j) \\
\dot{j}=R:(j-\alpha \omega)+\frac{1}{2}(\omega \times j),
\end{gathered}
$$

where $D$ is the elliptical strain matrix, $R$ the rotation matrix, and $\alpha$ is the value of the ratio of current to vorticity of the basic state. Bush showed that parallel disturbances, $\omega$ and $j$ which were orthogonal to the basic vorticity are exponentially growing, exact solutions to the above equations. It is found here that this special solution is unstable to slight variations in the parallelness of $\boldsymbol{\omega}$ and $j$. The figure below exhibits the growth and equilibration in time of the component of $j$ antiparallel to the basic current. In contrast to the initial exponential growth of the other $j$ components, the antiparallel component grows super-exponentially, due to the non-linear term, until it cancels the basic current. Although the disturbance vorticity continues its growth unabated, this study establishes that the disturbance magnetic field has drawn its energy from the basic magnetic field and has not exhibited dynamo action. Further studies should include the role of boundaries. The effect of a bounded domain is known to couple the spin-over mode to its basic field, and may couple it to the magnetic field as well. 


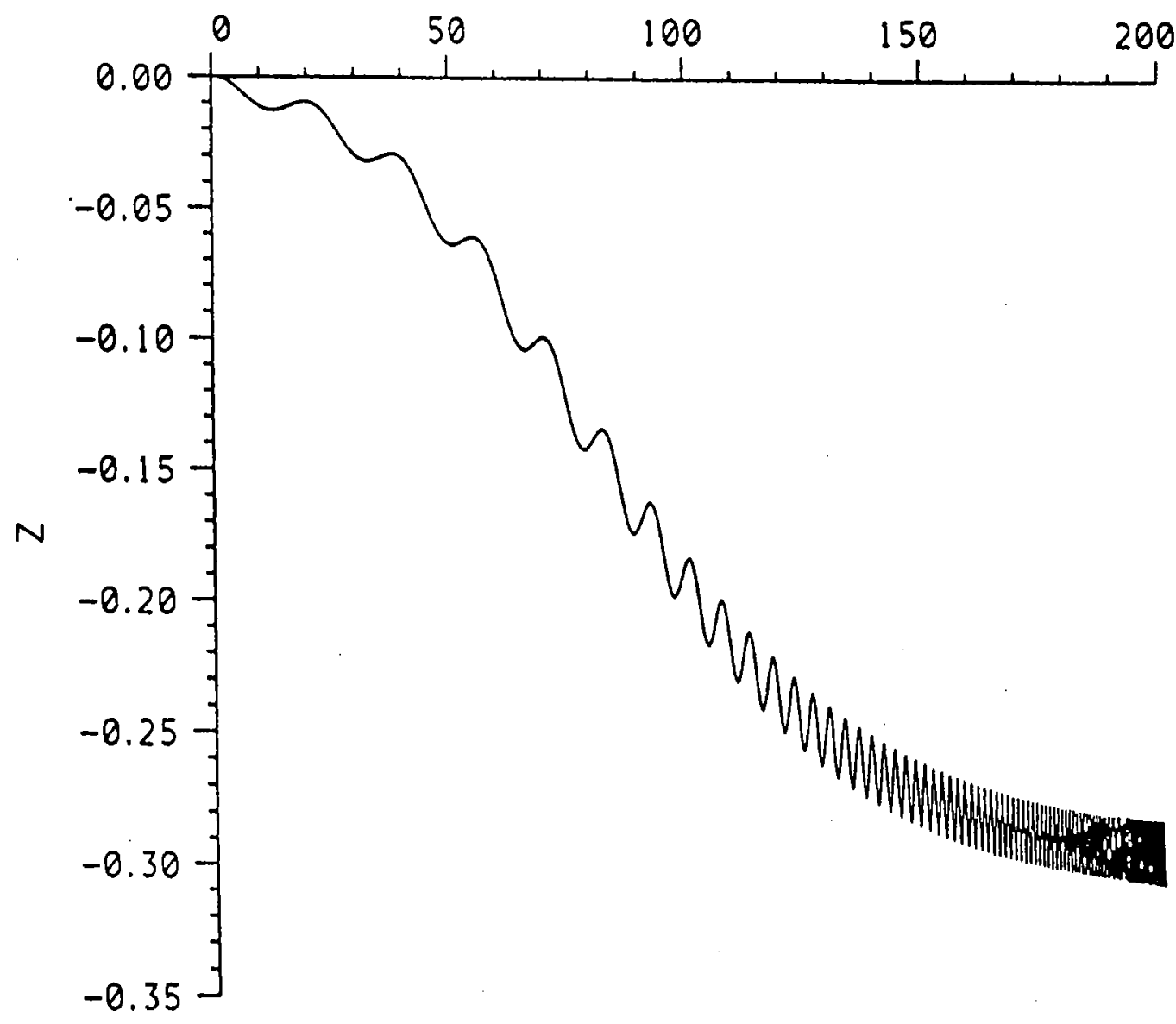

The super-exponential growth in time of the disturbance magnetic field, $Z$, antiparallel to the basic state.

\section{REFERENCES}

1. Bayly, B.J., "Three-dimensional instability of elliptical flow", Phys. Rev. Lett., 57, 2160, (1986).

2. Brazell, L., "The instability of an elliptical flow in the presence of a magnetic field", WHOI GFD (1987), Fellow's report.

3. Bush, J., "Hydromagnetic instability of elliptical flow", a M.I.T. 18.357 term paper, outlined in Malkus, 1989.

4. Craik, A.D.D., "A class of exact solutions in viscous incompressible magnetohydrodynamics", Proc. R. Soc. A, 417, 235 (1988).

5. Gledzer, Ye. B., Dolzhanskiy, F. V., Obukhov, A.M. and Pomonarev, V. M., "An experimental and theoretical study of the stability of motion of a liquid in an elliptical cylinder". Izv. Atmos. and Oceanic Phys., 11, 981 (1975).

6. Malkus, W. V. R., "An experimental study of global instabilities due to the tidal (elliptical) distortion of a rotating elastic cylinder", GAFD, 48, 123 (1989).

7. Waleffe, F., "3D instability of bounded elliptical flow", WHOI GFD (1988) Fellow's report.

8. Waleffe, F., "3D instability of unbounded elliptical flow", Physics of Fluids, A2(1), $76,(1990)$. 

The Free Energy "Principle". Negative Energy Modes
and Stability

\author{
P. J. Morrison
}

Department of Physics and Institute for Fusion Studies

University of Texas at Austin

Austin, TX 78712

The Free Energy "Principle" is a conjecture about stability in Hamiltonian dynamical systems ${ }^{1}$, such as those that describe the motion of ideal fluids and plasmas ${ }^{2}$. The conjecture proceeds from $\delta^{2} \mathrm{~F}$, a functional that measures the energy difference between a dynamically accessible perturbed state and an equilibrium state. This quantity is easily derived for all equilibria of ideal fluid and plasma models, e.g., Euler's equation, the ideal magnetohydrodynamics equations, and the Maxwell-Vlasov equations ${ }^{3}$. When $\delta^{2} \mathrm{~F}$ is definite an equilibrium is stable. This is a generalization of the Lagrange-Dirichlet theorem of mechanics, and differs from previous work regarding Liapunov stability 4,2 in that $\delta^{2} F$ is the energy difference restricted to the constancy of all of the kinematical or Casimir invariants. When $\delta^{2} \mathrm{~F}$ is indefinite, either the equilibrium is linearly unstable, or we have the interesting situation where a linearly stable equilibrium does not correspond to an energy extremum. In the latter case the system possesses a negative energy mode. This definition of a negative energy mode (NEM) is a generalization of that commonly used in plasma physics that is based on the dielectric function 5 . Finite degree-of-freedom Hamiltonian systems have NEM's when the linear normal form is stable with indefinite signature; for example, when the linear Hamiltonian in action-angle variables $(J, \theta)$ has the form $\delta^{2} F=\Sigma \omega_{i} J_{i}$, where some of the frequencies $\omega_{i}$ are negative. It is conjectured that systems with NEM's are generically nonlinearly unstable to infinitesimal perturbations, in spite of the fact that they are linearly stable. This is exemplified by an example due to Cherry ${ }^{6}$, which due to linear resonance demonstrates explosive growth. Three or more degree-of-freedom systems with NEM's are thought to be unstable without linear resonance, due to a mechanism known as Arnold diffusion. It is tempting to speculate that generically, inf inite degree-of-freedom systems with NEM's are also unstable. In 
addition to nonlinear instability, systems with NEM's are often structurally unstable when dissipation is added to the dynamical system. Physically this is an appealing intuitive idea since when energy is removed from an NEM its amplitude must grow. This is a generalization of the Kelvin-Tait theorem. In summary, the Free Energy "Principle" is the conjecture that systems with NEM's are generically unstable, either nonlinearly or structurally.

\section{References}

1. P. J. Morrison and M. Kotschenreuther in Proceedings of the 4th International Workshop on Nonlinear Turbulent Processes in Physics, Kiev, USSR, 1989 (World Scientific, Singapore, in press 1990); M. Kotschenreuther et al., in Plasma Physics and Controlled Nuclear Fusion Research 1986 (IAEA, Vienna, 1987), Vol. 2, p. 149.

2. P. J. Morrison and S. Eliezer, Phys. Rev. A 33, 4205 (1986); P. J. Morrison and D. Pfirsch, Phys. Rev. A 40, 3898 (1989).

3. P. J. Morrison and D. Pfirsch. Physics of Fluids B 2, 1113 (1990).

4. M. Kruskal and C. Oberman, Phys. Fluids 1, 275 (1958); C. S. Gardner, Phys. Fluids 6, 839 (1963); D. Holm. J. E. Marsden, T. Ratiu and A. Weinstein, Physics Reports 123, 1 (1985).

5. P. A. Sturrock, J. Appl. Phys. 31, 2052 (1960). See also R. C. Davidson, Methods in Nonlinear Plasma Theory. (Academic Press, New York, 1972).

6. T. M. Cherry, Trans. Camb. Phil. Soc. XXIII, 199 (1925). See E. T. Whittaker Analytical Dynamics, (Cambridge, London, 1937) Sec. 182. p. 142; and A. Wintner. The Analytical Foundations of Celestial Mechanics, (Princeton Univ., Princeton,1947) Sec. 136 bis, p. 101. 


\title{
Radiatively Driven Stellar Winds
}

\author{
Stan Owocki \\ Bartol Research Institute \\ University of Delaware \\ Newark, DE 19711
}

The massive winds from hot, luminous stars are thought to driven by line-scattering of the star's continuum radiation flux. This summary emphasizes the physics of this line-driving mechanism and what it implies for basic wind properties, as well as for wind structure and variability. Linear perturbation analyses of the basic, CAK model for a steady line-driven wind indicate that such winds are in fact highly unstable. Numerical simulations of the nonlinear evolution of small amplitude (1\%), periodic perturbations at the wind base show that this instability leads to high speed rarefactions which terminate in strong reverse shocks. Subsequent work indicates that such variability can have an intrinsic character that persists even in the absence of explicit perturbations, and it now seems that this is a direct consequence of a degeneracy of the steady-state solutions. Current efforts are aimed at determining how this degeneracy and intrinisic variability are affected by including the dynamical effects of the diffuse, scattered radiation field. Future work will focus on generalizing the model to 2-D (or 3-D) with rotation, and on modeling the unsteady flow energy and ionization balance with radiative terms.

A more complete discussion of these points can be found in my recent reviews, "Winds from Hot Stars" (1990; Reviews of Modern Astronomy, Vol. 3., Springer: Berlin) and "Theory Instrinsic Variability in Hot Star Winds", (1990; Proc. of IAU Colloq. \#143, K. Van de Hucht, ed.) 


\title{
CHAOS AND NOISE IN DYNAMICAL SYSTEMS WITH SLOW INVARIANT SUBSPACES.
}

Michael R. E. Proctor

Dept. of Applied Mathematics \& Theoretical Physics, University of Cambridge, Silver st., Cambridge CB3 9EW, England

\begin{abstract}
There are many dynamical systems of interest in mathematical physics which possess the feature that there is an invariant subspace in which one or more of the variables vanish. If parts of this subspace are attracting and parts repelling with respect to orthoginol perturbations, then the trajectory may return repeatedly to a neighbourhood of the subspace. If in addition the dynamics in the subspace takes place on an asymptotically long time scale, then it is possible to represent the dynamics by compositions of maps, all of which are independent of the time scale ratio. The method is particularly useful when the slow subspace is one dimensional. A particular example is given for the equations describing three-wave resonance, and it is shown that the dynamics may be reduced to a map of the interval, given in simple analytic form.
\end{abstract}

During the evolution the orthogonal variables can become extremely small, and so any small perturbation that destroys the invariant plane has a dramatic effect on the dynamics; In particular, if the ratio of time scales is $\sigma(\ll 1)$ then perturbations have an $O(1)$ effect when their size $\epsilon$ is such that $\mid$ oren $\in \mid=0$ (1). If $\epsilon$ is larger than this the dynamics is dominated by the noise, and the evolution takes the form of a 'noisy periodic orbit' whose (mean) amplitude and period depend crucialiy on $\epsilon$. Dynamical systems of this type may provide a new paradigm for intermittency in disordered flows.

References: D.W.Hughes and M.R.E. Proctor 1990. A loworder model of the shear instability of convection: chaos and the effect of noise. Nonlinearity $3,127-153$.

D.W.Hughes and M.R.E. Proctor 1990. Chaos and the effect of noise in a model of three-wave mode coupling, Physica D, in press. 


\title{
Calculating Transient Coronal Loops
}

\author{
David W. Rose
}

12 July 1990

\begin{abstract}
Magnetic flux tubes, which emerge and then re-enter the photosphere, occur on a wide range of length scales in the corona of the sun. Most are fairly stable and persistent, lasting on the order of days to weeks. In 1972, E. N. Parker proposed a mechanism by which the extraordinarily high temperature of the solar corona could be caused by Ohmic heating of electrical currents contained within these flux tubes. In order that sufficient heat to produce the temperature differential be produced, based upon rough measurements of magnetic field strengths and flux tube volumes, it was necessary that very intense concentrations of current be formed in these flux tubes. Parker surmised that very strong and highly local current sheets would form as the magnetic flux lines became twisted up through motions in the photosphere. He argued that while the magnetic field would be driven to a force-free state within the corona, that the condition of its flux lines tied to photosphere plasma motions would cause an increase in the amount of line braiding until force-free fields would cease to exist. Then current sheets would form, causing local reconnection of the magnetic field, and simultaneously coronal heating.

The second part of this hypothesis, that current sheets would form, is still unknown. The difficulties are that the mathematical system is difficult, being inherently a three-dimensional non-linear problem. Analytically, there is no theory for the formation of singularities from such boundary motions, and while it is true that force free fields cease to exist, there is no theory of what happens. Even numerically, the task is difficult. One may reduce the MHD equations into a simpler system which presumably retains the essential terms necessary to determine current sheet formation. This simplification was performed by $\mathbf{H}$. Strauss in 1975. In its simplest form, it is obtained by renormalizing the MHD equations with the density and rescaling the velocity and magnetic induction as

$$
\begin{aligned}
\boldsymbol{B} & \leftarrow \hat{\mathbf{z}}+\varepsilon \boldsymbol{B}(\boldsymbol{x}, y, \varepsilon z, \varepsilon t) \\
\boldsymbol{u} & \leftarrow \varepsilon \boldsymbol{u}(\boldsymbol{x}, y, \varepsilon z, \varepsilon t)
\end{aligned}
$$

and taking as equal the coefficients of powers of $\varepsilon$. The effect of this is to decouple components tangent and transverse to the $\mathrm{z}$-axis. It also has the effect of enforcing incompressibility on the transverse flow velocity.
\end{abstract}


At present, I have produced a numerical code which performs pseudospectral approximation in the transverse space variables and centered finite differences in z. With time advance using a two-step method, a numerical instability results in the transverse direction originating at the location of maximal gradient of the magnetic potential. For a simple case, this instability is effectively of the type due to decoupling in space, coupling in time. A proposed solution to this problem is to "split" the Jacobian terms of the form $[A, U]=A_{x} U_{y}-A_{y} U_{x}$ so that the second term occurs implicitly in the next time step, while the first occurs explicitly.

\section{Blocking A Barotropic Shear Flow \\ Melvin Stern \\ Florida State University}

The "upstream influence" on an inviscid shear flow around a semi-circular cape (radius $=\mathrm{A}$ ) is computed for an undisturbed $(\mathrm{x}=-\infty)$ velocity profile: $\mathrm{u}_{o}(\mathrm{y})=\mathrm{y}$ (for $0 \leq \mathrm{y}$ $\leq 1), \mathrm{u}_{0}(\mathrm{y})=1+\zeta_{2} \mathrm{y}-\zeta_{2}$ (for $\left.1 \leq \mathrm{y} \leq \infty\right)$, where $-\zeta_{2}$ is a constant vorticity. Linear theory for $\mathrm{A} \rightarrow 0, \zeta_{2}=0$, Time $=\mathrm{t} \rightarrow \infty$ gives a "weak" upstream influence, in which the upstream area of the boundary layer (vorticity $=-1$ ) increases as $t^{1 / 2}$. For $A>1$ contour dynamical numerical calculations for the piecewise uniform vorticity flow show "strong" blocking or "complete" blocking, in which either a fraction or none of the boundary layer flux passes around the cape. A semi-quantitative critical condition on $\left(\zeta_{2}, A\right)$ no upstream influence is developed: The relevance of this simple barotropic model to the control of oceanic coastal and strait currents is suggested. 


\section{A Fluid Mechanicist's Introduction to Lie Symmetry Groups and Partial Differential Equations by Rick Salmon}

Symmetry group methods are attractive because they apply to general nonlinear equations. Good references include the books by Bluman and Cole (1974), Olver (1986), and Bluman and Kumei (1989). The lectures summarized below are a gentle introduction to Olver's chapters 2 and 3.

\section{Lecture 1. Symmetry Groups}

Given a differential equation, the idea is to find transformations of the dependent and independent variables for which the equation is unchanged. For ordinary differential equations, each such transformation leads to a reduction, by 1 , in the order of the differential equation. That is, applied successively, transformation groups can lead to quadrature. For partial differential equations, the transformation groups lead to a general family of invariant (similarity) solutions.

Given a partial differential equation of (say) second order in $u(x, t)$,

$$
F\left(x, t, u, u_{x}, u_{t}, u_{x x}, u_{x}, u_{t}\right)=0
$$

we want to find a solution,

$$
G(x, t, u)=0
$$

Here, $F$ and $G$ are ordinary functions of their respective arguments. The general situation is that $F$ is a given function and $G$ must be found. From a geometric viewpoint, (1) is a 7-dimensional surface in the 8-dimensional jet space with coordinates

$$
x, t, u, u_{x}, u_{t}, u_{x x}, u_{x s}, u_{t s}
$$

A solution (2) is a 2-dimensional surface in the 3-dimensional base space with coordinates

$$
x, t, u
$$

The generalization to more variables and higher derivatives is obvious.

We consider transformations of the variables from "old" coordinates $(x, t, u)$ to "new" coordinates $\left(x^{\prime}, t^{\prime}, u\right)$. Under certain assumptions such transformations form a group. If the group depends continuously on a parameter $s$, then it is called a Lie group :

$$
\begin{aligned}
x^{\prime} & =f(x, t, u ; s) \\
t^{\prime} & =g(x, t, u ; s) \\
u^{\prime} & =h(x, t, u ; s)
\end{aligned}
$$

It is conventional to let $s=0$ correspond to the identity element of the group. Then 


$$
\begin{aligned}
& x=f(x, t, u ; 0) \\
& t=g(x, t, u ; 0) \\
& u=h(x, t, u ; 0)
\end{aligned}
$$

One way to generate such a group is as the solution to equations of the form

$$
\begin{array}{lrl}
\frac{d x^{\prime}}{d s}=\xi\left(x^{\prime}, t^{\prime}, u^{\prime}\right), & x^{\prime}(0)=x \\
\frac{d t^{\prime}}{d s}=\eta\left(x^{\prime}, t^{\prime}, u^{\prime}\right), & t^{\prime}(0)=t \\
\frac{d u^{\prime}}{d s}=\phi\left(x^{\prime}, t^{\prime}, u^{\prime}\right), & u^{\prime}(0)=u
\end{array}
$$

It is then useful to think of $\left(x^{\prime}, t^{\prime}, u^{\prime}\right)$ as the "location" at "time" $s$ of a particle initially at $(x, t, u)$ that moves always with the "velocity"

$$
\mathbf{v}=[\xi(x, t, u), \eta(x, t, u), \phi(x, t, u)]
$$

The simplifying feature is that the "velocity field" (8) is "steady", i.e. s -independent. Thus, (8) is everywhere tangent to the base-space trajectories that define the transformation.

The "velocity field" (8) determines a corresponding "velocity field"

$$
\operatorname{pr} \mathbf{v}=\left[\xi, \eta, \phi, \frac{d\left(u_{x}\right)}{d s}, \frac{d\left(u_{t}\right)}{d s}, \frac{d\left(u_{x x}\right)}{d s}, \frac{d\left(u_{x t}\right)}{d s}, \frac{d\left(u_{n}\right)}{d s}\right]
$$

in the jet space with coordinates (3). (The notation $p r \mathbf{v}$ stands for "prolongation of $\mathbf{v}, " \mathbf{a}$ good terminology.) The first three components of (9) are the same as (8). The last five components of (9) can be expressed in terms of $\xi, \eta, \phi$ and their derivatives. It is obvious that such expressions must exist, from the simple fact that the formula for the tranformation of a function implicitly determines formulas for the transformations of all its derivatives. As an example, we will calculate $d\left(u_{t}\right) / d s$.

The Taylor expansion of (7) is

$$
\begin{aligned}
& x^{\prime}=x+s \xi(x, t, u)+\cdot \cdot \\
& t^{\prime}=t+s \eta(x, t, u)+\cdot \cdot \\
& u^{\prime}=u+s \phi(x, t, u)+\cdot \cdot
\end{aligned}
$$

and $(10 \mathrm{a}-\mathrm{b})$ revert to

$$
\begin{aligned}
& x=x^{\prime}-s \xi\left(x^{\prime}, t^{\prime}, u^{\prime}\right)+\cdots \\
& t=t^{\prime}-s \eta\left(x^{\prime}, t^{\prime}, u^{\prime}\right)+\cdots
\end{aligned}
$$

So 


$$
\begin{aligned}
\frac{\partial u^{\prime}}{\partial t^{\prime}} & =\left(\frac{\partial x}{\partial t^{\prime}} \frac{\partial}{\partial x}+\frac{\partial t}{\partial t^{\prime}} \frac{\partial}{\partial t}\right)[u+s \phi(x, t, u)]+O\left(s^{2}\right) \\
& =\frac{\partial u}{\partial t}+s\left\{D_{t} \phi-u_{x} D_{t} \xi-u_{t} D_{t} \eta\right\}+O\left(s^{2}\right)
\end{aligned}
$$

where

$$
D_{t} \equiv \frac{\partial}{\partial t}+u_{t} \frac{\partial}{\partial u}
$$

Therefore

$$
\frac{d\left(u_{t}\right)}{d s}=D_{t} \phi-u_{x} D_{t} \xi-u_{t} D_{t} \eta
$$

Returning to our differential equation (1), we seek transformations of the form (7) for which the equation takes the same form in new variables as in old, i.e.

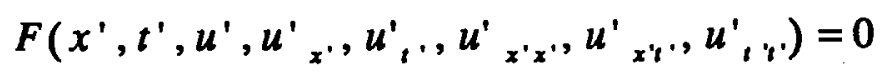

where $F(, \ldots,$,$) is the same function of its arguments in (15) as it is in (1). For small$ $s,(15)$ is

$$
F\left(x+\xi(x, t, u) s+O\left(s^{2}\right), t+\eta(x, t, u) s+O\left(s^{2}\right), \ldots \ldots \ldots . .\right)
$$

Thus, subtracting (1) from (16) and letting $s \rightarrow 0$,

$$
\xi(x, t, u) \frac{\partial F}{\partial x}+\eta(x, t, u) \frac{\partial F}{\partial t}+\cdots+\frac{d\left(u_{n}\right)}{d s} \frac{\partial F}{\partial u_{u}}=0
$$

(provided that the partial derivatives of $F$ are not all zero.) By changing the definitions (8) and (9) slightly to

$$
\mathrm{v} \equiv \xi(x, t, u) \frac{\partial}{\partial x}+\eta(x, t, u) \frac{\partial}{\partial t}+\phi(x, t, u) \frac{\partial}{\partial u}
$$

and

$$
\begin{gathered}
\operatorname{pr} \mathbf{v} \equiv \xi(x, t, u) \frac{\partial}{\partial x}+\eta(x, t, u) \frac{\partial}{\partial t}+\phi(x, t, u) \frac{\partial}{\partial u}+\frac{d\left(u_{x}\right)}{d s} \frac{\partial}{\partial u_{x}}+\cdots \\
\cdots+\frac{d\left(u_{u}\right)}{d s} \frac{\partial}{\partial u_{u}}
\end{gathered}
$$

we can rewrite (17) in the compact form

$$
(p r \mathbf{v}) \quad F=0
$$


The tangent vectors (18) and (19) are simply the "advective derivatives" associated with the "velocity fields" (8) and (9). Equation (20) just states that the jet-space "trajectory" must lie in the hypersurface (1) corresponding to the differential equation.

We can now solve (20) and (1) to determine the components of the "velocity field" that defines the transformation. This involves equating the coefficients of like powers of (3) to zero in (20), after using (1) to remove one of the coordinates. There results a set of linear differential equations in the functions $\xi, \eta$, and $\phi(x, t, u)$. These equations, which are called the determining system of the transformation, are linear (cf. eq. 14), even when the original differential equation (1) is nonlinear. This is what makes the method so useful. For high-order nonlinear equations, the determining system may contain hundreds of equations. Fortunately, there now exist symbolic manipulation programs that do nearly all the work of setting up and solving these systems.

As a simple example, we consider the heat equation,

$$
F=u_{t}-u_{x x}=0
$$

for which the general solution of the determining system turns out to be

$$
\begin{aligned}
& \xi(x, t, u)=c_{1}+c_{4} x+2 c_{5} t+4 c_{6} x t \\
& \eta(x, t, u)=c_{2}+2 c_{4} t+4 c_{6} t^{2} \\
& \phi(x, t, u)=\left(c_{3}-c_{5} x-2 c_{6} t-c_{6} x^{2}\right) u+\alpha(x, t)
\end{aligned}
$$

where the $c_{i}$ are arbitrary constants and $\alpha(x, t)$ is an arbitrary solution to the heat equation. We thus say that the heat equation is invariant to the transformations generated by

$$
\begin{aligned}
& \mathbf{v}_{1}=\frac{\partial}{\partial x}, \quad \mathbf{v}_{2}=\frac{\partial}{\partial t}, \quad \mathbf{v}_{3}=u \frac{\partial}{\partial u}, \quad \mathbf{v}_{4}=x \frac{\partial}{\partial x}+2 t \frac{\partial}{\partial t} \\
& \mathbf{v}_{5}=2 t \frac{\partial}{\partial x}-x u \frac{\partial}{\partial u}, \quad \mathbf{v}_{6}=4 t x \frac{\partial}{\partial x}+4 t^{2} \frac{\partial}{\partial t}-\left(x^{2}+2 t\right) u \frac{\partial}{\partial u} \\
& \mathbf{v}_{\alpha}=\alpha(x, t) \frac{\partial}{\partial u}
\end{aligned}
$$

The transformation generated by $\mathbf{v}_{1}$ is

$$
x^{\prime}=x+s, \quad t^{\prime}=t, \quad u^{\prime}=u
$$

Let

$$
u=f(x, t)
$$

be a particular solution of the heat equation. The $\mathbf{v}_{1}$-transform of (25) is

$$
u^{\prime}=f\left(x^{\prime}-s, t^{\prime}\right)
$$

But we already know that the primed variables also satisfy the heat equation. We therefore conclude that if $f(x, t)$ is a solution to the heat equation, then so must be $f(x-s, t)$ for any constant $s$. Repeating this logic for all of the generators in (23) we conclude that: 
If $u=f(x, t)$ is a solution to the heat equation, then so must be:

$$
\begin{array}{ll}
\mathbf{v}_{1}: & u=f(x-s, t) \\
\mathbf{v}_{2}: & u=f(x, t-s) \\
\mathbf{v}_{3}: & u=e^{s} f(x, t) \\
\mathbf{v}_{4}: & u=f\left(e^{-s} x, e^{-2 s} t\right) \\
\mathbf{v}_{5}: & u=e^{-s x+s^{2} t} f(x-2 s t, t) \\
\mathbf{v}_{6}: & u=\frac{1}{\sqrt{1+4 s t}} \exp \left(\frac{-s x^{2}}{1+4 s t}\right) f\left(\frac{x}{1+4 s t}, \frac{t}{1+4 s t}\right) \\
\mathbf{v}_{\alpha}: & u=f(x, t)+s \alpha(x, t)
\end{array}
$$

where $s$ is an arbitrary constant and $\alpha(x, t)$ is an arbitrary solution to the heat equation.

The ability to transform solutions into other solutions is sometimes useful by itself; the transformation of even trivial solutions (e.g. constants) can yield nontrivial results. However, it is in the determination and classification of invariant solutions that symmetry group methods really show their muscles.

\section{Lecture 2. Invariant Solutions}

In the first lecture we drew an analogy between a transformation group of a differential equation and the particle trajectories in a steady flow. The generators of the group are analogous to the velocity field of the flow. Knowing the generators is equivalent to knowing the transformation group, but, as the fluid mechanical analogy would suggest, it is usually much easier to deal with the generators than it is do deal with group.

The generators form a Lie algebra with mathematical properties that reflect the underlying group. The most interesting of these properties is this: If $\mathbf{v}_{\mathbf{a}}$ and $\mathbf{v}_{\mathbf{b}}$ are any two generators, then their Lie bracket defined as

$$
\left[\mathbf{v}_{a}, \mathbf{v}_{b}\right]=\mathbf{v}_{a} \mathbf{v}_{b}-\mathbf{v}_{b} \mathbf{v}_{a}
$$

is a linear combination of all the generators. For example, consulting (23), we find that

$$
\left[v_{2}, v_{6}\right]=4 v_{4}-2 v_{3}
$$

This closure property of the Lie algebra is a consequence of the correspondence between generators and transformation groups: It can easily be shown that the commutator (28) is the generator of the composite transformation consisting of an infinitesimal displacement along the trajectory corresponding to $v_{a}$, followed by an infinitesimal displacement in the direction of $\mathbf{v}_{\mathrm{b}}$, followed by backwards displacements in the directions of $\mathbf{v}_{\mathrm{a}}$, and then $\mathbf{v}_{\mathrm{b}}$. This composite transformation is certainly a member of the general group of transformations, and therefore its generator, (28), is some linear combination of the basis vectors $\mathbf{v}_{\boldsymbol{i}}$.

We now turn to invariant (similarity) solutions. Recall that $(p r v)$ is, by hypothesis, tangent to the solution surface $F=0$ in the eight-dimensional jet-space. However, $v$ is not 
necessarily tangent to an arbitrarily chosen solution surface $G=0$. That is, (pr v) $F=0$ but $v G \neq 0$. In fact, it is the "flow" across solution surfaces that carries solutions into other solutions, as in (27).

Now let $\mathbf{v}$ be a particular generator and consider the special solutions for which $\mathbf{y} G=$ 0 . The generator $\mathbf{v}_{1}$ in (23) offers a trivial but prototypical example. $\mathbf{v}_{1}$ has a component in the $x$-direction, but no components in the $t$ - or $u$ - directions. Thus $\mathrm{v}_{1} G=0$ only if $G=G(t, u)$. That is, solutions invariant to $v_{1}$ take the form $u=u(t)$ and are independent of $x$. (These solutions turn out to be trivial indeed: they are just constants!)

For an arbitrary generator $\mathbf{v}$, the invariant solutions are found by a method that amounts to finding the special coordinates in $(x, t, u)$-space for which that generator takes the canonical form (24). This is most easily done by the method of characteristics: one determines the functions $\mu_{1}(x, t, u)$ and $\mu_{2}(x, t, u)$, called differential invariants, whose isosurfaces intersect to form the trajectories of $\mathbf{v}$. The similarity solution then takes the form $\mu_{l}=g\left(\mu_{2}\right)$ where $g$ is a function to be determined by substitution into the original differential equation.

As an example, we calculate the similarity solutions corresponding to the generator $c v_{1}+v_{5}$ of the heat equation, with $c$ an arbitrary constant. The characteristic equations

$$
\frac{d x}{2 t+c}=\frac{d t}{0}=-\frac{d u}{x u}
$$

yield the differential invariants

$$
\mu_{1}=t, \quad \mu_{2}=u \exp \left(\frac{\frac{1}{2} x^{2}}{2 t+c}\right)
$$

so that the similarity solution takes the form

$$
u=g(t) \exp \left(\frac{-\frac{1}{2} x^{2}}{2 t+c}\right)
$$

with $g(t)$ left to be determined by substitution in the heat equation.

To study the most general similarity solution of the heat equation, we must use the general generator

$$
\mathbf{v}=c_{1} \mathbf{v}_{1}+c_{2} \mathbf{v}_{2}+c_{3} \mathbf{v}_{3}+c_{4} \mathbf{v}_{4}+c_{5} \mathbf{v}_{5}+c_{6} \mathbf{v}_{6}+\mathbf{v}_{\alpha}
$$

where $c_{i}$ are arbitrary constants. Unfortunately, the characteristic equations corresponding to (33) are very difficult to solve. However, this task can be circumvented by a procedure that forms the slickest part of the whole theory.

The essential idea is very well illustrated by the example (30-32). To obtain (32) we can use a combination of $v_{1}$ and $v_{5}$ as above; or we can use $v_{5}$ by itself to obtain (32) with $c=0$, and then use the property (27b), obtained from $v_{2}$, that any solution can be timetranslated. That is, we can use a more restricted generator to obtain our similarity solutions 
if we combine the results with the rules for transforming solutions into solutions. It can be shown, in fact, that if $c_{5} \neq 0$ in (33), then we can have $c_{l}=0$ with no loss in generality.

The special geometrical relationship between $\mathbf{v}_{1}, \mathbf{v}_{2}$ and $\mathbf{v}_{5}$ that allows this can be explained as follows: If the trajectories tangent to $\mathrm{v}_{5}$ are subjected to a coordinate transformation corresponding to $\mathbf{v}_{2}$, then the transformed trajectories are tangent to a linear combination of $v_{5}$ and $v_{1}$. We can regard the trajectories as material lines in a perfect fluid with velocity $\mathbf{v}_{2}$. The material lines are carried along by the fluid, and the tangents to these lines are Lie dragged by the velocity field $\mathrm{v}_{2}$ in the same way as the vorticity or magnetic field vectors in a perfect fluid. In complete analogy with the vorticity or induction equations, the evolution equation for the tangent, $v$, is

$$
\frac{d \mathbf{v}}{d s}=\left[\mathbf{v}_{2}, \mathbf{v}\right]
$$

with initial condition $\mathbf{v}(0)=\mathbf{v}_{5}$. The Taylor-series solution of (34) is

$$
\begin{aligned}
\mathbf{v}(s) & =\mathbf{v}_{5}+s\left[\mathbf{v}_{2}, \mathbf{v}_{s}\right]+\frac{1}{2} s^{2}\left[\mathbf{v}_{2},\left[\mathbf{v}_{2}, \mathbf{v}_{5}\right]\right]+\cdots \\
& =\mathbf{v}_{5}+s\left(2 \mathbf{v}_{1}\right)+\frac{1}{2} s^{2}\left[\mathbf{v}_{2}, 2 \mathbf{v}_{1}\right]+\cdots \\
& =\mathbf{v}_{5}+2 s \mathbf{v}_{1}
\end{aligned}
$$

Thus, a transformation ("advection") by $v_{2}$ drags $v_{5}$ into $v_{5}+2 s v_{1}$.

Starting with the general generator (33), we employ all possible draggings to eliminate as many components of (33) as possible. Each elimination requires an assumption about the arbitrary constants in (33) (typically, that a particular $c_{i}$ is nonzero), and the converse of each assumption must be separately examined. The final result is an optimal subset of generators, each very much simpler than (33). All the similarity solutions obtainable from (33) can then be obtained from this optimum subset (whose characteristic equations are much easier to integrate) plus the rules for transforming solutions into solutions.

For the heat equation, the optimal subset and corresponding forms of the similarity solutions turn out to be:

$$
\begin{array}{rlrl}
\mathbf{v}_{4}+c \mathbf{v}_{3} & u & =t^{c} g\left(\frac{x}{\sqrt{t}}\right) \\
\mathbf{v}_{2}+\mathrm{v}_{6}+c \mathbf{v}_{3} & u & =\left(1+4 t^{2}\right)^{-1 / 4} \exp \left\{-\frac{t x^{2}}{1+4 t^{2}}-\frac{c}{2} \arctan (2 t)\right\} g\left(\frac{x}{\sqrt{1+4 t^{2}}}\right) \\
\mathbf{v}_{2}-\mathrm{v}_{5} & u & =\exp \left\{x t+\frac{2}{3} t^{3}\right\} g\left(x+t^{2}\right) \\
\mathbf{v}_{2}+c \mathbf{v}_{3} & u & =e^{c t} g(x) \\
\mathbf{v}_{1} & u & =g(t)
\end{array}
$$

All the other similarity forms can be obtained from (36) by the transformations (27). The various cases in (36) yield ordinary differential equations for $g$ whose solutions can be written down as parabolic cylinder functions, Airy functions, or trigonometric functions.

Rainer Hollerbach and I have applied the above methods to the thermocline equation, a particularly gruesome fourth-order nonlinear partial differential equation of interest to oceanographers. The results, which include many previously unnoticed similarity forms, will be reported in a forthcoming publication. These lectures were a precursor to our summer on "Geometrical methods in fluid dynamics" in 1993. 


\title{
VORTICES IN TIGHT EMBRACE
}

\author{
by \\ E. L. Schucking, Department of Physics, New York University \\ and \\ E. A. Spiegel, Department of Astronomy, Columbia University
}

ABSTRACT. We study simple flows of an incompressible fluid of density $\rho$ and constant kinematic viscosity $v$ in a hypersphere $S^{3}$ of radius $\lambda$. The $S^{3}$ is imbedded into a Euclidean space of four dimensions and described by the equation

$$
\left|z_{1}\right|^{2}+\left|z_{2}\right|^{2}=\lambda^{2}, \quad z_{1}=x+\mathrm{i} y, \quad z_{2}=z+\mathrm{i} u
$$

We divide the $S^{3}$ into the three regions I, II, and III by

$$
\begin{array}{ll}
\text { I } & 0 \leq\left|z_{1}\right| \leq r_{1} \lambda \\
\text { II } & \lambda r_{1} \leq\left|z_{1}\right| \leq r_{2} \lambda \\
\text { III } & \lambda r_{2} \leq\left|z_{1}\right| \leq \lambda .
\end{array}
$$

The regions I and III are solid tori which show at time $t=0$ the velocity field of rigid rotation:

$$
\begin{array}{ll}
\text { I }\left.\quad \frac{d z_{1}}{d t}\right|_{t=0}=\mathrm{i} \omega_{1} z_{1},\left.\quad \frac{d z_{2}}{d t}\right|_{t=0}=0 ; \\
\text { III }\left.\quad \frac{d z_{1}}{d t}\right|_{t=0}=\mathrm{i} \omega_{3} z_{1},\left.\quad \frac{d z_{2}}{d t}\right|_{t=0}=0
\end{array}
$$

with $\omega_{1}$ and $\omega_{3}$ constant, i.e. independent of $r \equiv\left|z_{1}\right| / \lambda$.

In region II between the two vortex cores we assume at $t=0$ a pseudo-potential flow which has vanishing divergence of its shear with velocity field

$$
\left.\frac{d z_{1}}{d t}\right|_{t=0}=\mathrm{i} \omega(r) z_{1},\left.\quad \frac{d z_{2}}{d t}\right|_{t=0}=0, \quad \omega\left(r_{1}\right)=\omega_{1}, \quad \omega\left(r_{2}\right)=\omega_{2} .
$$

All three flows are stationary solutions of the Navier-Stokes equations at $t=0$ for a continuous velocity field. The jumps in velocity gradients at $r_{1}$ and $r_{2}$ lead to a timedependent solution of the Navier-Stokes equations for $\omega(t, r)$ in terms of Jacobi polynomials in $r$ with time-dependent coefficients.

\section{Flows in $S^{3}$}

The $S^{3}$ of radius $\lambda$ is given by the equation

$$
\left|z_{1}\right|^{2}+\left|z_{2}\right|^{2}=\lambda^{2}
$$


with

$$
z_{1} \equiv x+\mathrm{i} y, \quad z_{2} \equiv z+\mathrm{i} u
$$

where $x, y, z$, and $u$ are Cartesian coordinates of a Euclidean $\mathrm{R}^{4}$. to 1 ,

We parameterize the $S^{3}$ by the coordinates $r, \phi$ and $\psi$ such that, with $r$ ranging from 0

$$
z_{1}=\lambda r e^{i \phi}, \quad 0 \leq \phi, \psi \leq 2 \pi, \quad z_{2}=\lambda \sqrt{1-r^{2}} e^{i \psi}
$$

The differentials are

$$
d z_{1}=\lambda(d r+\mathrm{i} r d \phi) e^{\mathrm{i} \phi}, \quad d z_{2}=\lambda\left(\frac{-r d r}{\sqrt{1-r^{2}}}+\mathrm{i} \sqrt{1-r^{2}} d \psi\right) e^{\mathrm{i} \psi}
$$

We get then for the line element

$$
d s^{2}=\left|d z_{1}\right|^{2}+\left|d z_{2}\right|^{2}=\lambda^{2}\left\{\frac{d r^{2}}{1-r^{2}}+r^{2} d \phi^{2}+\left(1-r^{2}\right) d \psi^{2}\right\}
$$

We shall study flows for which $r$ and $\psi$ are constant. This gives for the velocity $v$

$$
\frac{d s}{d t}=v=\lambda r \frac{d \phi}{d t} \equiv \lambda r \omega
$$

The angular velocity $\omega$ may be a function of the two variables $r$ and $t$;:

$$
\omega=\omega(r, t)
$$

We first write down the condition that the flow has vanishing divergence. If we number coordinates $r, \phi, \psi$ as $x^{1}, x^{2}, x^{3}$ the velocity vector $\nu^{j}$ has the components $(j=1,2,3)$

$$
\frac{d x^{j}}{d t} \equiv v^{j}=\omega \delta_{2}^{j}=\frac{d \phi}{d t} \delta_{2}^{j}
$$

The formula for the divergence of a vector field $v j$ is given by

$$
v_{i j}^{j} \equiv \operatorname{div} \mathbf{v}=\frac{1}{\sqrt{g}}\left(\sqrt{g} v^{j}\right)_{, j}
$$

where the semicolon denotes the covariant derivative with respect to $x^{j}(j=1,2,3)$ and the comma the ordinary partial derivative. The determinant of the metric tensor is called $g$. In our case we have from (9) 


$$
\left\|g_{j k}\right\|=\lambda^{2}\left(\begin{array}{lll}
\frac{1}{1-r^{2}} & 0 & 0 \\
0 & r^{2} & 0 \\
0 & 0 & 1-r^{2}
\end{array}\right)
$$

and thus

$$
g=\operatorname{det}\left\|g_{j k}\right\|=\lambda^{6} r^{2} .
$$

The flow has vanishing divergence if

$$
v_{; j}^{j} \equiv \operatorname{div} \mathbf{v}=\frac{1}{\lambda^{3} r}\left(\lambda^{3} r \omega\right)_{.2}=0 .
$$

Since we assumed that $\omega$ did not depend on $x^{2} \equiv \phi$ the divergence vanishes.

The shear tensor for the flow is defined as

$$
s_{j k}=\frac{1}{2}\left(v_{j ; k}+v_{k ; j}\right)-\frac{1}{3} g_{j k} v_{; l}^{l}=\frac{1}{2}\left(v_{j ; k}+v_{k ; j}\right)
$$

where the last equality follows from the vanishing of the divergence in (12).

According to the definitions

$$
\begin{aligned}
& v_{j} \equiv g_{j k} v^{k}, \quad v_{j ; k} \equiv v_{j, k}-\Gamma_{j k}{ }^{l} v_{l}=v_{j, k}-\Gamma_{j k, l} v^{\prime}, \\
& \Gamma_{j k, l} \equiv \frac{1}{2}\left(g_{j l, k}+g_{k l, j}-g_{j k, l}\right)
\end{aligned}
$$

one derives that

$$
s_{j k}=\frac{1}{2}\left(g_{j k, l} \nu^{l}+g_{k k} \nu_{, j}+g_{j l} \nu_{, k}\right) .
$$

With (8) we get

$$
s_{j k}=\frac{1}{2}\left(g_{j k, 2} \omega+g_{2 k} \omega_{, j}+g_{j 2} \omega_{, k}\right) .
$$

As the first term vanishes since the metric is independent of $\phi \equiv x^{2}$, the only non-vanishing term of the shear for our flow (8) is

$$
s_{12}=s_{21}=\frac{1}{2} \lambda^{2} r^{2} \frac{\partial \omega}{\partial r}
$$

With an $\omega$ independent of $r$ we obtain thus rigid rotation. 
The viscous stresses enter the Navier-Stokes equations through the divergence of the shear tensor. We calculate

$$
s_{j k ;}^{k}=s_{j ; k}^{k}=\frac{1}{\sqrt{g}}\left(\sqrt{g} s_{j}^{k}\right)_{, k}-\frac{1}{2} g_{k l, j} s^{k l}
$$

from the definitions of covariant derivatives. The last term in this expression is zero since $g_{12}$ vanishes.

We have from (10) for the contravariant (inverse) metric

$$
\left\|g^{j k}\right\|=\frac{1}{\lambda^{2}}\left(\begin{array}{ccc}
1-r^{2} & 0 & 0 \\
0 & \frac{1}{r^{2}} & 0 \\
0 & 0 & \frac{1}{1-r^{2}}
\end{array}\right) .
$$

Thus from (17)

$$
s_{1}^{2}=s_{12} g^{2}=\frac{1}{2} \frac{\partial \omega}{\partial r}, \quad s_{2}{ }^{1}=s_{21} g^{11}=\frac{1}{2} r^{2}\left(1-r^{2}\right) \frac{\partial \omega}{\partial r}
$$

All other components vanish. We obtain thus from (18)

$$
s_{1 k ;}{ }^{k}=0, \quad s_{3 k ;}{ }^{k}=0, \quad s_{2 k ;}{ }^{k}=\frac{1}{2 r} \frac{\partial}{\partial r}\left[r^{3}\left(1-r^{2}\right) \frac{\partial \omega}{\partial r}\right] .
$$

The divergence of the shear tensor vanishes if

$$
\frac{\partial \omega}{\partial r}=-\frac{2 B}{r^{3}\left(1-r^{2}\right)}=-2 B\left(\frac{1+r^{2}}{r^{3}}+\frac{r}{1-r^{2}}\right), \quad B=\text { const. }
$$

This integrates to

$$
\omega=\omega_{0}+B\left(-\ln \frac{r^{2}}{1-r^{2}}+\frac{1}{r^{2}}\right), \quad \omega_{0}=\text { const }
$$

The flow consists of a rigid rotation with constant angular velocity $\omega_{0}$ and the "pseudopotential" flow given by the angular velocity

$$
\omega=B\left(\ln \frac{1-r^{2}}{r^{2}}+\frac{1}{r^{2}}\right)
$$

The expression"pseudo-potential" derives from the fact that in the limit $\lambda \rightarrow \infty$ the logarithmic term goes out and we obtain the potential flow

$$
v \sim \frac{1}{L}, \quad L=\lambda r
$$


where $L$ denotes the distance from the axis of rotation. However, while the potential flow in $\mathrm{R}^{3}$ has vanishing vorticity we have for the pseudo-potential flow in $S^{3}$ with (24)

$$
v_{1,2}-v_{2,1}=-\lambda\left(r^{2} \omega\right)_{, 1}=-B \lambda^{2} \frac{d}{d r} \ln \frac{1-r^{2}}{r^{2}}=\frac{2 B \lambda^{2}}{r\left(1-r^{2}\right)}
$$

while the other components of curl $\mathbf{v}$ vanish.

Going back now to the expression for the pseudo-potential flow in (24) we observe that $\omega$ becomes singular on the axis at $r=0$ and also at the largest distance from the axis at $r=1$. For positive $B$ the angular velocity $\omega$ decreases monotonously from $+\infty$ at $r=0$ to $-\infty$ at $r=1$.

\section{A model flow}

We want to study the development of a Couette flow without any free or rigid surfaces that is finite and has no singularities. To this end we sandwich the pseudo-potential flow between two rigidly rotating vortex cores.

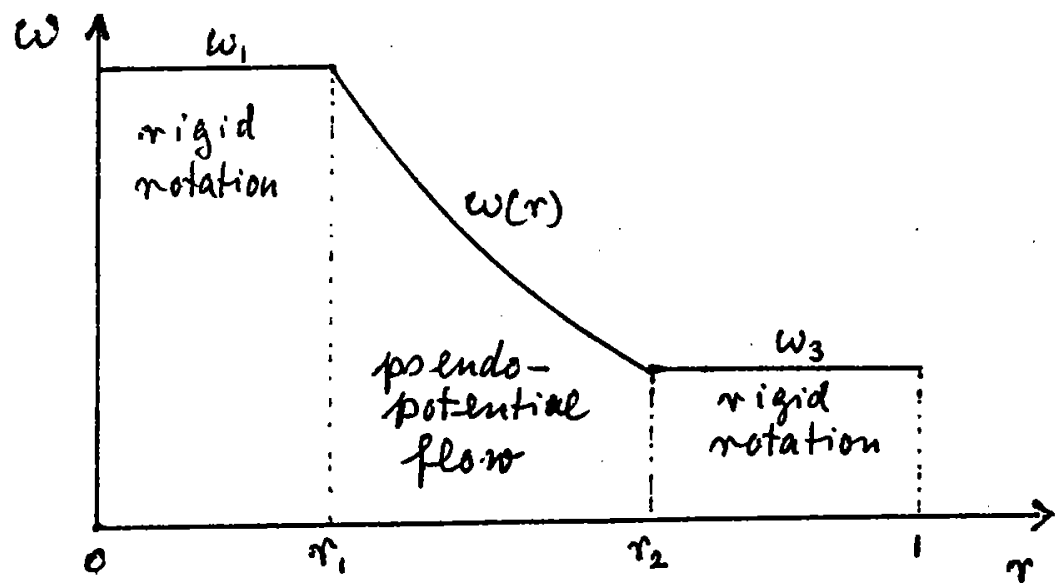

Figure 1. Initial distribution of angular velocity at time $t=0$ for $B>0$.

This eliminates the singularities at $r=0$ and $r=1$. The angular velocity -- and thus also the velocity -- is a continuous function of the radial distance $r$. At time $t=0$ we start with a stationary solution of the Navier-Stokes equations that has been pieced together at $r=r_{1}$ and $r=r_{2}$.. At these two surfaces we have finite jumps in the radial velocity gradients. These discontinuities will create a time dependence of the flow and it is to be expected that for $t \rightarrow \infty$ a uniform rigid rotation will result with an angular velocity determined by the initial (conserved) total angular momentum.

The Navier-Stokes equations are given by

$$
\frac{D v_{i}}{D t}=-\frac{1}{\rho} p_{, i}+2 v s_{i ; j}^{j}
$$


where $\rho$ is the constant density of the fluid, $p$ its pressure and $v$ its kinematic viscosity which is assumed to be constant here. The left-hand side of the equation is given by

$$
\frac{D v_{i}}{D t} \equiv \frac{\partial v_{i}}{\partial t}+v_{i ; k} v^{k}=\frac{\partial v_{i}}{\partial t}+\left(v_{i, k}-v_{k, i}\right) v^{k}+\frac{1}{2}\left(v_{k} v^{k}\right)_{, i}
$$

Since we take

$$
v_{1}=v_{3}=0
$$

for our flow we let the pressure $p$ depend only on $r$ and $t$. The " 3 "-component of the equation is then identically fulfilled as we see from (21) which also tells us that the "1"component becomes

$$
\frac{D v_{1}}{D t}=-v_{2,1} v^{2}+\frac{1}{2}\left(v_{2,1} v^{2}+v_{2} v_{, 1}^{2}\right)=-\lambda^{2} r \omega^{2}=-\frac{1}{\rho} \frac{\partial p}{\partial r}
$$

This means that the pressure gradient will balance the centrifugal force. The "2"-equation becomes with (21)

$$
\frac{D v_{2}}{D t}=\frac{\partial v_{2}}{\partial t}=\lambda^{2} r^{2} \frac{\partial \omega}{\partial t}=2 v \quad s_{2 k ;}{ }^{k}=\frac{v}{r} \frac{\partial}{\partial r}\left[r^{3}\left(1-r^{2}\right) \frac{\partial \omega}{\partial r}\right]
$$

We introduce now a new dimensionless time variable $\tau$ by

$$
\tau=\frac{4 v t}{\lambda^{2}}
$$

and indicate partial derivatives with respect to $\tau$ by a dot

$$
\dot{\omega} \equiv \frac{\partial \omega}{\partial \tau}
$$

Our equation (31) becomes

$$
r^{2} \dot{\omega}=\frac{1}{2 r} \frac{\partial}{\partial r}\left[r^{4}\left(1-r^{2}\right) \frac{1}{2 r} \frac{\partial \omega}{\partial r}\right]
$$

Instead of $r$ we introduce

$$
\xi \equiv r^{2}
$$

as a new variable also ranging from zero to one. We denote partial derivatives with respect to $\xi$ by a prime

$$
\omega^{\prime} \equiv \frac{\partial \omega}{\partial \xi}=\frac{1}{2 r} \frac{\partial \omega}{\partial r}
$$


Equation (34) becomes then

$$
\xi \dot{\omega}=\left[\xi^{2}(1-\xi) \omega^{\prime}\right]^{\prime}=\xi^{2}(1-\xi) \omega^{\prime \prime}+\xi(2-3 \xi) \omega^{\prime} .
$$

This gives the partial differential equation

$$
\dot{\omega}=\xi(1-\xi) \omega^{\prime \prime}+(2-3 \xi) \omega^{\prime} .
$$

Separation of variables gives

$$
\omega=f(\tau) g(\xi)
$$

and

$$
\frac{\dot{f}}{f}=\xi(1-\xi) \frac{g^{\prime \prime}}{g}+(2-3 \xi) \frac{g^{\prime}}{g}=-a
$$

with constant $a$. We obtain thus the equations

$$
\dot{f}+a f=0, \quad \xi(1-\xi) g^{\prime \prime}+(2-3 \xi) g^{\prime}+a g=0 .
$$

The second equation poses an eigenvalue problem with eigenvalue $a$ in the interval from 0 to 1 with the boundary conditions that $g$ should be finite. The solution is given by the Jacobi polynomials $J_{n}(2,2, x)$ which solve the hypergeometric differential equation

$$
\xi(1-\xi) g^{\prime \prime}+(2-3 \xi) g^{\prime}+n(2+n) g=0
$$

This means the eigenvalues $a$ are given by

$$
a=n(n+2), \quad n=0,1,2, \ldots .
$$

We write here

$$
J_{n}(2,2, x) \equiv \phi_{n}(x)
$$

and have as general solution

$$
\omega=\sum_{n=0}^{\infty} c_{n} \phi_{n}(x) e^{-n(n+2) \tau}
$$

The eigenfunctions $\phi_{n}$ are given by ${ }^{1}$

1 A simple formula is

$$
\phi_{n}(x)=\frac{(n+1) !}{x} \frac{d^{n}}{d x^{n}}\left[x^{n+1}(1-x)^{n}\right]
$$




$$
\phi_{n}(x)=1+\frac{1}{n+1} \sum_{k=1}^{n}(-1)^{k}\left(\begin{array}{l}
n \\
k
\end{array}\right)\left(\begin{array}{c}
n+k+1 \\
n
\end{array}\right) x^{k}
$$

The first ones are given by

$$
\begin{aligned}
& \phi_{0}=1, \quad \phi_{1}=1-\frac{3}{2} x, \quad \phi_{2}=1-4 x+\frac{10}{3} x^{2} \\
& \phi_{3}=1-\frac{15}{2} x+15 x^{2}-\frac{x}{4} x^{3} .
\end{aligned}
$$

The $\phi_{n}(x)$ form a complete system of orthogonal polynomials on the interval from zero to one with weight function $x$. We have

$$
\int_{0}^{1} x \phi_{m}(x) \phi_{n}(x) d x=\delta_{n m} \frac{1}{2(n+1)^{3}} .
$$

To solve the initial value problem we have to determine the coefficients $c_{n}$. We have from (45) for $\tau=0$

$$
\int_{0}^{1} x \omega(0, x) \phi_{m}(x) d x=\frac{c_{m}}{2(m+1)^{3}}
$$

The function $\omega(0, x)$ is given by

$$
\begin{array}{lll}
\omega(0, x)=\omega_{1} & \text { for } & 0 \leq x \leq r_{1}^{2} \\
\omega(0, x)=\omega_{2} & \text { for } & r_{1}^{2} \leq x \leq 1
\end{array}
$$

with constants $\omega_{1}$ and $\omega_{2}$. In the range

$$
r_{1}^{2} \leq x \leq r_{2}^{2}
$$

we have

$$
\omega(0, x)=B\left(\frac{1}{x}+\ln \frac{1-x}{x}\right)
$$

with

$$
\omega_{1}=B\left(\frac{1}{r_{1}^{2}}+\ln \frac{1-r_{1}^{2}}{r_{1}^{2}}\right), \quad \omega_{2}=B\left(\frac{1}{r_{2}^{2}}+\ln \frac{1-r_{2}^{2}}{r_{2}^{2}}\right)
$$

All integrals in (49) are elementary. A full discussion of the problem is still needed. 


\title{
$\propto \omega$ - GALACTIC DYNAMOS
}

A.M. Soward, Department of Mathematics and statistics, The University of Newcastle upon Tyne, NEl 7RU, U.K.

\begin{abstract}
A simple representatior of a galactic dynamo consists of a thin disc of electrically conducting fluid with the region external to it a vaccuum. Differential rotation in the disc provides the $\omega$-effect which stretches out meridional magnetic field into the azimuthal direction. small scale turbulent motions are responsible for diffusion but more importantly the $\omega$-effect, which produces meridional magnetic field from the azimuthal field so completing the dynamo process. When the $\alpha$-effect is antisymmetric about the equatorial plane, modes of two symmetries can be distinguished namely even (dipole) and odd (quadrapole). As the magnitude of Dynamo number, which provides a dimensionless measure of the product of the $\alpha$ and $\omega$-effects, is increased the growth rate of modes generally increases. Dynamo action often occurs when the real part of the complex growth rate vanishes. Four marginal modes can be distinquished. The are the steady dipole, steady quadrapole, oscillatory dipole and oscillatory quadrapole.
\end{abstract}

The galactic disc is characterisen by two length scales, the disc radius $R$ and the disc thickness $b$. Together they define the aspect ratio $\mathcal{E}=\mathrm{b} / \mathrm{R}$. The dynamo modes themselves have a length scale $L$, which is generally intermediate between the disc radius and its thickness; $b<<L<<$. In this limit the dynamo can be modelled locally by a slab model, first investigated extensively by Parker (See Parker, 1979). In this lecture multiple length scale procedures are described, which use solutions for the slab model as the first approximation to the complete solution. A test for the validity of the procedures is obtained by comparing results with stix's (1975, 1978) numerical results for a particular model in an oblate spheroid. The asymptotic results of soward (1977) for the first steady dipole (sometimes called the "forgotten" mode) are described. It is explained how the exterior potential magnetic field is a more potent mechanism for linking field amplitudes at distant parts of the disc than lateral diffusion within the disc, contary to the assumption made by some authors. New results are described for the oscillatory dipole. This model is unusual in as much as the radial dynamo length scale $L$ is comparable to the disc width $b$. A comparison is made with Stix's results. Local results for the oscillatory quadrapole have been obtained. In this case the most unstable mode occurs 
on a long length scale $\mathrm{L}(>>b)$. As yet, however, no satisfactory solution of the amplitude modulation problem on that length scale has been found.

REFERENCES :

Parker, E.N., 1979, Cosmical Magnetic Fieids,
Clarendon.
Soward, A. M., 1977, "A thin disc model of the
galactic dynamo", Astron. Nachr. 299, 25-33.
Stix, M. 1975, "The galactic dynamo", Astron, \&
Astrophys. $\underline{42}, 85-89$.
Stix, M. 1978, "The galactic dynamo", Astron. \&
Astrophys. $\underline{68}, 459$.

\section{THE CATASTROPHE STRUCTURE OF THERMOHALINE CONVECTION}

Olivier THUAL

NCAR, Po Box 3000, Boulder, Co, 80303

We apply a surface forcing to the 2D Boussinesq thermohaline convection in a rectangular box, by imposing a fixed cosine temperature and salinity flux. Multiple steady states are found numerically, allowing the competition between a thermally and a salinity driven symmetric circulations, as well as a one cell asymmetric circulation. In the control space of the two forcing parameters these equilibria form a double cusp catastrophe. This catastrophe can be mimiced on simple box models connecting stirred reservoir through pipes. These low order models might give hints for an asymptotic expansion which could catch the catastrophe phenomena on the Boussinesq equations. 


\title{
Simulations of Solar Convection
}

\author{
by Bob Stein and Ake Nordlund \\ Michigan State University
}

We have developed a three-dimensional hydrodynamic code for studying solar convection. The code is written in modular form. It solves the equations of mass, momentum, and internal energy conservation using $\ln \rho$, u, and $e$ as variables. This increases the accuracy of vertical hydrostatic equilibrium (since $\ln \rho$ is nearly linear while $\rho$ is nearly exponential). Derivatives are calculated using cubic splines and FFT's. The bottom boundary is a node for horizontally uniform motions (to facilitate analysis of $\mathrm{p}$ mode driving). Inflowing material has a uniform entropy and the pressure is uniform across the boundary layer. An extra large zone is placed at the top, across which the vertical derivative of the velocity and departure from hydrostatic equilibrium is zero and at the top of which the energy is held fixed.

A realistic, tabular, equation of state is used and radiative energy exchange is calculated by solving the non-grey, LTE transfer equation using a four-bin opacity distribution function.

The code has been tested for advection. Discontinuities less than six gridpoints produce ringing. An artificial viscosity, proportional to the fluid velocity, is used to spread such discontinuities and eliminate ringing.

Shock tubes with a pressure jump of 10 (Mach number 1.55) were run. A viscosity proportional to the velocity jump is used to spread shocks over four grid zones and dissipate their energy. Excellent agreement between numerical and theoretical profiles for velocity, energy, and $\ln \rho$ show that the code has good conservation properties.

Linear waves driven by a piston show that there is little damping and very small phase speed error for wavelength greater than four grid zones, and the damping and phase speed error increase sharply for smaller wavelengths. Two-dimensional acoustic and internal gravity wave pulses in a stratified atmosphere are dispersive, and we are checking the group and phase velocities against theory. Wave reflection is noticeable, but small.

Finally, we are comparing the results of a simulation of three-dimensional convection in a box of an ideal gas using our code and a ppm code. Preliminary results show that the spectrum of various quantities are the same at large scales, but our code is more diffusive at scales less than five grid zones.

We have found that the flow topology for convection in a stratified medium (sun) consists of a warm, smooth, slow upflow with embedded cool, filamentary, fast, downdrafts. This can be seen from the velocity field in two-dimensional slices, from following fluid parcels in time, and from transparent views of the vertical kinetic energy flux.

The horizontal flow pattern is cellular. At the surface the upflow breaks up into granules. Overpressure in the centers drives the flow horizontally, cooling radiatively as it goes. Eventually it runs into the expanding flow from neighboring granules and is stopped in intergranule lanes. In the process it has lost entropy, so gravity pulls it back down into the interior. As the downflows descend they converge into narrow filaments at the vertices between granules.

The horizontal flow at large depths is also cellular but with a larger scale -corresponding to meso-granule scales. There is often a swirling motion about the downdrafts, so there is vertical vorticity and helicity. We compared two runs, one with twice the number of gridpoints as the other, both starting from the same initial state after 1.5-2 largescale turnover times. The large scale upflow and downdraft structure is similar in the two runs and the high resolution run developed much more small scale structure. 
Finally, we have compared the emergent surface intensity with observed solar granulation. The size spectrum has similar shape. However, the simulation which has been smoothed by atmospheric seeing and a telescope modulation transfer function has more power at small scale than the observations. A video with superimposed observations from Pic du Midi and simulation results was shown, as well as a video showing how fluid parcels move tracking the changes in the granulation pattern.

Instability of Flow with TemperatureDependent viscosity:

A Model of Magma Dynamics

\title{
J. A. Whitehead and Karl R. Helfrich Woods Hole Oceanographic Institution Woods Hole, MA. 02543
}

\begin{abstract}
In a material whose viscosity is very temperature dependent, flow from a chamber through a cooled slot can develop a fingering instability or time dependent behavior, depending on the elastic properties of the chamber, the viscosity temperature relationship, and the geometry of the slot. A laboratory experiment is described where syrup flows from a reservoir through a tube immersed in a chilled bath to an exit hole at constant pressure. Flow is either steady, or periodic, depending on the temperature of the bath and the flow rate into the reservoir. A theory indicates that the transition from steady to periodic flow depends on nonlinearities in the steady state relation between pressure and flow rate and a general stabililty criterion is advanced. Parameters governing the oscillation period are determined. Theory also indicates that flow through a slot would develop finger-like instabilities under certain conditions. Qualitative laboratory experiments with paraffin spreading over a cold plate reveal the fingering.
\end{abstract}




\title{
A LONG IABORATORY SALT FINGER \\ by George Veronis \\ Kline Geological Laboratory \\ Yale University
}

\begin{abstract}
The initiation of salt fingers from an initial twolayer configuration is well understood and the essential physical balances necessary to maintain long quasi-steady salt fingers are also known. However, the evolution from linear stability to long fingers has received little attention, primarily because the process is very strongly non-linear and therefore not amenable to analysis. The best hope for developing a model for this evolution is to study the case of weakly driven salt fingers in the presence of strongly stable stratification. In two layers this case corresponds to $\mathrm{R}=\alpha \Delta \mathrm{T} / \beta \Delta \mathrm{S} \gg 1$, where $\alpha$ is the thermal expansion coefficient, $\beta$ the salinity contraction and $\Delta T, \Delta S$ the imposed temperature and salinity differences across the interface. $\Delta s$ must be small enough so that shear instabililty of adjacent rising and sinking fingers does not occur. this model is currently being analyzed in order to understand the evolution.
\end{abstract}

During a recent visit with Jorg Imberger at the Centre for Water Research of the University of Western Australia, I learned about a simple laboratory experiment, originally proposed by G. I. Taylor, which seems to be a simpler version of the same problem. A long tube filled with water opens up into a reservoir of salt water at the top. Taylor predicted that a long salt finger would penetrate downward to a finite depth and then stop. Except for slow molecular diffusion there would be no further penetration of salt. Imberger's experiment confirmed Taylor's prediction.

This seemed to be a nice simple problem for a project for one of the summer fellows at this year's GFD program but the latter had all started on their projects by the time that I arrived. I ended up talking to Joe Keller about the problem and out of curiosity we wrote down the basic balances and derived a solution for a long quasi-steady 2D finger. The model is very similar to the one for infinitely long steady salt fingers in Howard and Veronis (1987) with modified boundary conditions. We also reproduced Imberger's experiment.

A model for the downward penetration of the salt tongue assumes that the horizontal salt balance is between horizontal diffusion of salt and vertical advection of the mean salt gradient but that the amplitude of the concentration (or vertical velocity-the two are proportional) is a slowly varying function of time and the vertical coordinate. Two equations for this $t, z$ dependence emerge from the horizontally integrated vorticity balance and salt conservation. The horizontal structure in these integral 
balances is taken to be the same as in the zero-order model. Numerical integration of the equations leads to a relation between time and the vertical penetration of the salt tongue that is qualitatively the same as the behavior observed in the laboratory experiment, viz., a rapid penetration is followed by balance between penetration distance and the logarithm of the time and finally the tongue slows and stops.

\section{REFERENCES :}

Howard, L.N. and G. Veronis (1987) The salt finger zone. JFM, 183, pp. 1-24. 


\title{
Applications of compactly supported wavelets to
} the numerical solution of partial differential equations.

\author{
John Weiss \\ Aware, Inc. \\ Suite 310 \\ 124 Mount Auburn Street \\ Cambridge, MA 02138
}

\section{Introduction}

Compactly supported wavelets have several properties that are quite useful for representing solutions of PDEs. The orthogonality, compact support and exact representation of polynomials of a fixed degree allow the efficient and stable calculation of regions with strong gradients or oscillations. For instance, we have applied wavelets to problems of shock capture and turbulence. The general method is a straightforward adaptation of the Galerkin procedure with a wavelet basis. Among the equations studied so far are Burgers equation, the equations of Gas dynamics, and the NavierStokes/Euler equations for an incompressible fluid in two dimensions.

The compact wavelets have a finite number of derivatives and the derivatives, when they exist, can be highly oscillatory. This makes, say, the numerical evaluation of integrals difficult and unstable. We have found methods for the evaluation of functionals on wavelet bases. Comparison with standard numerical results demonstrates that these procedures are critical for the wavelet methods, especially as applied to nonlinear problems.

\section{Compactly supported wavelets}

Ingrid Daubechies defined the class of compactly supported wavelets [1,2]. Briefly, let $\varphi$ be a solution of the scaling relation

$$
\varphi(x)=\sum_{k=0}^{N} a_{k} \varphi(2 x-k) .
$$

The $a_{k}$ are a collection of coefficients that categorize the specific wavelet basis. The expression $\varphi$ is called the scaling function. 
The normalization $\int \varphi d x=1$ of the scaling function obtains the condition

$$
\sum a_{k}=2 \text {. }
$$

The translates of $\varphi$ are required to be orthonormal

$$
\int \varphi(x-k) \varphi(x-m)=\delta_{k, m} .
$$

From the scaling relation this implies the condition

$$
\sum_{k=0}^{N} a_{k} a_{k-2 m}=\delta_{0 m} .
$$

For coefficients verifying the above two conditions, the functions consisting of translates and dilations of the scaling function, $\varphi\left(2^{j} x-k\right)$, form a complete, orthogonal basis for square integrable functions on the real line, $L^{2}(R)$.

If only a finite number of the $a_{k}$ are nonzero then $\varphi$ will have compact support.

Smooth scaling functions arise as a consequence of the degree of approximation of the translates. The conditionm that the polynomials $1, x, \cdots, x^{p-1}$ be expressed as linear combinations of the translates of $\varphi(x-k)$ is implied by the condition

$$
\sum(-1)^{k} k^{m} a_{k}=0
$$

for $m=0,1, \cdots, p-1$.

\section{The Wavelet-Galerkin Method}

For a PDE of the form

$$
F\left(U, U_{t}, \cdots, U_{x}, U_{x x}, \cdots\right)=0
$$

define the wavelet expansion

$$
U=\sum U_{k} \varphi(x-k) .
$$

An approximation to the solution is defined by

$$
\hat{U}=\sum_{k=-M}^{N} \hat{U}_{k} \varphi(x-k) .
$$

In effect, the solution is projected onto the subspace spanned by

$$
\Phi(M, N)=\{\varphi(x-k): k=-M, \cdots, N\} .
$$

To determine the coefficients of this expansion we substitute into the equation and again project the resulting expression onto the subspace $\Phi(M, N)$. This uniquely determines the coefficients $U_{k}$. 
The projection requires $\hat{U}_{k}$ to verify the equations

$$
\int_{-\infty}^{\infty} \varphi(x-k) F\left(\hat{U}, \hat{U}_{t}, \hat{U}_{x}, \cdots\right) d x=0
$$

for $k=-M, \cdots, N$. To evaluate this expression we must know the coefficients of the form

$$
\int \varphi(x) \varphi_{x}\left(x-k_{1}\right) \cdots \varphi_{x x}\left(x-k_{2}\right) \cdots d x
$$

Our original expansion is over the space dependence of the solution. If the equation has a time dependence the resulting equations for the $\hat{U}_{k}$ will be a system of ordinary differential equations in $t$.

\section{Burgers' equation}

Burgers' equation is

$$
U_{t}+U U_{x}=\sigma U_{x x},
$$

where $U(x, 0)=U_{0}(x)$ and $\sigma$ is the viscosity.

We use the exact formula [3] to check our numerical results. The dynamics of the Burgers' equation for small viscosity cause the formation of steep gradients and, in the limit of null viscosity, jump discontinuities or shocks [3].

To apply the Wavelet-Galerkin method to Burgers' equation we use the D6 scaling relation

$$
\varphi(x)=\sum_{k=0}^{5} a_{k} \varphi(2 x-k)
$$

and the scaling function expansion

$$
\hat{U}=\sum_{k=-M}^{M} U_{k} \varphi(x-k) .
$$

The differential equations for $U_{k}$

$$
U_{k, t}-\sigma A_{k, m} U_{m}+B_{k, m, l} U_{m} U_{l}=0
$$

are defined from the coefficients

$$
\begin{gathered}
A_{k, m}=\int_{-\infty}^{\infty} \varphi_{x x}(x-k) \varphi(x-m) d x \\
\cdot \cdot \int_{k, m, l}=\int_{-\infty}^{\infty} \varphi_{x}(x-k) \varphi(x-m) \varphi(x-l) d x .
\end{gathered}
$$

The semi-implicit time differencing for Burgers' equation

$$
\left(U_{n+1}-U_{n}\right) / \Delta t+U_{n} U_{n+1, x}=\sigma U_{n+1, x x}
$$


is used throughout and the Wavelet-Galerkin equations are

$$
\left(U_{k, n+1}-U_{k, n}\right) / \Delta t-\sigma A_{k, m} U_{m, n+1}+B_{k, m, l} U_{m, n} U_{l, n+1}=0
$$

Here $U_{k, n}$ is the wavelet space and semi-implicit time discretization of solution for Burgers' equation.

We compare the finite-difference, spectral and wavelet-Galerkin methods. Each has the semi-implicit time differencing and 64 modes. These results were presented by Latto and Tenenbaum in reference [4].

After calculation the solutions are smoothed with a three point averaging

$$
\hat{U}_{k, n}=\left(U_{k-1, n}+2 U_{k, n}+U_{k+1, n}\right) / 4 .
$$

We compare the numerical solutions, smoothed and unsmoothed, with the exact solutions evaluated using the Cole-Hopf transformation.

In summary the results are [4]

1. The Wavelet-Galerkin (WG) method appears to be stable for all viscosities, including null viscosity.

2. The WG appears to be close to exact for large times and small viscosity.

3. The three term smoothing of the oscillatory WG appears to be close to the exact solution for all times and small viscosity.

4. The WG method appears to handle error in a way that the information content (the exact solution) is not destroyed and can be recovered from the approximation.

\section{Acknowledgement}

This research was supported in part by the Advanced Research Projects Agency of the Department of Defense and was monitored by the Air Force Office of Scientific Research under Contract No. F49620-89-C-0125. The United States Government is authorized to reproduce and distribute reprints for governmental purposes notwithstanding any copyright notation hereon.

\section{References}

1. I. Daubechies, "Orthonormal Bases of Compactly Supported Wavelets", Commun. Pure Appl. Math, 41 (1988) 909-996.

2. G. Strang, "Wavelets and Dilation Equations: A Brief Introduction", SIAM Review, 31, (1989), 614-627.

3. G. B. Whitham, Linear and Nonlinear Waves, John Wiley and Sons, New York, NY (1974).

4. A. Latto and E. Tenenbaum, "Les ondellettes à support compact et la solution numérique de l'équation de Burgers", Aware Tech, Report AD900307.1.2, March (1990). To appear, Compte rendus Acad. Sci. France. 
Traveling Waves and Oscillations in Compressible Magnetoconvection

\author{
Nigel Weiss \\ Department of Applied Mathematics and Theoretical Physics \\ University of Cambridge, England
}

Sunspots are dark owing to partial suppression of convection by a strong vertical magnetic field. A large sunspot occupies only $0.1 \%$ of the visible disk but starspots may cover $50 \%$ of the surface of more active stars. These features have motivated studies of Boussinesq and (more recently) compressible magnetoconvection (Proctor\& Weiss 1982; Hughes \& Proctor 1988). The pioneering paper Hurlburt \& Toomre (1988) dealt mainly with steady motion: this lecture describes studies of timedependent convection carried out by Derek Brownjohn, Neal Hurlburt, Michael Proctor and myself (Hurlburt et al.1989; Weiss et al. 1990).

We consider two-dimensional convection in a compressible layer with an imposed temperature difference $\Delta T$ in an initially uniform magnetic field Bo. The relevant parameters are the Rayleigh number $\mathrm{R} \propto \Delta T$, the plasma $\beta=\mathrm{p} /\left(\mathrm{B}_{o}{ }^{2} / 2 \mu_{o}\right) \propto \mathrm{Q}^{-1}$ and the ratio $\zeta$ of the magnetic to the thermal diffusivity. The system is assumed to be periodic in the horizontal direction with a dimensionless wavelength $\lambda$. As $\mathrm{R}$ is increased the static solution may lose stability either in a pitchfork bifurcation or (if $\zeta<1$ and $Q$ is sufficiently large) in a Hopf bifurcation. In the latter case branches of standing wave and traveling wave solutions emerge from the bifurcation. Normal form equations indicate which solution is preferred but stability may be transferred from one branch to the other via an intermediate branch of modulated wave solutions. With a horizontal field, for example, traveling waves are preferred in the Boussinesq limit but we find for $\beta=32$ that standing waves are stable near the Hopf bifurcation at $R^{(o)}$, while modulated waves appear for $4 R^{(o)} \leq R \leq 16 R$ (0) and traveling waves eventually gain stability at $R=32 R^{(0)}$.

In a shallow layer with a vertical magnetic field magnetic pressure fluctuations become important when $\beta=O(1)$. We find that standing waves are always stable near the Hopf bifurcation but for $\lambda=2$ and $32 \geq \beta \geq 6$ there is a transition from a 2-roll standing wave solution to a 4-roll traveling wave solution." The traveling waves have a triangular structure with a prograde jet-stream and propagate with a velocity $\mathrm{v} \approx 1 / 2 \lambda \mathrm{Va}$ as in the Boussinesq limit. Apparently the motion builds up fields so strong that magnetic pressure has to be balanced by inertial terms rather than gas pressure. For $\lambda=1$ there is a straightforward transition from standing waves to traveling waves but for $\lambda=2$ the change of scale is achieved via an intermediate mixed-mode solution that is quasiperiodic. At higher values of $\beta$ the steady solutions are unstable and periodic solutions with large-scale streaming motion can be found.

Traveling waves only appear when $\zeta \approx 0.1$. In a sunspot there is a transition from photospheric layers, where $\zeta \approx 0.003$, to levels below $2000 \mathrm{~km}$ depth where $\zeta$ 
$\approx 30$. We expect oscillatory behavior above and steady overturning motion below. This can be modelled by taking a deep stratified layer with a density contrast ${ }^{\rho}$ bottom $/ \rho$ top $=11$ and setting $\zeta \propto \rho$. For $0.2 \leq \zeta \leq 2.2$ and $\lambda=4 / 3$ we find that the initial bifurcation is a pitchfork leading to steady 2-roll convection. As $\mathrm{R}$ is increased counter-rolls appear and grow. Eventually a complicated sequence of secondary and tertiary bifurcations leads to stable periodic oscillations. In these solutions there are four rolls which are modulated periodically in space. At the base of the layer the sense of motion is unchanged throughout the oscillation but the velocities at the top reverse as alternate rolls become prominent and penetrate towards the upper boundary. This form of modulated oscillation provides a natural explanation for the sporadic bright features, called umbral dots, that are observed near the center of sunspots. Time-dependent convection of this type must be responsible for supplying the energy radiated from sunspots, which remains significant in spite of the magnetic field.

\section{$\underline{\text { References }}$}

Hughes, D.W. \& Proctor, M.R.E. 1988. Ann.Rev. Fluid Mech.20, 187

Hurlburt, N.E., Proctor, M.R.E., Weiss, N.O. \& Brownjohn, D.P. 1989. J.Fluid Mech. 207, 587

Hurlburt, N.E. \& Toomre, J. 1988. Astrophys. J.327, 920

Proctor, M.R.E. \& Weiss, N.O. 1982. Rep.Prog.Phys..45, 1317

Weiss, N.O., Brownjohn, D.P., Hurlburt, N.E. \& Proctor, M.R.E. 1990 Mon. Not. Roy. Astron. Soc., in press 
Modeling Mesogranules And Exploders On The Sun

\author{
Nigel Weiss \\ Department of Applied Mathematics and Theoretical Physics \\ University of Cambridge, England
}

Observations reveal three different scales of convection on the surface of the sun. Supergranules, with a characteristic diameter of $30 \mathrm{Mm}$, have horizontal velocities of around $0.5 \mathrm{Km} \mathrm{s}^{-1}$ which are correlated with the magnetic network. Granules have a typical diameter of $1 \mathrm{Mm}$ and velocities of $1 \mathrm{~km} \mathrm{~s}^{-1}$. By tracking the proper motions of granules it has become possible to derive intermediate-scale velocity patterns and the existence of mesogranules, with diameters around $5 \mathrm{Mm}$ and velocities of $0.5 \mathrm{~km} \mathrm{~s}^{-1}$, has been confirmed (Simon et al. 1988). High-resolution white-light observations also show exploding granules (exploders); these are granules that expand rapidly to a diameter of about $3 \mathrm{Mm}$, forming a ring-like structure with a dark core and then fragmenting. Nearby granules are swept aside with velocities of order $2 \mathrm{~km} \mathrm{~s}^{-1}$. The fact that exploders occur preferentially near the center of mesogranules raises the question: Are mesogranules just the time-averaged consequence of recurrent exploders (Title et al. 1989)?

George Simon, Alan Title and I have used a simple kinematic model of the horizontal velocity in the photosphere in an attempt to answer this question. Sources are represented by axisymmetric outflows that can be derived from potentials of the form

$$
\phi(r)=\frac{1}{2} V R \exp \left[-(r / R)^{2}\right]
$$

It has already been shown that such sources provide a good description of mesogranular flows observed from Spacelab 2 (Simon \& Weiss 1990). We now suppose that a mesogranule can be represented by sources distributed with a probability

$$
\psi\left(\mathrm{r}_{o}\right)=\left(\pi \rho^{2}\right)^{-1} \exp \left[-(\mathrm{r} / \rho)^{2}\right]
$$

that there is a source distant $r_{o}$ from the origin. Then the expected value of the potential has the same form as (1) with a radius $\bar{R}=\left(R^{2}+\rho^{2}\right)^{1 / 2}$ and a velocity $\overline{\mathrm{V}}=(\mathrm{R} / \overline{\mathrm{R}})^{3} \mathrm{~V}$.

We have compared mesogranules $\left(\mathrm{R}_{m}=2 \mathrm{Mm}, \mathrm{V}_{m}=1 \mathrm{~km} \mathrm{~s}^{-1}\right)$ with exploders $\left(\mathrm{R}_{g}=1 \mathrm{Mm}, \mathrm{V}_{g}=8 \mathrm{~km} \mathrm{~s}^{-1}\right.$, lifetime $\left.\Delta \mathrm{t}=10 \mathrm{~min}\right)$, distributed normally about the mesogranule center. As diagnostics of the flow we introduce passive test particles (corks) travelling with local fluid velocity. Then we compare the cork patterns produced by different flows after periods of 1-6 hr have elapsed. In addition we obtain a quantitative description of these patterns by computing their factal dimensions. Figure 1(a) shows cork patterns generated by randomly distributed mesogranules. 
The region shown has dimensions $40 " \times 40$ " (30 $\mathrm{Mm} \times 30 \mathrm{Mm})$ and the average distance between mesogranule centers is $7 \mathrm{Mm}$. After $2 \mathrm{hr}$ the mesogranule centers are cleared and corks move gradually into a network which is clearly apparent after $5 \mathrm{hr}$. The corresponding patterns produced by explodes are shown in Figure 1(b). The network forms more rapidly but has a ragged appearance. The patterns are qualitatively similar and it is not possible to distinguish between them from the observations. However it is possible to rule out other models, with exploders dropped randomly over the region or distributed randomly about their predecessor so as to follow a random walk.

Figure 2 illustrates the effects of combining mesogranular and supergranular velocities. We deposit these supergranules $\left(\mathrm{R}_{s}=10 \mathrm{Mm}, \mathrm{V}_{s}=1 \mathrm{~km} \mathrm{~s}^{-1}\right)$ in the region and allow mesogranules to travel with the local supergranular velocity; if mesogranules approach too closely or escape from the region they are replaced and the cork supply is replenished to compensate for those escaping from the region. The patterns produced by mesogranules in Figure 2(a) can be compared with those generated by exploders in figure 2(b). The magnetic network between the supergranules is distorted by the small-scale motion.

These results can be compared with a 3-hour observational run made at the Pic-du-Midi (Frank et al. 1989). This sequence shows mesogranules drifting across supergranules, with an apparent lifetime of $3 \mathrm{hr}$. A preliminary inspection suggests that exploders develop from granules that appear at the center of mesogranules and drift outwards as they expand. This supports a picture in which a systematic outflow combines with random exploders to provide the averaged mesogranular velocity. Further work is needed to relate these surface features to the underlying three-dimensional dynamics.

\section{$\underline{\text { References }}$}

Frank, Z. et al. 1989. Preprint.

Simon, G.W. et al. Astrophys. J. 327, 964.

Simon, G.W. and Weiss, N.O., 1989. Astrophys.J.345, 1060.

Title, A.M. et al. 1989. Astrophys.J. 336, 475. 

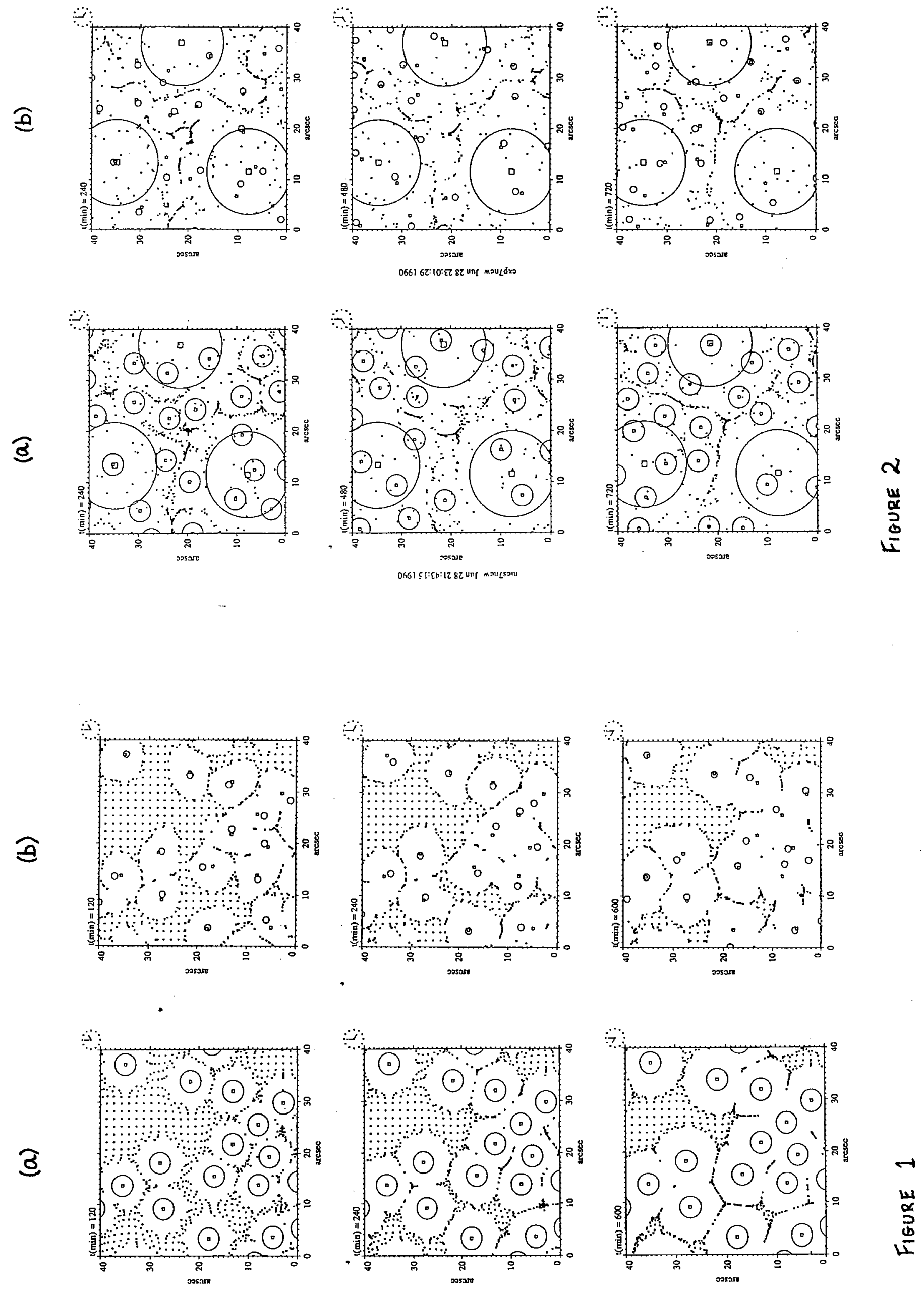


\section{Fluid Mechanics and Melting.}

Andrew W. Woods,

Scripps Institution of Oceanography.

In this presentation, we considered the role of melt convection upon the melting of a solid into a binary melt. At a horizontal interface six different convecting regimes may arise, depending upon the relative composition of the melt and the solid and whether the melt underlies or overlies the solid. These are summarised in the figure below.

First we considered the case of purely diffusion driven melting described by Woods (1990a). When the melt temperature far exceeds that of the melting temperature, the solid melts at a rate determined by the thermal diffusivity; in contrast, when the melt is of the same temperature or colder than the solid, but of a different composition, the solid may dissolve into the melt at a rate determined by the compositional diffusivity. The crucial difference between these two regimes is that in the melting regime, the compositional boundary layer becomes embedded in the melt, while in the dissolving regime, it is always located at the melt/solid interface; this difference can lead to some differences in the style of convection that results.

Next, we reviewed the situation in which the liquid which is melting the solid is in a state of thermal convection, as discussed by Huppert and Sparks (1988). This situation arises when the liquid underlies the relatively buoyant melting solid; owing to the density difference due to composition, the molten solid remains above the liquid, and each layer may convect thermally, as in a double-diffusive system (Turner, 1979).

We then considered the problem of melting a relatively buoyant solid by an overlying layer of dense liquid (Woods (1990b)). The molten solid is buoyant and rises into the liquid, producing vigorous convection which mixes the melt uniformly. A global model of the melting is able to predict the evolution of the melt temperature and composition as a function of the depth of melted solid. Experimental data suggest that in this well-mixed convection regime, the flux of solute at the interface scales as $F_{c} \sim \Delta C^{5 / 2}$. This is a result of the complex coupling of the convective heat flux, which drives the melting and thereby drives the convection; this non-linear coupling produces a compositional flux which 
exceeds that which would arise due to Raleigh-Benard type compositional convection, in which the convective flux would scale as $\Delta T^{4 / 3}$ when the boundary compositions are fixed. The difference arises because the convection is driven by the compositional flux at the boundaries whose magnitude is determined by the rate of melting.

We considered application of these results in the geological context of magma chambers; hot, dense molten basalt may intrude a crustal chamber and melt the walls, floor and roof. Typically the molten crust is less dense and therefore compositional convection in the chamber due to the floor melting will arise, in conjunction with thermal convection from the melting at the roof.

\section{References.}

Huppert, H.E. and Sparks, R.S.J., 1988, Melting the roof of a turbulently convecting chamber, J.F.M.

Woods, A.W., 1990a, Melting and dissolving a binary alloy, submitted.

Woods, A.W., 1990b, Fluid mixing during melting, submitted. 


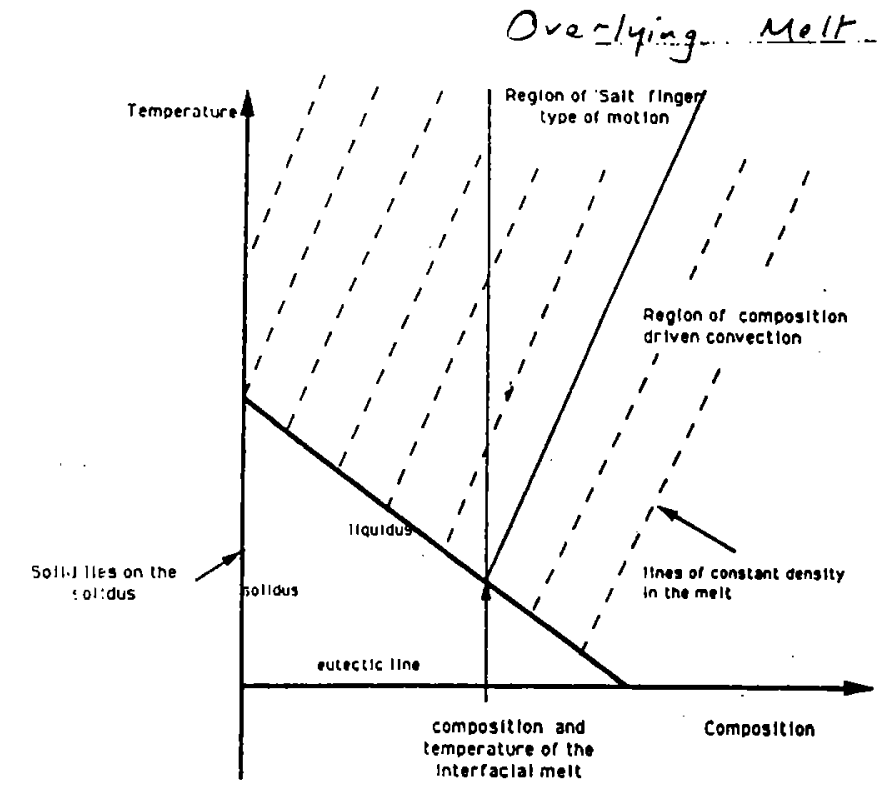

The composition and temperature of the overlying melt determ ine the nature of the convection driven by the melting of pure ice (composition - 0). Note the interface composition Is lower than that in the par-field.

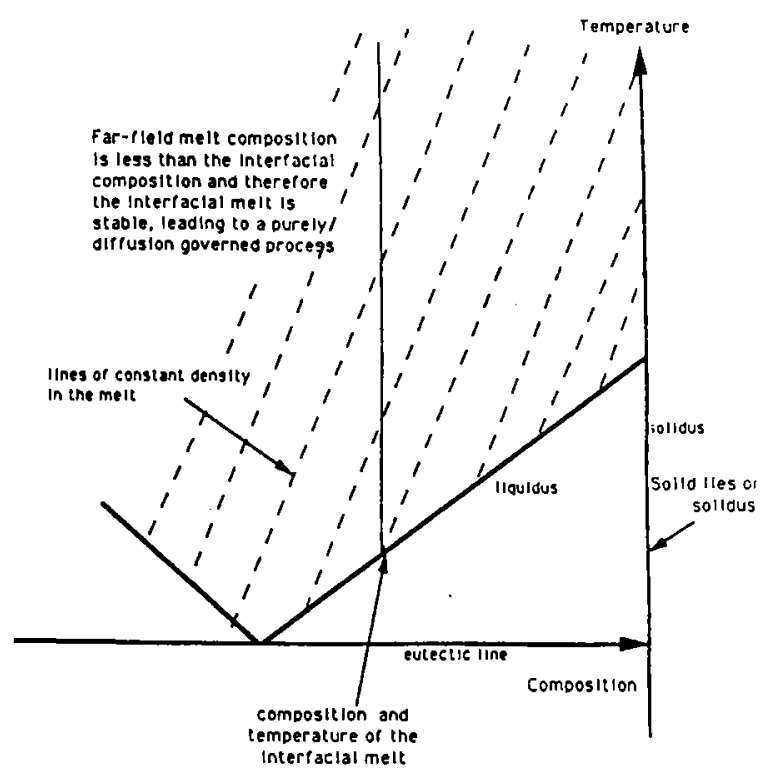

The composition and temperature of the overlying mett determine the nature of the convection ariven by the melting of pure lee (composition $=0$ ).

\section{Linderlying Melt}

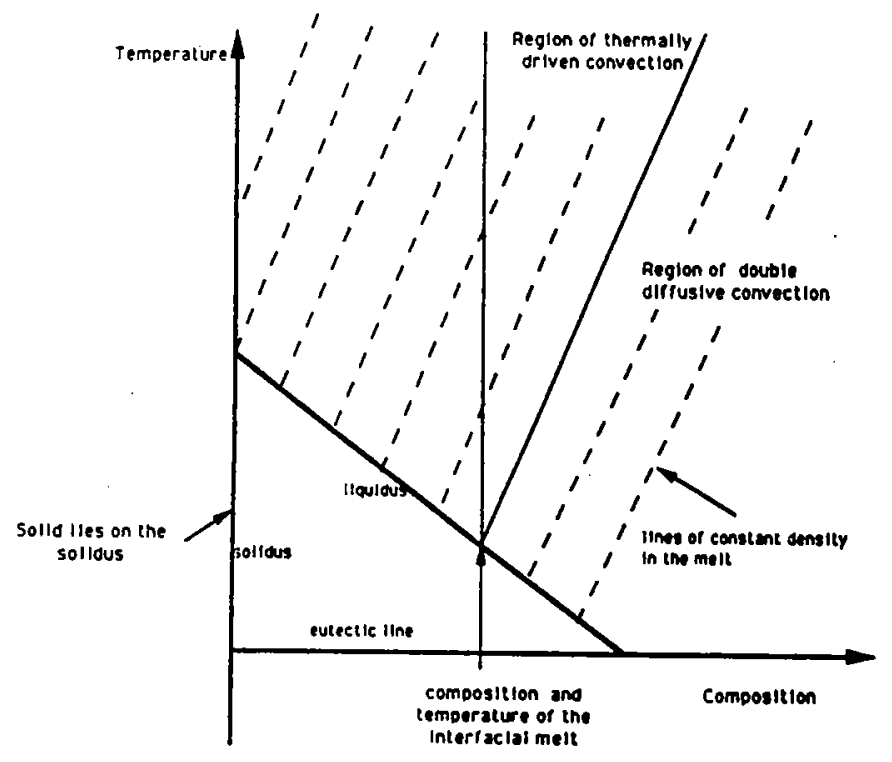

The composition ano temperature of the underlying melt determine the nature of the convection driven oy the melting of pure Ice (composilion - 0 ). Note the Interface composition is lower than that in the far-pleld.

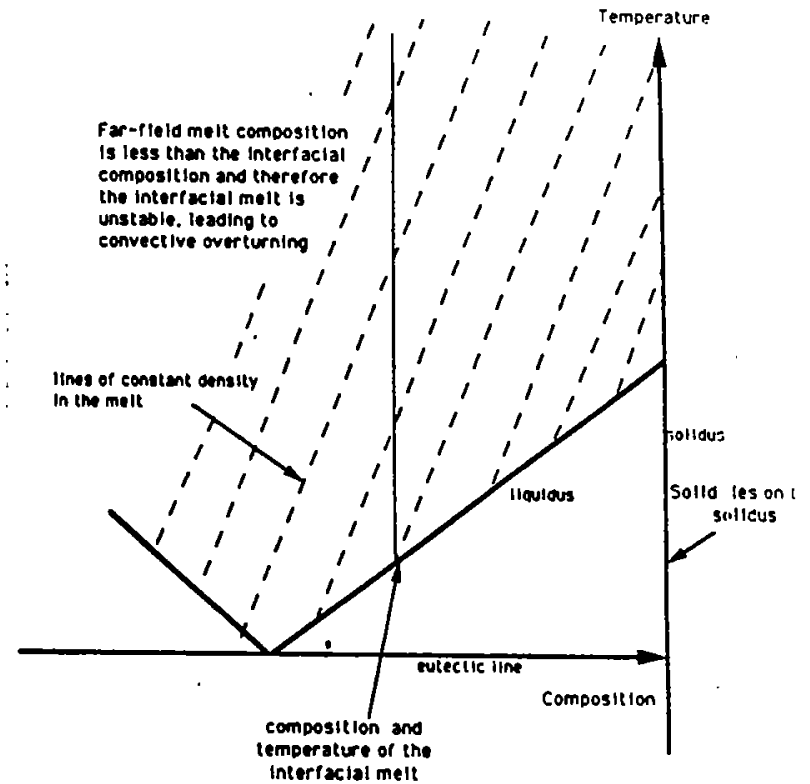

The composition and temperature of the underlying melt determine the nature or the convection driven by the melting of pure ice (composition - 0 ). 


\title{
CONVECTIVE PENETRATION IN STELLAR INTERIORS
}

\author{
Jean-Paul Zahn
}

\section{Introduction}

It has long been recognized that the penetration of motions beyond the classical boundary of a convective core set by the Schwarzschild criterion (i.e. where the temperature gradient becomes subadiabatic again) would have a crucial impact on stellar evolution. Such penetration would also modify substantially the temperature profile at the base of a convective envelope. But stellar structure models rarely include that effect, and there is still debate on whether such penetration even occurs.

One reason is that astrophysicists too often neglect to confront their problems with those of their colleagues in geophysics and fluid dynamics, who study similar cases, and often decades ahead. Another reason is the failure of the mixing-length treatment, which proved so powerful when dealing with convective envelopes (largely because it reduced one's ignorance to a single parameter), but cannot come to grips with penetrative convection.

Our purpose (in a Paper submitted to Astron. Astrophys.) is to convince the astrophysicists that convective penetration indeed exists, by recalling the relevant observations, experiments and computer simulations that are available. Furthermore, we give an estimate for the extent of penetration that is inspired by such observations and calculations, and does not rely on the mixing-length treatment.

\section{The evidence for penetrative convection}

There is overwhelming evidence for penetrative convection: in the atmosphere, the oceans, the laboratory. The dynamics of the planetary boundary layer, for instance, is entirely governed by it: every morning the nocturnal stable stratification is replaced from below by a growing unstable layer due to surface heating.

In the laboratory, penetration has been observed whenever a convectively unstable layer is imbedded in a stable stratification of fluid. One famous example is the ice-water experiment, which uses the peculiar property of water having a 
density maximum at $4^{\circ} \mathrm{C}$; it was suggested by $\mathrm{W}$. Malkus and performed here in Woods Hole by Furumoto and Roth (1961), and was later repeated by Townsend (1966) and by Adrian (1975). In all experiments, one observes a substantial amount of penetration, sometimes comparable with the thickness of the unstable layer.

What are the theoretical predictions? According to linear theory, some weak overshooting should occur outside the unstable region, but that result cannot be extrapolated into the non-linear regime, since the functional form of the solutions is deeply modified by their feed-back on the thermal stratification. This has been recognized already by Veronis (1963), who did the first non-linear analysis of penetrative convection (and published it in the Astrophys. Journal)! Since, various fully non-linear simulations have been carried out. All those which are based on firm physical ground confirm that there is indeed much more penetration than what could be inferred from the linear theory (Musman 1968; Moore and Weiss 1979; Zahn, Toomre and Latour 1982; Massaguer, Latour, Toomre and Zahn 1984).

The early investigations dealt with severely truncated equations, and as a result the solutions were stationary. The penetration depth was found comparable with the thickness of the unstable layer; it depended on the degree of stability of the outer medium and on the aspect ratio of the cells. Furthermore, three-dimensional cells were seen to penetrate much more than horizontal rolls, in the direction of their net kinetic energy flux.

More recently, penetrative convection has been simulated in two dimensions with much better spatial resolution, in a compressible fluid (Hurlburt, Toomre and Massaguer 1986). The solutions are no longer stationary, and they show vigorous concentrated downdrafts which penetrate rather deep into the stable region below. Those incursions strongly couple with gravity waves, as was observed by Townsend (1966) in the laboratory. Such downdrafts appear to be a genuine and important property of stratified convection, since they are also present in all three-dimensional simulations (Graham 1977, Nordlund 1984, Stein and Nordlund 1989, Cattaneo, Hurlburt and Toomre 1989), where they behave more like long-lived plumes than as thermals which would just traverse the domain.

To summarize, all numerical simulations exhibit substantial penetration of the convective layer into its stable environment, irrespective of the approximations made, provided the stratification is almost adiabatic in the unstable zone; that nearly adiabatic stratification extends well beyond the limits of the unstable region. Moreover, the flows show very similar behavior in the two parts of the domain: the only obvious manifestation of the change in the entropy slope, from an unstable to the stable one, is the reversal of the correlation between the vertical velocities and the temperature fluctuations, which switches the direction of convective heat flux from upwards to downwards. 


\section{Subadiabatic penetration at the bottom of a convective envelope}

We consider convection that is efficient enough to establish an adiabatic stratification and to transport most of the thermal energy produced in the core of the star. This requires that the Péclet number $w \ell / K$ be substantially larger than unity $(\ell$ and $w$ are the typical size and velocity of the convective motions, $K=\chi / \rho C_{P}$ the thermal diffusivity, and $\chi$ the radiative conductivity).

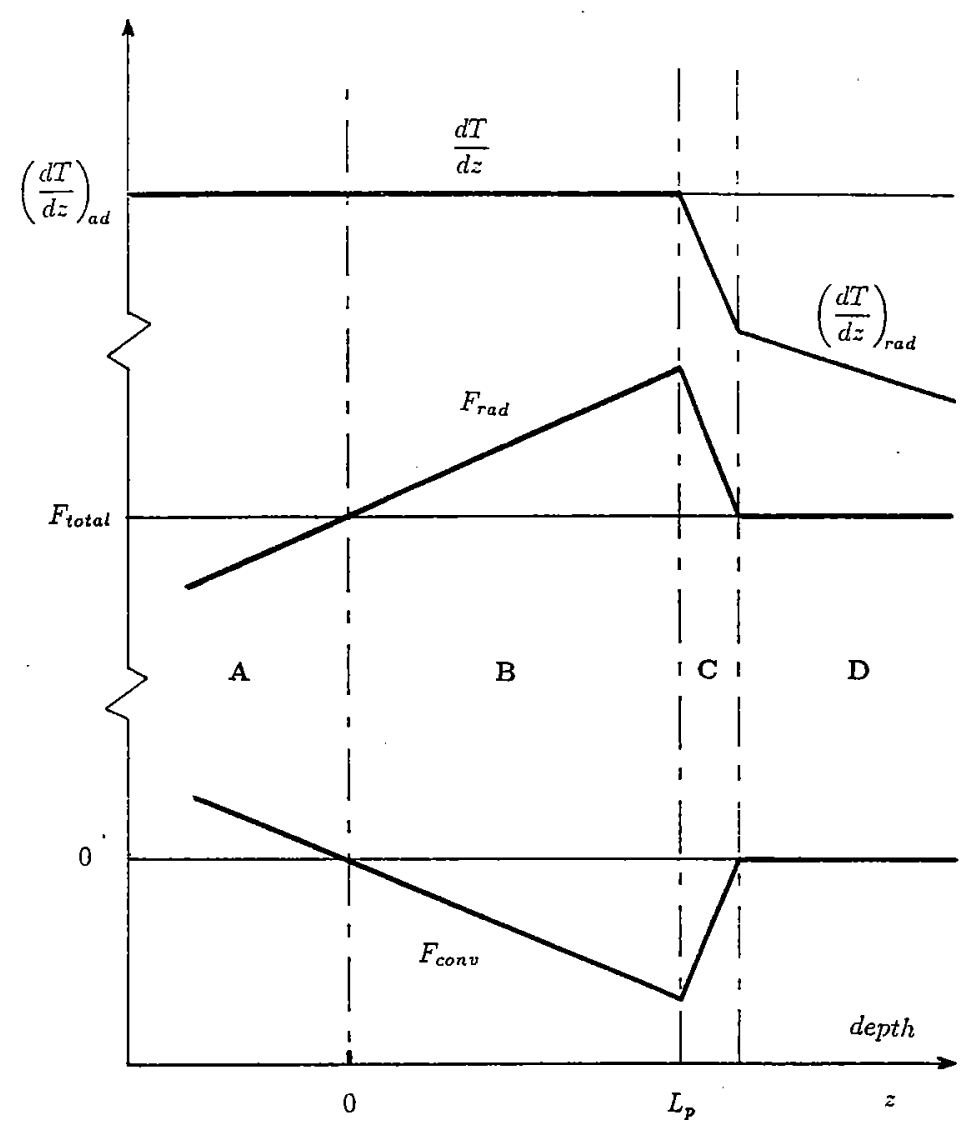

Figure 1

The structure of a star at the bottom of a convective envelope is sketched in Fig. 1. Due to the increase of the conductivity with depth, the radiative flux $F_{\text {rad }}$ rises until it equals the total flux $F_{\text {total }}$ at the level $z_{0}$. If there were no convective penetration, this would be the edge of the convection zone, as predicted by the Schwarzschild criterion; below, the energy flux would be carried only through radiation, and the temperature gradient would then be $(d T / d z)_{\text {rad }}=F_{\text {total }} / \chi$. But the motions penetrate into the stable region and they render it nearly adiabatic, provided their velocity $w$ is larger than some critical value (given below in Eq. 5.5). The motions decelerate through buoyancy until thermal diffusion becomes more 
important than advection, in a thin boundary layer where the temperature profile settles from the adiabatic to the radiative slope.

We thus identify four regions at the bottom of the convection zone. The first two have a quasi-adiabatic stratification: the superadiabatic domain, which is the seat of the convective instability (A), followed by the stable subadiabatic region (B). Below, after a shallow thermal boundary layer (C), heat is transported only through radiation in the radiative interior (D).

We now use this schematical picture to estimate the extent $L_{p}$ of the subadiabatic penetration region (B). In that region, the convective flux is, to a fairly good approximation,

$$
F_{\text {conv }}=-\left(\frac{d \chi}{d z}\right)_{0}\left(\frac{d T}{d z}\right)_{a d} z,
$$

where $z$ is counted from the depth $z_{0}$ where the $F_{\text {conv }}=0$ (the subscript 0 refers to that level).

We shall assume that the fraction $f$ of the area is occupied by downwards directed motions which transport most of the convective flux, as observed in the laboratory and in the computer simulations. We express that convective flux to lowest order in terms of the horizontal temperature fluctuations and the vertical velocity of these motions, which we assume strongly correlated:

$$
F_{\text {conv }}=-f \rho C_{P} W \delta T
$$

To estimate the penetration depth, we follow the downdrafts from $z=0$, where their velocity is $W_{0}$, until they stop at the base of the penetration zone, at $z=L_{p}$. Their deceleration is described to first order by

$$
\frac{1}{2} \frac{d W^{2}}{d z}=g \frac{\delta \rho}{\rho}=-g Q \frac{\delta T}{T},
$$

with the usual assumption of pressure equilibrium, and $Q=-(\partial \log \rho / \partial \log T)_{P}$ being the expansion coefficient at constant pressure. Elimination of $\delta T$ yields the following expression for the depth $L_{p}$ of the subadiabatic region

$$
\frac{L_{p}}{H_{P}}=W_{0}^{3 / 2} f^{1 / 2}\left[\frac{3}{2} g Q K \chi_{P} \nabla_{a d}\right]^{-1 / 2}
$$

with $\chi_{P}=(\partial \log \chi / \partial \log P)_{a d}$. We can go a step further by using the property that in all convection theories the velocity scales as $F_{\text {conv }}=$ const $\rho W^{3}$, in the fully developed regime; the proportionality coefficient is of order unity, and several prescriptions are available for it, which are roughly equivalent. Ours is

$$
F_{\text {conv }}=\frac{2 f^{*}}{3} \frac{H_{P}}{\Lambda} \frac{\rho W^{3}}{Q \nabla_{a d}},
$$


involving again a filling factor $f^{*}$ (which could a priori be different from that in the penetration zone), and $\Lambda$ being the mixing-length. Since the convective flux nearly equals the total flux over most of the unstable zone, we may approximate it by $F_{\text {total }}$. We thus obtain an alternate form of expression (3.4):

$$
\frac{L_{p}}{H_{P}}=\left[\frac{f}{\alpha f^{*}} \frac{1}{\chi_{P}}\right]^{1 / 2}=\left[\frac{\phi}{\chi_{P}}\right]^{1 / 2},
$$

with $\alpha=\Lambda / H_{p}$ and $\phi=f / \alpha f^{*}$. Let us stress that this estimate can only be applied to stars possessing a deep enough convective envelope, in which most of the heat is carried by convection.

As could be anticipated, the penetration depth (in units of the pressure scale height) only depends on the steepness of the conductivity gradient $\chi_{P}$, and on the ratio of the efficiencies of the convection in the unstable and stable regions, as measured here by $\phi=f / \alpha f^{*}$. Judging by the computer simulations that have been performed, this parameter $\phi$ should be close to unity, reflecting the similar behavior of the flows in the stable and unstable parts of the convective domain.

Much in the same spirit, it is possible to evaluate the thickness of the thermal boundary layer $(\mathrm{C})$ :

$$
L_{t} \approx\left(\phi \chi_{P}\right)^{-1 / 8}\left(K t_{d}\right)^{1 / 2} \quad \text { with } \quad t_{d}=\left(\frac{H_{P}}{g}\right)^{1 / 2} .
$$

Below the solar convection zone, where the dynamical time scale is $t_{d} \approx 300 \mathrm{~s}$ and the thermal diffusivity $K=\chi / \rho C_{P} \approx 210^{7} \mathrm{~cm}^{2} / \mathrm{s}$, the thermal boundary layer which terminates the penetration zone has a thickness of the order of $1 \mathrm{~km}$. That thin boundary layer plays no role in the dynamics: the convective motions have already been slowed down to a velocity $W_{t} \approx 1 \mathrm{~m} / \mathrm{s}$ when entering the layer. Thus the boundary of the whole convection zone (regions A and B) is sharply defined, although it probably ondulates somewhat due to the velocity dispersion of the impinging downdrafts (as illustrated in our planetary boundary layer when it is delineated by clouds).

\section{Penetration of a convective core}

The derivation of the extent of penetration of a convective core into its stable surroundings is very similar to that for a convective envelope. The only difference is the cause of the convective instability: in a stellar core, it is the steep increase of the nuclear energy generation rate $\varepsilon$, as one approaches the center of the star, which is mainly responsible for the onset of convection.

The penetration length scales as

$$
\frac{L_{p}}{H_{P}}=W_{0}^{3 / 2} f^{1 / 2}\left(\frac{2}{9} \frac{r_{0}}{H_{P}}\right)^{1 / 2}\left[g Q K \nabla_{a d}\left(\frac{\rho}{\bar{\rho}}-\frac{\rho \varepsilon}{\overline{\rho \varepsilon}}+\frac{1}{3} \chi_{P} \frac{r_{0}}{H_{P}}\right)\right]^{-1 / 2}
$$


with the same notations as before, and $\bar{\rho}, \overline{\rho \varepsilon}$ being the mean $\rho, \rho \varepsilon$ in the superadiabatic core of radius $r_{0}$.

We shall again use the value of $W_{0}$ given by the mixing-length treatment (Eq. 3.6), with

$$
\Lambda=r_{0} \min \left(1, \alpha H_{P} / r_{0}\right),
$$

and assume that most of the energy is carried by convection in the superadiabatic core. Then the alternate form of expression (4.1) is

$$
\frac{L_{p}}{r_{0}}=\left(\frac{\phi}{6} \min \left(1, \alpha H_{P} / r_{0}\right)\right)^{1 / 2}\left[\frac{\rho}{\bar{\rho}}-\frac{\rho \varepsilon}{\overline{\rho \varepsilon}}+\frac{1}{3} \chi_{P} \frac{r_{0}}{H_{P}}\right]^{-1 / 2},
$$

$\phi=f / f^{*}$ being here the ratio between the filling factors in the stable and unstable regions. It predicts an increase of $L_{p} / H_{P}$ with the size of the unstable core, and such a trend has actually been noticed by Maeder and Mermilliod (1981).

\section{The temperature gradient in the subadiabatic penetration region}

In our derivation of the penetration depth, we have assumed that the convective motions are efficient enough to enforce a nearly adiabatic temperature gradient when penetrating into the subadiabatic domain. We shall now establish the condition which must be fulfilled for this to occur.

We start from the specific entropy equation

$$
\rho T \frac{\partial s}{\partial t}+\rho T u_{i} \frac{\partial s}{\partial x_{i}}=\eta_{i j} \frac{\partial u_{i}}{\partial x_{j}}-\frac{\partial F_{i}}{\partial x_{i}},
$$

where $u_{i}$ is the velocity field, $F_{i}=\chi \partial T / \partial x_{i}$ the radiative heat flux and the first term on the r.h.s. represents the heat produced by viscous friction, which we shall neglect from now on. We restrict ourselves to quasi-stationary motions, i.e. to flows whose organization lives longer that the travel time across the penetration region, and we linearize the variables around their horizontal mean

$$
s=\bar{s}+s_{1}, \quad T=\bar{T}+T_{1} \quad \text { and } \quad F_{i}=F_{\text {rad }}+\left(F_{i}\right)_{1} .
$$

Assuming again that the fluctuations $T_{1}, s_{1}$ of temperature and entropy are strongly correlated with the vertical velocity $w$, at least in the downdrafts which occupy a fraction $f$ of the area, we get after some manipulation and horizontal averaging

$$
-c \frac{d}{d z} F_{\text {rad }}+C_{P}\left[\left(\frac{d T}{d z}\right)_{a d}-\frac{d T}{d z}\right] \rho W=\chi\left(\frac{d^{2}}{d z^{2}}-\frac{1}{a^{2}}\right) \delta T .
$$

Here $W$ is the r.m.s. of the vertical velocity in the downdrafts, and $c$ the triple

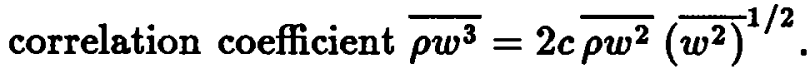


Depending on the strength and of the geometry of the motions, the temperature fluctuations will be controlled either by diffusion or by advection. In the advective case, the r.h.s. of Eq. (5.2) is negligible, and we have

$$
c \frac{d}{d z}\left(\chi \frac{d T}{d z}\right)=\rho C_{P} W\left[\left(\frac{d T}{d z}\right)_{a d}-\frac{d T}{d z}\right] .
$$

Assuming that the temperature gradients $\nabla=\partial \ln T / \partial \ln P$ are approximatively constant, we obtain the following approximate expression for the subadiabatic gradient in the penetration region:

$$
\frac{\nabla}{\nabla_{a d}-\nabla}=\frac{f}{c \chi_{P}} \frac{W H_{P}}{K} .
$$

We see that, although the temperature gradient is less adiabatic when it is achieved by downdrafts that do not fill the space, as one may expect, the departure from adiabacy will still be negligible as long as

$$
\frac{f}{c \chi_{P}} \frac{W H_{P}}{K} \gg 1 \text {. }
$$

This defines a critical value which the vertical velocity $W$ must exceed to enforce such a quasi-adiabatic stratification.

At the base of the solar convection zone, where $W H_{P} / K=210^{6}$ and $\chi_{P}=1.3$, a filling factor of about $10^{-5}$ is sufficient for quasi-adiabatic penetration to occur (for $c \approx 1 / 10$ ).

At the boundary of the convective core of a $9 \mathrm{M}_{\odot}$ ZAMS star, where the Péclet number $W r_{0} / K$ is of the order of $310^{6}$, filling factors larger than about $10^{-5}$ will again ensure such quasi-adiabatic penetration.

\section{Conclusion}

Our main result is that the conditions for subadiabatic convection are certainly fulfilled in the interior of star, both at the bottom of a deep convective envelope and at the edge of a convective core, due to the high efficiency of the convective heat transport. Even if that flux is carried by a rather sparse network of plumes, as suggested by the recently performed three-dimensional simulations, the stratification is nearly adiabatic throughout the convection zone, including the region of subadiabatic penetration, and the departures from adiabacy are confined within in a very narrow thermal boundary layer.

This property allows the use of the integral constraint that has been proposed by Roxburgh $(1978,1989)$ to calculate the size of a convective core. He showed that, provided the departures from the adiabatic gradient are sufficiently small, one 
can integrate Eq. (5.1) (divided bt $T$ ) over a whole convective core, to reach the condition

$$
\int_{0}^{r}\left(L_{\text {rad }}-L_{\text {total }}\right) \frac{1}{T^{2}} \frac{d T}{d r} d r=0
$$

(with $L=4 \pi r^{2} F$ ). The applicability of his result to actually predict the extent of penetration was seriouly questioned on the ground that the entropy gradient is not necessarily small everywhere (Baker and Kuhfuß 1987). But since the departures from adiabacy are restricted to a very thin boundary layer, provided condition (5.5) is fulfilled, the integral condition above is satisfied to a fairly good degree of approximation.

It is more problematic to use Roxburgh's prescription to predict the extent of penetration below a convective envelope. The reason is that the integral above would have to start at the surface of the star, that it would include a region where the departures from adiabacy are high and whose weight would be very large in the integrant, due to the strong temperature contrast between top and bottom. It then seems preferable to use our - admittedly much cruder - estimate of Eq. (3.6), and to turn to the observations for the calibration of the parameter $\phi$.

Let us stress that our results have been obtained by making several simplifying assumptions. An implicit assumption was that we ignored the Coriolis force, although it plays an important role in all convective cores and at the base of the convective envelope of most stars. It is not clear whether our basic scalings will be affected by rotation; presumably the law $F_{\text {conv }} \propto \rho W^{3}$ still holds, but the proportionality coefficient will be somewhat modified by the rotation, and hence also the penetration depth.

We believe that stellar structure theory has matured to a point where convective penetration must now be taken in account when constructing interior models. One may object that this would require yet an other parameter $\phi$, which cannot be derived from first principles. But with the advent of helioseismology, the diagnostic of the solar interior has improved so much that one will be able in the near future to evaluate the extent of penetration at the bottom of the convective envelope, by measuring the value of the temperature gradient at the edge of the adiabatic zone, and to derive from it the value of $\phi$, which should not vary much from star to star. In the case of a convective core, the situation is even simpler, since one can use Roxburgh's prescription to predict the depth of penetration.

\section{References}

Adrian, R.J.: 1975, J. Fluid Mech. 35, 7

Baker, N.H. and Kuhfuß, R.: 1987, Astron. Astrophys. 185, 117 
Cattaneo, F., Hurlburt, N.E. and Toomre, J. 1989, in Stellar and Solar Granulation, ed. R.J. Rutten and G. Sverino (Kluwer, Dordrecht), p. 415

Furumoto, A. and Rooth, C.: 1961 Geophys. Fluid Dynamics. Woods Hole Oceanographic Institution Rep.

Graham, E.: 1977, in IAU Coll. 38, Problems of Stellar Convection, eds. E.A. Spiegel and J.-P. Zahn (Springer, Berlin), p. 689

Hurlburt, N.E., Toomre, J. and Massaguer, J.M.: 1986, Astrophys. J. 311, 563

Latour, J., Toomre, J. and Zahn, J.-P.: 1981, Astrophys. J. 248, 1081

Maeder, A. and Mermilliod, J.C.: 1981, Astron. Astrophys. 83, 136

Massaguer, J.M., Latour, J., Toomre, J. and Zahn, J.-P.: 1984, Astron. Astrophys. 140, 1

Moore, D.R. and Weiss, N.O.: 1973, J. Fluid Mech. 61, 553

Musman, S.: 1968, J. Fluid Mech. 31, 342

Nordlund, A: 1984, in Small Scale Dynamical Processes in Quiet Stellar Atmospheres, ed. S.L. Keil (National Solar Observatory, Sunspot), p. 181

Press, W.H.: 1981, Astrophys. J. 245, 303

Renzini, A.: 1987, Astron. Astrophys. 188, 49

Roxburgh, I.W.: 1978, Astron. Astrophys. 65, 281

Roxburgh, I.W.: 1989, Astron. Astrophys. 211, 361

Schmitt, J.H.M.M., Rosner, R. and Bohn, H.U.: 1984, Astrophys. J. 282, 316

Stein, R. and Nordlund, Å: 1989, Astrophys. J. Letters 342, L95

Townsend, A.A.: 1966, Quart. J. Roy. Met. Soc. 80, 248

Veronis, G.: 1963, Astrophys. J. 137, 641

Zahn, J.-P., Toomre, J. and Latour, J.: 1982, Geophys. Astrophys. Fluid Dyn. 22, 159 


\section{N.J. Balmforth}

Galaxies such as the Milky Way appear to have a very thin disc-like structure. In many instances this disc is the setting for very beautiful spiral features. These "spiral arms" seem to be the location of amplified star formation and presumably have an enhanced density over the rest of the disc.

For the Milky Way itself the disparity of the radius of the disc with its thickness is typical: the disc radius is of the order of 10,000 parsecs, whilst its thickness is only about 500 parsecs. If we take the ratio of these two numbers and call the result the dimensionless number $N$, then we find

$$
N \sim 20 .
$$

For bodies that are infinitely thin $N \rightarrow \infty$, whilst stars and elliptical galaxies are characterized by values of $N$ that are of order unity. We have defined this parameter $N$ in anticipation of a characteristic, large parameter that shall occur in the equations governing the structure and stability of the disc. We shall exploit the magnitude of this parameter to develop the solution by an asymptotic technique.

If the disc of a galaxy contains all of its mass then the highly flattened aspect of the galaxy must indicate that the gas has been spun out by rotation and that there is a balance between the self-gravity and the centrifugal acceleration. In the direction of the rotation axis, however, the effects of the centrifuge are not felt; the structure is determined by the stratification of the gas under the balance of self-gravity and pressure. This leads to a characteristic thickness that is the Jeans length, $k_{J}^{-1}=4 \pi G \rho / c^{2}$, of the configuration, if it has the density $\rho$ and sound speed $c$.

The Jeans length is more well known from studies of the instability to gravitational collapse of large expanses of gas (termed the Jeans instability; Jeans, 1928). There, the gas is unstable to disturbances with wavelengths that exceed the Jeans length. In fact, this forms the basis for the idea that the mass will undergo a successive hierarcy of fragmentations until the characteristic length scale is less than $k_{J}^{-1}$. This illustrates how localized clumps of matter could form in an initially uniform universe.

The Jeans length is therefore singularly important for the structure and breakup of selfgravitating gaseous masses.

Clearly this conclusion is modified when one introduces angular momentum into the gas, and as we have noted, the gas can be spun out and become extremely elongated in the directions perpendicular to the axis. This is presumably the reason why many galaxies form discs.

The detailed equation of state of the matter in the disc will presently be considered unimportant. Instead a barotropic, and in particular a polytropic equation of state,

$$
p=K \rho^{\Gamma}=K \rho^{(n+1) / n},
$$

where $p$ is the pressure, $\rho$ is the density, and $K$ and $\Gamma$ are constants, shall be assumed.

The work here is based largely on an unpublished manuscript by Howard and Spiegel (1970). That paper develops the asymptotic technique and solves the equations for the equilibrium structure of the disc. Since this paper is unpublished, section 1 gives a brief account of the work, and illustrates some of the results. The linear stability analysis of these structures is then detailed in section 2. 


\section{THE EQUILIBRIUM STRUCTURES}

\subsection{The equations of galactic structure}

A disc is characterized by two different kinds of dynamical balance. In the vertical we have hydrostatic balance between the pressure support and the self gravity. If we use cylindrical coordinates then this balance is

$$
\frac{\partial p}{\partial z}+\rho \frac{\partial \Phi}{\partial z}=0
$$

where $\Phi$ is the gravitational potential. Horizontally this is modified by the centrifugal acceleration

$$
\frac{\partial p}{\partial r}+\rho \frac{\partial \Phi}{\partial r}=\rho \frac{V^{2}}{r}
$$

but at leading order, the pressure gradient must disappear. $V$ is the swirling velocity. Finally we have Poisson's equation

$$
\nabla^{2} \Phi=4 \pi G \rho
$$

We now nondimensionalize the equations of motion. We let $\rho_{c}$, the central density, be the unit of density, and $K p_{c} \Gamma$ the unit of pressure. As a unit of speed we adopt $c=\left(\Gamma p_{c} / \rho_{c}\right)^{1 / 2}$ and as a unit of length, we choose the radius of the disk, $a$. With these units equations (1.2) and (1.3) remain unchanged except for the appearance of a factor $\Gamma^{-1}$ in the pressure derivatives (though strictly a different notion is called for). Equations (1.4) becomes

$$
\frac{\partial^{2} \Phi}{\partial r^{2}}+\frac{1}{r} \frac{\partial \Phi}{\partial r}+\frac{\partial^{2} \Phi}{\partial z^{2}}=N^{2} \rho
$$

where

$$
N^{2}=\frac{4 \pi G \rho_{c}}{c^{2}} a^{2}
$$

This is just the parameter that we have already alluded to: the radius of the disk measured in units of a Jeans length $k_{c}^{-1}$, where

$$
k_{c}^{2}=\frac{4 \pi G \rho_{c}}{c^{2}}
$$

The equations of motion have the integral

$$
n \rho^{1 / n}+\Phi=F(r)
$$

where $F(r)$ is the centrifugal potential, and, in terms of $F$, the swirling velocity is given by

$$
V^{2}=r F^{\prime}(r)
$$

\subsection{Solutions for $N \rightarrow \infty$}

We shall now consider asymptotic solutions for large $N$. Since the disc is thin, vertical changes must occur very rapidly. Therefore, it is the vertical derivatives in Poisson's equation that balance the right hand side of equation (1.5) at leading order. This simply reflects the fact that $a$ is not a natural unit for $z$ and so it is convenient to introduce the stretched coordinate

$$
\zeta=N Z
$$


where $\zeta$ is $O(1)$ inside the disk. Equation (1.5) then becomes

$$
\frac{\partial^{2} H}{\partial \zeta^{2}}+\frac{H^{n}}{n^{n}}=\frac{1}{N^{2}} \frac{\partial}{\partial r}\left[r \frac{\partial(F-H)}{\partial r}\right]
$$

where the enthalphy, $H$, is given by

$$
H=n \rho^{1 / n}=F-\Phi .
$$

Our notation here might at first seem a little strange. In particular the specific enthalpy is normally referred to as $h$. To avoid confusion, however, we have chosen to use upper case lettering for the quantities describing the equilibrium structure and lower case lettering to represent the perturbations to it. The density and pressure will be the only exceptions.

We now consider the asymptotic expansions

$$
H=H_{1}+\frac{1}{N} H_{2}+\ldots, \Phi=N \Phi_{0}+\ldots \Phi_{1}+\ldots, F=N F_{0}+F_{1}+\ldots .
$$

The leading order terms in equations (1.12) and (1.11) give

$$
F_{0}=\Phi_{0}
$$

and

$$
\frac{\partial^{2} H_{1}}{\partial \zeta^{2}}+\frac{H_{1}^{n}}{n^{n}}=0
$$

The boundary conditions are that $H_{1}(r, 0)=H_{10}(r)$ and $\left(\partial H_{1} / \partial \zeta\right)_{\zeta=0}=0$, where $H_{10}$ is an arbitrary function whose importance in characterizing the structure of the disc shall become apparent. A first integral of equation (1.15) is then

$$
\left(\frac{\partial H_{1}}{\partial \zeta}\right)^{2}=\frac{2}{(n+1) n^{n}}\left(H_{10}^{n+1}-H_{1}^{n+1}\right)
$$

The solution of this equation can be obtained by elementary quadratures or it can be expressed as incomplete Beta-functions. It is convenient, however, to express it in terms of a function $C_{n}$ defined by

$$
x=\int_{C_{n}(x)}^{1} \frac{d s}{s^{\frac{n-1}{n}}\left(1-s^{\frac{n+1}{n}}\right)^{1 / 2}} .
$$

Then,

$$
\rho_{1}(r, \zeta)=\rho_{10}(r) C_{n}[\chi(r) \zeta]
$$

where

$$
\chi(r)=\left[\frac{2 n \rho_{10}^{\frac{n-1}{n}}}{n+1}\right]^{1 / 2}
$$

The value $x_{0}$ such that $C_{n}\left(x_{0}\right)=0$ is

$$
x_{0}=\int_{0}^{1} \frac{d s}{s^{\frac{n-1}{n}}\left(1-s^{\frac{n+1}{n}}\right)^{1 / 2}}=\left(\frac{n \pi^{1 / 2}}{n+1}\right) \frac{\Gamma\left(\frac{1}{n+1}\right)}{\Gamma\left(\frac{n+3}{2 n+2}\right)},
$$


The matching conditions then give

$$
F_{0}=\int_{0}^{\infty} \psi_{0}(k) J_{0}(k r) k d k
$$

and

$$
\left(\frac{2 n}{n+1}\right)^{1 / 2} \rho_{10}^{\frac{n+1}{2 n}}(r)=-\int_{0}^{\infty} k \psi_{0}(k) J_{0}(k r) k d k
$$

Eliminating the function $\psi_{0}$ from these equations leads to the integral equation relating $F_{0}(r)$ and $\rho_{10}(r)$,

$$
F_{0}=-\int_{0}^{1}\left(\frac{2 n}{n+1}\right)^{1 / 2} \rho_{10}^{\frac{n+1}{2 n}}(s) K(s, r) s d s
$$

where the kernel

$$
K(s, r)=\int_{0}^{\infty} J_{0}(k r) J_{0}(k s) d k
$$

is expressible as an elliptic integral. For future reference we also note that

$$
K(r, s)=\sum_{k=0}^{\infty}(2 k+1) \lambda_{k} P_{2 k}\left(\sqrt{1-r^{2}}\right) P_{2 k}\left(\sqrt{1-s^{2}}\right), \quad \lambda_{k}=\left[\frac{(2 k) !}{\left(2^{2 k} k !\right)^{2}}\right]^{2} \frac{\pi}{2},
$$

where the $P_{2 k}(x)$ are Legendre polynomials.

At leading order, then, we are left with only one independent arbitrary function, which may be either $\rho_{10}$ or $F_{0}$.

The surface density of the disc is

$$
\Sigma_{1}(r)=2 \int_{0}^{\Theta} \rho_{1}(r, \zeta) d \zeta=2\left[\frac{2 n}{n+1} \rho_{10}^{(n+1) / n}\right]
$$

which is just twice the function in the integrand of equation (1.31). We shall choose to use this quantity instead of $\rho_{10}$ to parameterize the radial structure of the disc.

\section{Some illustrative density and rotation laws}

The solution can be characterized by the surface density, $\Sigma_{1}$, and the function $F_{0}$ which are related by

$$
F_{0}(r)=-\frac{1}{2} \int_{0}^{1} \Sigma_{1}(s) K(r, s) s d s
$$

where the kernel, $K(s, r)$, is given by equation (1.32).

The models we shall consider here have the form

$$
\Sigma_{1}=2\left(\frac{2 n}{n+1}\right)^{1 / 2}\left(1-r^{2}\right)^{M+\frac{1}{2}},
$$

where $M$ is an integer. Near $r=0$ this form gives $\Sigma_{1} \sim 1-\left(M+\frac{1}{2}\right) r^{2}$ and so $M$ is a measure of the central concentration. For this surface density

$$
F_{0}(r)=-\left(\frac{\pi n}{n+1}\right)^{1 / 2} \frac{\Gamma\left(M+\frac{3}{2}\right)}{(M+1) !} \cdot 2 F_{1}\left(\frac{1}{2},-(M+1) ; 1 ; r^{2}\right)
$$

The surface density and rotation rate $\Omega_{0}=V / r$ for the indices $M=0,1,2,3,4,5$ and 10 are shown in figure 1. 
where $\Gamma$ is the Gamma function. Therefore the half thickness, $\Theta$ of the polytropic disk is

$$
\Theta(r)=\frac{\Gamma\left(\frac{1}{n+1}\right)}{\Gamma\left(\frac{n+3}{2 n+2}\right)}\left(\frac{\frac{1}{2} \pi n}{n+1}\right)^{1 / 2} \rho_{10}^{\frac{1-n}{2 n}}(r)
$$

Typically $\rho_{10}$ will decrease from the centre to the edge of the disc, and from equation (1.21) we see the curious shapes of polytropes of different indices. For $n=1(\Gamma=2)$, the models are discs of uniform thickness; for $n>1(\Gamma<2)$ they flare toward their edges, while for $n<1(\Gamma>2)$ they are lenticular.

\subsection{Matching to the external potential fleld}

To complete the solution to leading order we must consider the potential outside the polytrope, $\Phi^{\text {ext. }}$. This satisfies Laplace's equation

$$
\nabla^{2} \Phi^{e x t}=0
$$

The solution of this equation must match smoothly onto the potential in the interior, hence

$$
\Phi^{\text {ext }}\left(r, N^{-1} \Theta\right)=\Phi\left(r, N^{-1} \Theta(r)\right), \quad \Phi_{z}^{\text {ext }}\left(r, N^{-1} \Theta(r)\right)=\Phi_{z}\left(r, N^{-1} \Theta\right)
$$

where subscript $z$ denotes differentiation with respect to $z$.

The asymptotic sequences for the interior solution suggest that we seek further sequences of the form

$$
\Phi^{\text {ext }}=N \Phi_{0}^{\text {ext }}+\Phi_{1}^{\text {ext }}+\ldots, \quad \Theta=\Theta_{1}+\frac{1}{N} \Theta_{2}+\ldots,
$$

hence, at the upper edge of the disc,

$$
\begin{aligned}
& \Phi^{\text {ext }}\left(r, N^{-1} \Theta\right)=N \Phi_{0}^{\text {ext }}\left(r, 0^{+}\right)+\Theta_{1} \Phi_{0 z}^{\text {ext }}\left(r, 0^{+}\right)+\Phi_{1}^{\text {ext }}\left(r, 0^{+}\right)+\ldots \\
& \Phi^{\text {ext }}\left(r, N^{-1} \Theta\right)=N \Phi_{0}^{\text {ext }}\left(r, 0^{+}\right)+\Theta_{1} \Phi_{0 z z}^{\text {ext }}\left(r, 0^{+}\right)+\Phi_{1 z}^{\text {ext }}\left(r, 0^{+}\right)+\ldots
\end{aligned}
$$

The potential in the disk is given as a function of $\zeta$, and at $\zeta=0$, since $\partial / \partial z=N \partial / \partial \zeta$, we have

$$
\Phi=N \Phi_{0}\left(r, \Theta_{1}\right)+\Theta_{2} \Phi_{0 \zeta}\left(r, \Theta_{1}\right)+\Phi_{1}\left(r, \Theta_{1}\right)+\ldots
$$

and

$$
\Phi_{z}=N^{2} \Phi_{0 \zeta}\left(r, \Theta_{1}\right)+N \Theta_{2} \Phi_{0 \zeta \zeta}\left(r, \Theta_{1}\right)+N \Phi_{1 \zeta}\left(r, \Theta_{1}\right)+\ldots
$$

According to equation (1.14), we have $\Phi_{0}=F_{0}$, and so the leading terms of the matching give

$$
F_{0}(r)=\Phi^{\text {ext }}\left(r, 0^{+}\right), \quad \Phi_{1 \zeta}(r, \theta)=\Phi_{0 z}^{\text {ext }}\left(r, 0^{+}\right)
$$

To complete the matching we need only to solve equation (1.22) whose general axisymmetric solution for $z>0$,

$$
\Phi^{\mathrm{ext}}=\int_{0}^{\infty} \psi(k) e^{k z} J_{0}(k r) k d k
$$

where we may write the unknown function $\psi$ as

$$
\psi=N \psi_{0}+\psi_{1}+\ldots
$$


In figure 2 the shapes of the equilibrium configurations are illustrated for four different values of the polytropic index $(n=0.5,1,1.5$ and 3$)$ and for the model with the surface density index $M=2$. The edge evidently flares outwards to infinity for the models with the larger values of $n$ as the density in the midplane becomes vanishingly small. This irregularity occurs because towards the disc's outer edge, the radial derivatives in the Poisson equation (1.5) become large and so the asymptotic expansion breaks down. This indicates the presence of a boundary layer at the edge that, when treated correctly, will modify this singular behaviour. Nevertheless, to some extent the flare will still survive. An alternative method to avoid this flare is to recognize that outside the disc the density may not be zero, just very small. The edge then occurs at a finite density at which the flare is less pronounced. The external gravitational potential is still approximately given by equation (1.29), and so the explicit form of the solution is not appreciably modified. This procedure bears some resemblance to the "patching" technique of Monaghan and Roxburgh (1965).
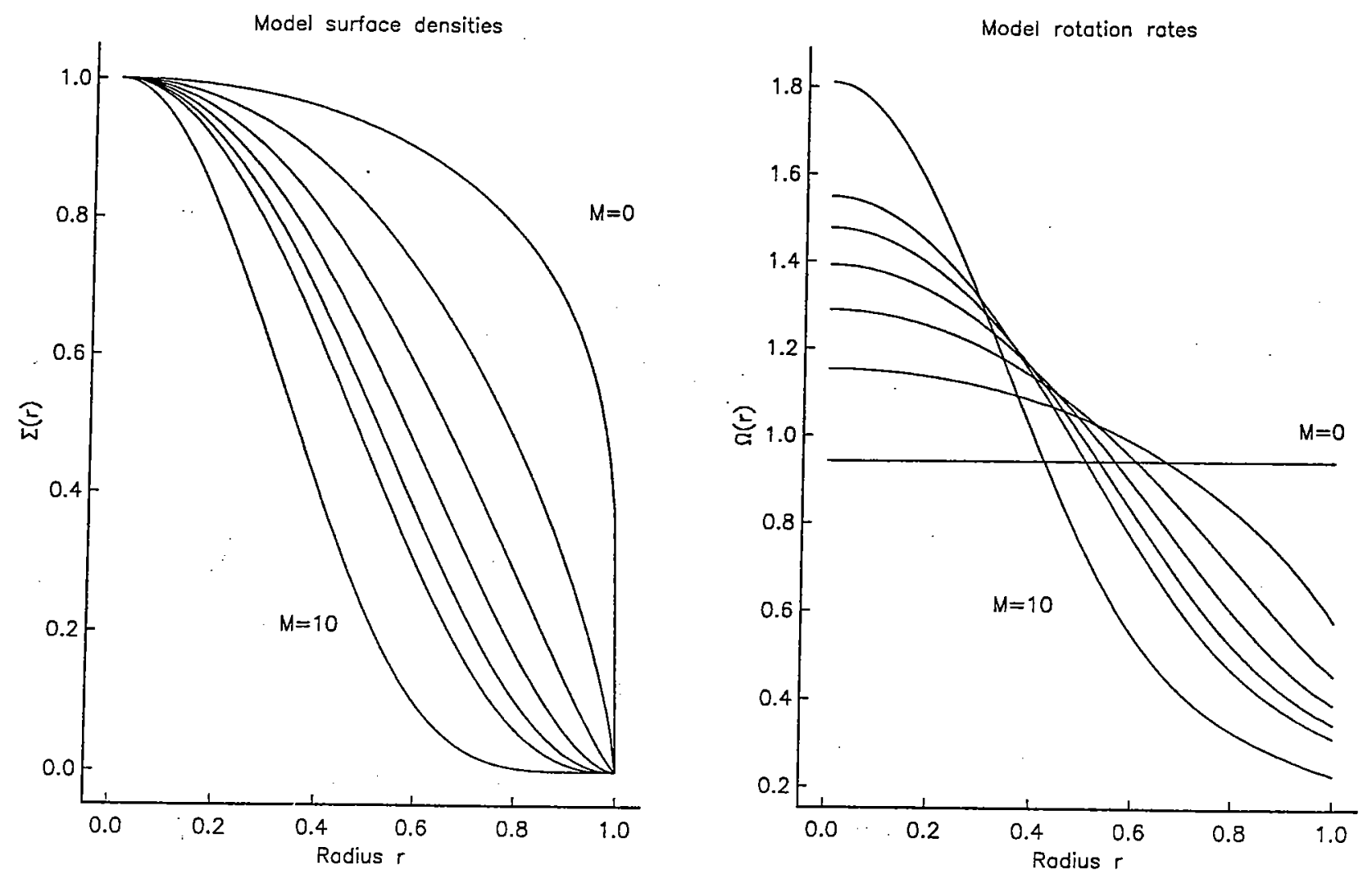

Figure 1: The surface density and rotation rate of polytropic models characterized by the index $M$ of the surface density law given by equation (1.37). The six curves correspond to the indices $M=0,1,2,3,4,5$ and 10 . Since the polytropic index $n$ appears only in a multiplicative factor in the surface density and rotation laws, the curves are exactly those for any polytropic index provided this factor is included. The curves are drawn for the case $n=1$. 
A very different way to avoid this behaviour is to introduce an external, halo potential of a parabolic form $\frac{1}{2} \alpha N^{2} \zeta^{2}$, where $\alpha$ is an arbitrary parameter (it is actually the ratio of the halo density to the central density of the disc), at the outset. This modifies the stratification from that predicted by equation (1.18) to

$$
\rho_{1}(r, \zeta)=\rho_{10}(r) S_{n}[\chi(r) \zeta ; \mu(r)]
$$

where the integral

$$
x=\int_{S_{n}(x ; \mu)}^{1} \frac{d s}{s^{\frac{n-1}{n}}\left[1-s^{\frac{n+1}{n}}+\mu\left(1-s^{\frac{1}{n}}\right)\right]^{1 / 2}}, \quad \mu(r)=\alpha\left[\frac{(n+1)}{\rho_{10}(r)}\right]^{1 / 2}
$$

defines the function $S_{n}(x ; \mu)$. This changes the shape of the polytrope and substantially reduces the flare. The dotted and dashed lines in figure 2 indicate the shape of the polytropes for the values of $\alpha$ of 0.1 and 1 .

Vertical structure of the $n=0.5$ polytrope Vertical structure of the $n=1$ polytrope
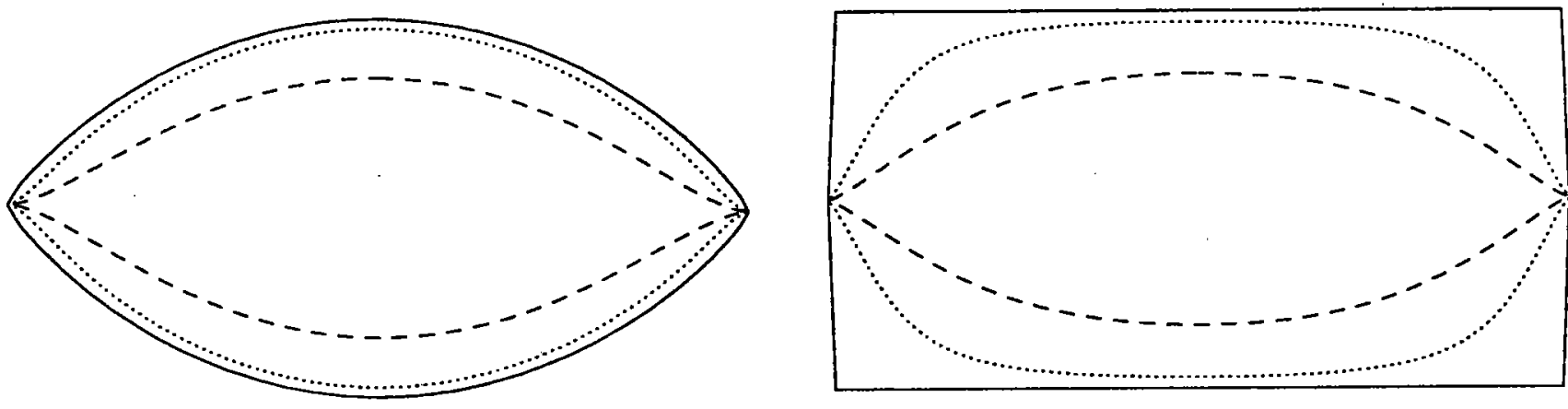

Vertical structure of the $n=1.5$ polytrope

$$
\text { Vertical structure of the } n=3 \text { polytrope }
$$
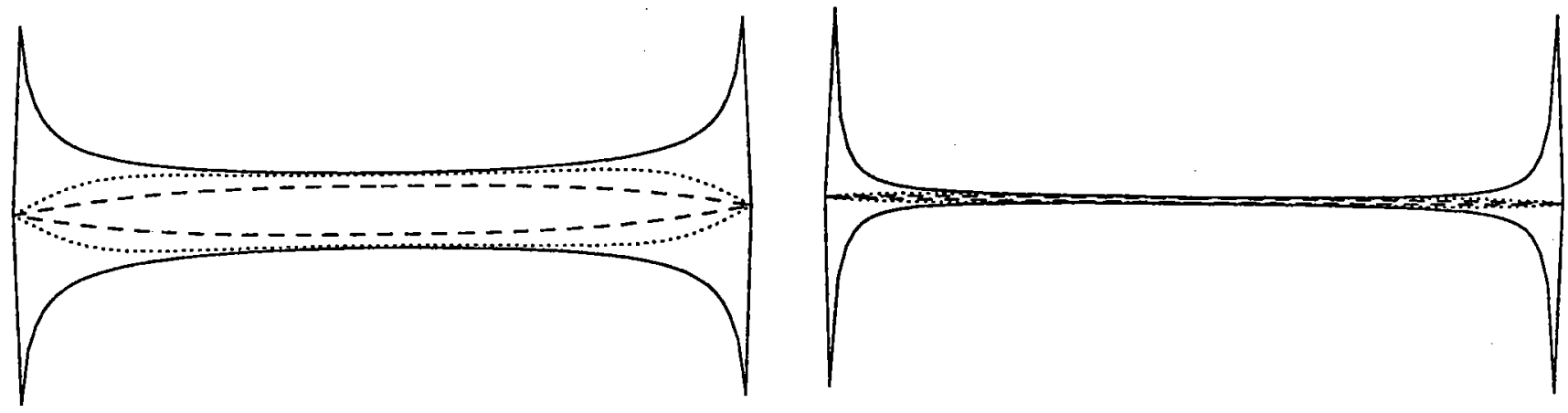

Figure 2: The shapes of the polytropes of index $n=0.5,1,1.5$ and 3 . The index of the surface density law is $M=2$. The dotted and dashed lines indicate the shapes when an external potential field $\frac{1}{2} \alpha N^{2} \zeta^{2}$ is also present. The dotted line has $\alpha=0.1$ whilst the dashed line is for $\alpha=1$. 


\section{LINEAR STABILITY ANALYSIS}

Spiral arms seem to be the manifestations of local enhancements of density and amplifications of star formation within the disc of the galaxy. They are not material arms since radial spokes of matter become very quickly sheared out and wound up on short timescales because of the relatively large shearing flow of rotation. Therefore they may be rotating patterns of gravitationally induced instabilities at finite amplitude. Such speculations have been asserted in the past, notably by Goldreich and Lynden-Bell (1965) in the context of gaseous discs, and by Shu (1970) in the theory of the stellar dynamics of sheets. Toomre (1969) has pointed out that such linear instabilities simply propagate towards the centre or edge of the disc and become anihilated very quickly. Here, however, we take the point of view that this may not happen at finite amplitude; nonlinear waves may form a steady rotating structure without dissipating. This view seems also to have been taken by Norman (1978), Qian, Spiegel and Proctor (1990) and Qian and Spiegel (1990).

\subsection{The equations in the limit of rapid rotation}

The linear, non-axisymmetric perturbations of the disc satisfy the equations of motion,

$$
\begin{gathered}
\left(\frac{\partial}{\partial t}+i m \Omega\right) u-2 \Omega v=-\frac{\partial}{\partial r}(\phi+h), \\
\left(\frac{\partial}{\partial t}+i m \Omega\right) v-2 B u=-\frac{i m}{r}(\phi+h)
\end{gathered}
$$

and

$$
\left(\frac{\partial}{\partial t}+i m \Omega\right) w=-\frac{\partial}{\partial z}(\phi+h)
$$

where the velocity of the perturbation is $(u, v, w)$, the fluctuations in the enthalpy and gravitational potential are $h$ and $\phi$ and the angular dependance $e^{i m \theta}$ has been assumed. The angular velocity of the mean undisturbed motion is $\Omega$ and

$$
B=\Omega+\frac{1}{2} r \frac{d \Omega}{d r}
$$

is one of Oort's constants.

The continuity equation is

$$
\left(\frac{\partial}{\partial t}+i m \Omega\right) \rho^{\prime}+\frac{1}{r} \frac{\partial}{\partial r}(r \rho u)+\frac{i m}{r} \rho v+\frac{\partial}{\partial z}(\rho w)=0
$$

and Poisson's equation becomes

$$
\frac{1}{r} \frac{\partial}{\partial r}\left(r \frac{\partial \phi}{\partial r}\right)-\frac{m^{2}}{r^{2}} \phi+\frac{\partial^{2} \phi}{\partial z^{2}}=N^{2} \rho^{\prime}
$$

The density fluctuation $\rho^{\prime}$ is related to the enthalpy perturbation by

$$
\left(\frac{H}{n}\right)^{n-1} h=\rho^{\prime} .
$$


The equations of motion (2.1) to (2.3) can be combined to yield

$$
\begin{gathered}
u=-\left[\left(\frac{\partial}{\partial t}+i m \Omega\right)^{2}+4 \Omega B\right]^{-1}\left[\left(\frac{\partial}{\partial t}+i m \Omega\right) \frac{\partial f}{\partial r}+\frac{2 i m \Omega}{r} f\right] \\
v=\left[\left(\frac{\partial}{\partial t}+i m \Omega\right)^{2}+4 \Omega B\right]^{-1}\left[2 B \frac{\partial f}{\partial r}-\frac{i m}{r}\left(\frac{\partial}{\partial t}+i m \Omega\right) f\right]
\end{gathered}
$$

and

$$
w=-\left(\frac{\partial}{\partial t}+i m \Omega\right)^{-1} \frac{\partial f}{\partial z}
$$

where $f=h+\phi$. When these equations are substituted into the continuity equation, this new equation and Poisson's equation become two coupled, second-order equations for the perturbations.

In accordance with the asymptotic solution of the equlibrium model, we again rescale the vertical coordinate $z=N \zeta$. Moreover, it is evident from the equations of motion (2.1) to (2.3) that, if the time derivatives are not to vanish at leading order, the characteristic timescale for the motions is just the rotation period, which is short in the limit of rapid rotation. Therefore we shall use the rescaled time $\tau=N^{1 / 2} t$.

We shall consider the normal modes of the disc, for which the dependance on time is of the form $e^{\eta \tau}$, where $\eta$ is the characteristic growth rate. The two equations determining the perturbations are then

$$
\partial_{\zeta}^{2} \phi-\left(\frac{H}{n}\right)^{n-1}(f-\phi)=-\frac{1}{N^{2}}\left[\frac{1}{r} \partial_{r}\left(r \partial_{r} \phi\right)-\frac{m^{2}}{r^{2}} \phi\right]
$$

and

$$
\begin{aligned}
& N^{2} \partial_{\zeta}\left(\rho \partial_{\zeta} f\right)=N \sigma^{2} \rho^{\prime}-\frac{\sigma}{r} \partial_{r}\left\{\frac{\rho r}{\kappa^{2}}\left[\sigma \partial_{r} f+\frac{2 i m\left(N^{-1 / 2} \Omega\right)}{r} f\right]\right\} \frac{i m \sigma \rho}{r \kappa^{2}}\left[2\left(N^{-1 / 2} B\right) \partial_{r} f-\frac{i m \sigma}{r} f\right] \\
& =N \sigma^{2} \rho^{\prime}-\frac{\rho \sigma^{2}}{\kappa^{2}}\left\{\frac{1}{r} \partial_{r}\left(r \partial_{r} f\right)+\partial_{r}\left(\log \frac{\rho}{\kappa^{2}}\right) \partial_{r} f+\left[\frac{2 i m(N-1 / 2 \Omega)}{r \sigma} \partial_{r}\left(\log \frac{\rho \Omega}{\kappa^{2}}\right)-\frac{m^{2}}{r^{2}}\right] f\right\},(2.1
\end{aligned}
$$

where

$$
\sigma=\eta+i m N^{-1 / 2} \Omega \text {. }
$$

If $\eta$ were purely imaginary, the motion would have the charactistic epicyclic frequency $i \kappa$, with

$$
\kappa^{2}=\sigma^{2}+4 N^{-1} \Omega B=\gamma+\Delta,
$$

where $\Delta$ is what could be described as the Rayleigh discriminant of the mean flow of rotation. Provided $\eta$ is real, $\kappa$ does not in general vanish exactly.

\subsection{Asymptotic expansion and solution}

The asymptotic development of the variables into a series follows simply from that used for the equilibrium state: we set

$$
\phi=N \phi_{0}+\phi_{1}+\ldots \quad f=N f_{0}+f_{1}+\ldots
$$


for the perturbations,

$$
\eta=\eta_{1}+N^{-1} \eta_{2}+\ldots, \quad \gamma=\gamma_{1}+N^{-1} \gamma_{2}+\ldots
$$

for the characteristic growth rate and its square, and

$$
\Omega=N^{1 / 2}\left(\Omega_{0}+N^{-1} \Omega_{1}+\ldots\right), \quad \sigma=\sigma_{1}+N^{-1} \sigma_{2}+\ldots
$$

and

$$
\kappa=\kappa_{1}+N^{-1} \kappa_{2}+\ldots, \quad \Delta=4 N^{-1} \Omega B=\Delta_{1}+N^{-1} \Delta_{2}+\ldots
$$

for the other subsidiary variables.

If we introduce these expansions into the equations (2.11) and (2.12) then the leading order equations are trivially satisfied by

$$
f_{0}=f_{0}(r) \quad \phi_{0}=\phi_{0}(r) \quad \phi_{0}=f_{0} .
$$

At next order equation (2.12) gives

$$
\partial_{\zeta}\left(\rho_{1} \partial_{\zeta} f_{1}\right)=0
$$

which has the integral $\rho_{1} \partial_{\zeta} f_{1}=$ constant. However, $\rho_{1}$ vanishes at the edge of the disc, and so if $f_{1}$ is bounded, then

$$
f_{1}=f_{1}(r) \text {. }
$$

The equation of the corresponding order from equation (2.11) gives

$$
\partial_{\zeta}^{2} h_{1}+\left(H_{1} / n\right)^{n-1} h_{1}=0
$$

This equation can be reduced to quadrature. For the moment, however, we shall ignore the vertical structure of the disturbance since we are more interested in its linear stability.

The $O(N)$ terms of equation (2.12) give

$$
\partial_{\zeta}\left(\rho_{1} \partial_{\zeta} f_{2}\right)=\sigma_{1}^{2} \rho_{1}^{\prime}-\frac{\sigma_{1}^{2}}{r}\left\{\partial_{r}\left(\frac{r \rho_{1}}{\kappa_{1}^{2}} \partial_{r} f_{0}\right)+\left[\frac{2 i m}{\sigma_{1}} \partial_{r}\left(\frac{\Omega_{0} \rho_{1}}{\kappa_{1}^{2}}\right)-\rho_{1} \frac{m^{2}}{\kappa_{1}^{2} r}\right] f_{0}\right\},
$$

Integrating this equation vertically, from the centre to the edge of the disc gives,

$$
\left[\rho_{1} \partial_{\zeta} f_{2}\right]_{0}^{\Theta_{1}}=\sigma_{1}^{2} \Sigma_{1}^{\prime}-\frac{\sigma_{1}^{2}}{r}\left\{\partial_{r}\left(\frac{r \Sigma_{1}}{\kappa_{1}^{2}} \partial_{r} f_{0}\right)+\left[\frac{2 i m}{\sigma_{1}} \partial_{r}\left(\frac{\Omega_{0} \Sigma_{1}}{\kappa_{1}^{2}}\right)-\Sigma_{1} \frac{m^{2}}{\kappa_{1}^{2} r}\right] f_{0}\right\},
$$

where $\Sigma_{1}$ is once more the surface density of the disc, and

$$
\Sigma_{1}^{\prime}=2 \int_{0}^{\Theta_{1}} \rho_{1}^{\prime} d \zeta
$$

is the perturbation induced in it.

A thin layer of fluid, symmetric about the plane $z=0$, can support motions that are either symmetric or asymmetric about this plane. The latter are sinuous modes and correspond to corrugations of the layer. A simple corrugation does not lead to a local enhancement in the density, and consequently the destabilizing effect of self-gravity is smaller for these modes than 
for the symmetric or varicose modes. If we particularize to the varicose modes, then $\partial_{\zeta} f_{2}=0$ at $\zeta=0$, and so the left hand side of equation (2.22) vanishes. Thus

$$
\Sigma_{1}^{\prime}=\frac{1}{r} \partial_{r}\left(\frac{r \Sigma_{1}}{\kappa_{1}^{2}} \partial_{r} f_{0}\right)+\left[\frac{2 i m}{\sigma_{1} r} \partial_{r}\left(\frac{\Omega_{0} \Sigma_{1}}{\kappa_{1}^{2}}\right)-\Sigma_{1} \frac{m^{2}}{\kappa_{1}^{2} r^{2}}\right] f_{0}
$$

Moreover, integrating equation (2.18) over the vertical extent of the disc gives

$$
\left[\partial_{\zeta} \phi_{1}\right]_{\zeta=\Theta_{1}}=\frac{1}{2} \Sigma_{1}^{\prime}
$$

These two equations, together with the formula derived from the boundary conditions upon the perturbed gravitational potential (see the following section), constitute an eigenvalue problem for $\sigma_{1}$, with the eigenfunction $f_{0}(r)$.

We can derive analogous equations to (2.23) and (2.24) for the terms of $O(1)$. These give the $O\left(N^{-1}\right)$ corrections to the eigenvalues. Explicitly they are

$$
\begin{gathered}
\Sigma_{2}^{\prime}=\frac{1}{r} \partial_{r}\left[\left(\frac{\Sigma_{2}}{\Sigma_{1}}+\frac{\partial_{r} f_{1}}{\partial_{r} f_{0}}-\frac{\gamma_{2}+\Delta_{2}}{\kappa_{1}^{2}}\right) \frac{r \Sigma_{1} \partial_{r}}{\kappa_{1}^{2}} f_{0}\right] \\
+\frac{2 i m \Omega_{0}}{\sigma_{1} r}\left\{\left(\frac{f_{1}}{f_{0}}-\frac{\sigma_{2}}{\sigma_{1}}+\frac{\Omega_{1}}{\Omega_{0}}\right) \partial_{r}\left(\frac{\Sigma_{1} \Omega_{0}}{\kappa_{1}^{2}}\right)+\partial_{r}\left[\left(\frac{\Sigma_{1} \Omega_{0}}{\kappa_{1}^{2}}\right)\left(\frac{\Sigma_{2}}{\Sigma_{1}}+\frac{\Omega_{1}}{\Omega_{0}}-\frac{\gamma_{2}+\Delta_{2}}{\kappa_{1}^{2}}\right)\right]\right\} \\
-\frac{m^{2} \Sigma_{1}}{r^{2} \kappa_{1}^{2}}\left(\frac{f_{1}}{f_{0}}+\frac{\Sigma_{2}}{\Sigma_{1}}-\frac{\gamma_{2}+\Delta_{2}}{\kappa_{1}^{2}}\right),
\end{gathered}
$$

and

$$
\left[\partial_{\zeta} \phi_{2}\right]_{\zeta=\Theta_{1}}=\frac{1}{2} \Sigma_{2}^{\prime}-\frac{\Theta_{1}}{r} \partial_{r}\left(r \partial_{r} f_{0}\right)
$$

\subsection{Matching the solution}

Outside the disc the perturbation to the gravitational potential $\phi^{\text {ext }}$ is once more a solution to Laplace's equation:

$$
\phi^{e x t}=\int_{0}^{\infty} \psi^{\prime}(k) J_{m}(k r) e^{-k z+i m \theta} k d k .
$$

To match this solution to the perturbation inside the disc we develop the function $\psi^{\prime}$ into a series of powers of $N^{-1}$, just as in the case of the equilibrium model. The continuity of the potential perturbation and its derivative then yields

$$
f_{0}=\int_{0}^{\infty} \psi_{0}^{\prime}(k) J_{m}(k r) k d k
$$

and

$$
\left[\partial_{\zeta} \phi_{1}\right]_{\zeta=\Theta_{1}}=-\int_{0}^{\infty} \psi_{0}^{\prime}(k) J_{m}(k r) k^{2} d k
$$

at leading order.

Eliminating $\psi_{0}^{\prime}(k)$ results in the equation

$$
f_{0}(r)=-\int_{0}^{\infty}\left[\partial_{\zeta} \phi_{1}\right]_{\zeta=\Theta_{1}} K_{m}(r, s) s d s
$$


where the kernel is now

$$
K_{m}(s, r)=\int_{0}^{\infty} J_{m}(k r) J_{m}(k s) d k .
$$

At the following order, we have

$$
\begin{gathered}
f_{1}(r)=-\int_{0}^{1}\left[\partial_{r} \phi_{2}\right]_{\zeta=\Theta_{1}} K_{m}(s, r) s d s \\
+\frac{1}{2}\left[\Theta_{1} \Sigma_{1}^{\prime}-\int_{0}^{1} s d s \int_{0}^{1} t d t \Sigma_{1}^{\prime}(t) \Theta_{1}(s) K_{m}(s, r) \int_{0}^{\infty} k^{2} d k J_{m}(k t) J_{m}(k s)\right] .
\end{gathered}
$$

If $\Theta_{1}$ were independent of $s$ (as it is in the case $n=1$ ), then the final term in square brackets would vanish exactly.

\subsection{The eigenvalue problem}

Equations (2.23), (2.24) and (2.28) can now be combined to give an integral equation for $f_{0}$ :

$$
f_{0}=-\frac{1}{2} \int_{0}^{1} \Sigma_{1}^{\prime} K_{m}(s, r) s d s
$$

or

$$
f_{0}=-\int_{0}^{1}\left\{\frac{1}{s} \partial_{s}\left[\frac{s \Sigma_{1} \partial_{s} f_{0}}{2 \kappa_{1}^{2}}\right]+\left[\frac{i m}{s \sigma_{1}} \partial_{s}\left(\frac{\Omega_{0} \Sigma_{1}}{\kappa_{1}^{2}}\right)-\frac{m^{2} \Sigma_{1}}{2 s^{2} \kappa_{1}^{2}}\right] f_{0}\right\} K_{m}(s, r) s d s .
$$

These equations are clearly analogous to equation (1.55) of the static problem, and reflect the leading order balance between the galactic centrifuge and the self gravity of the configuration.

Equation (2.33) is an integral, eigenvalue equation. The eigenvalue $\eta_{1}$ is explicitly contained in $\sigma_{1}$ and $\kappa_{1}$, and $f_{0}$ is the eigenfunction. These correspond to the varicose normal modes of the disc.

In principle, the $O\left(N^{-1}\right)$ corrections to the eigenvalue and eigenfuction $\left(\gamma_{2}\right.$ and $f_{1}$, respectively) can be derived from equation (2.30). In practice, however, it is difficult to treat such a complicated equation for general polytropic index and nonaxisymmetric disturbances. The final term on the right hand side appears to be an unnecessary complication: It vanishes for $n=1$ and must be small for high-order modes (for which $\Theta_{1} \simeq$ constant). If we assume that it is negligible, that equation becomes

$$
f_{1}(r)=-\frac{1}{2} \int_{0}^{1} \Sigma_{2}^{\prime}(s) K_{m}(s, r) s d s+\int_{0}^{1} \frac{\Theta_{1}(s)}{s} \partial_{s}\left(s \partial_{s} f_{0}\right) K_{m}(r, s) s d s
$$

with equation (2.25) determining the $O\left(N^{-1}\right)$ correction to the perturbation to the surface density $\Sigma_{2}^{\prime}$.

\subsection{Axisymmetric modes}

If we particularize to the axisymmetric case, $m=0$ and equation (2.30) becomes somewhat easier to analyse. We have

$$
f_{0}(r)=-\int_{0}^{1} \partial_{s}\left[\frac{s \Sigma_{1} \partial_{s} f_{0}}{2 \kappa_{1}^{2}}\right] K_{0}(s, r) d s
$$


where $K_{0}(s, r)$ is identical to the kernel considered in the static model. This kernel can be decomposed into an infinite sum of Legendre polynomials and so

$$
f_{0}(r)=-\sum_{k=0}^{\infty} \lambda_{k}(4 k+1) P_{2 k}\left(\sqrt{1-r^{2}}\right) \int_{0}^{1} \partial_{s}\left[\frac{s \Sigma_{1} \partial_{s} f_{0}}{2 \kappa_{1}^{2}}\right] P_{2 k}\left(\sqrt{1-s^{2}}\right) d s
$$

In addition

$$
\kappa_{1}^{2}=\eta_{1}^{2}+4 \Omega_{0} B_{0}=\gamma_{1}+\Delta_{1}
$$

If we define the function

$$
g_{1}(r)=\frac{\left(1-r^{2}\right)^{1 / 2} \partial_{r} f_{0}(r)}{\gamma_{1}+\Delta_{1}(r)}
$$

and change variables to $x=\left(1-r^{2}\right)^{1 / 2}$ and $y=\left(1-s^{2}\right)^{1 / 2}$, then the eigenvalue equation can be written in the more obvious form,

$$
\left[\gamma_{1}+\delta_{1}(x)\right] G_{1}(x)=\sum_{k=0}^{\infty} \lambda_{k}(4 k+1) P_{2 k}^{1}(x) \int_{0}^{1} \frac{\Xi(y)}{2 y} G_{1}(y) P_{2 k}^{1}(y) d y
$$

where $\Xi(y)=\Sigma(s), G_{1}(x)=g_{1}(r), \delta(x)=\Delta(r)$ and $P_{2 k}^{1}(x)$ is an associated Legendre polynomial.

In the previous section, a selection of equilibrium models were computed with the surface density law $\Sigma_{1}(r)=\left(1-r^{2}\right)^{M+1 / 2}$. If we introduce this parameterization of $\Sigma_{1}$ into equation (2.38) then the eigenvalue equation equation becomes

$$
\left[\gamma_{1}+\delta_{1}(x)\right] G_{1}(x)=\sum_{k=0}^{\infty} \lambda_{k}(4 k+1) P_{2 k}^{1}(x) \int_{0}^{1} y^{2 M} G_{1}(y) P_{2 k}^{1}(y) d y
$$

If we further decompose the functions $G_{1}(x)$ and $\delta_{1}(x)$ into a sum over the even associated Legendre polynomials,

$$
G_{1}(x)=\sum_{j=0}^{\infty} G_{1, j} P_{2 j}^{1}(x), \quad \delta_{1}(x)=\sum_{j=0}^{\infty} \delta_{1, j} P_{2 j}^{1}(x),
$$

then equation (2.34) is reduced to an infinite-dimensional matrix eigenvalue problem:

$$
\begin{gathered}
\gamma_{1} \sum_{k=0}^{\infty} G_{1, k} P_{2 k}^{1}(x)+\sum_{i=0}^{\infty} \sum_{j=0}^{\infty} G_{1, i} \delta_{1, j} P_{2 i}^{1}(x) P_{2 j}^{1}(x)= \\
\sum_{k=0}^{\infty} \lambda_{k}(4 k+1) P_{2 k}^{1}(x) \sum_{j=0}^{\infty} G_{1, j} \int_{0}^{1} y^{2 M} P_{2 j}^{1}(y) P_{2 k}^{1}(y) d y
\end{gathered}
$$

Setting $P_{2 i}^{1}(x) P_{2 j}^{1}(x)=\sum_{k=0}^{\infty} \alpha_{i j k} P_{2 k}^{1}(x)$, and comparing the coefficients of $P_{2 k}^{1}(x)$ yields

$$
\gamma_{1} G_{1, k}+\sum_{i=0}^{\infty} \sum_{j=0}^{\infty} \alpha_{i j k} G_{1, j} \delta_{1, i}=\sum_{j=0}^{\infty} \lambda_{k}(4 k+1) G_{1, j} \int_{0}^{1} y^{2 M} P_{2 j}^{1}(y) P_{2 k}^{1}(y) d y .
$$

This is an equation that is of the form

$$
\gamma_{1} \mathbf{G}_{1}=A_{1} \mathbf{G}_{1}
$$


where

$$
A_{1, k j}=\lambda_{k}(4 k+1) \int_{0}^{1} y^{2 M} P_{2 j}^{1}(y) P_{2 k}^{1}(y) d y-\sum_{i=0}^{\infty} \alpha_{i j k} \delta_{1, i}
$$

( $\alpha_{i j k}$ can be seen to be the third rank tensor that describes the coupling, in the presence of a mean differential rotation, between the eigenfunctions of a uniformly rotating disc.)

This final equation can be solved explicitly in the case of the rigid rotator $(M=0)$, and this is detailed in the following section. It can also be solved numerically.

When $m=0$, the $O\left(N^{-1}\right)$ corrections determined by equation (2.34) satisfy the simpler equation

$$
\begin{gathered}
f_{1}(r)=-\int_{0}^{1}\left\{\partial_{s}\left(\frac{s \Sigma_{1}}{2 \kappa_{1}^{2}} \partial_{s} f_{1}\right)+\partial_{s}\left[\left(\frac{\Sigma_{2}}{\Sigma_{1}}-\frac{\gamma_{2}+\Delta_{2}}{\kappa_{1}^{2}}\right) \frac{s \Sigma_{1}}{2 \kappa_{1}^{2}} \partial_{s} f_{0}\right]\right\} K(s, r) d s \\
+\int_{0}^{1} \Theta_{1} \partial_{s}\left(s \partial_{s} f_{0}\right) K(s, r) d s .
\end{gathered}
$$

The correction to the surface density $\Sigma_{2}(r)$ is determined by specifying the equilibrium configuration. If there are to be no corrections to the surface density law $\Sigma=\left(1-r^{2}\right)^{M+1 / 2}$ at $O\left(N^{-1}\right)$, then this function is identically zero. Thus

$$
\begin{gathered}
f_{1}(r)=-\int_{0}^{1} \partial_{s}\left(\frac{s \Sigma_{1}}{2 \kappa_{1}^{2}} \partial_{s} f_{1}\right) K(s, r) d s+\int_{0}^{1} \partial_{s}\left[\left(\frac{\gamma_{2}+\Delta_{2}}{\kappa_{1}^{4}}\right) \frac{s \Sigma_{1}}{2} \partial_{s} f_{0}\right] K(s, r) d s \\
+\int_{0}^{1} \Theta_{1} \partial_{s}\left(s \partial_{s} f_{0}\right) K(s, r) d s .
\end{gathered}
$$

Just as for the leading order equation, we shall define the function

$$
G_{2}(x)=g_{2}(r)=\frac{\left(1-r^{2}\right)^{1 / 2} \partial_{r} f_{1}(r)}{\gamma_{1}+\Delta_{1}(r)}
$$

Then equation (2.46) becomes

$$
\begin{gathered}
\left(\gamma_{1}+\delta_{1}\right) G_{2}(x)=\sum_{k=0}^{\infty} \lambda_{k}(4 k+1) P_{2 k}^{1}(x) \\
\left\{\int_{0}^{1} y^{2 M} G_{2}(y) P_{2 k}^{1}(y) d y-\int_{0}^{1}\left[y^{2 M}\left(\frac{\gamma_{2}+\delta_{2}}{\gamma_{1}+\delta_{1}}\right)+\frac{\theta_{1}}{y}\left(\gamma_{1}+\delta_{1}\right)\right] G_{1}(y) P_{2 k}^{1}(y) d y\right\},
\end{gathered}
$$

with $\theta_{1}(y)=\Theta_{1}(s)$. Decomposing the functions $G_{1}, G_{2}$ and the $\delta_{1}$ on the left hand side of equation (2.48) into sums of the $P_{2 i}^{1}(x)$ gives

$$
\left(\gamma_{1}-A_{1}\right) \mathbf{G}_{2}=\left(A_{2}+\gamma_{2} B\right) \mathbf{G}_{1}
$$

where

$$
A_{2, k j}=\lambda_{k}(4 k+1) \int_{0}^{1}\left[\frac{\delta_{2} y^{2 M}}{\gamma_{1}+\delta_{1}}+\frac{\theta_{1}}{y}\left(\gamma_{1}+\delta_{1}\right)\right] P_{2 j}^{1}(y) P_{2 k}^{1}(y) d y
$$

and

$$
B_{k j}=\lambda_{k}(4 k+1) \int_{0}^{1} \frac{y^{2 M}}{\gamma_{1}+\delta_{1}} P_{2 k}^{1}(y) P_{2 j}^{1}(y) d y
$$


The matrix $A_{1}$ can be symmetrized by using the vector $G_{1, i}^{\prime}=\sqrt{(4 k+1) \lambda_{k}} G_{1, i}$ instead of $G_{1, i}$ as teh eigenfunction. Therefore there must exist a vector $\mathbf{G}_{1}^{*}$ that satisfies the equation

$$
\left(\mathrm{G}_{1}^{*}\right)^{T} A_{1}=\left(\mathrm{G}_{1}^{*}\right)^{T} \gamma_{1}
$$

Taking the product of this vector with equation (2.49) gives an expression for the $O\left(N^{-1}\right)$ correction to the eigenvalue

$$
\gamma_{2}=-\frac{\left(\mathbf{G}_{1}^{*}\right)^{T} A_{2} \mathbf{G}_{1}}{\left(\mathbf{G}_{1}^{*}\right)^{T} B \mathbf{G}_{1}}
$$

In fact, since there is an infinite spectrum of these eigenvalues, there is an infinite set of equations (2.51).

\subsection{Axisymmetric instabilities of the rigid rotator}

In the case of a rigidly rotating disc, $M=0$ and $\delta_{1}=\pi$. In this case the eigenvalue equation (2.43) becomes

$$
\begin{gathered}
\left(\gamma_{1}+\pi\right) G_{1, k}=\sum_{j=0}^{\infty} \lambda_{k}(4 k+1) G_{1, j} \int_{0}^{1} P_{2 j}^{1}(y) P_{2 k}^{1}(y) d y \\
=\lambda_{k} 2 k(2 k+1) G_{1, k}
\end{gathered}
$$

Thus the $\mathrm{k}^{\text {th }}$ eigenvalue is

$$
\gamma_{1, k}=\eta_{1, k}^{2}=\pi\left\{2 k(2 k+1)\left[\frac{(2 k) !}{\left(2^{k} k !\right)^{2}}\right]^{2}-1\right\}
$$

This eigenvalue increases monotonically with $k$, and so it appears that, at this order, there is no high wavenumber cut-off to the range of unstable modes. This is not true of the Jean's instabilities of an infinite, plane, polytropic slab of gas (Ledoux, 1951; Qian, Spiegel and Proctor, 1990). Therefore, it is clear that there must be some stabilizing effect that enters into the equations at $O\left(N^{-1}\right)$ and eventually, for large enough $k$, dominates the instability determined at first order to create a finite range of unstable wavenumbers. This is physically plausible since at next order the effects of pressure support must enter, and these oppose a local collapse of the disc.

The eigenfunctions of the uniformily rotating disc are simply the associated Legendre polynomials $P_{2 k}^{1}\left(\sqrt{1-r^{2}}\right)$. The $k=1$ solution is a toroidal perturbation of the disc, whilst higher order solutions will look like concentric annuli of finite thickness. For asymptotically large $k$, the eigenfunction is

$$
G_{k} \sim\left(\frac{4 k}{\pi r}\right)^{1 / 2} \cos \left(2 k \sin ^{-1} r+\pi / 4\right)
$$

well away from $r=0$. As the order increases, the amplitude of the mode becomes concentrated to the centre of the disc; towards the edge, it oscillates strongly. Some eigenfunctions are sketched in figure 3. Equation (2.51) determines the $O\left(N^{-1}\right)$ correction to $\gamma_{1}$. For the rigid rotator this equation becomes

$$
\gamma_{2, k}=-\frac{(4 k+1)\left(\gamma_{1, k}+\pi\right)^{2}}{2 k(2 k+1)} \int_{0}^{1}\left[\frac{\theta_{1}(y)}{y}+\frac{\delta_{2}}{\left(\gamma_{1}+\delta_{1}\right)^{2}}\right] P_{2 k}^{1}(y) P_{2 k}^{1}(y) d y
$$

for the correction to the $i^{\text {th }}$ eigenvalue. 


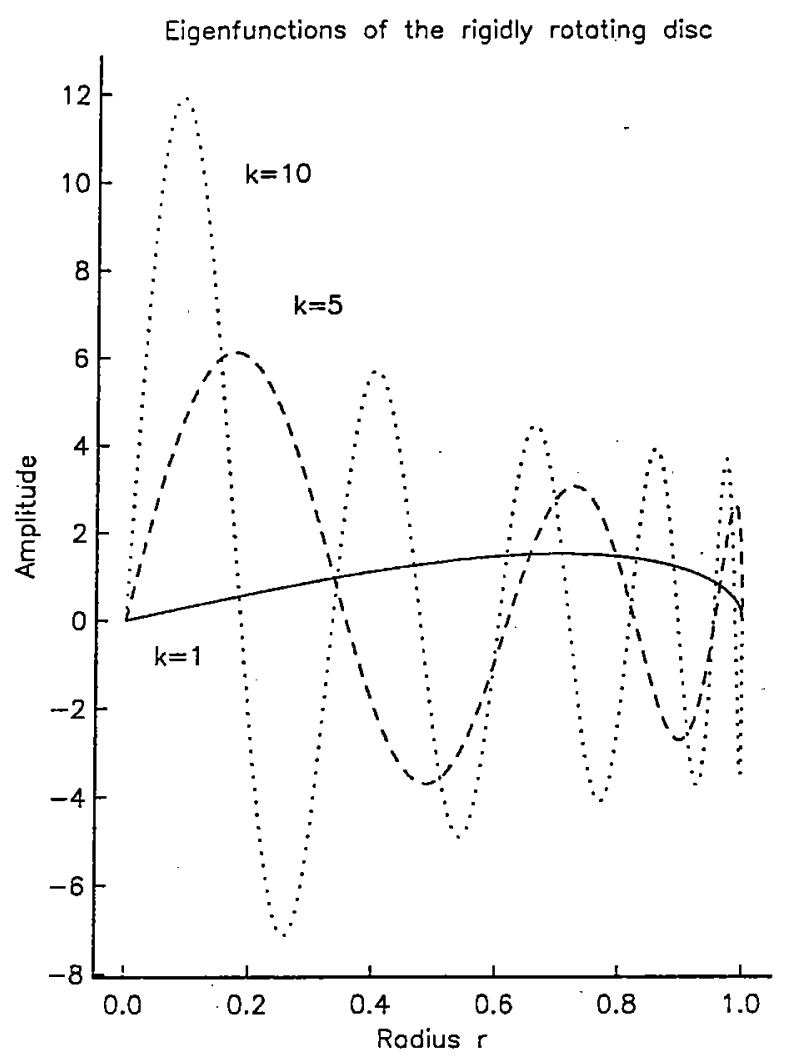

Figure 3: The axisymmetric eigenfunctions or ring modes of the rigidly rotating disc. In particular the modes of radial order $k=1,5$ and 10 are shown.

The function $\delta_{2}(x)$ can be expressed as a sum of the basis functions $P_{2 k}^{1}(x)$. Nevertheless, when $\gamma_{1}$ is large $(k>>1)$ it is a small correction. If we ignore it, then

$$
\gamma_{2, k} \simeq-\frac{(4 k+1)\left(\gamma_{1, k}+\pi\right)^{2}}{2 k(2 k+1)} \int_{0}^{1} \frac{\theta_{1}(y)}{y} P_{2 k}^{1}(y) P_{2 k}^{1}(y) d y
$$

For the polytrope with $n=1, \theta=\pi / 2$ and so

$$
\gamma_{2, k} \simeq-\frac{(4 k+1)\left(\gamma_{1, k}+\pi\right)^{2}}{2 k(2 k+1)} \frac{\pi}{2} I_{k}
$$

where

$$
I_{k}=\int_{0}^{1} P_{2 k}^{1}(y) P_{2 k}^{1}(y) \frac{d y}{y}=2\left[\frac{(2 k-1) ! !}{2^{k} k !}\right]^{2} \sum_{j=1}^{\infty} \sum_{i=1}^{k} \frac{(-k)_{j}(-k)_{i}\left(k+\frac{1}{2}\right)_{j}\left(k+\frac{1}{2}\right)_{i}}{(i+j-1)(i+j)\left(\frac{1}{2}\right)_{j}\left(\frac{1}{2}\right)_{i}(i-1) !(j-1) !}
$$

and $(2 k-1) ! !=(2 k-1)(2 k-3) \ldots 3.1$.

The lowest eigenvalues, to $O\left(N^{-2}\right)$, for various values of the large parameter $N$ are plotted in figure 4 for the polytrope with this value of $n$. It is clear that as the rotation of the disc grows 
it becomes increasingly susceptible to instabilities. This is not because rotation promotes the instability, but rather that as the rotation increases, the effects of pressure support which provide the stabilizing influence become more and more negligible.

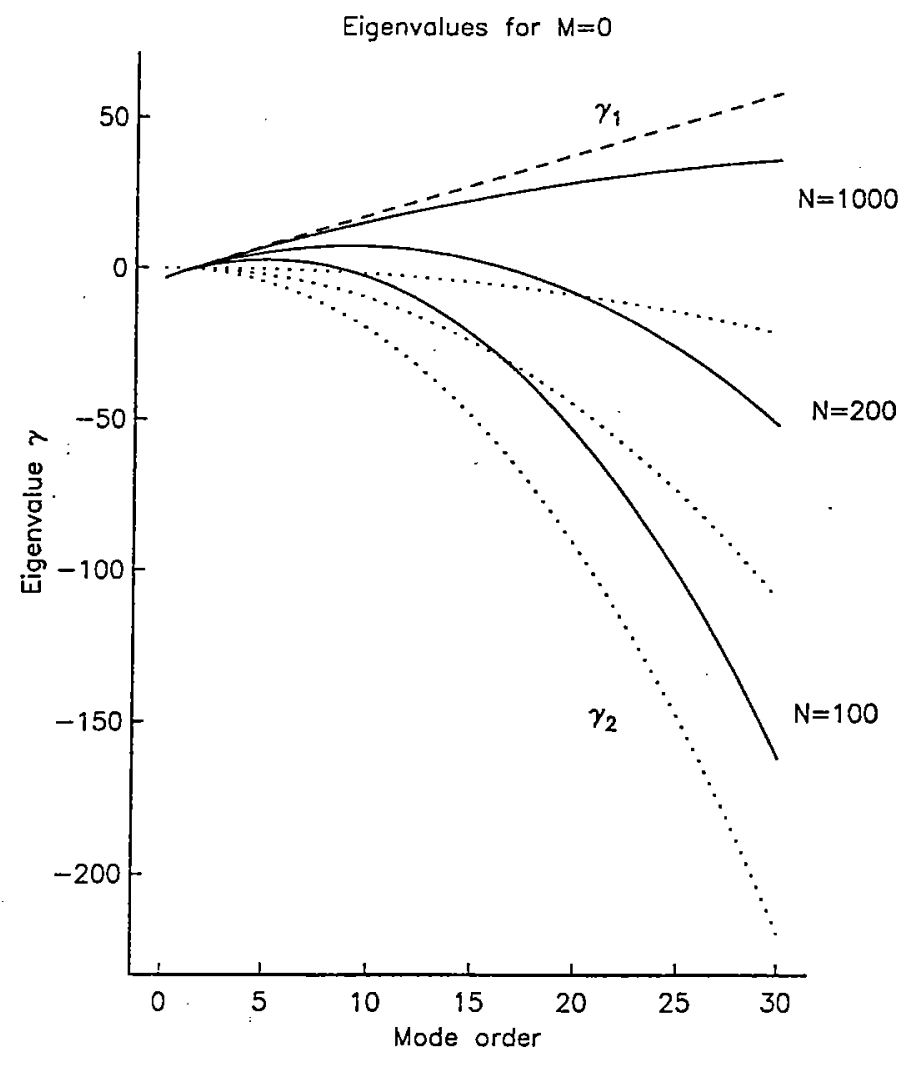

Figure 4: The axisymmetric eigenvalues for the rigid rotator. The eigenvalue to first order, $\gamma_{1}$, is indicated by the dashed line and the $O\left(N^{-1}\right)$ correction to it, $\gamma_{2}$, by the dotted lines for three values of $N$. The solid line is the eigenvalue correct to $O\left(N^{-2}\right)$, i.e. $\gamma_{1}+N^{-1} \gamma_{2}$.

\subsection{Nonaxisymmetric Instabilities}

For the nonaxisymmetric modes, the analysis presented above becomes more complicated. In particular, the kernel $K_{m}(r, s)$ no longer has the eigenvectors $P_{2 n}(x)$. Instead we have

$$
K_{m}(r, s)=\sum_{n=0}^{\infty} \lambda_{n}^{m} \varphi_{n}^{m}(x) \varphi_{n}^{m}(y)
$$

where the orthonormal eigenvectors

$$
\varphi_{n}^{m}(x)=\Gamma\left(m+\frac{1}{2}\right)\left[\frac{(2 n) !}{\pi} \frac{\left(n+m+\frac{1}{2}\right)}{\Gamma(2 n+2 m+1)}\right]^{1 / 2}\left(1-x^{2}\right)^{m / 2} C_{2 n}^{m+1 / 2}(x)
$$


and

$$
\lambda_{n}^{m}=\frac{2}{(2 n) !} \Gamma(2 n+2 m+1)\left[\frac{\Gamma\left(n+\frac{1}{2}\right)}{\Gamma(n+m+1)}\right]^{2} .
$$

The functions $C_{n}^{m}(x)$ in equation (2.59) are Gegenbauer or ultraspherical polynomials of index $m$ and order $n$ (e.g. Magnus, Oberhettinger and Soni, 1966).

The construction of the matrix eigenvalue equation now proceeds as before. For the rigidly rotating disc the matrix is once more diagonal indicating that the eigenfunctions, $G_{k}^{m}$, are just the functions $\varphi_{k}^{m}(x)$. The dispersion relation is now more complicated. It reduces to a pair of cubic equations for every $\sigma_{1, k}$, and each cubic in the pair corresponds to one of the two possible choices for $m$, namely $\pm|m|$. The solutions to these cubics are

$$
\begin{gathered}
\sigma_{1, k}^{(1)}= \pm \frac{2 i}{\sqrt{3}} \sqrt{\Psi(m, k)-\pi} \sinh \beta, \\
\sigma_{1, k}^{(2,3)}= \pm \sqrt{\Psi(m, k)-\pi} \cosh \beta\left[1 \pm \frac{i}{\sqrt{3}} \tanh \beta\right],
\end{gathered}
$$

where

$$
\Psi(m, k)=2 \frac{(2 k+2 m) !}{(2 k) !}\left[\frac{\Gamma\left(k+\frac{1}{2}\right)}{(k+m) !}\right]^{2}[2 k(2 k+2 m+1)+m]
$$

and

$$
\beta=\frac{1}{3} \tanh ^{-1}\left[\frac{\lambda_{k}^{m} m \Omega_{0}}{\sqrt{(\Psi-\pi)^{3} / 27+\left(\lambda_{k}^{m} m \Omega_{0}\right)^{2}}}\right] .
$$

When $[(\Psi-\pi) / 3]^{3 / 2}>>\left|\lambda_{k}^{m} m \Omega_{0}\right|$, these become

$$
\begin{gathered}
\sigma_{1, k}^{(1)} \simeq \pm \frac{2 i \lambda_{k}^{m} m \Omega_{0}}{\sqrt{3}(\Psi-\pi)} \\
\sigma_{1, k}^{(2,3)} \simeq \pm \sqrt{\Psi-\pi}\left[1 \pm \frac{i \lambda_{k}^{m} m \Omega_{0}}{\sqrt{3}(\Psi-\pi)^{3 / 2}}\right] .
\end{gathered}
$$

These is related to the growth rates by

$$
\eta_{1, k}^{(i)}=\sigma_{1, k}^{(i)}-i m \Omega_{0}
$$

The modes now oscillate in time in addition to the exponential growth or decay. This oscillation is caused in part by the simple precession of the mode (as indicated by the second term in equation [2.66]) and also because of the azimuthal motion itself (which leads to the imaginary parts of equation [2.65]).

The presence of six eigenvalues is not inconsistent with the results of the axisymmetric case in which we have noted only one eigenvalue. For the $m=0$ case the eigenvalue that we have plotted in figure 4 is actually a pair: $\sigma_{k}= \pm\left|\gamma_{k}\right|^{1 / 2}= \pm|\Psi(m=0, k)-\pi|^{1 / 2}$. In addition, we have omitted a possible solution $\gamma=0$. In actual fact all three of these eigenvalues are doubly degenerate, and when $m \neq 0$ all six modes appear with nontrivial time-dependence. This is shown in figure 5 . 


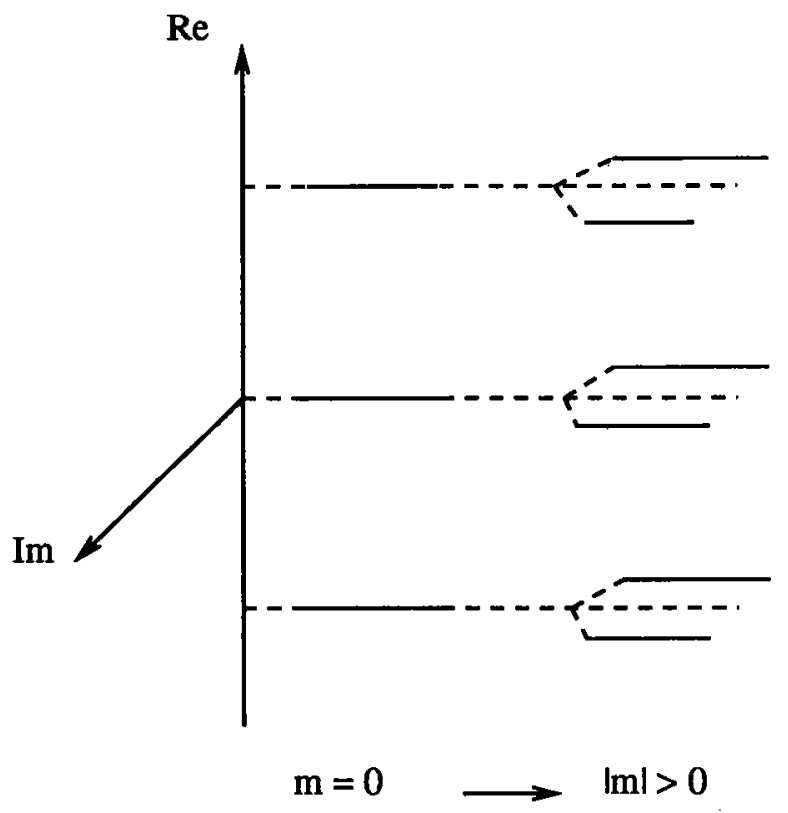

Figure 5: An illustration showing the double degeneracy of the eigenvalues for $m=0$, and how this is lifted when $m \neq 0$. The picture is an "energy level diagram" showing the real and imaginary parts of $\sigma_{1, k}$ for the six modes.

\section{CONCLUSIONS}

We have constructed models of polytropic gaseous masses using an asymptotic technique in which the disparity of the vertical and horizontal length scales led to a characteristic, large parameter that was exploited to develop the asymptotically valid solution. These models have intriguing shapes and vary substantially with the polytropic index.

Our analysis of the linear stability of the normal, varicose modes of the disc takes account of the global nature of the disturbances, instead of treating the perturbation purely locally. Moreover, by constructing the equilibrium structure initially, the linear stability analysis is completely internally consistent.

It is clear that we have uncovered a wide range of instabilities in the rotating disc. Fortunately, the disc is probably not catastrophically unstable, and the stabilizing influence of pressure support destroys the instability quickly at moderate wavenumber, provided the rotation is not excessively large. For the Milky Way, with $N \sim 20$, only the lowest order modes may be unstable.

The ring instabilities found here may not be purely mathematical artifacts: some galaxies do indeed possess ring-like structures, suggesting, perhaps, the presence, at finite amplitude, of these $m=0$ modes.

The detailed analysis of the stability of the non-axisymmetric modes is currently underway. Perhaps this will shed light on the selection mechanism behind spiral structure.

Acknowledgements: I would like to thank Prof. E. A. Spiegel without whom this project would have been unsurmountably difficult. I would also like to thank the fellowship committee for an enjoyable summer at Woods Hole. 


\section{REFERENCES}

1. Goldreich, P., and Lynden-Bell, D. (1965). Mon. Not. R.A.S., 130, 125.

2. Howard, L.N., and Spiegel, E.A. (1970). Unpublished manuscript.

3. Jeans, J.H. (1928). Astronomy and Cosmogony (Cambridge university press).

4. Ledoux, P. (1951). Annales d'Astrophys., 14, 438.

5. Magnus, W., Oberhettinger, F., and Soni, R.P. (1966). Formulas and theorems for the special functions of mathematical physics (Springer-Verlag, New York).

6. Monaghan, J.J., and Roxburgh, I.W. (1965). Mon. Not. R.A.S., 131, 13.

7. Norman, C.A. (1978). Mon. Not. R.A.S., 182, 457.

8. Qian, Z., and Spiegel, E.A. (1990). Preprint.

9. Qian, Z., Spiegel, E.A., and Proctor, M.R.E. (1990). Instability and Applied Analysis of Continuous Media, 1 , in press.

10. Shu, F. (1970). Ap. J., 160, 99.

11. Toomre, A. (1969). Ap. J., 158, 899. 


\title{
The Nonlinear Evolution of a
}

\section{Perturbed Axisymmetric Eddy}

\author{
George I. Bell \\ Woods Hole Oceanographic Institute \\ Woods Hole, MA 02543
}

\section{Introduction}

Numerical simulations of two dimensional and geostrophic turbulence (Babiano et al. 1986; McWilliams, 1984) demonstrate that an initially turbulent vorticity distribution evolves into one where the vorticity is concentrated into approximately circular patches surrounded by regions where the vorticity distribution is filamentary and on average an order of magnitude lower. Robust eddies, relatively isolated from the surrounding fluid, are also commonly observed in geophysical flows (eg. Gulf Stream rings), as well as laboratory experiments.

The problem of the linear and nonlinear evolution of a nearly axisymmetric eddy is therefore of considerable interest. This research is motivated, in part, by the numerical simulations of Carton \& McWilliams (1989). In the context of a two layer model, they considered an initially axisymmetric eddy with potential vorticity in the i'th layer, $q_{i}$, a given function of radius. The evolution of a slightly perturbed and linearly unstable eddy was calculated using a pseudospectral numerical code. They found that the instability was often nonlinearly stabilized at finite amplitude, and that the eddy appeared to undergo a complicated oscillation about a nonlinear, steadily rotating eddy which was approximately elliptical. The suggestion that the nonlinear dynamics of a perturbed axisymmetric eddy are quite complex, possibly even chaotic, is the driving force for the present research.

Before we embark on our search for chaos we should remark about the recent discovery of Polvani \& Wisdom (1990) about one type of chaos which occurs in the vicinity of a Kida vortex. A Kida vortex is simply an ellipse, filled with a uniform distribution of vorticity and placed in a background strain field. When the strain is zero, the ellipse rotates uniformly, a fact that was discovered by Kirchoff (1876). 
When a straining flow is added, Kida (1981) showed that the problem is still solvable exactly. For small strain rates the ellipse merely oscillates periodically. Although the flow at any fixed point is also periodic, particle trajectories outside the ellipse may be chaotic (Polvani \& Wisdom, 1990). This type of chaos has been termed chaotic advection (Aref, 1984), or Lagrangian chaos. In contrast, our search is for a vortex whose actual dynamics are chaotic. By this we mean that the ellipticity of the vortex (or some measure of its degree of distortion from the axisymmetric state) varies chaotically with time. Since the ellipticity of any stable Kida vortex is a periodic function of time, clearly Kida vortices do not have chaotic dynamics.

In Section 2, we introduce the simple two-contour eddy, and in Section 3 calculate it's linear stability properties. Finite amplitude behavior is calculated using a contour dynamics code (courtesy of Steven Meacham). In Section 4 it is demonstrated that an eddy which begins in a linearly unstable region of parameter space either breaks up catastrophically or equilibrates. In Section 5 we document the behavior of large amplitude perturbations to a linearly stable eddy, and by focussing in on a specific example we demonstrate that it is possible to duplicate the nonlinear interactions using a four degree of freedom Hamiltonian system. This reduced Hamiltonian system appears to show chaotic behavior in this parameter regime. The implications of this work are presented in Section 6, together with the suggestion that by uncovering the Hamiltonian structure of contour dynamics it may be possible to derive the nonlinear theory directly from the contour dynamics algorithm.

\section{The two-contour eddy}

The problem may be simplified considerably by supposing that the distribution of vorticity with radius is piecewise constant. The geometry of our model is depicted in Figure 1. For the unperturbed problem, let the potential vorticity be $q_{1}+q_{2}$ inside an inner circle of radius $r_{1}, q_{2}$ inside an outer circle of radius $r_{2}$, and zero outside the outer circle. The reason for including two circles, or contours, is that it is the simplest model which allows for linear instability.

Flierl (1988) showed that a barotropic two-contour eddy with $q>0$ everywhere is always stable to elliptical $(m=2)$ perturbations. This suggests that a two layer model is necessary for the complicated dynamics observed by Carton and McWilliams (1989) near an unstable $m=2$ mode. However, we can avoid the complication of a two layer model by allowing the lower layer to be infinitely deep and stationary (a so called equivalent barotropic model). The reason for using such a model, as we shall see, is that the elliptical mode can be unstable (even when $q>0$ everywhere).

If the circular contour $r=r_{i}$ is perturbed into $r(\theta, t)=r_{i}+\eta_{i}(\theta, t)$, then the 


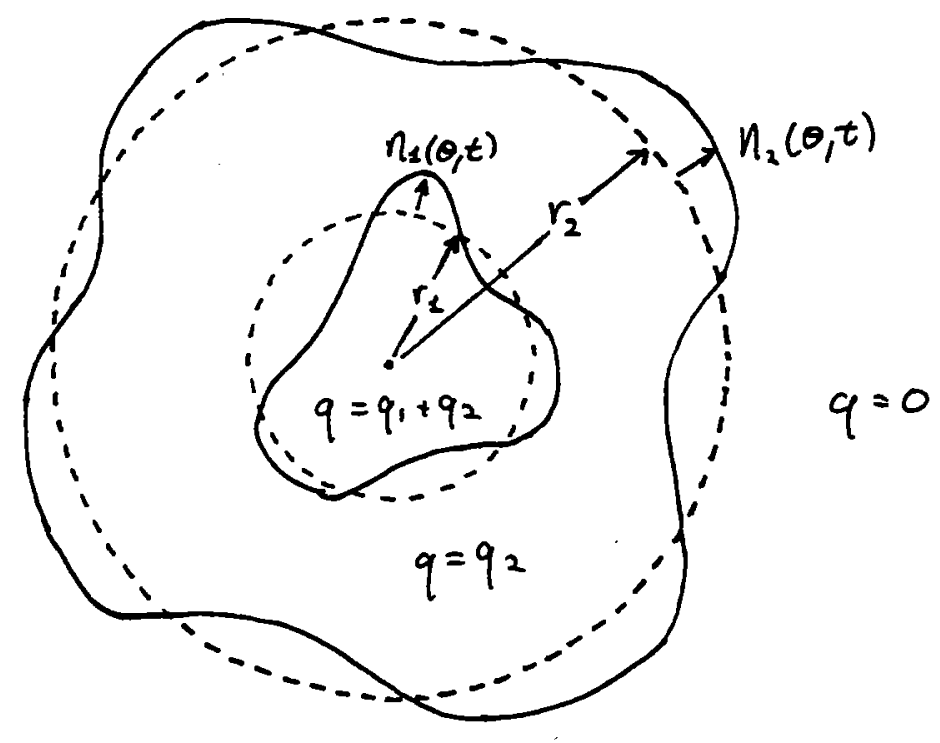

Figure 1: The perturbed two-contour eddy.

stream function $\psi$ for the flow in the upper layer is given by

$$
\mathcal{L} \psi=\sum_{j=1,2} q_{j} \Theta\left(r_{j}+\eta_{j}(\theta, t)-r\right)
$$

where $\Theta$ is the Heaviside step function and $\mathcal{L} \equiv \nabla^{2}-1$ is the operator connecting the stream function and potential vorticity distribution. Equation (1) is in nondimensional form, where time is measured in units of $q^{-1}$ and distance is measured in units of Rossby deformation radius.

The motion of each contour is specified by the kinematic condition

$$
\frac{\partial \eta_{j}}{\partial t}=-\frac{1}{r_{j}+\eta_{j}} \frac{d}{d \theta} \psi\left(r_{j}+\eta_{j}(\theta, t), \theta, t\right)
$$

which specifies that the contour $\eta_{j}$ moves inward or outward in response to the radial fluid velocity at its location.

\section{Linear theory}

The linear theory for problems of this type has been performed for similar specific cases (Childress 1984; Flierl 1988). The perspective presented below was chosen because it extracts the mathematical structure of the general case (with $n$ 
potential vorticity interfaces), and contains most previous results as special cases involving other choices for the operator $\mathcal{L}$.

We suppose first that the perturbation is small (compared to $r_{j}$ )

$$
\eta_{j}(\theta, t)=O(\epsilon)
$$

this implies by (1) that $\psi$ may be written as

$$
\psi=\psi_{0}(r)+\epsilon \psi_{1}(r, \theta, t)
$$

Substitution of (3) and (4) into (1) and (5) leads to.

$$
\begin{gathered}
\mathcal{L} \psi_{0}=\sum_{j=1,2} q_{j} \Theta\left(r_{j}-r\right) \\
\mathcal{L} \psi_{1}=\sum_{j=1,2} q_{j} \eta_{j} \delta\left(r_{j}-r\right) \\
r_{j} \frac{\partial \eta_{j}}{\partial t}=-\frac{\partial \psi_{1}}{\partial \theta}\left(r_{j}\right)-\frac{\partial \eta_{j}}{\partial \theta} \frac{\partial \psi_{0}}{\partial r}\left(r_{j}\right)
\end{gathered}
$$

A further reduction is achieved by Fourier transforming $\eta_{j}$ in azimuthal angle, in other words looking for normal modes

$$
\eta_{j}(\theta, t)=B_{j} e^{i m(\theta-\omega t)}
$$

The unperturbed azimuthal velocity $V_{0}=\partial \psi_{0} / \partial r$ and perturbed stream function $\psi_{1}$ can then be written in terms of similar operators:

$$
\begin{aligned}
& \left(\mathcal{L}-\frac{1}{r^{2}}\right) V_{0}=-\sum_{j=1,2} q_{j} \delta\left(r_{j}-r\right) \\
& \left(\mathcal{L}-\frac{m^{2}}{r^{2}}\right) \psi_{1}=\sum_{j=1,2} q_{j} \eta_{j} \delta\left(r_{j}-r\right)
\end{aligned}
$$

while the kinematic condition (7) reduces to

$$
\omega r_{j} \eta_{j}=\psi_{1}\left(r_{j}\right)+\eta_{j} V_{0}\left(r_{j}\right)
$$

Let $G_{m}\left(r_{j}, r\right)$ be the solution to

$$
\left(\mathcal{L}-\frac{m^{2}}{r^{2}}\right) G_{m}\left(r_{j}, r\right)=\delta\left(r_{j}-r\right)
$$


From the form of equations (9) and (10), we can see that $V_{0}$ and $\psi_{1}$ can be represented as sums over the Green's functions $G_{m}$. Substituting expressions for $V_{0}$ and $\psi_{1}$ in terms of the Green's functions into (11) yields an eigenvalue problem for the frequency $\omega$. The possible values of $\omega$ are the eigenvalues of the matrix $M$, where the elements of $M$ are defined by

$$
M_{i j}=\frac{\delta_{i j}}{r_{i}} \underbrace{\left[\sum_{j=1,2} q_{j} G_{1}\left(r_{j}, r_{i}\right)\right]}_{V_{0}\left(r_{i}\right)}-\frac{1}{r_{i}} \sum_{j=1,2} q_{j} G_{m}\left(r_{j}, r_{i}\right)
$$

Note that for the equivalent barotropic case we are considering, we have

$$
G_{m}(a, b)= \begin{cases}-a I_{m}(b) K_{m}(a) & \text { if } b<a \\ -a I_{m}(a) K_{m}(b) & \text { if } b \geq a\end{cases}
$$

where $I_{m}$ and $K_{m}$ are modified Bessel functions of order $m$. Because the matrix $M$ defined in (13) is 2 by 2 , it is easy to write down a criterion for the stability of a particular azimuthal perturbation.

Figure 2 shows the regions of linear instability for azimuthal modes $m=2,3$ and 4. Higher modes have similar instability regions which pile up against the line $r_{2}=1=r_{1}$. Perturbations in the $m=0$ mode are not allowed because they change the area enclosed by one of the contours, violating vorticity conservation. The $m=1$ mode is equivalent to a displacement of one of the initial circles, is always linearly stable here and gives rise to translations of the eddy (see Flierl 1984). If both of the potential vorticity jumps have the same sign, or $q_{2} / q_{1}>0$, then a form of the well known Rayleigh stability criterion may be invoked to verify that the perturbation is neutrally stable (we'll not discuss the dynamics of such eddies further).

One interesting aspect of Figure 2 is that there are large regions of parameter space where the eddy is linearly stable to all modes. This is rather surprising if one considers the analogous planar problem-a barotropic shear layer with nonzero vorticity within a strip $-d<y<d$. Such a shear layer is always unstable to a perturbation of wavenumber $O(1 / d)$. In the limit where $r_{2}$ is near $r_{1}$, we would expect that the curvature of the two interfaces is negligible compared with $\left(r_{2}-r_{1}\right)^{-1}$ and the planar shear layer would be approximated. From Figure 2, we can see that in this region, the eddy is likely to be unstable to a perturbation with a large $m$, confirming our intuition that it must always be unstable. As $r_{2}$ increases, it is the quantization of $m$ that leads to the linearly stable regions. For large $d$, unstable waves in the shear layer have very long wavelengths and an integral number of wavelengths do not fit around the perimeter of the circular eddy. This explains why the eddy is linearly stable to all modes when $r_{2}$ is large enough. 


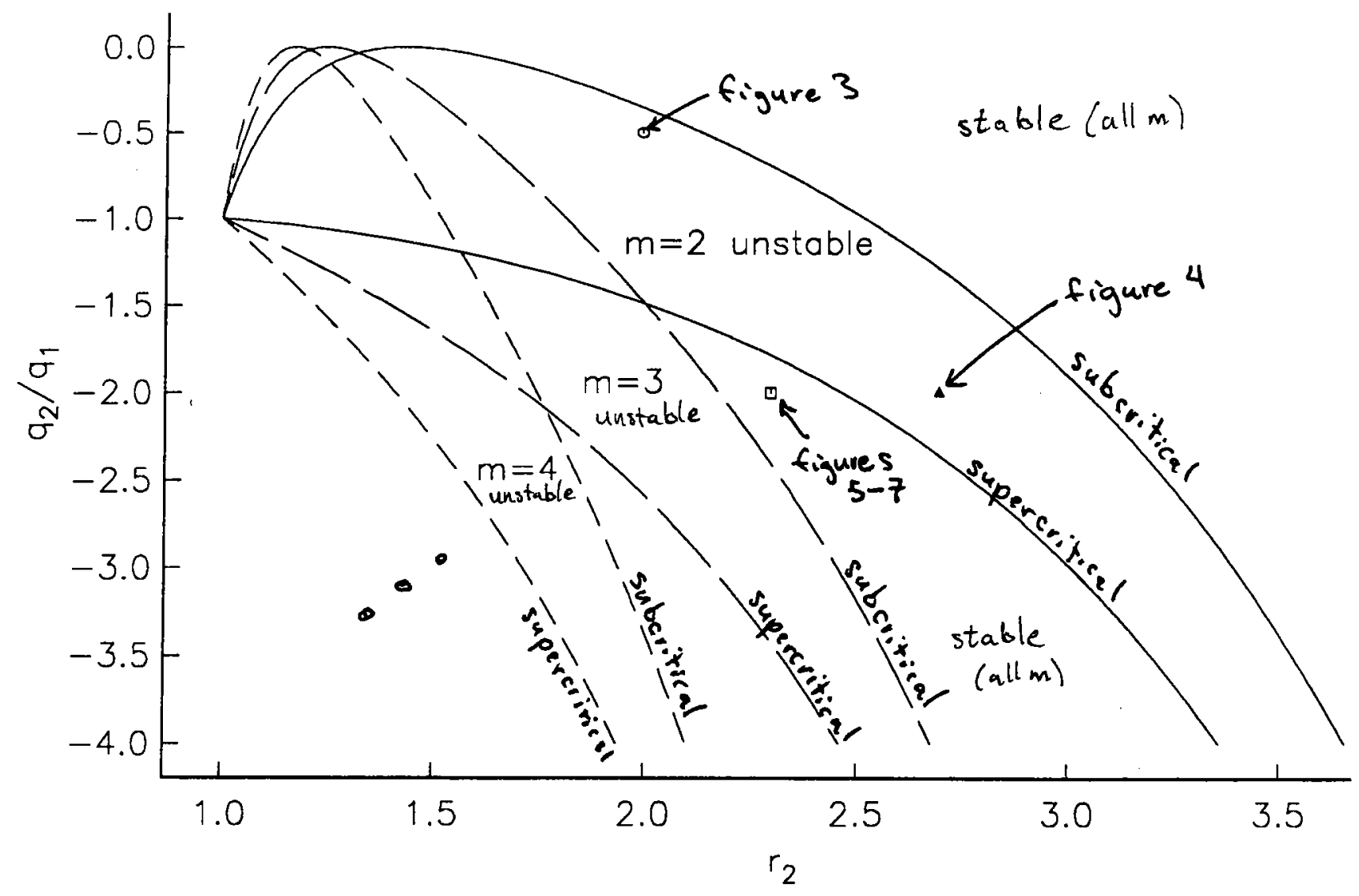

Figure 2: Regions of linear instability for the perturbed two-contour eddy $\left(r_{1}=1\right)$.

\section{Finite amplitude behavior in the region of lin- ear instability}

The weakly nonlinear calculations of Flierl (1984), for the barotropic case, indicate the type of bifurcation that occurs when the parameter $\boldsymbol{r}_{2}$ moves from a stable region into an unstable region. Although Flierl proved the result for the barotropic case, and only at specific points in parameter space, from simulations of the full equations of motion (2) and (1) using the method of contour dynamics, the following hypothesis may be drawn. If the instability region is entered by an increase of $r_{2}$ (and $\left.\left|q_{2}\right|>\left|q_{1}\right|\right)$, then the bifurcation is supercritical, and the solution oscillates around a finite amplitude, steadily rotating solution. On the other hand, if the instability region is entered by decreasing $r_{2}$, then the bifurcation is subcritical, and the weak nonlinearity further destabilizes the eddy.

What happens when an eddy lying well inside one of the unstable regions is perturbed? It turns out that there are two basic types of scenario, depending on whether $\left|q_{2}\right|>\left|q_{1}\right|$ or $\left|q_{2}\right|<\left|q_{1}\right|$. A hint regarding the nonlinear development comes 
from the conservation of angular momentum. In terms of the potential vorticity $q$, conservation of angular momentum is equivalent to the statement that (1/2) $q r^{2}$ integrated over the potential vorticity distribution is constant in time. In other words,

$$
\frac{1}{2} \sum_{j=1,2} q_{j} \int_{0}^{2 \pi} \int_{r_{j}}^{r_{j}+\eta_{j}} r^{2} r d r d \theta=\frac{1}{8} \sum_{j=1,2} q_{j} \int_{0}^{2 \pi}\left(r_{j}+\eta_{j}\right)^{4}-r_{j}^{4} d \theta=c
$$

The idea is that for large deformations which have grown from small perturbations the integrals in (15) are positive, while $c$ itself is small. In order that the sum in (15) nearly cancel, it is necessary that the deformation of the interface with the smaller value of $\left|q_{i}\right|$ be greater. If the linearizing assumption of small $\eta_{j}$ is made together with the decomposition into normal modes (8), equation (15) may be rewritten

$$
e^{2 m \omega_{i} t} \sum_{j=1,2} q_{j} r_{j}^{2}\left|B_{j}\right|^{2}=c^{\prime}
$$

where $\omega_{i}$ is the imaginary part of the frequency $\omega$. If the mode is linearly unstable then the left hand side of (16) will grow exponentially unless the expression in the sum is identically zero. Evidently, $c^{\prime}=0$ and

$$
\frac{\left|r_{1} B_{1}\right|}{\left|r_{2} B_{2}\right|}=\sqrt{-\frac{q_{2}}{q_{1}}}
$$

Equation (17) is an exact identity satisfied by any linearly unstable mode, and specifies that the perturbation of the weaker vorticity interface is proportionately larger.

Dritschel (1988) has extended (17) into the nonlinear regime by looking at a linear combination of the conserved quantities angular momentum and area. Essentially, he is able to derive a conserved quantity similar to (15) where the integrand is positive definite in each $\eta_{j}$. His result demonstrates that even in a nonlinear sense, the deformation of the weaker contour must be greater than the deformation of the stronger contour.

\subsection{Break up of a two-signed eddy}

Unstable eddies with $\left|q_{2}\right|<\left|q_{1}\right|$ are composed of potential vorticity of both signs, and the potential vorticity jump across the outer contour is weaker. In the linearly unstable region of parameter space, by (17) we expect that the perturbation in the outer contour will be of larger amplitude than the inner, and will filament first. This is confirmed by a full numerical simulation (Figure 3), showing the break up of an eddy into a tripole-like structure. For some recent experimental results on the formation of tripoles and dipoles from nearly axisymmetric vorticity distributions, the reader is referred to the fascinating photos in Kloosterziel \& Van Heijst (1989). 

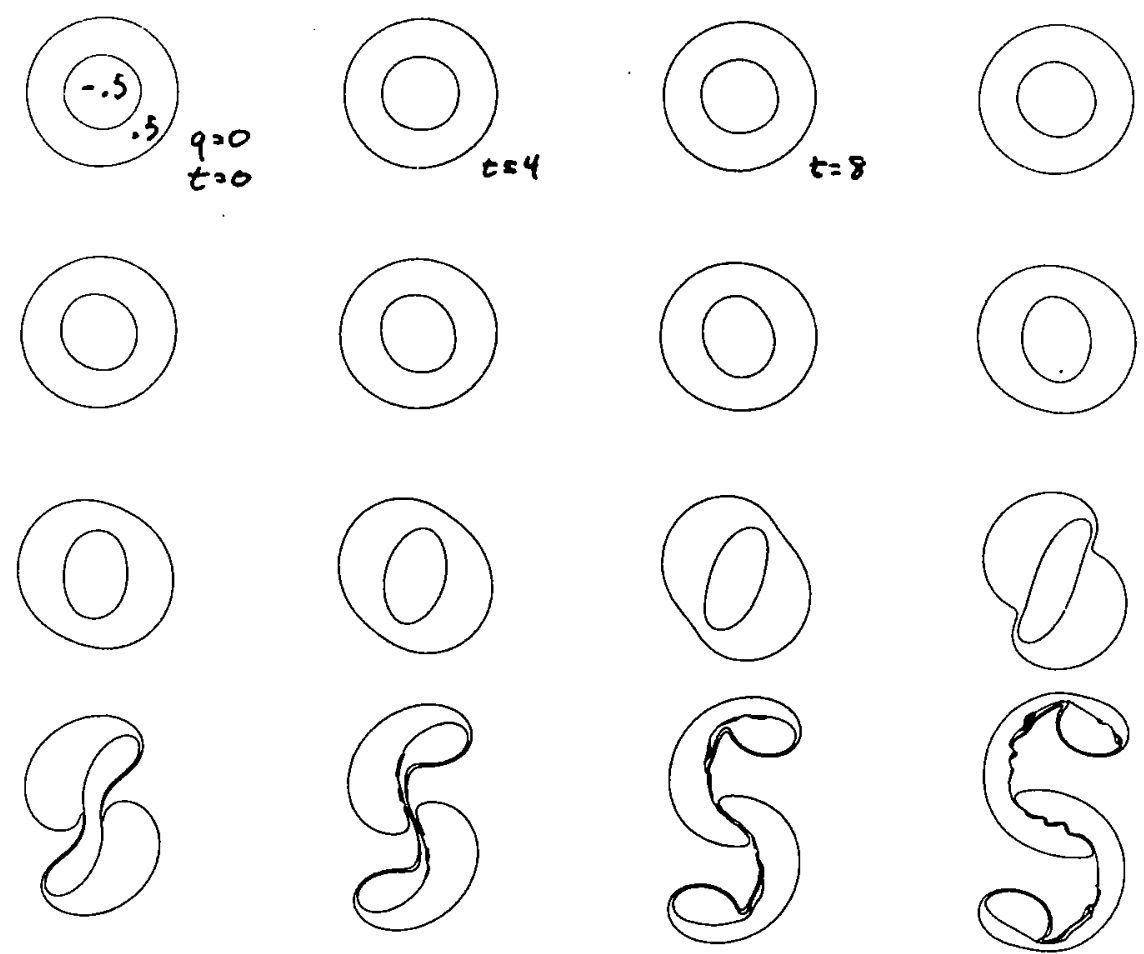

Figure 3: Nonlinear evolution of a linearly unstable eddy with $\left|q_{2}\right|<\left|q_{1}\right|$. Parameters $: r_{1}=1, r_{2}=2.0, q_{1}=-1$ and $q_{2}=.5$.
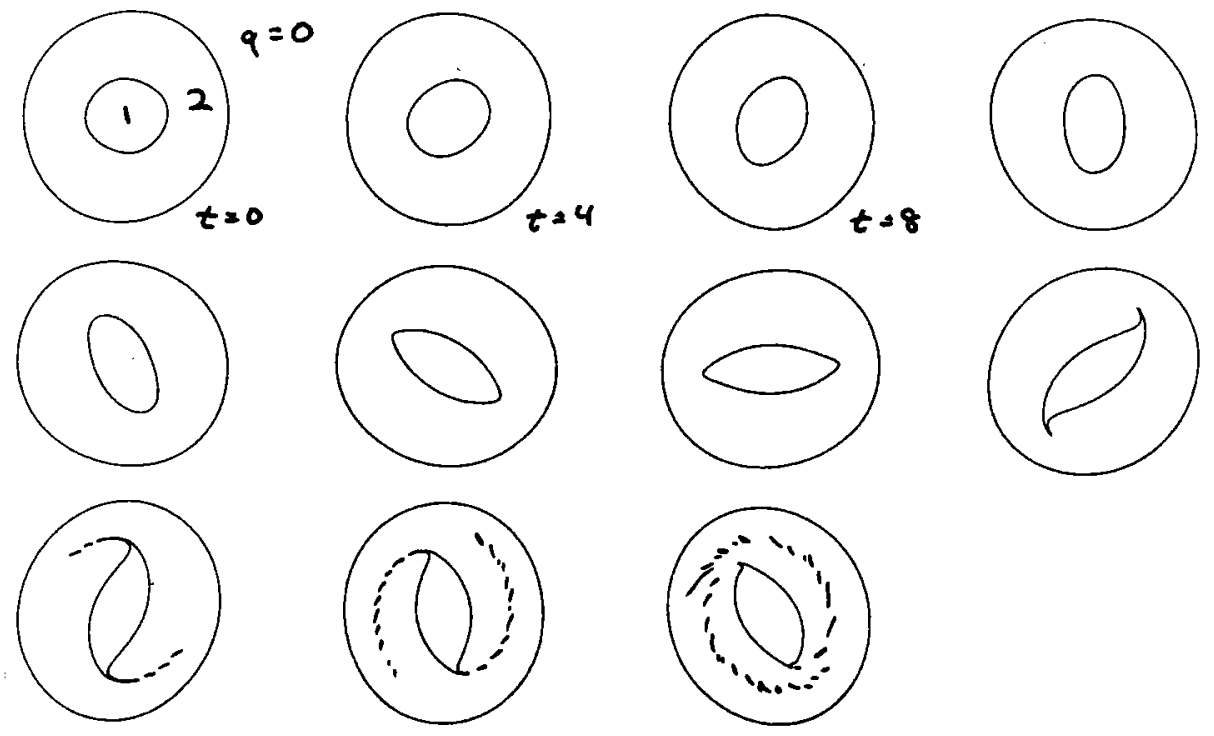

Figure 4: Equilibration of a linearly unstable eddy with $\left|q_{2}\right|>\left|q_{1}\right|$. Parameters : $r_{1}=1, r_{2}=2.7, q_{1}=-1$ and $q_{2}=2$. 


\subsection{Equilibration of a linearly unstable eddy}

The behavior of linearly unstable eddies with $\left|q_{2}\right|>\left|q_{1}\right|$ is quite different. Such eddies have an annular vorticity distribution, with potential vorticity gradients of both signs, but are composed entirely of potential vorticity of one sign. Since the inner contour is weaker, we expect filamentation to occur first in that contour. Figure 4 shows the evolution of such an eddy. The inner contour filaments and these filaments are then wrapped around the center of the eddy. In Figure 4, contour surgery has clipped off these filaments where they drift into the region between the two primary contours. The outer contour, in contrast, does not filament.

One can think of the behavior of Figure 4 as a relaxation of the eddy into a parameter regime where it is linearly stable, or at least marginally stable. The filamentation of the inner contour has two important consequences. First, it reduces the area of the inner contour. When $r_{1}$ decreases, by recalculating Figure 2 it may be shown that the instability regimes move upward. The effect is to move the eddy toward a parameter range where it is marginally stable. Second, if we imagine viscosity mixing the filament into the annular region between the contours, both $\left|q_{1}\right|$ and $\left|q_{2}\right|$ will be reduced and by the same amount. Since $\left|q_{2}\right|>\left|q_{1}\right|$, this will cause the ratio $\left|q_{2} / q_{1}\right|$ to increase. This also has the effect of moving the eddy into a region of parameter space where it is linearly stable.

\section{Finite amplitude behavior in the region of lin- ear stability}

The calculations of the previous section lead us to believe that an eddy which begins in the linearly unstable region with $\left|q_{2}\right|>\left|q_{1}\right|$ will evolve (in an approximate sense) into the linearly stable regions between the instability "spokes" of Figure 2. In this section we use the contour dynamics code to investigate finite amplitude behavior of eddies in this linearly stable region.

We initialize the run with a fairly large (10\% to $20 \%)$ perturbation in both the $m=2$ and $m=4$ modes. As the evolution proceeds the inner and outer contours deform, sometimes appearing nearly elliptical ( $m=2$ mode) and at other times more square ( $m=4$ mode). To view the nonlinear interactions, we decompose the result at each time $t$ into the normal modes dictated by the linear theory:

$$
\left[\begin{array}{l}
\eta_{1}(\theta, t) \\
\eta_{2}(\theta, t)
\end{array}\right]=\sum_{m=1}^{\infty}\left[A_{m 1}(t) \mathbf{u}_{m 1}+A_{m 2}(t) \mathbf{u}_{m 2}\right] e^{i m \theta}
$$

where $\mathbf{u}_{m 1}$ and $\mathbf{u}_{m 2}$ are the eigenvectors corresponding to the two normal modes (the 
eigenvectors of $M$ as defined in (13)). We then plot the amplitudes $\left|A_{i j}\right|$ versus time. If the linear theory were exact, the amplitude of each mode would be constant in time. Thus, any oscillations of the modes result from nonlinear interactions.

Figure 5 shows a typical plot of mode amplitudes versus time for a fairly large initial perturbation. The area enclosed by each contour (which should be constant
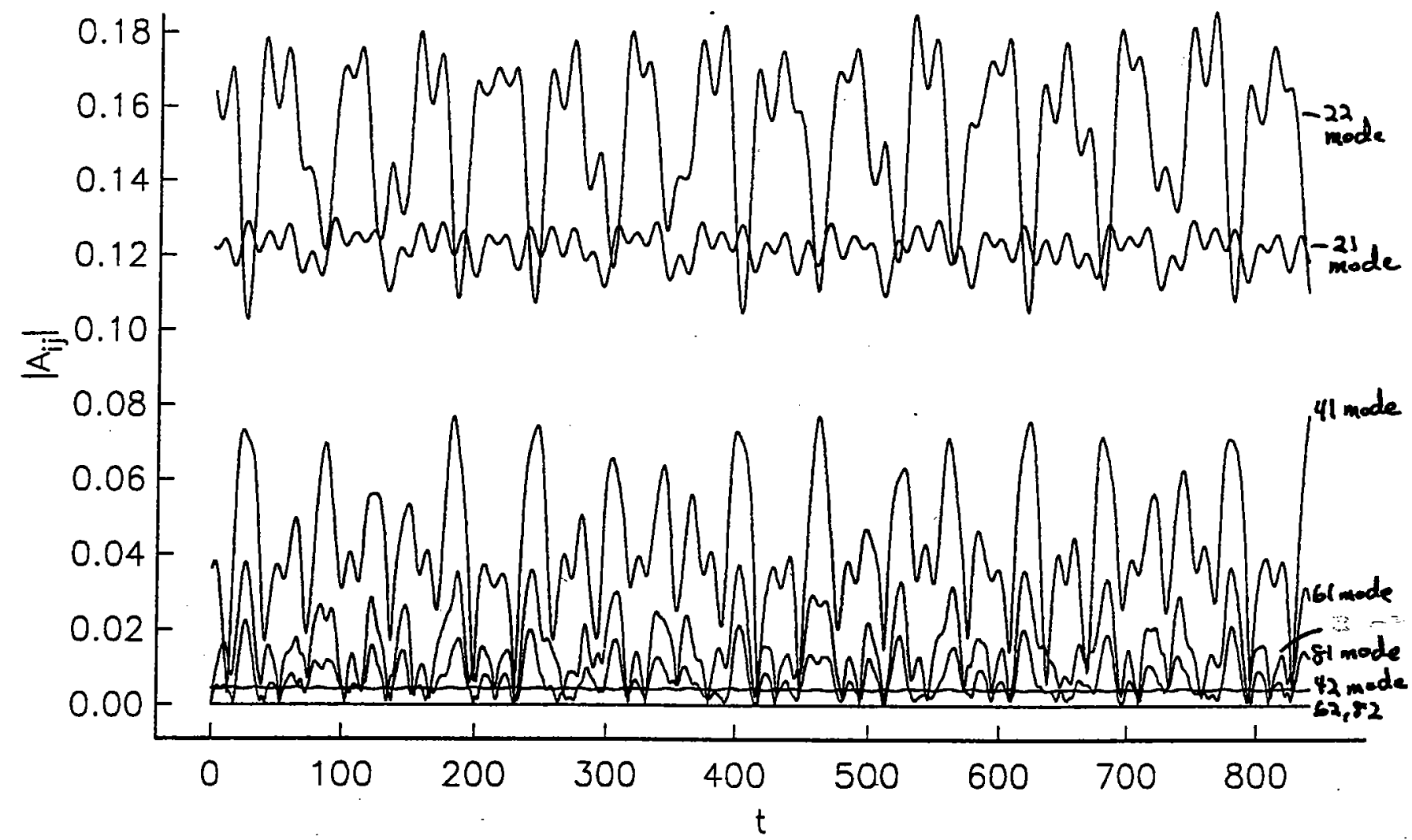

Figure 5: The nonlinear interaction between modes calculated from the output of the the contour dynamics simulation. Here $q_{1}=-1, q_{2}=2, r_{1}=1$ and $r_{2}=2.3$. The eddy turnover time is about 16 time units, so the entire run represents about 54 eddy turnovers.

by vorticity conservation) was monitored to ensure the accuracy of the calculation. In order to keep the changes in area under $1 \%$ over the course of a run, a small time step $(\Delta t=.03125)$ was necessary and the run of Figure 5 required nearly 24 hours of cpu time on a Sun Sparkstation. The primary interaction is between the two $m=2$ modes and one of the $m=4$ modes. However, one of the $m=6$ and $m=8$ modes are excited nonlinearly (and, as we shall see, cannot be ignored). The odd numbered modes are all nearly zero as there is no way they can be excited from even harmonics. Despite appearances, none of the curves in Figure 5 represent noise, as a reduction of 
the time step and increase in the number of nodes per contour produces only slight differences in every curve at the end of the run.

Although the evolution of the mode amplitudes in Figure 5 is complicated, it is difficult to determine if they are in fact chaotic. The Fourier transforms of the signals are quite broad banded, however the problem does not appear to be very sensitive to initial conditions. After shifting the initial phases of the modes by $10 \%$, the new mode amplitudes are still highly correlated with their old values through the entire run, with amplitude changes of only about $10 \%$ at the end. Surfaces of section may be produced from the output, however they contain at most 50 points which is far too few to uncover the dynamics. Effectively, we are solving a system of 500 differential equations (125 $x$ and $y$ coordinates for each of the two contours). What is evidently needed is a reduction in the number of degrees of freedom in the problem.

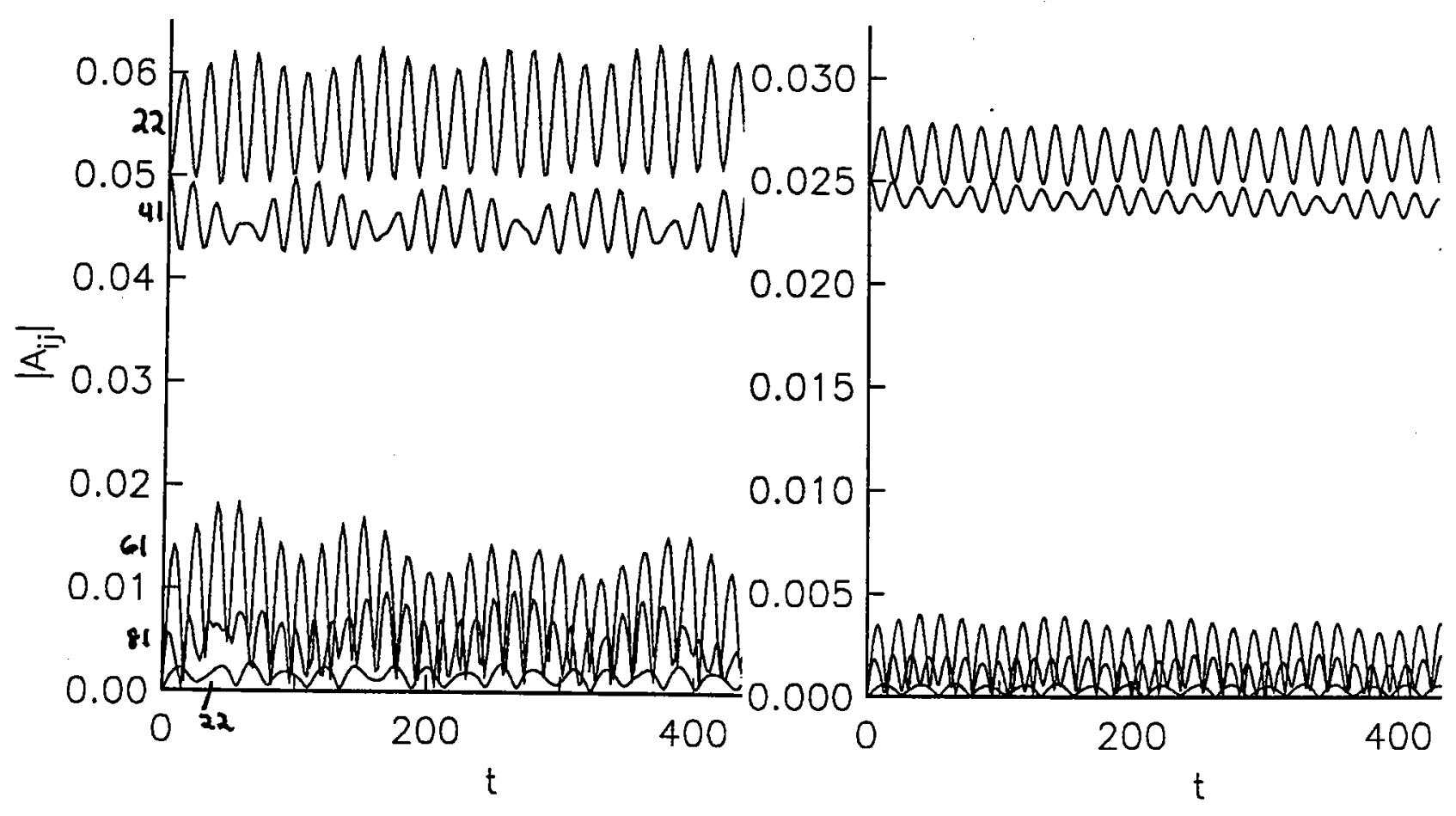

Figure 6: Mode amplitudes versus time where only the 22 and 41 modes are nonzero initially. Parameters are the same as in Figure 5, and initial amplitudes of .05 and .025 are shown.

We begin by simplifying the number of interacting modes in the contour dynamics output. In Figure 6, we begin with only the 22 and 41 modes (it is evident 
in Figure 5 that these modes interact strongly). Note that in Figure 6, if the initial amplitude of the modes decreases by a factor of two, the nonlinear oscillations decrease by a factor of four. Such a decrease indicates that the oscillations are due to a quadratic nonlinearity. A Fourier transform of the 41 mode (Figure 7) shows that four frequencies predominate, which may be written as sums and differences of the linear frequencies (see Table 1).

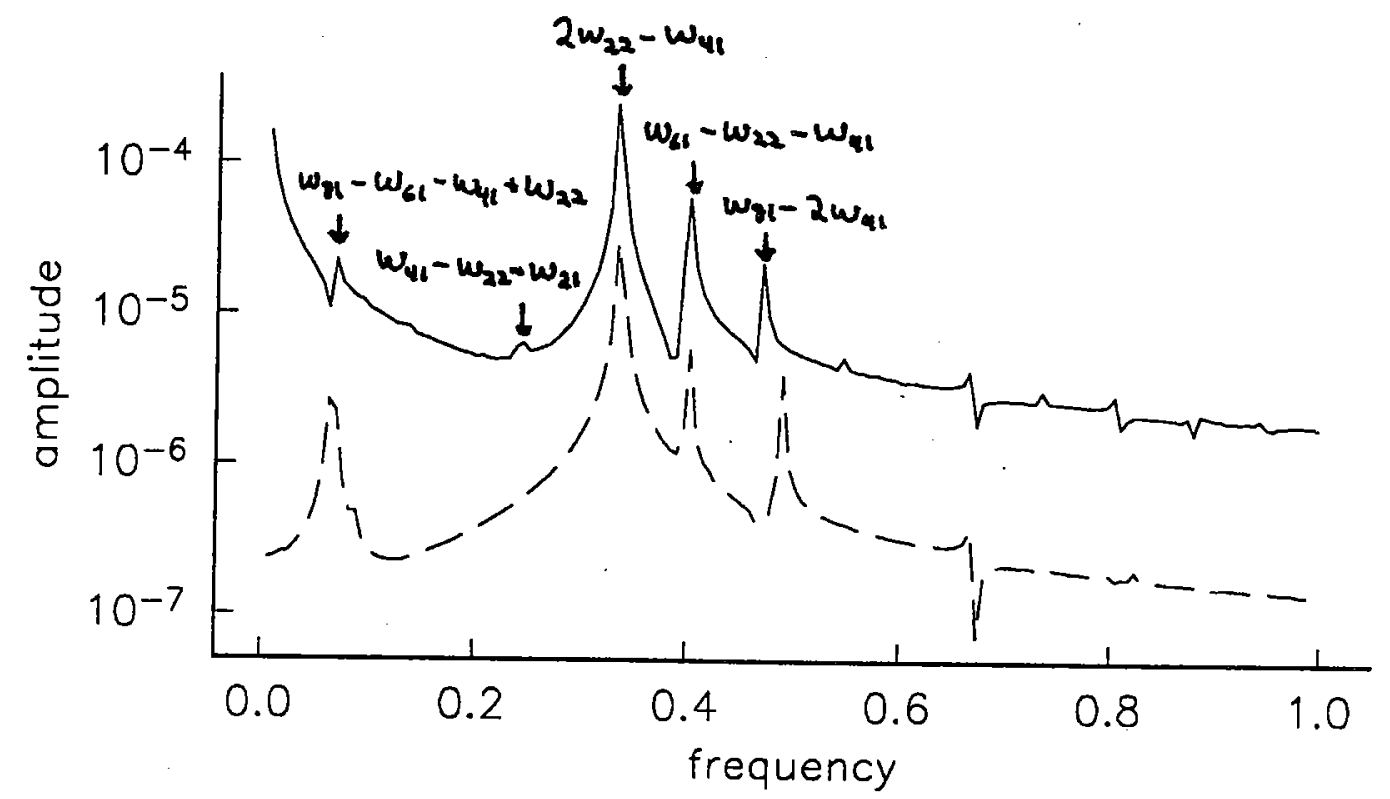

Figure 7: Fourier transform of the 41 mode of Figure 6 with initial amplitude .025 . Dotted curve is the Fourier transform of the 41 mode in the approximating Hamiltonian system (30) (Figure 8), displaced downward by a factor of 10 .

\begin{tabular}{|c|c|c|c||c|c|c|c|}
\hline Mode & Frequency & \multicolumn{2}{|c||}{ eigenvector } & Mode & Frequency & \multicolumn{2}{|c|}{ eigenvector } \\
$\mathrm{ij}$ & $\omega_{i j}$ & $B_{1}$ & $B_{2}$ & $\mathrm{ij}$ & $\omega_{i j}$ & $B_{1}$ & $B_{2}$ \\
\hline \hline 21 & .131104 & .853079 & .521782 & 22 & .223434 & .988270 & .152718 \\
\hline 41 & .111897 & .999946 & -.010361 & 42 & .636545 & .108970 & -.994045 \\
\hline 61 & -.066436 & 1.000000 & -.000851 & 62 & 1.318470 & .008994 & -.999960 \\
\hline 81 & -.249875 & 1.000000 & -.000107 & $\mathbf{8 2}$ & 2.040765 & .001094 & -.999999 \\
\hline
\end{tabular}

Table 1: Frequencies and eigenvectors for the normal modes for the linearly stable eddy $q_{1}=-1, q_{2}=2, r_{1}=1$ and $r_{2}=2.3$ 


\subsection{A low order nonlinear theory}

Although it would be nice to derive a nonlinear interaction theory directly from the equations of motion, we adopt a cruder approach by writing down the form of the nonlinear interaction, and choosing the free parameters to match the output from the contour dynamics program.

Consider the two normal modes $A_{22}(t) e^{2 i \theta}$ and $A_{41}(t) e^{4 i \theta}$ with small amplitude $\left(\left|A_{i j}\right|=O(\epsilon)\right)$, as in Figure 6. A quadratic nonlinearity may be introduced by multiplying pairs of normal modes together. However, there is only one product for each mode that has the proper phase (since the $m=4$ mode is an azimuthal harmonic of the $m=2$ mode). This suggests that a first approximation to the two mode interaction of Figure 6 is

$$
\begin{aligned}
& \dot{A}_{22}=i \omega_{22} A_{22}+\alpha_{1} A_{41} A_{22}^{*} \\
& \dot{A}_{41}=i \omega_{41} A_{41}+\alpha_{2} A_{22}^{2}
\end{aligned}
$$

where $\alpha_{1}$ and $\alpha_{2}$ are arbitrary complex constants. Equations (19) and (20) may be thought of as an asymptotic expansion in mode amplitudes $\left(\left|A_{i j}\right|=O(\epsilon)\right)$ which is truncated at order $\epsilon^{2}$. Note that we make no assumption about the size of the constants $\alpha_{i}$ (in fact, they will turn out to be $O(1)$ ).

Since the 2D Euler equations have a Hamiltonian formulation (Morrison 1982), it is reasonable to require that the equations (19) and (20) form a Hamiltonian system. The conversion to Hamiltonian form may be accomplished by the substitution

$$
A_{i j}=\sqrt{J_{i j}(t)} e^{i \theta_{i j}(t)}
$$

In terms of the J's, the system may be written

$$
\begin{array}{lll}
\dot{J}_{22}=-\frac{\partial H}{\partial \theta_{22}} & ; & \dot{\theta}_{22}=\frac{\partial H}{\partial J_{22}} \\
\dot{J}_{41}=-\frac{\partial H}{\partial \theta_{41}} & ; & \dot{\theta}_{41}=\frac{\partial H}{\partial J_{41}}
\end{array}
$$

where

$$
H=\omega_{22} J_{22}+\omega_{41} J_{41}+c_{1} J_{22} \sqrt{J_{41}} \cos \left(\theta_{41}-2 \theta_{22}+\phi_{1}\right)
$$

The complex constants $\alpha_{1}$ and $\alpha_{2}$ are related to the real constants $c_{1}$ and $\phi_{1}$ by the relations:

$$
\begin{gathered}
c_{1}=\left|\alpha_{1}\right|=2\left|\alpha_{2}\right| \\
e^{i\left(\phi_{1}+\pi / 2\right)}=\frac{\alpha_{1}}{\left|\alpha_{1}\right|}=-\frac{\alpha_{2}^{*}}{\left|\alpha_{2}\right|}
\end{gathered}
$$


Effectively, the requirement of a Hamiltonian system restricts the choices of the constants $\alpha_{i}$.

For $\left|J_{i j}(0)\right|$ small, the solution to the Hamiltonian system $(22)$ is $J_{i j}(t)=J_{i j}(0)$ and $\theta_{i j}(t)=\omega_{i j} t$. As $\left|J_{i j}(0)\right|$ increases, the effect of the third term in (22) is to introduce a modulation of $\left|J_{i j}(t)\right|$ with frequency $\omega_{41}-2 \omega_{22}$. Note that this frequency is exactly the dominant frequency that appears in Figure 7. From Figure 6, we can see that $\dot{J}_{22}=0$ at $t=0$, which implies that $\phi_{1}=0$. The constant $c_{1}$ determines the amplitude of the modulation in $\left|J_{i j}(t)\right|$, and we calculate from Figure 6 that $c_{1} \approx .35$.

With $\phi_{1}=0$ and $c_{1}=.35$, the Hamiltonian system (22) reproduces perfectly the nonlinear modulation of the 22 and 41 mode amplitudes. The system (22) admits an invariant,

$$
I=J_{22}+2 J_{41}
$$

and thus the two degree of freedom Hamiltonian system is integrable. To reproduce the higher order modulations present in Figure 6, we must include higher order nonlinear terms. This will lead to a more complex Hamiltonian system which is not integrable.

\subsection{A higher order nonlinear theory}

The idea behind the higher order nonlinear theory is simple: we proceed in our amplitude expansion (assuming $A_{22}, A_{41}=O(\epsilon)$ ), but now include terms up to order $\epsilon^{3}$. Unfortunately, there are a very large number of possible terms of order $\epsilon^{3}$. However, by looking at Figure 6, we can see that many of these terms are small. For example, we see that the 21 mode is excited to a much lesser extent than the 61 and 81 modes, and the 42,62 and 82 modes do not appear to be excited at all. The reason that these last three modes are not excited is that their eigenvectors are skewed very strongly towards the outer contour, while the 22 and 41 modes are skewed towards the inner contour (see Table 1 ). Thus, it seems reasonable to include only those $O\left(\epsilon^{3}\right)$ terms involving the 22, 41, 61 and 81 modes. Such a truncation yields

$$
\begin{aligned}
\dot{A}_{22}= & i \omega_{22} A_{22}+\alpha_{1} A_{41} A_{22}^{*}+\alpha_{3} A_{61} A_{41}^{*} \\
& +A_{22}\left(\alpha_{4}\left|A_{22}\right|^{2}+\alpha_{5}\left|A_{41}\right|^{2}\right)+\beta_{1} A_{81} A_{61}^{*} \\
\dot{A}_{41}= & i \omega_{41} A_{41}+\alpha_{2} A_{22}^{2}+\alpha_{6} A_{61} A_{22}^{*}+\alpha_{7} A_{81} A_{41}^{*} \\
& +A_{41}\left(\alpha_{8}\left|A_{41}\right|^{2}+\alpha_{9}\left|A_{22}\right|^{2}\right) \\
\dot{A}_{61}= & i \omega_{61} A_{61}+\alpha_{10} A_{22} A_{41}+\beta_{2} A_{81} A_{22}^{*} \\
\dot{A}_{81}= & i \omega_{81} A_{81}+\alpha_{11} A_{41}^{2}+\beta_{3} A_{22} A_{61}
\end{aligned}
$$


Note that the forced modes 61 and 81 have amplitudes $O\left(\epsilon^{2}\right)$. The terms $\beta_{i}$, although formally of order $\epsilon^{4}$, are included because they link the 61 and 81 modes together, and (with hindsight) the coefficients $\beta_{i}$ are large. Applying the transformation (21) we find the associated Hamiltonian

$$
\begin{aligned}
H= & \omega_{22} J_{22}+\omega_{41} J_{41}+\omega_{61} J_{61}+\omega_{81} J_{81}+a_{1} J_{22}^{2}+a_{2} J_{41}^{2}+a_{3} J_{22} J_{41} \\
& +c_{1} J_{22} \sqrt{J_{41}} \cos \left(\theta_{41}-2 \theta_{22}+\phi_{1}\right) \\
& +c_{2} \sqrt{J_{22} J_{41} J_{61}} \cos \left(\theta_{61}-\theta_{41}-\theta_{22}+\phi_{2}\right) \\
& +c_{3} J_{41} \sqrt{J_{81}} \cos \left(\theta_{81}-2 \theta_{41}+\phi_{3}\right) \\
& +c_{4} \sqrt{J_{22} J_{61} J_{81}} \cos \left(\theta_{81}-\theta_{61}-\theta_{22}+\phi_{4}\right)
\end{aligned}
$$

where once again the real constants $a_{i}, c_{i}$ and $\phi_{i}$ are all related to the complex constants $\alpha_{i}$ and $\beta_{i}$. The terms involving $a_{i}$ result in frequency shifts of the primary modes. The terms involving $c_{i}$ are responsible for the additional frequencies shown in Figure 7. The values of $c_{2}$ and $c_{3}$ may be determined by the amplitudes of the forced 61 and 81 modes. Figure 8 shows the result when we numerically integrate the Hamiltonian system (30) with $a_{i}=\phi_{i}=0, c_{1}=.35, c_{2}=1.0, c_{3}=.8$ and $c_{4}=2.1$, and use the same initial conditions as Figure 6 . Pictorially, the agreement with the contour dynamics results (Figure 6) is excellent, and their Fourier transforms (the two curves in Figure 7) are nearly identical.

\section{Conclusions and suggestions for additional work}

We have shown that for a certain amplitude range the nonlinear dynamics of the two-contour eddy can be very closely approximated by a four degree of freedom Hamiltonian system. Effectively, we have been able to reduce the number of degrees of freedom from 250 (for the contour dynamics algorithm) to 4 .

The next logical step is to investigate whether or not the Hamiltonian system (30) contains chaotic dynamics. The investigation into this question has just begun, and we present only some preliminary results. It has not known even if the system (30) is integrable or not, but there is strong evidence that it is not. As before, the system admits an invariant (possibly related to conservation of angular momentum),

$$
I=J_{22}+2 J_{41}+3 J_{61}+4 J_{81}
$$

however, it would appear that even the case $c_{3}=c_{4}=0$ is not integrable. To demonstrate this last statement, in Figure 9 we show two surfaces of section for 


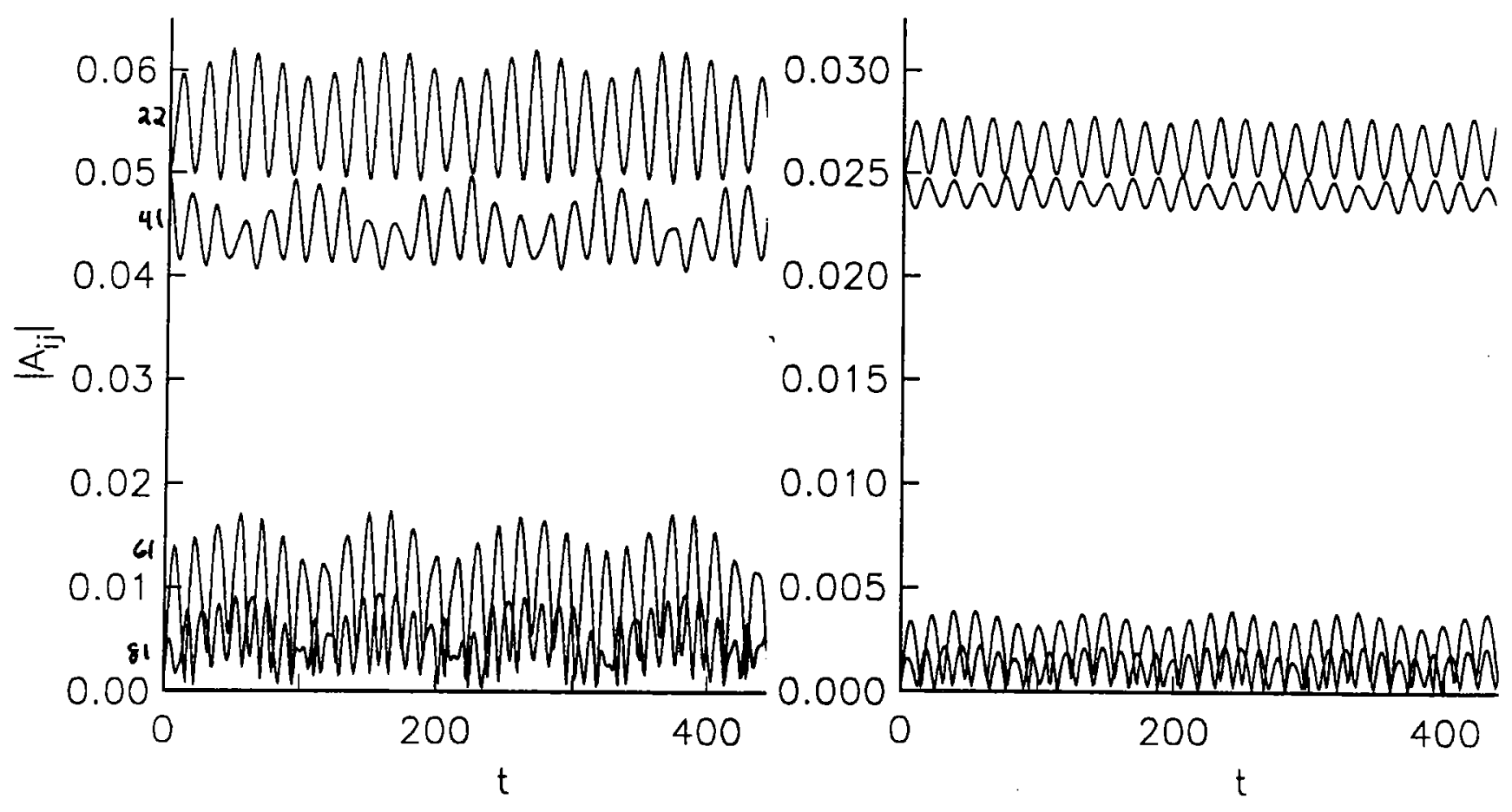

Figure 8: Mode amplitudes versus time for the Hamiltonian system (30) with $a_{i}=$ $\phi_{i}=0, c_{1}=.35, c_{2}=1.0, c_{3}=.8$ and $c_{4}=2.1$ (compare with Figure 6 )

slightly different initial amplitudes. The first case appears to be integrable, while the second does not.

One interesting aspect of the nonlinear theory of Section 5 is that it involves modes all of which have much higher amplitudes in the inner contour. In fact, a barotropic eddy consisting of but a single contour probably obeys similar dynamics. If in fact the Hamiltonian system (30) exhibits chaotic behavior in the parameter range of interest, then the ingredients for chaotic vortex motion are simpler than were thought at the beginning of this paper.

The nonlinear ansatz presented in Section 5 could be applied to a general nonlinear interaction between a wave and its spatial harmonics in any Hamiltonian system. For this particular case, however, the hole in the theory which needs to be filled in is a calculation of the coefficients in the Hamiltonian from first principles. This could be achieved by completing two steps. First, the equations of motion for the contours ((1) and (2)) must be written as a Hamiltonian system. As this report was being written, Phil Morrison completed this step for the case when $r(\theta)$ is sin- 

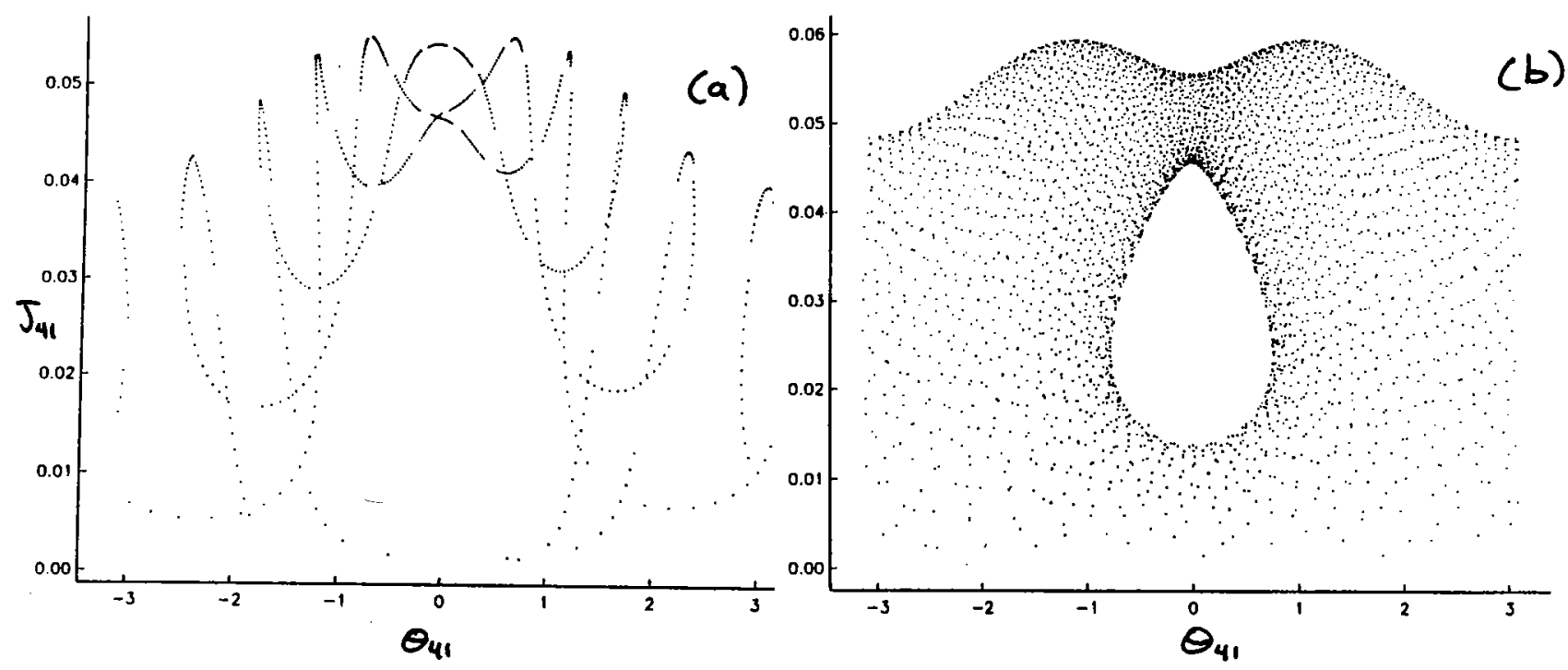

Figure 9: Two surfaces of section $J_{41}$ versus $\theta_{41}$ strobed by $\theta_{22}=0$ for the Hamiltonian system (30). (a) $c_{1}=.4, c_{2}=1$ (all other constants zero), $A_{22}(0)=.1, A_{41}(0)=.05$, $A_{61}(0)=.05$. (b) As in (a) but with $A_{61}(0)=.07$.

gle valued (this is always the case for the eddies of Section 5 ). Second, $r(\theta)$ must be expanded in normal modes and through knowledge of the Hamiltonian structure, action-angle variables extracted. This step is in process, but it must be that the nonlinear theory presented in Section 5 will emerge as some part of the Hamiltonian formalism. A Future project suggested by the Hamiltonian nature of contour dynamics is the investigation of negative energy states and resonances, and their possible connection to filamentation.

\section{Acknowledgements}

I would like to thank Glenn Flierl for a number of useful discussions in regard to this project, and Steven Meacham for giving out copies of his contour dynamics code so generously. Phil Morrison became interested in the project once Hamiltonian dynamics were mentioned, and I am grateful for the time he took to teach me about 
the subject. Thanks to all the GFD "dynamos", I had a great summer both in Walsh and on the softball field.

\section{References}

- Aref, H. 1984 Stirring by chaotic advection. J. Fluid Mech. 143. 1-21.

- Babiano, A., Basdevant, C., Legras, B., Sadourny, R. 1986 Vorticity and passive scalar dynamics of two-dimensional turbulence. J. Fluid Mech. 183 379390.

- Carton, X. and McWilliams, J. 1989 Barotropic and baroclinic instabilities of axisymmetric vortices in a quasigeostrophic model. Mesoscale/Synoptic Coherent Structures in Geophysical Turbulence Elsevier 225-248.

- Childress, S. 1984 A vortex-tube model of eddies in the inertial range. Geophys. Astrophys. Fluid Dyn. 29. 29-64.

- Dritschel, D. 1988 Nonlinear stability bounds for inviscid two dimensional flows, ...J. Fluid Mech. 191. 575-581.

- Flierl, G. 1988 On the instability of geostrophic vortices. J. Fluid Mech. 197. 349-388.

- Kida, S. 1981 Motion of an elliptic vortex in a uniform shear flow J. Phys. Soc. Jpn. 50 3517-3520.

- Kirchoff, G. 1876 Mechanik, Leipzig.

- Morrison, P. 1982 in Mathematical Methods in Hydrodynamics and Integrability in Dynamical Systems, AIP Conf. Proc. 8813.

- Polvani, L. and Wisdom, J. 1990 Chaotic Lagrangian trajectories around an elliptical patch embedded in a constant and uniform background shear flow. Phys. Fluids A 2 123-126.

- Kloosterziel, R. and Van Heijst, G. 1989 On tripolar vortices. Mesoscale Synoptic Coherent Structures in Geophysical Turbulence Elsevier 609-625. 


\title{
The Rise and Fall \\ of Buoyant Plumes
}

\author{
C. P. Caulfield \\ D. A. M. T. P. \\ University of Cambridge
}

\begin{abstract}
We generalize the theory of Morton, Taylor, and Turner (1956) to plumes of nonzero initial mass flux. We classify plumes with respect to the balance between their initial mass and momentum fluxes. We explain the effect first described by Morton (1959) of a reduction in plume height in a stratified environment with increased momentum flux in terms of unstratified behaviour. The Morton effect does not occur for all initial mass fluxes. However, for any initial mass flux, there exists an initial momentum flux with minimum height of rise. The behaviour of a plume rising from a source of fluid that exhibits nonmonotonic density variation with mixing is investigated as a model for volcanic eruptions, and a condition for collapse is found.
\end{abstract}

\section{Introduction}

Localised sources of fluid with density different from the density of the ambient occur in a wide range of geophysical, astrophysical and industrial contexts. Flows generated from such sources are modelled using the entrainment assumption, first discussed by Taylor (1945) (see Turner (1986) for a comprehensive review). The inflow velocity of ambient fluid into the fluid rising from the source is assumed to be proportional to some characteristic vertical velocity of the source fluid. The constant of proportionality is assumed to be the same at all heights above the initial release height. This steady state model assumes that the turbulence and velocity structures are preserved at all heights. Though this model is very simplified, since it assimilates all the effects of turbulence into one constant of proportionality (see List (1982) and Turner (1986) for discussion), the description and predictions of the model appear to be valid in many situations, and the results of interest are relatively robust with respect to variations in the exact value of this constant of prportionality.

Using this model the evolution of the behaviour of the fluid from a source can be modelled by considering the conservation of three quantities, namely mass flux, momentum flux, and buoyancy flux, i.e. the flux of density deficiency of the input fluid. Following the seminal paper of Morton, Taylor, and Turner (1956), we define the halfwidth of the plume arising from a source of buoyancy at a certain height as $b$ and its reduced gravity $g^{\prime}$,

$$
g^{\prime} \equiv g\left(\frac{\rho_{a}-\rho_{p}}{\rho_{\text {ref }}}\right)
$$


where $\rho_{a}$ is the density of the ambient fluid, $\rho_{0}$ is the density of the source, and $\rho_{\text {ref }}$ is a characteristic density of the system. We also identify a characteristic vertical velocity $w$. at each height, and assume that

$$
u_{\text {inflow }}=\alpha w \text {, }
$$

where $\alpha$ is known as the entrainment constant. The equation describing the evolution of mass flux is

$$
\frac{d}{d z}\left(\rho \pi b^{2} w\right)=2 \rho \pi b \alpha w
$$

This equation reflects the fact that the rate of increase of mass flux with height is balanced by the entrainment of ambient fluid. The rate of increase of momentum flux is balanced by the gravitational acceleration of the source fluid due to density differences, i.e.

$$
\frac{d}{d z}\left(\rho \pi b^{2} w^{2}\right)=\rho g^{\prime} \pi b^{2}
$$

The rate of change of buoyancy flux with height is balanced by the entrainment of fluid with density characteristic of that height, a balance described by

$$
\frac{g}{\rho_{\text {ref }}} \frac{d}{d z}\left[\pi b^{2} w\left(\rho_{a(0)}-\rho_{p}\right)\right]=-\pi N^{2} b^{2} w,
$$

where $N^{2}$ is the Brunt-Vaisala frequency, defined by

$$
N^{2} \equiv \frac{-g}{\rho_{\text {ref }}} \frac{d}{d z} \rho_{a},
$$

and $\rho_{a(0)}$ denotes the ambient density at $z=0$. If we make the further assumption that the Boussinesq approximation applies (i.e. $\rho_{a}-\rho_{p} \ll \rho_{\text {ref }}$ ) then the equations reduce to

$$
\begin{aligned}
& \frac{d}{d z} Q=2 \alpha M^{1 / 2} \\
& \frac{d}{d z} M=\frac{F Q}{M}, \\
& \frac{d}{d z} F=-Q N^{2}
\end{aligned}
$$

where

$$
\begin{aligned}
Q & =b^{2} w, & & \text { (volume flux) } \\
M & =b^{2} w^{2}, & & \text { (momentum flux) } \\
F & =g^{\prime} b^{2} w, & & \text { (buoyancy flux) }
\end{aligned}
$$

and we see that

$$
b=\frac{Q}{M^{1 / 2}} ; \quad w=\frac{M}{Q} ; \quad \text { and } \quad g^{\prime}=\frac{F}{Q}
$$


In the absence of stratification, Morton (1959) demonstrated, that since $F$ is constant, (4) and (5) may be combined as

$$
2 \alpha \frac{M^{3 / 2}}{F} \frac{d}{d z} M=Q \frac{d}{d z} Q
$$

which implies that

$$
\frac{8 \alpha}{5 F} M^{5 / 2}+c=Q^{2}
$$

where

$$
c=Q_{0}^{2}-\frac{8 \alpha}{5 F} M_{0}^{5 / 2}
$$

Thus (4-6) reduce to $F=F_{0}$ ( constant), (7), and

$$
\frac{d}{d z} Q=\left(20 \alpha^{4} F\right)^{1 / 5}\left(Q^{2}-c\right)^{1 / 3}
$$

In the case of initial mass flux and momentum flux zero, $c=0$, and (8) reduces to

$$
\frac{d}{d z} Q=\left(20 \alpha^{4} F\right)^{1 / 5} Q^{2 / 5}
$$

A similarity solution may then be found for $b, w$ and $g^{\prime}$ (see Turner (1973) for details), of the form

$$
\begin{aligned}
b & =\frac{6}{5} \alpha z \\
w & =\frac{5}{6 \alpha}\left(\frac{9}{10} \alpha F\right)^{1 / 3} z^{-1 / 3}, \\
g^{\prime} & =\frac{5 F}{6 \alpha}\left(\frac{9}{10} \alpha F\right)^{-1 / 3} z^{-5 / 3}
\end{aligned}
$$

These initial conditions characterize a well known and extremely common phenomenon in geophysics, namely the buoyant (pure) plume. We note, that in an unstratified environment, buoyant pure plumes rise without limit, with their mass flux and radius increasing with height as described by the similarity solution. However, in the geophysical context, ambient stratification is usually present. This has also been considered using this model (see Morton, Taylor and Turner (1956) and Morton (1959)), but care must be taken since energy may be radiated away in the form of internal waves when an intrusion occurs in a stratified environment (see List (1982)). In this case, $N$ is no longer zero, but we can still reduce the governing equations to two by noting that (5) and (6) combine:

$$
\frac{d}{d z} M^{2}=2 Q F=-\frac{1}{N^{2}} \frac{d}{d z} F^{2},
$$

that is

$$
M^{2}+\frac{F^{2}}{N^{2}}=c_{s}
$$


If the ambient stratification is statically stable, (since buoyancy flux decreases with height) (from (6) and see fig. 1), there will be a particular height at which the plume fluid density equals that of the ambient. At such a height, from (14), the plume will have maximum momentum flux. This is progressively,(and quite rapidly) lost, until at some later height, referred to as the final height of rise, the upward velocity goes to zero (see fig. 2). The final height of rise of a pure plume predicted by this model has been shown to apply very well to both experimental and geophysical plumes (see Turner (1973)), and indeed geophysically it appears that the pure buoyant plume is in some sense selected as a natural structure. Morton (1959) found that increasing the initial momentum flux from zero actually decreased the final height of rise for point source plumes of zero initial mass flux and finite buoyancy. Only plumes with very large momentum flux rose higher than a pure plume, which has zero initial momentum flux, an apparently paradoxical result. This report considers the effects of deviations from this classical simple model to more realistic source conditions.

In Section 2, we generalize this theory to arbitrary initial conditions of mass flux and momentum flux in the unstratified envoironment. We classify various plumes by the initial balance between momentum flux and mass flux quantified by the constant $c$ in (7), adopting a system of Lane-Serf et al (1989). We find that all plumes ultimately asymptote to a solution with $c=0$, that we call the pure plume solution. However, the effective origin, defined as the point at which the asymptotic pure plume similarity solution would have zero radius, is determined by the constant $c$.

In Section 3, we generalize the model to stratified environments. Although no similarity solutions exist, pure plumes (i.e. plumes with zero initial mass flux and momentum flux but finite buoyancy flux) have a well defined balance between momentum and mass flux at all heights. Also, for any initial mass flux $Q$, there is a particular associated momentum flux, $M=M(Q)$, that causes a minimum final height of rise for a plume with fixed initial buoyancy flux. For small initial mass flux, this minimum has an associated initial momentum flux greater than that of a pure plume, and this explains the Morton effect. For sufficiently large initial mass flux, this minimum has a momentum flux less than the pure plume. In this case, all jetlike sources penetrate further than a pure plume, and the Morton effect does not occur.This is likely to be important when a well developed plume passes through a region of varying stratification.

In Section 4, we consider the behaviour of a plume of fluid that exhibits nonmonotonic density variation during mixing. Such plumes may be considered models for the behaviour of several geophysical phenomena, e.g. volcanic eruption clouds, rain clouds etc. In the case where an initially dense fluid becomes buoyant after a certain amount of mixing, we derive a condition on the initial momentum flux such that the initial density difference is so large that the plume collapses at some height, which is calculated for several situations.

Section 5 presents conclusions, and details further work. 


\section{Unstratified Theory}

The governing equations are (7) and (9) above. We nondimensionalize on initial mass and buoyancy fluxes, nondimensional quantities having asterisks. Thus

$$
\begin{aligned}
& Q=Q_{0} Q^{*} \\
& z=\left(\frac{20 \alpha^{4} F}{Q_{0}^{3}}\right)^{-1 / 5} z^{*}, \\
& c=Q_{0}^{2} c^{*}
\end{aligned}
$$

Dropping the asterisks, (9) reduces to

$$
\frac{d}{d z} Q=\left(Q^{2}-c\right)^{1 / 5}
$$

Note that the plume with $M_{0}=Q_{0}=0$ has $c=0$. Now, modifying the concepts of Lane-Serff et al. (1989) we classify the plumes arising from all sources of buoyancy with initial mass flux and momentum flux in the following way:

i: if $c=0$ the plume is a pure plume;

ii: if $c<0$ then the plume has excess momentum flux, and is a jet;

iii: if $c>0$ then the plume has momentum flux deficiency, has too large initial radius, and is a distributed source.

By definition $-\infty<c \leq 1$, thus from (15) we see that mass flux always increases, and eventually, the behaviour must asymptote to

$$
\frac{d}{d z} Q \sim Q^{2 / 5}
$$

the solution for a pure plume.

The radius $b$ evolves according to

$$
\frac{d}{d z} b=\frac{6}{5} \alpha-\frac{F c}{2 M^{5 / 2}}
$$

Thus the rate of spread for a pure plume is $\frac{6 \alpha}{5}$, and increases for a jet, up to the well known maximum of $2 \alpha$ initially, when $Q_{0}=0$. For a distributed source, the spread is less than that of a pure plume, and indeed if

$$
Q_{0}^{2} \geq \frac{4 \alpha M_{0}^{5 / 2}}{5 F}
$$

the fluid actually initially contracts. Care must be taken in such cases (see Fischer et al. (1979) and List (1982) for a fuller discussion of the jetlike behaviour), since the actual value of the entrainment constant varies, and the assumptions of similarity in turbulent and velocity profiles may become more questionable, as does the assertion that mixing takes place all the way across a wide distributed source, right from the outset. However, 
a reduction in the intial entrainment in the case of a distributed source will increase the effect of the contraction described above. These effects are shown on fig. 3, where the evolution of radius for three different values of $c(-0.999,0.0$, and 0.999$)$ are plotted. Since each source has the same $Q_{0}=1$, the variation in $c$ is reflected by a variation in $b_{0}$, with the jet narrower, and the distributed source wider. The nondimensionalization is such that the pure plume solution has $b_{0}=1$ and angle of spread $3 / 5$. We see that the jet radius increases faster from a smaller $b_{0}$, and eventually crosses the pure plume solution, while the distributed source starts from a larger $b_{0}$, contracts and eventually crosses the pure plume solution also. Eventually, however, we see that all three asymptotically spread linearly with slope $3 / 5$, i.e. like pure plumes.

This approach to plume behaviour can also be seen in fig. 4 , where the evolution of $Q$ with height for these three different plumes is plotted. Note the larger increase in the jet's mass flux since its momentum flux requires a larger mass flux to balance it. The reverse is true for the distributed source.

The approach to a pure plume solution may be studied asymptotically. However, due to the nature of the problem, a numerical approach has been used. Since eventually the evolution of a source with arbitrary initial conditions is approximated by the evolution of a pure plume, we search for the "equivalent pure plume solution" of any plume. In dimensional terms, if we define the point where $Q_{0}=M_{0}=0$ (i.e. the point source) as $z_{0}$, then the pure plume similarity solution for the mass flux $Q$ is, from (11-13),

$$
Q=\frac{6 \alpha}{5}\left(\frac{9}{10} \alpha F\right)^{1 / 3}\left(z-z_{0}\right)^{5 / 3}
$$

or nondimensionally,

$$
Q=\left[\frac{3}{5}\left(z-z_{0}\right)\right]^{5 / 3}
$$

For the pure plume, which follows this evolution from $z=0$, where $Q=1$, we see the effective origin is $-5 / 3$. For all source conditions we can identify an effective origin similarly since eventually the mass flux evolves as above. Calculations of effective origin for the three values of $c$ are shown in fig. 5 , with the associated equivalent pure plume solution. Note that the distributed source approaches its equivalent pure plume from outside, with larger radii, while the jet approaches from inside, with smaller radii. Also, the effective origin of the distributed source is ahead of that for the pure plume, which is in turn ahead of that for the jet. The variation of effective origin with $c$ is shown in fig. 6 .

In summary, in an unstratified environment, sources with arbitrary initial conditions ultimately converge to a pure plume. However, the radius of an originally jetlike plume is larger than that of a pure plume, which in turn is larger than that of a distributed source, after an initial adjustment where the situation is reversed. This is a trivial consequence of the positioning of the effective origin. The implications of this in the stratified environment are investigated in the next section. 


\section{Stratifled Theory}

To recap, the equations that govern the behaviour in the stratified environment are

$$
\begin{aligned}
& \frac{d}{d z} Q=2 \alpha\left(c_{s}-\frac{F^{2}}{N^{2}}\right)^{1 / 4}, \\
& \frac{d}{d z} F=-Q N^{2},
\end{aligned}
$$

and

$$
c_{s}=M^{2}+\frac{F^{2}}{N^{2}},
$$

where $c_{s}$ is a constant.

We nondimensionalize these equations on the ambient stratification $N^{2}$ and the initial buoyancy flux $F_{0}^{2}$. As before, starred quantities are dimensionless.

$$
\begin{aligned}
F & =F_{0} F^{*}, \\
M & =2^{1 / 2} F_{0} N^{-1} M^{*}, \\
Q & =2^{5 / 8} \alpha^{1 / 2} F_{0}^{3 / 4} N^{-5 / 4} Q^{*}, \\
z & =2^{-5 / 8} \alpha^{-1 / 2} F_{0}^{1 / 4} N^{-3 / 4} z^{*},
\end{aligned}
$$

and the equations reduce to (once again dropping asterisks),

$$
\begin{aligned}
\frac{d}{d z} Q & =\left(c_{a}-\frac{1}{2} F^{2}\right)^{1 / 4}, \\
\frac{d}{d z} F & =-Q, \\
c_{a} & =M_{0}^{2}+\frac{1}{2} F_{0}^{2} .
\end{aligned}
$$

The point source pure plume solution has $c_{a}=1 / 2$ in this nondimensionalization. Since it only reaches a finite height (see fig. 2) a similarity solution analogous to that described in Section 2 does not exist. Nevertheless, there is a clearly defined evolution of mass flux and momentum flux. We generalize the approach of Morton (1959) to allow for the possibility of initial nonzero momentum flux. We define two parameters, $\sigma_{M}$ and $\sigma_{Q}$ as follows, with nondimensional quantities being asterisked:

$$
\begin{aligned}
\sigma_{M} & =\frac{N^{2} M^{2}}{N^{2} M^{2}+F^{2}} \\
& =\frac{2 M^{* 2}}{2 M^{* 2}+F^{* 2}} \\
\sigma_{Q} & =\frac{N^{5 / 2} Q^{2}}{N^{5 / 2} Q^{2}+2^{5 / 4} F_{0}^{-1 / 2} F^{2}} \\
& =\left(1+g^{* 2}\right)^{-1}
\end{aligned}
$$


These parameters are, respectively, a measure of the importance of momentum flux and mass flux compared to the natural momentum and mass flux scales determined by the initial buoyancy flux and stratification. The evolution of $\sigma_{Q}$ and $\sigma_{M}$ for a point source pure plume is shown in figs. 7 and 8. Initially $\sigma_{M}$ and $\sigma_{Q}$ are zero, and then they increase to a maximum (which is 1 ) when the plume ceases to be buoyant. Subsequently, they decrease, $\sigma_{M}$ to zero, when $w$ goes to zero (cf. fig. 2), and $\sigma_{Q}$ to a finite value, since the model breaks down, and $b \rightarrow \infty$.

Combining these data, we obtain a locus in $\sigma_{M}-\sigma_{Q}$ space for a pure plume solution of the equations, which is plotted in fig. 9. From fig. 7 , we know that every $\sigma_{Q}$ is associated with a specific height. Therefore, each point on the locus may be considered to specify a height, $h$. For given initial mass flux, a plume from a source with momentum flux and buoyancy flux calculated from $\sigma_{M}$ and $\sigma_{Q}$ at that point on the locus subsequently rises like a point source pure plume that had started from $z=-h$.

Thus in this context, we define pure plumes as plumes arising from sources that lie on this locus, for arbitrary initial mass fluxes. For a given mass flux, all points to the right of this curve correspond to sources with too much momentum flux, whose plumes shall be described as jets, analogously to before. Similarly, all points to the left correspond to sources with too little momentum flux, whose plumes shall be called distributed sources.

Morton (1959) considered the behaviour of plumes with $\sigma_{Q}=0$ and $\sigma_{M} \neq 0$. He found that the height of rise actually decreased for $\sigma_{M}>0$, reaching a minimum at around $\sigma_{M} \sim 0.7$ and reaching heights higher than the pure plume for $\sigma_{M} \geq 0.99$ (see fig. 10). We investigate this counterintuitive behaviour, which we shall refer to as the Morton effect, for general initial $Q_{0}$. For $\sigma_{Q}=0.12$ (see fig. 11), there is an essentially similar progression. In this case we see that the effect is generalized into the distributed source region of parameter space. Distributed sources go higher than the pure plume solution (which is marked with a cross) and there is a minimum rise height in the jet region. However, on increasing $\sigma_{Q}$ further to 0.27 , (see fig. 12), the plume solution rises to a height greater than all distributed sources with finite initial area, in the jet region the height of rise monotonically increases with $\sigma_{M}$, and there now is a minimum rise height in the distributed source region.

Fig. 13 shows the locus of minimum rise height for all possible $\sigma_{M}$ and $\sigma_{Q}$, and the region where the generalized Morton effect (i.e. jets go lower, distributed sources go higher than the pure plume) takes place. This effect may be explained as follows. If the evolution is largely unaffected by the stratification, the unstratified behaviour dominates until such time as $g^{\prime} / b$ becomes small enough relative to $N^{2}$ to allow stratified effects to be significant. Thus if $g^{\prime} / b$ remains large compared to $N^{2}$ for a sufficient height, jets will cross the pure plumes in radius, and asymptote to a pure plume with effective origin behind that of a pure plume with the same initial mass flux. If the stratification becomes important after this crossing event, the jet will rise to a lower height, provided that its momentum flux is not too much larger than that of a pure plume. (But we remember from figs. 1 and 2 that after $g^{\prime}$ goes negative, $M$ and $w$ rapidly decay to zero.) Similarly a distributed source will cross to a radius less than that of a pure plume, and provided that its momentum flux is not too much smaller than that of a pure plume when $g^{\prime}$ goes through zero, it will rise to a greater height. There is thus a balance between the competing effects of increasing. 
momentum flux "pushng" the effective origin further back when stratification is "weak", and yet, when stratification is strong, "pushing" weakly buoyant or even dense fluid higher. We thus obtain a minimum rise height where the relative effect of receding effective origin is strongest.

If the stratification is very strong, any source with nonzero initial mass flux will have a plume whose height of rise is substantially determined by the initial momentum flux of the source. (In this case, one is high up the $\sigma_{M}-\sigma_{Q}$ curve.) Solutions of this type can occur when a pure plume solution, rising through a region of low stratification passes into a region of high stratification. If the transition occurs at a height where $g^{\prime} / b$ is relatively small, the change in $N^{2}$ may trigger a significant displacement from the pure plume locus, and hence quite large variation (from the simple pure plume model prediction averaging the stratification in some way) in the final height of rise. This is the subject of further investigation.

A further effect, that must be remembered at all times, is that a nonzero initial mass flux may change the value of $\sigma_{Q}$ significantly. In the classical Morton, Taylor, Turner model of point source pure plumes, $Q_{0}=0$ and $F_{0}$ is finite (which without loss of generality we take to be $O(1)$ ), and so, at $z=0, g^{\prime}, w \rightarrow \infty$ (see fig. 2). Formally, we have the scaling for $\epsilon \ll 1$,

$$
g_{0}^{\prime}, w \sim O\left(\frac{1}{\epsilon}\right), \quad b_{0} \sim o(\epsilon) \Rightarrow F_{0}, M_{0} \sim O(1), \text { but } Q_{0} \sim o(\epsilon)
$$

and

$$
\frac{g_{0}^{\prime}}{b_{0}} \gg N^{2}
$$

We also see that the initial intrusion height is infinite, and the Boussinesq approximation breaks down. On the other hand, if $Q_{0}$ is finite, $g_{0}^{\prime}$ is also finite, and decreasing as $Q$ increases, thus increasing $Q_{0}$ decreases initial intrusion height. In fig. 14 we show that this effect, (equivalent to moving vertically in $\sigma_{M}-\sigma_{Q}$ space in fig. 15) can quite dramatically reduce final height of rise.

Thus, in certain situations, it appears to be important to take into account finite initial mass flux from a source, especially when the initial density difference between source fluid and and the ambient is small. 


\section{Nonmonotonic Density Variation during Mixing}

In several geophysical situations of interest, fluids exhibit nonmonotonic variation of density with mixing. In a plinian-style volcanic eruption, the efflux from the volcano is hot but dense, due to the particulate suspension within the cloud. As the very hot erupted material entrains and heats the ambient, the bulk density decreases, and eventually the column may become buoyant. In this case we have a density that evolves with mixing as shown in fig. 15. A different nonmonotonic density variation (see fig. 16) arises in moist convective clouds (see Squire and Turner (1962) for a fuller description).

We can model the essential fluid mechanical effects of both of these situations using a simplified model. We assume that the density of the fluid can be described by

$$
\rho=\rho_{\text {ext }}+\left(\lambda-\frac{Q_{0}}{Q}\right)^{2} \frac{\left(\rho_{0}-\rho_{\text {ext }}\right)}{(\lambda-1)^{2}} .
$$

Thus, when $Q_{0} / Q=\lambda, \rho=\rho_{\text {ext }}$, the extremal density, and when $Q_{0}=Q, \rho=\rho_{0}$, the input density: $\lambda$ may be considered to be a measure of the structure of the nonlinearity of the fluid mixing. In the limit $Q_{0} / Q=0$, the fluid is so well mixed that it must tend to the density of the ambient. Thus

$$
\rho_{a}=\rho_{e x t}+\frac{\lambda^{2}\left(\rho_{0}-\rho_{e x t}\right)}{(\lambda-1)^{2}} .
$$

If we now concentrate on the situation shown in fig. 15 , we see that

$$
\rho_{\text {ext }}=\rho_{\min },
$$

and the following regimes exist:

i: $\lambda=0 \Rightarrow \rho_{a}=\rho_{\min }$

ii: $0<\lambda<1 / 2 \Rightarrow \rho_{\min }<\rho_{a}<\rho_{0}$;

iii: $\lambda=1 / 2 \Rightarrow \rho_{\min }<\rho_{0}<\rho_{0}$;

iv: $\lambda \rightarrow 1 \Rightarrow \rho_{a} \rightarrow \infty$.

We see that

$$
g^{\prime}=\frac{g}{\rho_{\text {ref }}}\left(\frac{\left(\rho_{0}-\rho_{\min }\right)}{(\lambda-1)^{2}}\right)\left(\frac{2 \lambda Q_{0}}{Q}-\frac{Q_{0}^{2}}{Q^{2}}\right),
$$

and the other two governing equations are

$$
\frac{d}{d z} Q=2 \alpha M^{1 / 2},
$$

and

$$
\frac{d}{d z} M=\frac{g^{\prime} Q^{2}}{M} .
$$

As in the unstratified case, the system may be integrated to reduce the order. We arrive at

$$
\frac{d}{d z} Q=\left(\frac{40 \alpha^{4} F_{0}}{(2 \lambda-1)}\right)^{1 / 5}\left(\lambda Q^{2}-Q_{0} Q-c_{m}\right)^{1 / 5}
$$


where

$$
c_{m} \equiv \lambda Q^{2}-Q_{0} Q-\frac{4 \alpha(2 \lambda-1) M^{5 / 2}}{5 F_{0}}
$$

and

$$
(2 \lambda-1) / F_{0} \equiv(2 \lambda-1) /\left(g_{0}^{\prime} Q_{0}\right)>0
$$

at all times, by the definition of $\lambda$ above. Once again $c_{m}$ is a constant.

Now let us make the following nondimensionalization

$$
Q=\frac{Q_{0} Q^{*}}{\lambda}\left(\Rightarrow Q^{*}(0)=\lambda\right) ; c_{m}=\frac{Q_{0}^{2} c^{*}}{\lambda}
$$

Then

$$
c_{m}^{*}=Q^{2}-Q-\frac{4 \alpha \lambda(2 \lambda-1) M^{5 / 2}}{5 F_{0} Q_{0}^{2}}
$$

We now obtain a condition to determine when $w=0$. Clearly, from initial conditions

$$
c<\lambda(\lambda-1)
$$

$Q$ increases initially, and $\lambda(\lambda-1)$ goes through a minimum when $\lambda=1 / 2$. Thus if $c_{m}<$ $-1 / 4, M$ will be positive always . Also, since $Q$ is initially increasing, if $\lambda>1 / 2, Q^{*}\left(Q^{*}-1\right)$ increases also, and thus $M$ can never be zero in this case either. This is to be expected, since $\lambda>1 / 2$ corresponds to fluid that remains buoyant at all times. However, if $\lambda<1 / 2$, increasing $Q$ actually decreases $Q^{*}\left(Q^{*}-1\right)$, and thus if $c_{m}>-1 / 4$, we will always have at some height, (which may be calculated)

$$
c_{m}=Q^{* 2}-Q^{*},
$$

i.e. $M=0$. Thus the condition for stopping of the plume is

$$
\lambda\left((1-\lambda)+\frac{4 \alpha(2 \lambda-1) M_{0}^{5 / 2}}{5 F_{0} Q_{0}}\right)<\frac{1}{4} .
$$

We see that this occurs for small initial momentum flux, and large initial density anomaly or mass flux, all of which require that large amounts of mixing must take place before the plume can become buoyant. The height of rise for several different values of $\lambda$ are plotted in fig. 17. We note that the curves go off to infinity when $-c_{m}=1 / 4$, and that the solution is only defined for $-c_{m}>\lambda-\lambda^{2}$. We must remember that after these heights, the simple model of entrainment, with similarity of velocity and turbulence structures, breaks down. The process of mixing is likely to be extremely complicated about such a height. However, jump conditions on mass flux and radius may be applied across such a region between two areas of approximately plumelike behaviour. This is the subject of further investigation, as is the behaviour of a plume of fluid that starts off buoyant, and then becomes dense. This is analogous to the behaviour in a stratified environment, and preliminary results suggest that in this situation, for given initial mass flux, there also exists a well defined minimum height of rise with associated initial momentum flux. 


\section{Conclusions and Future Directions}

In this report we have described a generalization of the classical Morton, Taylor, Turner model to nonzero initial mass flux, and investigated the effect of this generalization on the final height of rise in a stratified environment. The relatively large variations predicted occur if the initial mass and momentum fluxes are finite when the stratification becomes important. This model is likely to be applicable to the behaviour of plumes that pass through a region of varying $N^{2}$. As an example volcanic eruption clouds can rise to heights of tens of kilometres. Since the Brunt-Vaisala frequency approximately doubles at about $10 \mathrm{~km}$, i.e. at the tropopause, (the boundary between the troposphere and the stratosphere), where the plume would be likely to have low $g^{\prime} / b$ and large $M, Q$, perturbations from a pure plume balance may have significant effect on the final height of rise, according to this model. The more realistic complicating factors of nonmonotonic mixing behaviour should be included, especially the interaction of nonmonotonicity with an ambient stratification. The limitations, and assumptions of the model should also be investigated more deeply, and to this end, experiments should be conducted to test its predictions.

\section{Acknowledgements}

This work was suggested by, and done with Andy Woods, who brought new meaning to the word collaboration. His unfailing enthusiasm, penetrating insight and availability for discussion (particularly while driving) were greatly appreciated. I would like to thank the Steering Committee, and in particular Rick Salmon, for organising such a stimulating and enjoyable summer, Jack Whitehead and W. H. O. I. for giving us access to their laboratory for some preliminary experiments, and Bob Frazel, for all his varied help. Finally, I would like to acknowledge the support of H. S. E. Sheffeld, U. K. and the contributions towards my travel expenses of D. A. M. T. P. and Churchill College, University of Cambridge.

\section{References}

Fischer, H. B., List, E. J., Koh, R. C. Y., Imberger, J. \& Brooks, N. H. 1979 Mixing in Inland and Coastal Waters. Academic. 483pp.

Lane-Serf, G. F., Linden P. F., \& Hillel, M. 1989 Forced, Angled Plumes. private communication.

List, E. J., 1982 Turbulent Jets and Plumes. Ann. Rev. Fluid Mech. 14, 189-212.

Morton, B. R. 1959 Forced Plumes. J. Fluid Mech. 5, 151-163.

Morton, B. R., Taylor G. I. \& Turner, J. S. 1956 Turbulent Gravitational convection from maintained and instantaneous sources. Proc. $R$. Soc. Lond. A 234, 1-23.

Squires, P. \& Turner, J. S. 1962 An entraining jet model for cumulonimbus updraughts. Tellus 14, 422-434.

Taylor, G. I. 1945 Dynamics of a mass of hot gas rising in air. U. S. Atomic Energy Commission MDDC 919. LADC 276.

Turner, J. S. 1973 Buoyancy Effects in Fluids. Cambridge University Press, 367 pp.

Turner, J. S. 1986 Turbulent entrainment: the development of the entrainment assumption, and its application to geophysical flows. J. Fluid Mech. 173, 431-471. 

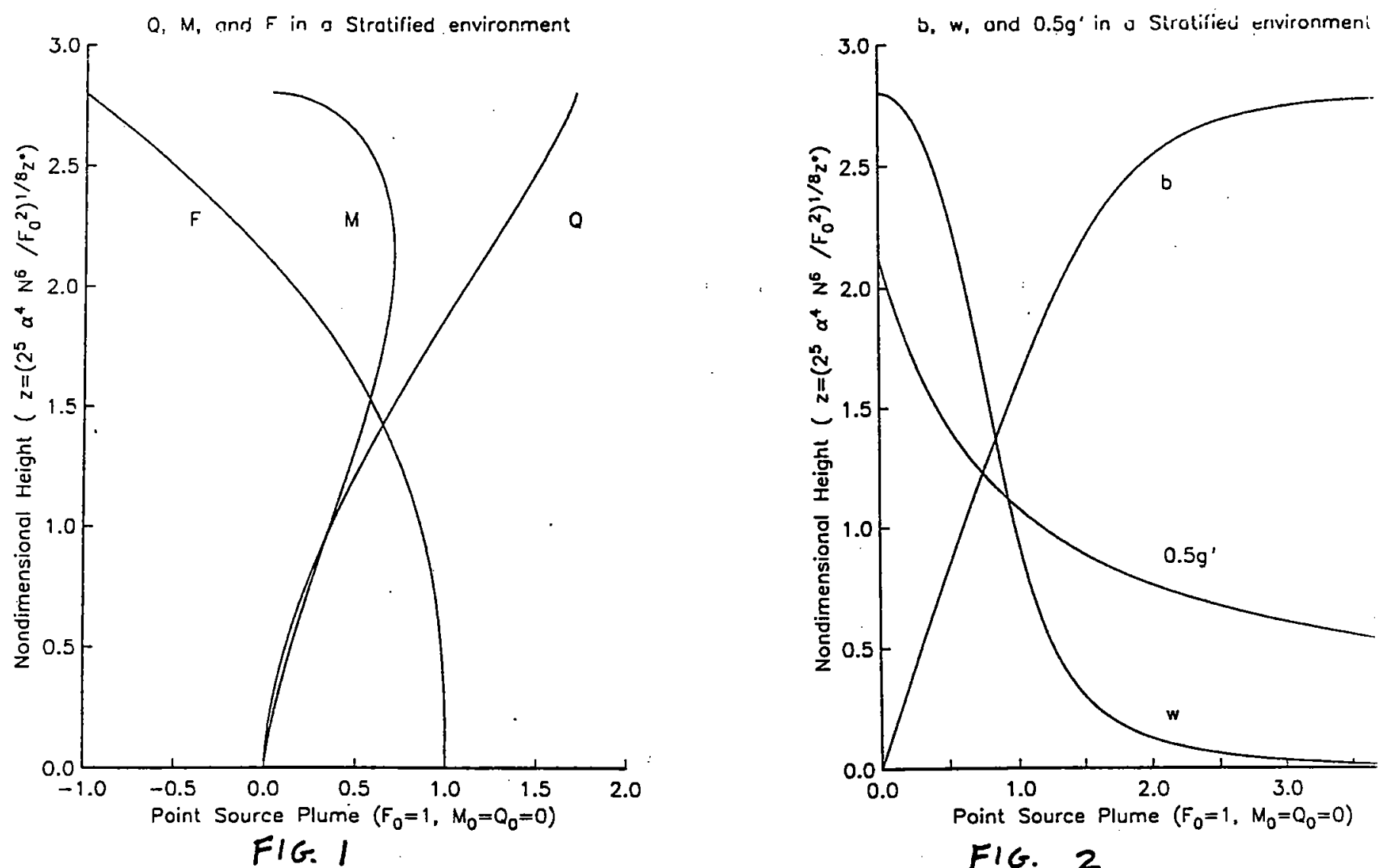

FIG. I

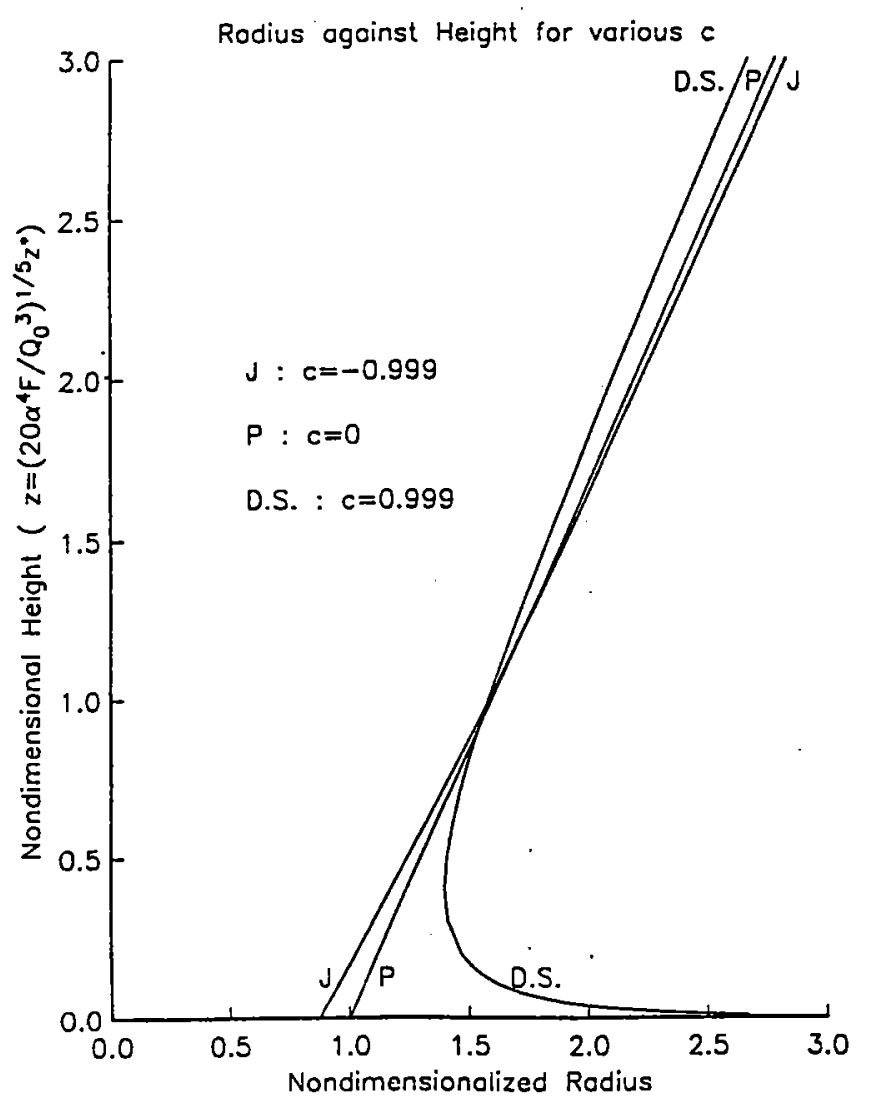

FIG. 3

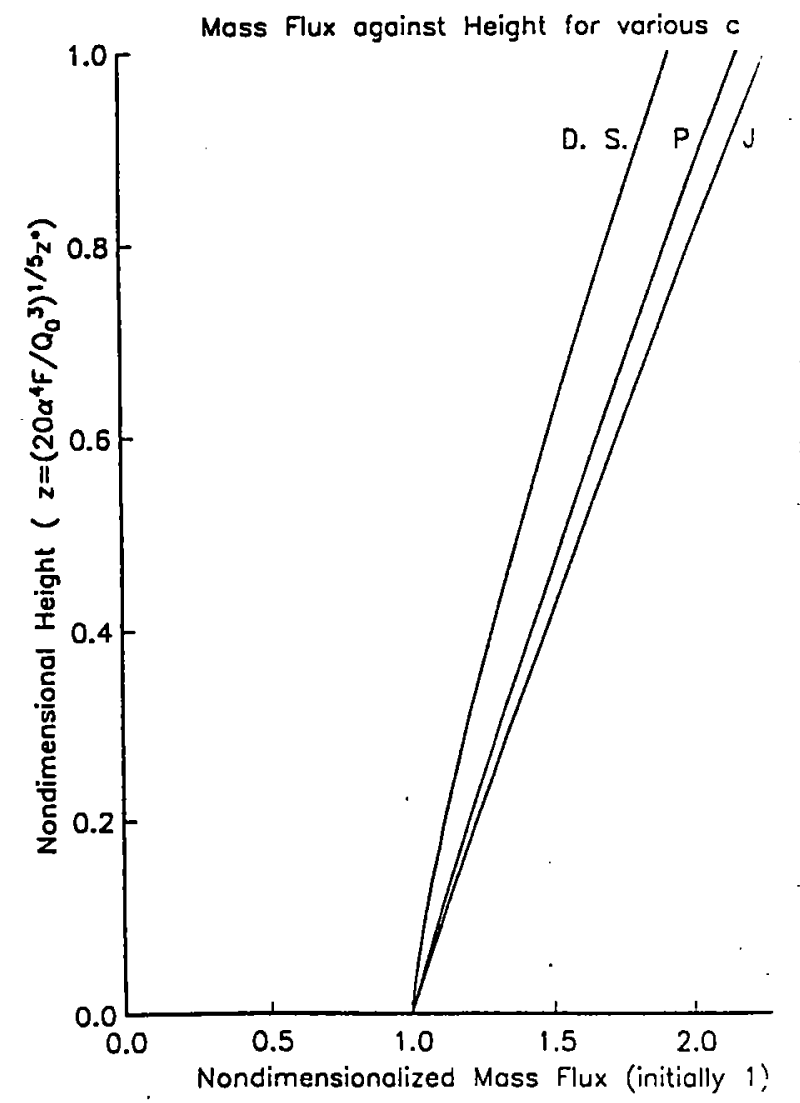

FIG. 4 

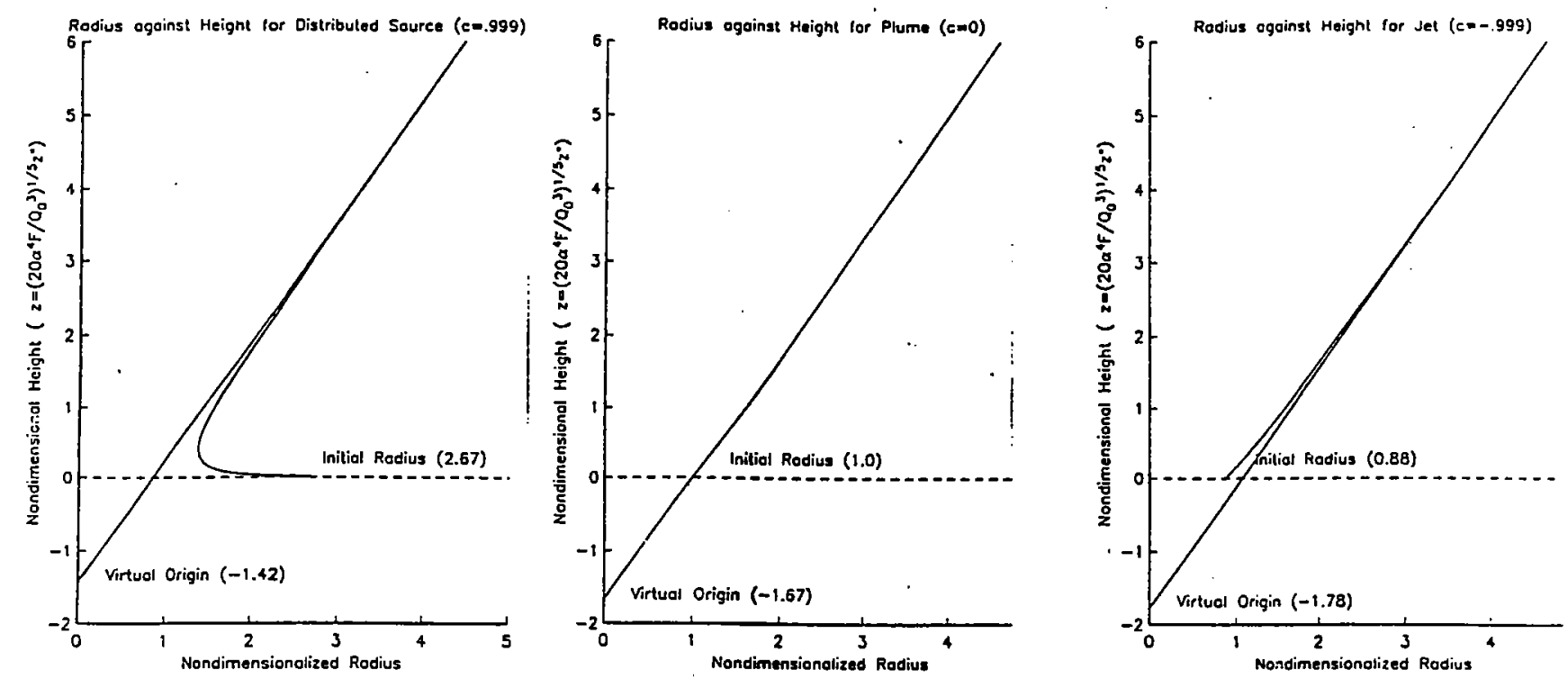

FIG. 5

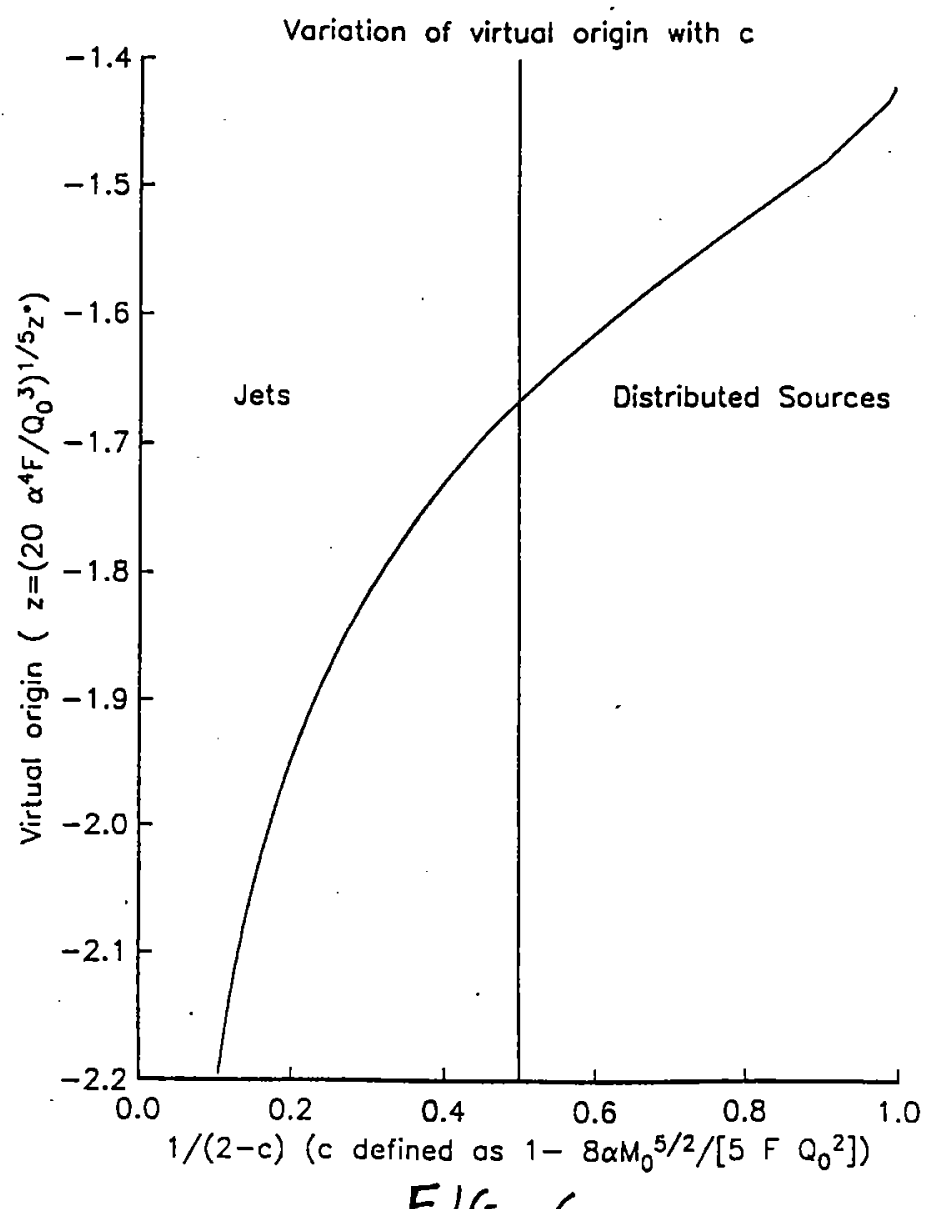

FIG. 6 


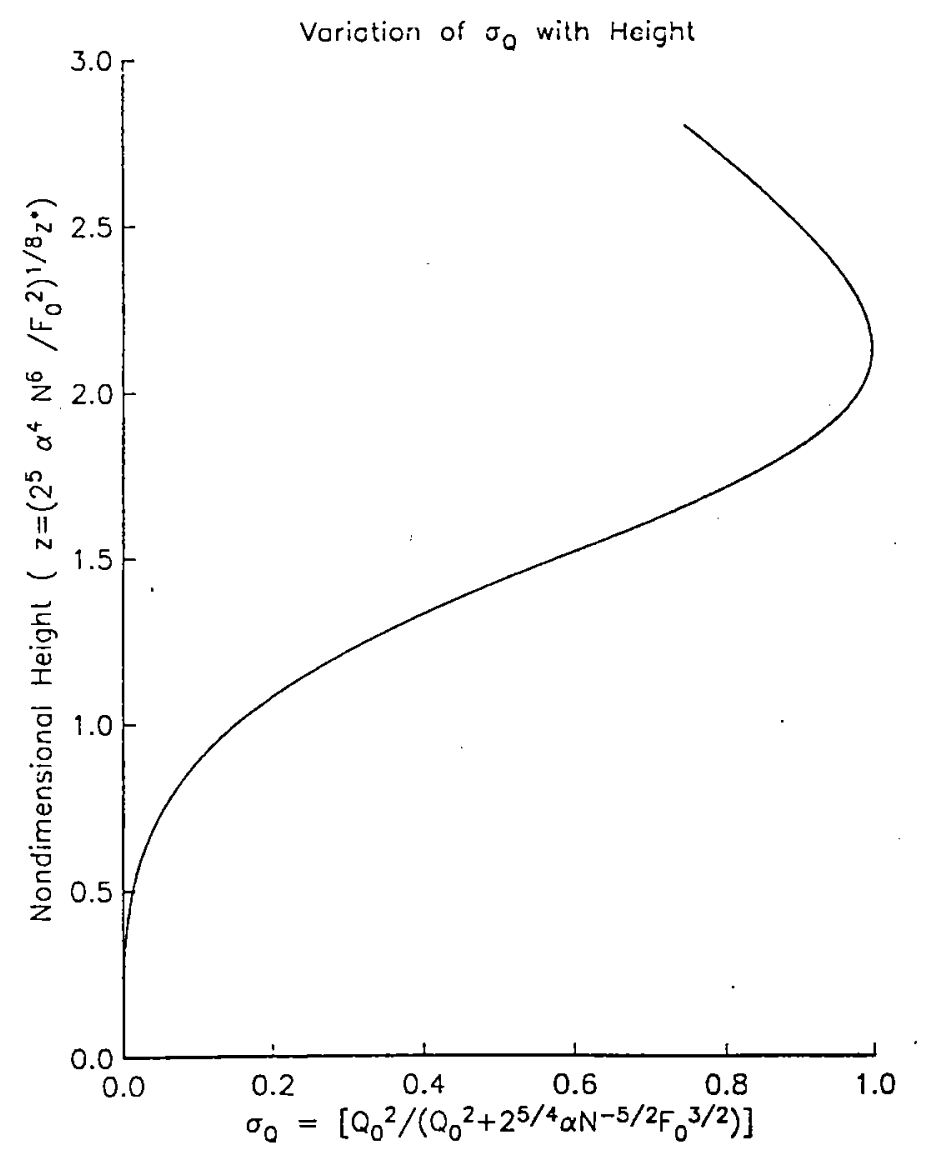

FIG. 7

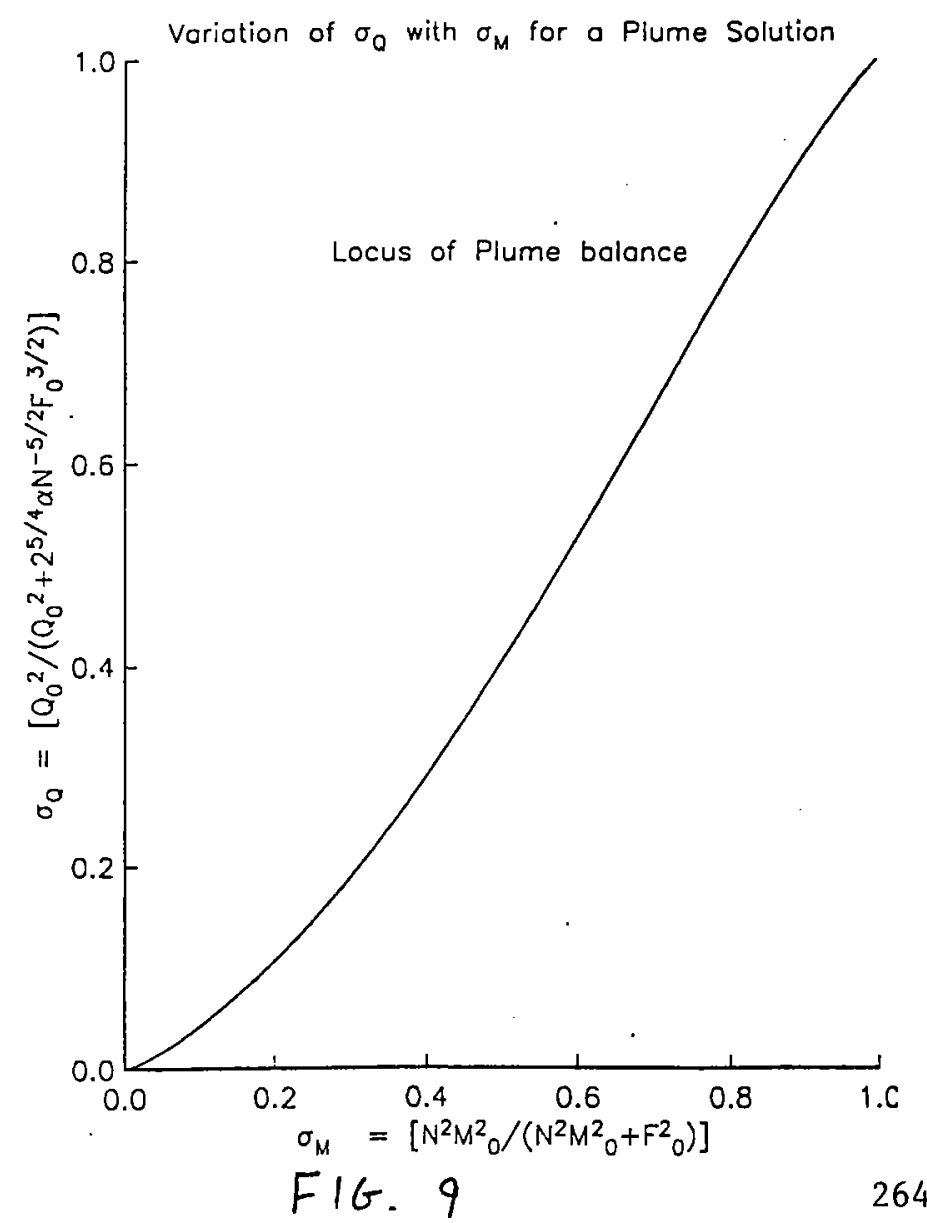

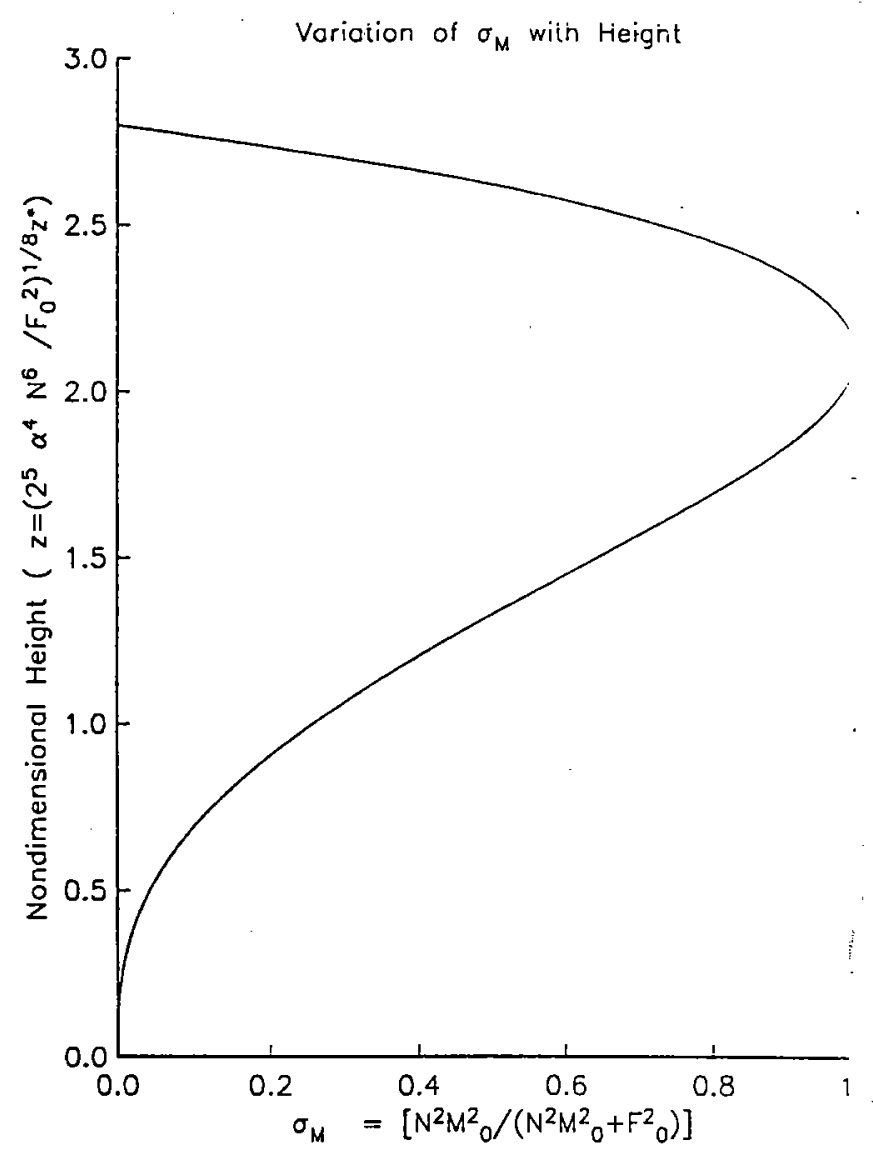

FIG 8

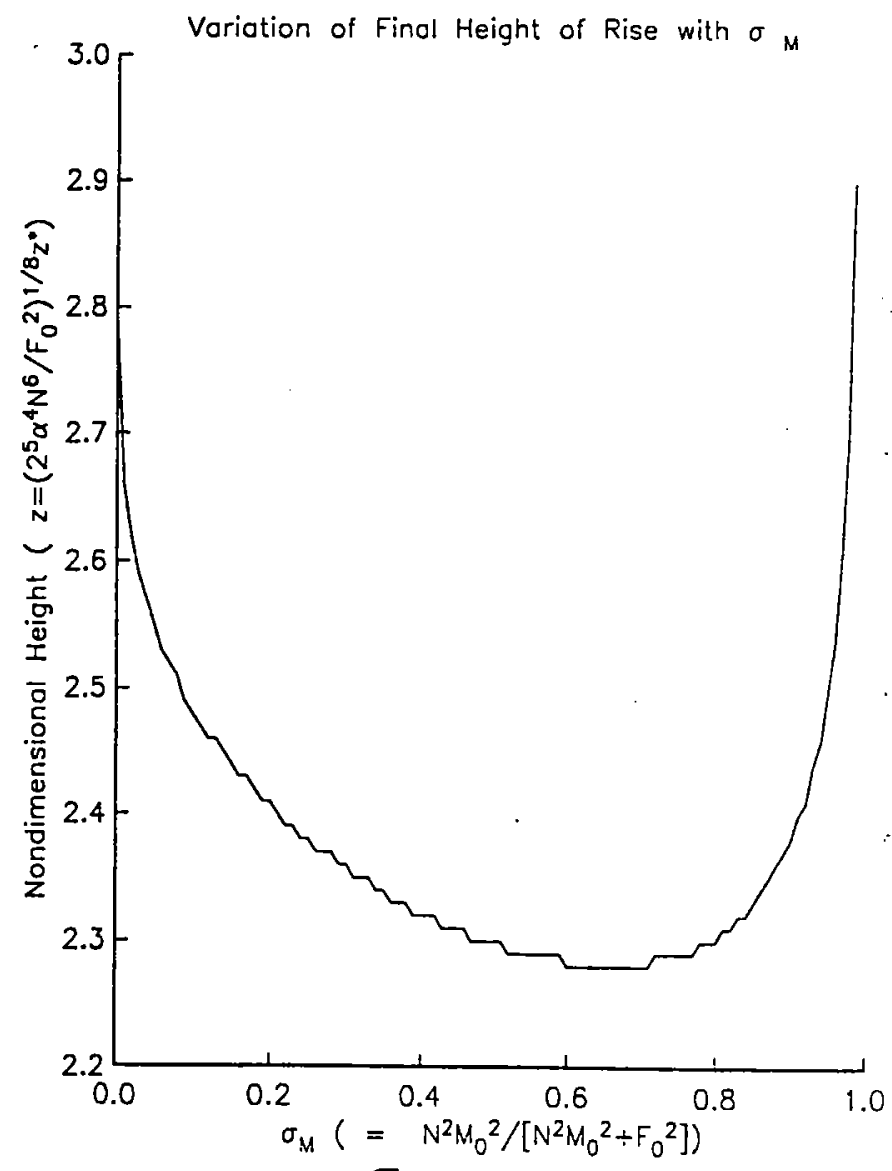

$F / G .10$ 

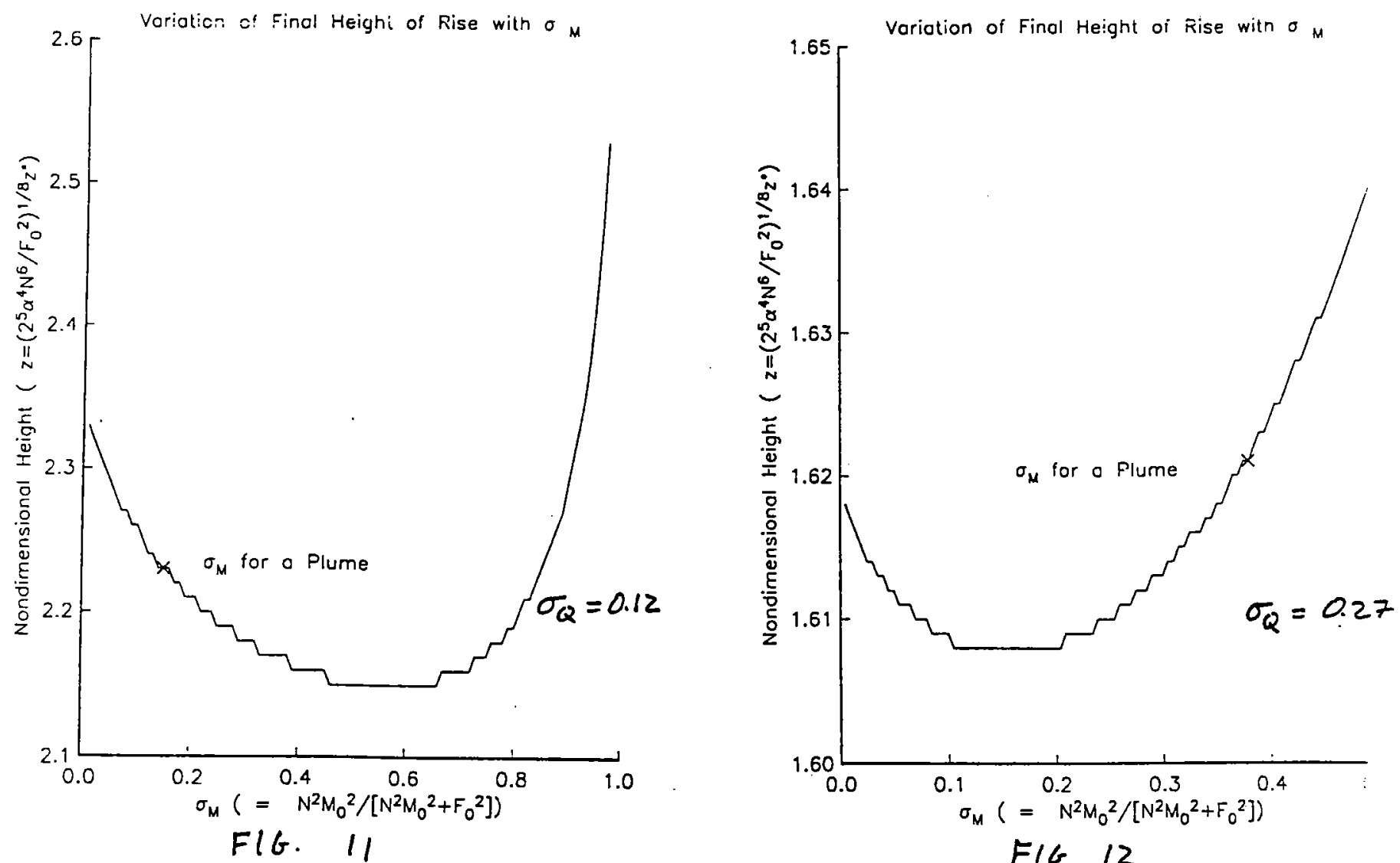

FIG. 12

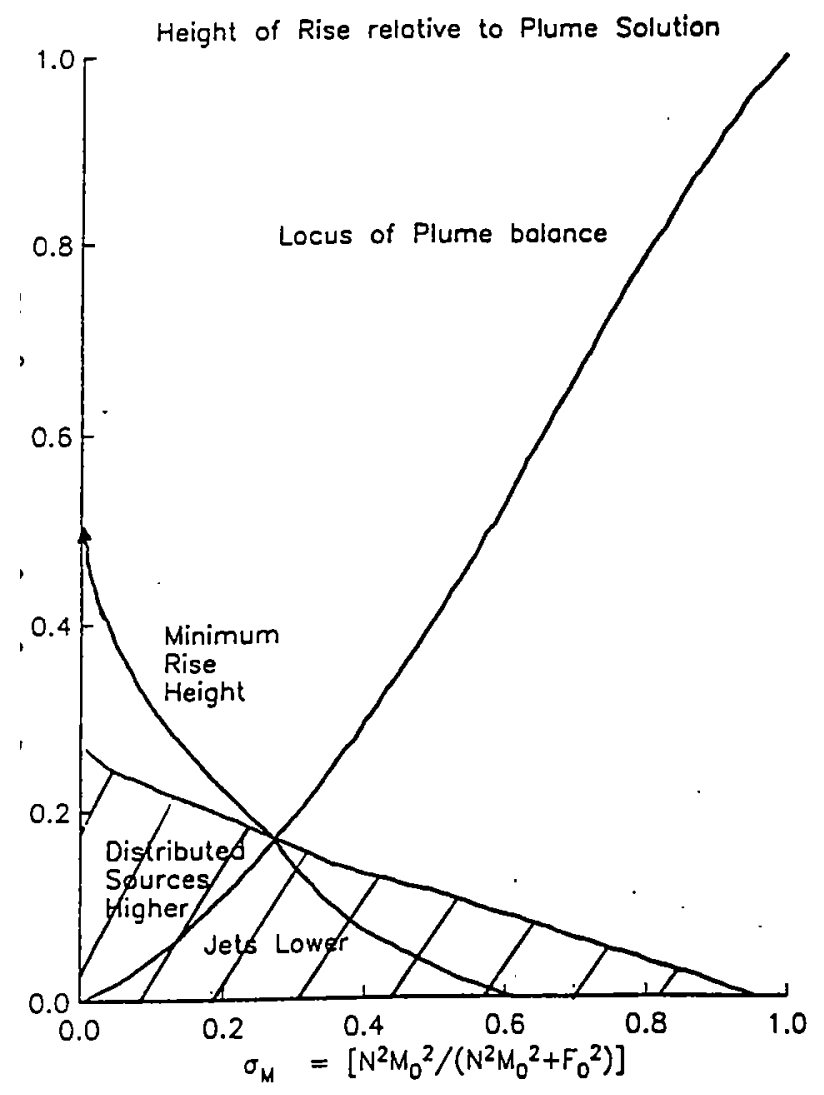

$F / G .13$

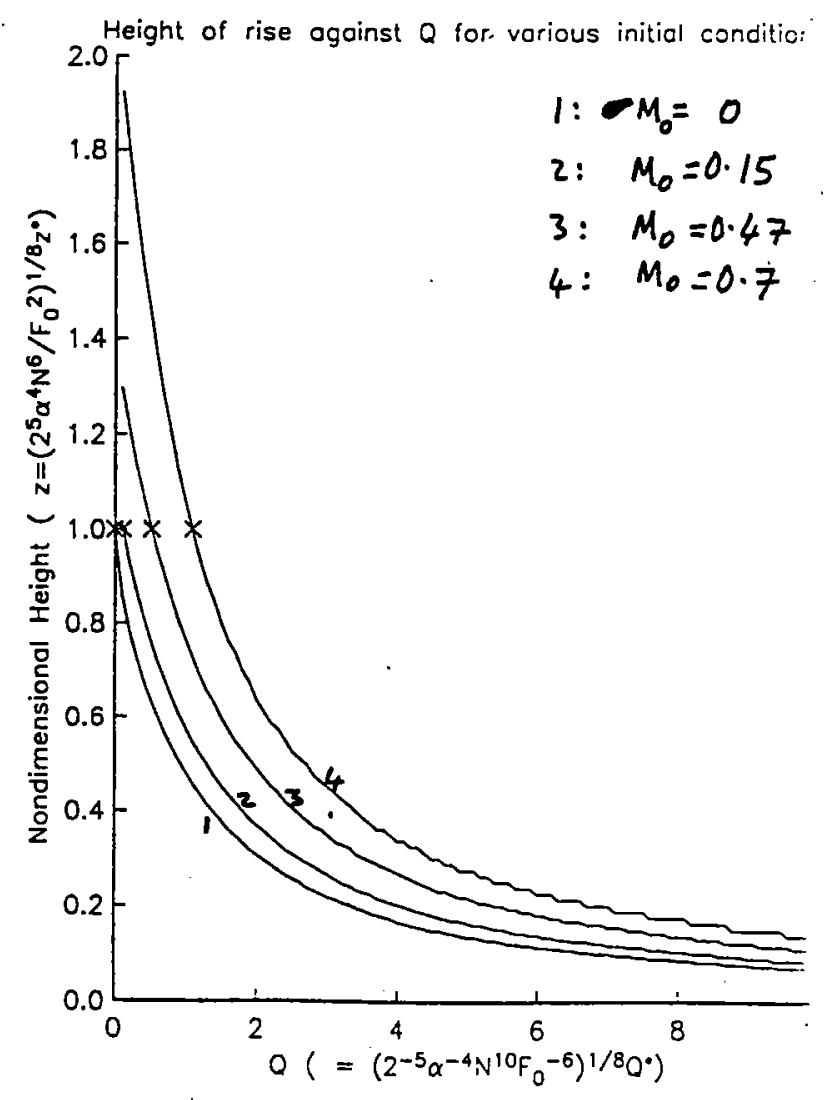

FIG. 14 


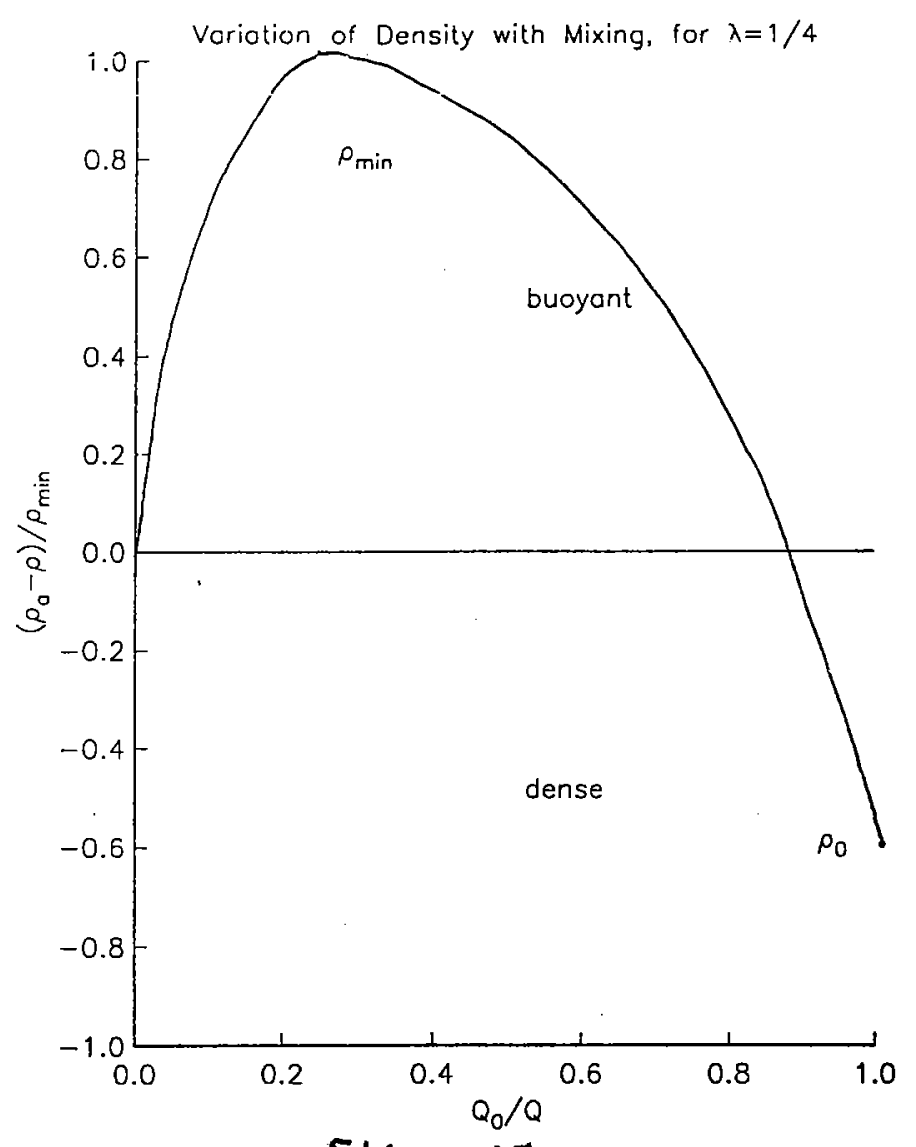

FIG. 15

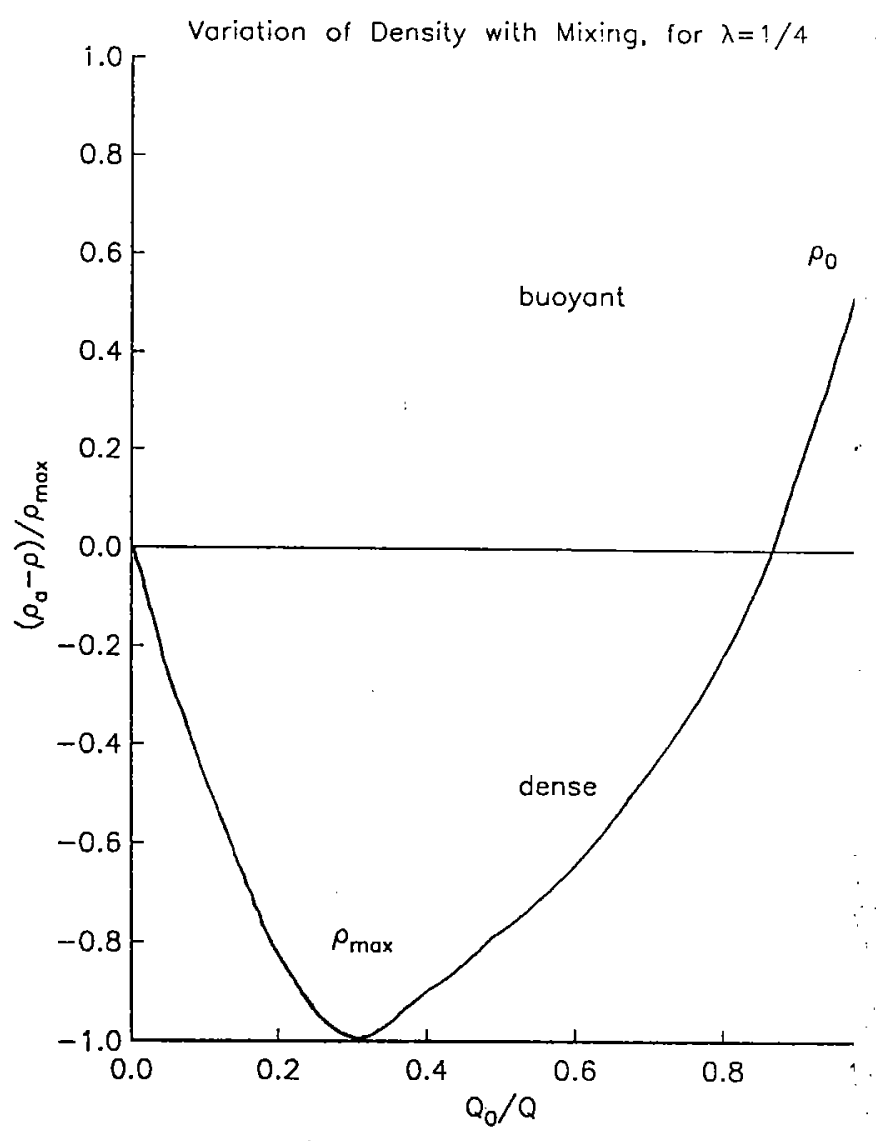

FIG. 16

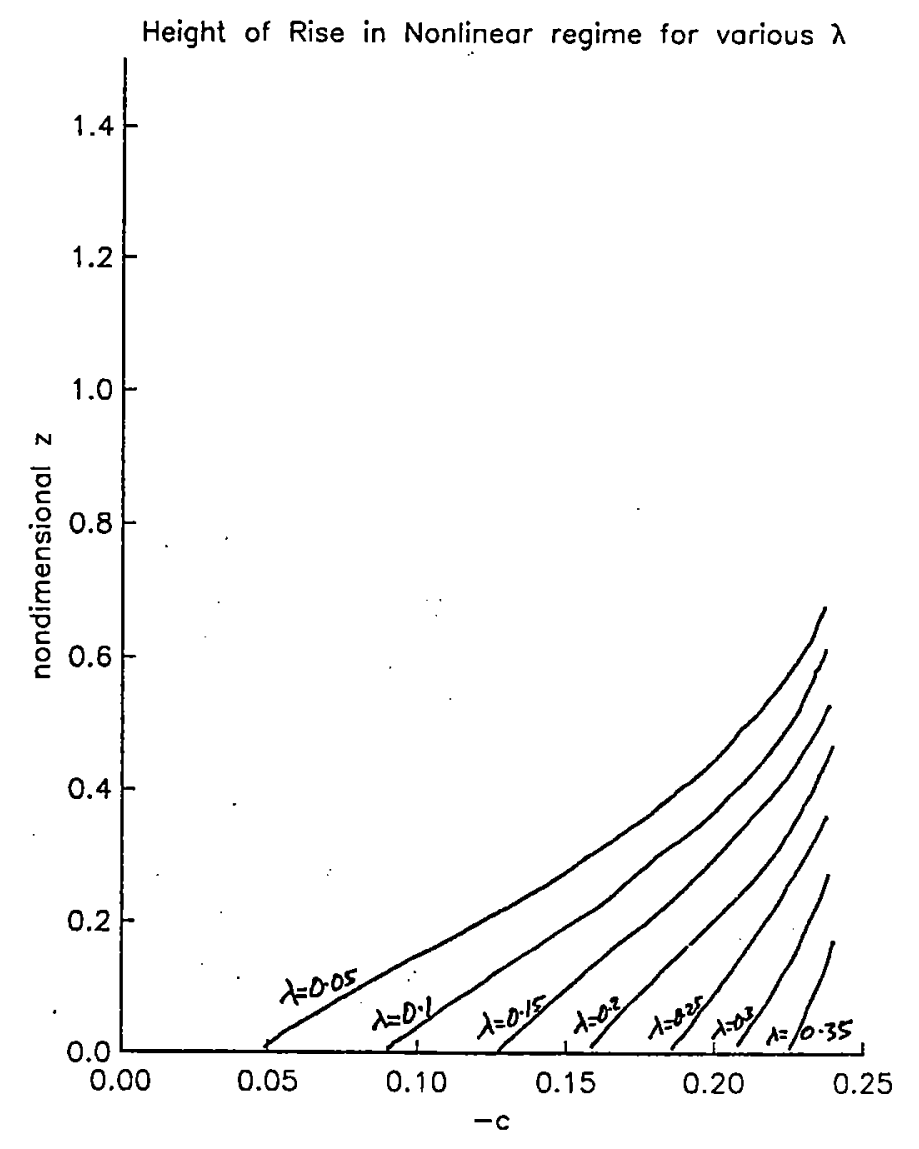

FIG. 17 


\title{
Transport of a Chemical
}

\section{in Stellar Radiative Zones}

\author{
Brian Chaboyer
}

\begin{abstract}
In this report, we examine under what conditions it is appropriate to treat the transport of a chemical in a stellar radiative zone due to a large scale velocity field as a pure diffusion process. We obtain an expression for this diffusion coefficient in terms of this velocity field and the turbulent diffusivities. This diffusive transport may be slower than the transport due to advection by the velocity field. We show that our results are consistent with observations of $\mathrm{Li}$ in the surface of stars, and may explain the work of Charbonneau, Michaud and Proffitt (1989) who found that some process was inhibiting the advection of Li by Eddington-Sweet circulation.
\end{abstract}

\section{Introduction}

In the convectively stable regions of a star, chemicals can be transported by large spatial scale (slow time scale) circulations and by molecular diffusion. Large scale meridional circulations, such as Eddington-Sweet circulation, are induced in a star by thermal instabilities (Eddington 1925, Sweet 1950) and may be the dominant cause of the transport of chemicals within a star, as the molecular diffusion coefficients are very small.

In attempting to model the evolution of a rotating star, the transport of chemicals due to the large scale motions is sometimes treated as diffusion process with a turbulent diffusion coefficient determined from the advection velocity, (see, for example Endal and Sofia 1978, Pinsonneault et al. 1989, Charbonneau and Michaud 1990) even though the equation which describes the transport of a chemical (see equation 1) includes an advection term. In this paper, we outline under what conditions it is appropriate to treat the vertical transport of a chemical as a simple diffusion process, and find an expression for that diffusion coefficient.

Another motivation for this work are the results of Charbonneau, Michaud and Proffitt (1989) who examined the depletion of $\mathrm{Li}$ in giant stars due to the advection of chemicals by Eddington-Sweet circulation on the main sequence (MS). The effects of turbulent diffusion were neglected. For the youngest cluster they studied, no Li depletion is observed, contrary to what would be expected from the Eddington-Sweet circulation. In order to account for this fact, Charbonneau et al. stated: 'one must investigate mechanisms that could have reduced the expected transport through meridional circulation'. An obvious candidate for 
such a mechanism is horizontal turbulent diffusion, which hinders the meridional advection. Such turbulent diffusion may also be able to account for observations of $\mathrm{Li}$ in field dwarfs by Boesgaard and Tripicco (1986b).

In section 2 of this report, we show under what conditions it is valid to treat the transport of a chemical as a diffusion process. Our approach is very similar to that used by G.I. Taylor (1953) who examined the dispersion of a chemical in a pipe with a shear flow. Section 3 compares our work with that of Charbonneau et al. and uses observations of giant stars in open clusters, and of field dwarfs to estimate the average diffusion coefficient which appears in our equations. Our conclusions are presented in section 4.

\section{Transport of a Chemical}

The transport of a chemical with concentration $c$ by both advection and diffusion is given by

$$
\frac{\partial}{\partial t}(\rho c)+\nabla \cdot(\rho c \vec{u})=\nabla \cdot(\rho \mathrm{D} \cdot \nabla c)
$$

where $\rho=$ density, $D=$ turbulent diffusion tensor, and $\vec{u}$ is the velocity. The microscopic diffusivities are very small, and so do not enter into our equations. We are assuming that the turbulent diffusion is due to small scale, turbulent motion which is excited by the strong differential rotation within a star which is induced by the large scale laminar velocity field $\vec{u}$. We are using a tensor for the turbulent diffusion in order to take into account the possibility that the small scale motions are anisotropic. We will assume that $\rho$ and $D$ are functions of the radius only (in spherical polar coordinates). This assumption is valid for stars (such as the Sun) in which the rotation does not introduce a significant departure from spherical symmetry. We shall expand take the radial velocity component in spherical functions

$$
u_{r}=\sum_{n} U_{n}(r) P_{n}(\cos \theta)
$$

where $P_{n}(\cos \theta)$ are the Legendre polynomials of order $n$, with $n$ non-zero. The classical Eddington-Sweet circulation (Eddington 1925, Sweet 1950) is described by a single $P_{2}(\cos \theta)$. We will assume axial symmetry, so there will be no dependency on the longitudinal direction.

It is convenient to express the concentration as

$$
c=c_{o}(r)+\delta c(r, \theta)
$$

where the horizontal average of $\delta c$ (denoted by $\langle\delta c\rangle$ ) is zero and the horizontal average of a function $f$ is defined as:

$$
<f>\equiv \frac{1}{2} \int_{0}^{\pi} f \sin \theta d \theta .
$$


Equation (1) may be expanded as

$$
\begin{aligned}
\rho \frac{\partial}{\partial t} c_{o}+\rho \frac{\partial}{\partial t} \delta c & +\rho u_{r} \frac{\partial}{\partial r} c_{o}+\rho \vec{u} \cdot \nabla \delta c \\
& =\nabla \cdot\left[\rho \mathrm{D} \cdot \nabla\left(c_{o}+\delta c\right)\right]
\end{aligned}
$$

where we have assumed that $\rho$ does not vary in time, so that

$$
\nabla \cdot(\rho c \vec{u})=\rho u_{r} \frac{\partial c_{o}}{\partial r}+\rho \vec{u} \cdot \nabla \delta c
$$

Taking the horizontal average in spherical polar coordinates of equation (5) we obtain

$$
\rho \frac{\partial}{\partial t} c_{o}+\frac{1}{r^{2}} \frac{\partial}{\partial r}\left[r^{2} \rho<\delta c u_{r}>\right]=\frac{1}{r^{2}} \frac{\partial}{\partial r}\left[r^{2} \rho D_{V} \frac{\partial c_{o}}{\partial r}\right]
$$

where we have separated the diffusion tensor into vertical $\left(D_{V}\right)$ and horizontal $\left(D_{H}\right)$ components.

In order to calculate the advective flux $<\delta c u_{r}>$, we need to determine $\delta c$. Assuming that

$$
\frac{\partial c_{o}}{\partial r}>>|\nabla \delta c|
$$

then the term $\rho \vec{u} \cdot \nabla \delta c$ may be ignored in equation (5). Essentially, we are assuming that the concentration of a chemical varies much more strongly in the radial direction than in the horizontal. This will be true if the horizontal diffusion coefficient is much greater than the vertical diffusion coefficient (see equation (10) and discussion thereafter). Subtracting equation (7) from (5) yields

$$
\begin{aligned}
\rho \frac{\partial}{\partial t} \delta c+\rho u_{r} \frac{\partial c_{o}}{\partial r} & =\frac{1}{r^{2}} \frac{\partial}{\partial r}\left[D_{V} \rho r^{2} \frac{\partial \delta c}{\partial r}\right] \\
& +\frac{1}{r^{2} \sin \theta} \frac{\partial}{\partial \theta}\left[\sin \theta D_{H} \rho \frac{\partial \delta c}{\partial \theta}\right]
\end{aligned}
$$

We now assume that

$$
D_{H}>D_{V} \frac{\ell_{H}^{2}}{\ell_{V}^{2}}
$$

where $\ell_{H}\left(\ell_{V}\right)$ is the distance over which $\delta c$ changes in the horizontal (vertical) direction, so that the first term on the right hand side of equation (10) may be ignored. As $D_{H}$ and $D_{V}$ are turbulent diffusion coefficients, equation (10) requires that the small scale motions are much more vigorous in the horizontal than in the vertical. This will be easily satisfied within the radiative regions of a star, in which the vertical velocities are inhibited by the gravitational force ( $\mathrm{Zahn} 1983$ ). In addition, we will assume that we are in a steady state, which is established when

$$
\frac{1}{t} \leq \frac{D_{H}}{r^{2}}
$$


Under the above two assumptions, and replacing $u_{r}$ by expression (2), equation (9) becomes

$$
\sum_{n}\left[\frac{r^{2}}{D_{H}} U_{n}(r) \frac{\partial c_{o}}{\partial r}\right] P_{n}(\cos \theta)=\frac{1}{\sin \theta} \frac{\partial}{\partial \theta}\left[\sin \theta \frac{\partial \delta c}{\partial \theta}\right] .
$$

The Legendre polynomials are solutions of the equation

$$
-n(n+1) P_{n}(\cos \theta)=\frac{1}{\sin \theta} \frac{\partial}{\partial \theta}\left[\sin \theta \frac{\partial}{\partial \theta} P_{n}(\cos \theta)\right]
$$

thus, the solution of equation (12) is

$$
\delta c=\sum_{n} \frac{-r^{2} U_{n}(r)}{n(n+1) D_{H}} \frac{\partial c_{o}}{\partial r} P_{n}(\cos \theta)
$$

where we have imposed the condition $\langle\delta c\rangle=0$.

We may now calculate the average advected flux

$$
\begin{aligned}
<\delta c u_{r}> & \equiv \int_{0}^{\pi} \delta c u_{r} \sin \theta d \theta \\
& =\sum_{n}-\frac{r^{2} U_{n}^{2}(r)}{2 n(n+1) D_{H}} \frac{\partial c_{o}}{\partial r} \int_{0}^{\pi}\left|P_{n}(\cos \theta)\right|^{2} \sin \theta d \theta \\
& =\sum_{n} \frac{-1 / 2}{n(n+1)(n+1 / 2)} \frac{r^{2} U_{n}^{2}(r)}{D_{H}} \frac{\partial c_{o}}{\partial r} .
\end{aligned}
$$

Thus, subject to the conditions given in equations (8), (10) and (11), the transport of a chemical within the radiative region of a star can be described as a pure diffusion process:

$$
\rho \frac{\partial}{\partial t} c_{o}=\frac{1}{r^{2}} \frac{\partial}{\partial r}\left[r^{2} \rho D_{t} \frac{\partial c_{o}}{\partial r}\right]
$$

with

$$
D_{t}=D_{V}+D_{*}
$$

where

$$
D_{*}=\sum_{n} \frac{1 / 2}{n(n+1)(n+1 / 2)} \frac{r^{2} U_{n}^{2}(r)}{D_{H}} .
$$

In particular, if we consider Eddington-Sweet circulation ( $n=2$ only), we see that

$$
D_{t}=D_{V}+\frac{r^{2} U_{o}^{2}(r)}{30 D_{H}}
$$

\section{Comparison to Li Observations}

In order to complete this work, it is necessary to obtain expressions for the turbulent diffusion coefficients in the vertical and horizontal directions. Unfortunately, this is a difficult 
problem, for which we have not been able to obtain a solution based on the principles of fluid dynamics. The only path left to us to estimate the diffusion coefficients is to consider observations of the surface abundances of stars.

Lithium is an important probe of stellar transport processes as it is destroyed when $T>2.6 \times 10^{6} \mathrm{~K}$ and this temperature is generally located in the radiative region of a star. Thus, a star which has an initially homogeneous chemical content, will arrive on the zero age main sequence (ZAMS) with a radial $\mathrm{Li}$ profile which is essentially a step function and defines a $\mathrm{Li}$ front. Below some critical radius $\left(r_{\mathrm{Li}}\right)$ there will be no $\mathrm{Li}$, above $r_{\mathrm{Li}}$ there will be the initial, so called cosmic $\mathrm{Li}$ abundance. This critical radius occurs in the radiative zone of most stars. If no chemical transport processes occur within the radiative zone of a star, then the amount of and location of $\mathrm{Li}$ in the star would remain constant at its ZAMS value.

Let us examine the effects of a large scale meridional circulation. Advection by EddingtonSweet circulation, if it occurres, distorts the $\mathrm{Li}$ front. At the poles (where $u_{r}$ is positive), the $\mathrm{Li}$ front will be moved upward. Near the equator (where $u_{r}$ is negative) Li will be carried below $r_{\mathrm{Li}}$ and so will be burned. Thus, Eddington-Sweet circulation will cause a continual decrease in the total amount of Li present in the star. However, as the star evolves on the MS, the radius at which $\mathrm{Li}$ is destroyed will move downward (in mass fraction) (Charbonneau, Michaud and Proffitt 1989, hereafter CMP), with a velocity of $u_{\mathrm{Li}}$. Thus, only when $\left|u_{r}\right|>\left|u_{\mathrm{Li}}\right|$ will $\mathrm{Li}$ be progressively destroyed on the MS due to advection by EddingtonSweet circulation. The velocity of Eddington-Sweet circulation depends critically on the rotation rate, $u_{r o t} ; u_{r} \propto u_{\text {rot }}^{2}$. Assuming solid-body rotation, CMP (who studied stars with $1.2 \leq M / M_{\odot} \leq 2.0$ ) found that no MS Li depletion occurres when $u_{r o t} \leq 20 \mathrm{~km} / \mathrm{s}$. When $u_{\text {rot }} \geq 35 \mathrm{~km} / \mathrm{s}$, essentially the full amount of $\mathrm{Li}$ depletion due to advection by EddingtonSweet circulation will occur. For the mass range of interest here, rotational velocities are typically $100 \mathrm{~km} / \mathrm{s}$, and so the full amount of $\mathrm{Li}$ depletion is expected for most stars.

If the Eddington-Sweet circulation penetrates the surface convection zone (as appears likely and was implicitly assumed by CMP), then the full amount of Li depletion will occur rather quickly. From Figure 3 in CMP, we see that a $1.5 M_{\odot}$ with $u_{\text {rot }}=50 \mathrm{~km} / \mathrm{s}$, will have no $\mathrm{Li}$ at its surface after just $0.286 \mathrm{Gyr}$. However, Balachandran (1990), who observed $199 \mathrm{~F}$ dwarfs, found numerous stars with $v \sin i \geq 50 \mathrm{~km} / \mathrm{s}$ in which the Li abundances were near cosmic. This is a sign that the destruction of $\mathrm{Li}$ on the surface of a star due to Eddington-Sweet circulation is being inhibited. CMP suggest that a boundary layer may form between the radiative zone and the surface convection zone, which prevents the Eddington-Sweet circulation from reaching the surface, so that no $\mathrm{Li}$ depletion will occur on the MS. The formation of such a boundary layer is unlikely, as there is no physical reason for its existence. If it did form, this would imply that no MS Li depletion would be observed. This is contrary to many observations of MS F stars (e.g. Balachandran 1990 and Boesgaard and Tripicco 1986b) which show significant Li depletion.

After a star has exhausted the $H$ in its core, it evolves off the MS onto the giant branch. During the rapid post-MS phase of evolution, a very deep convective zone will develop. 
This will mix $\mathrm{Li}$ depleted matter with $\mathrm{Li}$ rich matter, causing a dilution of the surface $\mathrm{Li}$ abundance, which is easily observable. If Eddington-Sweet circulation has caused $\mathrm{Li}$ to be destroyed on the MS, then the observed abundances in giant stars will be lower than that due to dilution during the post-MS evolution. CMP compared observations of $\mathrm{Li}$ in giants in three clusters $\left(\mathrm{M} 67\right.$ age $\approx 5 \mathrm{Gyr}$, turnoff mass $\approx 1.3 M_{\odot}$, NGC 752 age $\approx 2.2$ Gyr, turnoff mass $\approx 1.6 M_{\odot}$ and NGC 7789 age $\approx 1.6 \mathrm{Gyr}$, turnoff mass $\approx 1.8 M_{\odot}$ ) to that which would would be expected from standard stellar evolution with the advection of chemicals due to Eddington-Sweet circulation (diffusion was ignored). A major difficulty with this work is that the MS rotation velocity (and its evolution) for a particular star is not known - there is a spread in rotation velocities for stars with the same mass. Thus, one must examine a large enough sample of stars in order to use statistical arguments. CMP found that Li observations of M67 and NGC 752 were consistent with theoretical values computed including the effects of advection. However, in NGC 7789, there appeared to be no Li depletion in giant stars as would be expected from advection by EddingtonSweet circulation. Furthermore, observations by Pilachowski (1986) found normal (comic) $\mathrm{Li}$ abundances in stars near the turn off in NGC 7789 .

We may take this as a sign that horizontal diffusion is inhibiting the effects of the advection. This will occur when the time scale of advection is less than the time scale of the diffusion, i.e. we are in a steady state, which requires that equation (11) be satisfied. The time scale of diffusion is given by $t_{\text {diff }}=L^{2} / D_{t}$, where $L$ is the distance over which $\mathrm{Li}$ must travel in order to reach the surface, while the time scale of advection by EddingtonSweet circulation is $t_{\text {advec }}=L / U_{E S}$, where $U_{E S}$ is the Eddington-Sweet velocity. Hence, we require that $D_{t}<L U_{E S}$, which, from equation (19) implies that the horizontal diffusion coefficient must satisfy

$$
D_{H}>\frac{1}{30} \frac{U_{E S} R^{2}}{L}=\frac{1}{30} \frac{R^{2}}{t_{\text {advec }}} .
$$

By assuming that $L i$ is being diffused in the radiative zones of $F$ stars, we are able to estimate a time scale for the diffusion for stars with $1.6 \leq M / M_{\odot} \leq 1.8$. Clearly, the effects of diffusion must show up somewhere between 1.6 Gyr and 2.2 Gyr. For our rough estimate of the total diffusion coefficient, we shall take the time scale of diffusion to be $2 \mathrm{Gyr}$. Note that by postulating that diffusion is the principal means of transport of $\mathrm{Li}$ on the MS, we require that the depletion of $\mathrm{Li}$ be observable on the MS in stars which are older than 2 Gyr. Further evidence that the time scale for diffusion is about 2 Gyr may be found in Boesgaard and Tripicco (1986b) who observed $\mathrm{Li}$ in 75 field dwarfs. They found that $62 \%$ of stars younger than $2 \mathrm{Gyr}$ had the cosmic abundance of $\mathrm{Li}$, while $64 \%$ of stars older than 2 Gyr were $\mathrm{Li}$ depleted, with $\log \left(c / c_{0}\right) \approx-2$. This is in agreement with Pilachowski's $(1986)$ observations of no $\mathrm{Li}$ depletion at the MS turn off in NGC 7789. In addition, Hobbs and Pilachowski (1986) found that stars near the turnoff in NGC 752 (age $\approx 1.7 \mathrm{Gyr}$ ) had not suffered any depletion in their surface $\mathrm{Li}$ abundance.

In order to illustrate the expected behaviour from diffusion, we will solve the simplified 


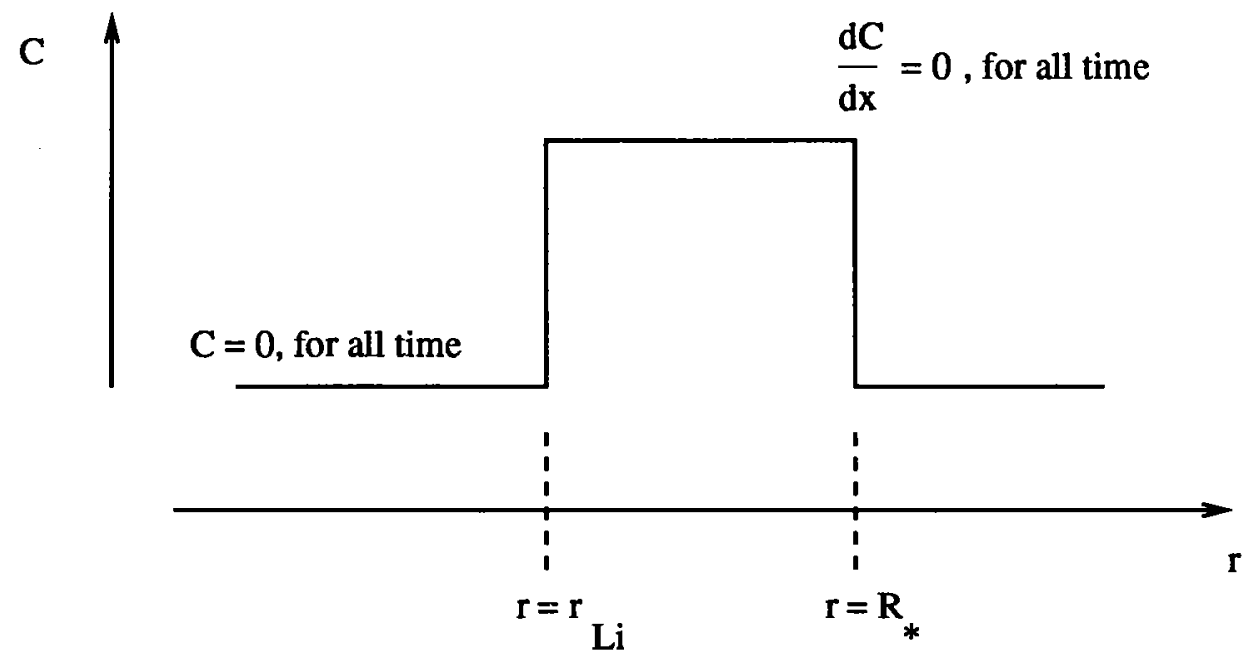

Figure 1: Initial conditions on the Li concentration

equation (c.f. equation (16)) via

$$
\frac{\partial}{\partial t} c=D \frac{\partial^{2}}{\partial r^{2}} c
$$

where we have ignored the effects of sphericity and of a variable diffusion coefficient. We have the initial condition that the $\mathrm{Li}$ concentration is $c=c_{o}$ when $r_{\mathrm{Li}} \leq r \leq R_{*}$. The boundary conditions are that $\mathrm{Li}$ gets destroyed at $r=r_{\mathrm{Li}}$, so that $c=0$ when $r<r_{\mathrm{Li}}$ and that there is no loss of $\mathrm{Li}$ at the surface of the star, so $\partial c / \partial r=0$ at $r=R_{*}$. A illustration of these conditions is shown in Figure 1. Although $r_{\mathrm{Li}}$ is a function of time, we will assume it to be a constant. This is a fairly reasonably assumption, as for a $1.5 M_{\odot}$ star, $L$ will change by less than $3 \%$ in $500 \mathrm{Myr}$.

The solution of equation (21) may be found by fourier decomposing $c$ :

$$
c=c_{0} \sum_{n} a_{n}(t) \sin \left[\left(\frac{\pi}{2}+n \pi\right)\left(\frac{r-r_{\mathrm{Li}}}{R_{*}-r_{\mathrm{Li}}}\right)\right]
$$

which leads to

$$
\frac{\partial a_{n}}{\partial t}=-\frac{\pi^{2}}{4} \frac{D}{\left(R_{*}-r_{\left.\mathrm{Li}_{\mathrm{i}}\right)^{2}}\right.}(2 n+1)^{2} a_{n}
$$

The solution for $c$ is

$$
c=c_{0} \frac{4}{\pi} \sum_{n} \frac{1}{(2 n+1)} \exp \left[-\frac{\pi^{2}}{4}(2 n+1)^{2} \frac{D t}{\left(R_{*}-r_{\mathrm{Li}}\right)^{2}}\right] \sin \left[\left(\frac{\pi}{2}+n \pi\right)\left(\frac{r-r_{\mathrm{Li}}}{R_{*}-r_{\mathrm{Li}}}\right)\right] .
$$

Observers determine the abundance of a chemical on the surface of a star on a decimal logarithmic scale. After a short time, the abundance at the surface will be given by the slowest decaying mode, $n=0$ and so the surface abundance will be given by

$$
\log \left(c / c_{o}\right) \simeq \log \left[\frac{4}{\pi} \exp \left(-\frac{\pi^{2}}{4} \frac{D}{L^{2}} t\right)\right]
$$




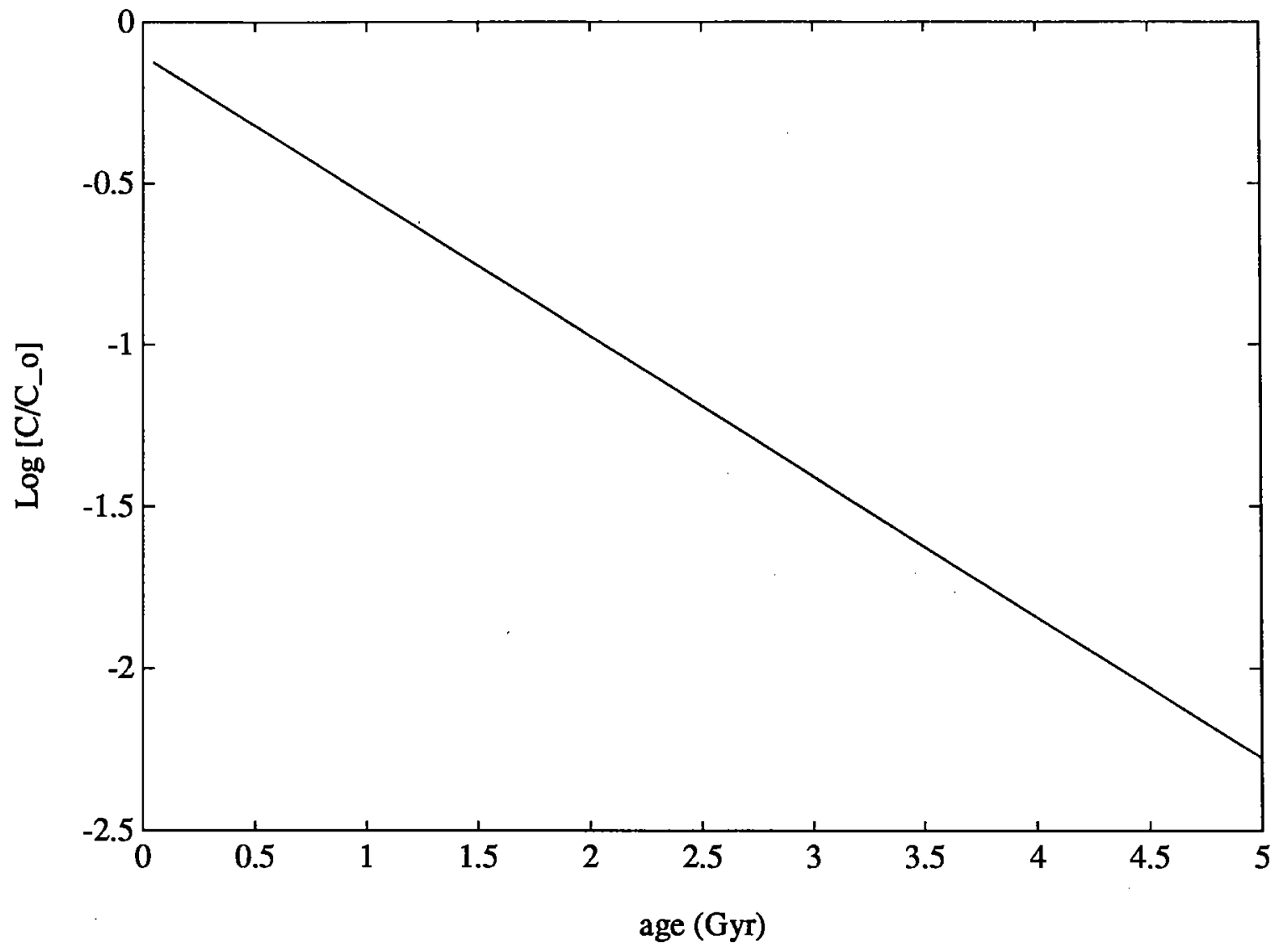

Figure 2: Expected Li depletion due to the transport by turbulent diffusion

where $L \equiv R_{*}-r_{\mathrm{Li}}$. When $x=2, \log \left(4 e^{-x} / \pi\right) \simeq-0.76$, which is the level at which observers consider $\mathrm{Li}$ depleted. Thus, we may associate the time scale of diffusion (2 Gyr) with

$$
2=\frac{\pi^{2}}{4} \frac{D}{L^{2}} t_{D} .
$$

From the Yale evolution code (Prather 1976, Seidel, Demarque, and Weinberg 1987), a $1.5 M_{\odot}$ star with the Hyades metallicity will have $L \simeq 4 \times 10^{8} \mathrm{~m}$ and so we may estimate $D$ for a $1.5 M_{\odot}$ star as

$$
D \simeq 6.5 \times 10^{7} \mathrm{~m}^{2} / \mathrm{yr} \sim 2 \mathrm{~m}^{2} / \mathrm{s} .
$$

A plot of the surface Li abundance (as given by equation (21), with the diffusion coefficient given above) as a function of time is shown in figure 2.

It addition, it is also possible to obtain a lower limit to the value of $D_{H}$ from the fact that the time scale of diffusion must be greater than the time scale of advection. From equations (20) and figure 3 in CMP we see that for a $1.5 M_{\odot}$ star with $u_{r o t}=50 \mathrm{~km} / \mathrm{s}$

$$
D_{H}>2 \mathrm{~m}^{2} / \mathrm{s} \text {. }
$$




\section{Conclusion}

In this paper, we have shown under what conditions a pure diffusion equation (as opposed to an equation which included an advection term) may be used to model the transport of chemicals due to large spatial scale, slow time scale motions, such as those induced by thermal instabilities in the radiative zone of a rotating star. Thus, we have given a theoretical justification for the work of such people as Pinsonneault et al. (1989) and Charbonneau and Michaud (1990) who use a pure diffusion equation to model the transport of chemicals by Eddington-Sweet circulation in a stellar evolution code. However, we are unable to obtain an expression for the turbulent diffusivities which occur in our diffusion equation.

In addition, we have shown that including the effects of turbulent diffusion may explain the results of CMP, who found that $\mathrm{Li}$ was transported on a slower time scale than would be expected from Eddington-Sweet circulation. As horizontal diffusion inhibits the effect of vertical advection, we can explain this result by assuming that the transport of $\mathrm{Li}$ was due to turbulent diffusion, as given by equation (16). This allows us to an averaged diffusion coefficient (c.f. equation (21)) to be $D=2 \mathrm{~m}^{2} / \mathrm{s}$ for early $\mathrm{F}$ stars. This requires that the horizontally turbulent diffusion coefficient be greater than $2 \mathrm{~m}^{2} / \mathrm{s}$. These value are consistent with the observations of Boesgaard and Tripicco (1986b) who found Li depletion occurred in dwarf stars older than 2 Gyr, and observations by Pilachowski (1986) who found no $\mathrm{Li}$ depletion in MS turn off stars in NGC 7789 (age $\approx 1.6 \mathrm{Gyr}$ ). The Li gap present in the Hyades cluster (Boesgaard and Tripicco 1986a), which occurres for stars slightly less massive than those considered here, clearly shows that the diffusion coefficient is very sensitive to stellar parameters.

\section{Acknowledgements}

I would like to thank my supervisor Jean-Paul Zahn who made this project possible. He conceived this project and guided me throughout the summer. He helped to make this an interesting and enjoyable summer. I would also like to thank Andy Woods and Rick Salmon with whom I had numerous enlightening discussions. Finally, I would like to thank all the fellows and staff of GFD who made this a very enjoyable summer.

\section{References}

Balachandran, S. 1990, Ap. J., 354, 310.

Boesgaard, A.M. and Tripicco, M.J. 1986a, Ap. J. (Letters), 302, L49.

Boesgaard, A.M. and Tripicco, M.J. 1986b, Ap. J., 303, 724.

Charbonneau, P. and Michaud, G. 1990, Ap. J., 352, 681. 
Charbonneau, P., Michaud, G. and Proffitt, C. R. 1989, Ap. J., 347, 821.

Eddington, A.S. 1925, Observatory, 48, 78.

Endal, A.S. and Sofia, S. 1978, Ap.J., 220, 279.

Hobbs, L.M and Pilachowski, C. 1986, Ap.J. (Letters), 309, L17.

Landau, L.D. and Lifshitz, E.M., 1987, Fluid Mechanics, 2nd English Edition (Oxford: Pergamon Press).

Pilachowski, C. 1986, Ap.J., 300, 289.

Pinsonneault, M.H., Kawaler, S.D., Sofia, S. and Demarque, P. 1989, Ap.J., 338, 424.

Prather, M. 1976, Ph.D. thesis, Yale University.

Seidel, E., Demarque, P. and Weinberg, D. 1987, Ap.J. Supp., 63, 917.

Sweet, P.A. 1950, M.N.R.A.S., 110, 548.

Taylor, G.I. 1953, Proc. Roy. Soc. London, A, $219,186$.

Zahn, J.-P. 1983, Astrophys. Processes in Upper Main Sequence Stars (Genèeve: Publ. Obseratoire Genève), 253. 


\title{
MAGNETIC FLUX TUBES AND CONVECTION
}

\author{
Richard Kerswell
}

August, 1990

\begin{abstract}
Three-dimensional cellular convection concentrates magnetic flux into ropes and sheets when the magnetic Reynolds number is large. We examine the equilibrium axisymmetric flux ropes sustained by cellular convection. The case of a compressible, electrically conducting fluid is studied and boundary layer analysis is found to yield a self-consistent solution for an externally driven convection field. Both kinematic and dynamic regimes may be examined and a scaling for the maximum value of the amplified field may be deduced.
\end{abstract}

\section{Introduction}

Magnetoconvection is a complex process common in the astrophysical context. Here a particular aspect is explored, motivated by observations of the solar surface. On the sun, photospheric granules seem to concentrate the magnetic flux into regions with intense local magnetic field. These flux tubes tend to form at granular boundaries where local downflows exist. This expulsion of magnetic field lines from convective eddies is a well known consequence of large magnetic Reynolds number flow (Parker 1963, Weiss $1966 \&$ 1967, Clark \& Johnson 1967, Busse 1975). When the total flux is small, concentration is limited only by magnetic diffusion. As the field strengthens, further effects become dynamically important and both induction equation and the equation of motion must be solved together. Galloway, Proctor \& Weiss 1978 [1] construct a model of this process in the case of an incompressible fluid and via boundary layer analysis are able to examine the transition from kinematic to the dynamic regime. Here the sole opposition produced by the field buildup in the rope is the Lorentz force. In an idealised example driven by horizontal temperature gradients, they observe a maximum field strength in the rope proportional to the square root of the ratio of the viscous to magnetic diffusivity. This study endeavours to extend their model in order to capture more of the "physics" of the actual process. The Sun is a complex mixture of partially or fully ionised gases and hence as such represents a highly compressible fluid. In addition it is clear that the energy equation, and hence the temperature field, requires a more careful treatment. Although the precise structure of sunspots is unknown, it is clear that they consist of one or many flux tubes erupting through the photosphere together. The observed cooling within a sunspot is suggestive of a more generic cooling inside flux tubes. This is confirmed by compressible magnetoconvection simulations conducted for example by Weiss and coworkers.

As a consequence of including these aspects of the observed process, additional 
forces play a role in the dynamic regime. The magnetic pressure, produced by the field lines, causes a localised drop in the fluid pressure. As a result the density tends to decrease and the fluid experiences an increased buoyancy-magnetic buoyancy. This is found to reduce the magnetic field in the rope. In contrast and as a consequence of downwellings in the tube, the temperature is found to decrease in the presence of the field and as a result amplifies the flux concentration. The crucial coupling between magnetic and temperature fields occurs via a magnetic-field-dependent thermal diffusivity. This produces cooling within the rope and forces the presence of a thermal boundary layer enclosing the flux tube The net effect is to produce an enriched selfconsistency relation ( $\mathrm{cf}$ [1]) for the axial velocity as well as a backreaction equation for the axial temperature field. The model assumes scalings which ensure that the modifications to temperature, pressure and density in the tube are all small. While this undoubtedly oversimplifies the structure ( particularly in pressure and density) it is a reasonable starting point for assessing the effects of compressibility in an analytical model. That such perturbations can be dynamically important is a consequence of the axis being a singularity in the equations of motion. Normally the regular solution is chosen, however the presence of a boundary layer around the axis allows the possibility of matching onto the singular solution. It will be shown later that this can allow small perturbations in the tube to produce $O(1)$ effects.

It should be noted that in pursuit of this self-consistent model, the main assumption made is that the tube is only slightly evacuated by the magnetic field. The model suggests that an equilibrated state is reached long before the alfvén speed within the tube reaches the sound speed. This is contrary to the findings of numerical simulations and observations of the above speeds on the solar surface. However so little is known about the magnetic field strengths below the photosphere that such a scenario may indeed exist deeper within the sun. On a similar note, the Boussinesq approximation is used when it is only sensibly applicable in the solar interior. The flux tubes are envisaged as extending at least 3 photospheric depths into the convection zone.

\section{The Sun}

The basic convective unit on the solar surface, a granule, has a length scale of $\approx 10^{3} \mathrm{~km}$. It's aspect ratio is unknown but commonly supposed to be $O(1)$. The photosphere has a depth of $500 \mathrm{~km}$ and hence granular convection can be taken as entering the convection zone which makes up the outer third of the sun and extends $\approx 2 \times 10^{5} \mathrm{~km}$ radially. Supergranules are coherent structures commonly comprising of $\approx 30$ granules. Their scale is $3 \times 10^{4} \mathrm{~km}$ and are thought to exist mainly in the convective zone. Sunspots have dimensions of supergranules and are thought to extend at least $10^{4} \mathrm{~km}$ deep. On the solar surface the temperature is $6000 \mathrm{~K}$ giving an acoustic speed of $\approx 12 \mathrm{kms}^{-1}$. In the flux tubes, field magnitudes are $1000-2000 \mathrm{G}$ producing surface alfvén speeds of order $\approx 5 \mathrm{kms}^{-1}$ and an Alfvén Mach number $\approx 0.5$. Typical fluid velocities are observed as $\approx 2 \mathrm{kms}^{-1}$ and the acceleration due to gravity at the surface is $274 \mathrm{~ms}^{-2}$. For calculation of dimensionless parameters the following scales are adopted:- $L \approx 2000 \mathrm{~km}, T \approx 6000 \mathrm{~K} \& v \approx 2 \mathrm{kms}^{-1}$. This gives estimates for the 
Peclet and Magnetic Reynolds numbers as follows:-

$$
\begin{array}{rl}
R_{m} & =\frac{L v}{\eta}=2 \times 10^{-9} T^{3 / 2} L v \approx 10^{6} \\
\text { Taking } \kappa \approx 10^{5} & P e=\frac{L v}{\kappa} \approx 10^{4}
\end{array}
$$

Hence $1 \ll P e \ll R_{m}$ The thermal and magnetic diffusivities are shown below (Priest 1982 p 312 [2]) -all figures are subject to debate (main source Priest 1982).

\section{Problem Formulation}

\subsection{Exterior Flow and Scalings}

A cylindrical convection cell of aspect ratio 1 is considered and a steady flow sought in which the magnetic and velocity fields are assumed purely meridional and azimuthal dependence is ignored:-

$$
\vec{q}=\left(q_{r}(r, z), 0, q_{z}(r, z)\right)
$$

The magnetic Reynolds number $R_{m}$ is taken as large and as a result the field is assumed concentrated in a central rope of radius $R_{m}^{-1 / 2}$. (The magnetic field is also flung outwards to the cell perimeter forming a true boundary layer. However in terms of magnetic field intensity this layer is dominated by the central rope and may be ignored.) The region outside the rope is labelled the exterior and is considered free of flux. Implicit in the formation of the flux tube is the presence of convection eddies sweeping the field into the axis. These are envisioned as toroidal with downflow at the centre. The external boundary conditions are assumed such as to produce this flow and no attempt is made to solve the exterior problem. Convection is assumed highly turbulent and this is modelled using a turbulent viscosity. Inertial terms are neglected in preference to the viscous terms. The Energy equation is considered in its simplified form of the steady temperature equation. The efficient turbulent mixing present in the exterior allows temperature advection to be replaced approximately by an adhanced thermal diffusivity i.e. $\vec{u} . \nabla T=\kappa_{m} \nabla^{2} T$ becomes $0 \approx\left(\kappa_{m}+\kappa_{e}\right) \nabla^{2} T$ where $\kappa_{m}$ is the molecular thermal diffusivity and $\kappa_{e} \gg \kappa_{m}$ represents an eddy diffusivity. The fluid is taken as a perfect gas whose state of ionisation remains constant.

The relevant equations then become for the exterior:-

$$
\begin{aligned}
0 & =-\nabla P+\rho \vec{g}+\rho \nu_{T}\left(\nabla^{2} \vec{u}+1 / 3 \nabla \nabla \cdot \vec{u}\right) \\
P & =\rho \Re T \\
0 & =\nabla \cdot(\rho \vec{u}) \\
0 & =\nabla^{2} T
\end{aligned}
$$

The exterior flow is scaled by balancing pressure and viscosity:-

$$
\frac{\rho c^{2}}{L} \sim \rho \nu_{T} \frac{v_{c}}{L^{2}}
$$

giving an estimate for the velocity

$$
v_{c} \sim \frac{c^{2} L}{\nu_{T}}
$$



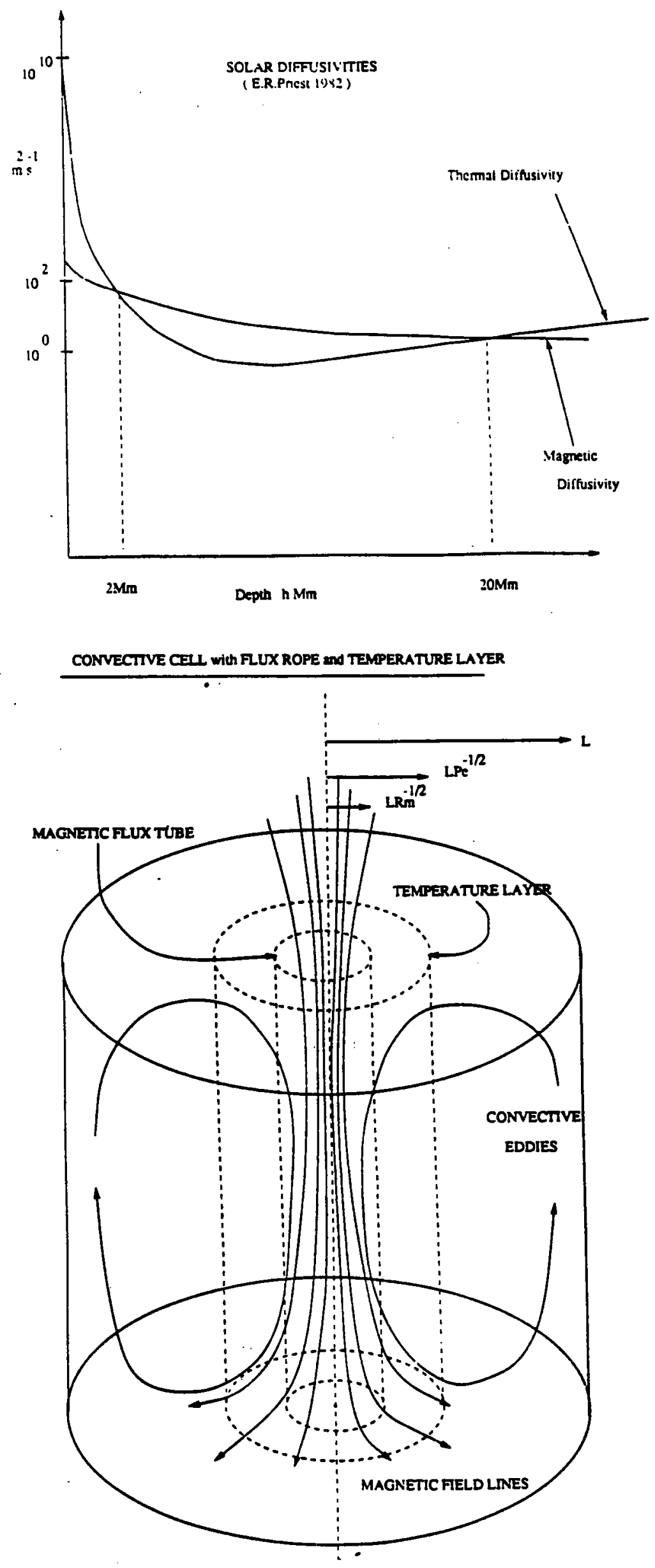
The magnetic Reynolds number is then $R_{m}=L v_{c} / \eta$. If the initial constant magnetic field permeating the cell is $B_{0}$, conservation of flux requires the field in the rope to have size $O\left(R_{m} B_{0}\right)$. The alfvén speed is then $v_{a}=R_{m} \frac{B_{0}}{\sqrt{\mu \rho}}$. Therefore

$$
\frac{v_{a}}{v_{c}}=\frac{L}{\eta} \frac{B_{0}}{\sqrt{\mu \rho}}
$$

\subsection{The Magnetic Flux Tube}

Assuming the exterior flow field is given, the Induction equation is now solved exactly.

$$
\nabla \times(\vec{u} \times \vec{B})=\eta \nabla^{2} \vec{B}
$$

Define $\chi$ such that

$$
\nabla \times\left(0, \frac{\chi(r, z)}{r}, 0\right)=\vec{B}=\left(-\frac{\chi_{z}}{r}, 0, \frac{\chi_{r}}{r}\right)
$$

Non-dimensionalizing

$$
\begin{aligned}
\vec{u} . \nabla \chi & =R_{m} D^{2} \chi \\
\text { with } D^{2} & =r \frac{\partial}{\partial r}\left(\frac{1}{r} \frac{\partial}{\partial r}\right)+\frac{\partial^{2}}{\partial z^{2}} \\
\text { as } \quad \nabla^{2}\left(\frac{\chi}{r} \overrightarrow{\hat{\theta}}\right) & =\vec{u} \times\left(\nabla \times\left(\frac{\chi}{r} \overrightarrow{\hat{\theta}}\right)\right.
\end{aligned}
$$

Introduce the boundary layer variable $\xi=R_{m}^{1 / 2} r$

$$
\vec{u} . \nabla \chi \approx \xi \frac{\partial}{\partial \xi}\left(\frac{1}{\xi} \frac{\partial \chi}{\partial \xi}\right)
$$

Assume an asymptotic form for $\vec{u}$

$$
\vec{u} \sim\left(\frac{1}{2} r g(z), 0, f(z)\right)
$$

Then

$$
\frac{1}{2} g \xi \chi_{\xi}+f \chi_{z} \approx \xi\left(\frac{\chi_{\xi}}{\xi}\right)_{\xi}
$$

It is helpful to adopt the Von-Mises coordinates of incompressible boundary layer theory. Here the temperature and magnetic field equations are linear for the given velocity field and the utility of these coordinates is to remove the variable coefficients. Let $\phi=1 / 2 \xi^{2} F(z)$ be a modified streamfunction and then choosing $g F+f \frac{d F}{d z}=0$ leads to

$$
\chi_{z}=2(F / f) \phi \chi_{\phi \phi}
$$

This has solution

$$
\text { with } \begin{aligned}
\chi & =\chi_{0}\left(1-e^{-1 / 2 \xi^{2} h(z)}\right) \\
\phi & =1 / 2 \xi^{2} F(z) \\
F(z) & =e^{-\int^{z} g / f d z} \\
h(z) & =\frac{F(z)}{2 \int^{z} F / f d z}
\end{aligned}
$$

Thus the magnetic field has Gaussian structure. 


\subsection{Temperature}

Intense magnetic field within the tube can be expected to reduce turbulence and in these circumstances a diffusion-dominated temperature field is unrealistic. We are forced to incorporate the effects of advection near the flux tube due to the highly anisotropic velocity field there. However rather than return to the exact temperature equation with only molecular thermal diffusivity, we imagine that the diffusivity is still enhanced by isotropic turbulent mixing. This allows us to speculate that the thermal diffusivity depends in some inverse way on the field strength which provides more intense coupling between the fields. Due to the strongly unidirectional field in the rope we should expect the thermal diffusivity to be anisotropic, however for simplicity this is ignored and $\kappa$ is written as $\kappa(B)$.

$$
\vec{u} . \nabla T=\nabla \cdot(\kappa(B) \nabla T)
$$

The exterior temperature field is unaware of the flux tube and may be expanded asymptotically for $r \rightarrow 0$ as follows:-

$$
T_{\text {ext }}=T_{0}(z)+r^{2} T_{1}(z)+\ldots
$$

and the interior perturbation field $T_{\text {int }}(\xi, z)$, which does feel the field, is superimposed on this. Defining the Peclet number as $P e=L v_{a} / \kappa_{\infty}$, the above equation becomes, after dropping small terms:-

$$
\begin{array}{cc}
P e\left[f(z) \frac{d T_{0}}{d z}+f(z) \frac{\partial T_{i n t}}{\partial z}=\right. & \kappa\left(\frac{d^{2} T_{0}}{d z^{2}}+4 T_{1}\right)+\left.\frac{\partial \kappa}{\partial z}\right|_{\xi}\left(\frac{d T_{0}}{d z}+\frac{\partial T_{i n t}}{\partial z}\right) \\
\left.+1 / 2 \xi g(z) \frac{\partial T_{\text {int }}}{\partial \xi}\right] & +R_{m} \frac{1}{\xi} \frac{\partial}{\partial \xi}\left(\xi \kappa \frac{\partial T_{\text {int }}}{\partial \xi}\right)
\end{array}
$$

Where $\kappa$ is now non-dimensionalized by its value at infinity. The exterior temperature field does not see a spatially dependent thermal diffusivity and must therefore satisfy the reduced equation:-

$$
\operatorname{Pe} f(z) \frac{d T_{0}}{d z}=\frac{d^{2} T_{0}}{d z^{2}}+4 T_{1}
$$

It is the interior perturbation field which adapts to the variable diffusivity.

$$
\begin{aligned}
& P e\left[(1-\kappa) f(z) \frac{d T_{0}}{d z}+f(z) \frac{\partial T_{i n t}}{\partial z}+=\left.\frac{\partial \kappa}{\partial z}\right|_{\xi}\left(\frac{d T_{0}}{d z}+\frac{\partial T_{i n t}}{\partial z}\right)+R_{m} \frac{1}{\xi} \frac{\partial}{\partial \xi}\left(\xi \kappa \frac{\partial T_{i n t}}{\partial \xi}\right)\right. \\
& \left.+1 / 2 \xi g(z) \frac{\partial T_{i n t}}{\partial \xi}\right]
\end{aligned}
$$

Here the ordering of parameters $1 \ll P e \ll R_{m}$, adopted above, is motivated by solar observations and the equation then naturally describes two regimes. Within the interior, $\kappa$ is different from 1 and hence the dominant balance is between first and last terms. In contrast, outside the flux tube $\kappa=1$, and the second term replaces the first in importance. To allow progress, we postulate a simple structure for $\kappa(B)$.

$$
\begin{aligned}
1-\kappa & =\alpha B^{\beta} \\
\text { or rather } \quad 1-\kappa & =\alpha\left(\chi_{0}^{*}\right)^{\beta} e^{-1 / 2 \beta \xi^{2} h(z)}
\end{aligned}
$$


with $\chi_{0}=\chi_{0}^{*} / R_{m}$ and $\alpha$ dimensionless although proportional to $B_{0}^{\beta}$. This choice conveniently allows immediate integration.

$$
\alpha\left(\chi_{0}^{*} h\right)^{\beta}\left(\frac{P e}{R_{m}}\right) f \frac{d T_{0}}{d z} \frac{1}{h \beta}\left(1-e^{-1 / 2 \beta \xi^{2} h(z)}\right)=\xi \kappa \frac{\partial T_{\text {int }}}{\partial \xi}
$$

using the boundary condition $\xi=0 \Rightarrow \frac{\partial T_{\text {ins }}}{\partial \xi}=0$. Notice the LHS is +ve, therefore $T_{\text {int }}$ increases as it leaves the tube-as desired. Also note that as $\xi \rightarrow \infty$

$$
T_{\text {int }} \sim \frac{\alpha}{\beta} \frac{P e}{R_{m}} \chi_{0}^{* \beta} h^{\beta-1} f \frac{d T_{0}}{d z} \ln \xi
$$

Such logarithmic behaviour is impossible to match directly with the exterior where the perturbation field must decay to zero. An intermediary layer is necessary and naturally appears from within the equations:-

$$
\operatorname{Pef}(z) \frac{\partial T_{i n t}}{\partial z} \simeq R_{m} \frac{1}{\xi} \frac{\partial}{\partial \xi}\left(\xi \frac{\partial T_{i n t}}{\partial \xi}\right)
$$

Outside the rope $\kappa=1$ a constant and $\left(\frac{P e}{R_{m}}\right) \ll 1$. Rescaling $\nu=\left(\frac{P e}{R_{m}}\right)^{1 / 2} \xi$ and defining $y=\int_{-1 / 2}^{z} d q / f(q)$ gives

$$
\frac{\partial T_{\text {int }}}{\partial y}=\frac{1}{\nu} \frac{\partial}{\partial \nu}\left(\nu \frac{\partial T_{\text {int }}}{\partial \nu}\right)
$$

( Recall $f(z)<0$ and so $z \in[-1,0]$ maps onto $y \in[+\infty,-\infty]$ ). A solution is needed of this equation which can match onto a logarithmic singularity as $\nu \rightarrow 0$ and which decays to zero as $\nu \rightarrow \infty$. Explicitly a solution $T(\nu, y)$ is required such that

$$
\begin{aligned}
& T(\nu, y) \sim A(y) \ln \nu \text { as } \nu \rightarrow 0 \\
& T(\nu, y) \rightarrow 0 \quad \text { as } \quad \nu \rightarrow \infty
\end{aligned}
$$

The inner condition represents a distribution of sinks along the $y$ axis. Hence the thermal layer is the adjustment region in which fluid flowing downwards accommodates the inner cool tube. The well-known fundamental solution

$$
T(\nu, y)=\frac{1}{y} e^{-\nu^{2} / 4 y}
$$

represents the effect of a $\delta$-fn source of heat placed at $\nu=0$. Hence the required solution is a convolution over the source distribution with the fundamental solution:-

$$
T=-\frac{1}{2} \int_{-\infty}^{y} \frac{A(w)}{y-w} e^{-\nu^{2} / 4(y-w)} d w
$$

Notice the lower limit is $w=-\infty$ which corresponds to $z=0$ i.e. the top of the cell. For convergence we assume $A(w) \rightarrow 0$ as $w \rightarrow-\infty$ which is clear physically and can be seen to hold later.

We proceed to match these layers to leading order. Call $\mathrm{T}$ inside the thermal boundary layer $\bar{T}_{\text {int }}$ to distinquish from that within the flux rope $T_{\text {int }}$. For leading order matching

$$
\lim _{\xi \rightarrow \infty} T_{\text {int }}=\lim _{\nu \rightarrow 0} \bar{T}_{\text {int }}
$$




$$
\begin{aligned}
\Rightarrow A(y) & =\frac{\alpha}{\beta} \frac{P e}{R_{\tau n}} \chi_{0}^{* \beta} h^{\beta-1} f \frac{d T_{0}}{d z} \\
\Rightarrow \bar{T}_{i n t}^{p a r t} & =-\frac{1}{2} \frac{\alpha}{\beta} \frac{P e}{R_{m}} \chi_{0}^{* \beta} \int_{-\infty}^{y} \frac{h^{\beta-1}(w)}{y-w} \frac{d T_{0}}{d w} e^{-\nu^{2} / 4(y-w)} d w
\end{aligned}
$$

The superscript part (-particular integral) is used to indicate a solution forced to exist by the dynamics as opposed to $c f$ (-complementary function) which exists as a consequence of boundary conditions. In the case of the temperature field, there exist the complementary solutions $F(z)$ and it is this degree of freedom which accommodates boundary layer matching. Above we have deduced the form $\bar{T}_{i n t}^{\text {part }}$, however full matching must take into account the complementary functions generated. In the exterior the perturbative temperature is zero and hence $\bar{T}_{\text {int }}=\bar{T}_{\text {int }}^{\text {part }}$ via matching at $\nu \rightarrow \infty$. Turning attention to the $\nu \rightarrow 0$ matching we have in the Interior:-

$$
\begin{aligned}
\frac{1}{\xi} \frac{\partial}{\partial \xi}\left(\xi \kappa \frac{\partial T_{\text {int }}}{\partial \xi}\right) & =(1-\kappa) f \frac{d T_{0}}{d z} \frac{P e}{R_{m}} \\
T_{i n t}^{\text {part }} & \sim \frac{\alpha}{\beta} \frac{P e}{R_{m}} \chi_{0}^{* \beta} h^{\beta-1} f \frac{d T_{0}}{d z} \ln \xi \text { as } \xi \rightarrow \infty \\
T_{\text {int }}^{c f} & =F(z)
\end{aligned}
$$

In the Middle layer:-

$$
\frac{1}{\nu} \frac{\partial}{\partial \nu}\left(\nu \frac{\partial \bar{T}_{i n t}}{\partial \nu}\right)=\frac{\partial \bar{T}_{i n t}}{\partial y}
$$

Asymptotically as $\nu \rightarrow 0, \bar{T}_{\text {int }}$ can be written most generally as

$$
\bar{T}_{i n t} \sim \alpha(y) \ln \nu+\beta(y)+\gamma(y) \nu^{2} \ln \nu+\delta(y) \nu^{2}+\ldots
$$

In terms of $\xi=\left(\frac{P e}{R_{m}}\right)^{-1 / 2} \nu$

$$
\begin{aligned}
\bar{T}_{i n t} & \sim \alpha(y) \ln \left(\frac{P e}{R_{m}}\right)^{1 / 2} \xi+\beta(y)+\ldots \\
& \sim \alpha(y) \ln \xi+\alpha(y) \ln \left(\frac{P e}{R_{m}}\right)^{1 / 2}+\beta(y)+\ldots
\end{aligned}
$$

The first 2 terms dominate and must match onto $T_{\text {int }}$ such that

$$
\alpha(y)=\frac{\alpha}{\beta} \frac{P e}{R_{m}} \chi_{0}^{* \beta} h^{\beta-1} f \frac{d T_{0}}{d z}
$$

As a consequence $T_{i n t}^{c f}=\alpha(y) \ln \left(\frac{P_{e}}{R_{m}}\right)^{1 / 2}$ and a temperature change is induced in the inner boundary layer of size $\ln \left(\frac{P_{e}}{R_{m}}\right)^{1 / 2}$ larger than the original temperature perturbation. Explicitly in the rope $T_{i n t}^{\text {part }}=o\left(T_{i n t}^{c f}\right)$. The complete temperature field can be written

$$
T_{\text {int }} \sim \alpha(y)\left[\ln \left(\frac{P e}{R_{m}}\right)^{1 / 2}+\ln \xi\right]
$$

as $\xi \rightarrow \infty$ with the first term dominating. The self-consistent relation for the temperature field can now be derived. Writing $\tilde{T}(z)$ as the axial temperature, the back reaction is $T_{\text {int }}^{c f}=\tilde{T}-\tilde{T}_{0}$ i.e.

$$
\tilde{T}-\tilde{T}_{0}=\frac{\alpha}{\beta} \frac{P e}{R_{m}} \ln \frac{P e^{1 / 2}}{R_{m}} \chi_{0}^{* \beta} h^{\beta-1} f(z) \frac{d \tilde{T}}{d z}
$$


Notice the backreaction is negative indicating tube cooling and that the order of the backreaction is $O\left(\alpha \frac{P e}{R_{m}} \ln \left(\frac{P e}{R_{m}}\right)^{1 / 2}\right)$, where $O(\alpha)=\Delta \kappa / \kappa_{\infty}$.

\subsection{The Equations of Motion}

We now turn attention to the equations of motion. Ignoring the inertial terms reduces the problem to a linear one and allows decomposition of the velocity field into its basic and perturbative components. We non-dimensionalize $\mathrm{P}$ with $\rho_{\infty} c^{2}, B^{2} / \mu \rho$ with $v_{a}^{2}$, external velocity field $\vec{u}_{0}$ by $v_{c}$ and interior solution $\vec{u}_{1}$ by $v_{a}$.

$$
\begin{aligned}
\frac{L c^{2}}{\nu v_{a}} \nabla^{*}\left(P^{*}+\left(\frac{v_{a}}{c}\right)^{2} \frac{\vec{B}^{*^{2}}}{2}\right) \simeq & \frac{g L}{v_{a}} \frac{L}{\nu}\left(-\rho^{*} \overrightarrow{\hat{z}}\right)+\frac{L v_{a}}{\nu} \vec{B}^{*} \cdot \nabla^{*} \vec{B}^{*}+\nabla^{*^{2}}\left[\frac{v_{c} \nu_{T}}{v_{a} \nu} \vec{u}_{0}^{*}+\vec{u}_{1}^{*}\right] \\
& +1 / 3 \nabla^{*}\left[\frac{v_{c} \nu_{T}}{v_{a} \nu} \nabla^{*} \cdot \vec{u}_{0}^{*}+\nabla^{*} \cdot \vec{u}_{1}^{*}\right]
\end{aligned}
$$

Here $*$ represents a non-dimensional quantity and is hereafter dropped. The dimensionless parameter $Q=\frac{\eta}{\nu}\left(\frac{v_{a}}{v_{c}}\right)$ is introduced,

$$
\begin{aligned}
Q R_{m}\left(\frac{c}{v_{a}}\right)^{2} \nabla\left(P+\left(\frac{v_{a}}{c}\right)^{2} \frac{B^{2}}{2}\right)= & Q R_{m} \frac{g L}{v_{a}^{2}} \rho \overrightarrow{\hat{z}}+Q R_{m} \vec{B} \cdot \nabla \vec{B} \\
& \nabla^{2}\left[\frac{v_{c} \nu_{T}}{v_{a} \nu} \vec{u}_{0}+\vec{u}_{1}\right]+1 / 3 \nabla\left[\frac{v_{c} \nu_{T}}{v_{a} \nu} \nabla \cdot \vec{u}_{0}+\nabla \cdot \vec{u}_{1}\right](28)
\end{aligned}
$$

This represents only two equations due to the absence of angular dependence and swirl components in $\vec{u}$ and $\vec{B}$. The radial component is as follows:-

$$
\begin{aligned}
Q R_{m}\left(\frac{c}{v_{a}}\right)^{2} \frac{\partial}{\partial r}(P+ & \left.\left(\frac{v_{a}}{c}\right)^{2} \frac{B^{2}}{2}\right) \simeq Q R_{m} \vec{B} \cdot \nabla \vec{B}_{r}+ \\
& \overrightarrow{\hat{r}} \cdot\left\{\nabla^{2}\left[\frac{v_{c} \nu_{T}}{v_{a} \nu} \vec{u}_{0}+\vec{u}_{1}\right]+1 / 3 \nabla\left[\frac{v_{c} \nu_{T}}{v_{a} \nu} \nabla \cdot \vec{u}_{0}+\nabla \cdot \vec{u}_{1}\right]\right\}
\end{aligned}
$$

The Alfven speed is that in the tube and hence $B_{z}=\mathrm{O}(1)$. As a consequence of $\nabla \cdot \vec{B}=0, B_{r}=O\left(R_{m}^{-1 / 2}\right)$ and then the scale of terms in the above equation reads

$$
O\left(Q R_{m}^{3 / 2}\right)+O\left(Q R_{m}^{1 / 2}\right)+O\left(R_{m}\right) \simeq 0
$$

Clearly the first term must vanish

$$
\begin{gathered}
\frac{\partial}{\partial \xi}\left[P+\left(\frac{v_{a}}{c}\right)^{2} \frac{B^{2}}{2}\right]=0 \\
\Rightarrow P(\xi, z)=P_{e x t}(r, z)-\left(\frac{v_{a}}{c}\right)^{2} \frac{B^{2}}{2} \\
P=P_{e x t}-\frac{1}{2}\left(\frac{v_{a}}{c}\right)^{2} \chi_{0}^{* 2} h^{2} e^{-\xi^{2} h(z)}
\end{gathered}
$$

we use the ideal gas equation of state $P=\rho T$ to produce

$$
\Rightarrow \rho=\frac{P_{e x t}}{T_{e x t}+T_{i n t}}-\frac{1}{2}\left(\frac{v_{a}}{c}\right)^{2}\left(\chi_{0}^{*} h\right)^{2} \frac{e^{-\xi^{2} h(z)}}{T_{e x t}+T_{i n t}}
$$


Using the earlier assumption that $T_{i n t} \ll T_{e x t}$ and neglecting second order changes in the density allows the two distinct density changing processes to be separated:-

$$
\rho \simeq \rho_{e x t}-\frac{P_{e x t}}{T_{e x t}}\left(\frac{T_{i n t}}{T_{e x t}}\right)-1 / 2\left(\frac{v_{a}}{c}\right)^{2}\left(\chi_{0}^{*} h\right)^{2} \frac{e^{-\xi^{2} h(z)}}{T_{e x t}}
$$

The second term represents the buoyancy due to the temperature perturbation and the third term is the magnetic buoyancy. The vorticity equation ( $\overrightarrow{\hat{\theta}} \cdot \nabla \times$ the momentum eq.) reads as follows

$$
0 \simeq Q R_{m} \frac{g L}{v_{a}^{2}}\left(\frac{\partial \rho}{\partial r}\right)-Q R_{m} \frac{\partial\left(\chi, \frac{1}{r^{2}} D^{2} \chi\right)}{\partial(r, z)}+\frac{1}{r} D^{2}\left[r \frac{v_{c} \nu_{T}}{v_{a} \nu} \omega_{0}+r \omega_{1}\right]
$$

Substitution of the density expression into this gives

$$
\begin{aligned}
\frac{1}{r} D^{2}\left[r \frac{v_{c} \nu T}{v_{a} \nu} \omega_{0}+r \omega_{1}\right]= & Q R_{m} \frac{g L}{v_{a}^{2}}\left(-\frac{\partial \rho_{\text {ext }}}{\partial r}+\frac{R_{m}^{1 / 2} \rho_{\text {ext }}}{T_{\text {ext }}} \frac{\partial T_{\text {int }}}{\partial \xi}\right. \\
& \left.-\left(\frac{v_{a}}{c}\right)^{2} \chi_{0}^{* 2} h^{3} \frac{R_{m}^{1 / 2}}{T_{e x t}} \xi e^{-\xi^{2} h(z)}\right)+Q R_{m} \frac{\partial\left(\chi, \frac{1}{r^{2}} D^{2} \chi\right)}{\partial(r, z)}
\end{aligned}
$$

This divides naturally as follows:-

Exterior : - $\quad \frac{1}{r} D^{2}\left[r \frac{v_{c} \nu_{T}}{v_{a} \nu} \omega_{0}\right]=-Q R_{m} \frac{g L}{v_{a}^{2}}\left(\frac{\partial \rho_{e x t}}{\partial r}\right)$

Interior Correction : $-\frac{1}{r} D^{2}\left[r \omega_{1}\right]=Q R_{m} \frac{g L}{v_{a}^{2}}\left(\frac{R_{m}^{1 / 2} \rho_{\text {ext }}}{T_{\text {ext }}} \frac{\partial T_{\text {int }}}{\partial \xi}\right.$

$$
\begin{aligned}
& \left.-\left(\frac{v_{a}}{c}\right)^{2} \chi_{0}^{* 2} h^{3} \frac{R_{m}^{1 / 2}}{T_{e x t}} \xi e^{-\xi^{2} h(z)}\right) \\
& +Q R_{m} \frac{\partial\left(\chi, \frac{1}{r^{2}} D^{2} \chi\right)}{\partial(r, z)}
\end{aligned}
$$

Upon simplification the interior correction equation reads

$$
\begin{aligned}
\frac{\partial}{\partial \xi}\left(\frac{1}{\xi} \frac{\partial}{\partial \xi}\left(\xi \omega_{1}\right)\right)= & -Q R_{m}^{1 / 2} \chi_{0}^{* 2}\left[2 h^{2} h^{\prime}+\frac{g L}{c^{2}} \frac{h^{3}}{T_{\text {ext }}}\right] \xi e^{-\xi^{2} h(z)} \\
& +Q R_{m} \frac{g L}{v_{a}^{2}} \frac{\rho_{\text {ext }}}{T_{\text {ext }}} \frac{\partial T_{\text {int }}}{\partial \xi}
\end{aligned}
$$

which can be integrated twice and with the boundary condition $\omega_{1}=0$ at $\xi=0$ yields

$$
\begin{aligned}
\xi \omega_{1}= & \frac{1}{2} Q R_{m}^{1 / 2} \chi_{0}^{* 2}\left[A(z) \xi^{2}+1-e^{-\xi^{2} h(z)}\right]\left(h^{\prime}+\frac{g L}{c^{2}} \frac{h}{2 T_{e x t}}\right) \\
& +Q R_{m} \frac{g L}{v_{a}^{2}} \frac{\rho_{e x t}}{T_{e x t}} \int_{0}^{\xi} \zeta T_{i n t}(\zeta, z) d \zeta
\end{aligned}
$$




\subsection{Vorticity Matching}

The perturbation vorticity can be divided into two parts which are clearly distinct in structure and generating process. The first arises from Lorentz and magnetic buoyancy forces and as a consequence sees only the flux tube. In contrast the vorticity due to perturbative cooling sees both magnetic and thermal layers. To clarify notation $\bar{\omega}$ is used to indicate vorticity in the flux rope, $\bar{\omega}$ in the thermal layer and $\hat{\omega}$ loosely to describe vorticity in the exterior. (Notice the subscript 1 is dropped as only perturbation vorticity is considered.) Consider the Lorentz and magnetic buoyancy generated vorticity.

$$
r \tilde{\omega}=\frac{1}{2} Q \chi_{0}^{* 2}\left[A(z) \xi^{2}+1-e^{-\xi^{2} h(z)}\right]\left(h^{\prime}+\frac{g L}{c^{2}} \frac{h}{2 T_{e x t}}\right)
$$

This generates a velocity field $\tilde{u}_{z}^{\text {part }}$ via

$$
r \tilde{\omega}=r\left(\frac{\partial \tilde{u}_{r}^{p a r t}}{\partial z}-\frac{\partial \tilde{u}_{z}^{p a r t}}{\partial r}\right) \simeq-r \frac{\partial \tilde{u}_{z}^{\text {part }}}{\partial r}
$$

The complementary velocity field corresponds to some arbitary function of $z$ to be later specified by matching requirements.

$$
\tilde{u}_{z}^{c f}=\tilde{F}(z)
$$

Outside the flux rope, the vorticity experiences no forcing and hence satisfies the homogeneous equation

$$
D^{2}(r \hat{\omega})=0
$$

Near the axis $r \rightarrow 0$, this vorticity can be expanded generally by

$$
\begin{aligned}
r \hat{\omega} & \sim \alpha(z)+\beta(z) r^{2} \ln r+\gamma(z) r^{2}+\ldots \\
& \sim \alpha(z)+\beta(z)\left(\frac{\xi^{2}}{R_{m}} \ln R_{m}^{-1 / 2}+\frac{\xi^{2}}{R_{m}} \ln \xi\right)+\gamma(z) \frac{\xi^{2}}{R_{m}}+\ldots
\end{aligned}
$$

We match vorticity fields at the flux rope boundary to leading order

$$
\lim _{\xi \rightarrow \infty} r \tilde{\omega}=\lim _{r \rightarrow 0} r \hat{\omega}
$$

which produces

$$
\begin{aligned}
\alpha(z) & =\frac{1}{2} Q \chi_{0}^{* 2}\left(h^{\prime}+\frac{g L}{c^{2}} \frac{h}{2 T_{e x t}}\right) \\
\beta(z) \frac{\ln R_{m}^{-1 / 2}}{R_{m}} & =\frac{1}{2} Q A(z) \chi_{0}^{* 2}\left(h^{\prime}+\frac{g L}{c^{2}} \frac{h}{2 T_{e x t}}\right)
\end{aligned}
$$

All the expansion functions $\alpha, \beta, \ldots$ can be taken as being the same order in $R_{m}$ as they are implicitly coupled in the expansion. If $\alpha=O(Q)$ then this implies $A(z)=$ $O\left(\frac{\ln R_{m}^{-1 / 2}}{R_{m}}\right)$. The $A(z) \xi^{2}$ term is therefore subdominant to 1 within the rope and the vorticity can be taken as radially independent to leading order.

$$
r \bar{\omega} \sim \frac{1}{2} Q \chi_{0}^{* 2}\left(h^{\prime}+\frac{g L}{c^{2}} \frac{h}{2 T_{e x t}}\right) \quad \text { as } \quad r \rightarrow 0
$$


This drives a motion just outside the flux tube of

$$
\hat{u}_{z}^{\text {part }} \simeq-\frac{1}{2} Q \chi_{0}^{* 2}\left(h^{\prime}+\frac{g L}{c^{2}} \frac{h}{2 T_{\text {ext }}}\right) \ln r
$$

The complementary function $\hat{u}_{z}^{c f}=\hat{F}(z)$ is subdominant to the driven flow as $r \rightarrow 0$ and hence can be neglected during the axial velocity matching. Continuity demands

$$
\lim _{\xi \rightarrow \infty}\left(\tilde{u}_{z}^{\text {part }}+\tilde{u}_{z}^{c f}\right)=-\frac{1}{2} Q \chi_{0}^{* 2}\left(h^{\prime}+\frac{g L}{c^{2}} \frac{h}{2 T_{e x t}}\right)\left(\ln r=\ln \xi-\ln R_{m}^{-1 / 2}\right)
$$

When the rope vorticity is expanded asymptotically as $\xi \rightarrow \infty$ only integral powers of $R_{m}$ can arise. Certainly no $\ln R_{m}^{-1 / 2}$ terms are available and so the driven velocity field can only accommodate the $\ln \xi$ term. Hence we must have

$$
\lim _{\xi \rightarrow \infty} \tilde{u}_{z}^{c f}=\tilde{u}_{z}^{c f}=\frac{1}{2} Q \chi_{0}^{* 2}\left(h^{\prime}+\frac{g L}{c^{2}} \frac{h}{2 T_{e x t}}\right) \ln R_{m}^{-1 / 2}
$$

This represents the backreaction at the axis due to Lorentz and magnetic buoyancy forces. The complementary solution has an order $O\left(\ln R_{m}^{-1 / 2}\right)$ larger than the driven flow. This boundary layer magnification mechanism is crucial to the existence of the self-consistent model presented here. Such magnification allows backreaction forcing to be treated as perturbative, but ensures that the resulting flows in the rope are $O(1)$ and hence capable of equilibrating the system.

For the temperature-forced vorticity two layers must be fitted to each other and to the exterior. In the Inner flux layer, the vorticity is forced by a functional of the temperature along the axis.

$$
r \tilde{\omega}^{T}=Q \frac{g L}{v_{a}^{2}} \frac{\rho_{e x t}}{T_{e x t}} \int_{0}^{\xi} \zeta T_{i n t}(\zeta, z) d \zeta
$$

At the edge of the flux tube, in the limit as $\xi \rightarrow \infty$

$$
\begin{aligned}
T_{i n t} & \sim \frac{\alpha}{\beta} \frac{P e}{R_{m}} \chi_{0}^{* \beta} h^{\beta-1} f(z) \frac{d T}{d z}\left[\ln \left(\frac{P e}{R_{m}}\right)^{1 / 2}+\ln \xi\right] \\
r \tilde{\omega}^{T} & \sim Q \frac{g L}{v_{a}^{2}} \frac{\rho_{e x t}(z)}{T(z)} \frac{\alpha}{\beta} \frac{P e}{R_{m}} \chi_{0}^{* \beta} h^{\beta-1} f(z) \frac{d T}{d z}\left[\frac{1}{2} \xi^{2}\left(\ln \left(\frac{P e}{R_{m}}\right)^{1 / 2}+\ln \xi\right)\right] \\
\tilde{u}_{z}^{\text {part }} & \sim Q \frac{g L}{v_{a}^{2}} \frac{\rho_{\text {ext }}(z)}{T(z)} \frac{\alpha}{\beta} \frac{P e}{R_{m}} \chi_{0}^{* \beta} h^{\beta-1} f(z) \frac{d T}{d z}\left[-\frac{1}{4} \xi^{2}\left(\ln \left(\frac{P e}{R_{m}}\right)^{1 / 2}+\ln \xi\right)\right] \\
\tilde{u}_{z}^{c f} & \sim \tilde{F}^{T}(z)
\end{aligned}
$$

In the Middle thermal layer

$$
\begin{aligned}
r \bar{\omega}^{T} & =Q \frac{g L}{v_{a}^{2}} \frac{R_{m}}{P e} \frac{\rho_{\text {ext }}}{T(z)} \int_{0}^{\nu} \zeta \bar{T}_{\text {int }}(\zeta, z) d \zeta \\
\text { and } \quad \bar{T}_{i n t}(\zeta, z) & =-\frac{\alpha}{2 \beta} \chi_{0}^{* \beta} \frac{P e}{R_{m}} \int_{-\infty}^{y(z)} \frac{h^{\beta-1}(w)}{y-w} \frac{d T}{d w} e^{-\zeta^{2} / 4(y-w)} d w
\end{aligned}
$$

Vorticity matching at the inner edge of the boundary layer is automatically accomplished by ensuring correct temperature matching treated earlier. Matching axial velocities as $\nu \rightarrow 0$ trivially produces

$$
\vec{u}_{z}^{c f}=\tilde{u}_{z}^{c f}
$$


We look for boundary layer magnification at the external barrier of the thermal boundary layer.

As $\nu \rightarrow \infty$

$$
\begin{aligned}
r \bar{\omega}^{T} & \sim-\frac{1}{2} \frac{\alpha}{\beta} Q \frac{g L}{v_{a}^{2}} \frac{\rho_{\text {ext }}}{T(z)} \chi_{0}^{* \beta} \int_{0}^{\infty} \zeta d \zeta \int_{-\infty}^{y(z)} \frac{h^{\beta-1}(w)}{y-w} \frac{d T}{d w} e^{-\zeta^{2} / 4(y-w)} d w \\
\Rightarrow r \bar{\omega}^{T} & \sim \frac{\alpha}{\beta} Q \frac{g L}{v_{a}^{2}} \frac{\rho_{\text {ext }}}{T(z)} \chi_{0}^{* \beta} \int_{0}^{z}-h^{\beta-1}(\bar{z}) \frac{d T}{d \bar{z}} d \bar{z}
\end{aligned}
$$

In the exterior, as before, the unforced vorticity has the general asymptotic expansion, as $r \rightarrow 0$

$$
r \hat{\omega}^{T} \sim \alpha(z)+\beta(z) r^{2} \ln r+\gamma(z) r^{2}+\ldots
$$

Matching $r \hat{\omega}^{T}$ and $r \bar{\omega}^{T}$ in the respective limits reveals that

$$
\alpha(z)=-\frac{\alpha}{\beta} Q \frac{g L}{v_{a}^{2}} \frac{\rho_{e x t}}{T(z)} \chi_{0}^{* \beta} \int_{0}^{z} h^{\beta-1}(\bar{z}) \frac{d T}{d \bar{z}} d \bar{z}
$$

The resulting axial velocity has asymptotic form $\hat{u}_{z}^{\text {part }} \sim-\alpha(z) \ln r$ and dominates the complementary field $\hat{u}_{z}^{c f}$ for $r \rightarrow 0$. Matching to this leads to

$$
\begin{aligned}
\bar{u}_{z}^{\text {part }} & \sim-\alpha(z) \ln \nu \\
\bar{u}_{z}^{c f} & =\alpha(z) \ln P e^{1 / 2} \quad \text { as } \quad \nu \rightarrow \infty
\end{aligned}
$$

As a result the backreaction flow at the axis due to the temperature cooling is:-

$$
\tilde{u}_{z}^{c f}=\bar{u}_{z}^{c f}=-\frac{\alpha}{\beta} Q \frac{g L}{v_{a}^{2}} \ln P e^{1 / 2} \frac{\rho_{\text {ext }}}{T(z)} \chi_{0}^{* \beta} \int_{0}^{z} h^{\beta-1}(\bar{z}) \frac{d T}{d \bar{z}} d \bar{z}
$$

This velocity is negative and thus the temperature effect is amplifying on the convective flow. Within the dynamic regime the axial velocity backreaction is $O(1)$ and if this is not to disrupt the asymptotic form of the flow field within the tube, the backreaction must possess the same limiting form i.e.

$$
\vec{u}_{1}=\left(\frac{1}{2} r g_{1}(z), 0, f_{1}(z)\right)
$$

This requires the condition

$$
\frac{\xi}{\rho} \frac{\partial \rho}{\partial \xi} \ll 1
$$

within the tube via the continuity equation $\nabla \cdot(\rho \vec{u})=0$, and motivates our restriction that density changes in the tube are small.

\subsection{Self-Consistency Equations}

The self-consistency relations are

$$
\begin{aligned}
\left(f-f_{0}\right) v_{c}= & {\left[-\frac{\alpha}{\beta} Q \frac{g L}{v_{a}^{2}} \ln P e^{1 / 2} \frac{\rho_{e x t}}{T(z)} \chi_{0}^{* \beta} \int_{0}^{z} h^{\beta-1}(\bar{z}) \frac{d T}{d \bar{z}} d \bar{z}\right.} \\
& \left.+\frac{1}{2} Q \chi_{0}^{* 2} \ln R_{m}^{1 / 2}\left(h^{\prime}+\frac{g L}{c^{2}} \frac{h}{2 T_{e x t}}\right)\right] v_{a} \\
T-T_{0}= & \frac{\alpha}{\beta} \frac{P e}{R_{m}} \ln \frac{P e^{1 / 2}}{R_{m}} \chi_{0}^{* \beta} h^{\beta-1} f(z) \frac{d T}{d z}
\end{aligned}
$$


Recall

$$
h(z)=\frac{e^{-\int g / f d z}}{2 \int F / f d z}
$$

And from continuity

$$
\begin{aligned}
& g(z)=\frac{-(\rho f)^{\prime}}{\rho} \\
& h>0 \quad \Rightarrow \quad \int^{z} \rho(\hat{z}) d \hat{z}<0
\end{aligned}
$$

Then we solve

$$
\begin{aligned}
\frac{2 \int_{0}^{z} \rho_{\text {ext }}(\hat{z}) d \hat{z}}{\rho_{\text {ext }}(z)}\left(h-h_{0}\right)= & {\left[-\frac{\alpha}{\beta} Q \frac{g L}{v_{a}^{2}} \ln P e^{1 / 2} \frac{\rho_{\text {ext }}}{T(z)} \chi_{0}^{* \beta} \int_{0}^{z} h^{\beta-1}(\bar{z}) \frac{d T}{d \bar{z}} d \bar{z}\right.} \\
& \left.+\frac{1}{2} Q \chi_{0}^{* 2} \ln R_{m}^{1 / 2}\left(h^{\prime}+\frac{g L}{c^{2}} \frac{h}{2 T_{\text {ext }}}\right)\right] \frac{v_{a}}{v_{c}} \\
T-T_{0}= & \frac{\alpha}{\beta} \frac{P e}{R_{m}} \ln \frac{P e^{1 / 2}}{R_{m}} \chi_{0}^{* \beta} h^{\beta-1} f(z) \frac{d T}{d z}
\end{aligned}
$$

over $z \in[-1,0]$ with boundary conditions $h(-1)=0$ and $h(0)<\infty$.

\subsection{Scalings}

We now list all the scalings implicitly taken in the model

- For density changes in the tube to be small

$$
\left(\frac{v_{a}}{c}\right)^{2} \ll 1
$$

- Velocity backreactions to be $O(1)$

1. Temperature

$$
O\left(\alpha \frac{v_{a}}{v_{c}} \ln P e^{1 / 2} Q \frac{g L}{v_{a}^{2}}\right) \approx 1
$$

2. Magnetic buoyancy

$$
O\left(\frac{v_{a}}{v_{c}} Q \ln R_{m}^{1 / 2} \frac{g l}{c^{2}}\right) \approx 1
$$

3. Lorentz

$$
O\left(\frac{v_{a}}{v_{c}} Q \ln R_{m}^{1 / 2}\right) \approx 1
$$

and for the driving effects to be small

1. $O\left(\alpha Q \frac{g L}{v_{a}^{2}}\right) \ll 1$

2. $O(Q) \ll 1$

3. $O\left(Q \frac{g L}{c^{2}}\right) \ll 1$

- With regards Temperature backreaction

$$
O\left(\alpha \ln \left(\frac{P e}{R_{m}}\right)^{1 / 2} \frac{P e}{R_{m}}\right) \approx 1
$$


- The exterior flow should be $O(1)$

$$
O\left(Q R_{m} \frac{g L}{v_{a}^{2}} \frac{\nu}{\nu^{T}} \frac{v_{a}}{v_{c}}\right) \approx 1
$$

- The form taken for the thermal diffusivity $1-\kappa=\alpha B^{\beta}$ imposes the restriction

$$
\alpha=O(1)
$$

A typical set of parameters which allow all effects to be felt by the system except the temperature backreaction is

$$
\begin{aligned}
\frac{g L}{c^{2}} & \approx 1 \\
Q & \approx \frac{1}{\ln R_{m}^{1 / 2}} \frac{v_{c}}{v_{a}} \\
\left(\frac{v_{a}}{c}\right)^{2} & \approx \alpha \frac{\ln P e^{1 / 2}}{\ln R_{m}^{1 / 2}} \\
\frac{\nu}{\nu^{T}} & \approx \frac{\alpha \ln P e^{1 / 2}}{R_{m}} \\
\frac{v_{c}}{v_{a}} & =o\left(\ln P e^{1 / 2}\right)
\end{aligned}
$$

Within this model, as $\alpha=O(1)$, the temperature backreaction is always negligible.

\section{An Illustrative Example}

In order to analyse the Self-Consistency equations ( eqns $51 \& 52$ ), the functional forms of the leading fields $\rho_{\text {ext }}, T_{0} \& f_{0}$ are required. Ideally we would like to consider asymptotic forms of realizable exterior flows, however this is another problem in itself and will not be attempted. Rather, we take the simplest forms possible and just demand that they satisfy the appropriate limiting exterior equations.

$$
\begin{aligned}
& 0 \simeq-\nabla p-\rho \hat{\bar{z}}+\nabla^{2} \vec{u} \\
& 0=\nabla \cdot(\rho \vec{u}) \\
& 0 \simeq \nabla^{2} T
\end{aligned}
$$

These lead to the restriction that

$$
\frac{d}{d z}\left(T_{0} \rho_{e x t}\right)+\rho_{e x t}=\frac{d^{2} f_{0}}{d z^{2}}
$$

The simplest double-zeroed function is $f_{0}=z(z+1)$ ( the axial velocity must vanish at top \& bottom of the cell ) and an associated linear family of fields.

$$
\begin{array}{cl} 
& T_{0}=C-\frac{1}{2} z \\
\text { and } \quad \rho_{\text {ext }}=A+\frac{\left(2-\frac{1}{2} A\right) z}{C}
\end{array}
$$


In what follows we have taken $\mathrm{A}=6$ and $\mathrm{C}=1$ so that both $T_{0}$ and $\rho_{\text {ext }}$ increase with depth.

$$
\begin{aligned}
T_{0} & =1-\frac{1}{2} z \\
\& \quad \rho_{\text {ext }} & =6-z
\end{aligned}
$$

We concede that, with the model as it stands, the temperature backreaction is negligible and so $T(z) \approx T_{0}(z)$ is taken. Equation 51 becomes:-

$$
\begin{aligned}
& \begin{array}{ll}
\frac{1-\frac{1}{2} z}{6-z}\left[\frac{12 z-z^{2}}{6-z} h-\right. & z(z+1)] \\
& =\frac{1}{2} a b^{\beta} \int_{0}^{z} h^{\beta-1}(q) d q+e b^{2} \frac{1-\frac{1}{2} z}{6-z}\left(\frac{d h}{d z}+\frac{h d}{2-z}\right) \\
\text { subject to } h(-1)= & 0 \\
\qquad \quad \frac{d h}{d z}+\frac{h d}{2}= & 0 \text { at } z=0
\end{array}
\end{aligned}
$$

Here

$$
\begin{gathered}
a=\bar{\alpha} \ln P e^{1 / 2} \frac{\eta}{\nu} \frac{g L}{v_{c}^{2}} \frac{\chi_{0}^{* \beta}}{\beta} \\
e=\frac{1}{2} \ln R_{m}^{1 / 2} \chi_{0}^{* 2} \frac{R_{m} L}{c \nu \mu \rho} \\
d=\frac{g L}{c^{2}} \\
b=B_{0}
\end{gathered}
$$

This parameterization is chosen to isolate the initial flux $B_{0}$. By varying this and only this parameter, we can examine the equilibrium state reached in both kinematic and dynamic regions. The value of $d$ is largely unimportant in the solution and so is set to 1 . The solution for $h$ can then be written as

$$
h=h\left(a^{1 / \beta} b, \frac{e}{a^{2 / \beta}}\right)
$$

and hence the natural dimensionless parameter to adjust is $\frac{e}{a^{2 / \beta}}$, and the plot to examine is $a^{1 / \beta} b h$ vs $a^{1 / \beta} b$. However in keeping with the original paper by Galloway, Proctor \& Weiss, we plot $b h$ vs $b$ which represents the magnetic field at the axis ( at some $\mathrm{z}$ value-we take $\mathrm{z}=-1 / 2$ ), divided by $R_{m}$ verses the initial uniform field. We consider various sizes of the control parameter $\frac{e}{a^{2 / \beta}}$ which allows the relative effects of Temperature and Lorentz backreactions to be studied, small values implying small Lorentz forces.

\subsection{Results}

The first 3 plots show the effect of varying $\beta$ on the solution. All have the same qualitative form:- a linear kinematic growth of magnetic field followed by a maximum as the dynamic regime is reached. The effect of the Temperature term governed by $a$ is to amplify the flux tube compression and hence increase the axis field. The 4 th 
plot shows the field on the axis as a function of $z$. Note that the field is strongest near the top of the cell, modelling the field entering the Photosphere and decreases to zero at the base. That the maximum is slightly displaced into the cell is a consequence of the magnetic buoyancy. Plot 5 reiterates the similarity between various powers of $\beta>1$. The last two plots show the effect of gradually letting the temperature backreaction dominate. We expected to find qualitatively the same results as before but with perhaps increased maxima, and indeed this is what is observed for $e \geq 1$ ( The bottom curve, $e=6$, is that in plot 2 with $a=1$ ). However for $e<1$ there are runaway solutions i.e. the solution does not equilibrate and the model appears to break down. In this case no solution exists for an initial field exceeding some critical finite value.

\subsection{Scaling Results}

From the plots, we see that a maximum magnetic field strength is achieved for a finite value of the initial field. Typically

$$
\begin{gathered}
B=O\left(R_{m} B_{0}\right) \\
B=O\left(\frac{\nu R_{m}}{L} \sqrt{\mu \rho} Q\right)
\end{gathered}
$$

The scaling of $Q$ depends on the dominant backreaction. If the Lorentz force provides the balance

$$
\begin{gathered}
\Rightarrow Q \sim \sqrt{\frac{\eta}{\nu \ln R_{m}^{1 / 2}}} \\
\Rightarrow B_{a x i s}=O\left(\frac{R_{m}}{L} \sqrt{\frac{\eta \mu \nu \rho}{\ln R_{m}^{1 / 2}}}\right)
\end{gathered}
$$

which agrees with Galloway, Proctor \& Weiss 1978 (1). If the temperature backreaction dominates then

$$
B_{a x i s}^{T}=O\left(\frac{R_{m}}{L} \frac{v_{a}}{c} \sqrt{\frac{\eta \mu \nu \rho}{\alpha \ln P e^{1 / 2}} \frac{c^{2}}{g L}}\right)
$$

Note that

$$
\frac{B_{a x i s}^{T}}{B_{a x i s}}=O\left(\frac{v_{a}}{c} \sqrt{\frac{\ln R_{m}^{1 / 2}}{\alpha \ln P e^{1 / 2}} \frac{c^{2}}{g L}}\right)
$$

and on the sun this seems $O(1)$. Using the first estimate of order with the following parameters:-

$$
\begin{gathered}
B_{a x i s}=2000 \mathrm{Gauss}=0.2 \text { Tesla } \\
\mu=1.257 \times 10^{-6} \mathrm{Hm}^{-1} \\
\eta=10^{9} / T^{3 / 2} \mathrm{~m}^{2} \mathrm{~s}^{-1} \\
L=10^{6} \mathrm{~m} \\
T=10^{4} \mathrm{~K}
\end{gathered}
$$




$$
\rho=3 \times 10^{-4} \mathrm{kgm}^{-3}
$$

gives

$$
\Rightarrow R e=\frac{4 R_{m}^{2}}{10^{8} \ln R_{m}}
$$

Taking $R_{m} \approx 10^{6}$ implies $R e \approx 5000$.

\section{Comments}

- We feel that the energy equation requires a still more careful treatment. Modelling Eddy diffusivities involves entropy gradients as opposed to the rather simplistic approach adopted here of using only temperature gradients. Introducing entropy will complicate the system by coupling the energy equation and equation of state. The effect is to be investigated.

- The stability of the flux rope equilibrium state is an obvious point of interest and one which we would like to study. However a lack of an exterior convective flow solution to base the analysis upon severely limits the utility of any such effort. Moreover, interaction with this flow would appear to be an essential point of this stability problem.

- The model, as it stands, can accommodate only small perturbations of pressure within the flux tube. We would like to extend this to finite amplitude modifications as seems more appropriate in the solar context.

- Plot 7 appears to show that the model merely breaks down for sufficiently small $e$. However one can't help speculate whether this might indicate a critical bound on the initial flux for such a convective state to exist, which might provide a selection mechanism for the size of flux tubes. It is not out of the question that such a breakdown mechanism could be associated with fibrillation of flux. 

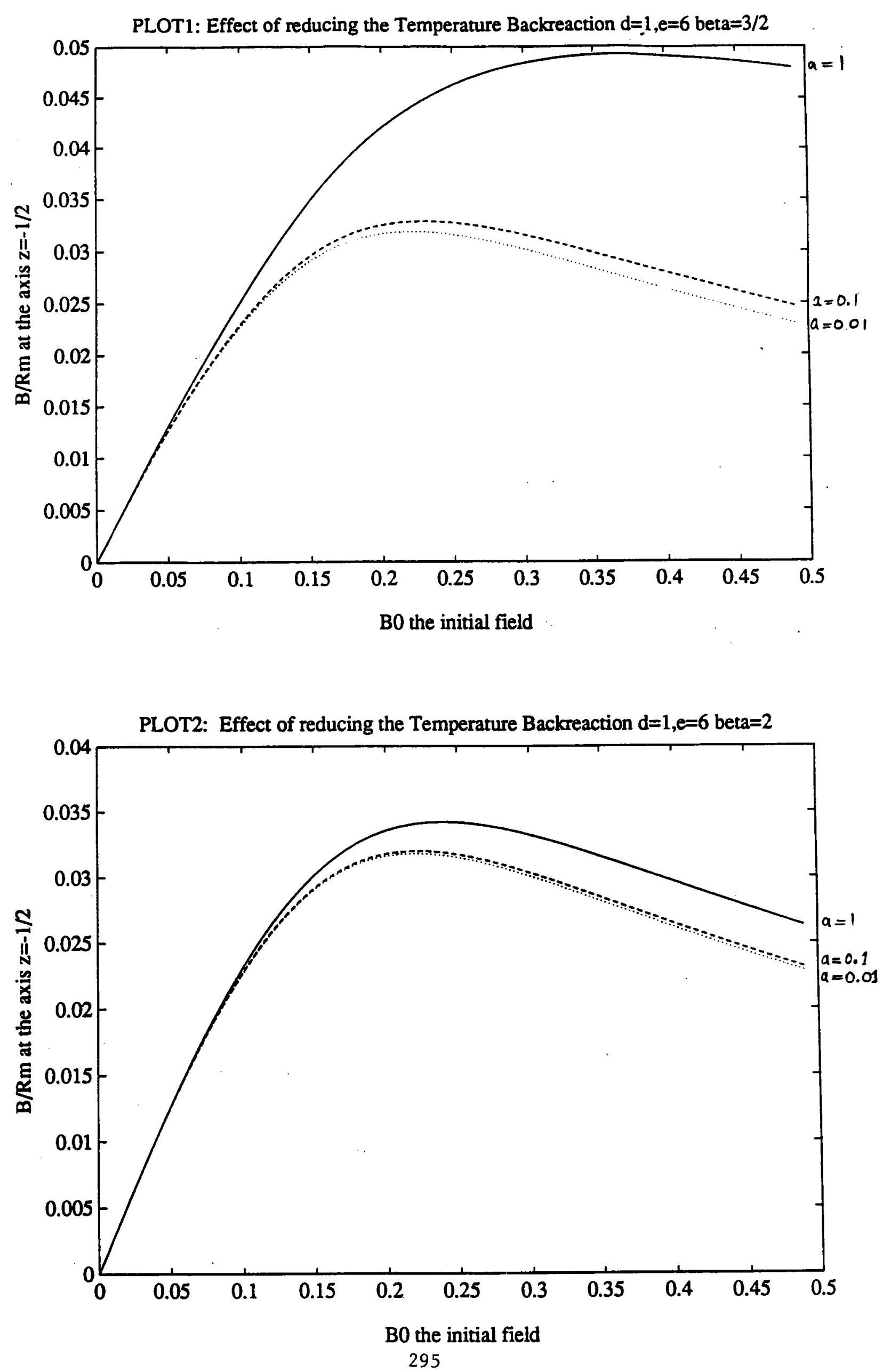

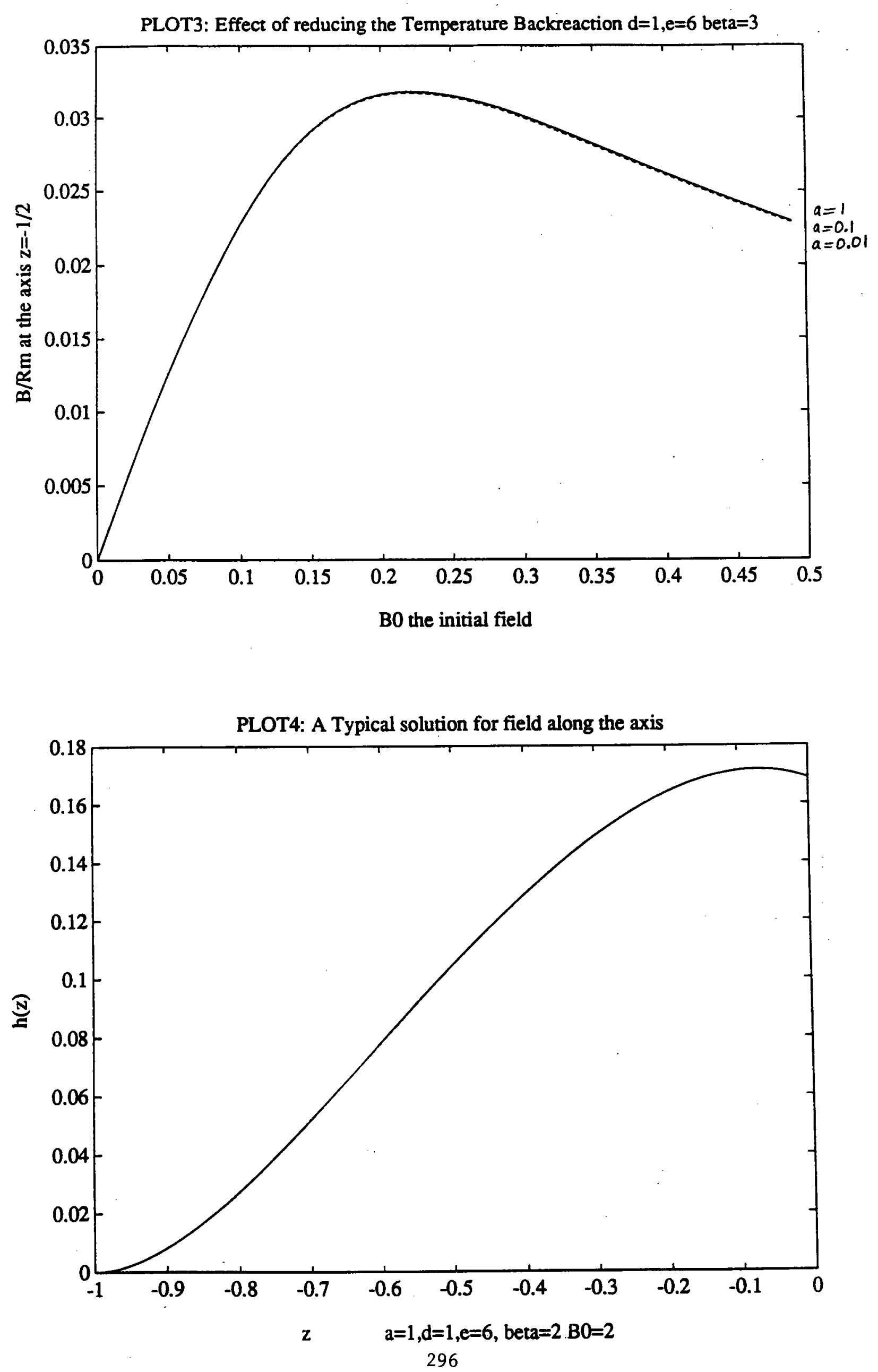

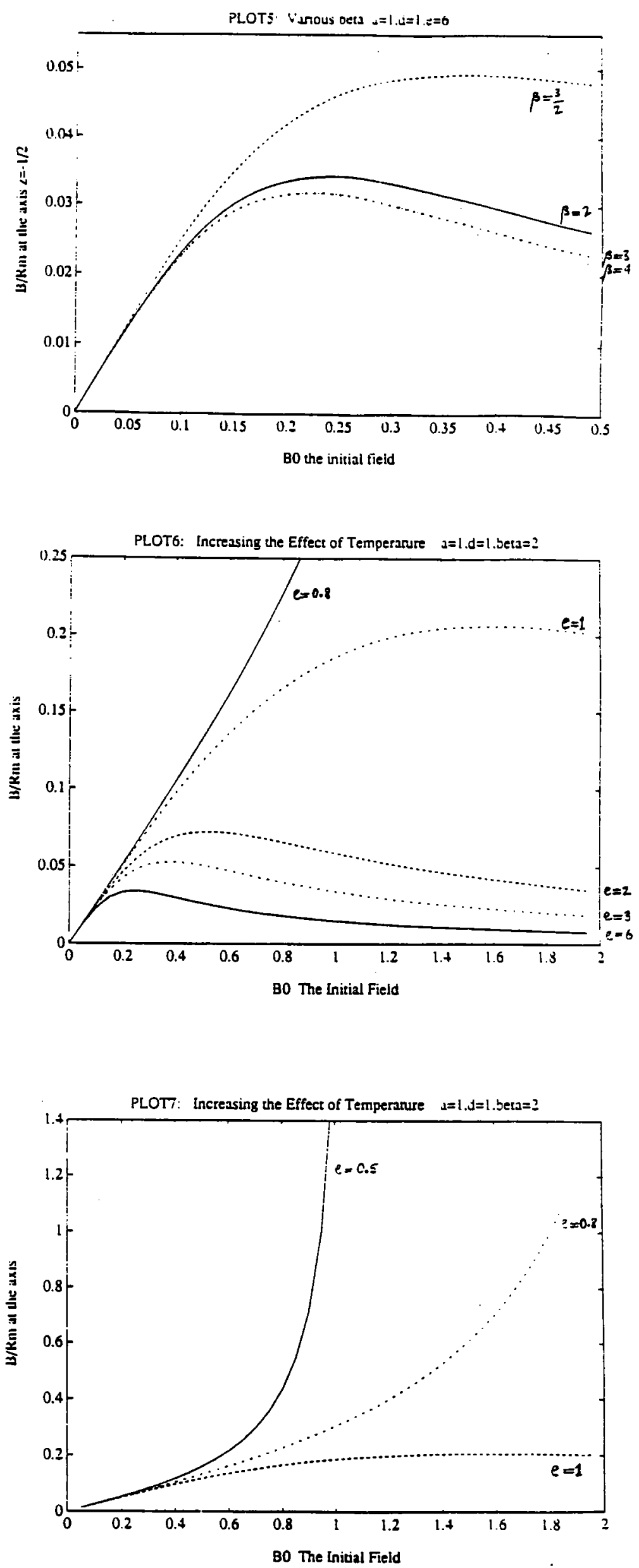

297 


\section{Acknowledgements}

I am indebted to Steve Childress for suggesting and supervising this work. His obvious interest in science and physical insight have been inspirational and I leave here with his words ringing in my ears:- "Now Rich, just think about the physics and the rest will take care of itself..." Hey, thanks, Steve. I must also thank Nigel Weiss, Mike Proctor and particularly Bob Rosner for invaluable advice and encouragement. Thanks also to Ed Spiegel, Phil Morrison and Andrew Soward for sharing their thoughts and opinions and to Andy Woods for always being ready to argue the physics and drive his car very fast. Of course, this summer has been much more than just this project and for this I must thank all the fellows and faculty. Rick Salmon deserves a special mention for making the summer run so smoothly and Ed Spiegel and Jean-Paul Zahn for introducing a bunch of applied mathematicians to astrophysics.

\subsection{References}

1. Galloway, D.J., Proctor, M.R.E. \& Weiss, N.O. 1978 Magnetic flux ropes and convection J. Fluid Mech. 87 243-261

2. Priest, E.R. 1982 Solar Magnetohydrodynamics

3. Clark, A. \& Johnson, A.C. 1967 Magnetic field accumulation in supergranules Solar Phys.2 433-440

4. Parker, E.N. 1963 Kinematic hydrodynamic theory and its application to the low solar photosphere Astrophys. J. $138552-575$

5. Busse, F.H. 1975 Nonlinear interaction of magnetic field and convection J.Fluid Mech.71 193-206

6. Weiss, N.O. 1966 The expulsion of magnetic flux by eddies. Proc.Roy.Soc. A 293 310-328

7. Weiss, N.O. 1967 Magnetic fields and convection. In Problems of Stellar Convection (ed E.A.Spiegel \& J.P.Zahn), pp176-187 Springer. 


\title{
NATURALLY DRIVEN DISPERSION IN TLLTED POROUS LAYERS
}

\author{
Stefan J. Linz \\ FR.11.1 Theoretische Physik \\ Universität des Saarlandes \\ D-6600 Saarbrücken \\ Germany
}

\begin{abstract}
:
We consider convective motion in a tilted porous layer which is driven by a vertical temperature gradient and an additional temperature gradient between the boundaries of the layer. We find an exact stationary flow solution in that system. We discuss Taylor dispersion of passive particles in this flow and emphasize its geophysical implications. The existence of a zero wave number instability in this system is shown. We discuss the stability of the no flow solution in the system without the additional temperature gradient between the boundaries. For the vertical layer we derive nonlinear evolution equations determining the behavior above the convective threshold.
\end{abstract}

\section{INTRODUCTION}

Convection in layers of fluids enclosed between impermeable boundaries seems to be one of the most fascinating aspects in fluid dynamics (cf. the book Platten and Legros (1984) for a recent revlew). The paradigm of these systems, the Rayleigh-Benard system (a horizontal fluid layer subject to a vertical temperature gradient) and its geophysical modification ( porous layer filled with fluid) have be discussed in the past in great detail (see e.g. Chandrasekhar (1961) and for the porous medium case Beck(1972)). However little attention has been given to the situation in which the layer is inclined. In the case of porous media Caltagirone and Bories (1985) and Riley and Winters (1990) discussed fixed temperature boundary conditions while Sen et al (1988) studied fixed heat flux boundary conditions acting on the tilted layer. This report forms a generalization and extension of their work.

Our work is motivated by the following geophysical situation: Suppose there is an inclined sandstone layer between layers of shale. The gravita tional field acts vertically downwards while a geothermic temperature gradient acts upwards. If the thermal diffusivity in shale and the porous medium filled with fluid would be the same the isotherms would be horizontal. However. 
in practice the thermal diffusivities can differ. Continuity of the normal component of the heat flux requirs a declination of the isotherms at the boundaries between shale and sandstone which is analogous to an additional temperature gradient between the boundaries. To model this situation we study convection in a tilted porous layer which is subject to two driving forces: (i) a lateral temperature gradient along the boundaries and (ii) a temperature difference between the boundaries.

Our report is organized as follows: In section 2 we describe our model system. Section 3 contains the basic hydrodynamic equations in nondimensionalized form. In Section 4 we present the most simple solutions of the system and in particular we discuss the structure of the non-trivial flow solution. A study of the "mean" motion of injected passive particles in this flow (Taylor dispersion) and its geophysical significance will be given in Section 5. In Section 6 we find an infinite wave number instability of the flow solution. Up to that point the full system with both gradlents is discussed. Section 7 contains some linear and nonlinear results if no additional forcing between the boundaries is present. In Section 8 we discuss the flow solution in the geophysical problem mentioned above. Section 9 summarizes our findings.

\section{THE SYSTEM}

We consider a porous layer of thickness $d$ enclosed between two parallel boundaries. Gravitational acceleration acts vertically. The porous layer is tilted about the horizontal axis with tilting angle $\varphi$. The layer is subject to a vertical temperature gradient $T_{0} G$ which varies linearly with $z . T_{0}$ is the reference temperature at the origin of the frame of coordinates (cf. Fig.1 ). In addition there is fixed temperature difference $\Delta T$ between the boundaries.

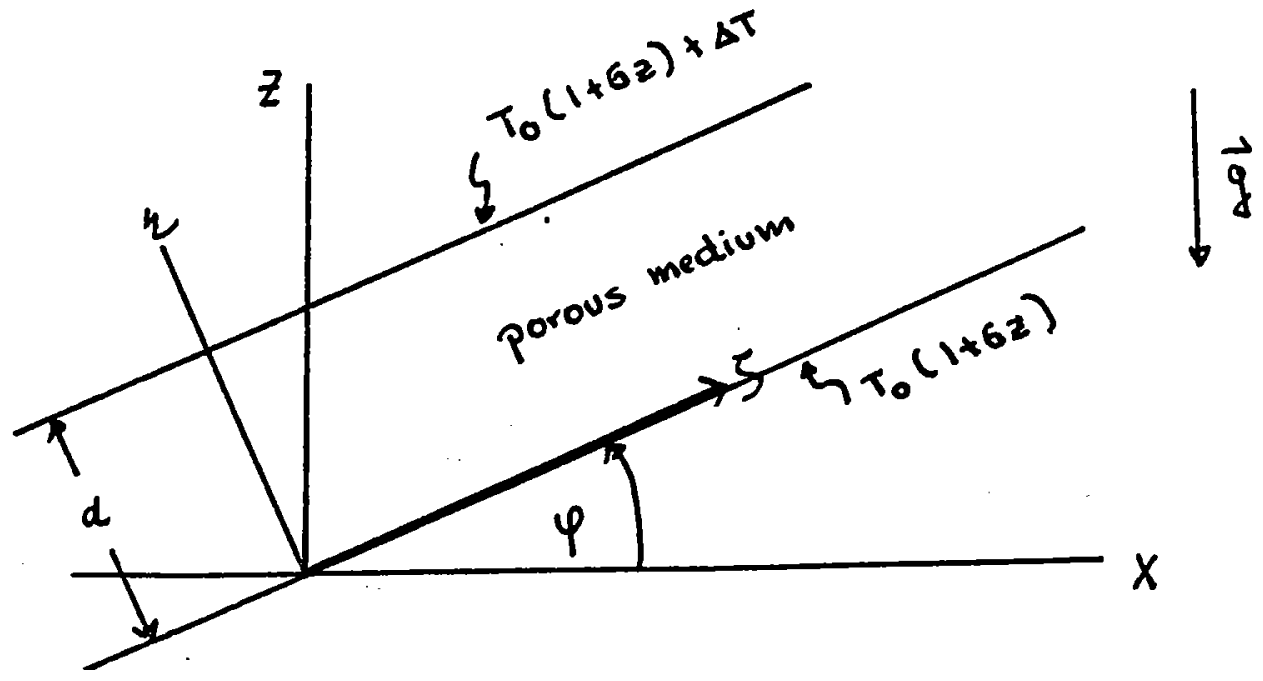

Fig.1 The geometry. 
We assume that the lateral extension is very large in comparison to its thickness. By the way one should not be worried about the fact that for large enough negative $z$ the temperature may become negative. This is only an artifact of our model. In practice this problem does not arise since the layer is long but not infinite. We also require mass conservation in the layer.

In principle there are three parameters which can be varied independently: The gradient along the boundaries, the temperature difference between the boundaries, and the tilting angle.

\section{BASIC EQUATIONS AND BOULNDARY CONDITIONS}

Following D.D. Joseph (1972) we use the generalized Darcy equation to describe the evolution of the seepage velocity $u$ of the fluid inside of the porous layer given by

$$
(\rho / \varepsilon) \partial_{t} \boldsymbol{\alpha}=\nabla p+\rho g-(\mu / K) \mathbf{g}
$$

where $\mu$ is the viscosity of the fluid, $\varepsilon$ the porosity ( i.e. the ratio of void volume to the total volume of the porous medium), $p$ the pressure field, $\mathbf{g}=-\mathrm{ge}_{\mathrm{Z}}$ the downwards acting gravity acceleration, $\mathrm{K}$ the permeability of the porous medium, $\rho$ the mass density field of the fluid and $p_{0}=\rho(x=0, z=0)$. Note that Darcy's equation is linear in $\boldsymbol{a}$ and is valid only if $\boldsymbol{u}$ is small.

The evolution equation for the temperature fleld reads

$$
\left(\partial_{\mathrm{t}}+\mathbf{u} \cdot \nabla\right) \mathrm{T}=\varkappa \nabla^{2} \mathrm{~T},
$$

where $x$ is the thermal diffusivity in the porous medium (Joseph 1972). We assume the density varies linearly with temperature

$$
\rho=\rho_{0}\left[1-\alpha\left(T-T_{0}\right)\right]
$$

where $\alpha$ is the thermal expansion coefficient, $T_{0}$ the temperature at $x=0$ and $z=0$, and $\rho_{0}=\rho\left(T=T_{0}\right)$. We also assume incompressibility

$$
\nabla \cdot \mathbf{u}=0
$$

We have used the Oberbeck-Boussinesq approximation,that all thermal and transport quantities are assumed to be constant and density variations enter only via bouyancy. We non-dimensionalize as follows: length with $d$, time with $d^{2} / x$, velocity with $x / d$, temperature with $x v / \alpha g d K$ and $p / \rho_{0}$ with 
$x^{2 / d^{2}}$ to arrive at the following set of nondimensionalized equations

$$
\begin{aligned}
& \left(\partial_{t}+\varepsilon \sigma\right) \mathbf{u}=-\varepsilon \nabla \hat{\mathbf{p}}-\varepsilon\left(\rho / \rho_{0}\right) \hat{\mathbf{g}} \mathbf{e}_{\mathbf{z}} \\
& \nabla \cdot \mathbf{u}=0 \\
& \left(\partial_{\mathbf{t}}+\mathbf{u} \cdot \nabla\right) \mathbf{T}=\nabla^{2} \mathrm{~T} \\
& \rho / \rho_{0}=1-(\sigma / \hat{\mathbf{g}})\left(\mathrm{T}-\mathrm{T}_{0}\right) .
\end{aligned}
$$

Here $\hat{p}$ corresponds to the nondimensionalized ratio $\left(p / \rho_{o}\right), \sigma=v d^{2} / x K$ is the Darcy-Prandtl number which differs by $d^{2} / K$ from the bulk Prandtl number, and $\hat{\mathbf{g}}=\mathrm{gd}^{3} / \mathrm{x}^{2}$ is the nondimensionalized gravity acceleration. $e_{z}$ denotes the unit vector in $z$ direction. We impose are fixed temperatures, i.e. $T=T_{0}(1+G z)$ at the lower and $T=T_{0}(1+G z)+\Delta T$ at the upper boundaries. $T_{0} G$ denotes the temperature gradient along the boundaries. There is no flow through the boundaries; a consequence of this and the constraint of incompressiblity is that there is no mean flow through the cell. Since the friction in the Darcy equation is proportional to $a$ we have no boundary condition on the I component along the boundaries.

For convenience in the present study we use a tilted coordinate system $(\zeta, \eta)$ (compare Fig.1). In that system $z=\zeta \sin \varphi+\eta \cos \varphi$ and $e_{z}=\sin \varphi e_{\zeta}+\cos \varphi e_{\eta}$. Thus we can write the boundary conditions as

$$
\begin{aligned}
& T(\zeta, \eta=0)=T_{0}(1+G \zeta \sin \varphi) \\
& T(\zeta, \eta=1)=T_{0}(1+G \zeta \sin \varphi)+T_{0} G \cos \varphi+\Delta T \\
& w(\eta=0)=w(\eta=1)=0 .
\end{aligned}
$$

Writing $\nabla=\left(\partial_{\zeta}, \partial_{\eta}\right), \boldsymbol{u}=\mathbf{u}(\zeta, \eta)=(u, w)$, and $T=T(\zeta, \eta)$ the governing equations of our system in the tilted frame read

$$
\begin{aligned}
& \left(\partial_{t}+\varepsilon \sigma\right) \mathbf{u}=-\varepsilon \nabla \hat{p}-\varepsilon\left[g-\sigma\left(T-T_{0}\right)\right]\left(\sin \varphi e_{\zeta}+\cos \varphi e_{\eta}\right) \\
& \left(\partial_{t}+\mathbf{u} \cdot \nabla\right) T=\nabla^{2} T \\
& \nabla \cdot \mathbf{u}=0 .
\end{aligned}
$$


These equations are the starting point of the following work. Let us note several special cases which have been discussed already by other authors:

1) No tilting, $\varphi=0$ : Our system reduces to the Rayleigh-Benard problem in a porous medium with an effective temperature difference $\Delta T_{\text {eff }}=-\left(\Delta T+T_{0} G\right)$ between the boundaries. This case was already studied by Lapwood (1948).

2) No lateral gradient, i.e. $G=0$, but $\Delta T \neq 0$ and $\varphi$ arbitrary: This corresponds to a tilted Rayleigh-Benard system which has been discussed just recently by Caltagirone and Bories (1985) and Rlley and Winters(1990).

3) Vertical layer without lateral gradient, 1.e. $G=0$. In that case Gill (1969) proved that the basic flow solution cannot be destabilized by any $\Delta T$.

To our knowledge the system we consider has not been studied before.

The Darcy-Prandtl number: If the porous medium consists of closely packed sphere-like objects with a mean diameter $d_{0}$, e.g. sand, then Kozeny's formula ( Joseph 1972 ) gives an empirical relation between porosity $\varepsilon$ and permeability $K$

$$
K=\varepsilon^{3}\left[150(1-\varepsilon)^{2}\right]^{-1} \mathbf{d}_{0}^{2}
$$

Typical values of $\varepsilon$ for porous media are $\varepsilon=0.35$ implying that $K$ is about $6.710^{-4} \mathrm{~d}_{0}^{2}$. Thus the Darcy-Prandtl number is given by

$$
0=1500 \sigma_{\text {bulk }}\left(d / d_{0}\right)^{2} \text {. }
$$

The bulk Prandtl number of the fluid, $\sigma_{b u l k}$, is typically of the order 1 . Since we use the seepage concept for the structure of the velocity fleld eq., $d_{0}$ must be very small in comparison to $d$, for sand $d_{0}$ is about $10^{-2} \mathrm{~cm}$. The layer widths $d$ we focus on are typically of the order $1 \mathrm{~cm}$ or much bigger. Thus $\sigma>1.510^{6} \sigma_{\text {bulk }}$ and the limit $\sigma \rightarrow \infty$ seems to be a reasonable appro$x$ imation. Beyond this we note that for fixed $d_{0}$ the thicker the layer the larger the Darcy-Prandtl number.

\section{BASIC SOLUTIONS}

We now derive simple stationary solutions of the equations (12) and (13).

\subsection{No Flow Solution}

The purely conductive solution has a stationary horizontally uniform temperature field and no fluid motion, i.e.

$$
\mathbf{u}_{\mathrm{ml}}=\partial_{\mathbf{t}} \mathbf{u}_{\mathrm{ml}}=\partial_{t} \mathbf{T}_{\mathbf{m l}}=0
$$


everywhere in the layer. Here the index $\mathrm{ml}$ refers to the motionless state. The temperature field equation reduces to

$$
\left(\partial_{\zeta}^{2}+\partial_{\eta}^{2}\right) T_{m l}=0
$$

Using the boundary conditions we easily find the temperature profile in the layer

$$
T_{m l}(\zeta, \eta)=T_{0}+T_{0} G \zeta \sin \varphi+\left(T_{0} G \cos \varphi+\Delta T\right) \eta
$$

Inserting this profile into the velocity field eq. again a condition

$$
\left(T_{0} G \cos \varphi+\Delta T\right) \sin \varphi=T_{0} G \cos \varphi \sin \varphi .
$$

Hence the conductive solution can only exist if either (a) there is no tilting, i.e. $\varphi=0$ or $\varphi=\pi$, one recovers the Lapwood (1948) result, or (b) there is no boundary forcing, i.e. $\Delta \mathrm{T}=0$ and for fixed $\mathrm{z}$ the temperature variations at the plates are the same. Thus as long as there is only a lateral temperature gradient at the boundaries the conductive solution is the "trivial" solution of our system.

\subsection{Stationary Unidirectional Flow Solution}

Let us next try to find a stationary flow solution of the field equations (12) and (13) supposing that there is only a flow solution in $\zeta$ direction, $i$. e.

$$
\partial_{t} \bar{u}_{s}=\partial_{t} T_{s}=0 \text { and } \mathbf{u}_{s}=\mathbf{u}_{s}(\zeta, \eta)=\left(u_{s}, 0\right)
$$

Incompessibility implies that $u_{s}$ can depend on $\eta$ only. Thus we have to solve

$$
\begin{aligned}
& \partial_{\eta} \mathrm{u}_{\mathbf{s}}=\sin \varphi \partial_{\eta} \mathrm{T}_{\mathbf{s}}-\cos \varphi \partial \zeta \mathrm{T}_{\mathbf{s}} \\
& \mathrm{u}_{\mathbf{s}} \partial \zeta \mathrm{T}_{\mathbf{s}}=\left(\partial \frac{2}{\zeta}+\partial_{\eta}^{2}\right) \mathrm{T}_{\mathbf{s}} .
\end{aligned}
$$

where the index s refers to the stationary flow solution. To find this solution we write the temperature fleld as

$$
T_{s}(\zeta, \eta)=T_{m l}(\zeta, \eta)+f(\eta)
$$

where $f(\eta)$ is the "disturbance" of the temperature field caused by the nonzero velocity $u_{s}(\eta)$. Insertion of this ansatz into eqs. (21) and (22) leads immediately to the following relations between the velocity fleld and $f(\eta)$ : 


$$
\begin{aligned}
& u_{s}=\left(T_{0} G \sin \varphi\right)^{-1} \partial_{\eta}^{2} f \\
& \partial_{\eta} u_{s}=\Delta T \sin \varphi+\sin \varphi \partial_{\eta} f .
\end{aligned}
$$

Both can be combined to an inhomogeneous third order equations for $f(\eta)$ which takes the form

$$
\partial_{\eta}^{3} f-a \partial_{\eta} f=b
$$

where $a=T_{0} G \sin ^{2} \varphi$ and $b=T_{0} G \Delta T \sin ^{2} \varphi$. The general solution of (27) is

$$
f(\eta)=f_{1} \exp (\gamma \eta)+f_{2} \exp (-\gamma \eta)+(b / a) \eta+c / a
$$

$f_{1}, f_{2}$, and $c$ are determined by the three boundary conditions

$$
f(\eta=0)=f(\eta=1)=\int_{0}^{1} d \eta \partial_{\eta}^{2} f=0
$$

corresponding to no temperature fluctuations at the boundaries and no lateral mean flow. After some algebra one finds

$$
\begin{aligned}
f(\eta)= & (1 / 2) \Delta T[\exp (\gamma / 2)-\exp (-\gamma / 2)]^{-1}[\exp (\gamma(\eta-1 / 2))-\exp (-\gamma(\eta-1 / 2))] \\
& +\Delta \mathrm{T}(\eta-1 / 2)
\end{aligned}
$$

where the abbreviation $\gamma^{2}=T_{0} G \sin ^{2} \varphi$ was introduced. Note that depending on the sign of G, $\gamma$ is real or imaginary. Combining this solution with (25) and (26) one obtains the desired flow solution as

$$
\begin{aligned}
& u_{s}(\eta)=(1 / 2) \Delta T \sin \varphi H(\gamma, \eta) \\
& T_{S}(\zeta, \eta)=T_{0}+T_{0} G(\zeta \sin \varphi+\eta \cos \varphi)+(1 / 2) \Delta T(1+H(\gamma, \eta))
\end{aligned}
$$

where $H(\gamma, \eta)=\sinh (\gamma(\eta-1 / 2)) / \sinh (\gamma / 2)$ if $G>0$

$$
H(\gamma, \eta)=\sin (|\gamma|(\eta-1 / 2)) / \sin (|\gamma| / 2) \quad \text { if } G<0 .
$$

Let us note the following properties of $H(\gamma, \eta)$

1) $H(\gamma \rightarrow 0, \eta)=2(\eta-1 / 2)$

2) $H(\gamma, \eta=0)=-1$

3) $\mathrm{H}(\gamma, \eta=1)=1$

4) $\mathrm{H}(\gamma, \eta=1 / 2)=0$

5) antisymmetry of $\mathrm{H}$ about midplane: $\mathrm{H}(\gamma, 1-\eta)=-H(\gamma, \eta)$. 
Since the basic flow is stationary $\mathrm{a}_{s}$ and $\mathrm{T}_{\boldsymbol{s}}$ do not depend on the DarcyPrandtl number. In the limit $\Delta T \rightarrow 0$ we recover the no flow solution (17) and (19). Thus the flow solution grows continuously from the no flow solution when $\Delta T$ is increased from zero and grows linearly with $\Delta T$. In the limit $\gamma \rightarrow 0$ $(G \rightarrow 0)$ we recover the solution of Caltagirone and Borles (1985).

An interesting property of our solution is that variation across the layer is determined by the sign of $\mathrm{G}$, the flow direction in the upper/lower half of the layer is determined by the sign of $\Delta T$ : There is upflow in the part bordering on the hotter boundary and downflow in the other part.

In Fig. 2 we sketch the velocity profiles for different $G$ and $\Delta T$. Positive $G$ lead to a suppression of flow near to the midplane in comparison to the case $G=0$. As $G$ increases the flow becomes more concentrated near to the boundaries (boundary layer flow).In contrast if $\gamma$ is negative the flow becomes enhanced near to the middle of the layer. For negative $G$ the flow solution shows anomalies whenever $|\gamma|$ is an even multiple of $\pi$. Then $u_{s}$ diverges inside of the layer. This seems to indicate that our flow solution is unphysical near to these points and that the flow solution is unstable below the first divergence. We attribute this phenomenon to a breakdown of the validity of the Darcy's equation which holds only for small velocity fields.

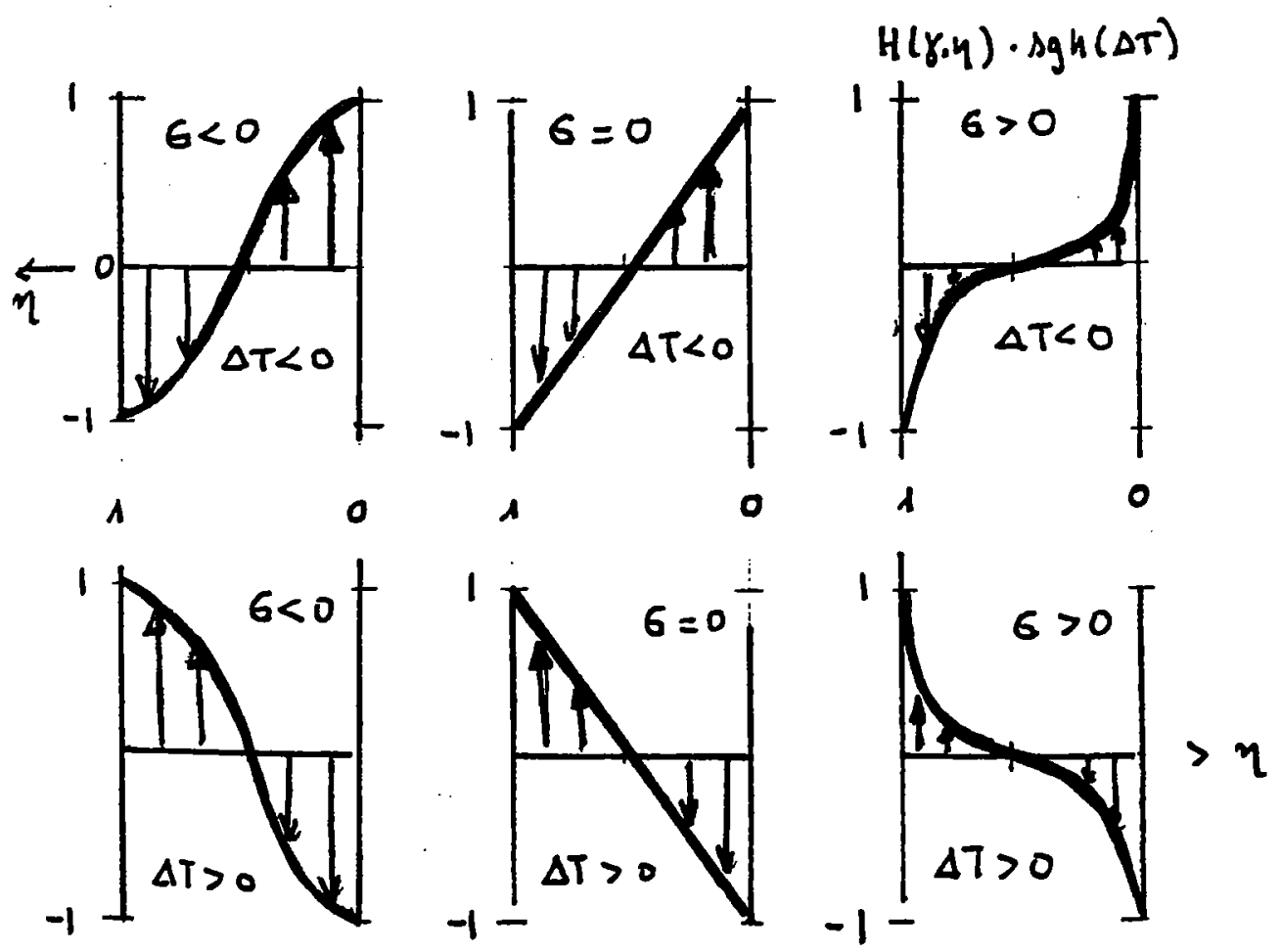

Fig.2 $H(\gamma, \eta) \operatorname{sgn}(\Delta T)$ as function of $\eta$ for several combinations of $G$ and $\Delta T$. 


\section{S TAYLOR DISPERSION IN TILTED LAYERS}

In a seminal paper G.I: Taylor (1953) discussed the the motion of passive particles injected in flows. In particular he considered the long time behavior of dye in a Poiseuille flow and showed that due to the combined action of molecular diffusion and shear a particle distribution initially concentrated at a fixed position diffuses about the center of mass as a Gaussian and is advected with the mean flow.

Following the approach of G.I. Taylor; we now derive the dispersion associated with this flow. Note however the differences from Taylor's case :

our flow is generated by the boundary conditions on the temperature fleld ditions on the temperature field and there is no mean flow.

The diffusion equations for the tracer particles in our flow solution reads

$$
\partial_{t} c+u_{s}(\eta) \partial \zeta^{c}=D \nabla^{2} c
$$

where $D$ is the molecular diffusivity (for reasons of nondimensionalization scaled with $x$ ). Let us assume that variations of $c$ in time occur slower than variations of $c$ caused by diffusion and advection, as will be the case for enough downstream from the initial state. Then

$$
u_{s}(\eta) \partial \zeta^{c}=D \partial_{\eta}^{2} c
$$

Splitting $c$ into a mean concentration $\bar{c}(D)$ which is nothing but the average of $c$ over the layer width and a fluctuating part $c^{\prime}(\zeta, \eta)$, i.e.

$$
c(\zeta, \eta)=\bar{c}\left(\zeta+c^{\prime}(\zeta, \eta)\right.
$$

and noting that one can neglect $\zeta$ variations of the fluctuations $c$ if these are small in comparison to the $\zeta$ variations of mean concentration, one arrives at

$$
\partial_{\eta}^{2} c^{\prime}=D^{-1} u_{s}(\eta) \partial \zeta^{\bar{c}}
$$

With these approximations, in the spirit of G.I. Taylor, we have transformed the fully nonlinear advection-diffusion problem to a linear inhomogeneous differential equation for the concentration fluctuations which can be solved analytically. Inserting of our flow profile and using that

yields

$$
\partial_{\eta}^{2} H(\gamma, \eta)=\gamma^{2} H(\gamma, \eta)
$$




$$
\partial_{\eta}^{2}\left[c^{\prime}-A(\zeta) \gamma^{-2} H(\gamma, \eta)\right]=0
$$

where $A(\emptyset)(\Delta T / 2 D) \partial_{\zeta} \bar{C} \sin \varphi$ is not $\eta$ dependent. This differential equation can be readily solved to give

$$
c^{\prime}=A(\zeta) \gamma^{-2}\left[H(\gamma, \eta)-\left(\partial_{\eta} H(\gamma, \eta)\right)_{\eta=0} \eta+\text { const }\right]
$$

where we have imposed impermeable boundary conditions (no transport of tracer particles through the boundaries): $\partial_{\eta} c^{\prime}=0$ at $\eta=0$ and $\eta=1$.

The constant in $c^{\prime}$ is in general undetermined and will drop out in the following calculation.

The mean concentration flux in $\zeta$ direction is given by

$$
J_{C}=\int_{0}^{1} c u_{s} d \eta=\int_{0}^{1} c\left(\zeta u_{s} d \eta+\int_{0}^{1} c^{\prime} u_{s} d \eta .\right.
$$

Since our flow has no mean flow, the first term on the r.h.s. vanishes. Inserting $c^{\prime}$ into the second term on the r.h.s. yields

$$
J_{C}=(1 / 4 D) \Delta T^{2} \gamma^{-2} \sin ^{2} \varphi F(\gamma) \partial \zeta^{\bar{c}}
$$

where $F(\gamma)$ is given by

$$
F(\gamma)=\int d \eta\left[H^{2}(\gamma, \eta)-\left(\partial_{\eta} H(\gamma, \eta)\right)_{\eta=0} H(\gamma, \eta) \eta\right]
$$

and has to be calculated for both types of the flow profile.

The mean concentration obeys a diffusion law in the long time limit, since

$$
\partial_{t} \bar{c}(\varphi)=-\partial \zeta J_{c}=D_{T} \partial \zeta \bar{c}(\zeta)
$$

From (39) we can read off immediately the value of the Taylor dispersion coefficient which looks formally like a diffusion constant, but is in fact caused by advection of the tracer particles. One finds

$$
D_{T}=-B(\varphi, \Delta T) \gamma^{-2} F(\gamma)
$$

where

$$
B(\varphi, \Delta T)=(1 / 4 D) \Delta T^{2} \sin ^{2} \varphi
$$

and

$$
\begin{aligned}
& F(\gamma)=-\left[2 \gamma \sinh ^{2}(\gamma / 2)\right]^{-1}(2 \gamma+\gamma \cosh \gamma-3 \sinh \gamma) \text { if } G>0 \\
& F(\gamma)=\left[2|\gamma| \sin ^{2}(|\gamma| / 2)\right]^{-1}(2|\gamma|+|\gamma| \cos |\gamma|-3 \sin |\gamma|) \text { if } G<0 .
\end{aligned}
$$




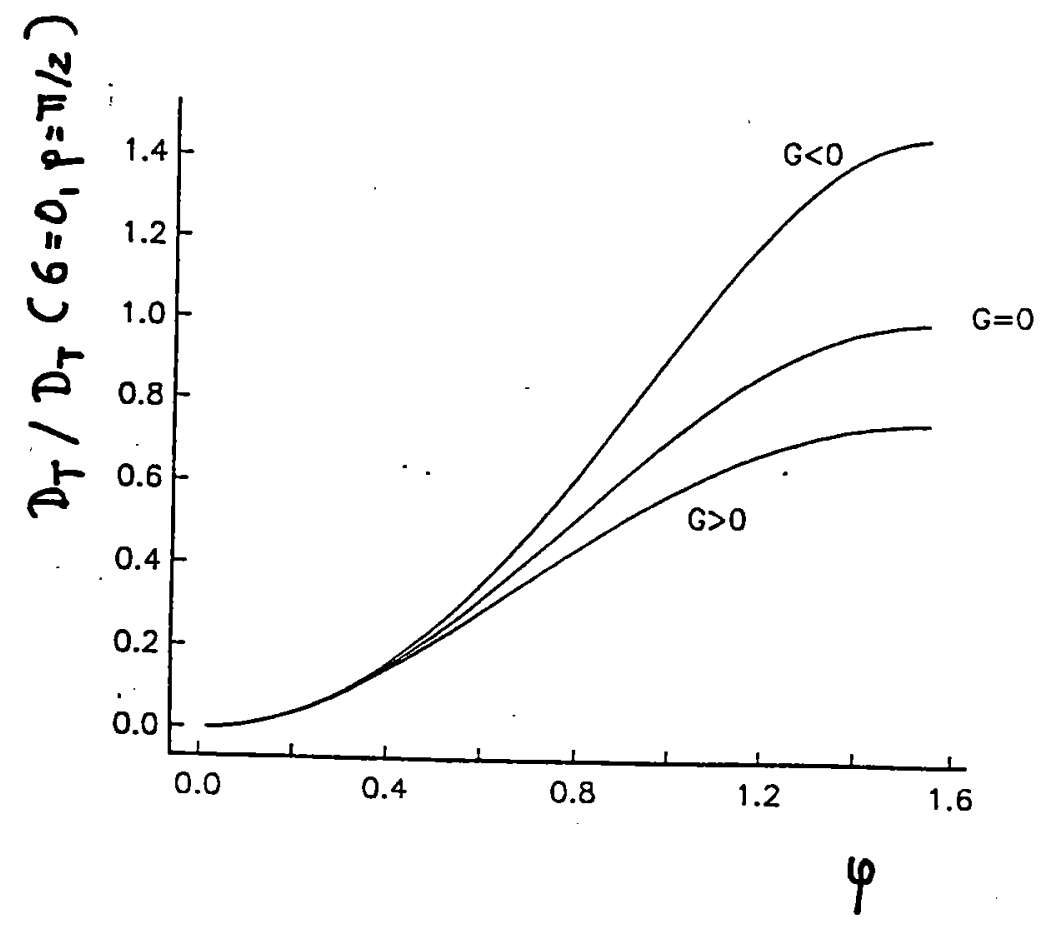

Fig.3 Variation of $D_{T} / D_{T}(G=0, \varphi=\pi / 2)$ with tilting angle $\varphi$.the curves are for $\gamma=3, \gamma=0$, and $\gamma=31$.

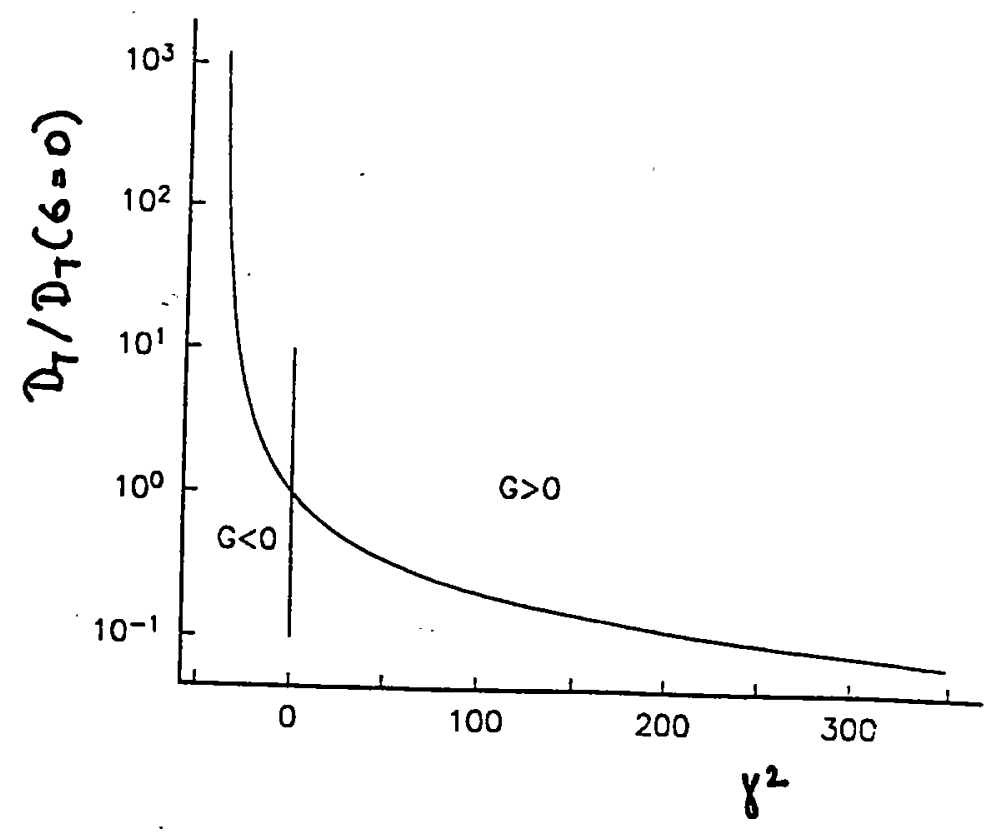

Fig.4 Variation of $D_{T} / D_{T}(G=0)$ as function of $\gamma^{2} \sim G$. Here $\varphi=\pi / 3$. 
Thus the Taylor coefficient consists of a part determined by tilting and $\Delta T$, i.e. $B(\varphi, \Delta T)$ and a part which describes changes of $D_{T}$ due to the temperature variation at the boundaries. One reads off immediately that tilting and temperature gradient across the layer are necessary for a nonvanishing of $D_{T}$. Beyond this $D_{T}$ is invariant with respect to $\Delta T \rightarrow-\Delta T$, depends only quadratically on $\Delta T$ and is symmetric about $\varphi=\pi / 2$. In the limit $\gamma \rightarrow 0(G \rightarrow 0)$ both branches approach the value

$$
D_{T}=(1 / 120 D) \Delta T^{2} \sin ^{2} \varphi \text {. }
$$

Here the maximum value of $D_{T}$ is reached for the vertical layer.

As in Taylor's case $D_{\mathrm{T}}$ is proportional to the inverse of the molecular diffusivity. Let us now study the $\varphi$ and $G$ dependence of $D_{T}$. In Fig. 3 we present the the variation of $D_{T}$ normalized by $D_{T}(0)$ which represents the value of $D_{T}$ with $\mathrm{G}=0$ and $\varphi=\pi / 2$. Of course $D_{T} / D_{T}(0)$ drops to zero quadratically with $\varphi$ if $\varphi \rightarrow 0$. For nonzero $G$ the reduced Taylor coefficlent is similar as shown in Fig.3. The main difference is that negative $G$ enhances its magnitude whereas positive $G$ lowers its magnitude. This behavior can be understood physically by remembering that the r.m.s. flow is bigger (smaller) in comparison to $G=0$ if $G<0(G>0)$. The bigger the mean flow in a layer half the more effective is dispersion. This behavior can also be seen from Fig.4 where we present $\mathrm{D}_{\mathrm{T}} / \mathrm{D}_{\mathrm{T}}(0)$ as function of $\gamma^{2}$. For positive $\gamma^{2}$ the Taylor dispersion coefficient decreases to zero in the limit $\gamma \rightarrow 00$, for negative $\gamma^{2}$ it grows rapidly and diverges at $\gamma^{2}=-4 \pi^{2}$. This divergence is caused by the singular behavior of the flow field for that $\gamma$ value. As we shall see in the next section this $\gamma$ value is the lower bound of stability of our flow solution (29).

Geophysical implications: To estimate the order of magnitude of the Taylor dispersion coefficient we return to dimensional quantities $\bar{D}=\mathrm{D} x$ and $\overline{\mathrm{D}}_{\mathrm{T}}=\mathrm{D}_{\mathrm{T}} x$. For $\mathrm{G}=0$, the Taylor dispersion coefficient with dimensions is

$$
\overline{\mathrm{D}}_{\mathrm{T}}=(1 / 120) x^{2} \overline{\mathrm{D}}^{-1} \Delta \mathrm{T}^{2} \sin ^{2} \varphi
$$

Since in general $\Delta \mathrm{T}$ is of the order 1 or smaller the Taylor dispersion coefficient is typically

$$
\overline{\mathrm{D}}_{\mathrm{T}} \sim \mathrm{O}(x(x / \overline{\mathrm{D}}))
$$

Here $x / \bar{D}$ is an inverse Lewis number being typically bigger than one.

We deduce from (43) that the Taylor dispersion coefficient is of the order of the thermal diffusivity of the transporting fluid. This seems to be the main result of our theory. Since in general $\bar{D}$ is much smaller than the 
thermal diffusivity $x$ (typical values $\bar{D}=10^{-5} \mathrm{~cm}^{2} / \mathrm{sec}, \quad x=10^{-3} \mathrm{~cm}^{2} / \mathrm{sec}$ ) it follows that the Taylor dispersion coefficient is much bigger than the molecular diffusivity. Our theory can also be applied in the following ways: 1) The molecular diffusivity of a given passive tracer can be determined by experiment using the above theory.

2) Given the molecular diffusivity one can estimate the amount of material which would be transported by Taylor dispersion through the layer. Assuming that at time $t=0$ all particles are concentrated at $\zeta=0$ the solution of the "diffusion" equation (39) is a Gaussian with respect to $\zeta$ of the form

$$
\bar{c}(\zeta, t) \approx\left(4 \pi \bar{D}_{\mathrm{T}} \mathrm{t}\right)^{-1 / 2} \exp \left(-\zeta^{2} / 4 \bar{D}_{\mathrm{T}} \mathrm{t}\right)
$$

Here we have already used that $\bar{D} T$ » $\bar{D}$.

The width of the Gaussian is given by $l=2\left(\bar{D}_{\mathrm{T}} t\right)^{1 / 2}$. Thus assuming a $\Delta \mathrm{T}$ of order $1, x=10^{-3} \mathrm{~cm}^{2} / \mathrm{sec}$, and $\bar{D}=10^{-5} \mathrm{~cm}^{2} / \mathrm{sec}$ one can estimate the spread of the particles due to dispersion: Supposing $\varphi=\pi / 2$ after $1 \mathrm{~h} l=3.3 \mathrm{~cm}$, after $1 \mathrm{~d} l=16.6 \mathrm{~cm}$, and after $10 \mathrm{a} l=1 \mathrm{~km}$.

The final question is the non-dimensionalized value of the temperature difference $\Delta T_{\text {dim }}$ between the boundaries corresponding to $\Delta T$. Since we scaled temperature by $x v / \alpha g d K$ the value of $\Delta \mathrm{T}_{\text {dim }}$ depends on fluid properties and layer width. For water $\left(x \approx v \approx 10^{-3} \mathrm{~cm}^{2} / \mathrm{sec}, \alpha \approx 310^{-4} 1 / \mathrm{grd}\right)$ in sandstone $\left(\mathrm{K} \approx 2 \cdot 10^{-5} \mathrm{~cm}^{2}\right)$ one finds that $\Delta \mathrm{T}$ dim $=(1 / 6 \mathrm{~d}) \Delta \mathrm{T}[\mathrm{grd} \mathrm{cm}]$. Thus the thicker the layer the smaller the external temperature difference necessary to obtain a fixed $\Delta T$.

A final comment to our theory: We have assumed that the porous medium is homogeneous and isotropic and ignored changes of $\bar{D}$ due the structure of the porous medium.

\section{BOUND FOR THE STABILITY OF THE FLOW SOLUTION}

If the layer is horizontal, $\varphi=0$, the results of Lapwood (1948) can be recovered: There is an stationary instability at $C_{s}(k)=-\left(\Delta T+T_{0} G\right)=-\left(\pi^{2}+k^{2}\right)^{2} / k^{2}$ where the critical wave number (which minimalizes the absolute value of $C_{s}$ ). is $k_{c}=\pi$ and the critical temperature gradient there is $C_{c r i t}=4 \pi^{2}$. In that case there is no instability present if $k=0$. These results are concerned with the stability of the conductive solution.

The situation is different if the layer is tilted. Then the linearized equations for disturbances $u-\left(u_{s}, 0\right)$ and $\theta=T-T_{s}$ of the basic flow solution read

$$
\begin{aligned}
& \left(\partial_{t}+\varepsilon \sigma\right) \nabla^{2} \Psi=-\varepsilon \sigma\left(\sin \varphi \partial_{\eta} \Theta-\cos \varphi \partial \zeta^{\Theta}\right) \\
& \left(\partial_{t}-\nabla^{2}\right) \Theta=-\left(\partial_{\eta} \mathrm{T}_{s} \partial \zeta^{\Psi}-\partial \zeta^{\mathrm{T}}{ }_{s} \partial_{\eta} \Psi+u_{s} \partial \zeta^{\Theta}\right) .
\end{aligned}
$$


Here we have introduced the stream function $\Psi$ which is related to the velocity field by $\boldsymbol{u}-\left(\mathrm{u}_{s}, 0\right)=\left(-\partial_{\eta} \Psi, \partial \zeta \Psi\right)$ if the flow is two-dimensional and incompressible. The general solution of the above stability problem is difficult, in particular since via $T_{s}$ and $u_{s}$ these equations are explicitely $\eta$ dependent and for arbitrary tilting angles $\varphi$ there is a crosscoupling of derivatives. However we can find an upper bound for the instability of the basic flow solution analytically. To do this we consider the $k=0$ limit of the above equations at the stability threshold $C_{t h}=T_{0} G_{t h}$ which are given by

$$
\begin{aligned}
& \left(\partial_{t}+\varepsilon \sigma\right) \partial_{\eta}^{2} \Psi=-\varepsilon \sigma \sin \varphi \partial_{\eta} \theta \\
& \left(\partial_{t}-\partial_{\eta}^{2}\right) \theta=C_{\text {th }} \sin \varphi \partial_{\eta} \Psi .
\end{aligned}
$$

Exact eigenfunctions of these equations are

$$
\begin{aligned}
& \Psi(\eta, t)=a(t)+b(t) \sin \lambda \eta+e(t) \cos \lambda \eta \\
& \Theta(\eta, t)=c(t)+d(t) \sin \lambda \eta+f(t) \cos \lambda \eta
\end{aligned}
$$

where the boundary conditions $\Psi=\theta=0$ at $\eta=0$ and 1 imply that $\lambda=2 \pi n, e=-a$, and $f=-c$. Here $n$ is positive and integer. Note that $\lambda=\pi n$ with odd $n$ do not fullfil the boundary conditions. This is significantly different from the nontilted case. The time dependence of $a, b, c$, and $d$ at $C_{t h}$ is $\exp (\Lambda t)$ with $\operatorname{Re}(\Lambda)=0$ and in general $\operatorname{Im}(\Lambda)=\omega$.

Inserting these expressions into the $k=0$ equations leads to a characteristic equation

$$
-\omega^{2}+\left(4 \pi^{2} n^{2}+\sigma \varepsilon\right) i \omega+4 \pi^{2} n^{2} \sigma \varepsilon+\sigma \varepsilon C_{t h} \sin ^{2} \varphi=0
$$

Equating imaginary and real parts implies that $\omega=0$. Thus the instability is stationary and there is no oscillatory instability for $k=0$. From the real parts one finds that stability thresholds are located at

$$
C_{\text {th }}=-4 \pi^{2} n^{2} \sin ^{-2} \varphi
$$

for $n=1,2, \ldots$. The stability threshold with the smallest absolute value is the one with $n=1$. Thus a lower bound for the the instability of $u_{s}$ is given by

$$
C_{S}(k=0)=-4 \pi^{2} \sin ^{-2} \varphi
$$

Whenever $\mathrm{C}<\mathrm{C}_{\mathbf{s}}(\mathrm{k}=0)$ our flow solution is unstable at least against infinite 
wave length perturbations. Note that the stability threshold is independent of the applied $\Delta \mathrm{T}$. This is caused by the fact that all terms containing $\Delta T$ enter via $\zeta$ derivatives which drop out if $k=0$ instabilities are discassed. Thus the lateral gradient is the only destabilizing force. The zero wave number instability exists whenever the tilting angle is nonzero.

From (52) it follows that in the limit $\varphi \rightarrow 0$ this instability vanishes. Its minimal value is reached if the layer is vertical. Gill (1969) has shown that if $G=0$ the basic flow solution cannot be destabilized by any $\Delta T$ if the layer is vertical. We have shown with (52), that an additional lateral gradient can destabilize this solution. We present $C_{s}(k=0)$ reduced by its maximal value as function of the tilting angle $\varphi$ in Fig.5.

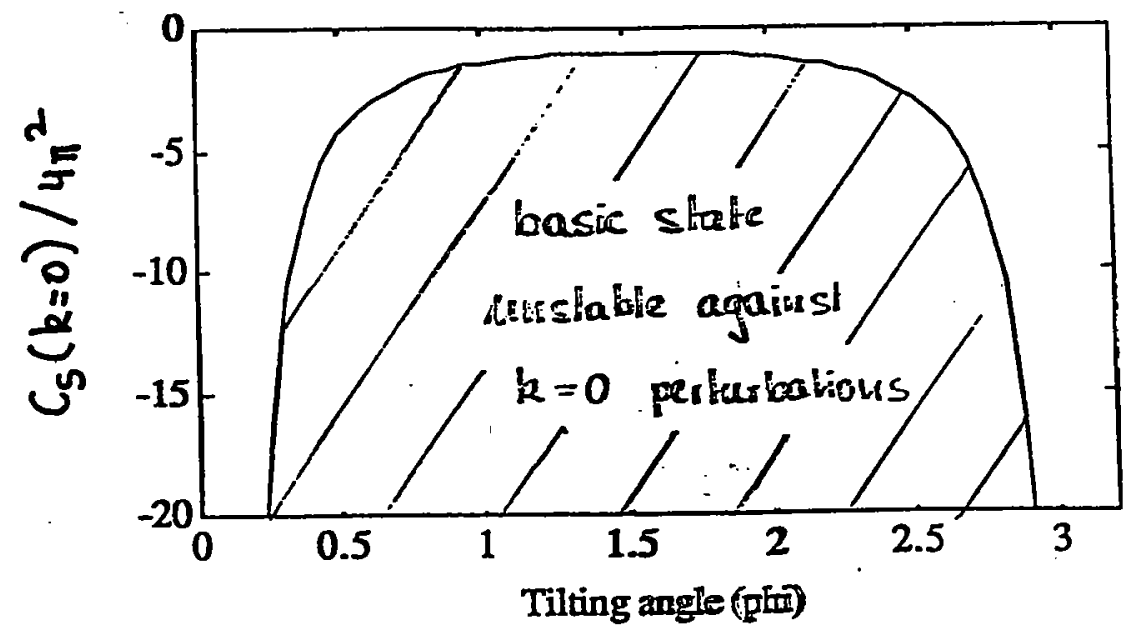

Fig.j Variation of $C_{S}(k=0) / 4 \pi^{2}$ with $\varphi$

Up to now we have not discussed the arbitrary wave number case. A search for exact analytical eigenfunctions would be difficult. Thus numerical investigations based on a Galerkin method have to be performed. This is planned in the future.

\section{THE LAYER WITH $\Delta T=0$}

We discuss in this section the stability of the the no flow solution with $\Delta \mathrm{T}=0$ and develop nonlinear evolution equations. We suppose that the DarcyPrandtl number is infinite. 


\subsection{Stability of the conductive state for a vertical layer}

Here we want to calculate how the stability threshold bifurcates out of the $k=0$ instability. Thus we have to study the stationary case only. For the vertical layer $(\varphi=\pi / 2)$ the equations linearized about the conductive state read after insertion of a lateral Fourier ansatz $\sim \exp (i \mathrm{k} \emptyset$ at threshold

$$
\begin{aligned}
& \left(\partial_{\eta}^{2}-k^{2}\right) \Psi=-\partial_{\eta} \Theta \\
& \left(\partial_{\eta}^{2}-k^{2}\right) \Theta=-C_{s} \partial_{\eta} \Psi,
\end{aligned}
$$

or up to the second order in $k$

$$
\left[\partial_{\eta}^{4}-\left(2 k^{2}+C_{s}\right) \partial_{\eta}^{2}\right] \Psi=0
$$

where the boundary conditions $\Psi=\Theta=0$ have to be fullfilled. Since these equations are invariant with respect to $k \rightarrow-k$ the following perturbation ansatz of $\Psi$ and $\mathrm{C}_{S}$ holds

$$
\begin{aligned}
& \Psi=\Psi_{0}+\Psi_{2} k^{2}+O\left(k^{4}\right) \\
& C_{s}=C_{o}+C_{2} k^{2}+O\left(k^{4}\right) .
\end{aligned}
$$

The order $k=0$ solution was found already in Section 6 . In order $k^{2}$ we have to solve the equation

$$
\begin{aligned}
\left(\partial_{\eta}^{2}+4 \pi^{2}\right) \Psi_{2} & =\left(C_{2}+2\right) \partial_{\eta}^{2} \Psi_{0} \\
& =4 \pi^{2}\left(C_{2}+2\right)[a \cos 2 \pi \eta-b \sin 2 \pi \eta]
\end{aligned}
$$

where $C_{0}=-4 \pi^{2}$ and $\Psi_{0}$ in form of eq.(48) was used. The inhomogeneous solution of $\Psi_{2}$ is given by

$$
\Psi_{2, \text { inh }}=K_{1} \eta(1-\cos 2 \pi \eta)+K_{2} \eta \sin 2 \pi \eta
$$

with $K_{1}=(b / 4 \pi)\left(C_{2}+2\right)$ and $K_{2}=-(a / 4 \pi)\left(C_{2}+2\right)$. A solvability condition follows from the boundary condition on $\theta$. One finds

$$
\left.\dot{\partial}_{\eta} \Psi_{2}\right|_{0} ^{1}=\left(C_{2}+2\right)(-a / 4 \pi)=0 \text {. }
$$

Thus $C_{2}=-2$ and the stability threshold up to order $k^{2}$ reads

$$
C_{s}(k)=-4 \pi^{2}\left[1+k^{2} / 2 \pi^{2}+O\left(k^{4}\right)\right] .
$$


From eq. $(60)$ we infer that $C_{0}$ is in fact at least for small $k$ the critical value of the stability threshold. Beyond we note that $\Psi_{2}$ is zero.

\subsection{Stability threshold for the tilted layer}

In the tilted case things are somewhat different. The equations linearized around the conductive state read

$$
\begin{aligned}
& \left(\partial_{\eta}^{2}-k^{2}\right) \Psi=1 k \cos \varphi \theta-\sin \varphi \partial_{\eta} \theta \\
& \left(\partial_{\eta}^{2}-k^{2}\right) \theta=1 k C \cos \varphi \Psi-C \sin \varphi \partial_{\eta} \Psi
\end{aligned}
$$

which are in general no longer invariant with respect to $k \rightarrow-k$. Thus the the perturbation ansatz in that case must also incorporate terms proportional to $\mathrm{k}$

$$
\begin{aligned}
& \Psi=\Psi_{0}+\Psi_{1} k+O\left(k^{2}\right) ; \theta=\theta_{0}+\theta_{1} k+O\left(k^{2}\right) \\
& C_{s}=C_{0}+C_{1} k+O\left(k^{2}\right)
\end{aligned}
$$

Performing a calculation similar to the one in Section 7.1. one finds

$$
C_{1}^{2}=32 \pi^{2} \cot \varphi / \sin ^{2} \varphi \text {. }
$$

Thus there are two stability thresholds bifurcating out of $C_{0}$ given by

$$
C_{S}=-4 \pi^{2} \sin ^{-2} \varphi\left[1 \pm 2^{1 / 2} \pi^{-1} \cot \varphi k\right]
$$

where the one with the plus sign is the minimal one for positive $k$. (64) tells us in the tilted layer in general the $k=0$ instability is not critical. The relevant first correction in $k$ is linear in $k$ and always destabilising with the exception of the vertical layer. Thus one has to expect a behavior as sketched in Fig.6.

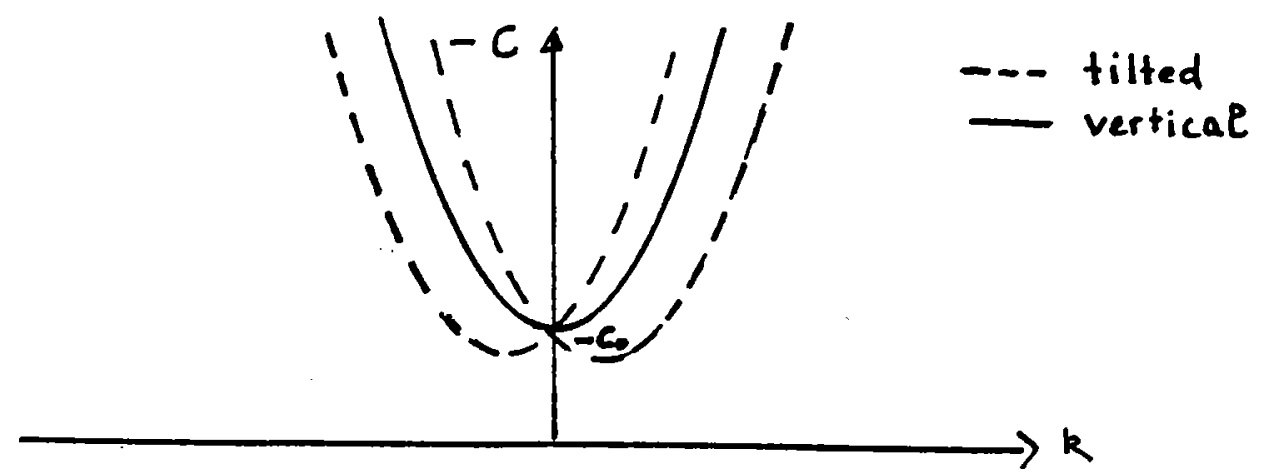

Fig.6 Stability thresholds of the tilted and the vertical layer as function of k. 


\section{3. nonlinear amplitude equations for the vertical layer}

Here we want to derive two coupled amplitude equations governing the convective state above threshold. In the case of the vertical layer we know from Section 7.1. that the $k=0$ instability is critical. Thus an approach similar to the one performed by Chapman and Proctor (1980) can be applied. The basic difference between their work ( done for Rayleigh-Benard convection with fixed heat flux at the boundaries) and our work consists in the different types of scaling.

To study long wave number instabilities one scales length in $\zeta$ directions with $\mathrm{L}$ where $\mathrm{L}$ is assumed to be the lateral extension of the layer. $\mathrm{L}$ has to be very large in comparison to $d$. Defining $\varepsilon=d / L$ the rescaling is $\zeta \rightarrow \varepsilon^{-1} \zeta$ and $\partial \zeta \rightarrow \varepsilon \partial_{\zeta}$. Wave numbers are rescaled according to $k \rightarrow \varepsilon k$. Time will be scaled with $\varepsilon^{-2}$. The time scaling is motivated by the scale on which growth temperature fluctuations can take place. Beyond this we scale $\Psi$ and $\theta$ with $\varepsilon$.

Motivated by the stability analysis of Section 7.1 we set

$$
C=C_{0}+\mu \varepsilon^{2}
$$

where we assume that $\mu=O(1)$. Thus $\mu$ measures for a given $\varepsilon$ the magnitude of the the actual temperature gradient $C=T_{0} G$.

Then the rescaled equations read

$$
\begin{aligned}
& \left(\varepsilon^{2} \partial_{\zeta}^{2}+\partial_{\eta}^{2}\right) \Psi=-\partial_{\eta} \theta \\
& \left(\varepsilon^{2} \partial_{\zeta}^{2}+\partial_{\eta}^{2}-\varepsilon^{2} \partial_{t}\right) \theta=-\left(C_{0}+\mu \varepsilon^{2}\right) \partial_{\eta} \Psi-\varepsilon^{2}\left(\partial_{\eta} \Psi \partial_{\zeta} \Theta-\partial_{\zeta} \Psi \partial_{\eta} \theta\right) .
\end{aligned}
$$

Note that the nonlinearities (which appear in the temperature field equation only) are proportional to $\varepsilon^{2}$ according to our scaling. Since (66) and (67) are invariant with respect to $\varepsilon \rightarrow-\varepsilon$, an appropriate ansatz for $\Psi$ and $\theta$ in terms of $\varepsilon$ is

$$
(\Psi, \theta)=\left(\Psi_{0}, \theta_{0}\right)+\varepsilon^{2}\left(\Psi_{2}, \theta_{2}\right)+O\left(\varepsilon^{4}\right)
$$

The zeroth order in $\varepsilon$ was already calculated in Section 7.1. The only difference is the interpretation of the amplitudes, which are now in general $\zeta$ and $t$ dependent. We find

$$
\begin{aligned}
& \Psi_{0}(\zeta, t)=A(\zeta, t)(1-\cos 2 \pi \eta)+B(\zeta, t) \sin 2 \pi \eta \\
& \Theta_{0}(\zeta, t)=2 \pi B(\zeta, t)(1-\cos 2 \pi \eta)-2 \pi A \sin 2 \pi \eta .
\end{aligned}
$$

Here again $C_{0}=-4 \pi^{2}$. Our intention in the following is the derivation of evolution equations for the amplitudes $\mathrm{A}$ and $\mathrm{B}$. To do this we have to solve the order $\varepsilon^{2}$ which takes the form 


$$
\begin{aligned}
\left(\partial_{\eta}^{3}+4 \pi^{2} \partial_{\eta}\right) \Psi_{2}=-4 \pi^{2}\left(A \partial_{\zeta^{A}}+B \partial \zeta^{B}\right)+2 \pi\left(\partial_{\zeta}^{2} B-\partial_{t} B\right)+ \\
+\left(4 \pi^{2}\left(A \partial \zeta^{B}-B \partial \zeta^{A}\right)+2 \pi \mu A-4 \pi \partial_{\zeta}^{2} A+2 \pi \partial_{t} A\right) \sin 2 \pi \eta \\
+\left(4 \pi^{2}\left(A \partial \zeta^{A}+B \partial \zeta^{B}\right)+2 \pi \eta B-4 \pi \partial_{\zeta}^{2} B+2 \pi \partial_{t} B\right) \cos 2 \pi \eta
\end{aligned}
$$

Solving the inhomogeneous contribution of $\Psi_{2}$ and using the boundary conditions $\Psi_{2}(\eta=1)-\Psi_{2}(\eta=0)=0$ and $\partial_{\eta} \Psi_{2}(\eta=1)-\partial_{\eta} \Psi_{2}(\eta=0)=0$, which follow from the velocity field and the temperature boundary condition respectively, leads finally to the following coupled amplitude equations

$$
\begin{aligned}
& \partial_{t} B=(4 / 3) \partial_{\zeta}^{2} B-(\mu / 3) B-2 \pi\left(A \partial \zeta^{A}+B \partial_{\zeta} B\right) \\
& \partial_{t} A=2 \partial_{\zeta}^{2} A-\mu A-2 \pi\left(A \partial \zeta^{B}-B \partial \zeta^{A}\right) .
\end{aligned}
$$

Let us discuss some properties of these equations. 1) Setting $B=0$ the nonlinearity in the A equation vanishes. The contrary is not true.

2) The equations are invariant with respect to $\zeta \rightarrow-\zeta, B \rightarrow-B, A \rightarrow-A$, with respect to $\zeta \rightarrow-\zeta, \mathrm{B} \rightarrow-\mathrm{B}, \mathrm{A} \rightarrow \mathrm{A}$, and with respect to $\zeta \rightarrow \zeta, \mathrm{B} \rightarrow \mathrm{B}, \mathrm{A} \rightarrow-\mathrm{A}$. They reflect three invariances (i) $\zeta \rightarrow-\zeta, \eta \rightarrow \eta, \Psi \rightarrow-\Psi, \theta \rightarrow-\theta$, (ii) $\zeta \rightarrow-\zeta$, $\eta \rightarrow(1-\eta) . \Psi \rightarrow \Psi, \Theta \rightarrow-\Theta$, and (iii) $\zeta \rightarrow \zeta, \eta \rightarrow(1-\eta), \Psi \rightarrow-\Psi, \Theta \rightarrow \Theta$ of the basic equations (66) and (67), respectively.

3) The linear stability analysis of the conductive state $A=B=0$ yields that the amplitudes $A$ and $B$ become unstable at different control parameter values: $A$ at a squared reduced wave number $q^{2}=-\mu / 4$ and $B$ at $q^{2}=-\mu / 2$. Going back to the non-rescaled variables this implies that $\mathrm{A}$ becomes unstable at at $C_{A}(k)=-4 \pi^{2}\left(1+k^{2} / 2 \pi^{2}\right)$ and $B$ at $C_{B}(k)=-4 \pi^{2}\left(1+k^{2} / \pi^{2}\right)$. Thus for $C<C_{A}$ the amplitude $A$ becomes unstable first and drives via nonlinearities the amplitude $B$ to finite values.

Work on the nonlinear solutions of these equations is in progress.

\section{ANALOGY TO A GEOPHYSICAL PROBLEM.}

Let us now turn to the geophysical problem mentioned in the introduction which is motivated by considerations of Davis et al. (1985): a tilted water-filled sandstone layer embedded in shale under the influence of a vertical temperature gradient. Now we show how the difference in the thermal diffusivities of shale, $x_{s}$, and fluid-filled sandstone, $x$, are in the case of stationary flow analogous to an external temperature difference between the boundaries sandstone-shale. In that case the temperature gradient along the boundaries is $\partial_{\zeta} T=T_{0} G \sin \varphi$ while the one normal to the boundaries is $\partial_{\eta} T=\varepsilon T_{0} G \cos \varphi$. Here $\varepsilon=x_{s} / x$. These boundary conditions come from the fact that the temperature and the normal component of the heat fluxes have to be equal at the boundary standstone-shale. 
Using these boundary conditions and eqs. (11) - (14) one finds the following stationary flow solution

$$
u_{s}(\eta)=(\varepsilon-1) \cot \varphi E(\gamma, \eta)
$$

where

$$
\begin{array}{ll}
E(\gamma, \eta)=\gamma \sinh (\gamma(\eta-1 / 2)) / \cosh (\gamma / 2) & \text { if } G>0 \\
E(\gamma, \eta)=-|\gamma| \sin (|\gamma|(\eta-1 / 2)) / \cos (|\gamma| / 2) & \text { if } G<0,
\end{array}
$$

and $\gamma^{2}=T_{0} G \sin ^{2} \cdot \rho$. We remark that this flow solution vanishes, i.e. $a_{s}=0$, if either the thermal diffusivities $x$ and $x_{s}$ of the fluid-filled sandstone and the shale are equal or the porous layer is vertical.

Comparing this stationary flow solution with the one found in eq. (29) one finds as condition for equivalence of both flows

$$
\begin{array}{ll}
\Delta \mathrm{T} \tan \varphi \sin \varphi=-2|\gamma|(\varepsilon-1) \tan (|\gamma| / 2) & \text { if } \mathrm{G}<0 \\
\Delta \mathrm{T} \tan \varphi \sin \varphi=2 \gamma(\varepsilon-1) \tanh (\gamma / 2) & \text { if } G>0 .
\end{array}
$$

Via (75) and (76) we can map the results for the geophysical problem to the system with externally applied $\Delta T$ whenever the flow is stationary and the layer is tilted ( $\varphi$ nonzero and not equal to $\pi / 2$ ). This holds in particular for our theory of Taylor dispersion. In nature $\varepsilon$ is typically between .8 and 1.2 (see e.g. Davis et al.(1985). Thus the sign of the equivalent $\Delta T$ depends on the direction of the geothermal gradient and the value of $\varepsilon-1$.

\section{CONCLUSIONS AND PERSPECTIVES.}

To summarize our findings:

1) For the system described in Section 2 we calculated a nonlinear flow solution and gave an upper bound for the stability of this solution.

2) We studied Taylor dispersion in that flow and found that the dispersion coefficient is of the order of the thermal diffusivity of the fluid filled porous medium.

3) We discussed for the case of zero $\Delta T$ the stability of the conductive solution and found that the vertical layer has a critical zero wave number instability. This is no longer the case for the inclined layer. Beyond this nonlinear evolution equations for the vertical layer were given.

To the future work:

1) We have also derived nonlinear evolution equations for the tilted case. Then a different scaling is used, since the nonlinear basic equations are no longer invariant with respect to $k \rightarrow-k$. These results will be discussed in 
detail elsewhere. In particular the problem of the distinguished limit for $\varphi \rightarrow \pi / 2$ is of interest.

2) The stability analysis of the flow solution for the case of nonzero $\Delta T$ has to be carried out.

3) An extension our theory of Taylor dispersion to the case of bulk fluids (without the porous medium) has to be discussed ( Linz and Woods, in preparation).

\section{ACKNOWLEDGMENTS:}

I would like to thank Andy Woods for introducing me into this subject, his continuous interest, advice, and encouragement. It was a pleasure to work with him.

Gratefully acknowledged are also interesting discussions with Joe Keller, Ed Spiegel, and George Veronis. Special thanks also to Rick Salmon for perfect organization of the GFD summer program. It was a great summer in every way.

\section{REFERENCES:}

J.L. Beck (1972), Phys. Fluids 15,1377.

J.P.Caltagirone and S.A.Bories (1985), JFM 155,267.

S. Chandrasekhar (1981), Hydrodynamic and Hydromagnetic Stability.

C. Chapman and M.R.E. Proctor (1980), JFM 199,760.

S.H.Davis, S. Rosenblat, J.R. Wood, and T.A. Hewitt (1985), Am.J.Sc. 285,207. A.E. Gill (1969), JFM 35,545.

D.D. Joseph (1972), Stability of Fluid Motions II,Ch. X, Springer-Verlag,Berlin.

E.R. Lapwood (1948), Proc. Camb. Phil. Soc. 44,508.

J.K. Platten and J.C. Legros (1984), Convection in Fluids,Springer Verlag.

D.S.Riley and K.H. Winters (1990), JFM 215,309.

M.Sen, P. Vasseur, and L. Robillard (1988), Phys. Fluids 31,3480.

G.I. Taylor (1953), Proc.Roy.Soc.London 219,186. 


\title{
Behavior of a Fifth Order System of ODE's with Intermittency
}

by

\author{
N. Platt
}

\begin{abstract}
A fifth order system of ordinary differential equations is studied as control parameters are varied. The system displays an intermittency akin to type II intermittency. The intermittent behavior of one of the variables is characterized by random switching on and off of the activity. Fixed points of the system are found and their linear stability is studied both numerically and analytically. Different characteristics of the chaotic attractor are presented through time series, phase plots and Poincare sections. Numerical study of the statistics of the intermittency include the average length of the laminar phase near the intermittency threshold, histograms of the length of the laminar phases near the onset of the intermittency and the lacunarity of the autocorrelation function.
\end{abstract}

\section{Introduction}

In this presentation we are interested in a dynamical system which exhibits a peculiar behavior characterized by some variables intermittently turning themselves off and staying inactive for a considerable amount of time and later having a burst of activity. In general, intermittency in a dynamical system is characterized by a random burst of activity in a system already undergoing some periodic oscillations. Figure 1 shows a typical time signal obtained in a dynamical system with intermittency. This is to be contrasted with Figure 2 showing a time signal obtained in a dynamical system under consideration. 


\section{Governing Equations}

We follow the presentation given by Spiegel $^{1}$ to obtain a dynamical system with intermittency shown in Figure 2 . Consider a mechanical system with 2 degrees of freedom driven by a force derived from a time-dependent potential and subject to a drag force. Then equations governing the motion are

$$
\begin{aligned}
& \ddot{x}=-\frac{\partial V}{\partial x}-\epsilon \nu \dot{x} \\
& \ddot{y}=-\frac{\partial V}{\partial y}-\epsilon \nu \dot{y}
\end{aligned}
$$

where $\epsilon, \nu$ are constants. The time dependence of $V$ enters through a time dependent parameter $z, V=V(x, y, z)$. We take the following differential equation for $z$

$$
\dot{z}=-\epsilon\left(z+a\left(x^{2}+y^{2}-1\right)\right)
$$

where $a$ is a constant. We take a special case of the generic potential given by $\mathrm{Thom}^{2}$ and obtain the following parabolic umbilic

$$
V=\frac{1}{4}\left(x^{4}+y^{4}\right)+y x^{2}-\frac{1}{2} z\left(x^{2}+y^{2}\right)
$$

Thus, we arrive at the fifth order system of differential equations

$$
\begin{aligned}
& \dot{x}=p \\
& \dot{p}=-x^{3}-2 x y+z x-\epsilon \nu p \\
& \dot{y}=q \\
& \dot{q}=-y^{3}-x^{2}+z y-\epsilon \nu q \\
& \dot{z}=-\epsilon\left(z+a\left(x^{2}+y^{2}-1\right)\right)
\end{aligned}
$$

Here, $a, \nu, \epsilon$ are constants and we restrict them to be positive. If we set $x=0, p=0$, then the third order system in $(q, y, z)$ separates out. This system is equivalent to a Lorenz system ${ }^{3}$ under a suitable transformation of variables $^{4}$. If $\epsilon=0$ then the $\dot{z}$ equation simplifies to $z=$ const and thus we have a Hamiltonian system with 2 degrees of freedom where $H$ is given by

$$
H=V+\frac{1}{2}\left(p^{2}+q^{2}\right)
$$




\section{$3 \quad$ Fixed Points}

In preparation for the study of the linear stability of the system, we look for the fixed points of the dynamical system. They satisfy

$$
\begin{aligned}
p & =0 \\
q & =0 \\
x\left(-x^{2}-2 y+z\right) & =0 \\
-y^{3}-x^{2}+z y & =0 \\
z+a\left(x^{2}+y^{2}-1\right) & =0
\end{aligned}
$$

Two cases arise:

Case 1: $x \neq 0$

Then the equations of the fixed points can be written as

$$
\begin{aligned}
x & = \pm \sqrt{z-2 y} \\
z & =-a\left(x^{2}+y^{2}-1\right) \\
-(1+2 a) y^{3}+3 a y^{2}+(2+a) y-a & =0
\end{aligned}
$$

These equations were solved numerically using Mathematica for various values of the parameter $a>0$ and four real fixed points I-IV were found with fixed points I, IV and II, III related by equation 16 .

Case 2: $x=0$

In this case we obtain the following fixed points

$$
\begin{array}{ll}
\left(x=0, y=\sqrt{\frac{a}{1+a}}, z=\frac{a}{1+a}\right) & \text { Fixed Point V } \\
\left(x=0, y=-\sqrt{\frac{a}{1+a}}, z=\frac{a}{1+a}\right) & \text { Fixed Point VI } \\
(x=0, y=0, z=a) & \text { Fixed Point VII }
\end{array}
$$




\section{Linear Stability Analysis}

To study linear stability of the fixed points we obtain the Jacobian of the system

$$
\nabla_{\mathbf{x}} F=\left[\begin{array}{ccccc}
0 & -3 x^{2}-2 y+z & 0 & -2 x & -2 a \epsilon x \\
1 & -\epsilon \nu & 0 & 0 & 0 \\
0 & -2 x & 0 & -3 y^{2}+z & -2 a \epsilon y \\
0 & 0 & 1 & -\epsilon \nu & 0 \\
0 & x & 0 & y & -\epsilon
\end{array}\right]
$$

and examine its eigenvalues at various fixed points $x_{\mathbf{0}}$.

If $x=0$ then the eigenvalues $\lambda$ satisfy

$$
\left(\lambda(\epsilon \nu+\lambda)-z_{0}+2 y_{0}\right)\left((\epsilon+\lambda)\left(-3 y_{0}^{2}+z_{0}-\lambda(\lambda+\epsilon \nu)\right)\right)=0
$$

Substituting for the fixed point VII, we obtain

$$
(\epsilon+\lambda)(\lambda(\epsilon \nu+\lambda)-a)^{2}=0
$$

Thus,

$$
\begin{aligned}
\lambda_{1} & =-\epsilon \\
\lambda_{2,3} & =\frac{-\epsilon \nu-\sqrt{\epsilon^{2} \nu^{2}+4 a}}{2} \\
\lambda_{2,3} & =\frac{-\epsilon \nu+\sqrt{\epsilon^{2} \nu^{2}+4 a}}{2}
\end{aligned}
$$

Hence, for $a>0$ fixed point VII is always unstable. Similarly, it can be shown that fixed point VI is always unstable if $a>0$.

Stability of the rest of the fixed points was determined numerically by Mathematica for various $a>0, \nu>0, \epsilon>0$ and is summarized below:

- Fixed points II, III are always unstable

- There exists $\epsilon_{1}, \epsilon_{2}$ with $\epsilon_{2}<\epsilon_{1}$ such that

\begin{tabular}{l|l|l}
\multicolumn{2}{c}{ Fixed Points I, IV unstable $\left.\right|_{1} ^{\epsilon_{1}}$ Fixed Points I, IV stable } \\
Fixed Pt V unstable & $\epsilon_{2} \ldots \quad$ Fixed Pt V stable
\end{tabular}


Numerical experiments on the dynamical system with initial conditions $x=0.01$ and the rest of the variables set to zero produce the following diagram:

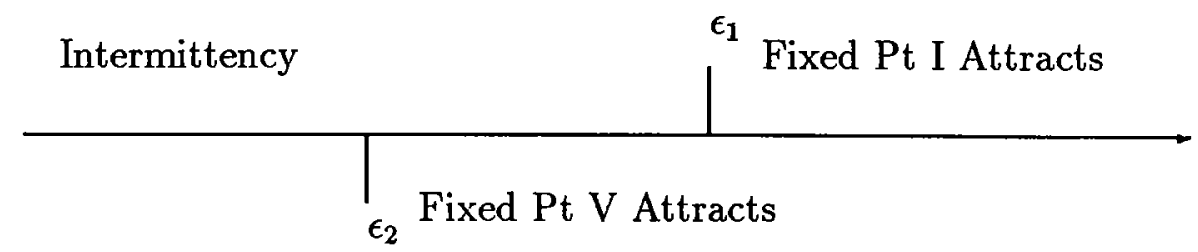

Thus, the threshold of the intermittency in the dynamical system is controlled by the stability of the fixed point $\mathrm{V}$ and the parameter $\epsilon$ when the fixed point $V$ is losing stability is going to be denoted by $\epsilon_{c}$.

\section{Intermittent Regime}

For further studies we fix $a=6.5$ and $\nu=4.125$ and study the resulting system in more detail near the intermittency threshold $\epsilon_{c}$.

Numerically, we obtain the following values for the fixed points and critical values of $\epsilon$ :

$$
\begin{array}{cc}
\multicolumn{2}{c}{\epsilon_{1}=0.825} \\
\epsilon_{2}=\epsilon_{c}=0.441279 & \\
(x=-0.82, y=-0.65, z=-0.62) & \text { Fixed Point I } \\
(x=-0.76, y=0.45, z=1.47) & \text { Fixed Point II } \\
(x=0.76, y=0.45, z=1.47) & \text { Fixed Point III } \\
(x=0.82, y=-0.65, z=-0.62) & \text { Fixed Point IV } \\
(x=0, y=0.93, z=0.87) & \text { Fixed Point V } \\
(x=0, y=-0.93, z=0.87) & \text { Fixed Point VI } \\
(x=0, y=0, z=6.5) & \text { Fixed Point VII }
\end{array}
$$

Table 1 lists the dimensions of the unstable manifolds of various fixed points at the intermittency threshold $\epsilon_{c}=0.441279$. 


\begin{tabular}{|c|c|c|}
\hline Fixed Point & Dimension & Eigenvalue \\
\hline I,IV,V & 2D & complex conjugate \\
II, III & 1D & real \\
VI & 3D & complex conjugate + real \\
VII & 2D & both real \\
\hline
\end{tabular}

Table 1: Dimensions of the unstable manifolds at $\epsilon_{c}$

Since the threshold of the intermittency is determined by the fixed point $\mathrm{V}$ becoming unstable, and its occurs as a pair of complex conjugate eigenvalues cross the imaginary axis, we conclude that this dynamical system displays intermittency similar to type II intermittency. The main distinction is that in a standard definition of type II intermittency ${ }^{5}$ the limit cycle, not a fixed point, becomes unstable as a pair of complex conjugate Floquet multipliers leave the unit circle in the complex plane (This is equivalent to a couple of complex conjugate eigenvalues moving across the imaginary axis).

Figures 2-4 show the time series of $x, y, z$ and Figure 5 shows the broad band power spectrum of the signal $x$. The time series of $x$ is characterized by the intermittent switching on and off of the signal. Here, $x$ spends approximately 2/3 of its time in the laminar phase. From Figures 3 and 4 we conclude that in the $y-z$ plane the dynamical system exhibits a Lorenz-type chaotic behavior, spending most of the time circling around either fixed point $\mathrm{V}$ or VI with random jumps from one to another. Unlike a pure Lorenz system, we note that fixed point VI has an extra unstable manifold when compared to the fixed point $V$ with a positive real eigenvalue. Hence, the dynamical system spends most of its time in the vicinity of the fixed point $\mathrm{V}$.

Figure 6 shows the $x-p$ phase space plot. This plot is especially interesting since it describes the behavior of the system during intermittent bursts of activity (in the laminar phase, $x=p=0$ ). Figure 7 depicts the $y-z$ phase space plot. It shows two characteristic Lorenz type ears. Unlike the Lorenz attractor, one of the ears is traversed by the solution much longer than the other (see explanation in the previous paragraph). In addition, there are some extra curves of motion imposed on top of the Lorenz attractor. We believe that they correspond to the intermittent excursions of the dynamical system during bursts of activity in the $x-p$ plane. Figure 8 shows the phase 
space plot in the $x-z$ plane. Here, the laminar phase corresponds to the line $x=0$, and additional curves capture the dynamics of the chaotic phases.

Let us briefly describe the dynamics in the $x-p$ plane. Treating $z$ and $y$ as free parameters, we find the following governing equations:

$$
\begin{aligned}
& \dot{x}=p \\
& \dot{p}=-x^{3}+\left(z_{o}-2 y_{o}\right) x-\epsilon \nu p
\end{aligned}
$$

Stability of the fixed point $(x=p=0)$ is determined by the eigenvalues

$$
\lambda_{1,2}=\frac{-\epsilon \nu \pm \sqrt{\epsilon^{2} \nu^{2}+4\left(z_{o}-2 y_{o}\right)}}{2}
$$

Thus, $(x=p=0)$ is stable if and only if $z_{o}-2 y_{\circ} \leq 0$. Close to the fixed point $\mathrm{V}, z_{o}-2 y_{o}$ is negative. Thus, fixed point $(x=p=0)$ is stable. As the trajectory spirals out of the fixed point $\mathrm{V}$ along its $2 \mathrm{D}$ unstable manifold it reaches a point where $z_{o}-2 y_{o}$ becomes positive and thus $(x=p=0)$ is unstable. As the solution traverses the spiral, the farther away from the fixed point $\mathrm{V}$ the trajectory is located, then the longer $z_{0}-2 y_{0}$ remains positive. Eventually, the solution either jumps to another ear of the Lorenz attractor and the process starts all over again, except much faster, or there is an intermittent burst of activity in the $x-p$ plane. After that burst of activity, the trajectory is reinjected back close to the fixed point $\mathrm{V}$ (the least unstable fixed point), and the whole process starts all over again. Thus, the length of the laminar phase depends on how close to the fixed point $V$ the solution reinjects itself and the number of times the solution jumps from one ear of the Lorenz attractor to another without significantly departing from the fixed point $(x=p=0)$ in the $x-p$ plane.

Figure 9 shows a natural log-log plot of the average length of the laminar phase vs distance from the critical value of the parameter $\epsilon$. The most surprising feature of this plot is that it seems that the average length of the laminar phase does not approach infinity as $\epsilon \rightarrow \epsilon_{c}{ }^{5,6}$. One possible explanation for that phenomena is that as $\epsilon \rightarrow \epsilon_{c}$ the total length of the laminar phase diverges. Hence, it is possible that there is a range in the parameter space close to $\epsilon_{c}$ where the average length of the laminar phase stays almost constant, but the length of the chaotic phase is rapidly decreasing. The slope of the straight line in Figure 9 is approximately -0.64 . 
Figure 10 depicts a projection of the Poincare section of the attractor onto the $x-p$ plane at $\epsilon=0.44$. Here, the cutting plane is $y=0.93$ and $\dot{y}>0$. This Poincare section describes the behavior of the attractor during chaotic phases $(x=p=0$ in the laminar phase). Figure 11 depicts a projection of the Poincare section of the attractor onto the $y-z$ plane. Here, the cutting plane is $x=0.05$ and $\dot{x}>0$. This Poincare section also depicts the behavior of the system during chaotic phases. In Figure 12, we show the Poincare section projection onto the $x-p$ plane. Here, the cutting plane is $z=0.87$ and $\dot{z}>0$. The laminar phase corresponds to the dots located at the origin. Figures 13-16 show additional Poincare sections.

\section{Lacunarity}

We consider a statistical moment, $C$, on a fractal set that depends on a separation scale $l^{7}$. A self-similar fractal set may be expected to satisfy the scaling law

$$
C(l)=\sigma^{-1} C(\rho l)
$$

where $\rho, \sigma$ are real numbers. One of the particular solutions is

$$
C_{o}(l)=A l^{d}
$$

where $A$ is a constant, $d=\ln \sigma / \ln \rho$. The general solution is

$$
C(l)=l^{d} \chi\left(\frac{\ln l}{P}\right)
$$

where $P=\ln \rho$ and $\chi$ is a periodic function of period 1 .

We apply the above to the autocorrelation function of the time signal $x^{2}$ given by

$$
C(l)=\lim _{T \rightarrow \infty} \frac{1}{T} \int_{0}^{T} x^{2}(t) x^{2}(t+l) d t
$$

Figure 17 shows a $\ln -\ln$ plot of the autocorrelation function at $\epsilon=0.441279$ and Figure 18 is a blow-up of the same. The indicated line has a slope $d \sim-1.1$. Also, periodic oscillations are clearly present in the plot, but their period seems to decrease. Figures 19 and 20 show $\ln -\ln$ plots of the autocorrelation function as parameter $\epsilon$ is decreasing. The slope $d$ is increasing as parameter $\epsilon$ is moving away from the critical value $\epsilon_{c}$. 


\section{Histograms of the length of the laminar phase}

Figure 21 show ln-ln plot of the histogram of the length of the laminar phases vs. number of phases at the critical value $\epsilon=0.441279$. Smoothing average have been applied. The slope of a straight line is approximately $-3 / 2$. Figures 22-24 depict histograms of the lengths of the laminar phases at various values of the parameter $\epsilon$, as indicated. It is interesting to note that the slope is decreasing as $\epsilon$ is moving away from the critical value.

\section{Conclusions}

In this report a fifth order system of differential equations with intermittency has been investigated. The nature of the intermittency (type II) was identified. All fixed points of the system were found and linear stability analysis performed. Phase space plots and Poincare sections have been employed in an attempt to describe the physical nature of the intermittency and the structure of the chaotic attractor. An interesting feature of the dynamical system relating to the average length of the laminar phase was discovered. It seems that the average length of the laminar phase does not approach infinity as $\epsilon \rightarrow \epsilon_{c}$ (or at least it has an almost constant plateau for some range of parameter $\epsilon$ near $\epsilon_{c}$ ). Some analysis of the autocorrelation function of the signal and histograms of the lengths of the laminar phases has been performed.

Future work should resolve the point raised about the average length of the laminar phase near the threshold of the intermittency. Also, additional work is required for a closer examination of the lacunarity of the autocorrelation function. Another avenue worth investigating is to look for a statistical model describing the intermittency.

Acknowledgement: I would like to thank Edward Spiegel for his guidance and help in dealing with this problem. Most of the numerically intense computations were performed at the Center for Fluid Mechanics, Turbulence and Computation of Brown University, Providence, RI. 


\section{References}

[1] Spiegel, E.S., A class of ordinary differential equations with strange attractors, Annals of the New York Academy of Sciences, 357, p. 305-312, (1980)

[2] Thom, R., Structural stability and morphogenisis, W.A. Bemjamin, London, (1975)

[3] Lorenz, E.N., Deterministic nonperiodic flow, J. Atmos. Sci., 29, p. 5363, (1963)

[4] Marzec, C.J. and E.A. Spiegel, Ordinary differential equations with strange attractors, SIAM J. App. Math., 38, p. 387-421, (1980)

[5] Berge, P., Pomeau, Y., C. Vidal, Order within Chaos, John Wiley \& Sons, Inc., New York, (1986)

[6] Argoul, F. and A. Arneodo, A three-dimensional dissipative map modeling type-II intermittency, submitted for publication.

[7] Smith, L.A, Fournier, J.-D. and E.S. Spiegel, Lacunarity and intermittency in fluid turbulence, Physics Letters, 114A, No. 8-9, p. 465-468, (1986) 


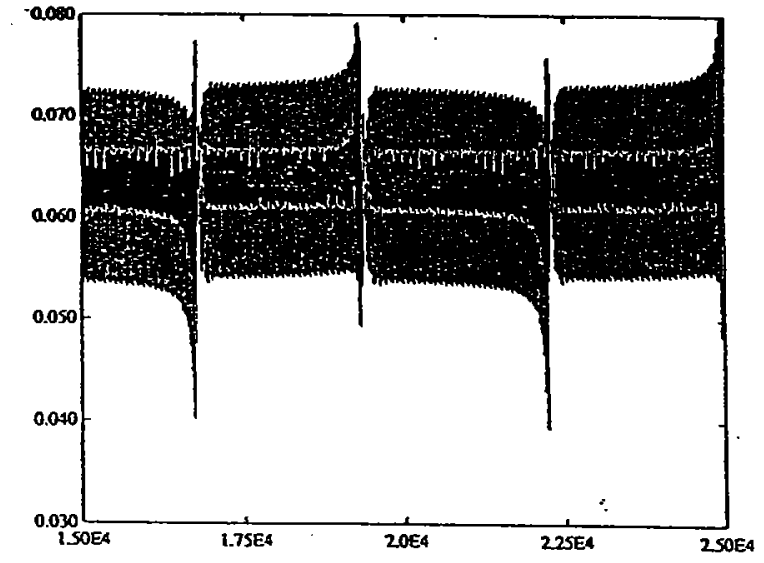

Figure 1: Time Series of a System with Intermittency

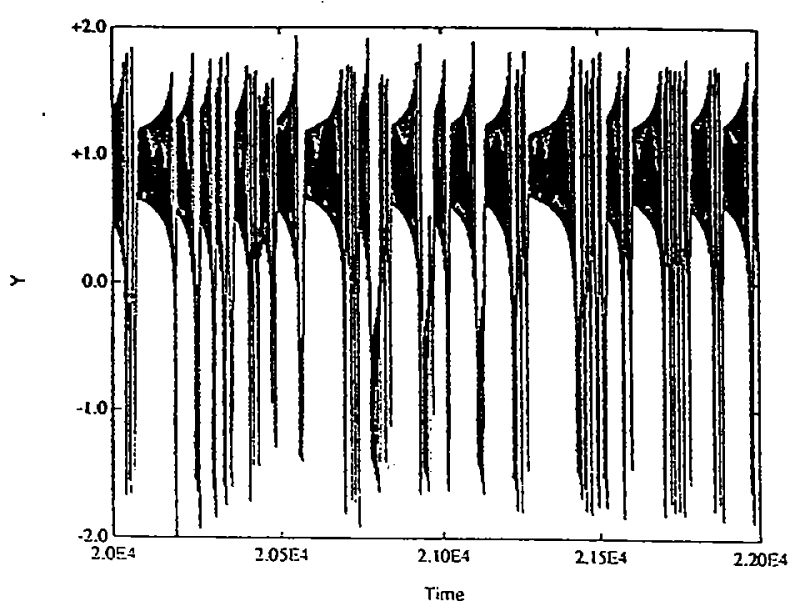

Figure 3: Time Series of $y$ at $\epsilon_{c}$

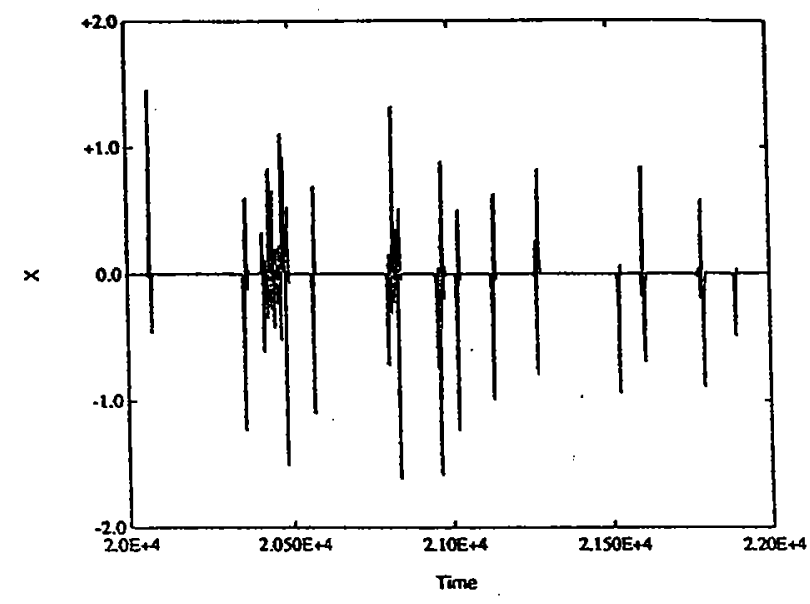

Figure 2: Time Series of $x$ at $\epsilon_{c}$

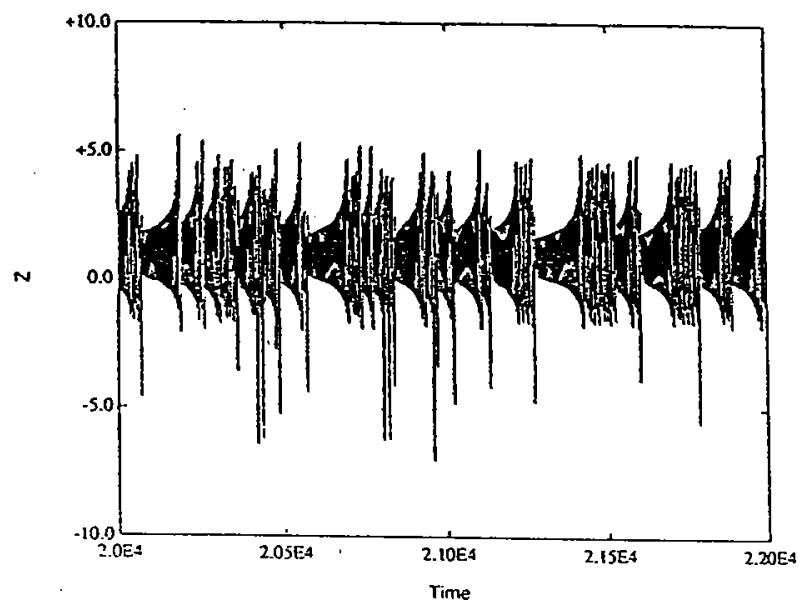

Figure t: Time Scries of $z$ at $\epsilon_{c}$ 


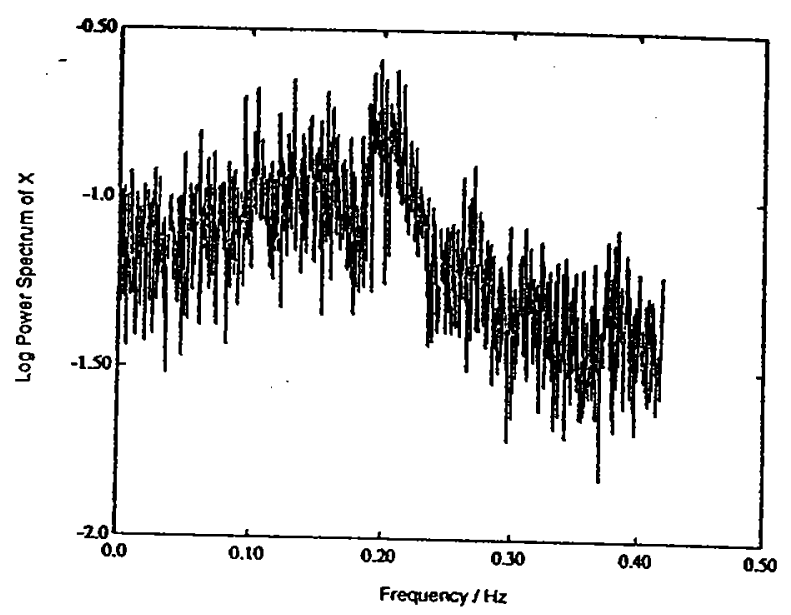

Figure 5: Power Spectrum of $x$ at $\epsilon_{c}$

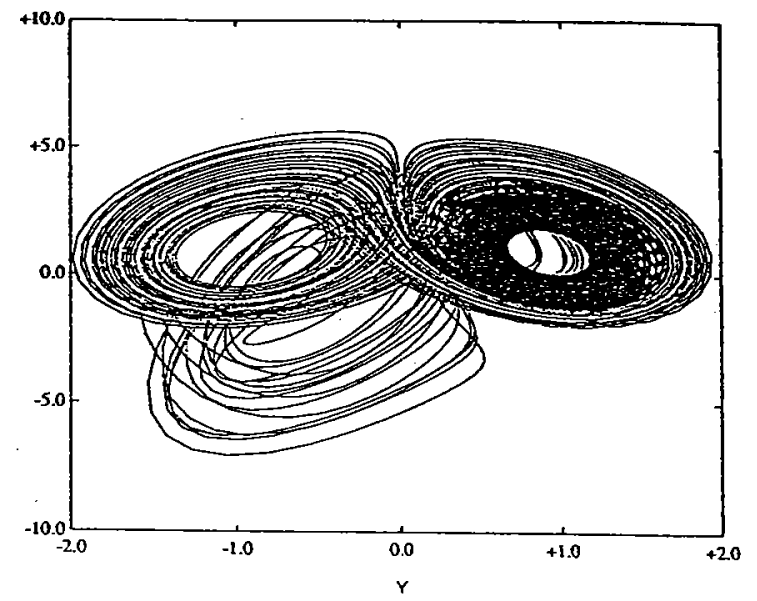

Figure 7: $y-z$ Phase Space Plot at $\epsilon_{c}$

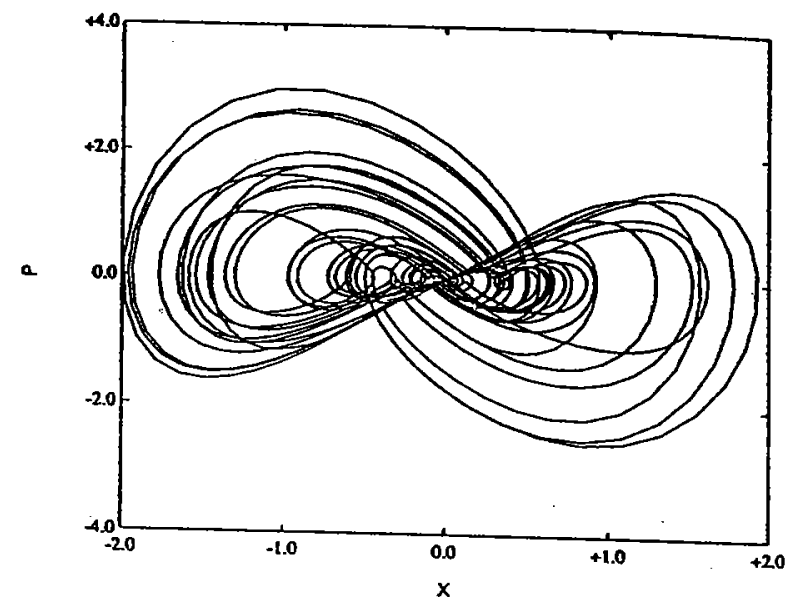

Figure 6: $x-p$ Phase Space Plot at $\epsilon_{c}$

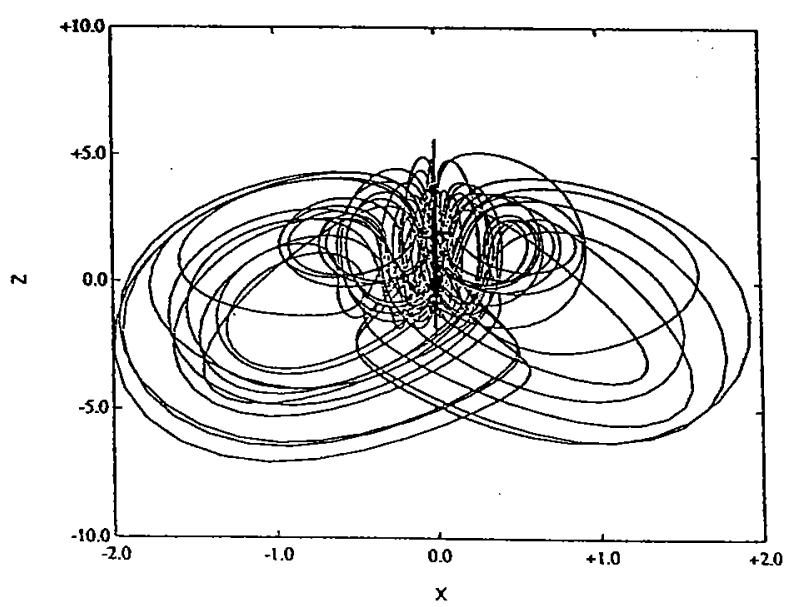

Figure S: $x-z$ Phase Space Plot at $\epsilon_{c}$ 


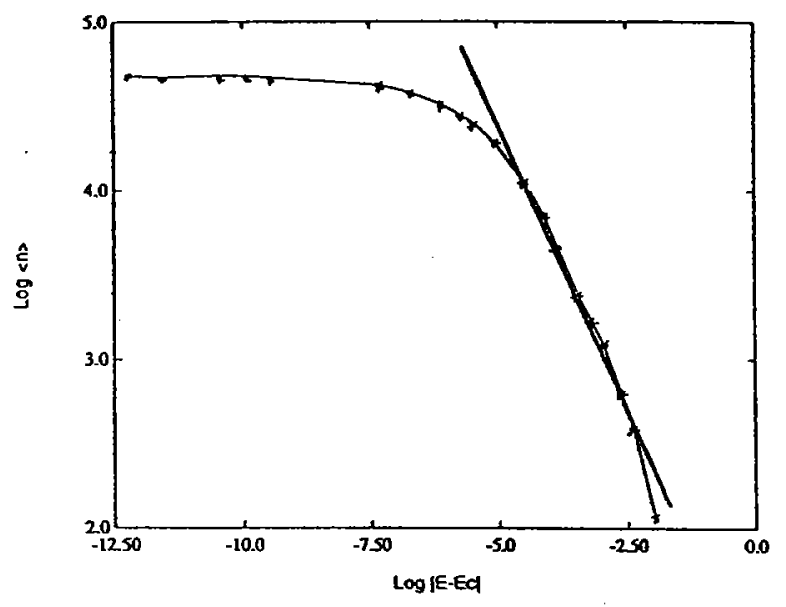

Figure 9: $\ln \langle n\rangle$ vs $\ln \left|\epsilon-\epsilon_{c}\right|$

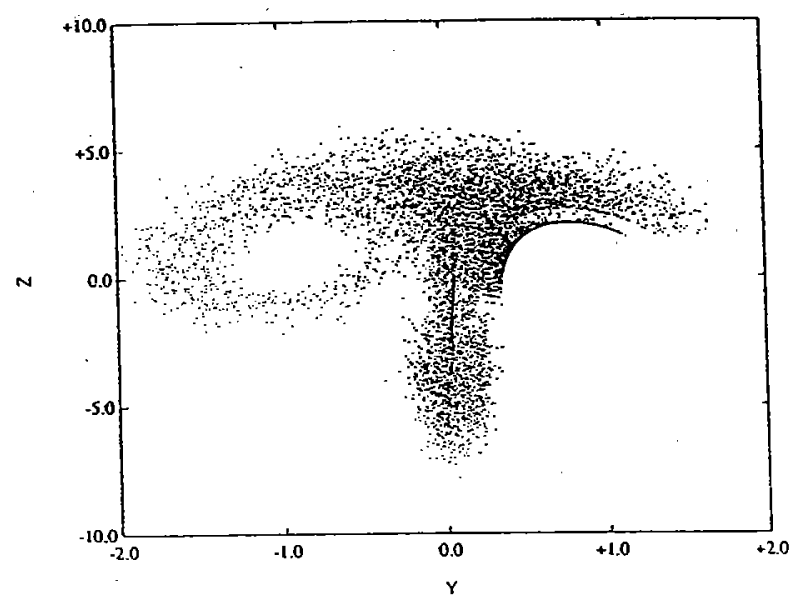

Figure 11: Poincare Section at $\epsilon=$ 0.44 . Cutting plane $x=0.05$

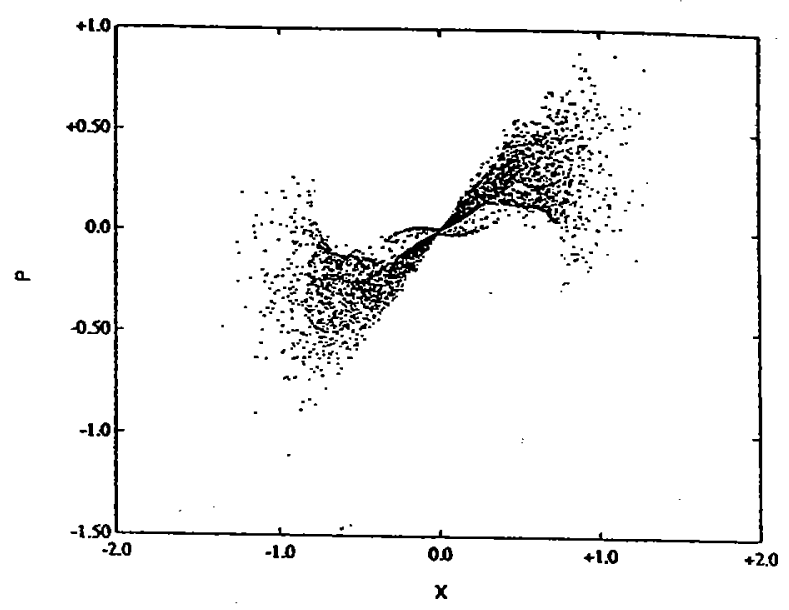

Figure 10: Poincare Section at $\epsilon=$ 0.44. Cutting plane $y=0.93$

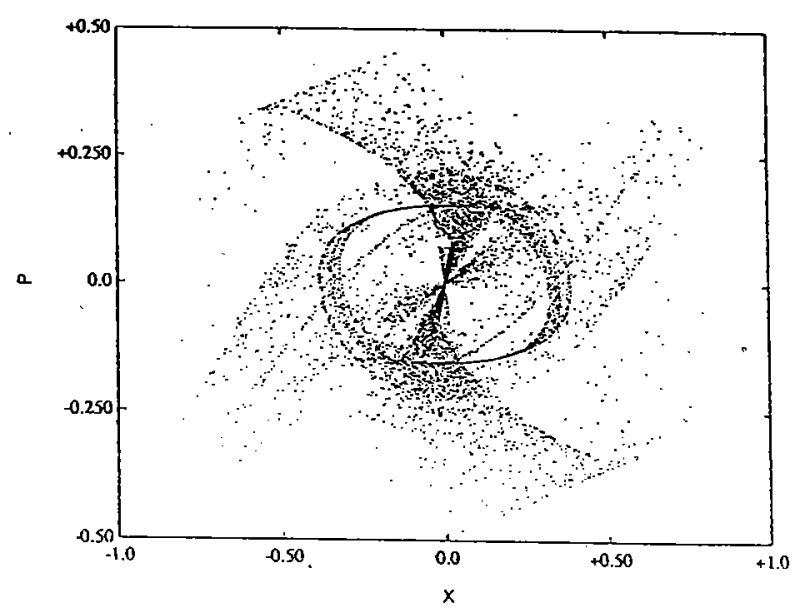

Figure 12: Poincare Section at $\epsilon=$ 0.44 . Cutting plane $z=0.87$ 


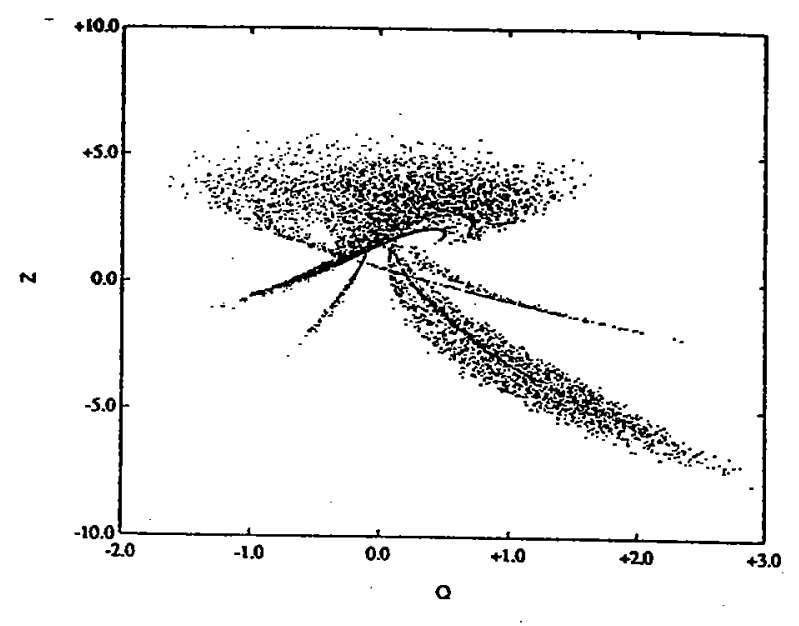

Figure 13: Poincare Section at $\epsilon=$ 0.44 . Cutting plane $x=0.05$

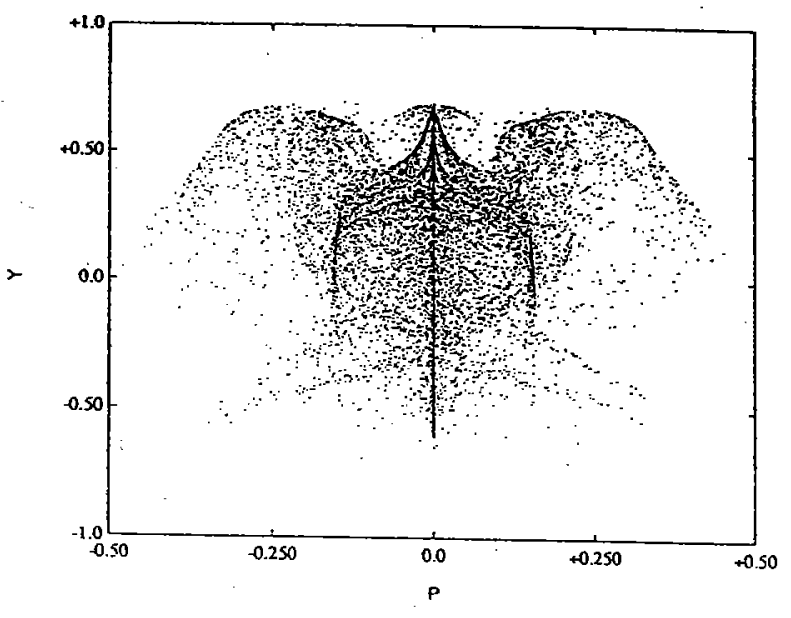

Figure 15: Poincare Section at $\epsilon=$ 0.44 . Cutting plane $z=0.87$

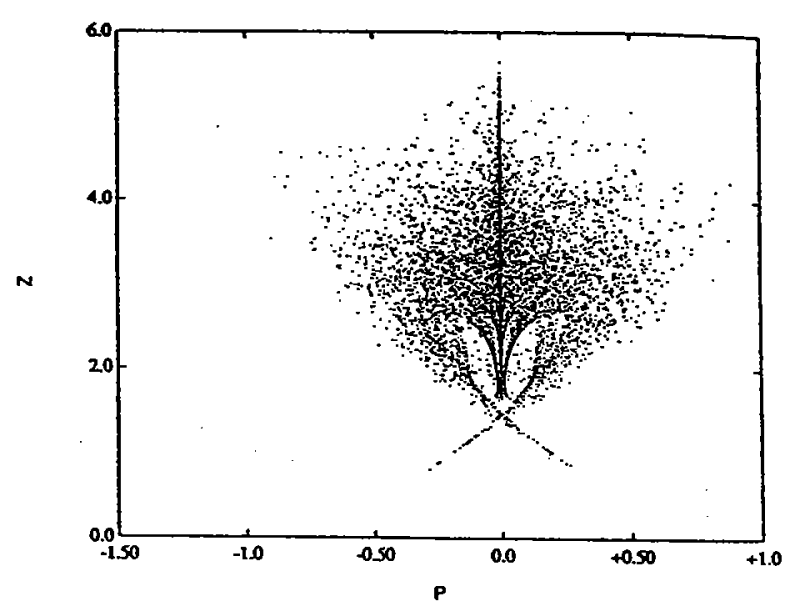

Figure 14: Poincare Section at $\epsilon=$ 0.44 . Cutting plane $y=0.93$

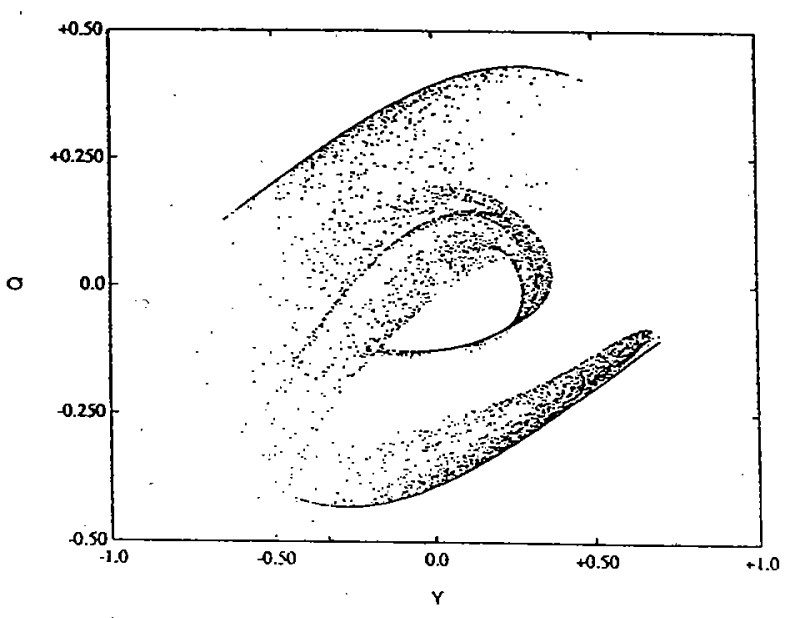

Figute 16: Poincare Section at $\epsilon=$ 0.44 . Cutting plane $z=0.87$ 


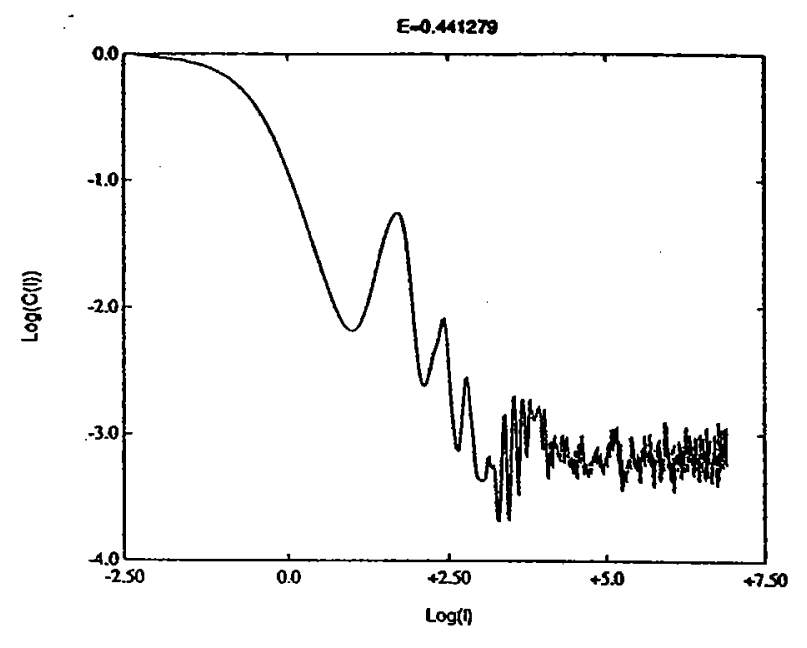

Figure 17: $\ln (C(l))$ vs $\ln (l)$ at $\epsilon=$ 0.441279

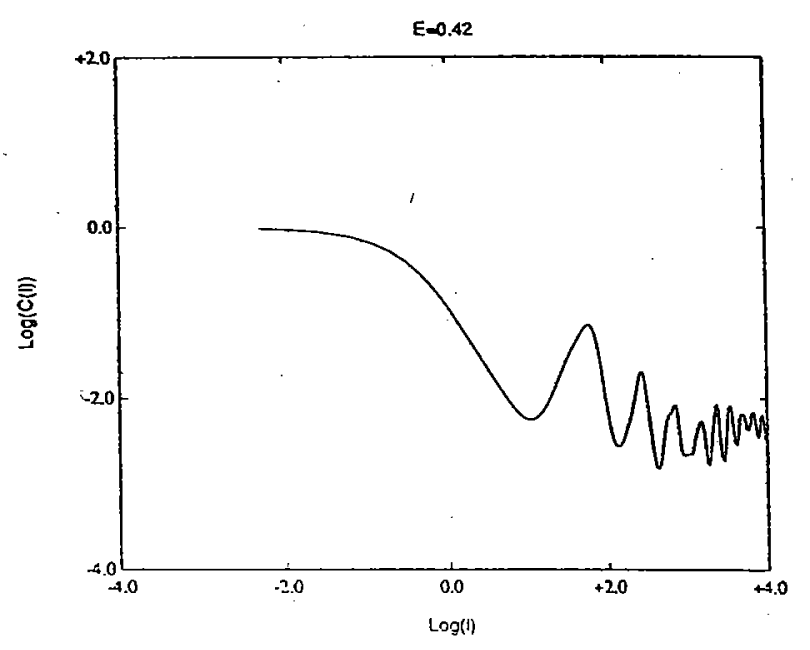

Figure 19: $\ln (C(l))$ vs $\ln (l)$ at $\epsilon=0.42$

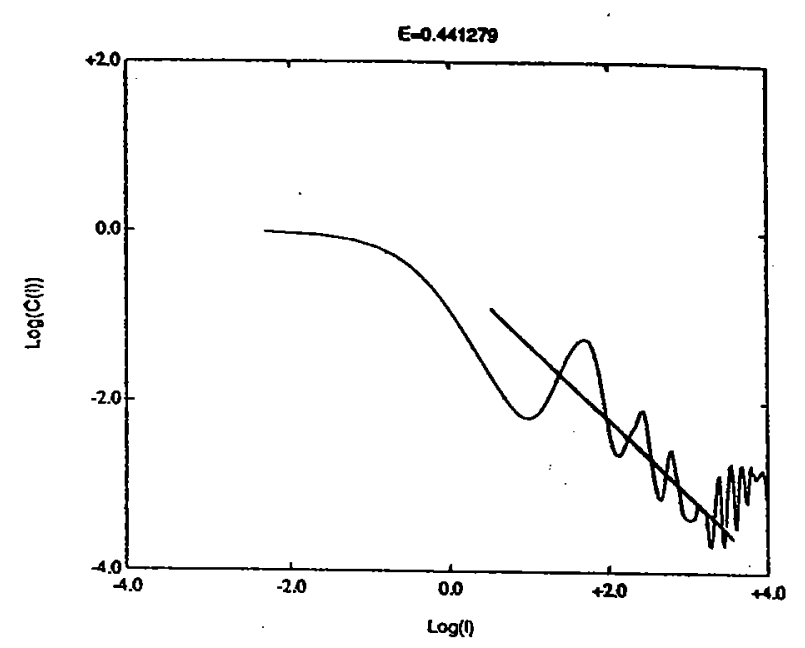

Figure 18: Blow up of Figure 17

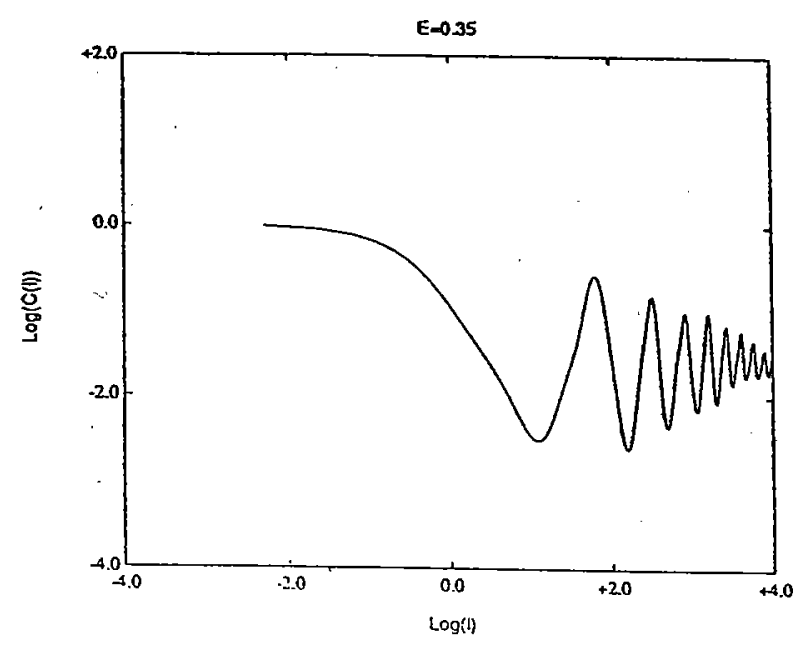

Figure 20: $\ln (C(l))$ vs $\ln (l)$ at $\epsilon=0.35$ 


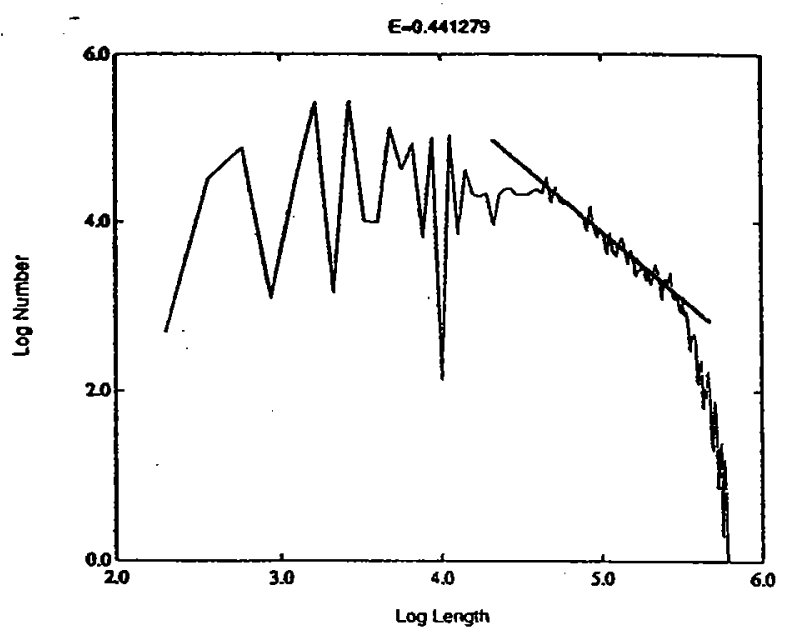

Figure 21: Histogram of $n$ vs length at $\epsilon=0.441279$

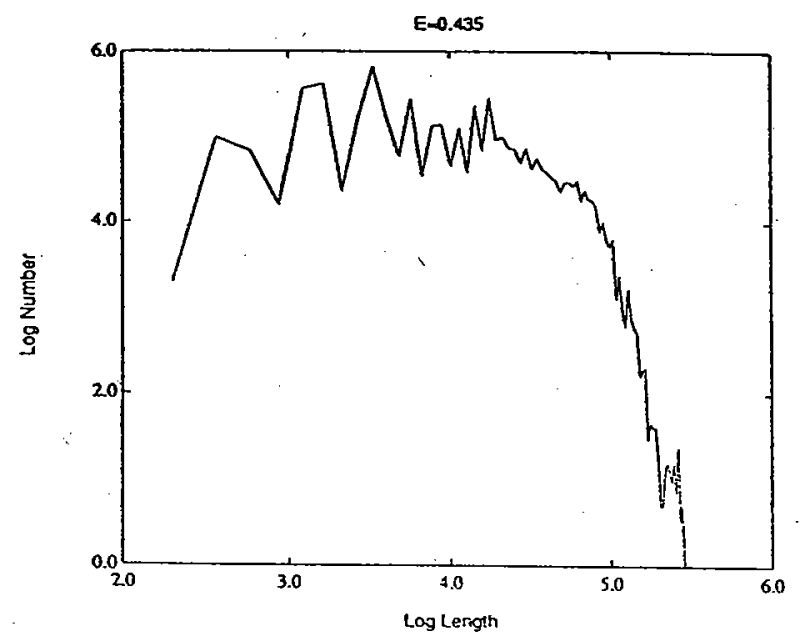

Figure 23: Histogram of $n$ vs length at $\epsilon=0.435$

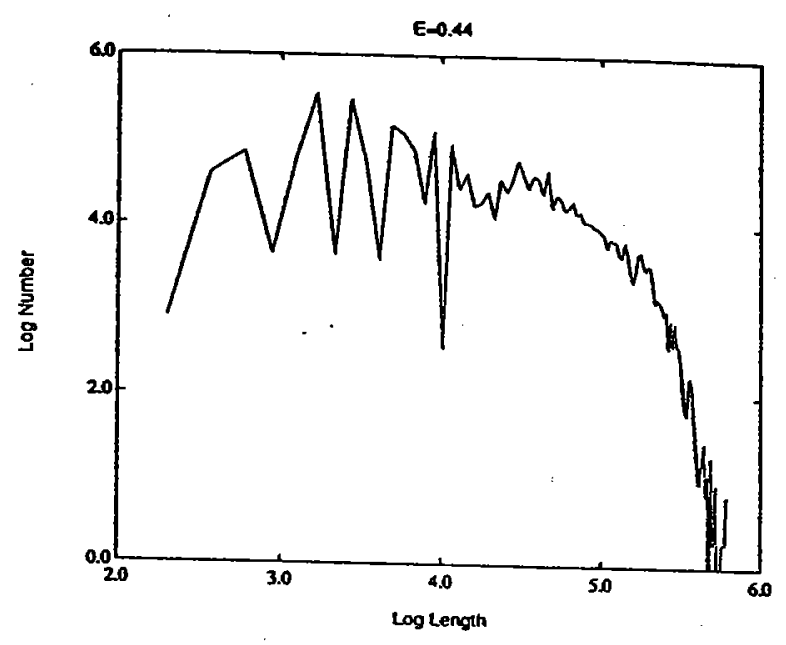

Figure 22: Histogram of $n$ vs length at $\epsilon=0.44$

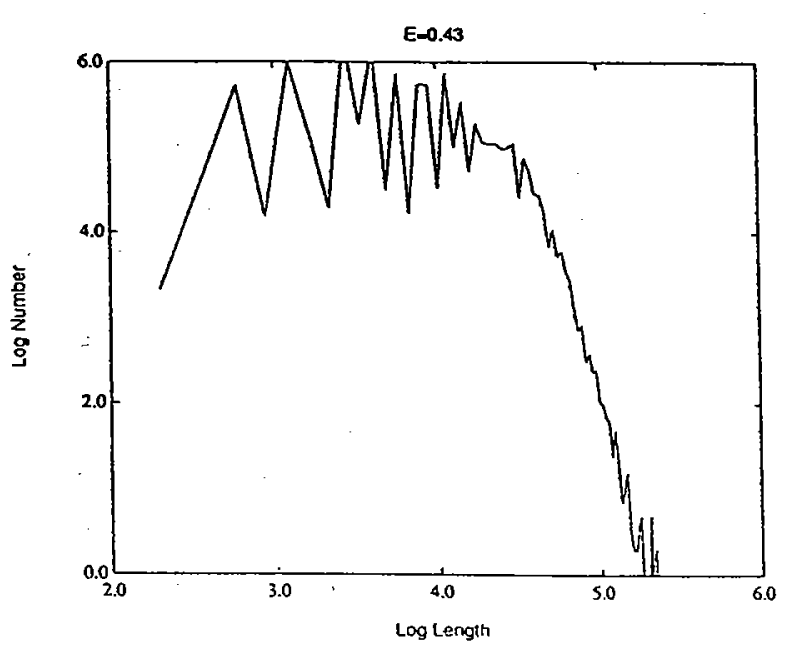

Figure 24: Histogram of $n$ vs length at $\epsilon=0.43$ 


\title{
DIFFUSION IN POISEUILLE FLOW
}

\author{
Wendell T. Welch \\ Department of Applied Mathematics \\ University of Washington \\ Seattle, WA 98195 \\ August, 1990
}

\begin{abstract}
Diffusion of a passive additive in Poiseuille flow in a pipe is studied. Exact and asymptotic equations are derived for $\bar{c}$, the average concentration over a cross-section. The asymptotic equations contain higher derivatives than the simple diffusion equation derived by Taylor. These higher order terms may explain the asymmetry in the concentration profile observed experimentally. The asymptotic equations are solved for $\bar{c}$ and the results compared with Taylor's solution.
\end{abstract}

\section{INTRODUCTION}

Using physical reasoning, Taylor $(1953,1954 \mathrm{a})$ derived and solved the following diffusion equation for $\bar{c}(x, t)$, the cross-sectionally averaged concentration of a passive additive diffusing in a laminar pipe flow:

$$
\left[\partial_{t}+\bar{u} \partial_{x}-C_{1} \partial_{x}^{2}\right] \bar{c}(x, t)=\bar{q}(x, t) \text {. }
$$

Here, $\bar{u}$ is the average fluid velocity in the $x$-direction, $C_{1}$ is a coefficient and $\bar{q}$ is a source term. Keller (1989) has rederived this result by a more systematic method which yields a generalization of (1) containing third derivatives. We shall present this derivation, evaluate the coefficients which occur in it, and solve it.

The basic mechanism is the combination of radial diffusion with advection due to a parabolic velocity profile, which spreads out the additive longitudinally. (Longitudinal diffusion is also present, but its effect is very slow.) Taylor assumed that the radial diffusion happens so quickly that the concentration across each cross-section could be considered uniform at any given instant, and thus he obtained analytically a concentration profile which is symmetric about the centroid of the fluid motion (a point travelling with the mean speed of the fluid). Such a Gaussian profile, however, is only observed experimentally after hundreds of pipe diameters down the pipe; at earlier times the profile is distinctly asymmetric. Thus Taylor's results seem to apply only at large times. 
By assuming that the time scales of radial diffusion and longitudinal advection overlap, we have relaxed Taylor's major assumption and derived an asymptotic equation governing $\bar{c}$. The distinctive feature of this equation is that it contains derivative terms of higher order than in (1); for example, to order $\epsilon^{3}$ (where $\epsilon$ is an aspect ratio of the flow) the asymptotic equation is:

$$
\left[\partial_{t}+\bar{u} \partial_{x}-C_{1} \partial_{x}^{2}-C_{2} \partial_{x}^{2} \partial_{t}-C_{3} \partial_{x}^{3}\right] \bar{c}(x, t)=\bar{q}(x, t)
$$

The coefficients $C_{1}, C_{2}$ and $\mathrm{C}_{3}$ depend on the variation in fluid velocity across the pipe. We have solved (2) for various cases which incorporate some or all of these higher derivative terms and have obtained an asymmetric concentration profile. This profile should agree with experiments at times much earlier than Taylor's solution.

\section{FUNDAMENTAL EQUATION}

Let us consider a pipe parallel to the $\mathrm{x}$-axis, with a cross-section $D$ of any shape in the $y, z$ plane. Let $u(y, z, t)$ be the $x$-component of the fluid velocity within the pipe, and let $c(x, y, z, t)$ be the concentration of a passive additive in the fluid. (Note: we have assumed that the fluid is incompressible by requiring $u$ to be independent of $x$.)

Suppose that $c$ satisfies the following diffusion equation and boundary condition:

$$
\begin{aligned}
\left(\partial_{t}+u \partial_{x}-\partial_{x} k_{x} \partial_{x}-\partial_{y} k_{y} \partial_{y}-\partial_{z} k_{z} \partial_{z}\right) c=q, \quad(y, z) \in D \\
\left(n_{y} k_{y} \partial_{y}+n_{z} k_{z} \partial_{z}\right) c=0, \quad(y, z) \in \partial D
\end{aligned}
$$

where $n_{y}$ and $n_{z}$ are the $y$ and $z$ components of the outward normal to $D$. In (3) $q$ is the sum of a source distribution $q_{s}$ and an initial concentration $c_{o}$ :

$$
q=q_{s}(x, y, z, t)+\delta(t) c_{o}(x, y, z)
$$

where $q_{s}$ is non-zero only for $t \geq 0^{+}$. We also assume that $c=0$ at $t=0^{-}$. Here $k_{x}, k_{y}$, and $k_{z}$ are the diffusion coefficients in the three directions and are functions of $(x, y, z, t) ; n_{x}$ and $n_{y}$ are the components of the unit outward normals to $\partial D$. We denote by $\bar{f}(x, t)$ the average over a cross section of any function $f(x, y, z, t)$, and by $f^{\prime}=f-\bar{f}$, the deviation from the mean. We also write $f^{\prime}=P f$, where $P$ is a projection operator.

We would first like to obtain an equation for $\bar{c}$, and thus we divide each of $u, k_{x}, c$, and $q$ in (3) into an average and a variable part. Thus we can rewrite (3) as:

$$
\left(L+V-\Delta_{T}\right)\left(\bar{c}+c^{\prime}\right)=\bar{q}+q^{\prime}
$$

where $L, V$, and the transverse diffusion operator $\Delta_{T}$ are defined by:

$$
L=\partial_{t}+\bar{u} \partial_{x}-\partial_{x} \overline{k_{x}} \partial_{x}, \quad V=u^{\prime} \partial_{x}-\partial_{x} k_{x}^{\prime} \partial_{x}, \quad \Delta_{T}=\partial_{y} k_{y} \partial_{y}+\partial_{z} k_{z} \partial_{z}
$$


Taking the cross-sectional average of (6) and simplifying yields:

$$
L \bar{c}+\overline{V c^{\prime}}=\bar{q}
$$

where the term $\Delta_{T}\left(\bar{c}+c^{\prime}\right)$ drops out because of the boundary condition (4). We can then subtract (8) from (6) to give the following equation for $c^{\prime}$ :

$$
\left(-\Delta_{T}+L+P V\right) c^{\prime}=q^{\prime}-V \bar{c} .
$$

(The operator $P$ always operates on everything to its right.) Solving (9) for $c^{\prime}$ and substituting into (8) finally gives an equation for $\bar{c}$ :

$$
\left[L+\overline{V\left(\Delta_{T}-L-P V\right)^{-1} V}\right] \bar{c}=\bar{q}+\overline{V\left(\Delta_{T}-L-P V\right)^{-1} q^{\prime}} .
$$

Note that (10) is an exact equation for $\bar{c}$. To use it, we make the approximation (verified through experiments) that $L+P V$ is small compared to $\Delta_{T}$. More specifically, for a circular pipe of radius $a$, let $l_{x}$ be a characteristic length of pipe travelled by the fluid. We are interested in times such that:

$$
\epsilon \equiv \frac{a}{l_{x}} \ll 1
$$

and we choose length and time scales such that $a=O(1)$ and $\bar{u}=O(1)$ (or equivalently, $\left.\partial_{t}=O(\epsilon)\right)$. Therefore, $\partial_{x}=O(\epsilon)$ and $\partial_{y}, \partial_{z}=O(1)$. If we also assume that $k_{x}, k_{y}$, and $k_{z}$ are all $O(1)$ and that all perturbation terms are $O(\delta)$ where $\delta<\epsilon$, we see that:

$$
L=O(\epsilon)+O\left(\epsilon^{2}\right), \quad V=O(\epsilon \delta)+O\left(\epsilon^{2} \delta\right)<O(\epsilon)+O\left(\epsilon^{2}\right), \quad \Delta_{T}=O(1) .
$$

Thus we can rewrite part of the coefficient of $\bar{c}$ in (10) and expand it in a binomial series:

$$
\begin{aligned}
& V\left(\Delta_{T}-L-P V\right)^{-1}=V\left[\left(1-L \Delta_{T}^{-1}-P V \Delta_{T}^{-1}\right) \Delta_{T}\right]^{-1} \\
& \quad=V \Delta_{T}^{-1}\left\{1+\left(L \Delta_{T}^{-1}+P V \Delta_{T}^{-1}\right)+\left(L \Delta_{T}^{-1}+P V \Delta_{T}^{-1}\right)^{2}+\ldots\right\}
\end{aligned}
$$

Substituting this expansion into (10) yields our basic asymptotic PDE. If we assume that $k_{x}^{\prime}=q^{\prime}=0$ and keep terms only to $O\left(\epsilon^{3}\right)$, we obtain:

$$
\begin{aligned}
& {\left[\partial_{t}+\bar{u} \partial_{x}-\partial_{x} \overline{k_{x}} \partial_{x}+\overline{u^{\prime} \partial_{x} \Delta_{T}^{-1} u^{\prime} \partial_{x}}\right.} \\
& \left.+\overline{u^{\prime} \partial_{x} \Delta_{T}^{-1}\left(\partial_{t}+\bar{u} \partial_{x}\right) \Delta_{T}^{-1} u^{\prime} \partial_{x}}+\overline{u^{\prime} \partial_{x} \Delta_{T}^{-1} P u^{\prime} \partial_{x} \Delta_{T}^{-1} u^{\prime} \partial_{x}}\right] \bar{c}=\bar{q}
\end{aligned}
$$


We can simplify this equation further by recalling that $u$ (and thus $u^{\prime}$ ) is independent of $x$ and by assuming that $u^{\prime}$ and $\overline{k_{x}}$ are independent of $t$ and $x$, respectively, to give:

$$
\begin{aligned}
{\left[\partial_{t}+\right.} & \bar{u} \partial_{x}-\left(\overline{k_{x}}-\overline{u^{\prime} \Delta_{T}^{-1} u^{\prime}}\right) \partial_{x}^{2} \\
& \left.+\left(\overline{u^{\prime} \Delta_{T}^{-1} \Delta_{T}^{-1} u^{\prime}}\right) \partial_{x}^{2} \partial_{t}+\left(\overline{u^{\prime} \Delta_{T}^{-1} \bar{u} \Delta_{T}^{-1} u^{\prime}}+\overline{u^{\prime} \Delta_{T}^{-1} P u^{\prime} \Delta_{T}^{-1} u^{\prime}}\right) \partial_{x}^{3}\right] \bar{c}=\bar{q}
\end{aligned}
$$

Because $\bar{u}$ is not affected by $\Delta_{T}^{-1}$, we can pull it outside the first coefficient for $\partial_{x}^{3}$. Then, introducing a moving coordinate system, defined by $\xi \equiv x-\bar{u} t$, we can write:

$$
\left[\partial_{t}-\left(\overline{k_{x}}-\overline{u^{\prime} \Delta_{T}^{-1} u^{\prime}}\right) \partial_{\xi}^{2}+\left(\overline{u^{\prime} \Delta_{T}^{-1} \Delta_{T}^{-1} u^{\prime}}\right) \partial_{\xi}^{2} \partial_{t}+\left(\overline{u^{\prime} \Delta_{T}^{-1} P u^{\prime} \Delta_{T}^{-1} u^{\prime}}\right) \partial_{\xi}^{3}\right] \bar{c}(\xi, t)=\bar{q}
$$

This coordinate system moves with the mean speed of the fluid, i.e. the speed in the $x$ direction averaged over a cross section.

Equation (16) is our basic asymptotic equation for $\bar{c}$ in pipe flow. From $c\left(x, y, z, 0^{-}\right)=$ 0 , we obtain the initial condition:

$$
\bar{c}\left(\xi, 0^{-}\right)=0 .
$$

Then we can solve (16) for $\bar{c}$ and substitute back into (9) to obtain $c^{\prime}$, again assuming $L+P V \ll \Delta_{T}$ and expanding as before.

Let us now apply (16) to the specific case of Poiseuille flow in a circular pipe of radius $a$. Thus $u(r)=u_{\max }\left(1-r^{2} / a^{2}\right)$ and we can calculate all the coefficients in (16) to obtain:

$$
\left[\partial_{t}-\left(\overline{k_{x}}+\frac{a^{2} u_{\max }^{2}}{192 k_{r}}\right) \partial_{\xi}^{2}+\frac{a^{4} u_{\max }^{2}}{2880 k_{r}^{2}} \partial_{\xi}^{2} \partial_{t}+\frac{a^{4} u_{\max }^{3}}{23,040 k_{r}^{2}} \partial_{\xi}^{3}\right] \bar{c}=\bar{q}
$$

Here we have assumed that:

$$
k_{y}=k_{z}=k_{r}, \quad k_{r} \text { independent of } z .
$$

As an example, let us calculate the coefficient of the second derivative in (16). We proceed from right to left, first calculating $u^{\prime}$ :

$$
u^{\prime}=u-\bar{u}=u_{\max }\left(1-\frac{r^{2}}{a^{2}}\right)-\frac{1}{\pi a^{2}} \int_{\theta=0}^{2 \pi} \int_{r=0}^{a} u(r) r d r d \theta=\frac{u_{\max }}{2}\left(1-2 \frac{r^{2}}{a^{2}}\right)
$$

Now let $\Delta_{T}^{-1} u^{\prime} \equiv w(y, z, t)$. Thus $w$ is that solution of

$$
\Delta_{T} w=u^{\prime}
$$


which satisfies:

$$
\frac{\partial w}{\partial r}=0 \text { at } r=a, \text { and } \bar{w}=0
$$

The first condition of (22) follows from the boundary condition (4); we require each of the coefficients in (16) to satisfy (4) so that $\bar{c}$ will. The second condition is due to the fact that, by definition, $\overline{u^{\prime}}=0$; thus, since $w$ is related to $u^{\prime}$, it must also satisfy $\bar{w}=0$.

Using (19), we can rewrite (21) in cylindrical coordinates as:

$$
k_{r}\left(\frac{\partial^{2} w}{\partial r^{2}}+\frac{1}{r} \frac{\partial w}{\partial r}\right)=u^{\prime}
$$

Integrating twice yields:

$$
w(r)=\frac{-1}{k_{r}}\left\{\int_{0}^{r} \frac{1}{\eta}\left[\int_{\eta}^{a} \xi u^{\prime}(\xi) d \xi\right] d \eta\right\}+K
$$

where the constant $K$ is determined by imposing the second condition of (22). Carrying out this integration yields:

$$
w(r)=\frac{-u_{\max }}{16 a^{2} k_{r}}+\frac{u_{\max }}{8 k_{r}} r^{2}-\frac{u_{\max } a^{2}}{24 k_{r}}
$$

The coefficient of $\partial_{\xi}^{2}$ in $(16)$ is thus:

$$
\overline{k_{x}}-\overline{u^{\prime} w}=\overline{k_{x}}-\frac{2}{a^{2}} \int_{0}^{a} u^{\prime}(r) w(r) r d r=\overline{k_{x}}+\frac{u_{m a x}^{2} a^{2}}{192 k_{r}}
$$

To simplify (18), let us introduce a new space variable $\tau \equiv\left(\overline{k_{x}}+\frac{a^{2} u_{\text {max }}^{2}}{192 k_{r}}\right) t$. Then we can rewrite (18) in the form:

$$
\bar{c}_{\tau}-\bar{c}_{\xi \xi}+\beta \bar{c}_{\xi \xi \tau}+\delta \bar{c}_{\xi \xi \xi}=\frac{\bar{q}}{\overline{k_{x}}+\alpha}
$$

where we define:

$$
\alpha \equiv \frac{a^{2} u_{\max }^{2}}{192 k_{r}}, \quad \beta \equiv \frac{a^{4} u_{\max }^{2}}{2880 k_{r}^{2}}, \quad \delta \equiv \frac{\frac{a^{4} u_{\max }^{3}}{23,040 k_{r}^{2}}}{\overline{k_{x}}+\frac{a^{2} u_{\max }^{2}}{192 k_{r}}}
$$




\section{SOLUTION METHOD}

We will seek a solution of (27) with $\bar{q}=0$ and with the initial condition

$$
\bar{c}(\xi, 0) \equiv \bar{c}_{0}(\xi) \equiv \frac{1}{\pi a^{2}} \delta(\xi)
$$

This corresponds to a unit amount of the additive distributed uniformly in a crosssection of infinitesimal thickness at $x=t=0$.

To solve this problem we seek $\bar{c}$ in the form:

$$
\bar{c}=\int_{-\infty}^{\infty} f(k) e^{i k \xi-\sigma(k) r} d k
$$

Then $f(k)$ is calculated from (29) via an inverse Fourier Transform as follows:

$$
f(k)=\frac{1}{2 \pi} \int_{-\infty}^{\infty} \bar{c}(\xi, 0) e^{-i k \xi} d \xi=\frac{1}{2 \pi^{2} a^{2}}
$$

\section{TAYLOR'S CASE}

The simplest form of (27) occurs when $\beta=\delta=0$, when it becomes the simple diffusion equation G.I.Taylor derived in $1953\left(\bar{c}_{r}=\bar{c}_{\xi \xi}\right)$. Its solution, with initial condition (29), is

$$
\bar{c}(\xi, \tau)=\frac{1}{2 \pi^{3 / 2} a^{2} \tau^{1 / 2}} e^{-\frac{\xi^{2}}{4 \tau}}
$$

By assuming $\overline{k_{x}}=0$, we can write (32) as Taylor did:

$$
\bar{c}(\xi, t)=\frac{1}{2 \pi^{3 / 2} a^{2} t^{1 / 2} \alpha^{1 / 2}} e^{-\frac{\xi^{2}}{4 \alpha t}}
$$

where $\alpha \equiv \frac{a^{2} u_{\max }^{2}}{192 k_{r}}$ is the effective dispersion coefficient of the additive in laminar pipe flow.

Physically, this corresponds to a Gaussian profile of $\bar{c}$ vs $x$, moving with the mean speed of the fluid and spreading out as $t$ increases. The symmetry of this profile about the centroid is of special interest. This is due to Taylor's assumption that $L+P V$ is negligible in comparison to $\Delta_{T}^{-1}$ in equation (10), i.e. that radial diffusion is much faster than either advection or longitudinal diffusion. He assumed that radial diffusion would act quickly to smooth out any differential concentration within a cross-section before advection had a chance to act, or equivalently, that diffusion and advection occur on such disparate time scales that they can be considered to act independently. Thus, in this idealized case, we can think of the additive as diffusing 
symmetrically about the stationary point $x=0$ with effective dispersion coefficient $\alpha$, and simultaneously (but independently) being advected by the fluid at the mean flow velocity. This last statement assumes that we can consider all the additive as moving with the average speed of the fluid. This is because we are dealing only with the average concentration over a cross-section, and since the radial diffusion is assumed to be instantaneous, we are effectively reducing the pipe down to a line with infinitesimal cross-sectional area, in which all of the fluid moves with the average speed of the flow.

Such a Gaussian profile is indeed observed experimentally, but only after hundreds of pipe diameters have been traversed by the fluid. The asymmetry of the concentration observed at earlier times can not be explained by formulas (32) and (33); it is precisely for this reason that we have considered the more general cases which follow, in which we include the third derivatives in (27).

\section{AIRY FUNCTION SOLUTION}

Next we solve (27) with $\beta=0$ and $\delta \neq 0$. Thus we must solve the following PDE:

$$
\bar{c}_{\tau}-\bar{c}_{\xi \xi}+\delta \bar{c}_{\xi \xi \xi}=0
$$

We note that this case is not motivated by a particular physical meaning, since in general $O(\beta)=O(\delta)$ and thus $\beta$ can not be considered negligible. However, the solution to (34) can be obtained exactly in terms of Airy functions, and thus we pursue this case for the qualitative insight it may yield regarding the solution to the more general equation (27).

Substituting (30) into (34) gives the dispersion relation:

$$
\sigma(k)=k^{2}-i \delta k^{3}
$$

Thus we can rewrite (30) in the form:

$$
\bar{c}=\frac{1}{2 \pi^{2} a^{2}} \int_{-\infty}^{\infty} e^{i\left[k \xi+i k^{2} \tau+\delta k^{3} \tau\right]} d k
$$

where we have also used the initial condition (31). This can be transformed to an Airy integral by letting $k^{\prime}=k-\frac{i}{3 \delta}$ to yield

$$
\begin{aligned}
\bar{c} & =\frac{1}{2 \pi^{2} a^{2}} e^{\left[\frac{1}{3 \delta} \xi+\frac{2}{27 \delta^{2}} \tau\right]} \int_{-\infty}^{\infty} e^{i\left[k\left(\xi+\frac{1}{3 \delta} \tau\right)+\delta \tau k^{3}\right]} d k \\
& =2 \frac{1}{2 \pi^{2} a^{2}} e^{\left[\tau \frac{2+2 \delta v}{27 \delta^{2}}\right]} \int_{0}^{\infty} \cos \left[k\left(\xi+\frac{1}{3 \delta} \tau\right)+\delta \tau k^{3}\right] d k
\end{aligned}
$$


where $v \equiv \frac{\varepsilon}{\tau}$. (The sine term cancels out because it is an odd function of $k$.) Thus, using the definition of an Airy function, we have:

$$
\bar{c}(\xi, \tau)=2 \frac{1}{2 \pi^{2} a^{2}} e^{\left[\tau \frac{2+96 \tau}{27 \delta^{2}}\right]} \frac{\pi}{(3 \delta \tau)^{\frac{1}{3}}} \mathrm{Ai}\left[\frac{\xi+\frac{1}{3 \delta} \tau}{(3 \delta \tau)^{\frac{1}{3}}}\right]
$$

This solution has been computed numerically and plotted together with Taylor's solution on the graphs which follow. These graphs correspond to different values of time, where time has been measured in terms of the number of pipe diameters traversed by the mean flow. In each graph, the horizontal axis indicates $\xi$ in pipe diameters, i.e. the number of pipe diameters away from the centroid, so that the centroid is always at 0 . A vertical line has been drawn to indicate the starting point of the additive (the plane $x=0$ ).

In this computation, the values used for the various parameters were taken from an experiment Taylor performed using $\mathrm{KMnO}_{4}$ in water:

$$
a=.0252 \mathrm{~cm}, \overline{k_{x}}=k_{r}=.7 \times 10^{-5} \mathrm{~cm}^{2} / \mathrm{sec}, \quad u_{m a x}=.527 \mathrm{~cm} / \mathrm{sec}
$$

We can notice several features of these graphs. (All except the last two have been plotted on the same scale to facilitate comparison.) First, the concentration in the Airy function solution reaches a maximum to the left of the centroid; this agrees with the asymmetry we expect from observational data. Second, the maximum concentration is slightly lower for the Airy case than for Taylor's. Both of these effects are due to the fact that we have assumed that $L+P V$ in (10) is small but not negligible in comparison to $\Delta_{T}^{-1}$. We expect radial diffusion to occur more slowly than in Taylor's case, so that advection is felt before radial diffusion has smoothed out all differential concentration over a cross-section. Thus, in this case, radial diffusion and advection are not independent, and we do not expect a symmetric concentration profile about the centroid. (Note that longitudinal diffusion occurs only in the coefficient of $\bar{c}_{\xi \xi}$ and has negligible effect, since usually $k_{x} \ll \frac{a^{2} u_{\max }^{2}}{192 k_{r}}$.) We also notice that the profiles do approach a Gaussian, but only after several hundred pipe diameters have been traversed by the fluid. This can be seen from the last two graphs at 500 and 1000 pipe diameters, which have been plotted on their own scales to put in perspective the difference between the Airy and Gaussian solutions. At 500 diameters, one can still see a difference between the two curves, but at 1000 diameters they are virtually identical on this scale.

A few comments on the computation are in order. $\operatorname{Ai}(\eta)$ was computed for each $\eta \equiv \frac{\xi+\frac{1}{36} \tau}{(3 \delta \tau)^{1 / 3}}$ by either an ascending series for "small" values of $\eta$, or by an asymptotic expansion for $\eta>3.7$. Since an Airy function oscillates above and below zero for negative values of its argument, the profile of $\bar{c}$ was ended as soon as it became zero. The physically meaningless negative concentration given by the solution is possible 

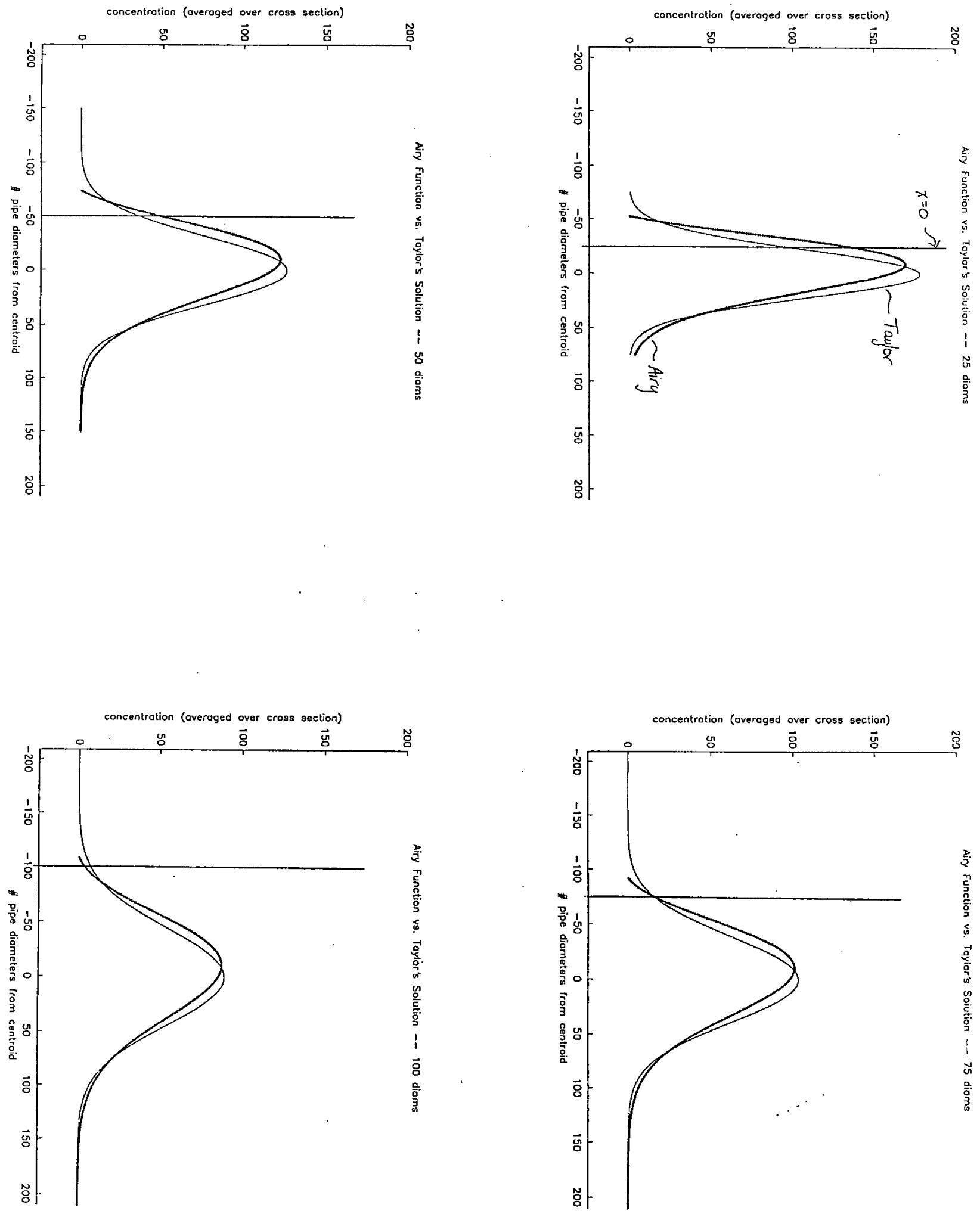

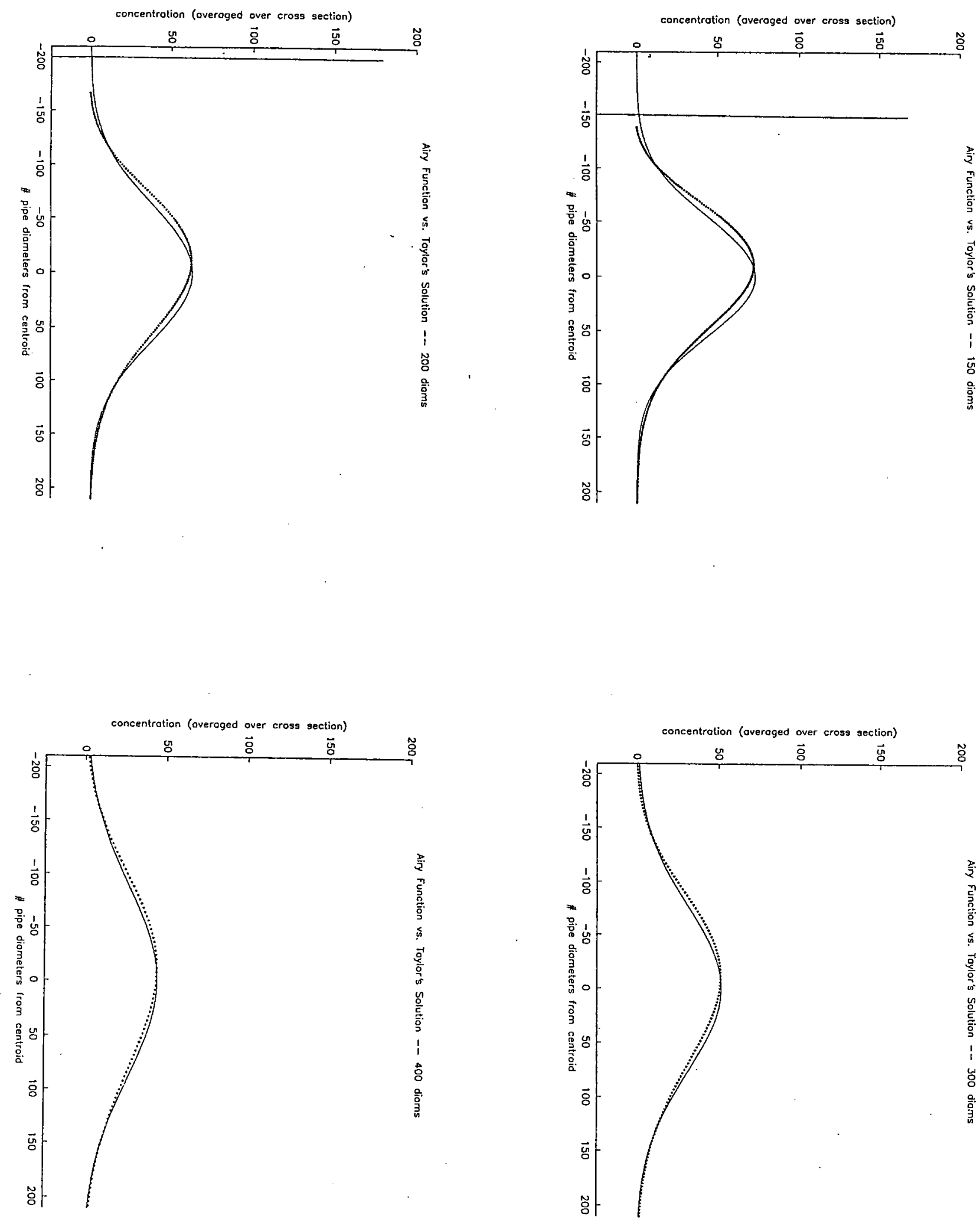

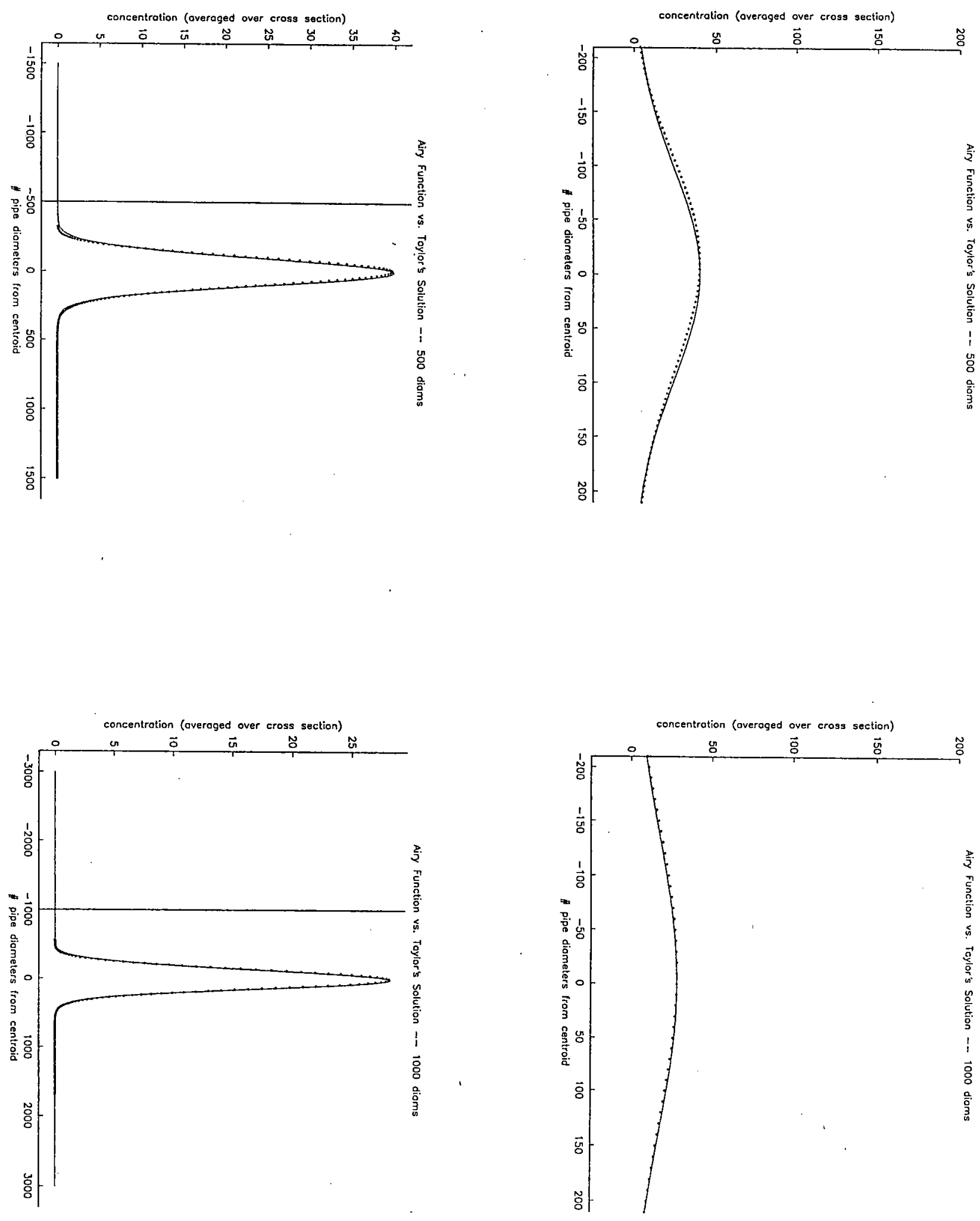
because our original PDE (27) is only approximate (only accurate to order $\epsilon^{3}$ ), and thus its solution will not necessarily retain all the physical aspects of the original problem.

\section{GENERAL CASE}

Lastly, we consider the full equation

$$
\bar{c}_{\boldsymbol{\tau}}-\bar{c}_{\xi \xi}+\beta \bar{c}_{\xi \xi \tau}+\delta \bar{c}_{\xi \xi \xi}=0
$$

(We do not pursue the case $\bar{c}_{\tau}-\bar{c}_{\xi \xi}+\beta \bar{c}_{\xi \xi \tau}=0$, because it seems to be no easier to solve than (40) and does not correspond to a particular physical situation.)

Substituting (30) into (40) gives the dispersion relation:

$$
\sigma(k)=\frac{k^{2}-i \delta k^{3}}{1-\beta k^{2}}
$$

Now let us rewrite our general solution (30) in the form:

$$
\bar{c}=\int_{-\infty}^{\infty} f(k) e^{-\tau \varphi(k)} d k
$$

Here

$$
\varphi \equiv \sigma(k)-\frac{i k \xi}{\tau}=\frac{k^{2}-i \delta k^{3}}{1-\beta k^{2}}-i k v
$$

\subsection{Method Of Steepest Descents}

We will first use the method of Steepest Descents to approximate this integral for large $\tau$. Thus, we will look for stationary points $k$, of $\varphi(k)$ at which the integrand of (42) is a maximum. We will then evaluate this integral along a contour on which the imaginary part of $\varphi(k)$ is constant.

To find the stationary points of $\varphi(k)$, we set $\varphi^{\prime}\left(k_{s}\right)=0$ and solve for $k_{s}$. This yields the following quartic equation for $k_{s}$ :

$$
\left(i \beta \delta-i \beta^{2} v\right) k_{s}^{4}+(2 i \beta v-3 i \delta) k_{s}^{2}+2 k_{s}-i v=0 .
$$

Notice that, for a given physical situation, these values of $k$, vary only with $v$; they do not depend on $\tau$. To simplify matters, we introduce a new variable $z$, defined by $z \equiv i k_{x}$, and rewrite (44) as a polynomial with all real coefficients:

$$
\left(\beta \delta-\beta^{2} v\right) z^{4}+(3 \delta-2 \beta v) z^{2}+2 z-v=0 .
$$


Thus in general there will be four complex stationary points for each value of $v$. These will be maxima or minima of $\operatorname{Re}(\varphi(k))$ depending on the contour of constant $\operatorname{Im}(\varphi)$ along which we choose to integrate; that is, each $k$, will in general be a saddle point of $\operatorname{Re}(\varphi(k))$.

We could use several methods to solve (45) for $\mathrm{z}$. Although we could use a formula to solve the quartic analytically, this would be complicated, since so doing requires decision-making based on the associated cubic and quadratic equations, and this would have to be done for each value of $v$. To get around this problem, we look for ranges of $v$ within which we can solve the quartic easily (without such manual decisions). In these ranges, then, the four $z$ will have distinctive characteristics (i.e. all complex, or two complex and two purely imaginary below the real axis, etc). We then develop a solution for (40) in a piecewise manner, treating each range of $v$ separately.

Once we have solved (45) for the stationary points, either numerically or analytically, we must investigate the contours of constant $\operatorname{Im}(\varphi)$ which go through these $k_{s}$ in the complex $k$ plane. Thus, we must find all values of $k$ for which $\operatorname{Im}\{\varphi(k)\}=$ $\operatorname{Im}\left\{\varphi\left(k_{\bullet}\right)\right\}$. Using (43) we have:

$$
\left(\frac{k^{2}-i \delta k^{3}}{1-\beta k^{2}}-i k v\right)=\left(\frac{k_{s}^{2}-i \delta k_{s}^{3}}{1-\beta k_{s}^{2}}-i k_{s} v\right)
$$

or, letting $k=a+i b$ :

$$
\frac{\beta \delta a^{5}-\delta a^{3}+2 a b+3 \delta a b^{2}+2 \beta \delta a^{3} b^{2}+\beta \delta a b^{4}}{\left(1-\beta a^{2}+\beta b^{2}\right)^{2}+4 \beta^{2} a^{2} b^{2}}-a v=\left(\frac{k_{s}^{2}-i \delta k_{s}^{3}}{1-\beta k_{s}^{2}}-i k_{s} v\right)
$$

In general there will be two perpendicular contours going through each $k_{s}$; we must pick the one to which we can deform the original contour (the real $k$-axis) of the solution (42). This will also turn out to be the contour along which $\operatorname{Re}(\varphi(k))$ has its minimum value at $k_{s}$ (and hence the integrand of (42) its maximum value at $k_{s}$ ). Further, we may be required to include the effects of more than one stationary point if two fall on the same contour.

If we know the stationary points and proper contours for a certain range of $v$, then we can expand $\varphi(k)$ about $k$, and approximate the concentration in that range by the following formula from the method of Steepest Descents:

$$
\bar{c}(\xi, \tau) \approx \frac{1}{\pi^{3 / 2} a^{2}\left[2 \tau \varphi^{\prime \prime}\left(k_{s}\right)\right]^{1 / 2}} e^{-\tau \varphi\left(k_{s}\right)}
$$




\subsection{Results}

The zeroes of equation (44) have been calculated for a range of $v$ and are plotted on the complex $k$ plane on the graph which follows. (This range of $v$ corresponds to $-100<\xi<+100$ pipe diameters at a time when the mean flow has traveled 100 diameters from the $x=0$ plane.) Arrows on these graphs indicate the direction of increasing $v$ or $\xi$.

There are two pairs of zeroes. Stationary points (i.e. zeroes) 1 and 2 are always complex and have the same imaginary part but opposite real parts. For $v<\delta / \beta$, they have positive imaginary parts, and for $v>\delta / \beta$, negative imaginary parts.

Stationary points 3 and 4 are more complicated. For $v \lessgtr-\delta / \beta$, both are complex and again have the same (negative) imaginary part and opposite real parts. Similarly, for $v \geqslant 2 \delta / \beta$, both are complex with the same (positive) imaginary part and opposite real parts. But for the middle range, $-\delta / \beta \leqslant v \leqslant 2 \delta / \beta$, stationary points 3 and 4 are purely imaginary. For $-\delta / \beta \leqslant v<0$ both are negative; for $0<v<\delta / \beta$ one is positive and one negative; and for $\delta / \beta<v \leqslant 2 \delta / \beta$ both are positive.

The remaining task to calculate an approximate solution for this general case is to investigate the contours of constant $\operatorname{Im}(\varphi(k))$ for the different stationary points in each range of $v$. One should then determine, for each range of $v$, those $k_{s}$ which are minima of $\operatorname{Re}(\varphi(k))$ and thus have contours which are deformable from the original contour (the real $k$ axis). Stationary points 1 and 2 will in general have different contours from those of points 3 and 4 . Thus they should be investigated separately. Then, with equation (48), a piecewise approximate solution to (40) can be obtained, using appropriate stationary points and contours.

\subsection{Numerical Integration}

The integral in (42) with $\varphi(k)$ given by (43) has been integrated numerically for various values of $\tau$, and this result is shown together with the Airy and Gaussian solutions on the graphs which follow. (These graphs are displayed in the same way as the previous set; all are on the same scale except the last one at 500 diameters, which has been shown on its own horizontal scale.)

To compute this solution, a cautious adaptive Romberg extrapolation method (also known as CADRE) was used. As the integrand of (42) will oscillate very quickly for certain values of $v$, i.e. those which yield a large magnitude of $\operatorname{Im}(\varphi(k))$, there can be errors due to cancellation of large terms, and thus these graphs should be regarded as premliminary only. (Such errors will be most significant at small values of time, when the exponential damping of the integrand due to $-\tau * \operatorname{Re}(\varphi(k))$ is also small.) 
Taking this into account, one can still derive some qualitative insight from the graphs. First, all three curves agree better as $\tau$ increases, which is expected, since the third order terms of (40) decrease in importance as $\epsilon$ of (11) decreases (i.e. as time and $\tau$ increase).

Second, the numerically integrated curve is indeed asymmetric, although this is difficult to discern from the graphs. For example, the maximum concentration occurs at approximately $-3,-7$, and -8 pipe diameters when the mean flow has traversed 25 , 100 , and 200 pipe diameters, respectively. This asymmetry decreases as $\tau$ increases, again as expected.

Lastly, the numerically integrated curve lies below both the other solutions for all $\tau$. By conservation of mass of the additive, this means that the additive must spread out more (i.e. faster) longitudinally than either the Gaussian or the Airy function theory predicts.

For small $\tau$, then, the Airy function solution has two features which are absent from the Gaussian profile: it lies under the Gaussian and it has an asymmetry about the centroid. These features yield some qualitative insight on the exact profile which the Taylor theory cannot predict.

\section{FURTHER WORK}

There are various extensions of this work which suggest themselves. One could perform finite differences on the truncated PDE (40) to obtain a numerical solution. A comparison with experimental data could be performed as well.

In addition to Poiseuille flow, there are other geometries and flows which would be interesting to investigate. Turbulent flow in a pipe is a natural extension of this work and was discussed by Taylor $(1954 \mathrm{~b})$. Laminar or turbulent flow in a layer, in which the velocity $u$ could, in general, have components in all three spatial directions, is also of interest and has applications to estruaries. This work could also be applied to a spherical geometry, with flow radially outward and/or along spherical shells, to model diffusion of chemicals within and out of a star (Chaboyer, 1990).

Finally, to obtain an exact solution to a problem with simultaneous advection and molecular diffusion (as opposed to the approximate solutions found above), a more simple model could be developed. The solution to such a problem might then illuminate the physics observed in the more complicated geometries and flows. 


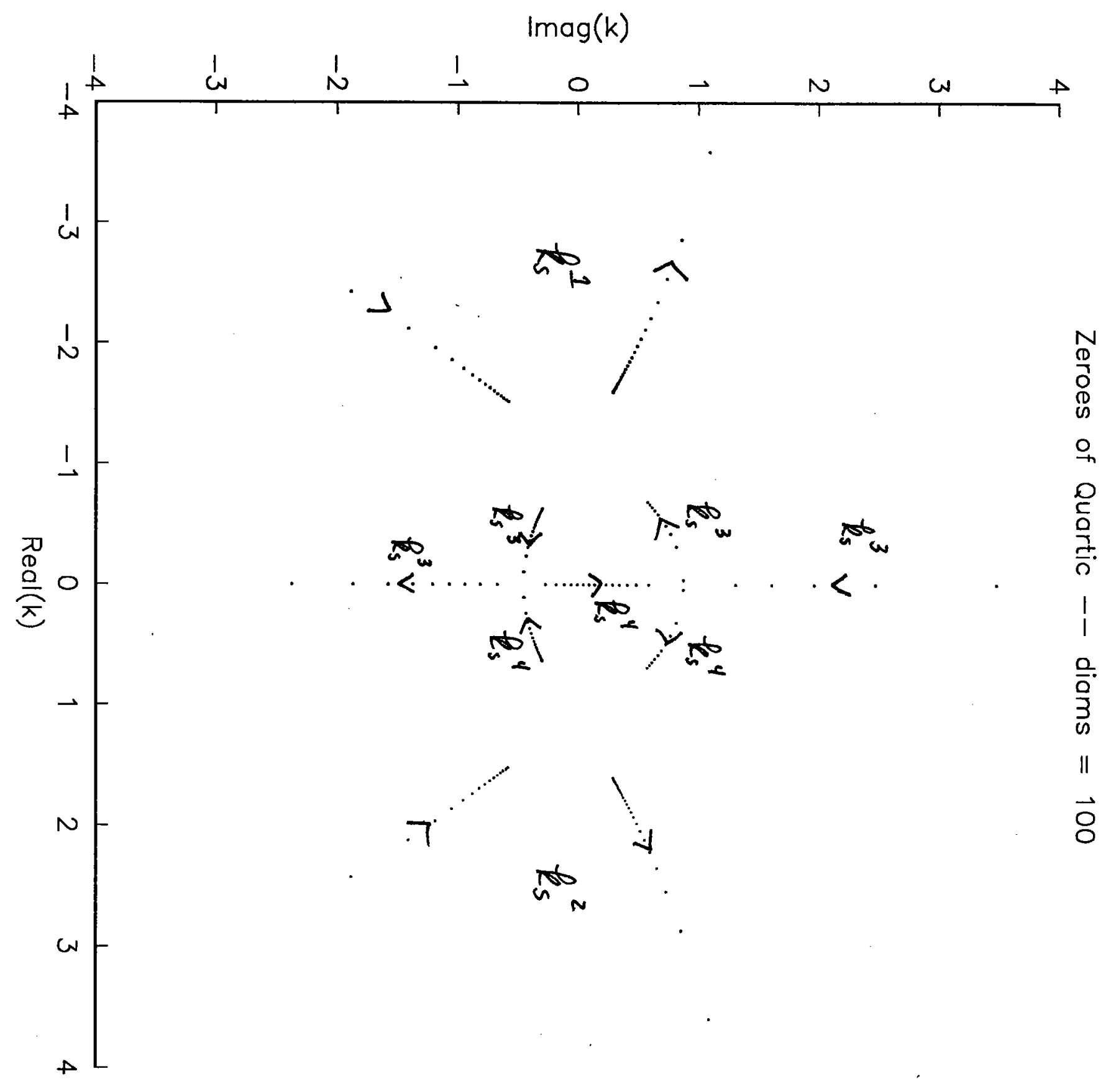

351 

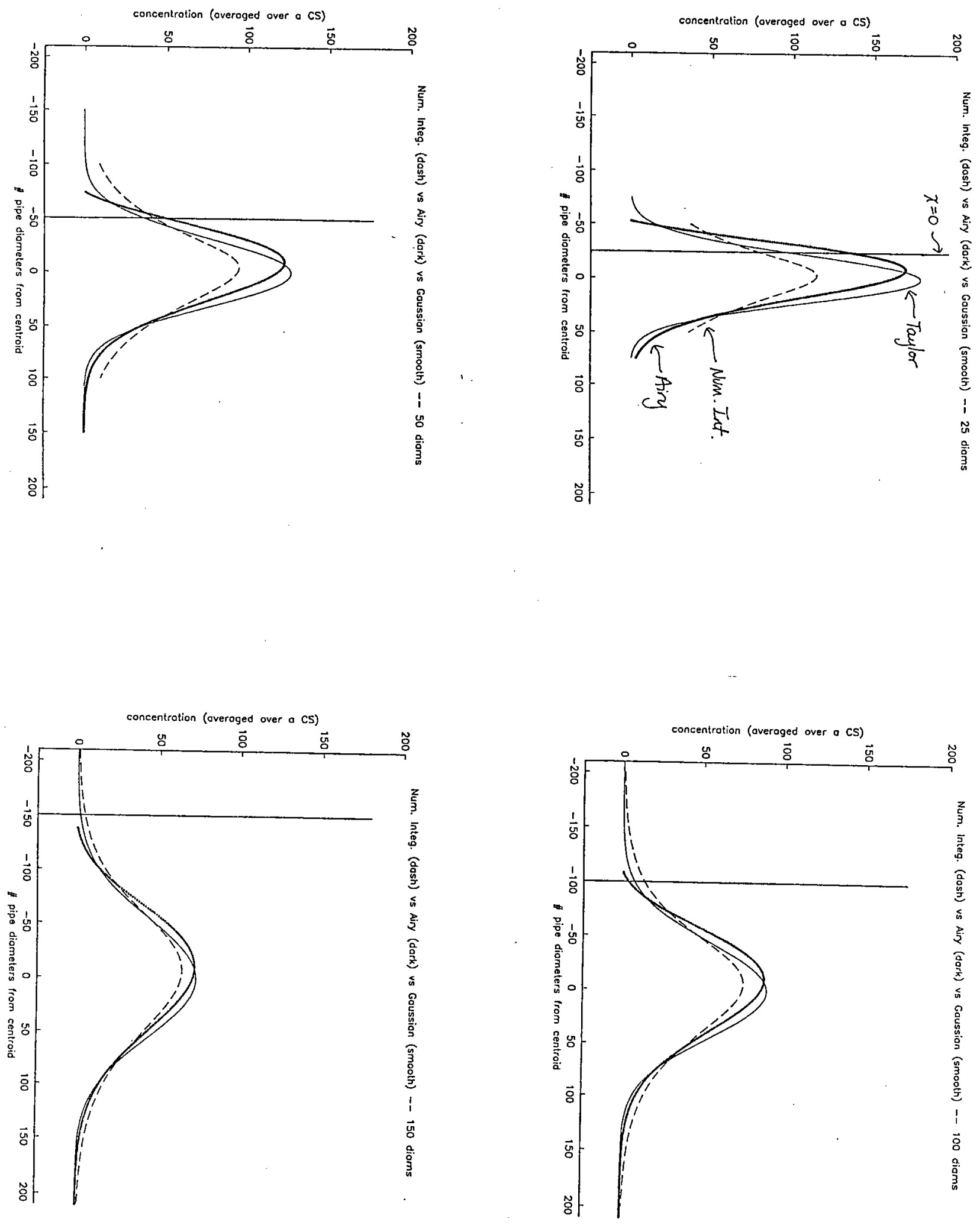

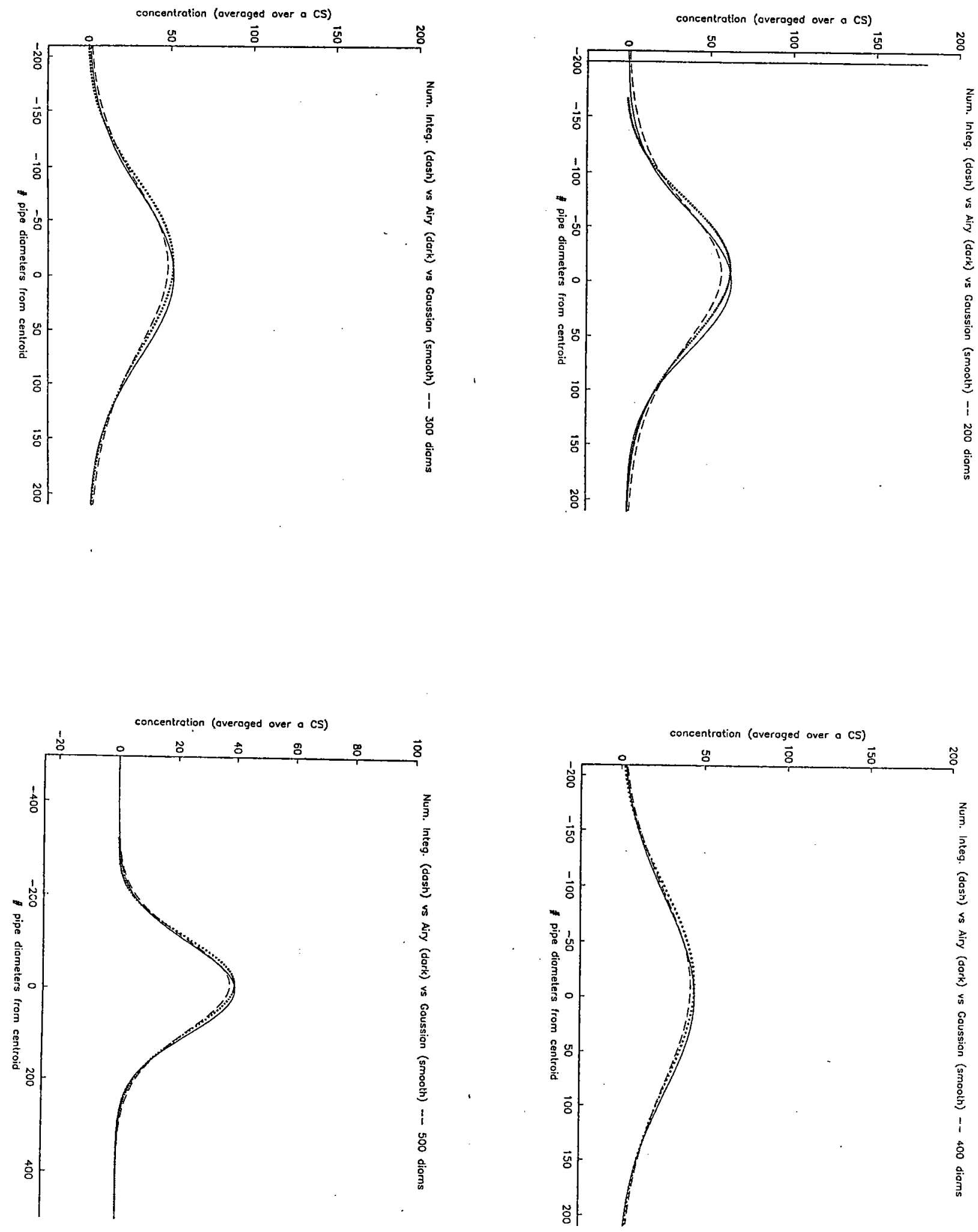


\section{ACKNOWLEDGEMENTS}

I would like to thank Joe Keller for his marvelous insight, instruction and patience; I thought and learned a lot, Joe! I also appreciate all that Rick Salmon did, especially behind the scenes, to organize this year's program and keep it running so smoothly. Thanks to all the professors and fellows for an intellectually stimulating and wonderful summer!

\section{REFERENCES}

1. Taylor, G.I., (1953), Dispersion of Soluble Matter in Solvent Flowing Slowly Through a Tube, Proceedings of the Royal Society of London, A219, p.186.

2. Taylor, G.I., (1954a), Conditions Under Which Dispersion of a Solute in a Stream of Solvent Can Be Used to Measure Molecular Diffusion, Proceedings of the Royal Society of London, A223, p.446.

3. Keller, J.B., (1989), Diffusion in a Shear Flow (preprint).

4. Taylor, G.I., (1954b), The Dispersion of Matter in Turbulent Flow, Proceedings of the Royal Society of London, A225, pp.473-477.

5. Chaboyer, B., (1990), Transport of a Chemical in Stellar Radiative Zones, Geophysical Fluid Dynamics Summer Program: Fellows' Project Reports, Woods Hole Oceanographic Institution.

6. Abramowitz, M. \& Stegun, I., (1970), Handbook of Mathematical Functions, 9th ed., Dover Publications, New York, pp.446-448. 


\title{
Classification of Similarity Solutions of the Two-Dimensional Convection Equations
}

\author{
Eric C. Won
}

\section{Introduction and Overview}

This project applied a method developed by Sophus Lie for systematically solving differential equations. E. Galois' work on solutions to polynomial equations (which started the theory of discrete groups) served as Lie's motivation. Well after mathematicians discovered the quadratic $\left[x=\left(-b \pm \sqrt{b^{2}-4 a c}\right) / 2 a\right]$, cubic, and quartic formulas, Abel proved that there is no general quintic formula. Galois' work, however, showed how to associate with any polynomial (of any order) a mathematical object which is now called the Galois group of the polynomial. The Galois group determines whether or not a polynomial can be solved "by radicals," i. e. certain polynomials of fifth degree and higher can be solved exactly. In addition to giving a "solvability" criterion, the structure of the Galois group of a solvable polynomial also tells one how to construct those roots. Galois theory however, is accessible only after one is quite familiar with the theory of discrete groups and the theory of algebraic fields.

Lie's work has led to a theory which is to differential equations as Galois' theory is to polynomials: to each (ordinary or partial) differential equation, one can associate a Lie group which shows one how to, in the case of an ODE (sometimes) reduce the order of the equation, and in the case of PDE's:

1. Transform a given solution to another solution, and

2. Find similarity solutions.

We are primarily concerned with similarity solutions to PDE's. Thus, confronted with a system of PDE's, one might naïevely use point 2 of the theory to find several similarity solutions and then use point 1 of the theory to (hopefully) discover new similarity solutions. This effort would certainly lead one to some similarity solutions of the system of PDE's, but if one has not solved all possible similarity equations, how does one know whether or not one has found all similarity solutions? One might also wonder, since point 1 allows one to transform a given solution into another solution, whether there is a minimal or optimal subset of similarity solutions on which one can then use point 1 to generate all possible similarity forms? It turns out that the structure of the Lie group allows one to find this optimal subset which greatly reduces one's work. Thus, the answer to the first question, roughly, is that if one properly analyses the structure of the Lie group of a system of PDE's, then one can find an optimal subset of similarity solutions on which one can apply point 1 to find all others.

Lie's theory, which is a theory of continuous groups, involves the base manifold of dependent and independent variables and prolongations of the base tangent in the jet manifold of the differential equation when applied to solving partial differential equations. The theory is 
powerful in the sense that the determination of the Lie group is essentially algorithmic and one who wishes to apply the theory to find similarity solutions to a system of PDE's need not master advanced topics in differential geometry. Writers of several symbolic manipulation programs (MACSYMA, Maple, and REDUCE) have taken advantage of the theory's algorithmic aspects and now have packages available which begin the analysis. These packages obviate the necessity for one to understand the subtleties of the theory. Rick's first lecture gives a brief summary of the theory upon which the packages are based for whose who do wish to have a sense of what the "black box" does. Part of the algorithm the packages perform resembles the multiplication of arbitrary polynomials, which is a programming problem assigned in undergraduate computer science courses.

Nevertheless, let us briefly review a few basics of the theory of Lie groups and Lie algebras and at the same time give a brief overview of the method before we begin the discussion of similarity solutions to the two-dimensional convection equations. A simple example of a Lie group is the $x-t$ plane with vector addition. It is a Lie group essentially since one can move up, down, left, and right by any amount one wishes and still remain within the space, and one can also not move at all $(+0)$. It is nice to first consider the plane with addition as a Lie group since it is easy to see its two subgroups, the $x$-axis and the $t$-axis, which are Lie groups as well-on the $x$-axis one can move up and down by any amount which includes 0 , and likewise for the $t$-axis. Note that if one allows other operations, such as rotation or scalar multiplication in addition to vector addition, the Lie group will have a richer subgroup structure. For the moment, though, let us continue to restrict our attention to the $x$ - $t$ plane with vector addition.

The transformations described by Lie groups are all finite. It turns out that it is often quite useful to consider the infinitesimal transformations related to a Lie group's finite transformations. It is not surprising that whenever derivatives are involved, it is often useful to consider these related infinitesimal transformations in addition to (and sometimes instead of) the finite transformations of the Lie group. The infinitesimal transformations for the Lie group we are considering are $\partial_{x}$ and $\partial_{t}$. The mathematical rules this collection of infinitesimal transformations obey are no longer those of a group. Instead, these infinitesimal transformations obey the rules of an algebra. It should come as no surprise, then, that the infinitesimal transformations related to the finite transformations of a given Lie group are called a Lie algebra. The infinitesimal transformations which comprise the Lie algebra are called generators. Thus the generators for the Lie algebra associated to the Lie group we are considering, the $x$ - $t$ plane with addition, are $\partial_{x}$ and $\partial_{t}$. It is important to remember that if one knows a Lie group one can determine its Lie algebra, and vice-versa. If one extends the operations allowed by our Lie group to include rotations and scalar multiplication, the generators of the Lie algebra will include $\left\{t \partial_{x}-x \partial_{t}\right\}$ and $\left\{x \partial_{x}, t \partial_{t}\right\}$ respectively.

The Lie group associated with a given system of PDE's is called the symmetry group of the system, its related Lie algebra is called the symmetry algebra, and the generators of the symmetry algebra are called symmetry generators. An arbitrary combination of symmetry generators will be called a generator.

It turns out that the theory's algorithm does not directly specify the symmetry group. Instead, given a system of PDE's, the algorithm shows one how to compute the symmetry generators. Thus, we are first given the symmetry algebra, from which we can deduce 
the symmetry group. Let us suppose that a given system of PDE's has the two symmetry generators $\mathbf{v}_{1}$ and $\mathbf{v}_{2}$. Each symmetry generator gives rise to a way of transforming solutions to solutions, say $t_{1}$ and $t_{2}$. Thus if $\mathbf{u}$ is a solution to the system, then so are $t_{1} \mathbf{u}$ and $t_{2} \mathbf{u}$. So for example, if the system were a single PDE for $u(x, t)$ and $\mathbf{v}_{1}=\partial_{x}$ and $\mathbf{v}_{2}=\partial_{t}$, then $t_{1} u=u(x-s, t)$ and $t_{2} u=u(x, t-s)$ where $s$ is an arbitrary real number. An important fact of the theory is that each possible generator gives rise to a similarity solution. So in general one would expect that $v_{1}$ gives similarity solution $u_{1}, v_{2}$ gives $u_{2}$, and the combination $\mathbf{v}_{1}+c \mathbf{v}_{2}$ gives $\mathbf{u}_{\star}$, where $c$ is an arbitrary constant. Thus since $\mathbf{u}_{1}, \mathbf{u}_{2}$ and $\mathbf{u}_{\star}$ are similarity solutions,

$$
t_{1} \mathbf{u}_{1}, \dot{t_{1}} \mathbf{u}_{2}, t_{1} \mathbf{u}_{\star}, t_{2} \mathbf{u}_{1}, t_{2} \mathbf{u}_{2}, t_{2} \mathbf{u}_{\star}
$$

are similarity solutions as well. The solutions may not all be distinct, though.

The crucial structural property of Lie algebras for the purposes of this project is that they are closed under Lie dragging, i. e. a given generator must carry/advect/Lie drag any other generator into some (possibly trivial) combination of generators. Lie dragging, or equivalently the adjoint of $\mathbf{v}_{j}$ by $\mathbf{v}_{\boldsymbol{i}}$ is defined by

$$
\operatorname{Ad}\left[\mathbf{v}_{i}, \mathbf{v}_{j}\right]=\mathbf{v}_{j}+s\left[\mathbf{v}_{i}, \mathbf{v}_{j}\right]+\frac{s}{2}\left[\mathbf{v}_{i},\left[\mathbf{v}_{i}, \mathbf{v}_{j}\right]\right]+\cdots
$$

where $[\cdot, \cdot]$ is the commutator or Lie bracket, and $s$ is the arbitrary adjoint parameter. Note that the adjoint is linear in its second argument, but not in its first. Now typically the Lie dragging or the adjoint of $\mathbf{v}_{j}$ by $\mathbf{v}_{i}$ is $\operatorname{Ad}\left[\mathbf{v}_{i}, \mathbf{v}_{j}\right]=\mathbf{v}_{j}, i=1,2$, i. e. Lie dragging or the adjoint of one by the other has no effect. In this case, the solutions (1) are all distinct, and one would need to explicitly solve for $\mathbf{u}_{1}, \mathbf{u}_{2}$, and $\mathbf{u}_{\star}$. If, however,

$$
\operatorname{Ad}\left[\mathbf{v}_{2}, \mathbf{v}_{1}\right]=\mathbf{v}_{1}+s \mathbf{v}_{2}
$$

then one does not need to solve for $u_{\star}$ since $u_{\star}=t_{2} u_{1}$. This is shown in

$$
\operatorname{Ad}\left[\mathbf{v}_{2}, \mathbf{v}_{1}+c \mathbf{v}_{2}\right]=\left(\mathbf{v}_{1}+s \mathbf{v}_{2}\right)+c \mathbf{v}_{2}
$$

since one can pick $s=-c$ which leaves $v_{1}$ only. Thus if (3) holds, then (1) becomes

$$
t_{1} \mathbf{u}_{1}, t_{1} \mathbf{u}_{2}, t_{1} t_{2} \mathbf{u}_{1}, t_{2} \mathbf{u}_{1}, t_{2} \mathbf{u}_{2},\left(t_{2} \mathbf{u}_{2}\right)
$$

where the group property that the product of two elements of the group is also within the group has been used. So in this case, one had only to solve two PDE's to find $\mathbf{u}_{1}$ and $\mathbf{u}_{2}$ to obtain all similarity solutions (by using point 1 of the theory on $\mathbf{u}_{1}$ and $\mathbf{u}_{2}$ ). Adjoint values like (3), therefore, mean that the optimal subset will be smaller than the full general set. Indeed in this example with (3) holding, optimal subset is $\left\{\mathbf{v}_{1}, \mathbf{v}_{2}\right\}$ instead of the most general $\left\{\mathbf{v}_{1}, \mathbf{v}_{2}, \mathbf{v}_{1}+c \mathbf{v}_{\star}\right\}$. The adjoint table which displays all adjoint pairs, manifests most clearly this structure. Thus, the structure evinced by the adjoint table helps one find the optimal subset of generators. More details can be found in Rick's second lecture. The appendix includes an explicit example. We will call this procedure of using the adjoint table to find the optimal subset of generators whose similarity solutions can be used determine all other similarity solutions the adjoint reduction and will call a generator belonging to the optimal subset an optimal generator. 


\section{Main Results}

The equations of two-dimensional convection are

$$
\begin{aligned}
\nabla^{2} \Psi_{t}+\mathrm{J}\left(\Psi, \nabla^{2} \Psi\right) & =-\rho_{x}+\operatorname{Pr} \nabla^{2} \nabla^{2} \Psi \\
\rho_{t}+\mathrm{J}(\Psi, \rho) & =\nabla^{2} \rho
\end{aligned}
$$

where $\Psi$ is the stream function and $\rho$ is the density. We consider an infinite fluid, and therefore will not be concerned with boundary conditions. In addition to the general convection problem, (4) and (5) above, we also examine the high Prandtl number case:

$$
\begin{aligned}
0 & =-\rho_{x}+\nabla^{2} \nabla^{2} \Psi \\
\rho_{t}+\mathrm{J}(\Psi, \rho) & =0
\end{aligned}
$$

and the inviscid case:

$$
\begin{aligned}
\nabla^{2} \Psi_{t}+\mathrm{J}\left(\Psi, \nabla^{2} \Psi\right) & =-\rho_{x} \\
\rho_{t}+\mathrm{J}(\Psi, \rho) & =0 .
\end{aligned}
$$

We used the SPDE package which was written for the REDUCE symbolic manipulation program to begin the computation of the symmetry generators for each case. After completing the calculation by hand, we found the general system, (4) and (5), has five symmetry generators and both special cases have seven each. The surprising result, however, is that the five symmetry generators of (4) and (5) are included in each special case, i. e. five of the seven symmetry generators of the high Prandtl number case and five of the seven symmetry generators of the inviscid case are the five symmetry generators of the general system. Furthermore, the remaining two symmetry generators in each special case are the same up to a scalar which multiplies the $\partial_{\rho}$ part of each symmetry generator.

Table 1 shows our findings for all three cases where $\alpha, \beta$, and $\gamma$ are arbitrary functions of $t$. The top five symmetry generators are the five which are common to all cases. The bottom two, which are enclosed by brackets, " $\{$ " and " $\}$," are the additional symmetry generators for the special cases. Note that the scalars $A$ and $B$ in the $\partial_{\rho}$ term are the only differences between the high Prandtl number case where $A=1$ and $B=-1$, and the inviscid case where $A=2$, and $B=1$. The right hand column of Table 1 shows the way to transform solutions to solutions, which is called the symmetry group action on solutions.

The next step is to perform the adjoint reduction. For the general case, the optimal subset consists of only two optimal generators: $\partial_{t}+c \partial_{\rho}$ and $c \partial_{\rho}+\left[\beta(t) \partial_{y}+x \dot{\beta}(t) \partial_{\Psi}\right]+$ $\left[\gamma(t) \partial_{x}-y \dot{\gamma}(t) \partial_{\Psi}\right]$. Their similarity forms and corresponding similarity differential equation sets are given in Table 2. The appendix gives the details of the computation for the first generator $\partial_{t}+c \partial_{\rho}$.

The adjoint reduction, similarity form, and similarity differential equation for the special cases are given in Tables 4 and 5 in the same format. The similarity forms are closely related. The last similarity differential equation set in the inviscid is not included since it is complicated. All systems in Tables 4 and 5 are nonlinear (except the one not included). S. Childress reports some colleagues are currently investigating Table 4 case B.1. with $\mathrm{c}=2$ numerically with a supercomputer. 


\begin{tabular}{c|cc} 
sym. generators & \multicolumn{2}{|c}{ group action on solutions } \\
\hline$\partial_{t}$ & $\Psi=F(x, y, t-s)$ & $\rho=G(x, y, t-s)$ \\
$\partial_{\rho}$ & $\Psi=F$ & $\rho=G+s$ \\
$\alpha(t) \partial_{\Psi}$ & $\Psi=F+\alpha(t)$ & $\rho=G$ \\
$\beta(t) \partial_{y}+x \dot{\beta}(t) \partial_{\Psi}$ & $\Psi=F(x, y-\beta, t)+x \dot{\beta}$ & $\rho=G(x, y-\beta, t)$ \\
$\gamma(t) \partial_{x}-y \dot{\gamma}(t) \partial_{\Psi}$ & $\Psi=F(x-\gamma, y, t)-y \dot{\gamma}$ & $\rho=G(x-\gamma, y, t)$ \\
$\left\{\partial_{t}-\Psi \partial_{\Psi}-A \rho \partial_{\rho}\right.$ & $\Psi=\lambda^{-1} F\left(x, y, \lambda^{-1} t\right)$ & $\left.\rho=\lambda^{-A} G\left(x, y, \lambda^{-1} t\right)\right\}$ \\
$\left\{x \partial_{x}+y \partial_{y}+2 \Psi \partial_{\Psi}+B \rho \partial_{\rho}\right.$ & $\Psi=\lambda^{2} F\left(\lambda^{-1} x, \lambda^{-1} y, t\right)$ & $\left.\rho=\lambda^{B} G\left(\lambda^{-1} x, \lambda^{-1} y, t\right)\right\}$
\end{tabular}

Table 1. Symmetry generators and corresponding group action on solutions. $\alpha, \beta$, and $\gamma$ are arbitrary functions of $t$, and $\lambda$ is a real constant. $\operatorname{Pr} \gg 1$ case: $A=1, B=-1$. Inviscid case: $A=2$, and $B=1$.

$$
\begin{aligned}
& \text { general case } \quad\left\{\begin{array}{cl}
\nabla^{2} \Psi_{t}+\mathrm{J}\left(\Psi, \nabla^{2} \Psi\right) & =-\rho_{x}+\nabla^{2} \nabla^{2} \Psi \\
\rho_{t}+\mathrm{J}(\Psi, \rho) & =\nabla^{2} \rho
\end{array}\right. \\
& \mathbf{v}=c_{1} \partial_{t}+c_{2} \partial_{\rho}+\alpha(t) \partial_{\Psi}+\left[\beta(t) \partial_{y}+x \dot{\beta}(t) \partial_{\Psi}\right]+\left[\gamma(t) \partial_{x}-y \dot{\gamma}(t) \partial_{\Psi}\right]
\end{aligned}
$$

A.1. $c_{1} \neq 0$. Adjoint reduction gives $\partial_{t}+c \partial_{\rho}$

$$
\begin{aligned}
& \left\{\begin{array}{l}
\Psi=f(x, y) \\
\rho=g(x, y)+c t
\end{array}\right. \\
& \left\{\begin{array}{cl}
\mathrm{J}\left(f, \nabla^{2} f\right) & =-g_{x}+\operatorname{Pr} \nabla^{2} \nabla^{2} f \\
c+\mathrm{J}(f, g) & =\nabla^{2} g
\end{array}\right.
\end{aligned}
$$

A.2. $c_{1}=0$. Adjoint reduction gives $c \partial_{\rho}+\left[\beta(t) \partial_{y}+x \dot{\beta}(t) \partial_{\Psi}\right]+\left[\gamma(t) \partial_{x}-y \dot{\gamma}(t) \partial_{\Psi}\right]$

$$
\begin{gathered}
\left\{\begin{array}{l}
\Psi=\frac{1}{2}\left(\frac{\dot{\beta}}{\gamma} x^{2}-\frac{\dot{\gamma}}{\beta} y^{2}\right)+f(\xi, t) \\
\rho=c\left(\frac{x}{\gamma}+\frac{y}{\beta}\right)+g(\xi, y)
\end{array}\right. \\
\begin{cases}\kappa f_{\xi \xi t}-\kappa \phi \xi f_{\xi \xi \xi}+\dot{\kappa} f_{\xi \xi}+\dot{\theta}=-\frac{g_{\xi}}{\gamma}-\frac{c}{\gamma}+P r \kappa^{2} f_{\xi \xi \xi \xi} \\
g_{t}-\phi \xi g_{\xi}+c\left(\frac{2}{\beta \gamma} f_{\xi}+\hat{\phi} \xi\right) & =\kappa g_{\xi \xi}\end{cases}
\end{gathered}
$$

where

$$
\begin{aligned}
\kappa(t) & =\frac{1}{\beta^{2}}+\frac{1}{\gamma^{2}} & \theta(t) & =\frac{\dot{\beta}}{\gamma}-\frac{\dot{\gamma}}{\beta} \\
\phi(t) & =\frac{d}{d t} \ln (\beta \gamma) & \hat{\phi}(t) & =\frac{d}{d t} \ln \frac{\beta}{\gamma}
\end{aligned}
$$

Table 2. Similarity forms and differential equations for the general case. 


$$
\begin{gathered}
\operatorname{Pr} \gg 1 \text { case : } \quad\left\{\begin{array}{l}
0=-\rho_{x}+\nabla^{2} \nabla^{2} \Psi \\
0=\rho_{t}+\mathrm{J}(\Psi, \rho)
\end{array}\right. \\
\mathbf{v}=c_{1} \partial_{t}+c_{2} \partial_{\rho}+\alpha(t) \partial_{\Psi}+\left[\beta(t) \partial_{y}+x \dot{\beta}(t) \partial_{\Psi}\right]+\left[\gamma(t) \partial_{x}-y \dot{\gamma}(t) \partial_{\Psi}\right] \\
+c_{6}\left[t \partial_{t}-\Psi \partial_{\Psi}-\rho \partial_{\rho}\right]+c_{7}\left[x \partial_{x}+y \partial_{y}+2 \Psi \partial_{\Psi}-\rho \partial_{\rho}\right]
\end{gathered}
$$

A. The cases where $c_{6}=c_{7}=0$ are exactly as in the general case.

B.1. $c_{6} \neq 0$. Adjoint reduction gives $\mathbf{v}_{6}+c \mathbf{v}_{7}$

$$
\begin{gathered}
\left\{\begin{array}{l}
\Psi=t^{2 c-1} f\left(\frac{x}{t^{c}}, \frac{y}{t^{c}}\right) \\
\rho=\frac{1}{t^{c+1}} g\left(\frac{x}{t^{c}}, \frac{y}{t^{c}}\right)
\end{array}\right. \\
\left\{\begin{array}{l}
0=-g_{\xi}+\nabla^{2} \nabla^{2} f \\
0=-(c+1) g-c\left(\xi g_{\xi}+\eta g_{\eta}\right)+f_{\xi} g_{\eta}-f_{\eta} g_{\xi}
\end{array}\right.
\end{gathered}
$$

2. $\mathbf{v}_{6}$, alone $(c=0)$

$$
\begin{gathered}
\left\{\begin{array}{l}
\Psi=\frac{f(x, y)}{t} \\
\rho=\frac{g(x, y)}{t}
\end{array}\right. \\
\left\{\begin{array}{l}
0=-g_{x}+\nabla^{2} \nabla^{2} f \\
0=-g+\mathrm{J}(f, g)
\end{array}\right.
\end{gathered}
$$

C.1. $c_{6}=0, c_{7} \neq 0$. Adjoint reduction gives $c \partial_{t}+\mathbf{v}_{7}$

$$
\begin{gathered}
\left\{\begin{array}{l}
\Psi=e^{2 c t} f\left(x e^{-c t}, y e^{-c t}\right) \\
\rho=e^{-c t} g\left(x e^{-c t}, y e^{-c t}\right)
\end{array}\right. \\
\left\{\begin{array}{l}
0=-g_{\xi}+\nabla^{2} \nabla^{2} f \\
0=-c\left(g+\xi g_{\xi}+\eta g_{\eta}\right)+\mathrm{J}(f, g)
\end{array}\right.
\end{gathered}
$$

2. $\mathbf{v}_{7}$ alone

$$
\begin{gathered}
\left\{\begin{array}{l}
\Psi=y^{2} f\left(\frac{x}{y}, t\right) \\
\rho=\frac{1}{y} g\left(\frac{x}{y}, t\right)
\end{array}\right. \\
\left\{\begin{array}{l}
0=-g_{\xi}+4(1-3 \xi) f_{\xi \xi}-4 \xi\left(1+4 \xi^{2}\right) f_{\xi \xi \xi}+\left(1-\xi^{4}\right) f_{\xi \xi \xi \xi} \\
0=g_{t}-f_{\xi} g-2 f g_{\xi}
\end{array}\right.
\end{gathered}
$$

Table 3. Similarity forms and similarity differential equations for $\operatorname{Pr} \gg 1$. 


$$
\begin{gathered}
\text { inviscid case }\left\{\begin{array}{c}
\nabla^{2} \Psi_{t}+\mathrm{J}\left(\Psi, \nabla^{2} \Psi\right)=-\rho_{x} \\
\rho_{t}+\mathrm{J}(\Psi, \rho)=0
\end{array}\right. \\
\mathbf{v}=c_{1} \partial_{t}+c_{2} \partial_{\rho}+\alpha(t) \partial_{\Psi}+\left[\beta(t) \partial_{y}+x \dot{\beta}(t) \partial_{\Psi}\right]+\left[\gamma(t) \partial_{x}-y \dot{\gamma}(t) \partial_{\Psi}\right] \\
+c_{6}\left[t \partial_{t}-\Psi \partial_{\Psi}-2 \rho \partial_{\rho}\right]+c_{7}\left[x \partial_{x}+y \partial_{y}+2 \Psi \partial_{\Psi}+\rho \partial_{\rho}\right]
\end{gathered}
$$

A. The cases where $c_{6}=c_{7}=0$ are exactly as in the general case.

B.1. $c_{6} \neq 0$. Adjoint reduction gives $\mathbf{v}_{6}+c \mathbf{v}_{7}$

$$
\begin{gathered}
\left\{\begin{array}{l}
\Psi=t^{2 c-1} f\left(\frac{x}{t^{c}}, \frac{y}{t^{c}}\right) \\
\rho=t^{c-2} g\left(\frac{x}{t^{c}}, \frac{y}{t^{c}}\right)
\end{array}\right. \\
\left\{\begin{aligned}
-\nabla^{2} f-c\left(\xi \nabla^{2} f_{\xi}+\eta \nabla^{2} f_{\eta}\right)+\mathrm{J}\left(f, \nabla^{2} f\right) & =-g_{\xi} \\
(c-2) g-c\left(\xi g_{\xi}+\eta g_{\eta}\right)+\mathrm{J}(f, g) & =0
\end{aligned}\right.
\end{gathered}
$$

2. $\mathbf{v}_{6}$ alone $(c=0)$

$$
\begin{gathered}
\left\{\begin{array}{r}
\Psi=\frac{f(x, y)}{t} \\
\rho=\frac{g(x, y)}{t^{2}}
\end{array}\right. \\
\left\{\begin{array}{cl}
\nabla^{2} f-\mathrm{J}\left(f, \nabla^{2} f\right) & =g_{x} \\
2 g-\mathrm{J}(f, g) & =0
\end{array}\right.
\end{gathered}
$$

C.1. $c_{6}=0, c_{7} \neq 0$. Adjoint reduction gives $c \partial_{t}+\mathbf{v}_{7}$

$$
\begin{gathered}
\left\{\begin{array}{c}
\Psi=e^{2 c t} f\left(x e^{-c t}, y e^{-c t}\right) \\
\rho=e^{c t} g\left(x e^{-c t}, y e^{-c t}\right)
\end{array}\right. \\
\left\{\begin{array}{cl}
c\left(\xi \nabla^{2} f_{\xi}+\eta \nabla^{2} f_{\eta}\right)-\mathrm{J}\left(f, \nabla^{2} f\right) & =g_{\xi} \\
c\left(g-\xi g_{\xi}-\eta g_{\eta}\right)+\mathrm{J}(f, g) & =0
\end{array}\right.
\end{gathered}
$$

2. $\mathbf{v}_{7}$ alone

$$
\left\{\begin{array}{l}
\Psi=x y f\left(\frac{x}{y}, t\right) \\
\rho=\sqrt{x y} g\left(\frac{x}{y}, t\right)
\end{array}\right.
$$

Table 4. Similarity forms and similarity differential equations for inviscid case. 


\section{Shear Flow, Waves, and Instabilities}

In this section we briefly solve special cases of one of the similarity differential equation sets of the general case and interpret the solution. We examine the system A.2. of Table 2 which is rewritten for convenience below:

$$
\begin{aligned}
\kappa f_{\xi \xi t}-\kappa \phi \xi f_{\xi \xi \xi}+\dot{\kappa} f_{\xi \xi}+\dot{\theta} & =-\frac{g_{\xi}}{\gamma}-\frac{c}{\gamma}+\operatorname{Pr} \kappa^{2} f_{\xi \xi \xi \xi} \\
g_{t}-\phi \xi g_{\xi}+c\left(\frac{2}{\beta \gamma} f_{\xi}+\hat{\phi} \xi\right) & =\kappa g_{\xi \xi}
\end{aligned}
$$

where $\kappa, \theta, \phi$, and $\hat{\phi}$ are also given in Table 2 A.2. We first neglect diffusion by setting the right most terms to zero and also set $c=0$. Recalling the definition of $\phi$, we see (11) becomes

$$
g_{t}-\frac{d}{d t} \ln (\beta \gamma) \xi g_{\xi}=0 .
$$

The method of characteristics suggests the new variable

$$
\eta \equiv \beta \gamma \xi=\beta(t) x-\gamma(t) y
$$

which is like a transformation to Lagrangian coordinates. If we now use $\eta$ instead of $\xi$ as a similarity variable so that the similarity forms are

$$
\begin{aligned}
\Psi & =\frac{1}{2}\left(\frac{\dot{\beta}}{\gamma} x^{2}-\frac{\dot{\gamma}}{\beta} y^{2}\right)+f(\eta, t) \\
\rho & =c\left(\frac{x}{\gamma}+\frac{y}{\beta}\right)+g(\eta, y)
\end{aligned}
$$

and insert these into (4) and (5), then the similarity differential equation set becomes

$$
\begin{aligned}
\partial_{t}\left[\tilde{\kappa} f_{\eta \eta}+\theta\right] & =-\beta g_{\eta}-\frac{c}{\gamma}+\operatorname{Pr} \tilde{\kappa}^{2} f_{\eta \eta \eta \eta} \\
g_{t}+c\left[\eta \frac{\hat{\phi}}{\beta \gamma}+2 f_{\eta}\right] & =\tilde{\kappa} g_{\eta \eta}
\end{aligned}
$$

where $\tilde{\kappa}=\beta^{2}+\gamma^{2}$. Again neglecting diffusion, we now consider two special cases of this system.

Setting $c=0$ for the first case, we have

$$
\begin{aligned}
\partial_{t}\left[\kappa f_{\eta \eta}+\theta\right] & =-\beta g_{\eta} \\
g_{t} & =0
\end{aligned}
$$

which we can directly integrate. The result is

$$
\begin{aligned}
\Psi= & \frac{1}{2 \tilde{\kappa}}\left[\left(x^{2}-y^{2}\right)(\dot{\beta} \gamma)+x y\left(\beta^{2}-\gamma^{2}\right)\right]-\frac{G(\beta(t) x-\gamma(t) y) \int^{t} \beta}{\tilde{\kappa}} \\
& \quad+\frac{H(\beta(t) x-\gamma(t) y)}{\tilde{\kappa}}+\frac{A(t)(\beta(t) x-\gamma(t) y)}{\bar{\kappa}} \\
\rho= & G^{\prime}(\beta(t) x-\gamma(t) y)
\end{aligned}
$$


where $G, H$, and $A$ are arbitrary functions of one argument and we have neglected an arbitrary function of time in the stream function $\Psi$ which gives no physics. We now set the arbitrary functions $A=G=H=\gamma=0$ which gives

$$
\begin{aligned}
\Psi & =a x y \\
\rho & =G^{\prime}\left(x e^{a t}\right)
\end{aligned}
$$

which describe a shear flow.

If we now restrict $\beta$ and $\gamma$ to be constants, (13) and (14) become

$$
\begin{aligned}
\tilde{\kappa} f_{\eta \eta} & =-\beta g_{\eta}-\frac{c}{\gamma} \\
g_{t}+2 c f_{\eta} & =0
\end{aligned}
$$

If we now differentiate (15) w.r.t. $t$ and (16) w.r.t. $\eta$, we have

$$
f_{\xi \xi \eta \eta}=\lambda^{2} f_{\eta \eta}, \quad \text { where } \quad \lambda^{2}=\frac{2 \beta c}{\bar{\kappa}}
$$

which we can integrate to

$$
\begin{aligned}
\Psi & =A(\beta(t) x-\gamma(t) y) e^{\lambda t}+B(\beta(t) x-\gamma(t) y) e^{-\lambda t}+D(t)(\beta(t) x-\gamma(t) y) \\
\rho & =-\frac{2 c}{\lambda}\left[A^{\prime}(\beta(t) x-\gamma(t) y) e^{\lambda t}-B^{\prime}(\beta(t) x-\gamma(t) y) e^{-\lambda t}+\int^{t} D\left(t^{\prime}\right)\right]+\frac{2 c}{\beta} y
\end{aligned}
$$

where again we have neglected an arbitrary function of $t$ in $\Psi$. If we adjust the arbitrary functions $\mathrm{A}, \mathrm{B}$, and $\mathrm{D}$ properly, they will be zero when $t=0$. Thus

$$
\rho_{y}=\frac{2 c}{\beta}
$$

Now if $(20)<0$ then as one moves upwards, density decreases. One would expect this to be a stable configuration. (20) $<0$ by (17) means the system (18) and (19) will exhibit waves. (20) $>0$ similarly shows possibilities of instability.

\section{Appendix The Essentials of Adjoint Reduction by Example}

Here we show how to proceed with part of the adjoint reduction of the general generator of the two-dimensional convection system. In particular we show how to go from the general generator

$$
\mathbf{v}=c_{1} \partial_{t}+c_{2} \partial_{\rho}+\xi(t) \partial_{\Psi}+\left[\eta(x) \partial_{y}+x \dot{\eta}(x) \partial_{\Psi}\right]+\left[\zeta(x) \partial_{x}-y \dot{\zeta}(x) \partial_{\Psi}\right]
$$

where $\xi, \eta$, and $\zeta$ are arbitrary functions of $t$, to $\partial_{t}+c \partial_{\rho}$ via the adjoint table, given for all cases studied in Table A. We will refer to the first five lines of its top two tables. 


\begin{tabular}{c|ccc} 
& \multicolumn{3}{|c}{$\mathbf{v}_{j}$} \\
$\mathbf{v}_{i}$ & $\partial_{t}$ & $\partial_{\rho}$ & $\xi(t) \partial_{\Psi}$ \\
\hline$\partial_{t}$ & $\partial_{t}$ & $\partial_{\rho}$ & $e^{s \partial_{t}} \xi \partial_{\Psi}$ \\
$\partial_{\rho}$ & $\partial_{t}$ & $\partial_{\rho}$ & $\xi \partial_{\Psi}$ \\
$\alpha(t) \partial_{\Psi}$ & $\partial_{t}-s \dot{\alpha} \partial_{\Psi}$ & $\partial_{\rho}$ & $\xi \partial_{\Psi}$ \\
$\beta(t) \partial_{y}+x \dot{\beta}(t) \partial_{\Psi}$ & $\partial_{t}-s\left(\dot{\beta} \partial_{y}+x \ddot{\beta} \partial_{\Psi}\right)$ & $\partial_{\rho}$ & $\xi \partial_{\Psi}$ \\
$\gamma(t) \partial_{x}-y \dot{\gamma}(t) \partial_{\Psi}$ & $\partial_{t}-s\left(\dot{\gamma} \partial_{x}-y \dot{\gamma} \partial_{\Psi}\right)$ & $\partial_{\rho}$ & $\xi \partial_{\Psi}$ \\
$\left\{\partial_{t}-\Psi \partial_{\Psi}-A \rho \partial_{\rho}\right.$ & $e^{-s} \partial_{t}$ & $e^{A s} \partial_{\rho}$ & $\left.e^{s\left(1+t \partial_{t}\right)} \xi \partial_{\Psi}\right\}$ \\
$\left\{x \partial_{x}+y \partial_{y}+2 \Psi \partial_{\Psi}+B \rho \partial_{\rho}\right.$ & $\partial_{t}$ & $e^{-B s} \partial_{\rho}$ & $\left.e^{-2 s} \xi \partial_{\Psi}\right\}$
\end{tabular}

\begin{tabular}{c|cc} 
& \multicolumn{2}{|c}{$\mathbf{v}_{j}$} \\
\hline $\mathbf{v}_{i}$ & $\eta(t) \partial_{y}+x \dot{\eta}(t) \partial_{\Psi}$ & $\zeta(t) \partial_{x}-y \dot{\zeta}(t) \partial_{\Psi}$ \\
\hline$\partial_{\rho}$ & $e^{s \theta_{t}} \eta \partial_{y}+x e^{s \partial_{t}} \dot{\eta} \partial_{\Psi}$ & $e^{s \theta_{t}} \zeta \partial_{x}-y e^{s \partial_{t} \dot{\zeta} \partial_{\Psi}}$ \\
$\alpha(t) \partial_{\Psi}$ & $\eta \partial_{y}+x \dot{\eta} \partial_{\Psi}$ & $\zeta \partial_{x}-y \zeta \partial_{\Psi}$ \\
$\beta(t) \partial_{y}+x \dot{\beta}(t) \partial_{\Psi}$ & $\eta \partial_{y}+x \dot{\eta} \partial_{\Psi}$ & $\zeta \partial_{x}-y \dot{\zeta} \partial_{\Psi}$ \\
$\gamma(t) \partial_{x}-y \dot{\gamma}(t) \partial_{\Psi}$ & $\eta \partial_{y}+x \dot{\eta} \partial_{\Psi}$ & $\zeta \partial_{x}-y \dot{\zeta} \partial_{\Psi}-s(\dot{\beta} \zeta) \partial_{\Psi}$ \\
$t \partial_{t}-\Psi \partial_{\Psi}-A \rho \partial_{\rho}$ & $\eta \partial_{y}+x \dot{\eta} \partial_{\Psi}+s(\dot{\eta} \gamma) \partial_{\Psi}$ & $\zeta \partial_{x}-y \dot{\zeta} \partial_{\Psi}$ \\
$\left\{x \partial_{x}+y \partial_{y}+2 \Psi \partial_{\Psi}+B \rho \partial_{\rho}\right.$ & $e^{s t \partial_{t}} \eta \partial_{y}+x e^{s \theta_{t}}(t \dot{\eta}) \partial_{\Psi}$ & $\left.e^{s t \partial_{t}} \zeta \partial_{x}-y e^{s \partial_{t}}(t \dot{\zeta}) \partial_{\Psi}\right\}$ \\
& $e^{-s}\left(\eta \partial_{y}+x \dot{\eta} \partial_{\Psi}\right)$ & $\left.e^{-s}\left(\zeta \partial_{x}-y \dot{\zeta} \partial_{\Psi}\right)\right\}$
\end{tabular}

\begin{tabular}{c|cc} 
& \multicolumn{2}{|c}{$\mathbf{v}_{j}$} \\
$\mathbf{v}_{i}$ & $t \partial_{t}-\Psi \partial_{\Psi}-A \rho \partial_{\rho} \equiv \mathbf{v}_{6}$ & $x \partial_{x}+y \partial_{y}+2 \Psi \partial_{\Psi}+B \rho \partial_{\rho} \equiv \mathbf{v}_{7}$ \\
\hline$\partial_{t}$ & $\mathbf{v}_{6}+s \partial_{t}$ & $\mathbf{v}_{7}$ \\
$\partial_{\rho}$ & $\mathbf{v}_{6}-s \partial_{\rho}$ & $\mathbf{v}_{7}-s \partial_{\rho}$ \\
$\alpha(t) \partial_{\Psi}$ & $\mathbf{v}_{6}-s(\alpha+t \dot{\alpha}) \partial_{\Psi}$ & $\mathbf{v}_{7}+s 2 \alpha \partial_{\Psi}$ \\
$\beta(t) \partial_{y}+x \dot{\beta}(t) \partial_{\Psi}$ & $\mathbf{v}_{6}-s\left[t \dot{\beta} \partial_{y}+(t \dot{\beta}) x \partial_{\Psi}\right]$ & $\mathbf{v}_{7}+s\left(\beta \partial_{y}+x \dot{\beta} \partial_{\Psi}\right)$ \\
$\gamma(t) \partial_{x}-y \dot{\gamma}(t) \partial_{\Psi}$ & $\mathbf{v}_{6}-s\left[t \dot{\gamma} \partial_{y}+(t \dot{\gamma}) x \partial_{\Psi}\right]$ & $\mathbf{v}_{7}+s\left(\gamma \partial_{x}-y \dot{\gamma} \partial_{\Psi}\right)$ \\
$t \partial_{t}-\Psi \partial_{\Psi}-A \rho \partial_{\rho}$ & $\mathbf{v}_{6}$ & $\mathbf{v}_{7}$ \\
$x \partial_{x}+y \partial_{y}+2 \Psi \partial_{\Psi}+B \rho \partial_{\rho}$ & $\mathbf{v}_{6}$ & $\mathbf{v}_{7}$
\end{tabular}

Table A. Adjoint table. General case: omit third table and lines enclosed by \{brackets\}. $\operatorname{Pr} \gg 1$ case: $A=1, B=-1$. Inviscid case: $A=2, B=1$. 
After first recalling the definition of the adjoint of two generators (2), one should then study the relevant portion of Table A paying particular attention to where the adjoint parameter appears. Having familiarized ourselves with the adjoint table, we reason as follows. Firstly, assume $c_{1} \neq 0$. Let us drag $\mathbf{v}$ by the arbitrary generator $\beta(t) \partial_{y}+x \dot{\beta}(t) \partial_{\Psi}$ where $\beta$ is an arbitrary function of $t$.

$$
\begin{aligned}
\operatorname{Ad} & {\left[\beta \partial_{y}+x \dot{\beta} \partial_{\Psi}, \frac{1}{c_{1}} \mathbf{v}\right] } \\
& =\left[\partial_{t}-s\left(\dot{\beta} \partial_{y}+x \ddot{\beta} \partial_{\Psi}\right)\right]+c \partial_{\rho}+\xi \partial_{\Psi}+\left[\eta \partial_{y}+x \dot{\eta} \partial_{\Psi}\right]+\left[\left(\zeta \partial_{x}-y \dot{\zeta} \partial_{\Psi}\right)-s(\dot{\beta} \zeta)\right] \\
& =\partial_{t}+c \partial_{\rho}+[\xi-s(\dot{\beta} \zeta)]+\left[\eta \partial_{y}+x \dot{\eta} \partial_{\Psi}-s\left(\dot{\beta} \partial_{y}+x \ddot{\beta} \partial_{\Psi}\right)\right]+\left[\zeta \partial_{x}-y \dot{\zeta} \partial_{\Psi}\right],
\end{aligned}
$$

where in (22) we have used the linearity of $\mathrm{Ad}[\cdot, \cdot]$ in its second argument, have let $c=c_{2} / c_{1}$, and also have absorbed $c_{1}$ into $\xi, \eta$, and $\zeta$. Now in (23) let us set the arbitrary function $\beta=\mathrm{Y}=\int^{t} \eta$ (so $\dot{\beta}=\eta$ ). If we now set the adjoint parameter $s=1$ and call $\hat{\xi}=\xi-(\dot{\mathrm{Y}} \zeta)$, then $v$ dragged by $\mathrm{Y} \partial_{y}+x \dot{Y} \partial_{\Psi}$, is

$$
\overline{\mathbf{v}}=\partial_{t}+c \partial_{\rho}+\tilde{\xi} \partial_{\Psi}+\zeta \partial_{x}-y \dot{\zeta} \partial_{\Psi} .
$$

If we now drag $\mathbf{v}$ by $Z \partial_{x}-y \dot{Z} \partial_{\Psi}$ where $Z=\int^{t} \zeta$ we have

$$
\hat{\mathbf{v}}=\partial_{t}+c \partial_{\rho}+\hat{\xi} \partial_{\Psi} \text {. }
$$

And if we drag $\hat{\mathrm{v}}$ by $\Xi(t) \partial_{\Psi}$ where $\Xi(t)=\int^{t} \alpha$ And adjust $s$ accordingly, we have our result. Note that the results are unchanged if either $\xi$, $\eta$, or $\zeta$ were 0 . The other optimal generator for the general two-dimensional convection equations is obtained in a similar manner, but with $c_{1}=0$ in (21). Finally, let us remark that it is helpful to have computed the commutator table before computing the adjoint table.

\section{References}

Olver, Peter J. Applications of Lie Groups to Differential Equations. Springer-Verlag GTM $107,1986$.

Salmon, Rick. "A Fluid Mechanicist's Introduction to Lie Symmetry Groups and Partial Differential Equations." GFD Special Seminar, 1990.

Schwarz, Fritz. "Symmetries of Differential Equations: From Sophus Lie to Computer Algebra," SIAM Rev. 30 (1988) p. 450.

\section{Acknowlegements}

Thanks to Rick Salmon for his encouragement and assistance throughout this project. I thank him for teaching me the theory and technique. His intuition amazed me many times, and his facility with calculation impressed me throughout our collaboration. Thanks also to Steve Childress both for suggesting this problem and for intermittent discussions along the way. He also seemed to "know" what was interesting. Thanks to the other GFD Fellows especially Stefan Linz and to the other staff members for making this a truly first-class

summer. And finally, thanks to the Eastman-Kodak Company for generously supporting me through this early part of my graduate studies, including this summer. 


\section{DOCUMENT LIBRARY}

January 17, 1990

\section{Distribution List for Technical Report Exchange}

Attn: Stella Sanchez-Wade

Documents Section

Scripps Institution of Oceanography

Library, Mail Code C-075C

La Jolla, CA 92093

Hancock Library of Biology \& Oceanography

Alan Hancock Laboratory

University of Southern California

University Park

Los Angeles, CA 90089-0371

Gifts \& Exchanges

Library

Bedford Institute of Oceanography

P.O. Box 1006

Dartmouth, NS, B2Y 4A2, CANADA

Office of the International

Ice Patrol

c/o Coast Guard R \& D Center

Avery Point

Groton, CT 06340

NOAA/EDIS Miami Library Center

4301 Rickenbacker Causeway

Miami, FL 33149

Library

Skidaway Institute of Oceanography

P.O. Box 13687

Savannah, GA 31416

Institute of Geophysics

University of Hawaii

Library Room 252

2525 Correa Road

Honolulu, HI 96822

Marine Resources Information Center Building E38-320

MIT

Cambridge, MA 02139

Library

Lamont-Doherty Geological

Observatory

Colombia University

Palisades, NY 10964

Library

Serials Department

Oregon State University

Corvallis, OR 97331
Pell Marine Science Library

University of Rhode Island

Narragansett Bay Campus

Narragansett, RI 02882

Working Collection

Texas A\&M University

Dept. of Oceanography

College Station, TX 77843

Library

Virginia Institute of Marine Science

Gloucester Point, VA 23062

Fisheries-Oceanography Library

151 Oceanography Teaching Bldg.

University of Washington

Seattle, WA 98195

Library

R.S.M.A.S.

University of Miami

4600 Rickenbacker Causeway

Miami, FL 33149

Maury Oceanographic Library

Naval Oceanographic Office

Bay St. Louis

NSTL, MS 39522-5001

Marine Sciences Collection

Mayaguez Campus Library

University of Puerto Rico

Mayagues, Puerto Rico 00708

Library

Institute of Oceanographic Sciences

Deacon Laboratory

Wormley, Godalming

Surrey GU8 5UB

UNITED KINGDOM

The Librarian

CSIRO Marine Laboratories

G.P.O. Box 1538

Hobart, Tasmania

AUSTRALIA 7001

Library

Proudman Oceanographic Laboratory

Bidston Observatory

Birkenhead

Merseyside L43 7 RA

UNITED KINGDOM 



\begin{tabular}{|c|c|c|c|}
\hline $\begin{array}{l}\text { REPORT DOCUMENTATION } \\
\text { PAGE }\end{array}$ & $\begin{array}{l}\text { 1. REPORT NO. } \\
\qquad \text { WHOI-91-03 }\end{array}$ & 2. & 3. Reclplent's Accossion No. \\
\hline \multirow{2}{*}{\multicolumn{3}{|c|}{$\begin{array}{l}\text { 4. Title and Subtitle } \\
\text { 1990 Summer Study Program in Geophysical Fluid Dynamics } \\
\text { STELLAR FLUID DYNAMICS }\end{array}$}} & $\begin{array}{l}\text { 5. Report Date } \\
\text { October } 1990 \\
\end{array}$ \\
\hline & & & 6. \\
\hline \multicolumn{3}{|c|}{$\begin{array}{l}\text { 7. Author(s) } \\
\text { Rick Salmon, Director, Edited by Barbara Ewing DeRemer }\end{array}$} & $\begin{array}{l}\text { 8. Performing Organization Rept. No. } \\
\text { WHOI-91-03 }\end{array}$ \\
\hline \multicolumn{3}{|c|}{ 9. Performing Organization Name and Address } & 10. Project/Task/Work Unit No. \\
\hline \multicolumn{3}{|c|}{$\begin{array}{l}\text { Woods Hole Oceanographic Institution } \\
\text { Woods Hole, Massachusetts } 02543\end{array}$} & $\begin{array}{l}\text { 11. Contract(C) or Grant(G) No. } \\
\text { (C) OCE } 8901012 \\
\text { (G) }\end{array}$ \\
\hline \multicolumn{3}{|c|}{ 12. Sponsoring Organization Name and Address } & $\begin{array}{l}\text { 13. Type of Report \& Period Covered } \\
\text { Technical Report }\end{array}$ \\
\hline \multicolumn{3}{|l|}{ National Science Foundation } & 14. \\
\hline
\end{tabular}

This report should be cited as: Woods Hole Oceanog. Inst. Tech. Rept., WHOI-91-03.

16. Abstract (LImit: 200 words)

The 1990 program in Geophysical Fluid Dynamics had as its special topic "Stellar Fluid Dynamics". Introductory lectures by Edward Spiegel and Jean-Paul Zahn paved the way for more specialized seminars on solar oscillations, neutron stars, stellar winds, solar convection, and flows with strong magnetic fields. As usual, the lectures ranged far beyond the special topic of the summer, with GFD filling its traditional role as a clearing house for information between the various fields that share an interest in rotating, differentially-heated flows. Under the supervision of the staff members, our nine student fellows completed original research projects. Their reports appear in the 1990 volume, along with the lecture notes of Spiegel and Zahn, and summaries of the other lectures.

17. Document Analysis a. Descriptors

stellar fluid dynamics

geophysical fluid dynamics

solar magnetohydrodynamics (MHD)

b. Identifiers/Open-Ended Terms

c. COSATI Field/Group

18. Availability Statement

Approved for public release; distribution unlimited.

\begin{tabular}{|l|l|}
\hline 19. Security Class (This Report) & $\begin{array}{l}\text { 21. No. of Pages } \\
369\end{array}$ \\
\hline 20. Security Class (This Page) & 22. Price \\
\hline
\end{tabular}


